\title{
Summary Environmental Site Assessment Report for the U.S. Department of Energy Oxnard Facility, Oxnard, California
}

February 1996

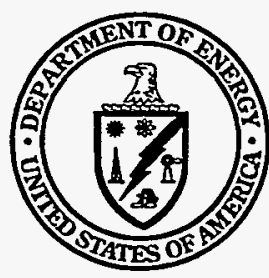

U.S. Department of Energy Grand Junction Projects Office

Approved for public release; distribution is unlimited.

\footnotetext{
Work Performed Under DOE Contract No. DE-AC04-94AL96907 for the U.S. Department of Energy
} 
This report was prepared as an account of work sponsored by an agency of the United States Government. Neither the United States Government nor any agency thereof, nor any of their employees, makes any warranty, express or implied, or assumes any legal liability or responsibility for the accuracy, completeness, or usefulness of any information, apparatus, product, or process disclosed in this report, or represents that its use would not infringe privately owned rights. Reference herein to any specific commercial product, process, or service by trade name, trademark, manufacturer, or otherwise, does not necessarily constitute or imply its endorsement, recommendation, or favoring by the United States Government or any agency thereof. The views and opinions of authors expressed herein do not necessarily state or reflect those of the United States Government or any agency thereof. 


\section{Summary Environmental Site Assessment Report for the U.S. Department of Energy Oxnard Facility Oxnard, California}

February 1996

Prepared for

U.S. Department of Energy

Albuquerque Operations Office

Grand Junction Projects Office

Grand Junction, CO

Prepared by

Rust Geotech

Grand Junction, Colorado

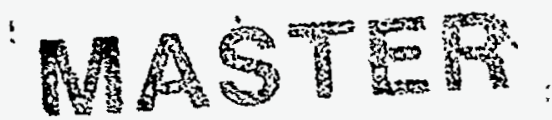

Work Performed Under Contract No. DE-AC04-94AL96907

FISTPEITION OF THS OOCURENT IS UHLIMITED 
This page intentionally blank

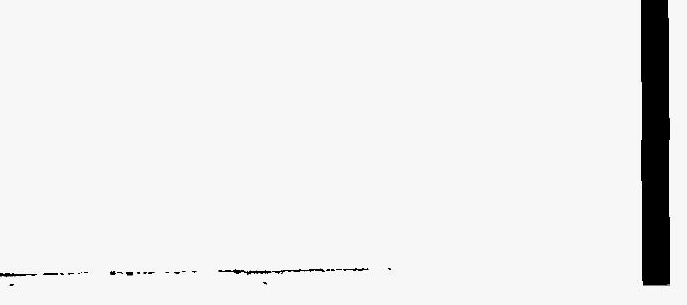




\section{Contents}

Page

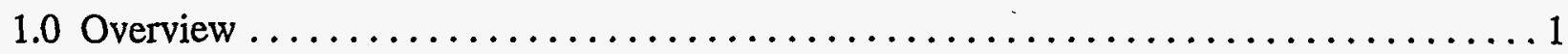

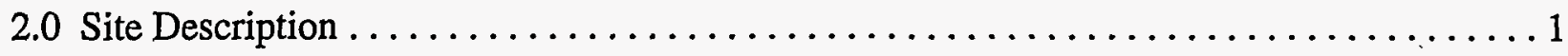

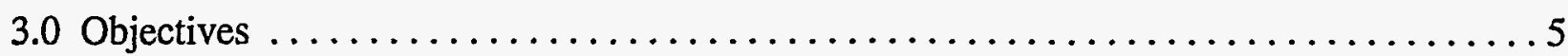

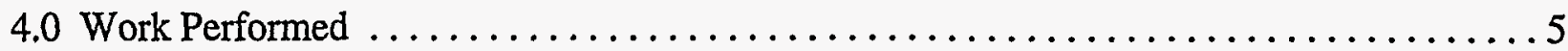

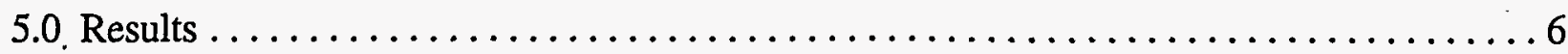

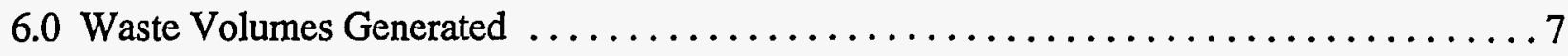

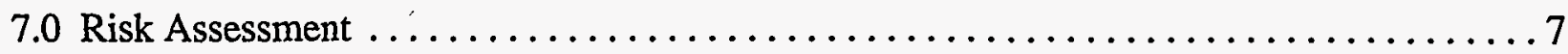

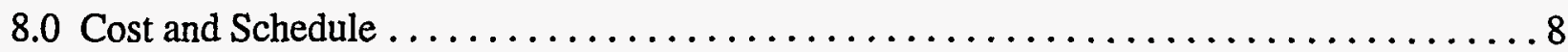

9.0 Conclusions and Limitations $\ldots \ldots \ldots \ldots \ldots \ldots \ldots \ldots \ldots \ldots \ldots \ldots \ldots \ldots \ldots \ldots \ldots \ldots \ldots \ldots \ldots$

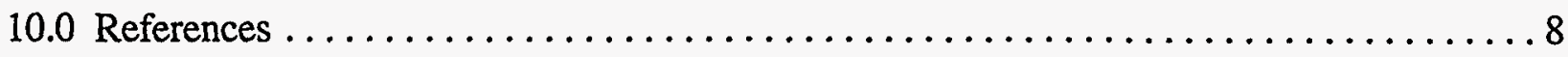

Figures

Figure 1. Location of the DOE Oxnard Facility, Oxnard, California $\ldots \ldots \ldots \ldots \ldots \ldots$

2. Site Layout of the DOE Oxnard Facility $\ldots \ldots \ldots \ldots \ldots \ldots \ldots \ldots \ldots \ldots \ldots \ldots \ldots \ldots$

\section{Table}

Table 1. ARARs for the DOE Oxnard Facility $\ldots \ldots \ldots \ldots \ldots \ldots \ldots \ldots \ldots \ldots \ldots \ldots \ldots \ldots \ldots$ 


\section{-Appendices}

Page

Appendix A. Phase I Environmental Site Assessment for the EG\&G Rocky Flats

Oxnard Facility

A-1

Appendix B. Phase II Environmental Site Assessment Report for the Kaiser-Hill

Company LLC Oxnard Facility, Oxnard, California

Appendix C. Phase III Environmental Site Assessment Report for the U.S. Department of Energy Oxnard Facility, Oxnard, California $\ldots \ldots \ldots \ldots \ldots \ldots \ldots \ldots \ldots \ldots \ldots \ldots$

Appendix D. Asbestos Operation and Maintenance Plan, DOE Oxnard Facility ........... D-1

Appendix E. Condition Assessment Survey for the Kaiser-Hill LLC Oxnard Facility ......... E-1 


\subsection{Overview}

This report summarizes the investigations conducted by Rust Geotech ${ }^{1}$ at the U.S. Department of Energy (DOE) Oxnard facility, 1235 East Wooley Road, Oxnard, California (Figure 1). These investigations were designed to locate, identify, and characterize any regulated contaminated media on the site. The effort included site visits; research of ownership, historical uses of the Oxnard facility and adjacent properties, incidences of and investigations for contaminants on adjacent properties, and the physical setting of the site; sampling and analysis; and reporting.

These investigations identified two friable asbestos gaskets on the site, which were removed, and nonfriable asbestos, which will be managed through the implementation of an asbestos management plan. The California primary drinking water standards were exceeded for aluminum on two groundwater samples and for lead in one sample collected from the shallow aquifer underlying the site; remediation of the groundwater in this aquifer is not warranted because it is not used. Treated water is available from a municipal water system. Three sludge samples indicated elevated heavy metals concentrations; the sludge must be handled as a hazardous waste if disposed. Polychlorinated biphenyls (PCBs) were detected at concentrations below remediation criteria in facility soils at two locations. In accordance with U.S. Environmental Protection Agency (EPA) and State of California guidance, remediation of the PCBs is not required. No other hazardous substances were detected in concentrations exceeding regulatory limits.

\subsection{Site Description}

The Oxnard facility occupies 13.75 acres (5.6 hectares) in an industrial park (zoned M-2 warehouse-industrial) within the incorporated city limits of Oxnard, California. Seven buildings on the Oxnard facility property enclose approximately 86,000 square feet (7,990 square meters) of covered floor space (Figure 2). Both paved and unimproved open land areas are located on the site. The legal description for the property is presented on reproductions of the property deeds in Appendix A, Section 3.0. Bordering the facility are industrial maintenance, manufacturing, and agricultural packaging operations.

The site was used as farmland until 1949, when Allis-Chalmers built a plant to manufacture farm implements. Activities at the site included founding (foundry casting), forging, machining, welding, cutting, sanding, grinding, painting, and coating. In 1982, DOE assisted Precision Forge (a private company providing specialty nonferrous metalworking products to DOE) in relocating to the Oxnard property. In 1984, DOE acquired Precision Forge and operated the facility through Rockwell International, DOE's Rocky Flats prime contractor. EG\&G succeeded Rockwell International as prime contractor at the Rocky Flats facility in 1989. Kaiser-Hill Company has operated the Oxnard facility since the second quarter of 1995 . Metalworking at the Oxnard facility has involved stainless steel, titanium, aluminum, copper alloys, tantalum, molybdenum, and tungsten.

\footnotetext{
${ }^{1}$ Rust Geotech is the prime contractor for DOE at the Grand Junction Projects Office.
} 


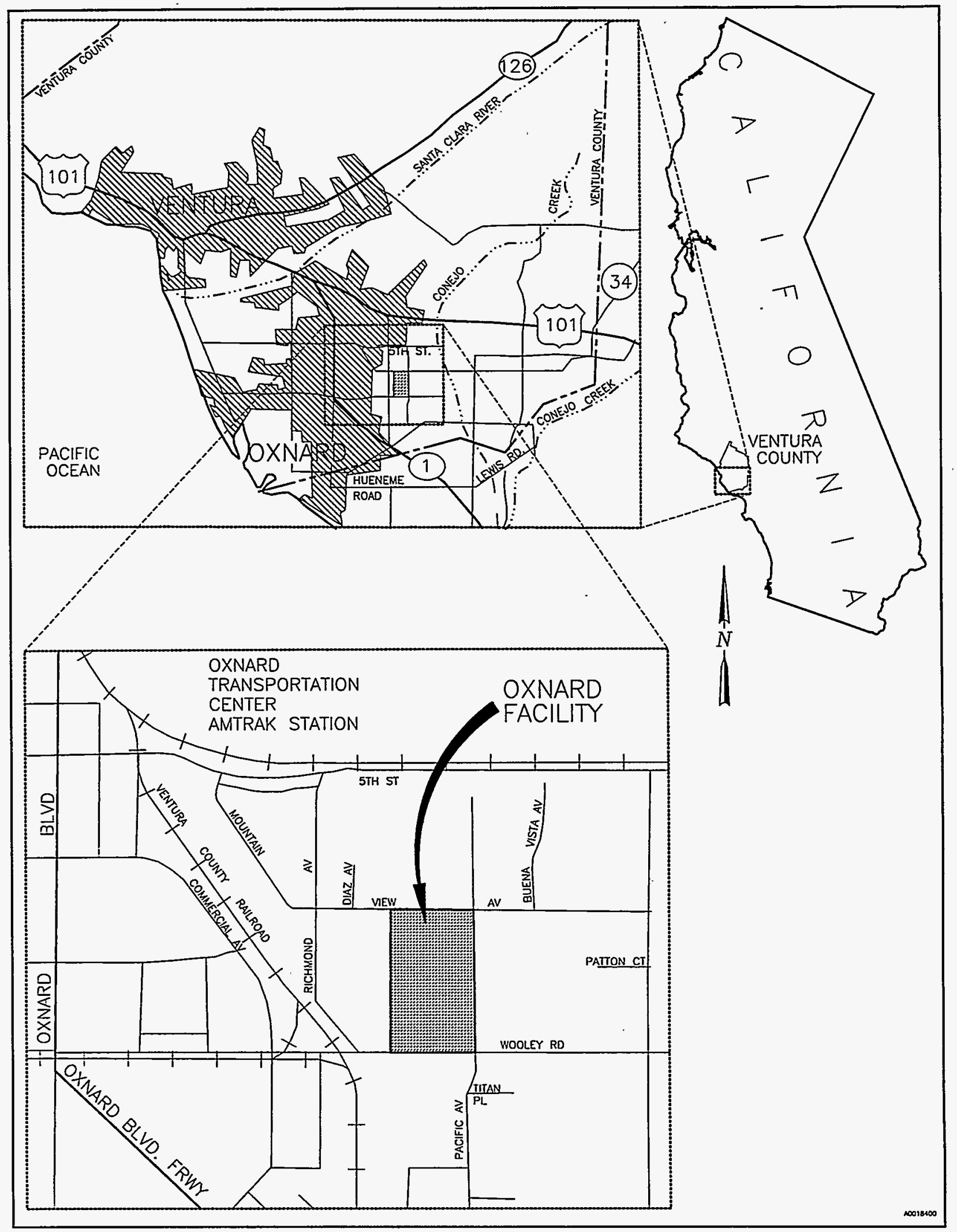

Figure 1. Location of the DOE Oxnard Facility, Oxnard, California 


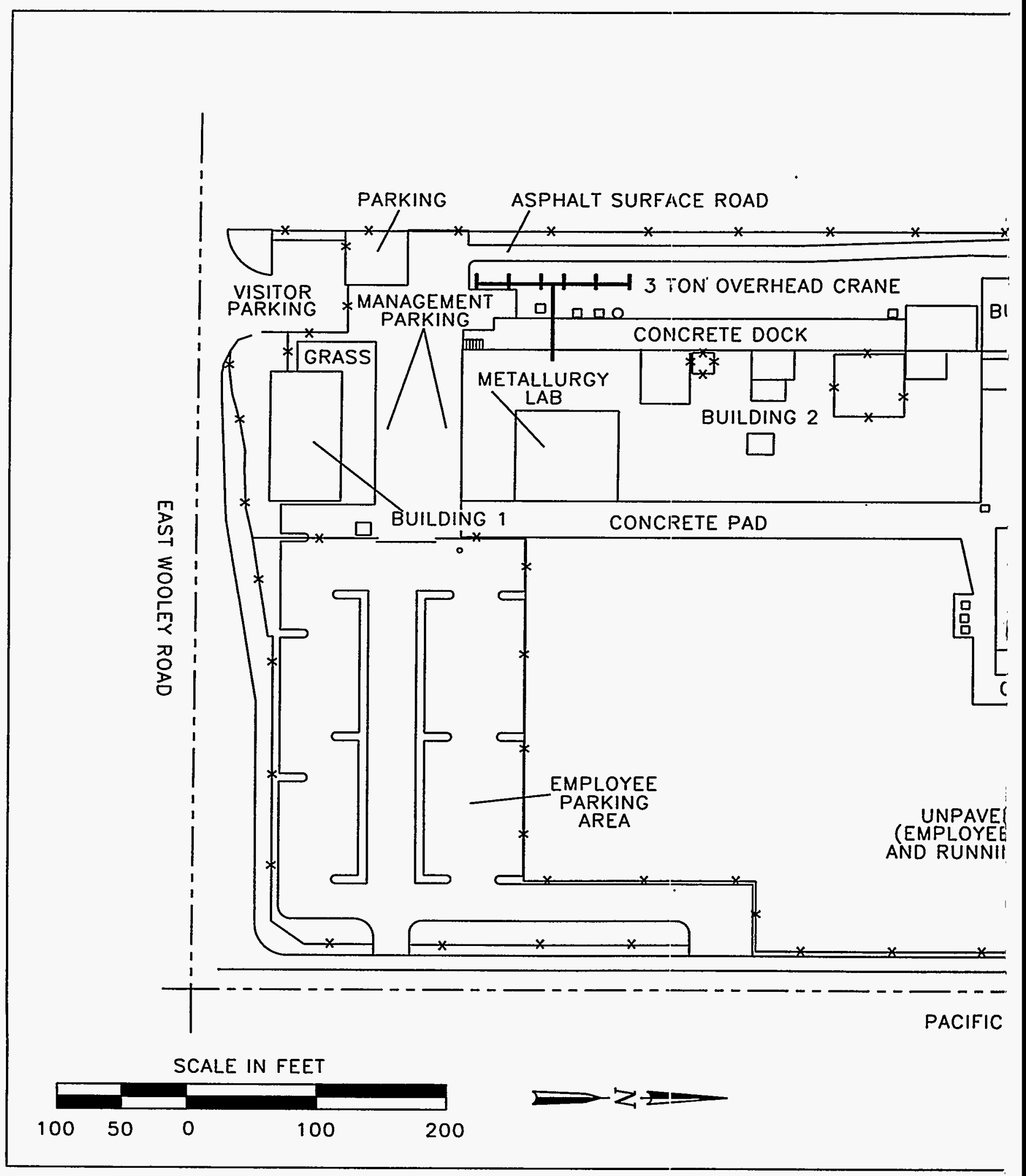

Figure 2. Site Layout o! 


\subsection{Objectives}

Potential hazardous wastes were identified on the basis of historical and current activities at the site. The applicable or relevant and appropriate requirements (ARARs) are shown in Table 1.

Table 1. ARARs for the DOE Oxnard Facility

\begin{tabular}{|l|l|}
\hline \multicolumn{1}{|c|}{ Medium } & \multicolumn{1}{c|}{ ARARs } \\
\hline Asbestos & $\begin{array}{l}\text { Toxic Substances Control Act , 40 CFR 763 } \\
\text { Title 8, CAL/OSHA, Sections 341.15, 341.6, and 1529 } \\
\text { Ventura County Air Pollution Control District Rule 62.7 }\end{array}$ \\
Groundwater & $\begin{array}{l}\text { Title 22, CCR } \\
\text { Title 22, CCRivion 4.5, Chapter 15, Section 66265.97 } \\
\text { Title 22, CCR, Division 4, Chapter 15, Section 64431 }\end{array}$ \\
Soil & Title 22, CCR, Division 4.5, Chapter 11, Article 3, Section 66261.24 3, Section 66261.24 \\
Sludge/Oil & Title 22, CCR, Division 4.5, Chapter 11, Article 3, Section 66261.24 \\
\hline
\end{tabular}

${ }^{\mathrm{a}} \mathrm{CCR}=$ California Code of Regulations

All activities conducted by Rust were reviewed for National Environmental Policy Act compliance and were determined to be categorically excluded from requirements to prepare an Environmental Assessment or Environmental Impact Statement.

\subsection{Work Performed}

Site investigations conducted by Rust began in June 1994 with an Environmental Site Assessment (ESA) Preliminary Evaluation site tour. Results of the Preliminary Evaluation indicated the need for a Phase I and Phase II ESA.

A nonintrusive site inspection and data gathering effort in August 1994 identified "areas of concern" at the facility (Appendix A, Table 6-1). The results of this investigation were presented in the Phase I Environmental Site Assessment for the EG\&G Rocky Flats Oxnard Facility (Appendix A). The findings of the Phase I ESA indicated the need to conduct sampling and analysis activities to determine whether asbestos was present in the physical plant and whether regulated contaminants were present in the soil, groundwater, and in the oil and sludge collection and treatment systems at the facility.

Results of the Phase II intrusive ESA investigation, conducted in January and February 1995, were presented in the Phase II Environmental Site Assessment Report for the Kaiser-Hill Company LLC Oxnard Facility, Oxnard, California (Appendix B). This assessment included sampling and analyses for asbestos, and for hazardous contaminants in groundwater, in the surface and subsurface soils, and in the sludge and oil. Sample locations were selected by identifying locations with the greatest potential for detecting the presence of hazardous substances. Additional investigation was required when detectable concentrations of PCBs were identified in two soil samples. 
suggested regulatory action level (Appendix C). Analytical results are presented in Appendix B, Tables D-2 and D-5, and Appendix C, Table 2.

A survey of the shipping and receiving area was conducted for beta-gamma contamination. No above-background activities were detected (Appendix B).

Analytical results of sludge and oil samples indicated levels of chromium and nickel (one sample), lead and zinc (one sample), and lead (one sample) exceeding soluble threshold limit concentrations when tested by the California Waste Extraction Test. The sludge from these locations could be classified as a hazardous waste if removed for disposal from site processes. Analytical results are presented in Appendix B, Tables D-3 and D-4.

\subsection{Waste Volumes Generated}

Results of analyses of samples collected of soil, groundwater, and monitoring well development water indicated that these materials did not contain hazardous substances in concentrations exceeding regulatory limits. All soil and water samples were disposed of on site following laboratory analysis. Samples collected for identification of asbestos and the associated personal protective equipment will be handled as asbestos-containing material and disposed of properly.

The oil and sludge samples were managed by Truesdail Laboratories.

\subsection{Risk Assessment}

A risk assessment, modeled after CERCLA guidance, was performed to determine the exposure of site workers to elevated metals detected in the oil/sludge samples and the PCB and metal concentrations in the soils. The pathways of exposure were ingestion, inhalation, and absorption. On-site exposure to groundwater was considered to be an incomplete pathway because treated municipal water is supplied. The risk of off-site exposure to groundwater was considered insignificant because of the low concentrations of detected metals and because the water in the shallow aquifer is not used for domestic purposes and is separated from the underlying potable water source by low-permeability clays.

The Hazard Index $(\mathrm{HI})$ for noncarcinogens was $8.9 \times 10^{-4}$. The risk to human health from carcinogens was $3.2 \times 10^{-6}$ (Appendix C, Section 4.0). These are conservative risk determinations. An $\mathrm{HI}$ for noncarcinogens below a value of 1 is considered insignificant. The requirement for action at a site with a risk from carcinogens of between $1 \times 10^{-4}$ and $1 \times 10^{-6}$ must be decided on a site-specific basis in concert with EPA. 
U.S. Department of Energy, 1995. Sampling and Analysis Plan for. the.Phase III Environmental Site Assessment of the Oxnard Facility, U.S. Department of Energy Oxnard, California, P-GJPO-2204, prepared by Rust Geotech for the U.S. Department of Energy Grand Junction Projects Office, Grand Junction, Colorado, September 1995. 


\title{
Appendix A
}

\section{Phase I Environmental Site Assessment}

\author{
for the
}

EG\&G Rocky Flats Oxnard Facility 
Phase I Environmental Site Assessment for the EG\&G Rocky Flats Oxnard Facility

September 30, 1994

Prepared By:

RUST Geotech Inc.

P.O. Box 14000

Grand Junction, Colorado 81502

Prepared for the

U.S. Department of Energy

Grand Junction Projects Office

under DOE Contract No. DE-AC04-86ID 12584 


\title{
Phase I Environmental Site Assessment for the EG\&G Rocky Flats Oxnard Facility
}

September 30, 1994

Prepared By:

RUST Geotech Inc.

P.O. Box 14000

Grand Junction, Colorado 81502

\author{
Prepared for the \\ U.S. Department of Energy \\ Grand Junction Projects Office \\ under DOE Contract No. DE-AC04-86ID12584
}


1.0 INTRODUCTION $\ldots \ldots \ldots \ldots \ldots \ldots \ldots \ldots \ldots \ldots \ldots \ldots \ldots \ldots \ldots$

2.0 SITE DESCRIPTION $\ldots \ldots \ldots \ldots \ldots \ldots \ldots \ldots \ldots \ldots \ldots \ldots$

2.1 Site Ownership .................... 3

2.2 Physical Location/Setting $\ldots \ldots \ldots \ldots \ldots \ldots \ldots \ldots \ldots$

2.3 Utilities ........................ 6

2.4 Geology ......................... 7

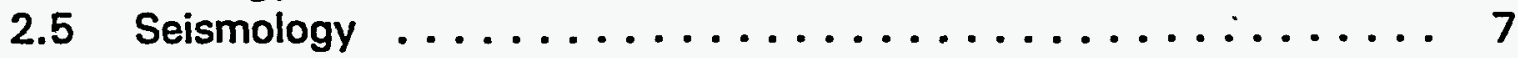

2.6 Groundwater ..................... 8

2.7 Surface Hydrology $\ldots \ldots \ldots \ldots \ldots \ldots \ldots \ldots \ldots$

2.8 Demography....................... 8

3.0 TITLE SEARCH $\ldots \ldots \ldots \ldots \ldots \ldots \ldots \ldots \ldots \ldots \ldots \ldots \ldots$

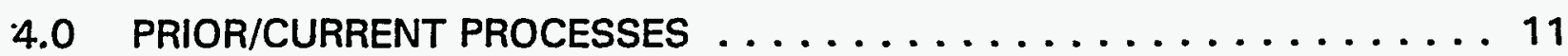

4.1 Historical Production Processes . . . . . . . . . . . . 11

4.2 Current Basic Production Process . . . . . . . . . . 11

4.3 Current Building Descriptions and Specific Functions ... . . . 12

5.0 REGULATORY AGENCY INFORMATION .............. 19

5.1 Listing of Agencies/Properties of Concern . . . . . . . . 19

5.2 EG\&G Rocky Flats Oxnard Facility . . . . . . . . . . 20

5.2.1 Solid/Hazardous Waste . . . . . . . . . . . . 20

5.2.2 Wastewater Discharge ................. 22

5.2.3 Underground Storage Tanks (USTs) $\ldots \ldots \ldots \ldots \ldots \ldots 23$

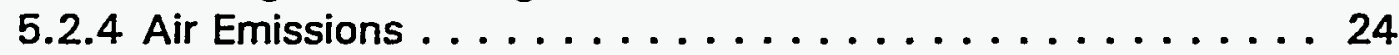

5.2 .5 Hazardous Materials . . . . . . . . . . . . 24

5.3 Kingstone Wheel Corp. (Formerly Del Manufacturing) . . . . . 25

5.3.1 Solid/Hazardous Waste . . . . . . . . . . . . 25

5.3.2 Wastewater Discharge ................ 25

5.3.3 Underground Storage Tanks (USTs) $\ldots \ldots \ldots \ldots \ldots 25$

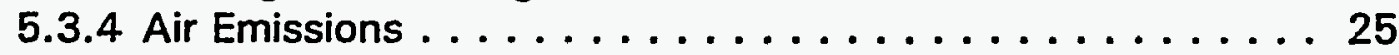

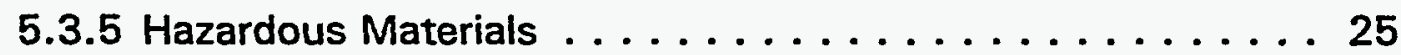

5.4 Deardorff-Jackson, Inc. . . . . . . . . . . . . 25

5.4.1 Solid/Hazardous Waste . . . . . . . . . . . 25

5.4.2 Wastewater Discharge ................ 26

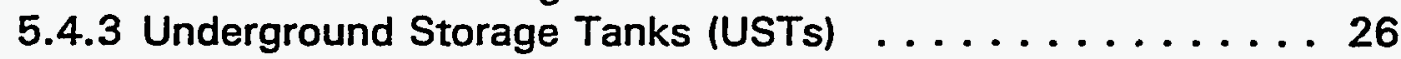

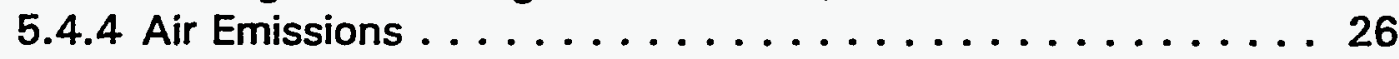

5.4.5 Hazardous Materials . . . . . . . . . . . 26

5.5 Boskovich Corp. . . . . . . . . . . . . . . 26

5.6 City of Oxnard Vehicle Maintenance Facility . . . . . . . . 27

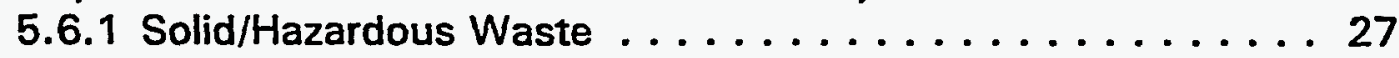

5.6.2 Wastewater Discharge ............... 27 
5.6.3 Underground Storage Tanks (USTs) $\ldots \ldots \ldots \ldots \ldots$

5.6.4 Air Emissions . . . . . . . . . . . . . . 28

5.6 .5 Hazardous Materials . . . . . . . . . . . . 28

5.7 Gold Coast Steel (formerly J\&S Engineering) . . . . . . . . 28

5.7.1 Solid/Hazardous Waste . . . . . . . . . . . 28

5.7.2 Wastewater Discharge ................. 29

5.7.3 Underground Storage Tanks (USTs) . . . . . . . 29

5.7.4 Air Emissions . . . . . . . . . . . . . . . 29

5.7 .5 Hazardous Materials . . . . . . . . . . . . 29

6.0 SUMMARY OF FINDINGS $\ldots \ldots \ldots \ldots \ldots \ldots \ldots \ldots \ldots \ldots \ldots$

6.1 . Geology/Hydrogeology $\ldots \ldots \ldots \ldots \ldots \ldots \ldots \ldots \ldots \ldots$

6.2 Off-Site Influences $\ldots \ldots \ldots \ldots \ldots \ldots \ldots \ldots \ldots \ldots$

6.3 On-Site Areas of Concern ................. 30

6.3.1 Basis for Areas of Concern . . . . . . . . . . 32

7.0 RECOMMENDATIONS $\ldots \ldots \ldots \ldots \ldots \ldots \ldots \ldots \ldots \ldots \ldots \ldots \ldots \ldots$

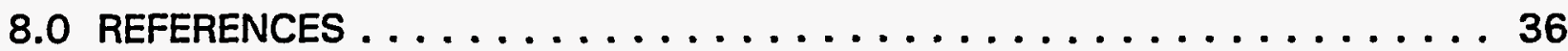

Figure $2-1$ Oxnard Vicinity $\ldots \ldots \ldots \ldots \ldots \ldots \ldots \ldots \ldots \ldots \ldots$

Figure $2-2$ Oxnard Vicinity ..................... 5

Figure 4-1 Building Plot Plan $\ldots \ldots \ldots \ldots \ldots \ldots \ldots \ldots \ldots$

Table 6-1 Oxnard Facility - Areas of Concern $\ldots \ldots \ldots \ldots \ldots$

Appendix A Environmental Site Assessment, Preliminary Evaluation Trip Report

Appendix B Construction Boring Logs (Buena Engineers, Inc. 1982)

Appendix C Summary of Hazardous Waste Streams 1989-1993

Appendix D Photo Documentation

Appendix E Design As-Built Drawings (Buildings 5 and 6)

Appendix F Hazardous Materials Map and Hazardous Materials

Chemical Descriptions 


\subsection{INTRODUCTION}

On June 8, 1994, an Environmental Site Assessment (ESA) Preliminary Evaluation (PE) was conducted at the EG\&G Rocky Flats Oxnard Facility (Oxnard Facility). (Note: A copy of the PE trip report is found in Appendix A.) The facility is located at 1235 East Wooley Road, Oxnard, California. This PE determined that information had been generated over various years, but that the potential or real presence of environmental contamination on the "real property" was not well documented. The PE trip report (Section 5.0) further recommended the preparation of a Phase I and Phase II ESA.

During the week of August 8, 1994, a "due diligence" Phase I ESA was performed. The Phase I included:

- Determination of site ownership and location

- Property title search

- Review of historical site photographs

- Plant site personnel interviews

- Detailed plant site tour

- Drive-by tour of surrounding facilities

- Record reviews at the following regulatory agencies

- City of Oxnard - Source Control Program

- Ventura County Air Pollution Control District (VCAPCD)

- Ventura County Department of Environmental Health Hazardous Waste \& UST (VCDEH)

- California Environmental Protection Agency Department of Toxic Substances Control (DTSC)

This Phase I ESA was conducted by RUST Geotech Inc., contractor to the U.S. Department of Energy Grand Junction Projects Office (DOE-GJPO). Participants included:

Sam Campbell
Donald Koch
Jack McCaslin
Environmental Sciences

Environmental Standards \& Oversight Technical Support

This Phase I ESA recommends that a full Phase II ESA be conducted to further reduce the uncertainties associated with the areas of concern identified in Section 6.0 of this report. This further investigation should resolve concerns pertaining to two abandoned underground storage tanks, a pit area beneath Building 6 previously used by Allis-Chalmers, asbestos-containing material in the 
seven on-site buildings, several previously polychlorinated biphenyl (PCB) contaminated areas, and a trench/sump collection system located in the forging and press shops. 


\subsection{SITE DESCRIPTION}

\subsection{Site Ownership}

The Oxnard facility is a nonferrous metalworking (forging, machining, welding, cutting, grinding, wheelabrating, and painting) facility. The facility is owned by the U.S. Department of Energy (DOE) and is operated by EG\&G Rocky Flats. .

\subsection{Physical Location/Setting}

The Oxnard facility occupies $\mathbf{1 3 . 7 5}$ acres and is located in an industrial park within the incorporated city limits of Oxnard and within the county of Ventura (Figure 2-1). The facility is bordered by industrial maintenance, manufacturing, and agricultural packaging facilities. The City of Oxnard operates a vehicle maintenance garage and trash receptacle storage yard on Pacific Avenue to the east. Deardorff-Jackson Inc. and Boskovich Corp. are vegetable/fruit packers located on Mountain View Avenue to the northwest and northeast, respectively. Kingstone Wheel Corp. (previously Del Manufacturing) is a metal wheel fabricator located on the southwest. A steel fabricator and machinist owned by Gold Coast Steel is located on the northwest. To the south, across Wooley Road, Told Corporation owns property which was previously used as farmland. This property is presently being marketed for commercial development.

The facility is comprised of seven buildings with approximately 86,000 square feet of covered floor space (Figure 2-2). The seven buildings include:

$\begin{array}{ll}\text { Building \#1 } & \begin{array}{l}\text { Administrative Offices } \\ \text { Machine Shop, Engineering, Quality Control, Metallurgical } \\ \text { Laboratory, Production Control Office, Maintenance } \\ \text { Offices, and lunch room }\end{array} \\ \text { Building \#3 } & \begin{array}{l}\text { Saw Shop } \\ \text { Building \#4 }\end{array} \\ \text { Guilding Shop } \\ \text { Building \#6 } & \text { Press Shop, Wheelabrator Room, Dye Penetrant Room } \\ \text { Building \#7 } & \text { Forge Shop } \\ & \text { Tank Farm/Lubrication Stores }\end{array}$




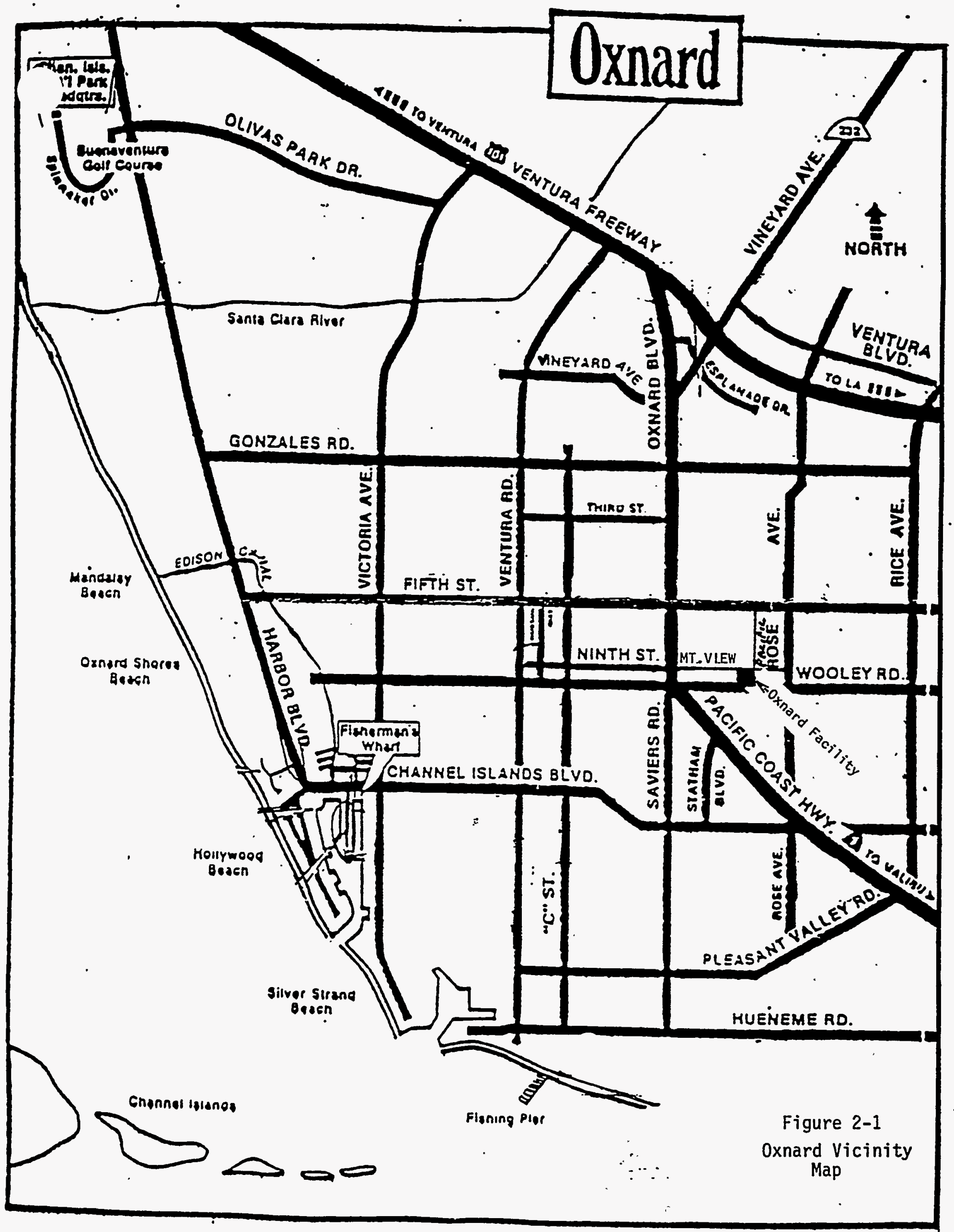




\subsection{Utilities}

The plant's utilities currently operate well below their design potential. Prime utilities were designed during construction to allow for future expansion. Capacity and current supply availability includes:

Electrical - two 2500 ampere switchboards. Systems exist in place to double this capacity in a short period of time.

Water - An eight-inch supply line furnishes water in excess of either current needs or any reasonable expansion requirements for this facility.

Natural gas - A 5 -inch main at 5 pounds per square inch (psi) fed by 223 psi from the street services the plant. The most significant limitation on gas availability and usage is the Ventura County Air Pollution Control District's emission regulations. The District currently permits an annual consumption not to exceed 50 million cubic feet (MMcf) of natural gas in all combustion equipment.

The Oxnard facility currently contracts services with the following utilities:

Electrical: $\quad$ Southern California Edison

P.O. Box 600

Rosemead, CA 91771-0001

Natural Gas: Southern California Gas Company

P.O. Box C

Monterey Park, CA 91756

Telephone: Cellular One

P.O. Box 78132

Phoenix, AZ 85062-8132

GTE California

P.O. Box 215

Newbury Park, CA 91319

Water: $\quad$ City of Oxnard

Water Resource Dept.

305 West Third Street

Oxnard, CA 93030

Sewer: $\quad$ City of Oxnard

Wastewater Division

6001 South Perkins Road

Oxnard, CA 93030 


\subsection{Geology}

\section{Regional Setting}

Oxnard is located in the Pacific border physiographic province. The predominant trend of mountain ranges in this province is north-northwest. Oxnard is situated on the west side of the Transverse Ranges, an anomalous east-west trending group of mountains. The bedrock in this region consists mostly of Mesozoic and Cenozoic sediments, volcanics, and metamorphic sequences, tectonically accreted to the western margin of North America.

\section{Local Setting}

The Oxnard facility is situated on a flat area of deep alluvial fill known as the Oxnard Plain. The Santa Clara River, and smaller streams draining the Santa Monica Mountains, have deposited alluvium in a prograding alluvial fan. This fan forms the westward bulge in the shoreline from Ventura to Point Mugu. The Quaternary alluvium (less than 2 million years) covers downfaulted rocks similar to those exposed in the adjacent Santa Monica Mountains.

Lithologically, the alluvium of the Oxnard Plain consists of an upper (less than 6 feet below land surface) unit of light brown, silty sand top soil overlying grey, course-grained sands and gravels interbedded with grey, fine-grain sand and clayey silts.

In 1982 Buena Engineers, inc. under contract with Precision Forge (former owner to the DOE) drilled three borings to identify the immediate geology for the proposed construction of Building 6. A sketch identifying the location of the three borings and the boring logs are provided in Appendix $B$. The logs show the presence of free water for the shallow unconfined zone to exist between 5 feet and 8.5 feet. Furthermore, boring No. 1 identified trash and debris in the top 5 feet. The trash and debris are believed to be part of a foundry cast disposal pit used by the former owner, Allis-Chalmers.

\subsection{Seismology}

There are a number of major, northeast-southwest trending faults exposed in the Mountains and hills in the vicinity of the Oxnard facility. These include the Oak Ridge Fault to the north, the Simi Fault to the east, and the Sycamore Canyon Fault to the southeast. The Simi Fault projects beneath the alluvium of the Oxnard Plain, and therefore is most relevant.

The Simi Fault is a north-dipping reverse fault that strikes northeast. Most of the 5,300 feet of displacement on this fault occurred between 16 million to 10,000 years ago. Although no recent $(<10,000$ years) displacement on the Simi Fault has been recognized, there have been small magnitude $(M<2.5)$ earthquakes recorded. 


\subsection{Groundwater}

The Oxnard facility lies about $\mathbf{4 0}$ feet above sea level on a silty, sand-soil base. The shallow unconfined groundwater is encountered at a depth of 5 to 10 feet. This shallow unconfined groundwater has been described as perched by previous consultants because of its limited extent and because it is underlain by low permeability clays. Recharge for this perched zone is provided by local precipitation and agricultural irrigation. The potable aquifer lies approximately 120 feet below ground surface. According to Underground Storage Tank (UST) investigations at Gold Coast Steel and the City of Oxnard, the deep aquifer is not in hydraulic communication with the perched zone; and the groundwater was not impacted by leaking underground tanks at both properties. The groundwater flow direction in the perched zone is to the southwest toward the Pacific Ocean.

No evidence exists which would indicate salt water intrusion of either the perched or the potable zone aquifers beneath the Oxnard facility.

\subsection{Surface Hydrology}

No surface waters are on or within 1,000 yards of the Oxnard facility.

\subsection{Demography}

The 1990 population census for the City of Oxnard exceeded 150,000 . The Ventura County population was about 700,000 including the City of Oxnard. 


\subsection{TITLE SEARCH}

The title search for the Oxnard facility involved a review of applicable files at the County of Ventura's Recorder's Office, Treasurer-Tax Collection Office and Assessor's Office. Research indicates the following:

- On March 20, 1946, Edwin L. Carty and Doris C. Carty granted all real property (Lots 1 and 8 and East 158.65 feet of Lots 2 and 7, Block C, Virginia Parks together with all and singular the tenements, hereditaments and appurtenances) to Allis-Chalmers Manufacturing Company, a Delaware Corporation.

- On May 3, 1946, Edwin L. Carty and Doris C. Carty granted a 6.25/130 interest in a water well, pumping plant and distribution system to AllisChaimers Manufacturing Company, a Delaware Corporation.

- On August 3, 1982, PERFCO Leasing Company granted a street right-of-way to the City of Oxnard, a Municipal Corporation.

- On December 3, 1983, PERFCO Leasing Company granted an exclusive easement for waterlines and related appurtenances to the City of Oxnard, a Municipal Corporation.

- On January 18, 1983, PERFCO Leasing Company granted an easement and right-of-way for electrical utility use to Southern California Edison Company, a California Corporation.

- On June 26, 1984, PERFCO Leasing Company granted the same property described in the Deed dated, March 20,1946, to Charles D. Pearce and $\mathrm{K}$. Thomas Rose as Trustees for Security Pacific Leasing Corporation.

- On June 24, 1984, Charles D. Pearce and K. Thomas Rose as Trustees for Security Pacific Leasing Corporation, granted the same property described by the Deed dated, June 26, 1984, to the United States of America. The above described Real Property was acquired for the Department of Energy.

Concerns noted during the title search include:

- No record could be found identifying a deed transaction between AllisChalmers and PERFCO, assuming PERFCO was the next title owner. 
- PERFCO Leasing Company could not be determined as a company owned and operated by Precision Forge.

- An unexplained change in the Assessor's Parcel Number occurred in the 1983-84 tax year. The previous number APN201-0-301-02 was changed to APN201-0-301-03. 

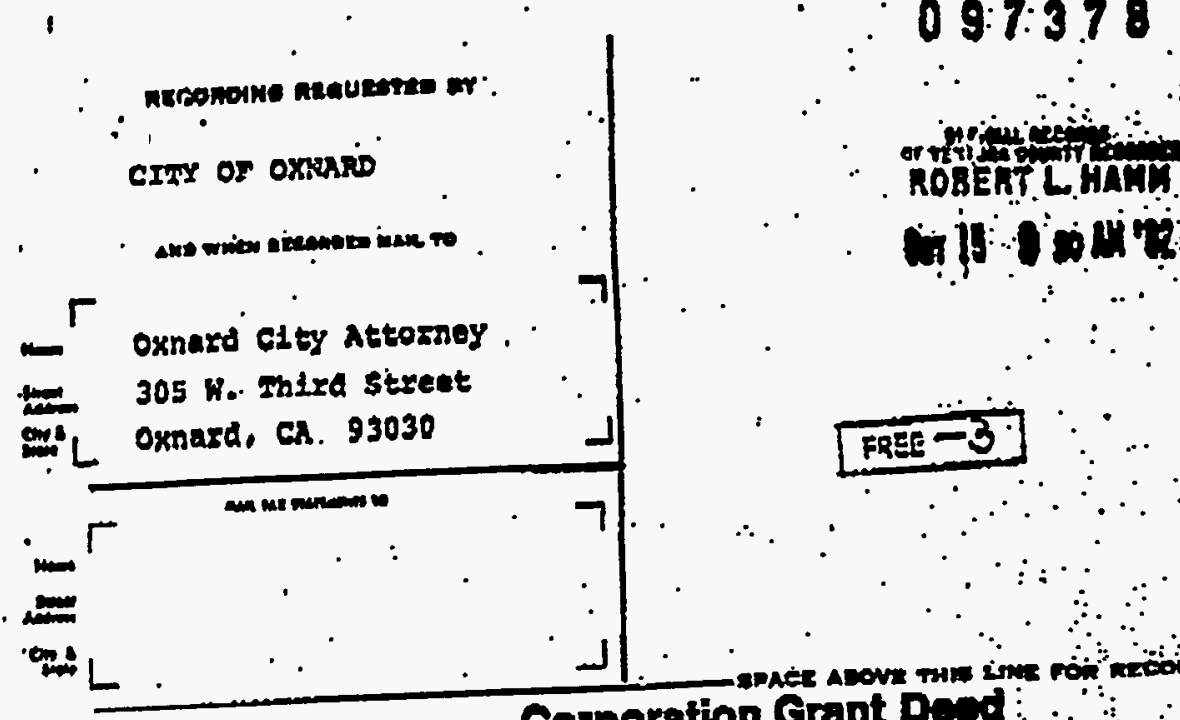

Corporation Grant Dyod

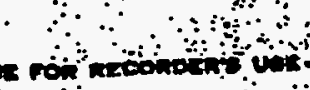

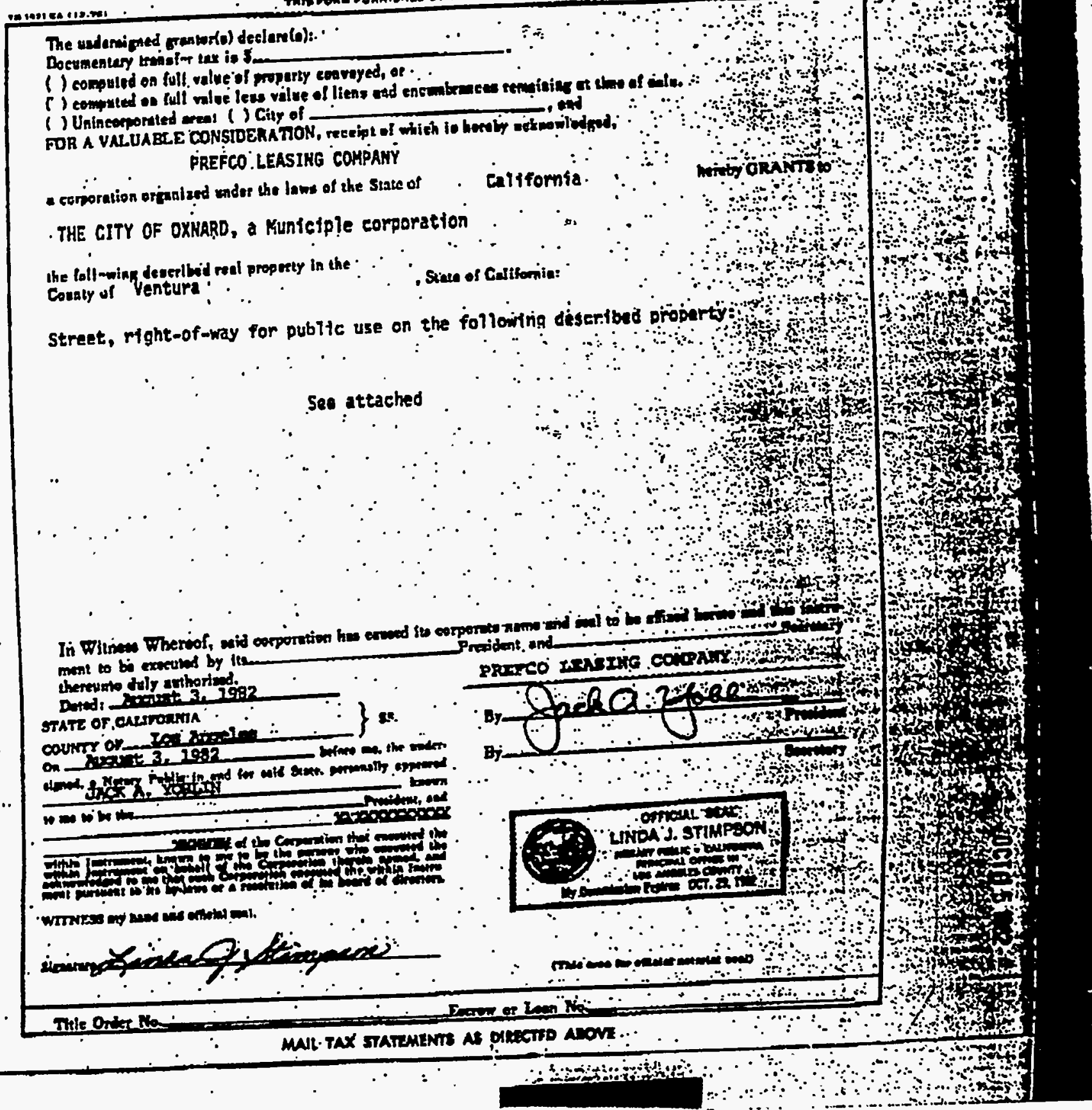




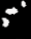

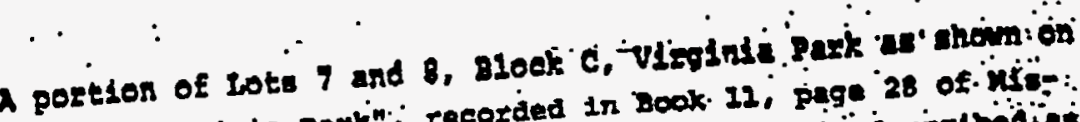

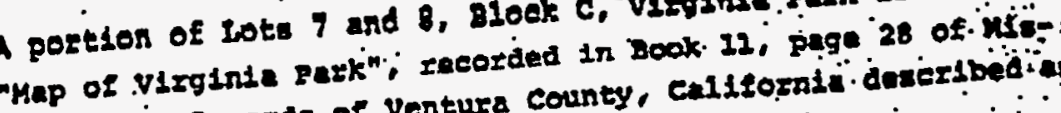

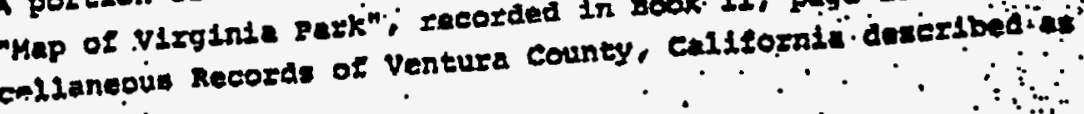
collow:

Beginning at the interatetion of the veteely. 2ine of: pacific

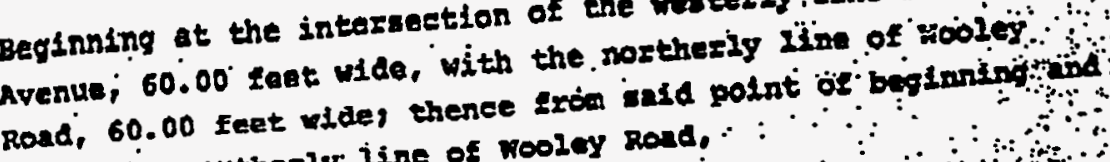
along the northerly ine of rooley rond. : $\because:$ :

1st: . Worth 89" '57' 00". West 105.03 tent: to the biogining DE a tangent curve coneave to ind noreiniest ind having tadios of 945.00 zeut thareer:

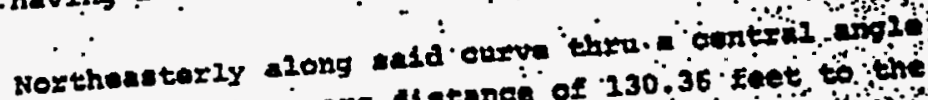
of 70 54' 14" an are distance of 130.36 enet to the

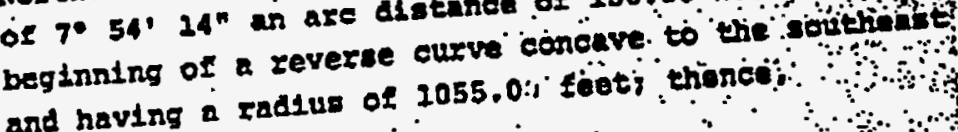

2nd:

ixt: Northeatterly along sald curve thru a cintrat ongle

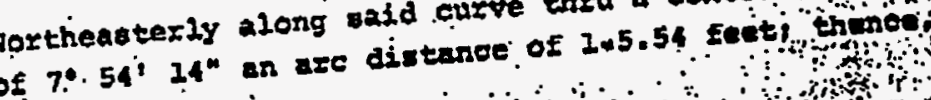

$$
\text { - }
$$

South $89^{\circ}$ 57. 00" sout 104 is7 soct to the boginging

itris of a tungant curve concave to the inorthrest and having a radius of 25.00 finet: triented,

$\therefore$

5th:

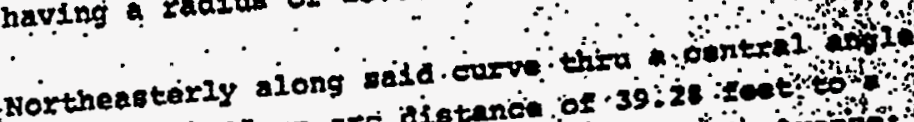

. 


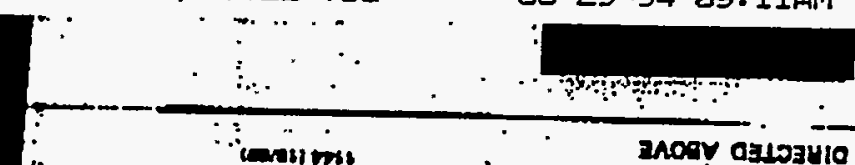

mogy ajloguio sN sewaralyLs XV1 714
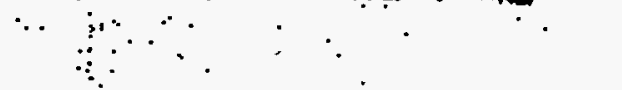
A portion of Lots $1,2, .7$ and 8, Block $C_{1}$ Virginia park, a shown on "Map of.Virginia Park," recorded in Book II, page 28 of Miscellaneous Records of Ventura county; Califernia, described ait follows:

Parcel 1: The westerily 20.00 feet of the easterly. 158.65 fect of lot 2 and the wasterly 20.00 feet of the northerly 479.62 feet of the easterly 158.65 feet of Iot 7 .

Parcel 2: The northerly 15.00 feet of the southerly $125.00 \%$. feat of the easterly 158.65 feet of Iot 7 and. the northerly 15.00 feet of the southerly 1.25.00. feat of lot 8 .

Parcel 3: The southerly 15.00 feet of tho northerly 424.59 feet of the easterly 170.00 feet of. Iot 1 :

Parcel 4: The weateriy 15.00 feet of the eastiorly $255.50^{\circ}$ feet of the northeriy 215.00 feet of the southeriy 340.00 feet of Iot 8 .

parcei 5: The weaterly 15.00 feet of the eanterly. 261.50 fent of the southerly 125.00 . Feet of . Lot 8. .

The grantor shall not birden the surface of the easement harian granted with any above ground fences, buildings or structures, which covenant and condition shall be observed by the grantor, its successors or assigns. 


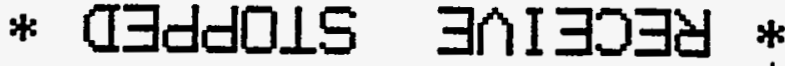

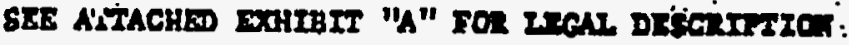

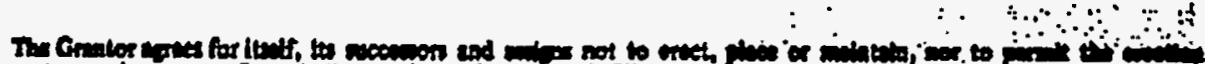

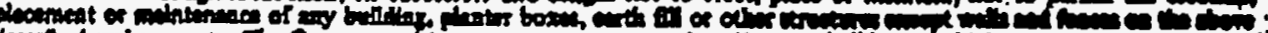

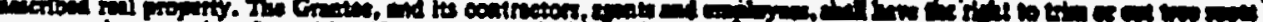

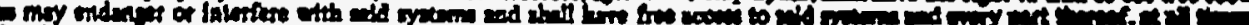

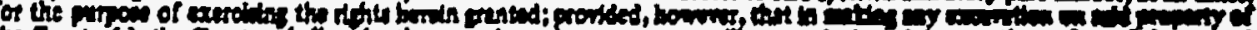

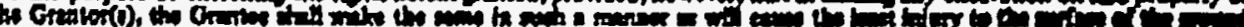

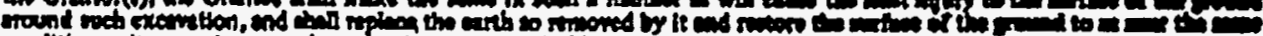

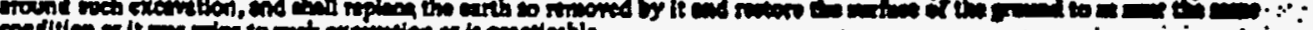

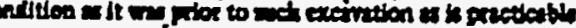

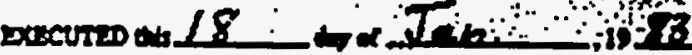

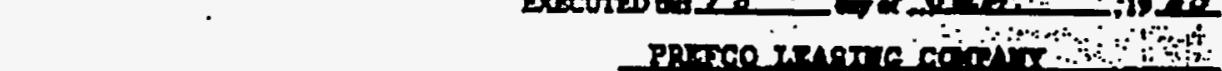
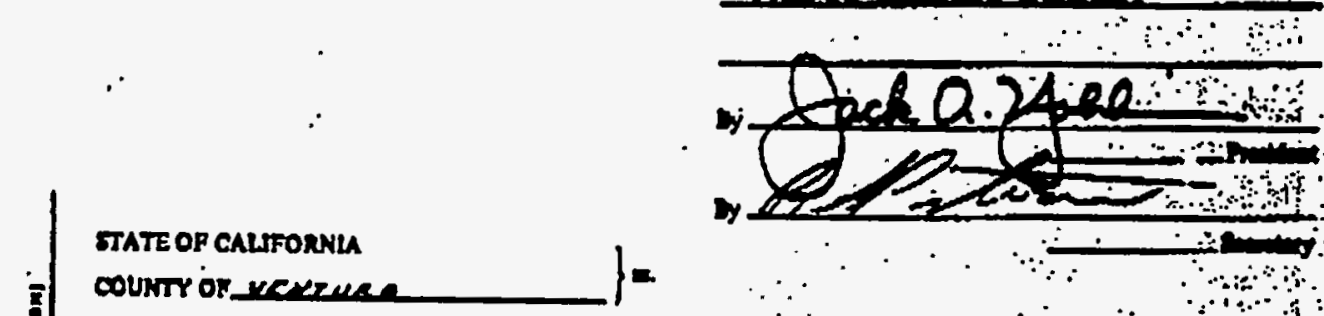

colvtr os vereruse

s.

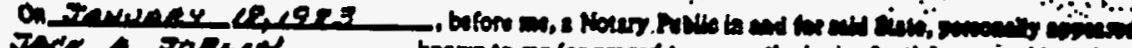
$\therefore \circ$ $\cdot \cdot$

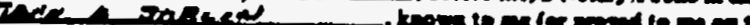

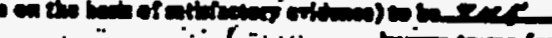

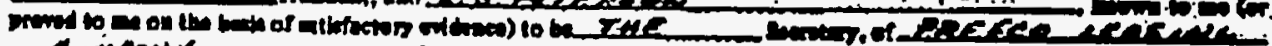

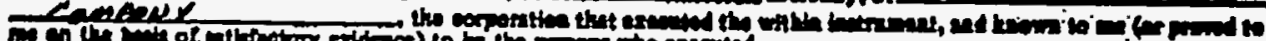

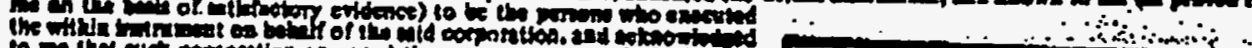

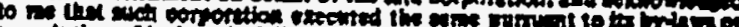

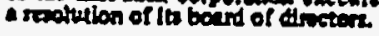

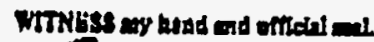

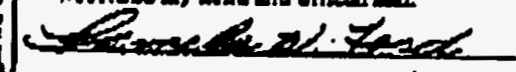

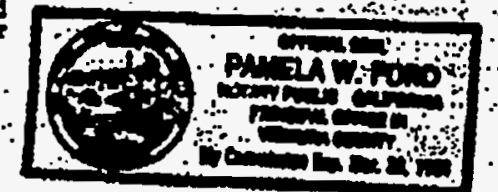


Grant of Bogemnnt $6639-2596,2-2524$

Page 2

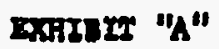

A otylp.of Iand 8 fait wide lying within lot 8 of zloek $\mathrm{C}$ of.

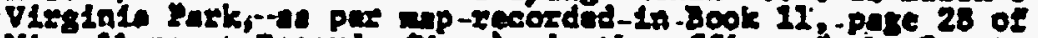

Mecallaneou Reeords (Nipn), In the office of the county

kecordes of uald County; the centerilaie of ald aterip of land 10 desartbed as Lollows:

Bopinnits at point in the eesterly 3 ine of cold fos. 8 the If 373 feet noztherly of the conferilne of pooley lond. 60 seot wide.

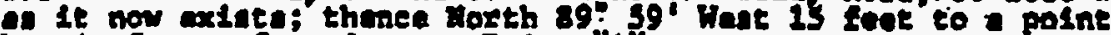
hereinnfter reforzed to an Polnt "A".

Aloo two (2) atrips of land, esch 6 feet wide; the cinterilma Are describad as Eollown:

BXRIP YO, 2: Baglmins at Polnt "A" herelnbeforie referzed to; .

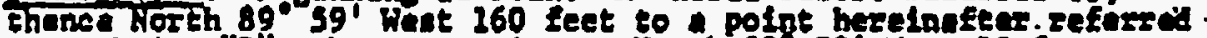

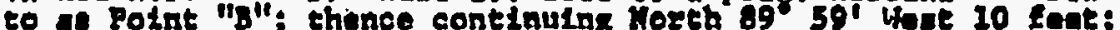
thence North $43^{3}$ 00' Want is faet; thence North $0^{\circ}$. ol ' Lat 190 fent to polnt hareinater referred. to as Polit "t"i thenea

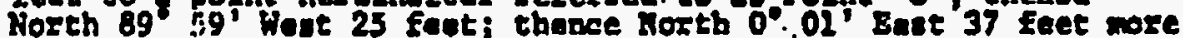
or 2008 to the coterlor wall of a bullding.

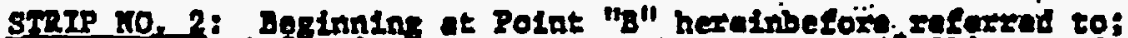

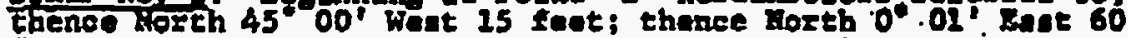
Eeat.

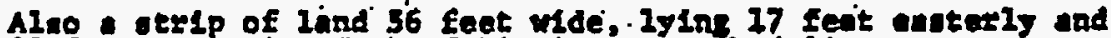
39 Lace weoteris of the followhs daserlbed ines

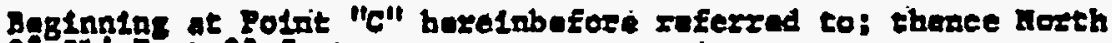
o 01 ' Eant 25. Euet. 
Grunt of Easemrnt $6639-2596,2-2524$ Pate 2

\section{EXIIBTY "A"}

A etrip of land 8 fuet wide lying withln Lot 8 of Block $C$ of Virginis Park, at per mop recorded in Book 11 , page 28 of MLecli aneous Recozds (Mape), in the offles of the County Incoxdur of ald Count; the centerline of gald ierip of land 10 doceribed at follows:

Bugtnning at a point In the earexiy ilne of sald Lot 8 thit is 373 feet northerig of the centerline of Wooley Rond, 60 feet wide a It now exinte: thence Norch 89 $59^{\circ}$ Weat is feet to a point harelnafte: raferred to a Point "A".

Also two (2) otripr of land, each 6 feet wide; the centerinas are dencribed at followe:

8rfy NO, I: Dagining at Point " $A$ " herelnbefore refarred to: thance iorth $89^{\circ} 59^{\prime}$ West 160 seet to point hereinaftar referred to al Point "B", thence continuing North 89" 59 " wont 10 faet: thance North $45^{\circ} 00^{\prime}$ Weat 15 Eeet; thence North $0^{\circ} 01^{\prime}$ East 190 fart to point herelanfter refarred. to 18 Point " $\mathrm{C}$ "; thence North $89^{\circ} 59^{\prime}$ Weat 25 feet; thonce North $0^{\circ} 01^{\prime}$ Bart 37 feet wore or lese to the exterios wall of a buliding.

Sym 10, 2: Beginning ot Point "B" herainbefore referred to:

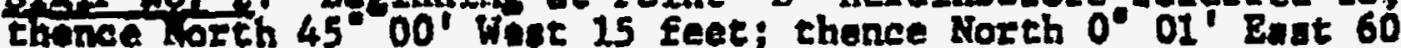
Eeat.

A1no atrip of land 56 feet wde, lylng 17 feet eartoriy and 39 Eact wateriy of the following degcribed IIne:

Becinning at Polnt " $C$ " hereinbeiore referred to; thence North 0 ol rant 25. feet. 
Fecurdty proftse saning cort|

- 8.0. $80 \times 9723$

w San rxenelteo, ex 94211

- Atent Sorn Vojivba. Ise.

at

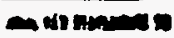

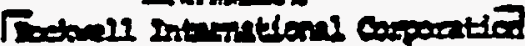

- 8.0.

- Colens, $\infty 80102$

int

Atari K., K.H. Shrzen

L.

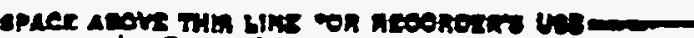

\section{Corporetlon Grant Dand}

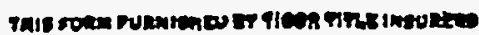

A.1. 201-201-020

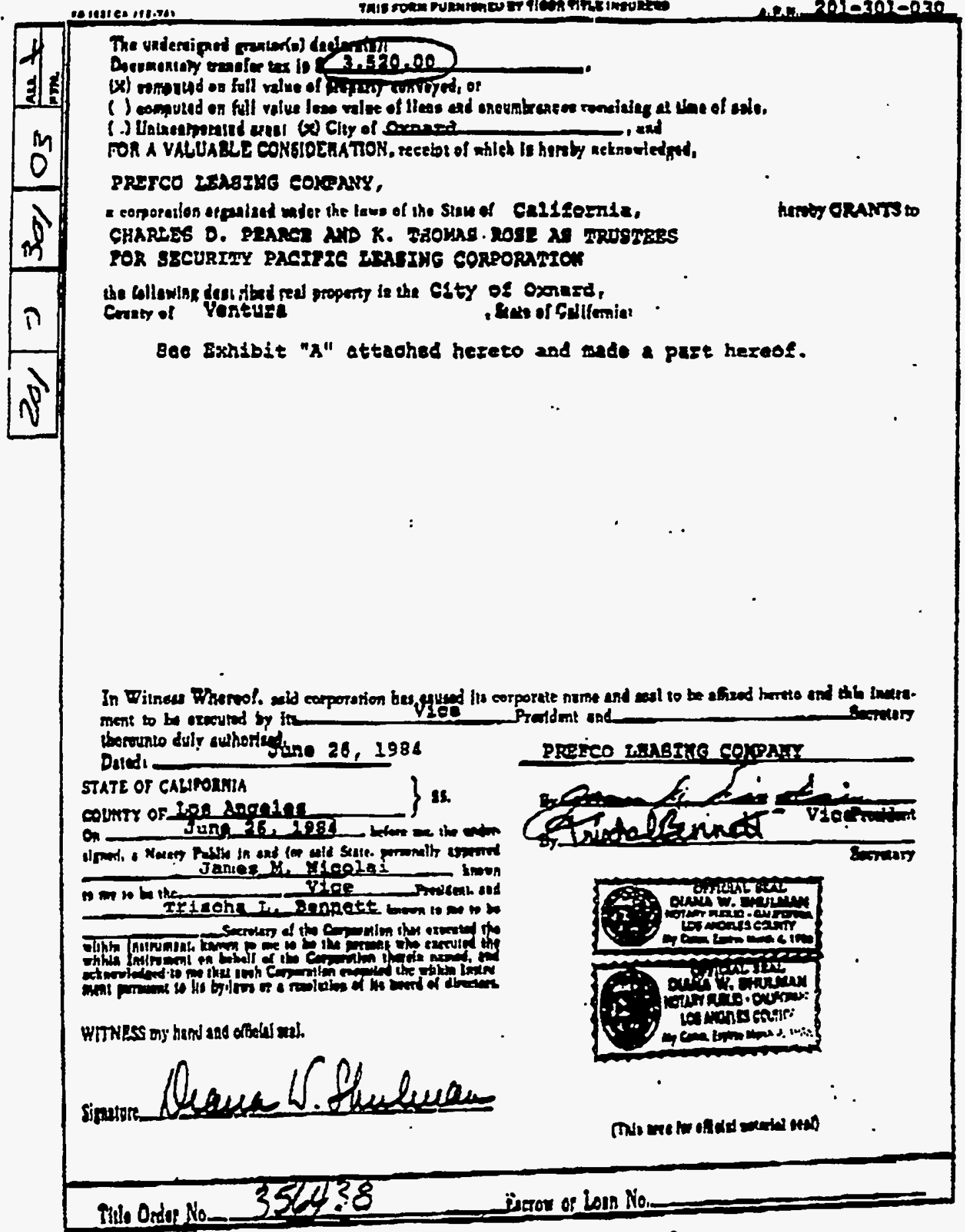

MAlB TAX STATEMHATS AS OIRETTEO ALOVE

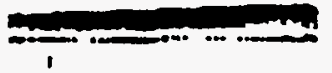




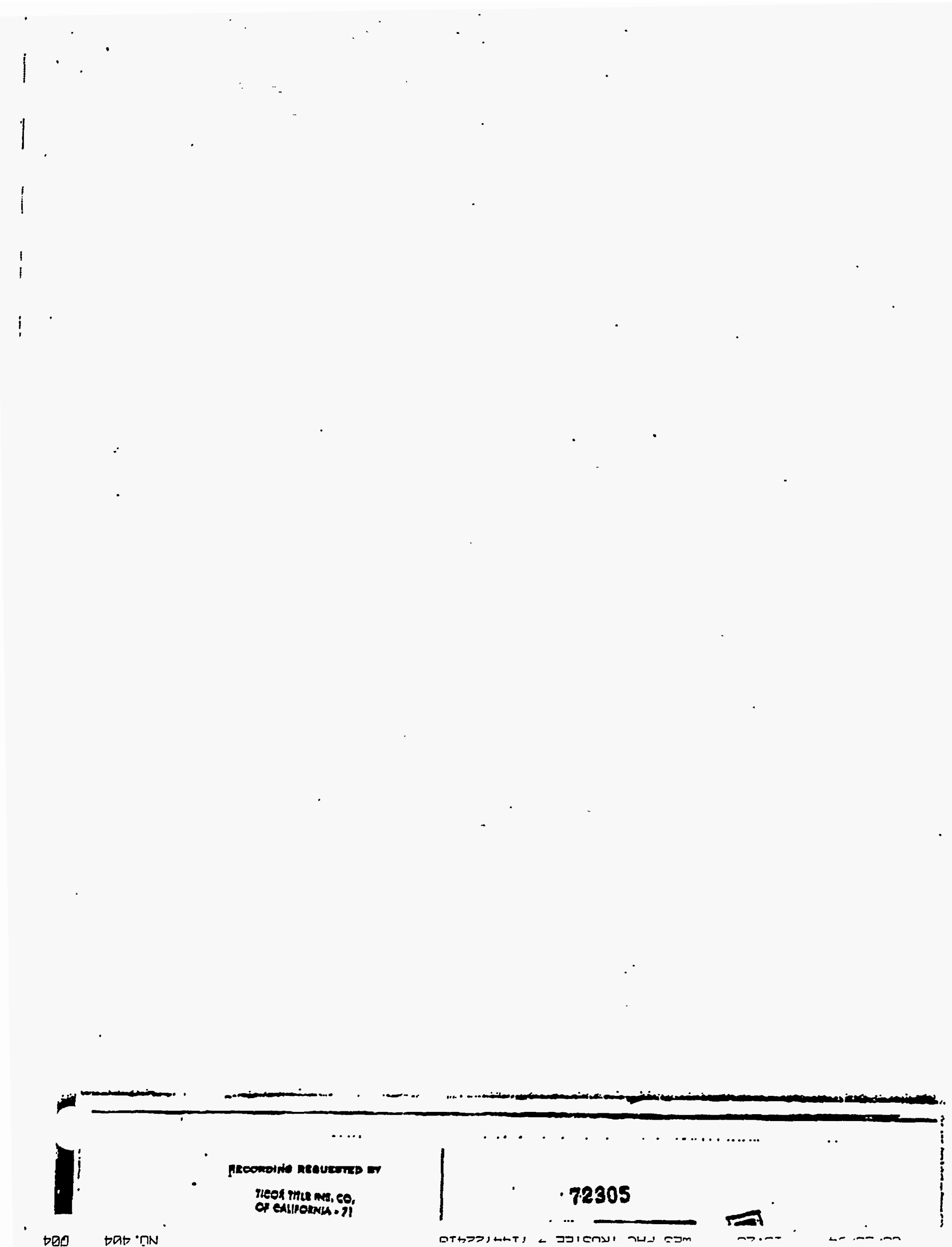




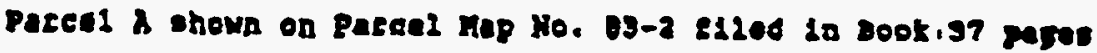
o4 ant 95 of pazeol maps in the ozelee of the. County incozen of Vóatura County.
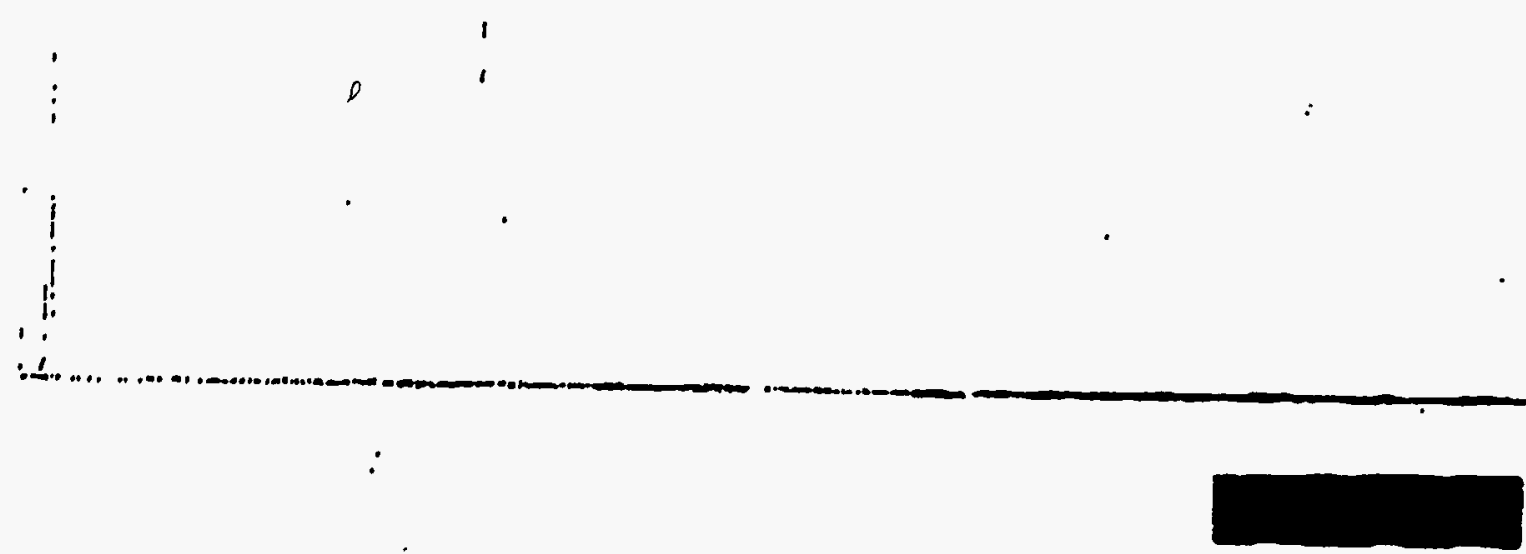

- \# Db9LOS96TLT

$\leftarrow$ 


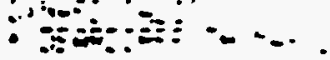

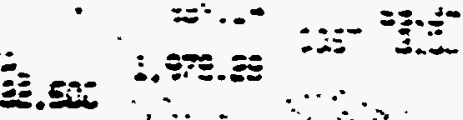

$\cdots \because \cdots, \because \because \because x^{2}$

$\therefore$ ind

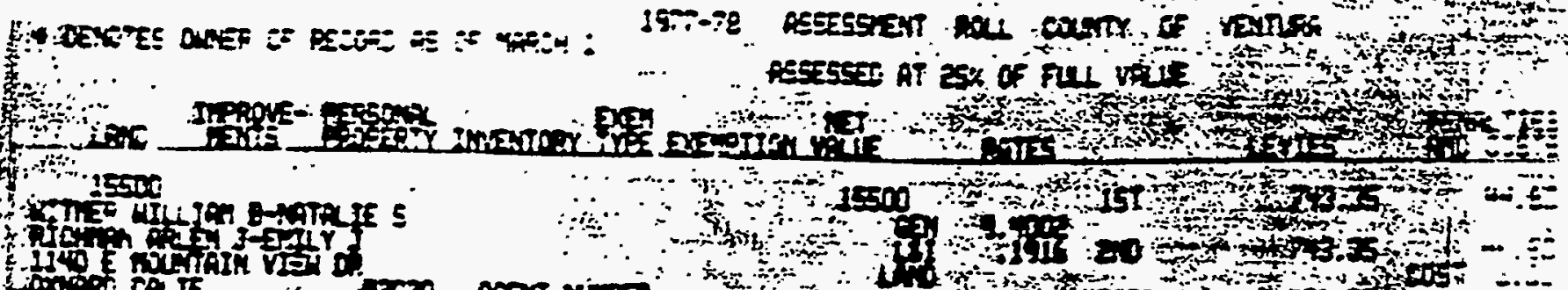
Il

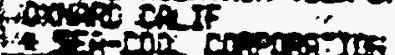

rc30 acar vis:

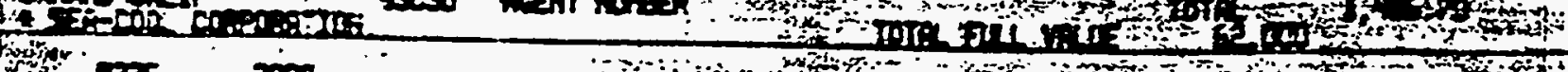

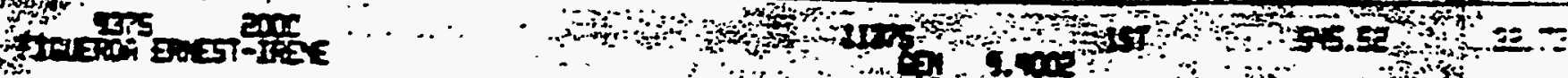

ire ExThate 5 :

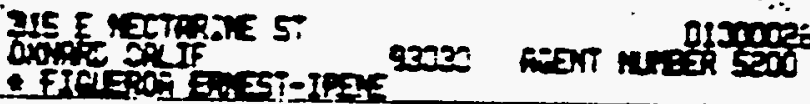

12750

Ixpes

of

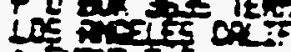

gote: navi MrajR

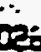

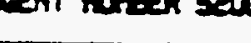

and

verer

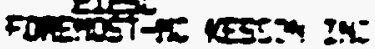

ore PS: 5

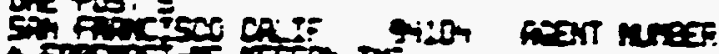

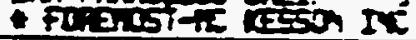
$x=20 \quad 312 x$

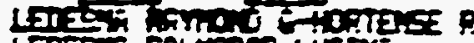

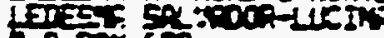

Po pox seg

orito calf

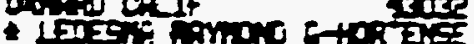

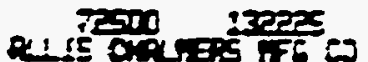

P. C. DOS 5E

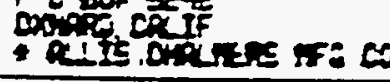

$3.4=00$

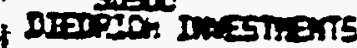

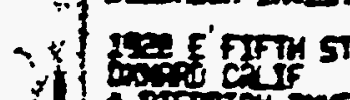

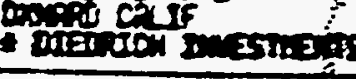

oxisich If

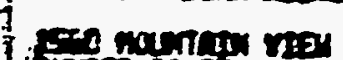

1)

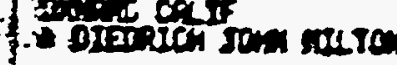

Exice

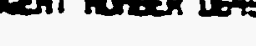

27450 In 138225 427450

臂 9.4009

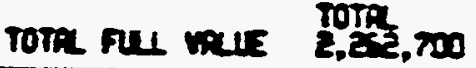

3050

Tota Fu vole : Tota

21203

ty

rom fri vive ToTh, 500

5750

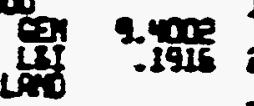

$157 \quad 2,75.63$

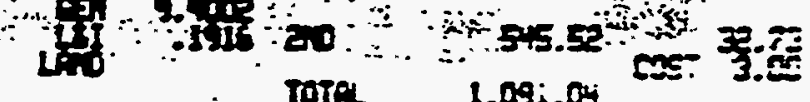
rore nut wis rota

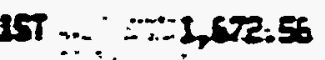

10

(1)

67.56

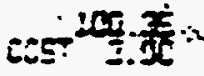

3.345.i2

1,019.12 B:..ं9

1.019 .12

2.032.24

Eis: $=\div$

2,75.63 co5.

$\lim _{x \rightarrow 10} 5,515.2$

$20, \mathrm{men}^{2}$

$20,326.50$

$1, \ldots .-3.20$

20

20.205

$=-\frac{1}{2}+217 \cdot \frac{25}{30}$

$151: \quad 1,459.59$

87.50 58

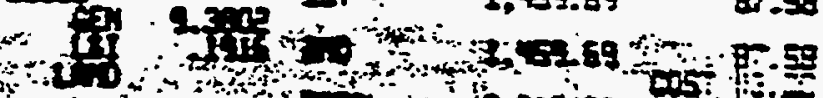

200 con neds

Wre. 3759

790 Them

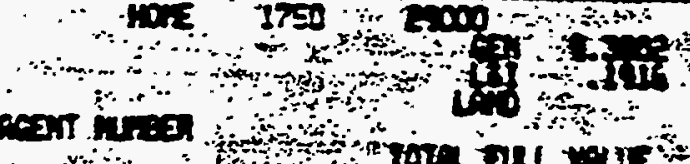

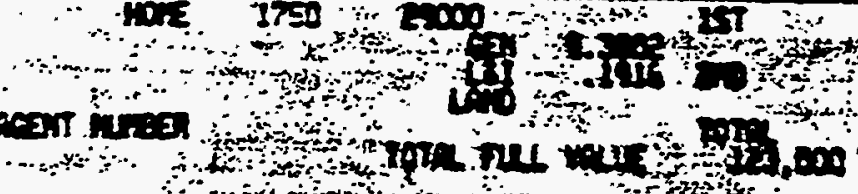

$\therefore$.

ing

$1,325 \times 10.30$

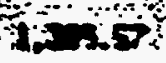

$\because 527$

2,7214

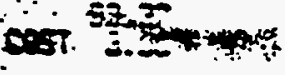

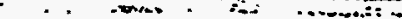

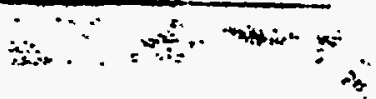

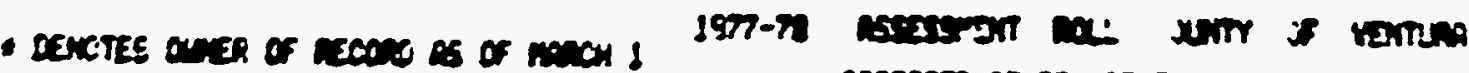

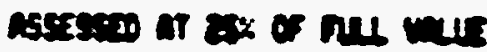

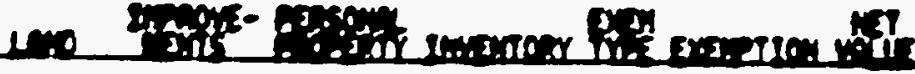

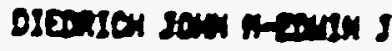

ISen menare vere

. 9.20

ivis

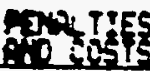




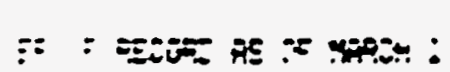

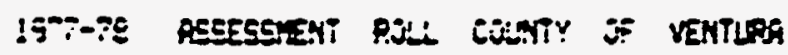

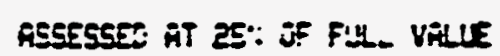

:

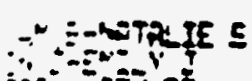

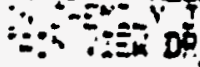

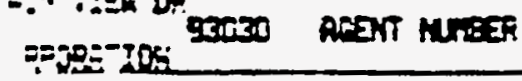

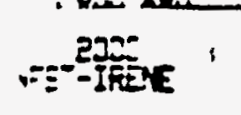

NE E-

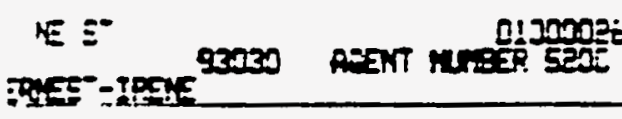

15000 Litres

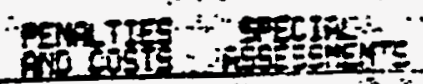

\begin{tabular}{|c|c|c|c|c|c|c|c|}
\hline 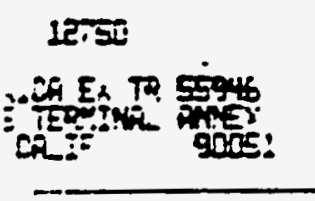 & part Muzes? & 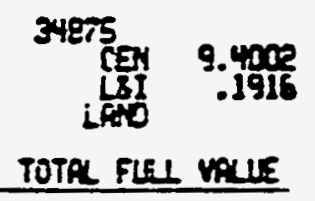 & $\begin{array}{l}15 \\
200 \\
1079 \\
139,500\end{array}$ & $\begin{array}{l}1,672.55 \\
1,672.50 \\
3,35.12\end{array}$ & $\begin{array}{r}100.35 \\
\cos ^{102 .} \frac{}{3.00}\end{array}$ & & 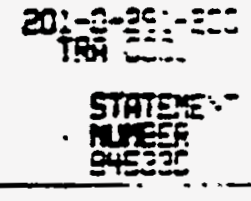 \\
\hline 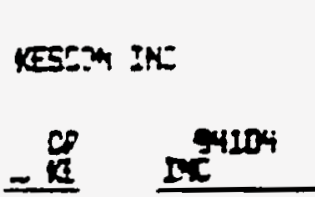 & AE्ET Mreax & 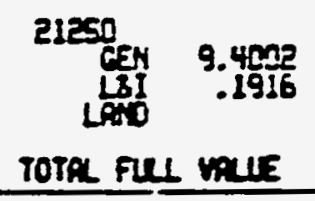 & $\begin{array}{l}\text { IST } \\
\text { aD } \\
\text { TOTR, } 100\end{array}$ & $\begin{array}{l}3,019.12 \\
1,019.12 \\
2,112.29\end{array}$ & $\begin{array}{r}61.14 \\
\cos \frac{61.14}{3.00}\end{array}$ & & 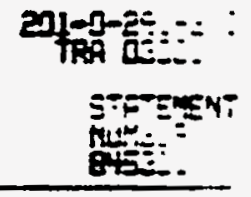 \\
\hline 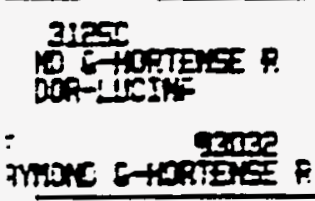 & 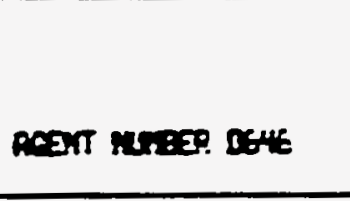 & 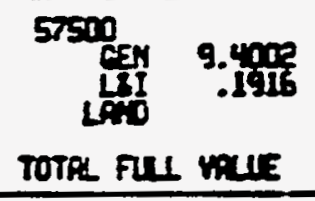 & $\begin{array}{l}15 T \\
20 \\
\text { rorge } \\
20,000\end{array}$ & $\begin{array}{l}.2,75.13 \\
.2,79.13 \\
5,515.36\end{array}$ & $\begin{array}{r}165.45 \\
\cos 15.5 .45\end{array}$ & & 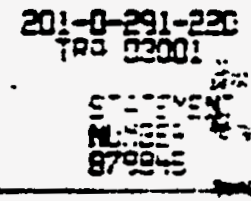 \\
\hline 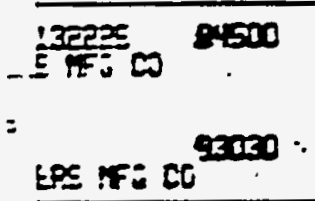 & 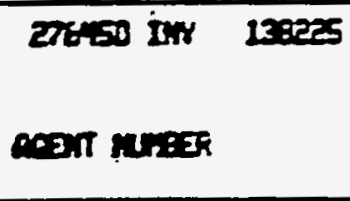 & 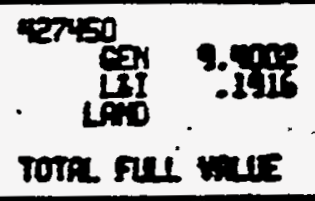 & $\begin{array}{l}15 T \\
201 \\
\text { Torte. }\end{array}$ & 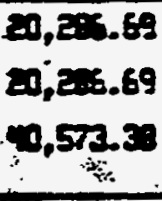 & 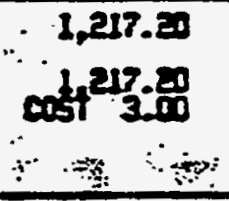 & $\begin{array}{c}\cdots \\
\cdots\end{array}$ & 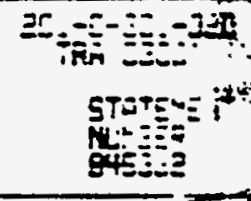 \\
\hline
\end{tabular}
3000 \%

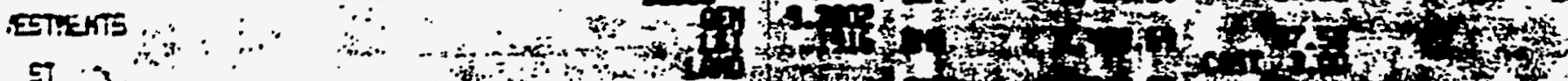

ET $\cdots$.

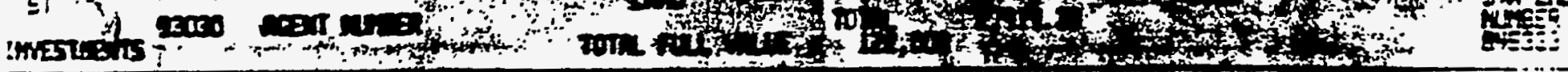

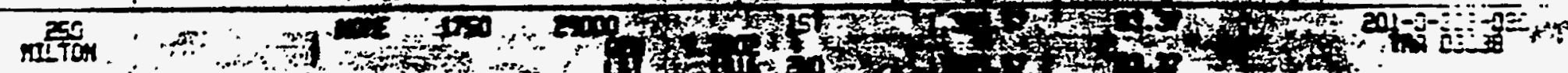
In viEx …

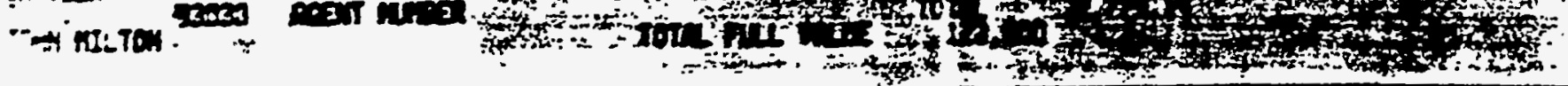

STaTE:-

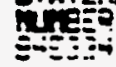
-

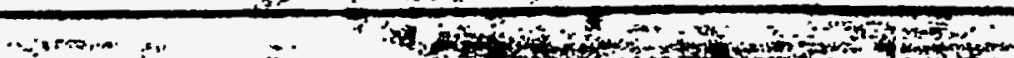
9

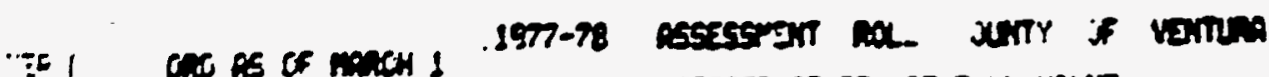
Pane assesesd at $2 \%$ of nul hats

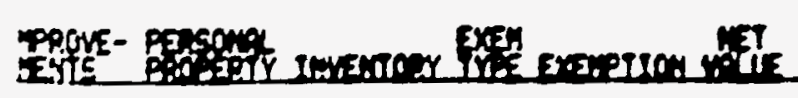
mares ISYIES Aived

a-Eming $\mathrm{J}$ art.

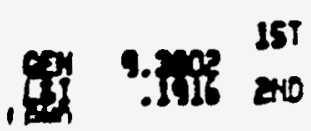




\subsection{PRIOR/CURRENT PROCESSES}

\subsection{Historical Production Processes}

Prior to the forging operations by Precision Forge or the DOE, the site was used as farm land until 1949. Allis-Chalmers, a farm implement manufacturing company, purchased the property in 1949 and built a plant consisting of 6 buildings. Production activities included founding (foundry casting), forging, machining, welding, cutting, sanding/grinding and painting/coating. These activities resulted in the manufacture, assembly, and testing of non-motorized farm implements (plows, discs, harrows, bailers, cutters, rakes). The area now used as an exercise track was used by Allis Chalmers as the testing track for the farm equipment. A historical concern is a suspect pit that existed in the area of Building \#6. This area allegedly received sand casts, slag and other debris during the Allis-Chalmers tenure. While drilling for geotechnical testing before the construction of Building \#6, Buena Engineers, Inc. recorded fill material containing foundry operation debris in the upper five feet of the core.

\subsection{Current Basic Production Process}

The Oxnard facility is a nonferrous metalworking (SIC 3462 and 3463 ) (forging, machining, welding, cutting, grinding, Wheelabrating and painting) facility. Historically, Oxnard facility provided metalworking in stainless steel, titanium, aluminum and copper alloys. Presently, all metal working is with stainiess steel, tantalum, molybdenum, and tungsten. The basic process involves the following:

- $\quad$ Cut stainless steel bars into specific lengths called billets.

- Grind and sand the billets to deburr the ends

- Wheelabrate to polish

- Coat with a dye lubricant-graphite lubricant or Delta glaze

- Heat with a furnace and forge by high energy rate forging hammers

- Water quench

- Wheelabrate and coat

- Re-forge/water quench

- Inspect 
- Grind, sand, and wheelabrate

- Saw forgings/inspect

- $\quad$ Trim press

- Grind, sand or wheelabrate

- Machine

- Pull and test (metallurgical lab)

- Mark per customer finish drawings

- Package/ship

\subsection{Current Building Descriptions and Specific Functions}

The Oxnard facility occupies 13.75 acres in an industrially zoned park (M-2 warehouse/industrial). The facility possesses seven buildings housing a total of approximately 86,000 square feet of floor space and approximately 14 million dollars in capital equipment (un-depreciated). Figure 4-1 is a sketch of the seven buildings and their respective areas. The specific building functions are as follows:

- Administration Offices (Building \#1)

The Administration Offices house technical and administrative staff (accounting and purchasing), a conference room, human resources services, and reception area. Hazardous substances and wastes are neither stored, manufactured, processed, or otherwise used in the building. The building may contain asbestos containing materials (ACM), including: vinyl asbestos tile/mastic, heating ventilation and air conditioning (HVAC) wrapping, roof tiles, and ACM concrete walls in the plant vault.

- Machine Shop (Building \#2)

Building \#2, the largest building, houses shipping and receiving, the production, planning, and control office, the 90-day hazardous waste generator accumulation area, the maintenance office, stock room, electrical maintenance room, lunch area, storage area, QA office, machining floor, metallurgical laboratory, and engineering/computer assisted drafting. Although not housed in Building \#2, the loading dock and fabrication shop are attached to the west side of the building. The building may contain ACM, including: vinyl asbestos tile/mastic, HVAC wrapping, and roof tiles. 


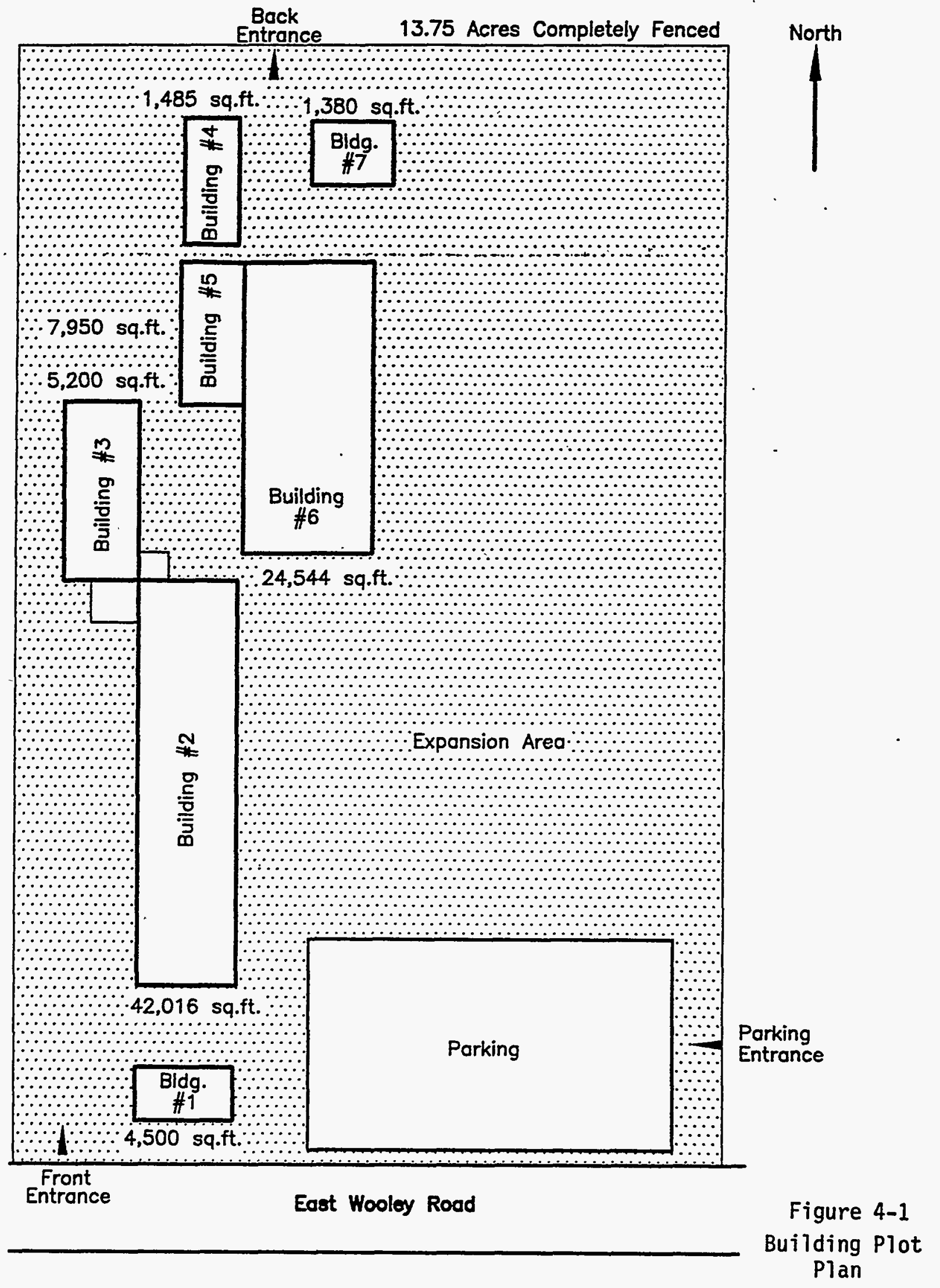


The 90-day generator accumulation area is operated under a VCDEH Waste Producer Permit to Operate issued February 1989 and in accordance with 40 CFR 262.34. The area is curbed and fenced and stores acids from the metallurgical lab, spent synthetic coolants/ lubricating oils from the machine shop, grinding shop cyclone fines, Wheelabrator baghouse fines, and various aerosol paint cans and paint wastes from several plant-wide locations. Waste is shipped off-site to Rollins Environmental, Deer Park, Texas, the Oil Process Co. (OPC), Los Angeles, California, a subsidiary of Rollins Environmental, and Petroleum Recycling Corporation in Signal Hill, California. The staging area for offsite pick-up is the south end of the loading dock. No known spills have been recorded in this area.

The fabrication shop contains a parts washer that uses a water-based detergent called Simple Green ${ }^{\mathrm{T}}$. The shop previously contained a cold solvent degreasing tank that was owned by the Oxnard facility and serviced (drained and refilled periodically) by Safety Kleen of California. The solvent used by Safety Kleen is believed to have been a Stoddard agent. This tank has been removed from service due to a leak and has been disposed appropriately off-site. The flooring immediately beneath the tank showed no sign of staining.

Used oil filters are drained of free flowing oil and then stored in 55-gallon drums located along the west outside wall of the fabrication shop. These filters are sent to Petroleum Recycling Corporation (PRC), Signal Hill, California, for recycling. Historically, Oxnard has also used a company called Black Gold (owner and location unknown). The area where these drums are stored shows no signs of staining; however, the drums were showing signs of deterioration.

The metallurgical lab uses various acids (1-2 liters per month of acetic, nitric, hydrochloric and hydrofluoric) for destructive testing (acid etching of fabricated metal components). Acids were historically sent to the sink drain, which contained an inline calcium carbonate neutralization system upstream of the sewer outfall. This elementary neutralization process was discontinued due to deterioration of the wastewater transfer lines. (Transfer lines were believed to be cast iron.) Known leaks have been recorded in the area surrounding the deteriorated lines.

The machining floor contains various lathes, drill presses, milling and tooling machines, and an optical fabrication inspection station. Many of these machines use two chemical substances that result in two waste streams. These chemical substances include Mobil lubricating oil and a synthetic coolant (hexylene glycol). Minor leaks and preventative maintenance on the machining equipment result in spent lubricating oils which are collected and sent to the 90-day generator accumulation area for off-site disposal. Spent synthetic coolant, generated when machine 
coolant becomes biologically contaminated, is drained into an appropriate storage container and sent to the 90-day generator accumulation area for off-site disposal. The machining floor showed no signs of spills or leaks. Additionally, machining and tooling generates metal cuttings. These cuttings are collected into recycling bins that sit on the loading dock ramp immediately west of Building \#2. Historically, Weston reported that these cuttings were coated with soluble cutting oils which appeared to collect at the bottom of the bins and leaked onto the cement ramp below. Immediately west of this ramp, opposite bay door \#13, is a storm water drain that services this area, the fabrication shop, and the area immediately north. During the Phase I tour a slag deposit was observed approximately 100 feet north of drain.

- Saw Shop (Building \#3)

The saw shop houses various band and utility saws used to saw off billets for forging and machining. This building contains a five-ton overhead bridge crane to transport raw materials between the steel stockyard and the shop. Historically, Building \#3 housed a 110-gallon capacity tank and three 55-gallon drums of 111-trichloroethane (TCA) for die cleaning. Used or spent TCA was placed into 55-gallon drums, stored in the 90-day generator accumulation area and sent to an off-site recycler. The Weston report states that the tank area had no secondary containment. This report further states that the tank and drums were removed in August 1989. This area was investigated during Phase $I$ and showed no signs of spillage.

- Grinding Shop (Building \#4)

The grinding shop houses numerous grinding stations with a vacuum collection system. This collection system is fitted with an air abatement particle separator (cyclone). This unit generates a very fine metal dust, which is collected in a drum beneath the cyclone. This metallic dust is a hazardous waste due to the presence of chromium (EPA DO07). The dust, following containerization, is stored at the 90-day generator accumulation area and sent to Rollins for off-site solidification/stabilization and land disposal. Signs of metallic dust (i.e., presence of fines) were noted along the south outside wall of Building \#4.

- Press Shop (Building \#5)

The press shop houses a 1600-ton mechanical press, a 3500-ton hydraulic press, a trim press, and three natural gas-fired furnaces. Beneath each press, is a press cellar pit in which oils (either leaked Shell Tellus-100 or Tellus-46) and metal fines accumulate. Spent oils from the 1600-ton press are containerized and stored in the 90-day generator 
accumulation area for off-site recycle. Spilled oils beneath the 1600-ton press are pumped out and sent to the oily wastewater treatment plant located at the tank farm and lube stores (Building 7). Oils in the 3500ton press are self-filtered. The filters are drained, containerized, and sent to the west wall of the saw shop for off-site recycle with PRC. Oils from the pit beneath this press are vacuum pumped and sent to the oily wastewater treatment plant. Because of the age and condition of the pits, no attempt was made to determine pit integrity. These pits and the soil interface beneath warrant further investigation. Design and as-built drawings for these press pits are found in Appendix $E$.

Located on the west side of the press shop is the Wheelabrator shop which houses four Wheelabrators. The Wheelabrators are enclosed rotating drums with small metallic balls used to polish metal components. Operationally, these machines create metallic fines that must be captured and properly disposed. The air emission/abatement system consists of two baghouse structures and stacks, each servicing two Wheelabrators. The baghouse dust (EPA DO07) is collected in drum containers and sent to the 90-day generator accumulation area prior to off-site solidification/stabilization by Rollins Environmental. Bags are replaced when breakthrough occurs and are containerized for off-site disposal. Signs of metallic dust (i.e. presence of fines) were noted along the south outside wall of Building \#4.

The dye penetrant and delta glaze room is also located on the west side of the press shop. The dye penetrant is used in quality assurance while the delta glaze is a lubricant coating applied prior to forging.

- Forge Shop (Building \#6)

The forge shop houses 6 High Energy Rate Forging (HERF) hammers with $40,000 \mathrm{ft}$. lb. maximum energy, 3 HERF hammers with 100,000 ft. lb. maximum energy rating, 4 HERF hammers with $225,000 \mathrm{ft}$. lb. maximum energy rating and $3 \mathrm{HERF}$ hammers with $300,000 \mathrm{ft}$. $\mathrm{lb}$. maximum energy rating. Additionally, 13 natural gas fired furnaces and 5 mechanical trim presses are housed. The cellars of each hammer and press take the form of a large underground concrete collection trench that slopes north. This trench collects leaking oils from the large presses and is also the repository for the quench water from Buildings \#5 and \#6. At the northern end, a sump pump is used to vacuum pump oily water to the oily wastewater treatment system located at the tank farm and Building \#7.

An investigation of the trench was conducted and photos were taken; however, due to the oily conditions within the trench, neither the integrity nor the construction of the trench could be ascertained. Review of the as-built drawings indicate the floor slab is 2-feet thick concrete, 
the side walls are 3-feet 7-inches thick concrete and the walls and floor are reinforced with \#5 rebar at 1-foot centers. The drawing showed no expansion joints and specifications required water-proofing of all side walls and floor. Design and as-built drawings for Buildings 5 and 6 are found in Appendix $E$.

- Tank Farm and Lubrication Stores

The tank farm is located north of Building 6. The tank farm consists of five tanks including:

Tank A

- $\quad$ Pit effluent tank (oil and water)

- $\quad$ Capacity $=10,000$ gallons

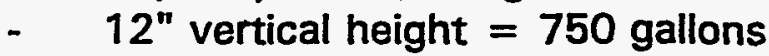

Tank B

- Back-up Pit effluent tank (oil and water)

- $\quad$ Capacity $=1,500$ gallons

- $12^{\prime \prime}$ vertical height $=150$ gallons

- Tank not in service

Tank C

- Dirty oil tank

- $\quad$ Capacity $=2,000$ gallons

- 12 " vertical height $=200$ gallons

Tank D

- Cleaned oil tank

- Capacity $=3,000$ gallons

- $12 "$ vertical height $=300$ gallons

Tank E

- Steam cleaner water tank (reusable)

- Capacity $=5,000$ gallons

- $12^{\prime \prime}$ vertical height $=200$ gallons

Hydraulic fluids are collected from the press pits in Building \#5 and the trench in Building \#6 and pumped to the tank farm (Tank A) for initial treatment (gravity separation). Following separation dirty oil is pumped to Tank $C$ for Treatment in Building \# 7 by cartridge filtration. The dirty water is sent to Tank $E$ along with steam cleaning waters for treatment in Building \#7 by ultra membrane filtration. The concentrate generated by ultra filtration is sent to the water "eater" evaporator for evaporation of free liquids under a DTSC Permit by Rule. The water is ultimately collected in two polyethylene tanks, tested and batch-released to the POTW. 
The tank farm is built on a concrete slab surrounded by a 3-foot high concrete berm for secondary containment. Previous consultant reports indicate no tank spills have been recorded or reported. Rainwater collects in the containment; however, its management was not determined. Historically, the western wall of the containment berm leaked at the wall/slab interface and was replaced and sealed in 1989.

Building \#7 contains the oil and wastewater filtration systems. These oil and water treatment systems receive oil from Tank $C$ and water from Tank $E$, respectively, through temporary flex hosing. Discussions with the operator indicate that no recorded or reported spills have occurred during hook-up, fluid transfer or detachment of the hosing, nor have any spills or overflow occurred at the treatment facilities located in Building \#7.

- Stockyard

The stockyard is located north of the saw shop near the western boundary of the site. The stockyard is an open air, concrete-paved storage facility that is used for the reception and storage of raw metal (stainless steel bars). No dry or liquid chemicals have been stored in this yard. During the Phase I tour, the concrete was observed to be ruststained in locations surrounding the legs of the five-ton overhead bridge crane and the billet storage racks. The concrete was in excellent condition showing no signs of cracking, etching or spalling.

- Boneyard

A fenced, unpaved boneyard exists in the northwest corner of the plant site. During the site visits, the boneyard contained numerous steel and poly tanks. All tanks were observed to be empty. Additionally, various pieces of scrap metal, old machinery and various assorted debris were being stored. Much of the old machinery was contaminated with oily residue and/or sludge. During the $\mathrm{PE}$, several areas of petroleum or discolored soils were observed, even though the yard had overgrown with weeds and grass. During the Phase 1 , it was observed that the yard had been bladed and that all vegetation had been removed. The determination of a soil herbicide application could not be verified. According to DOE-furnished documentation, the yard historically stored polychlorinated biphenyl containers or articles. Details of the storage could not be found in the plant's records.

Finally, the asphalt road leading to the boneyard was originally a dirt road that may have been periodically oiled for dust control. This yard and surrounding area warrants further investigation. 


\subsection{REGULATORY AGENCY INFORMATION}

\subsection{Listing of Agencies/Properties of Concern}

Environmental regulatory agencies with jurisdiction over the Oxnard facility were visited to determine and identify legally mandated permits, compliance violations, solid and hazardous material/waste inventories and active/closed corrective actions. The following agencies were visited to interview key personnel (permitting and inspection/enforcement) and review/copy records:

- City of Oxnard - Source Control Program 6001 Perkins Road Oxnard, CA 93033 Attention: Sally Mechuzak - File Search/Permitting

- Ventura County Air Pollution Control District (VCAPCD) 702 County Square Drive Ventura, CA 93030 Attention: Sue Megoalik - File Search

Peter Lawsón - Permits Dan Searcy - Inspection

- Ventura County Department of Environmental Health (VCDEH) Hazardous Waste \& UST. 800 South Victoria Avenue Venture, CA 93009

Attention: Heather Ebersole - File Search

Craig Cooper - Inspection

Greg Smith - EH Supervisor

- California Environmental Protection Agency

Department of Toxic Substances Control (DTSC)

Region 3 - Burbank 1011 North Grand Avenue Glendale, CA 91201

Attention: Brenda Alley - File Search Scott Simpson - Permitting Jim McCamon - Permitting

The Regional Water Quality Control Board refused to be interviewed stating that no files existed for the properties of concern. The agency further noted that they have no interest in the Oxnard facility unless groundwater contamination is discovered during Phase II of the ESA. Each agency was interviewed for information and records pertaining to the following properties: 
- EG\&G Rocky Flats Oxnard Facility

1235 East Wooley Road

Oxnard, CA 93030-7330

(805) $485-4881$

- Kingstone Wheel Corporation

(formerly Del Manufacturing)

950 Richmond Avenue

Oxnard, CA 93030-7330

(805) 486-2601

- City of Oxnard, Vehicle Maintenance Facility

1060 Pacific Avenue

Oxnard, CA

(805) 385-8051

- Gold Coast Steel

(formerly J\&S Engineering)

1140 Mountain View Avenue

Oxnard, CA

(805) 483-1560

- Deardorff-Jackson Company

1120 Mountain View Avenue

Oxnard, CA

(805) 487-7801

- Told Corporation

1701 Pacific Avenue

Oxnard, CA

(805) $487-4300$

This section is subdivided by property. Information existed for each property with the exception of Told Corporation and Boskovich Corp.

\subsection{EG\&G Rocky Flats Oxnard Facility}

\subsubsection{Solid/Hazardous Waste}

The Oxnard facility is a hazardous waste generator (CA9890090401) operating under the 90-day generator accumulation rule. The facility applied for and was granted a Hazardous Waste Producer Permit to Operate \#472 by the VCDEH. Under this permit, the facility generates, accumulates and manifests for off-site disposal or recycle the following identified streams: 
Waste Stream/Classification

- Waste Acids (hydrochloric, nitric, acetic) Waste Acid Liquid, N.O.S., Corrosive Material NA1760 CA Waste \#792, EPA Waste \#D002

- Spent Coolant Hazardous Waste Liquid, N.O.S., ORM-E NA9189 CA Waste \#132, EPA Waste \#D007

- Wheelabrator-Bag House.Dust Grinding shop cyclone fines Hazardous Waste Solid, N.O.S., ORM-E NA9189 CA Waste \#172, EPA Waste \#D007

- Oil (used) Hazardous Waste Liquid, N.O.S., ORM-E NA9189 CA Waste \#221

- Wastewater Evaporative Concentrate Hazardous Waste Liquid, N.O.S., ORM-E NA9189 CA Waste \#223

- Press Pit Sludge Hazardous Waste Liquid, N.O.S., ORM-E NA9189 CA Waste \#223, EPA Waste \#D007, D008

- Paint Debris Hazardous Waste Liquid, N.O.S., ORM-E NA9189 CA Waste \#352, EPA Waste \#D007, D008

- Paint Rinsate Hazardous Waste Liquid, N.O.S., ORM-E NA9189 CA Waste \#134, EPA Waste \#D007, D008

- Press Pit Oily Water

- Used Oil Filters

- Spray Cans Compressed Gas, N.O.S. Flammable Gas UN1954 CA Waste \#331, EPA Waste \#D001
Management

Neutralization.

Rollins Environmental

Incineration.

Rollins Environmental

Solidification/ stabilization

Rollins Environmental

Recycled

Petroleum Recycling Corporation .

Incineration. Rollins Environmental

Incineration

Rollins Environmental

Incineration

Rollins Environmental

Incineration

Rollins Environmental

Oily water treatment system

Oxnard Facility

Recycled

Petroleum Recycling

Corporation

Incineration

Rollins Environmental 
- Oxnard Recycling

- Eddie Recycling

- Standard Industries (carbon steel)

The DTSC authorized the Oxnard facility to operate the water "eater" -. evaporator, a fixed-treatment unit under the Permit by. Rule (PBR) (Title 22 CCR 67450.2(b)(5)). This unit removes water from the concentrate generated by the waste water ultra membrane filtration unit. Furthermore, DTSC approved Oxnard's conditional exemption for the treatment of empty containers $<110$ gallons capacity (HSC25200.3 and 25201.5).

In discussions with DTSC, it appears that the facility submitted an Application for Hazardous Waste Facility Permit Variance on July 27, 1989, for the manual neutralization of used acid before discharge to the sanitary sewer. This application was never acted upon by the state due to the developing PBR. After discussions with the state pertaining to the facility's current operation of 90-day accumulation/ off-site disposal of waste acids, the state suggested that the facility submit a PBR application and treat waste acids on site.

Three Notices of Violation (NOV) under the Permit to Operate \#472 were issued to the facility in 1986. The NOVs included violations pertaining to hazardous waste training, storage management (signing and secondary containment) and manifesting. These violations were corrected the same year. No other hazardous waste violations have been issued by VCDEH or DTSC.

\subsubsection{Wastewater Discharge}

The City of Oxnard, Source Control Program has issued the Oxnard facility Industrial Wastewater Discharge Permit \#38C per the Oxnard City Code, Chapter 25. This permit is transferrable provided the new owner submits an updated application, an Industrial User Baseline Monitoring Report, and transfer fee of $\$ 1,500$. The permit conditions/ provisions require that the facility comply with both local pretreatment standards and federal pretreatment standards (Aluminum Forming Point Source Category, 40 CFR 467 and Non-ferrous Metal Forming Point Source Category, 40 CFR 471). All samples for the selfmonitoring reports are collected from the sampling well located east of the tank farm and downstream of the gravity and membrane filtration system. 
NOVs have been issued in 1989, 1992, 1993 and 1994. One NOV in 1989 and two in 1993 were issued for late submittal of the selfmonitoring report. In December 1992 and July 1994, NOVs were issued for exceedence of the oil/grease standard $(100 \mathrm{mg} / \mathrm{L})$. This violation is believed to have occurred due to the use of soaps/detergents. The plant has reviewed its use of soaps/detergents and has discontinued their use. This corrective action appears to have alleviated the problem. In March 1994 the plant received a NOV for failure to quarterly report total toxic organics for December 1993. This violation was closed March 9, 1994, in a letter explaining that no discharge took place in December 1993 making it impossible to test.

\subsubsection{Underground Storage Tanks (USTs)}

The Oxnard facility previously managed two underground storage containers (USC) as defined by the California Water Resources Control Board. In December 1984 the facility filed two USC registration forms with the Board. One tank was identified as a $\mathbf{5 5 0}$ gallon, single-walled, diesel tank. This tank was constructed of unlined carbon steel and had no cathodic protection. The tank piping was suction only. The second tank was identified as a 1,000 gallon, single-walled concrete vault. No information existed on lining or protection. This second tank held rinse water that might have contained a dye penetrant called Dubl Chek HM406.

Following an application for abandonment in place, the VCDEH granted a permit to abandon the $\mathbf{5 5 0}$ gallon diesel fuel tank in place. The tank was closed by the following method:

"The tank was pumped empty, rinsed three times with a soapy water solution and vacuumed dry. An inert gas test was performed on the tank at 3-5 psi and found not to be leaking. This was observed by Greg Smith of the Resource Management Agency County of Ventura. All piping was removed from the tank and the tank was pumped full of concrete on March 26, 1986." (MHLoe Co., 1986)

On November 30,1992 , the VCDEH issued the facility a letter concurring with EG\&G's position that the concrete vault did not store a hazardous material as defined by the Leaking Underground Fuel Tank (LUFT) manual and therefore requires no formal abandonment. The tank was subsequently pumped dry, rinsed, and the fill pipe cement plugged. 


\subsubsection{Air Emissions}

The VCAPCD renewed a Permit to Operate, Number 1203 , on October 1, 1993. This permit allows the facility to operate 16 natural gas fired furnaces and one solvent cleaning operation. This permit limits the natural gas consumption for all combustion equipment to 50 MMcf per year.

This permit is fully transferrable as the permit applies to the plant's equipment and its usage. Should the permit not be transferred, a new owner would need to apply for Nitrogen oxide $\left(\mathrm{NO}_{\mathbf{x}}\right)$ and Reactive Organic Compounds (ROC) emission credits. Generally ROC credits are available from the community bank if plant emissions are less than 5 tons/year. $\mathrm{NO}_{x}$ credits are extremely difficult to acquire. Cost of a permit transfer is $\$ 200.00$.

A file search and discussions with the permitting section could identify no information pertaining to the two particulate $\left(\mathrm{PM}_{10}\right)$ abatement systems (the Wheelabrator baghouse and the grinding shop cyclonic particle separator) at the site. The VCAPCD suggested that the facility needs to submit a Letter of Permit Inquiry with an attached process and abatement description.

The Oxnard facility has received one NOV, dated June 7, 1990. This NOV cites the facility for failure to operate its Walker Box Aluminum Forging Furnace (Building \#5) within the permitted operating temperature of $1000^{\circ} \mathrm{F}$. This NOV was settled out of court per Rule 29.C. This required that a plan be developed and implemented to prevent firing temperatures from exceeding $1000^{\circ} \mathrm{F}$.

\subsubsection{Hazardous Materials}

The Oxnard facility has a hazardous materials permit from the City of Oxnard authorizing the storage, use or handling of hazardous materials/waste in accordance with AB 2185-2187; California Health and Safety Code, Chapter 6.95, §25504; and SARA Title III.

The Oxnard facility has filed a revised contingency plan and California Business Plan with the Fire Department, City of Oxnard. Appendix $\dot{F}$ provides a plant illustration identifying the location of hazardous material storage areas and copies of the hazardous materials chemical descriptions. 


\subsection{Kingstone Wheel Corp. (Formerly Del Manufacturing)}

\subsubsection{Solid/Hazardous Waste}

Kingstone Wheel Corp. is a hazardous waste generator operating under the 90-day generator accumulation rule. The facility has applied for a Hazardous Waste Producer Permit to Operate. No information exists as to the waste streams presently generated.

The DTSC has no record indicating that the facility presently or under previous ownership had a permitted treatment, storage, and disposal facility (TSDF) on site. Furthermore the site was not permitted under AB 1772 for tiered permitting including PBR, conditional authorization or conditional exemption.

\subsubsection{Wastewater Discharge}

Kingstone Wheel Corp. has an industrial wastewater discharge permit issued by the City of Oxnard, Source Control Programs. NOVs were issued to the previous owner, Del Manufacturing, for contaminant exceedants.

\subsubsection{Underground Storage Tanks (USTs)}

The site contains no registered underground containers including tanks, vaults, or sumps.

\subsubsection{Air Emissions}

The VCAPCD files show that Del Manufacturing operated under two Permits to Operate, Numbers 1120 and 1121 . These permits have been withdrawn and the emission inventory banked. The original permits and permitted emission calculation sheets were not available for review.

\subsubsection{Hazardous Materials}

Kingstone appears to maintain a large liquid chemical and gas tank storage facility based upon its California Business Plan. (Note: Time restraints did not permit the review of this Plan.)

\subsection{Deardorff-Jackson, Inc.}

\subsubsection{Solid/Hazardous Waste}

Deardorff-Jackson, Inc. is a hazardous waste generator operating under the 90-day generator accumulation rule. The facility has 
applied for and was granted a Hazardous Waste Producer Permit to Operator by the VCDEH. Under this permit, the facility generates, accumulates, and manifests for off-site disposal or recycle various solvents and/or mixed oils. The facility has received numerous NOVs since 1983 for storage, improper manifesting, lack of a contingency plan, and improper disposal to ground of solvent and mixed waste oils. The DTSC has no record indicating that the facility is a TSDF or that the facility is permitted under $A B \mathbf{1 7 7 2}$ for tiered permitting including PBR, conditional authorization or conditional exemption.

\subsubsection{Wastewater Discharge}

Deardorff has an industrial wastewater discharge permit issued by the City of Oxnard, Source Control Programs. No NOVs were identified in Deardorff's wastewater discharge file.

\subsubsection{Underground Storage Tanks (USTs)}

Deardorff presently operates two underground fuel tanks. The largest tank is registered as a 12,000 gallon premium unleaded motor vehicle fuel tank. The smaller tank is registered as a 6,000 gallon regular unleaded motor vehicle fuel tank. Both tanks are single-walled, unlined, and constructed of carbon steel. Tank piping is suction only. The tanks are monitored continuously by a vadose vapor probe system. No leakage or spills have been recorded.

Additional information in the file indicates that the perched zone lies approximately 7.5 feet below ground surface and has a TDS $>1000 \mathrm{mg} / \mathrm{L}$. The nearest pumping groundwater well is Well IN/22W-3R1 owned by Oxnard Frozen Foods.

\subsubsection{Air Emissions}

Although the site owns and operates two underground fuel storage tanks and dispensing islands, the VCAPCD has no record of a permit for either the tanks or the Phase I/II vapor control systems.

\subsubsection{Hazardous Materials}

No information existed in the VCDEH on Deardorff's Hazardous Materials Registration or California Business Plan.

\subsection{Boskovich Corp.}

No files existed at VCDEH, DTSC, the City of Oxnard or VCAPCD on this property. 


\subsection{City of Oxnard Vehicle Maintenance Facility}

\subsubsection{Solid/Hazardous Waste}

The City of Oxnard Vehicle Maintenance Facility (VMF) is a hazardous waste generator operating under the 90-day generator accumulation rule. VMF has applied for and was granted a Hazardous Waste Producer Permit to Operate. Under this permit, VMF generates, accumulates, and manifests for off-site disposal or recycle various solvents, paint wastes, and mixed oils. The DTSC has no record indicating that the facility is a TSDF or that the facility is permitted under $A B 1772$ for tiered permitting PBR, conditional authorization or conditional exemption.

The VCDEH has issued numerous notices of violations since 1990 citing inappropriate or lack of drum labelling, no contingency plan, no hazardous waste training program, no solvent/oil segregation, release of solvents and oils to the ground, and inadequate roofing for the 90-day accumulation area.

\subsubsection{Wastewater Discharge}

VMF has an industrial wastewater discharge permit issued by the City of Oxnard, Source Control Programs. NOV information could not be ascertained.

\subsubsection{Underground Storage Tanks (USTs)}

The City of Oxnard VMF operates four underground fuel tanks. These include a 12,000 gallon regular unleaded gasoline tank, a 10,000 gallon regular unleaded gasoline tank, a 5,000 gallon diesel fuel tank, and a $\mathbf{5 5 0}$ gallon waste oil tank. The three fuel tanks are singlewalled, unlined, and constructed of carbon steel. Corrosion protection varies from no protection for the 5,000 gallon diesel to fiberglass reinforced plastic lining for the 12,000 gallon gasoline tank and cathodic protection for the 10,000 gallon gasoline tank. All fuel tanks are monitored by a vadose zone probe system. In 1994 the VCDEH issued a warning notice to the city stating that the vadose zone probe system was inadequate as a vapor monitoring system. The warning further stated that the system was designed for direct liquid contact which would dissolve the wire insulation on the detector subsequently activating an audible and visual alarm. The $\mathrm{VCDEH}$ requested that the system be replaced or upgraded.

The $\mathbf{5 5 0}$ gallon used oil tank is single-walled, unlined, and constructed of carbon steel. The tank has no corrosion protection and monitoring is by tank inventory. 
In 1993 the piping to one of the gasoline tanks failed. The City of Oxnard hired Fugro-McClellen to investigate and remediate the spill. Records indicate that the line was repaired and that contaminated soils (approximately $700 \mathrm{yd}^{3}$ ) are being bioremediated. Groundwater sampling (7 quarters) indicate that total petroleum hydrocarbons (TPH), benzene, ethyl benzene, toluene, xylene (BETX), and total lead are below detectable levels. The consultant has determined that the leak did not impact either the perch zone or the deep water aquifer at 120 feet below ground surface.

\subsubsection{Air Emissions}

The City of Oxnard VMF has two air emission permits to operate. Permit $\mathbf{5 5 9 8}$ authorizes VMF to operate two underground gasoline storage tanks and Phase I/II vapor control systems. Permitted emissions include reactive organic compounds at a rate not to exceed .27 tons/year. Permit 0855 authorizes VMF to operate an automobile refinishing and metal parts cutting facility consisting of a Bleekers Brothers spray booth with low pressure or electrostatic spray guns. Permitted emissions include reactive organic compounds at a rate not to exceed 3.19 tons/year.

\subsubsection{Hazardous Materials}

No information existed at VCDEH on VMF's hazardous materials registration or California Business Plan. Information gleaned from other agencies indicate that the City stores considerable amounts of paints, solvents, cleaners, fuels, oils and lubricants.

\subsection{Gold Coast Steel (formerly J\&S Engineering)}

Gold Coast Steel has recently (within the last 2 months) purchased the property previously operated by J\&S Engineering. Prior to purchase the property was vacant for approximately six months. No records exist on Gold Coast Steel at any of the regulatory agencies interviewed. The following represents information on J\&S Engineering.

\subsubsection{Solid/Hazardous Waste}

Although it appears that J\&S Engineering was a generator of solid/hazardous waste, no records exist at the VCDEH or DTSC that would indicate J\&S Engineering as either a generator of solid/ hazardous waste or as a TSDF. 


\subsubsection{Wastewater Discharge}

Although it appears that J\&S Engineering discharged industrial wastewaters to the City of Oxnard, no records exist indicating submittal of either an industrial user baseline monitoring report or an application for an industrial wastewater discharge permit.

\subsubsection{Underground Storage Tanks (USTs)}

J\&S Engineering operated a 1,000 gallon diesel tank. The tank was manufactured in 1960 by Buehler Tank and Welding and was singlewalled, carbon steel constructed. The tank was unlined and had no corrosion protection. In August and September 1987, the tank was excavated and removed from service. The condition of the tank was identified as extremely poor with numerous holes. J\&S Engineering contracted Holquin \& Associates, a geotechnical consultant, to remediate the fuel spill. Remediation included sampling and analysis of the surrounding soils and shallow groundwater. Approximately $150 \mathrm{yd}^{3}$ of contaminated soils $(\approx 38 \mathrm{ppm}$ on a gasoline fingerprint analysis) were excavated and windrowed under the approval of the VCDEH and VCAPCD. Cleanup was to $<10 \mathrm{ppm}$ gasoline and $<100 \mathrm{ppm}$ diesel per the LUFT manual. Following aeration, the soil was replaced into the hole, clean fill was added to bring the level to grade and the soil compacted to $90 \%$ compaction. No groundwater remediation was necessary as sampling identified no detectable concentrations of TPH, BETX and the soluble lead concentration was $5 \mathrm{x}<$ the maximum contaminant level (MCL) for lead.

The VCDEH issued a letter dated July 6, 1989, indicating that all appropriate response actions have been completed and that the spill was considered fully remediated.

\subsubsection{Air Emissions}

Although J\&S Engineering previously owned and operated an underground fuel storage tank and dispensing island, the VCAPCD has no record of a permit for either the tank or the Phase I/II vapor control system.

\subsubsection{Hazardous Materials}

No information existed at the VCDEH indicating that J\&S Engineering had made an application for hazardous materials registration or that a California Business Plan had been developed and submitted per AB2185-87. 


\subsection{SUMMARY OF FINDINGS}

\subsection{Geology/Hydrogeology}

The Oxnard facility lies over unconsolidated sediments of the Oxnard Plain which consists of sands, silts, and clays. A shallow perched groundwater zone is encountered at a depth of 5 to 10 feet; groundwater flow direction is to the southwest. Water from this shallow zone is not used for any purpose. At a depth of approximately $120 \mathrm{ft}$., lies a potable aquifer that is separated from the shallow perched zone by clay units.

\subsection{Off-Site Influences}

Data gathering efforts from regulatory agencies were conducted to obtain information on surrounding properties and to determine potential environmental impacts on the Oxnard facility from off-site sources. This review of regulatory agency records showed that remedial activities in response to leaking USTs were conducted at two adjacent properties. Remediation at the Gold Coast Steel property has been completed, and remediation at the City of Oxnard Maintenance Yard is in progress. Records also indicated that degradation of the shallow groundwater system under these sites did not occur. There were no other documented environmental problems at the surrounding properties.

\subsection{On-Site Areas of Concern}

"Areas of concern" are locations at the facility that have documented historical contamination, suspected historical contamination, or a potential for contaminant release from historical or current operations. Areas of concern are summarized in Table 6-1 
Table 6-1. Oxnard Facility - Areas of Concern

\begin{tabular}{|c|c|c|}
\hline Area of Concern & Location & $\begin{array}{c}\text { Potential } \\
\text { Contaminants }\end{array}$ \\
\hline Allis-Chalmers pit & Beneath Building 6 & Metals, unknowns \\
\hline $\begin{array}{l}\text { Trench/sump and oil } \\
\text { collection system }\end{array}$ & Building 6 & Metals, hydraulic oil, PCBs \\
\hline Bioremediation area & East of Building 6 & Hydraulic oil, PCBs \\
\hline PCB soil area & East of Building 6 & PCBs \\
\hline PCE soil gas area & East side of facility & PCE \\
\hline PCE soil gas area & North side of facility & PCE \\
\hline Oil collection system & Building 5 & Metals, hydraulic oil, PCBs \\
\hline Tank farm & North of Building 6 & Metals, hydraulic oil, PCBs \\
\hline Abandoned diesel UST & $\begin{array}{c}\text { Outside of south side } \\
\text { of Building } 4\end{array}$ & Diesel fuel \\
\hline PCB soil area & East of the boneyard & PCBs \\
\hline Boneyard & $\begin{array}{l}\text { Northwest corner of the } \\
\text { facility }\end{array}$ & PCBs, metals \\
\hline Dirt road & $\begin{array}{c}\text { West side of facility and } \\
\text { east of the boneyard }\end{array}$ & Misc. oils, PCBs \\
\hline $\begin{array}{c}\text { Former TCA above ground } \\
\text { storage tank (AGST) }\end{array}$ & $\begin{array}{c}\text { Southwest corner of } \\
\text { Building } 3 \\
\end{array}$ & TCA \\
\hline Metal cuttings in recycling bins & West side of Building 2 & Metals, misc. oils \\
\hline Used oil filter storage area & West side of Building 2 & Metals, misc. oils \\
\hline Metal slag on ground & $\begin{array}{l}\text { West of dock next to } \\
\text { Building } 2\end{array}$ & Metals \\
\hline Loading dock & $\begin{array}{c}\text { Outside of Southwest } \\
\text { corner of Building } 2 \\
\end{array}$ & $\begin{array}{c}\text { Organics, hydraulic oils, } \\
\text { unknowns }\end{array}$ \\
\hline Metallurgical laboratory & South portion of Building 2 & Metals \\
\hline Cyclone and baghouse & Buildings 4 and 5 & Metals \\
\hline HVAC duct work & Building 1 & Asbestos \\
\hline Vault area & Building 1 & Asbestos \\
\hline
\end{tabular}




\subsubsection{Basis for Areas of Concern}

Following is a description of the evidence that was used to select the areas of concern listed in Table 6-1.

- Allis-Chalmers Pit Area

A 1973 aerial photograph (Appendix D) of the site shows a dark ring on the east side of Building 5 in the vicinity where the Allis-Chalmers Pit was located.

In 1982 Buena Engineers drilled three borings related to the foundation work during the construction of Building 6. Trash and debris were noted in the top 5 feet of boring No. 1and are believed to be part of a foundry cast disposal pit used by Allis-Chalmers.

A 1988 interoffice memorandum from the Roy F. Weston Company notes that a former pit/dump area was observed east of Building 5 in the vicinity of the current Building 6 . This area was noted to have received sand casts prior to the construction of Building 6 .

- Trench/Sump and Oil Collection System in Building 6

Piping, trenches, and a sump comprise a hydraulic oil collection system beneath the floor of Building 6 . Fluid is drained toward the sump where it is pumped to the tank farm for treatment. The potential for leakage from the concrete trench, concrete sump, and piping system make this an area of concern.

- Bioremediation Area

A known spill of hydraulic oil (verified by EG\&G) was removed, bioremediated, and returned to the spill site. This action was completed in April/May of 1994.

- PCB Soil Detections

In 1988, Rockwell collected soil samples from the boneyard, east of the boneyard, and east of Building 6. Concentrations of PCBs ranged nondetect to $221 \mathrm{ppm}$ in these areas.

- PCE Soil Gas Detections

A 1989 EBASCO, Inc. soil gas survey detected PCE in two areas at the Oxnard facility. One area was near the northern portion of the facility, and the other was near the east boundary of the facility. 


\section{- Oil Collection System in Building 5}

Building 5 contains a hydraulic oil collection system similar to Building 6. All fluids are drained to the cellar beneath the 3500-ton press, which are then pumped to the tank farm for treatment. Potential for leaks in the concrete cellar and the piping system make this an area of concern.

\section{- Abandoned Diesel UST}

Underground storage tanks have a potential for leakage, making this an area of concern.

\section{- Boneyard}

PCBs were detected in soil samples from this area, and visible oilstained soil was noted during the PE site tour.

\section{- Former Dirt Road}

The Weston memorandum indicates that the road on the west side of the facility was routinely sprayed with oil to minimize dust and that the road running north of Building 4 had several small areas that appeared to have oil dumped on them.

- Former TCA Storage Tank

The Weston memorandum reports the historic storage of a 110-gallon above-ground tank of TCA and three 55-galion drums of TCA in Building 3 (verified by EG\&G personnel). Because there was no secondary containment for the tank or drums, this is an area of concern.

\section{- Metal Cuttings in Recycling Bins}

The Weston memorandum reports that metal cuttings coated with cutting oils from the saw shop were drained and then placed in recycling bins on the dock west of Building 2. The collected oil subsequently leaked through the recycling bin.

\section{- Used Oil Filter Storage Area}

Used oil filters are drained of free flowing oil and stored in 55-gallon drums on the west side of Building 2. Because the drums showed signs of deterioration, there is a potential for oil to be released to the environment. 


\section{- Metal Slag on Ground}

A piece of metal slag on the ground west of the loading dock was noted during the site inspection. There is a potential for other materials to have been deposited here during past operations.

- Loading Dock

This is the loading/unloading area for all materials entering or leaving the facility. There is a potential for historical spills at this location.

- Metallurgical Laboratory

EG\&G personnel verified that the metal piping from the sink in the Metallurgical Laboratory was corroded because acids were historically dumped down the sink. During the site inspection, it was noted the lines beneath the sink had been replaced with plastic piping.

- Cyclone and Baghouse

Metal fines are generated in the grinding shop and from the wheelabrators in Buildings 4 and 5 . The metal fines from the grinding shop are collected by a cyclone, and fines from the wheelabrator are collected by a baghouse unit. Metal fines were noted between Buildings 4 and 5 during the site inspection.

- $\mathrm{ACM}$

Potential ACM in the HVAC system and in the walls of the vault in Building 1 was noted during the site inspection. 


\subsection{RECOMMENDATIONS}

This Phase I ESA identifies areas of concerns that warrant a Phase II ESA intrusive investigation. A drilling and sampling program designed to investigate the areas of concern is recommended. Analyses of representative soil, groundwater, and suspected ACM samples will be used to determine if environmental problems exist at the Oxnard Facility. The analytical program will focus on contaminants that have been or could potentially be released to the environment from past and current on-site operations. Analyses of soil and groundwater samples should include metals, petroleum hydrocarbons, PCBs, and chlorinated solvents.

The first step in the Phase II Environmental Site Assessment will be the preparation of a Sampling and Analysis Plan. Particulars of the Phase II investigation will be specified in the Sampling and Analysis Plan. This plan will designate sampling locations, rationale, well construction methods, sampling and analytical procedures, and the documentation protocol to be used. The Sampling and Analysis Plan will establish quality assurance requirements to ensure that all samples are representative of their original environment and that methods of collection and analyses result in scientifically valid data of known precision and accuracy. 


\subsection{REFERENCES}

The following summarizes the references used to prepare this Phase I ESA:

1. Koch, D. G. "Preliminary Evaluation Trip Report," July, 1994.

2. Noronha, Cliff. "DOE Oxnard Facility Trip Report," Booz. Allen \& Hamilton/Belfort, June 8, 1994.

3. "Final Report, Soil Gas Investigation at the Rockwell Oxnard Facility, Oxnard, California," EBASCO Services Incorporated, Newport, California, September $27,1989$.

4. "Tier Two Emergency and Hazardous Chemical Inventory," U.S. DOE, Oxnard, California, 1989-1994.

5. "Toxic Chemical Release Inventory Forms," U.S. DOE, Oxnard, California, 1987-1993. 


\section{Appendix A}

Environmental Site Assessment

\section{Preliminary Evaluation}

Trip Report

(July 28, 1994) 


\section{Oxnard Facility Trip Report}

\section{$1.0 \quad$ INTRODUCTION}

On June 8, 1994, a site visit was conducted at the EG\&G Rocky Flats Oxnard Facility. The primary purpose of the visit was to evaluate the potential presence of contamination on the property from current and/or historical uses, to interview key plant personnel, and to request additional EG\&G furnished information.

The site visit consisted of a plant-wide tour, including a photo investigation of the underground trench beneath the forging presses, and an exit meeting. This site visit constituted a preliminary evaluation at a screening level only and should not be construed as a Phase I Environmental Site Assessment satisfying "due diligence" requirements.

The participants at the site visit included representatives from the U.S. Department of Energy, RUST Geotech Inc., the Oxnard facility and Booz•Allen \& Hamilton. Individuals, personnel titles, organizations and phone numbers follow:

$\begin{array}{llll}\text { Holly Dumas } & \text { EG\&G Environmental Engineer } & \text { Oxnard Facility } & \text { (805) 486-4881 } \\ \text { Rick Dumas } & \text { EG\&G Support Operations Manager } & \text { Oxnard Facility } & \text { (805) 486-4881 } \\ \text { Carl Jacobson } & \text { RUST Program Manager } & \text { RUST Geotech Inc. } & \text { (303) 248-6568 } \\ \text { Donald Koch } & \text { RUST Environmental Engineer } & \text { RUST Geotech Inc. } & \text { (303) 248-6594 } \\ \text { Ron Kowalewski } & \text { DOE-HQ Project Manager } & \text { DOE-HQ (EM-451) } & \text { (301) 903-7652 } \\ \text { John Lyttle } & \text { RUST Program Development Manager } & \text { RUST Geotech Inc. } & \text { (303) 248-6395 } \\ \text { Cliff Noronha } & \text { Booz•Allen \& Hamilton Consultant } & \text { Booz Allen/Belfort } & \text { (301) 916-7207 } \\ \text { Joe Virgona } & \text { DOE-GJPO Project Manager } & \text { DOE-GJPO } & \text { (303) 248-6006 }\end{array}$

\subsection{OWNERSHIP AND SITE LOCATION}

The Oxnard Plant is a metalworking (forging, machining, welding, cutting, grinding, wheelabrating, and painting) facility. The facility is owned by the U.S. Department of Energy (DOE) and is operated by EG\&G Rocky Flats.

The Oxnard facility is located at 1235 East Wooley Road, Oxnard, California 93030-7330 (Figure 1). The facility is located in an industrial park and is bordered by industrial maintenance, manufacturing, and agricultural packaging facilities. The city of Oxnard operates a vehicle maintenance garage and trash receptacle storage yard on Pacific Avenue to the East. Deardorf-Jackson and Boskovich are fruit packers located on Mountain View Avenue to the Northwest and Northeast, respectively. Del Manufacturing, a subcontractor to the Department of Defense, is located on the Southwest. A steel fabricator owned by Gold Coast Steel is located on the Northwest. To the South, across Wooley Road, Told Corporation owns property which was previously used as farmland. This property is presently being marketed for commercial development (Figure 2).

The facility, occupying 13.75 acres, is comprised of seven buildings (approximately 86,000 square feet of covered floor space). The seven buildings include:

Building \#1 Administrative Offices

Building \#2 Machine Shop, Engineering, Quality Control, Metallurgical Laboratory, Production Control Office 


$\begin{array}{ll}\text { Building \#3 } & \text { Saw Shop } \\ \text { Building \#4 } & \text { Grinding Shop } \\ \text { Building \#5 } & \text { Press Shop, Wheelabrator Room, Dye Penetrant Room } \\ \text { Building \#6 } & \text { Forge Shop } \\ \text { Building \#7 } & \text { Tank Farm/Lubrication Stores }\end{array}$

Each building is described in Section 3.0, Site Description and Characterization.

\subsection{SITE DESCRIPTION AND CHARACTERIZATION}

The site description and characterization subsections are organized by building number. In addition to the buildings, the stockyard and boneyard are also described.

\subsection{Administration Offices (Building \#1)}

The Administration Offices house technical and administrative staff, a conference room, Human Resources Services, and reception area. Hazardous substances and wastes are neither stored, manufactured, processed, or otherwise used in the building. The building may contain asbestos containing materials (ACM), including: vinyl asbestos tile/mastic, heating ventilation and air conditioning (HVAC) wrapping, roof tiles, and ACM walls in the plant vault.

\subsection{Machine Shop (Building \#2)}

Building \#2 houses shipping and receiving, the production, planning, and control office, the 90-day hazardous waste generator accumulation area, the maintenance office, stock room, electrical maintenance room, lunch area, storage area, QA office, machining floor, metallurgical laboratory, and Engineering/Computer Assisted Drafting. Although not housed in Building \#2, the loading dock and fabrication shop are attached to the west side of the building. The building may contain ACM, including: vinyl asbestos tile/mastic, HVAC wrapping, and roof tiles.

The 90-day generator accumulation area is operated in accordance with 40 CFR 262.34. The area is curbed and fenced and stores acids from the Metallurgical Lab, spent synthetic coolants/lubricating oils from the machine shop, and various aerosol paint cans and paint wastes from several plant-wide locations. Waste is shipped off-site to Rollins Environmental, Deer Park, Texas, the Oil Process Co. (OPC), Los Angeles, California, a subsidiary of Rollins Environmental, and Petroleum Recycling Corporation. The staging area for off-site pick-up is the south end of the loading dock. Although no known spills have been recorded, the south loading dock area is suspect and warrants further investigation.

The fabrication shop contains a cold solvent degreasing tank that is owned by the Oxnard facility and was previously managed (drained and refilled periodically) by Safety Kleen of California. The solvent used by Safety Kleen is believed to have been a Stoddard agent. The tank is not in service due to a leak and is scheduled for off-site disposal. The flooring immediately beneath the tank showed no sign of staining; however, the area warrants further investigation. The plant has purchased and is using a parts washer that uses a water-based detergent called Simple Green ${ }^{\mathrm{m}}$. 
Used oil filters are drained of free flowing oil and then stored in $\mathbf{5 5}$ gallon drums located along the west outside wall of the fabrication shop. These filters are sent to Petroleum Recycling Corporation (PRC), Signal Hill, California, for recycling. Historically, Oxnard has also used a company called Black Gold (owner and location unknown). The area where these drums are stored shows no signs of staining; however, the drums were showing signs of deterioration. This area warrants further investigation.

The Metallurgical Lab uses various acids for destructive testing (acid etching of fabricated metal components). Acids were historically sent to the sink drain, which contained an inline calcium carbonate neutralization system upstream of the sewer outfall. This elementary-neutralization process was discontinued due to deterioration of the wastewater transfer lines. Known leaks have been recorded in the area surrounding the deteriorated lines and warrants further investigation.

The machining floor contains various lathes, drill presses, milling, and tooling machines, and an optical fabrication inspection station. Many of these machines use two chemical substances that result in two waste streams. These include Mobil lubricating oil and a synthetic coolant (hexylene glycol). Minor leaks and preventative maintenance on the machining equipment result in spent lubricating oils which are collected and sent to the 90-day generator accumulation area for off-site disposal. Spent synthetic coolant generated when machine coolant becomes biologically contaminated, is drained into an appropriate storage container and sent to the 90-day generator accumulation area for offsite disposal. The machining floor showed no signs of spills or leaks.

\subsection{Saw Shop (Building \#3)}

The saw shop houses various band and utility saws used to saw off the initial billets for forging and machining. Historically, Building \#3 housed a 110-gallon capacity tank and three 55-gallon drums of 111-trichloroethane (TCA) for die cleaning. Used or spent TCA was placed into 55-gallon drums, stored in the 90-day generator accumulation area and sent to an off-site recycler. The Weston report states that the tank area had no secondary containment. This report further states that the tank and drums were removed in August 1989. This area was not investigated during the trip, as it was occupied by supplies and equipment. This area is suspect and warrants further investigation.

\subsection{Grinding Shop (Building \#4)}

The grinding shop houses numerous grinding stations with a vacuum collection system. This collection system is fitted with an air abatement particle separator (cyclone). This unit generates a very fine metal dust, which is collected in a drum beneath the cyclone. This metallic dust is a hazardous waste due to the presence of chromium. The dust, following containerization, is stored at the 90-day generator accumulation area and sent to Rollins for off-site solidification/stabilization and land disposal. No signs of spillage (i.e., presence of fines) were noted in the area.

\subsection{Press Shop (Building \#5)}

The press shop houses a 1600-ton mechanical press, a 3500-ton hydraulic press, and three natural gas-fired furnaces. Beneath each press, in a press cellar pit, oils (either leaked Shell Tellus-100 or Tellus-46) and metal fines accumulate. Spent oils from the 
1600-ton press and/or spilled oils beneath the unit are vacuum pumped, containerized, and stored in the 90-day generator accumulation area for off-site recycle. Oils in the 3500-ton press are self-filtered. The filters are drained, containerized, and sent to the west wall of the saw shop for off-site recycle with PRC. Oils from the pit beneath the unit are vacuum pumped and sent to the oily wastewater treatment plant located at the tank farm and lube stores (Building \#7). Because of the age and condition of the pits, no attempt was made to determine pit integrity. Plant personnel indicate that groundwater intrusion into the pits may have occurred historically during high groundwater periods. These pits and the soil interface beneath warrant further investigation.

Located on the west side of the press shop is the Wheelabrator shop which houses four Wheelabrators. The Wheelabrators are enclosed rotating drums with small metalic balls used to polish metal components. Operationally', these machines create metallic fines that must be captured and properly disposed. The air emission/abatement system consists of three bag house structures and stacks, each servicing two Wheelabrators. The bag house dust (EPA D007) is collected in drum containers and sent to the 90-day generator accumulation area prior to off-site solidification/stabilization by Rollins Environmental. Bags are replaced when breakthrough occurs and are containerized for off-site disposal. No signs of spillage (i.e. presence of fines) were noted in the area.

\subsection{Forge Shop (Building \#6)}

The forge shop houses 6 High Energy Rate Forging (HERF) forging hammers with $40,000 \mathrm{ft}$. lb. maximum energy, $3 \mathrm{HERF}$ forging hammers with $100,000 \mathrm{ft}$. lb. maximum energy rating, $4 \mathrm{HERF}$ forging hammers with $225,000 \mathrm{ft}$. lb. maximum energy rating and $3 \mathrm{HERF}$ forging hammers with $300,000 \mathrm{ft}$. lb. maximum energy rating. Additionally, 13 natural gas fired furnaces and 5 mechanical trim presses are housed. The cellars of each hammer and press take the form of a large underground concrete collection trench that slopes north. This trench collects leaking oils from the large presses and is also the repository for the quench water from Buildings \#5 and \#6. At the northern end a sump pump is used to vacuum pump oily water to the oily wastewater treatment system located at the tank farm and building 7.

An investigation of the trench was conducted and photos were taken; however, due to time constraints, neither the integrity nor the construction of the trench could be ascertained. Photos of this trench are included as Appendix A. Similar to the press shop pits, plant personnel indicate that groundwater intrusion into the trench may have occurred historically during high groundwater levels. The trench and the soil interface beneath warrants further investigation.

\subsection{Tank Farm and Lubrication Stores}

The tank farm is located north of Building 6. The tank farm consists of five tanks including:

Tank A

- Pit effluent tank (oil and water)

- Capacity $=10,000$ gallons

- $12^{\prime \prime}$ vertical height $=750$ gallons 


\section{Tank B}

- Back-up Pit effluent tank (oil and water)

- Capacity $=1,500$ gallons

- $12^{n}$ vertical height $=150$ gallons

- Tank not in service

Tank C

- Dirty oil tank

- Capacity $=2,000$ gallons

- $12^{n}$ vertical height $=200$ gallons

Tank D

- Cleaned oil tank

- Capacity $=3,000$ gallons

- $12^{\prime \prime}$ vertical height $=300$ gallons

\section{Tank E}

- Steam cleaner water tank (reusable)

- Capacity $=5,000$ gallons

- $12^{\text {N }}$ vertical height $=200$ gallons

Hydraulic fluids are collected from the press pits in Building $\# 5$ and the trench in Building \#6 and pumped to the tank farm (Tank A) for initial treatment (gravity separation). A full description of the oily wastewater treatment process will be provided in the Phase I report.

The tank farm is built on a concrete slab surrounded by a 3-foot high concrete berm for secondary containment. Previous consultant reports indicate no tank spills have been recorded or reported. Rainwater collects in the containment; however, its management was not determined during the trip. Historically, the western wall of the containment berm leaked at the wall/slab interface and was replaced and sealed in 1989.

Building \#7 contains the oil and wastewater filtration systems. These oil and water treatment systems receive oil from Tank $\mathrm{C}$ and water from Tank $\mathrm{E}$ respectively through temporary flex hosing (no permanent piping exists between the tank farm and Building \#7). Discussions with the operator, Ed Sandoval, indicate that no recorded or reported spills have occurred during hook-up, fluid transfer or detachment of the hosing, nor have any spills or overflow occurred at the treatment facilities located in Building \#7.

\subsection{Stockyard}

The stockyard is located north of the saw shop near the western boundary of the site. The stockyard is an open air, concrete-paved storage facility that is used for the reception and storage of raw metal. No dry or liquid chemicals have been stored in this yard. The yard was not walked down during the site visit. 


\subsection{Bone Yard}

A fenced unpaved bone yard exists in the northwest comer of the plant site. During the site visit, the bone yard contained numerous steel and poly tanks. All tanks were observed to be empty. Additionally, various pieces of scrap metal, old machinery and various assorted debris were being stored. Much of the old machinery was contaminated with oily residue and/or sludge. Several areas of petroleum or discolored soils were observed even though the yard has overgrown with weeds and grass. According to DOE-furnished documentation, the yard historically stored polychlorinated biphenyl containers or articles.

Finally, the asphalt road leading to the bone yard was originally a dirt road that may have been periodically oiled for dust control. This yard and surrounding area warrants further investigation:

\subsection{MISCELLANEOUS ITEMS}

\subsection{Generated Wastes/Management}

During the exit interview, plant personnel identified the various waste streams generated at the site and the disposition of each. The following identifies the stream's classifications and their management.

\section{Waste Stream/Classification}

- Waste Acids (hydrochloric, nitric, acetic)

Waste Acid Liquid, N.O.S., Corrosive Material NA1760

CA Waste \#792, EPA Waste \#D002

- Spent Coolant

Hazardous Waste Liquid, N.O.S., ORM-E NA9189

CA Waste \#132, EPA Waste \#D007

- Wheelabrator Bag House Dust

Grinding shop cyclone fines

Hazardous Waste Solid, N.O.S., ORM-E NA9189

CA Waste \#172, EPA Waste \#D007

- Oil (used)

Hazardous Waste Liquid, N.O.S., ORM-E NA9189

CA Waste \#221

- Wastewater Evaporative Concentrate

Hazardous Waste Liquid, N.O.S., ORM-E NA9189

CA Waste \#223

- Press Pit Sludge

Hazardous Waste Liquid, N.O.S., ORM-E NA9189

CA Waste \#223, EPA Waste \#D007, D008

\section{Management}

Neutralization.

Rollins Environmental

Incineration.

Rollins Environmental

Solidification/

stabilization

Rollins Environmental

Recycled

-Petroleum Recycling

Corporation

Incineration.

Rollins Environmental

Incineration

Rollins Environmental 
- Paint Debris

Hazardous Waste Liquid, N.O.S., ORM-E NA9189

CA Waste \#352, EPA Waste \#D007, D008

- Paint Rinsate

Hazardous Waste Liquid, N.O.S., ORM-E NA9189

CA Waste \#134, EPA Waste \#D007, D008

- Press Pit Oily Water

- Used Oil Filters

Spray Cans

Compressed Gas, N.O.S. Flammable Gas UN1954

CA Waste \#331, EPA Waste \#D001

- Scrap Metal .

- Degreasing Solvent

Hazardous Waste Liquid, N.O.S., ORM-E NA9189 EPA Waste \#D001
Incineration

Rollins Environmental

Incineration

Rollins Environmental

Oily water treatment

system

Oxnard Facility

Recycled

-Petroleum Recycling

Corporation

Incineration

Rollins Environmental

On-site Bin Storage

Recycled with

- SOS Metals - LA

- Oxnard Recycling

- Eddie Recycling

- Standard Industries

(carbon steel)

Recycled

Safety Kleen

\subsection{Potentially Contaminated Areas}

The following lists potentially contaminated areas requiring further investigation. Delineation of these potential areas resulted from previous investigations or observations made during the on-site visit (June 8, 1994).

- Asbestos inspection including bulk analysis for ACM in all buildings.

- A pit/dump area in the vicinity of Building \#6 possibly containing sand casts, slag, oily residue, etc. Tierra Tech (1982) report/Weston memorandum (1988).

- Area(s) surrounding deteriorated piping from the sink drains exiting the metallurgical lab for potential $\mathrm{pH} /$ metals contamination.

- Loading dock (south end) for potential of spillage during off-site loading of hazardous waste. 
- Flooring in the areas of the solvent degreaser (Building $\$ 2$ - Fabrication shop) and previous 111 - TCA tank and drum storage (Building \#3) for potential solvent/metals contamination.

- Used oil filter drum storage area for potential petroleum contamination.

- Biological remediation area west of Building $\# 6$ for solvent, petroleum, PCB, and metals contamination.

- Bone yardldiscolored soils area east of the bone yard for solvent, petroleum, PCB - and metals contamination.. (Site visit and Rockwell 1988/89 soil samples.)

- Collection trench (Building \#6) and press cellars (Building \#5) containing metal shavings, hydraulic oils and quench waters.

\subsection{RECOMMENDATIONS}

This preliminary evaluation identifies the need to conduct a full Phase I Environmental Site Assessment (ESA) prior to any intrusive investigations, including analytical testing associated with a Phase II Site Characterization. The primary purpose of the Phase I will evaluate the potential or known presence of contamination on "real property" from current or historical uses within the legally defined property boundary. This phase will also define the level of intrusive investigations that should be performed prior to realty transfer. Previous studies appear fragmented providing insufficient information upon which to base either the quantity or location/depth of soil borings or monitoring wells or the indicator chemical(s) or chemical specific analysis to be considered.

The preparation of a Phase I and Phase II environmental assessments is recommended. The Phase I work would require $160-200$ hours and would involve the following:

- $\quad$ Planning and Research

- $\quad$ Site visit including environmental agency visits

- Data evaluation

- Preparation of the ESA Report

The proposed sequence of events for the Phase I environmental assessment includes:

1. Obtain and review DOE-furnished information, including a site map showing legal property boundaries.

2. Conduct regulatory list reviews: local, state, and federai. (See \#8)

3. Identify government data sources and make data requests. (See \#8)

4. Review historical aerial photographs of the site and site vicinity.

5. Arrange site access and interviews through the plant and perform site and site vicinity visual surveys. 
6. Visit local tax assessor's office to obtain a copy of chain-of-title information and the recorded legal site map.

7. Visit local building department to obtain copies of permitted building modifications.

8. Conduct site record reviews at the following offices, as required:

- U.S. Environmental Protection Agency (EPA) Region IX

- CAL-EPA, Dept. of Toxic Substances Control (Region IV, Long Beach, CA)

- State Water Resources Control Board (UST)

--- Regional Water. Quality Control-Board (Region IV, Los Angeles, CA)

- Integrated Waste Management Board

- City of Oxnard (Wastewater Division \& Water Management Division)

- Dept. of Health (Ventura County)

9. Evaluate data

10. Prepare the draft ESA report.

11. Schedule internal report reviews, if desired by the DOE.

12. Submit final report to the DOE. 


\section{Appendix B}

Construction Boring Logs

(Buena Engineers, Inc. 1982) 


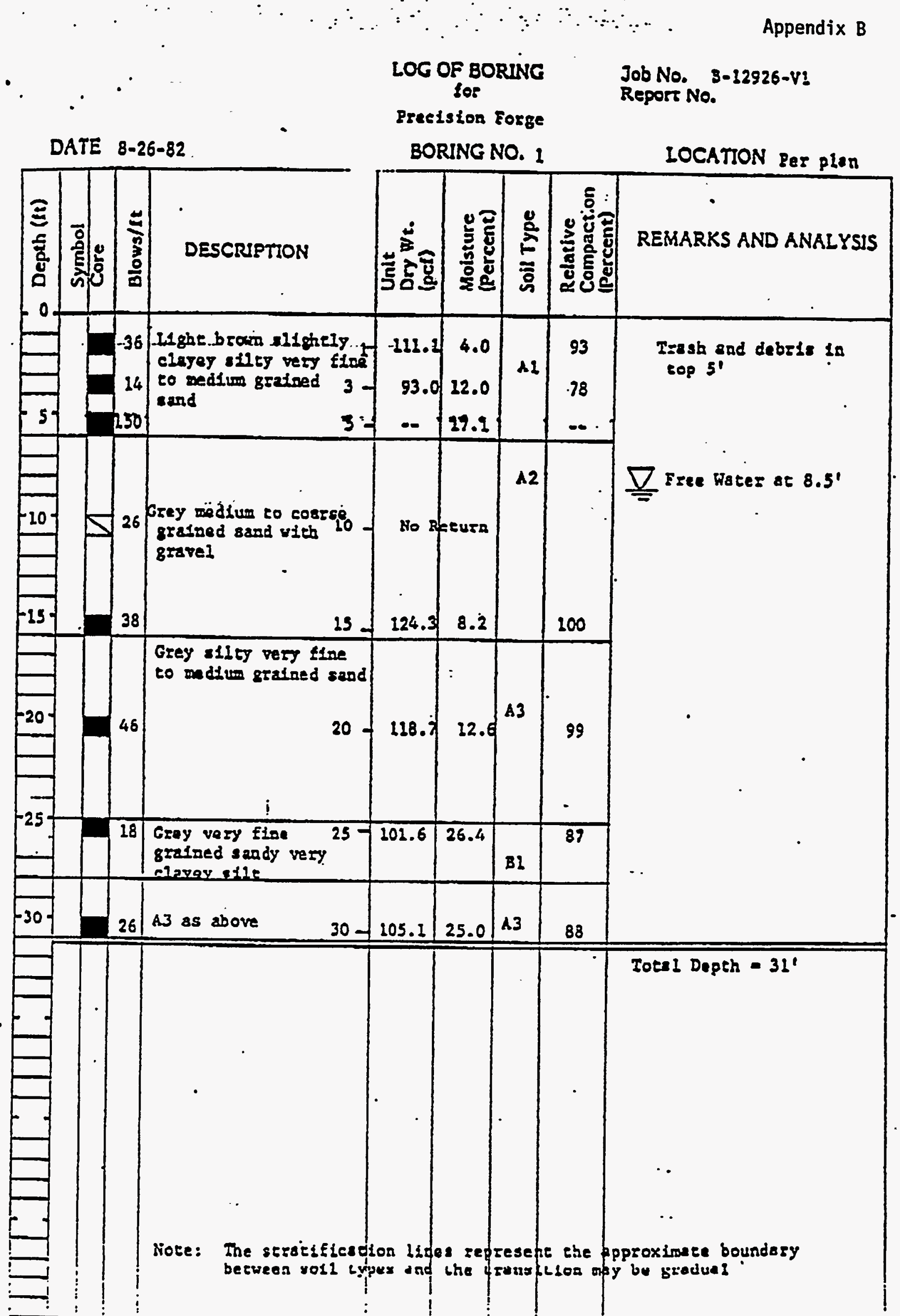


Appendix B

Lin wr suktria for

Preciston Forge

DATE 8-26-צ2

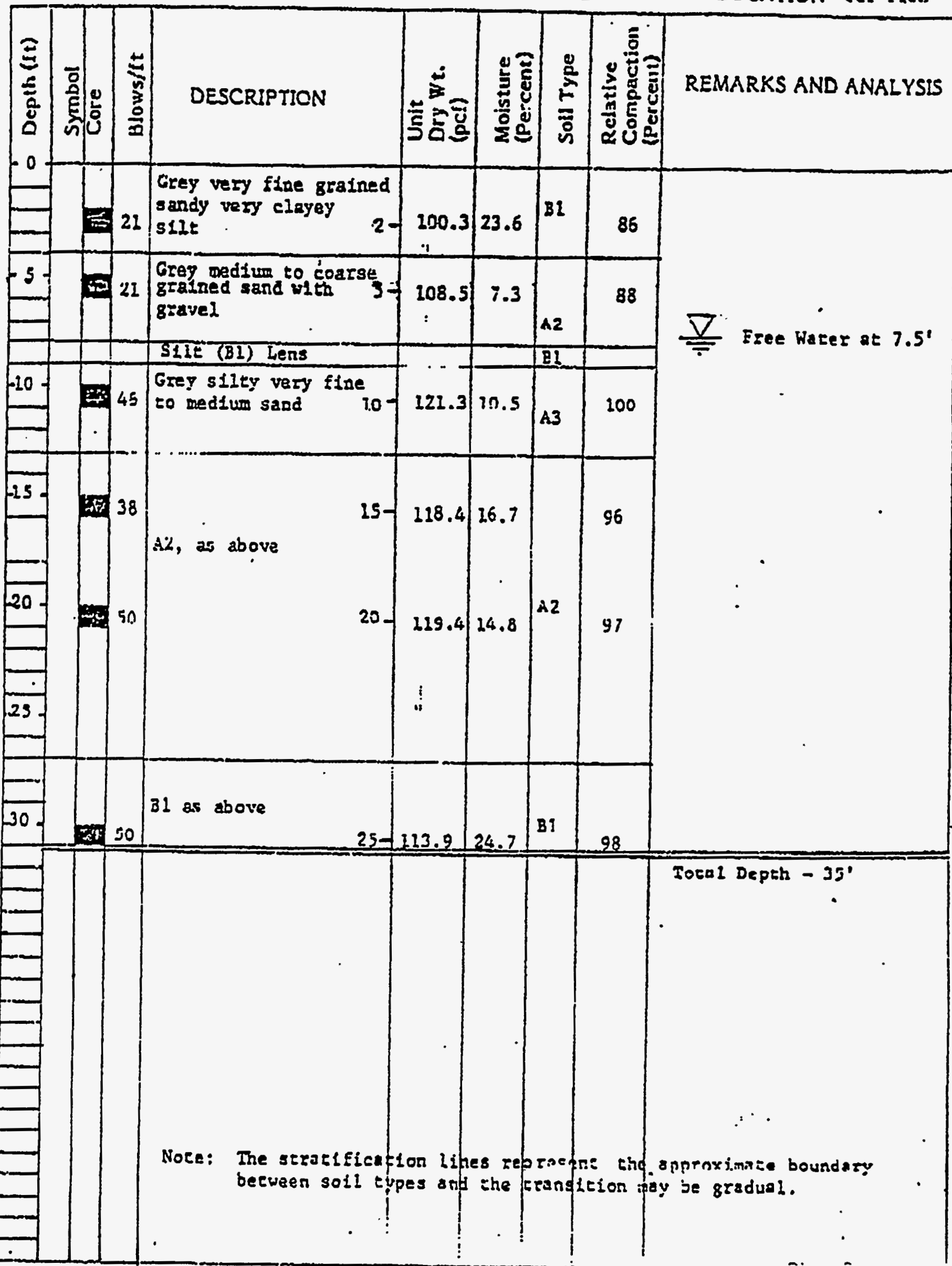

JOD NO. - 3-12926-:1

Report No.

LOCATION PET PLAn 
Report No. Appendix B

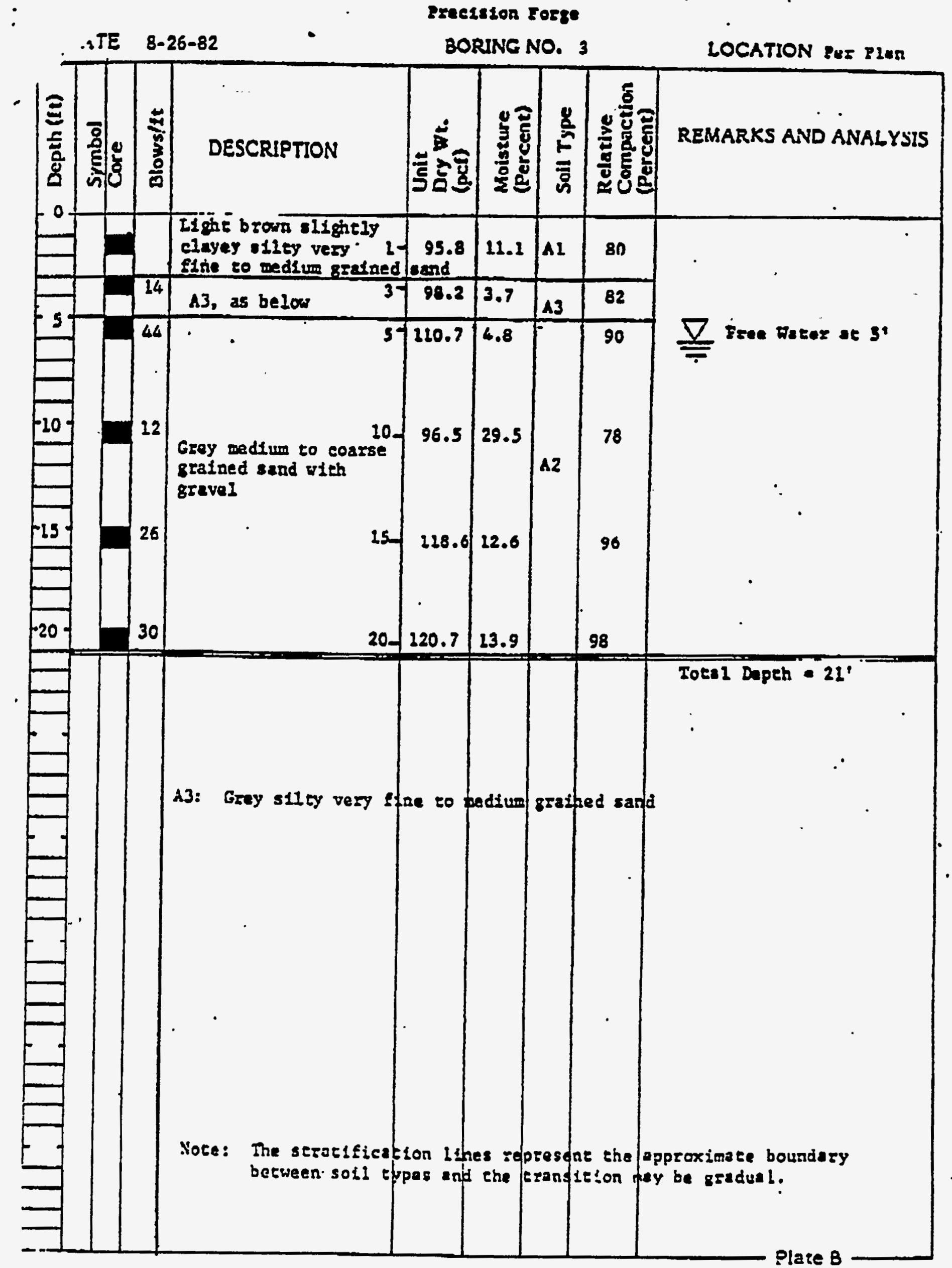




\section{Appendix C}

\section{Summary of Hazardous Waste Streams}

1989-1993 
YEAR: 1989

\begin{tabular}{|c|c|c|c|c|c|}
\hline WASTE STREAM & ROUTINE? & CWC & POUNDS & PERCENI & ROUTINE\% \\
\hline 1,1,1-Trichloroethane & YES & 211 & $2,270.4$ & $5.74 \%$ & $8.31 \%$ \\
\hline PCB Contaminated dirt & No & & $8,400.0$ & $21.23 \%$ & \\
\hline \multirow[t]{2}{*}{ PCB Contaminated materials } & No & & $1,900.0$ & $4.80 \%$ & \\
\hline & & 261 & $10,300.0$ & $26.03 \%$ & \\
\hline Sulfuric Acid & NO & 551 & 150.0 & $0.38 \%$ & \\
\hline Bunker Oil & YES & & $4,578.0$ & $11.57 \%$ & $16.76 \%$ \\
\hline \multirow[t]{2}{*}{ Soluble Oil } & YES & & $7,940.0$ & $20.06 \%$ & $29.07 \%$ \\
\hline & & 221 & $12,518.0$ & $31.63 \%$ & $45.84 \%$ \\
\hline Isopropyl Alcohol & NO & 212 & 64.0 & $0.16 \%$ & \\
\hline Deltaglaze (alcohol-base) & NO & & 78.0 & $0.20 \%$ & \\
\hline Pennforge 41 & NO & & 431.2 & $1.09 \%$ & \\
\hline Turco Protectoil & NO & & 385.0 & $0.97 \%$ & \\
\hline \multirow[t]{2}{*}{ Pb Free Al Forge Compound } & NO & & 372.7 & $0.94 \%$ & \\
\hline & & 331 & $1,266.9$ & $3.20 \%$ & \\
\hline Spray cans & YES & 343 & 250.0 & $0.63 \%$ & $0.92 \%$ \\
\hline Oil Filters & YES & & $1,400.0$ & $3.54 \%$ & $5.13 \%$ \\
\hline \multirow[t]{2}{*}{ Oil Sorbent } & YES & & $4,680.0$ & $11.83 \%$ & $17.14 \%$ \\
\hline & & 223 & $6,080.0$ & $15.36 \%$ & $22.26 \%$ \\
\hline Metal Dusts & YES & 172 & $4,600.0$ & $11.62 \%$ & $16.84 \%$ \\
\hline Waterforge 1211 & NO & 141 & 484.0 & $1.22 \%$ & \\
\hline D110 Developer & YES & 132 & $1,531.2$ & $3.87 \%$ & $5.61 \%$ \\
\hline Paint Wastes & YES & 461 & 60.0 & $0.15 \%$ & $0.22 \%$ \\
\hline
\end{tabular}

WASTE TOTALS: $39.574 .5 \quad 100.00 \%$ ROUTINE WASTE TOTALS: \begin{tabular}{lll}
27.309 .6 & $69.01 \% \quad 100.00 \%$ \\
\hline
\end{tabular} 
YEAR: 1990

\begin{tabular}{|l|r|r|r|r|r|}
\hline \multicolumn{1}{|c|}{ WASTE STREAM } & ROUTNE? & CWC & POUNDS & PERCENT & ROUTNE $\%$ \\
\hline \hline Waste Oil & YES & 221 & $1,950.0$ & $10.46 \%$ & $11.06 \%$ \\
\hline Spray cans & YES & 343 & 76.0 & $0.41 \%$ & $0.43 \%$ \\
\hline Miscellaneous off-spec chemicals & NO & 331 & 416.0 & $2.23 \%$ & \\
\hline Coolant & YES & & $4,590.0$ & $24.62 \%$ & $26.03 \%$ \\
\hline Neutralized acid solution & YES & & $1,325.0$ & $7.11 \%$ & $7.52 \%$ \\
\hline & & 132 & $5,915.0$ & $31.73 \%$ & $33.55 \%$ \\
\hline Oil Filters & YES & & 900.0 & $4.83 \%$ & $5.10 \%$ \\
\hline Daraclean 283 & YES & & $1,080.0$ & $5.79 \%$ & $6.13 \%$ \\
\hline Oil Sorbent & YES & & $6,330.0$ & $33.96 \%$ & $35.90 \%$ \\
\hline & & 223 & $8,310.0$ & $44.58 \%$ & $47.13 \%$ \\
\hline Ammonium Hydroxide & NO & 123 & 25.0 & $0.13 \%$ & \\
\hline Metal Dusts & YES & 172 & $1,200.0$ & $6.44 \%$ & $6.81 \%$ \\
\hline Miscellaneous off-spec chemicals & NO & 141 & 120.0 & $0.64 \%$ & \\
\hline PCB Samples & NO & 261 & 450.0 & $2.41 \%$ & \\
\hline Paint Wastes & YES & 461 & 180.0 & $0.97 \%$ & $1.02 \%$ \\
\hline
\end{tabular}

WASTE TOTALS: $18,642.0 \quad 100.00 \%$ 
YEAR: 1991

\begin{tabular}{|c|c|c|c|c|c|}
\hline WASTE STREAM & ROUTINE? & CWC & POUNDS & PERCENT & ROUTINE \% \\
\hline Waste Oil & YES & 221 & $3,147.0$ & $13.21 \%$ & $15.28 \%$ \\
\hline Spray cans & NO & 343 & 76.0 & $0.32 \%$ & \\
\hline Coolant & YES & & $3,907.0$ & $16.40 \%$ & $18.97 \%$ \\
\hline Neutralized Acid solution & YES & & 539.0 & $2.26 \%$ & $2.62 \%$ \\
\hline . & & 132 & $4,446.0$ & $18.66 \%$ & $21.59 \%$ \\
\hline Non-neutralized Acid solution & YES & 792 & 460.0 & $1.93 \%$ & $2.23 \%$ \\
\hline Oil Filters & YES & & 461.0 & $1.93 \%$ & $2.24 \%$ \\
\hline \multirow[t]{2}{*}{ Oil Sorbent } & YES & & 943.0 & $3.96 \%$ & $4.58 \%$ \\
\hline & & 223 & $1,404.0$ & $5.89 \%$ & $6.82 \%$ \\
\hline Metal Dusts & YES & 172 & $10,360.0$ & $43.48 \%$ & $50.30 \%$ \\
\hline Paint Debris & NO & 461 & 428.0 & $1.80 \%$ & \\
\hline Pallet contaminated w/ oil & NO & 223 & 138.0 & $0.58 \%$ & \\
\hline Pit Sludge/Debris & No & 223 & $2,516.0$ & $10.56 \%$ & \\
\hline RCF Blanket contaminated w/oil & NO & 223 & 72.0 & $0.30 \%$ & \\
\hline Solvent & YES & 213 & 778.0 & $3.27 \%$ & $3.78 \%$ \\
\hline
\end{tabular}

WASTE TOTALS: $23,825.0 \quad 100.00 \%$ ROUTINE WASTE TOTALS: $20,595.0 \quad 86.44 \%$ $100.00 \%$

NOTE: Routine waste information is in bold type. 
YEAR: 1992

\begin{tabular}{|l|r|r|r|r|r|}
\hline \multicolumn{1}{|c|}{ WASTE STREAM } & ROUTNE? & \multicolumn{1}{c|}{ CWC } & \multicolumn{1}{c|}{ POUNDS } & \multicolumn{1}{c|}{$\%$} & ROUTINE\% \\
\hline \hline Waste Oll & YES & 221 & 350.0 & $3.47 \%$ & $3.95 \%$ \\
\hline Coolant & YES & 132 & $1,001.0$ & $9.93 \%$ & $11.30 \%$ \\
\hline Acid solution & YES & 792 & 548.0 & $5.43 \%$ & $6.18 \%$ \\
\hline Oll Sorbent & YES & 223 & 655.0 & $6.50 \%$ & $7.39 \%$ \\
\hline Wastewater evap. effluent & YES & 223 & $1,465.0$ & $14.53 \%$ & $16.53 \%$ \\
\hline Discontinued die lubricant & NO & 223 & 653.0 & $6.48 \%$ & \\
\hline Oily Waste & NO & 223 & 544.0 & $5.39 \%$ & \\
\hline TOTAL FOR ALL CWC 223 WASTES & & & $3,317.0$ & $32.89 \%$ & \\
\hline Metal Dusts & YES & 172 & $3,975.0$ & $39.42 \%$ & $44.85 \%$ \\
\hline Solvent & YES & 213 & 868.0 & $8.61 \%$ & $9.79 \%$ \\
\hline Spray Paint Cans & NO & & 25.0 & $0.25 \%$ & \\
\hline
\end{tabular}

WASTE TOTALS:

$10,084.0 \quad 100.00 \%$ ROUTINE WASTE TOTALS:

$8,862.0 \quad 87.88 \%$
$100.00 \%$

NOTE: Routine waste information is in bold type.

92 HAZARDOUS WASTE IN TONS:

5.042 
YEAR: 1993

\begin{tabular}{|l|r|r|r|r|r|}
\hline \multicolumn{1}{|c|}{ WASTE STREAM } & ROUTNE? & \multicolumn{1}{c|}{ CWC } & \multicolumn{1}{c|}{ POUNDS } & \multicolumn{1}{c|}{$\%$} & ROUTNE $\%$ \\
\hline Waste Oil & YES & 221 & $1,890.0$ & $18.84 \%$ & $23.81 \%$ \\
\hline Coolant & NO & 132 & 454.0 & $4.53 \%$ & \\
\hline Acid Solution & YES & 792 & 756.0 & $7.54 \%$ & $9.52 \%$ \\
\hline Wastewater Evap. Effluent & YES & 223 & $1,811.0$ & $18.06 \%$ & $22.81 \%$ \\
\hline Oil Sorbent & NO & 223 & 973.0 & $9.70 \%$ & \\
\hline Pit Sludge & NO & 223 & 170.0 & $1.69 \%$ & \\
\hline SP400 (oily waste-outdated chem) & NO & 223 & 375.0 & $3.74 \%$ & \\
\hline TOTAL FOR ALL CWC 223 WASTES & & & & $33.19 \%$ & \\
\hline Metal Dusts & YES & 172 & $2,442.0$ & $24.35 \%$ & $30.76 \%$ \\
\hline Solvent & YES & 213 & $1,039.0$ & $10.36 \%$ & $13.09 \%$ \\
\hline Paint Debris & NO & & 70.0 & $0.70 \%$ & \\
\hline Spray Paint Cans & NO & & 50.0 & $0.50 \%$ & \\
\hline
\end{tabular}

WASTE TOTALS: $10,030.0 \quad 100.00 \%$ ROUTINE WASTE TOTALS: \begin{tabular}{lll}
$7,938.0 \quad 79.14 \%$ & $100.00 \%$ \\
\hline
\end{tabular}

NOTE: Routine waste information is in bold type. 
Appendix D

Photo Documentation 


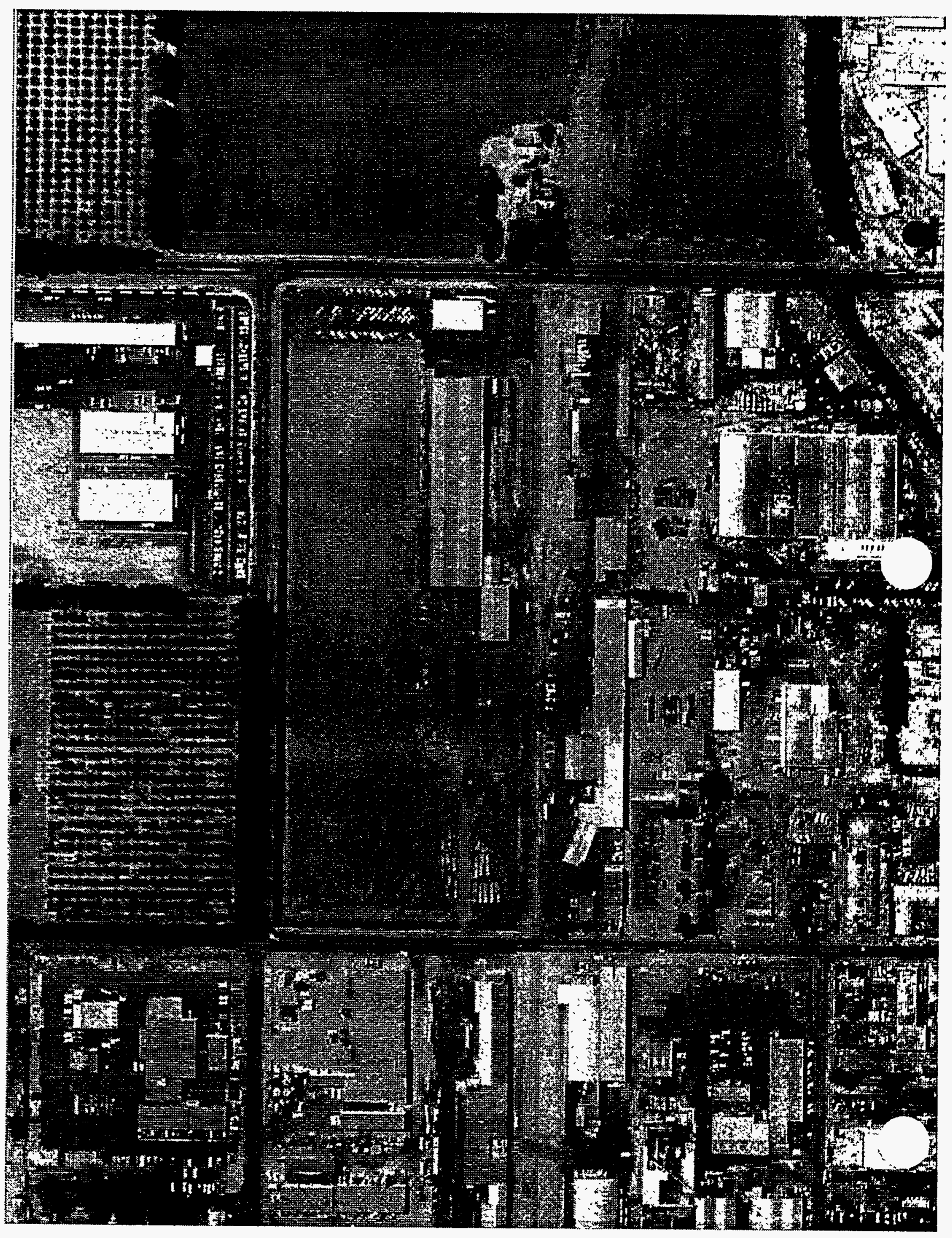


Building \#5

3500 ton Press Pit

Pump Suction Line
Building \#5

3500 ton Press Pit

Side Wall Showing

Metal Hydraulic Oif Sludge
Building \#6

Forging Hammer Trench

Hydrautic Hosing

\&

Hammer Dampeners
Building \#6

Forging Hammer Trench

View Looking North 

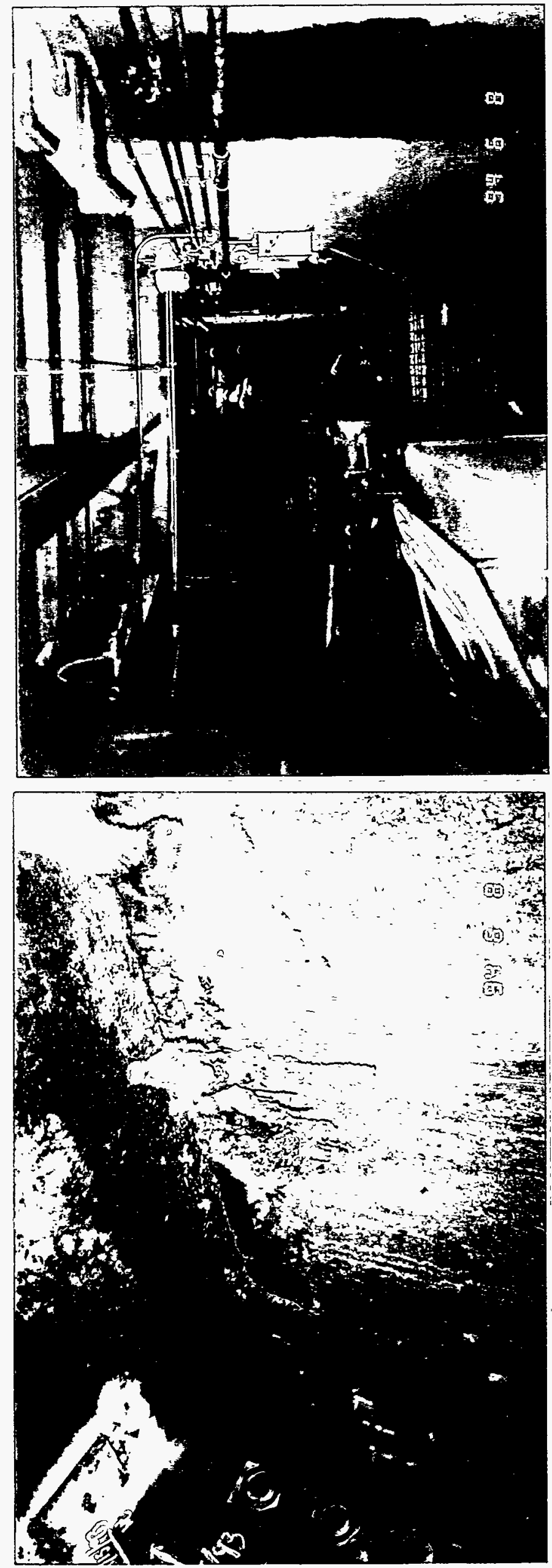

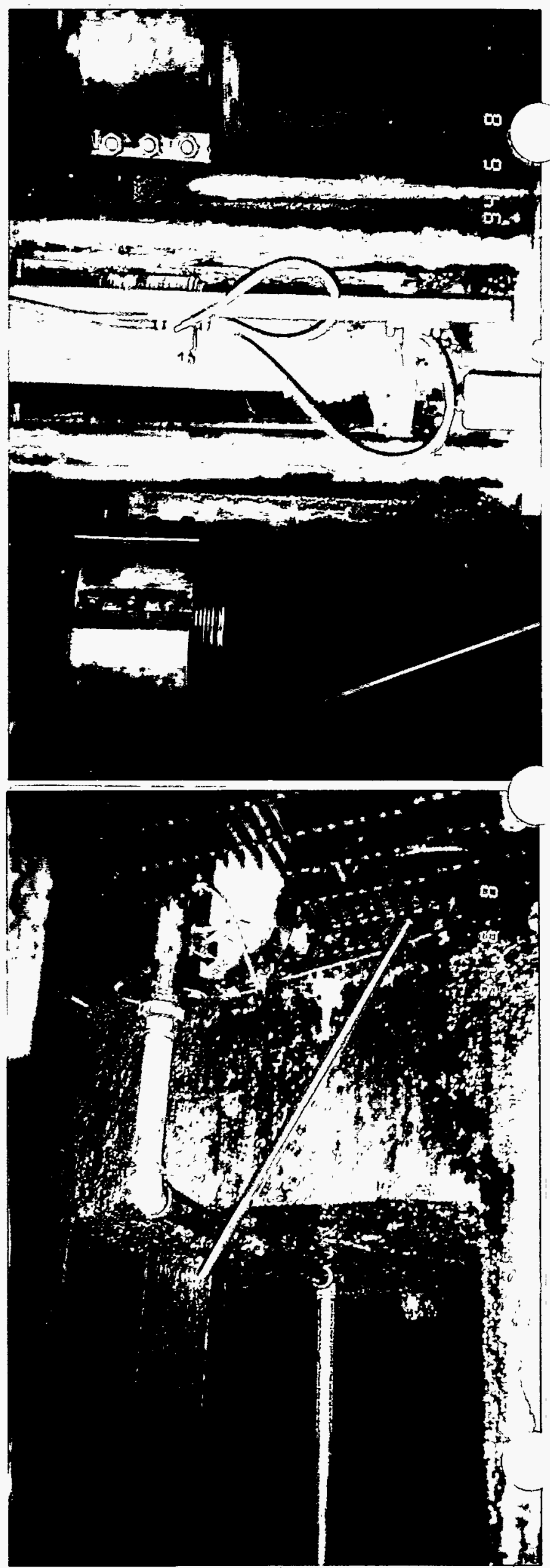


Building \#6

Forging Hammer Trench

Forging Hammer Pedesta]
Building \#6

Forging Hammer Trench

Suction Piping to

$0 \mathrm{i} /$ Water Tank Farm
Building \#6

Forging Hammer Trench

oily Water Beneath

Trench Grating
Building \#6

Forging Hammer Trench

View Looking North 

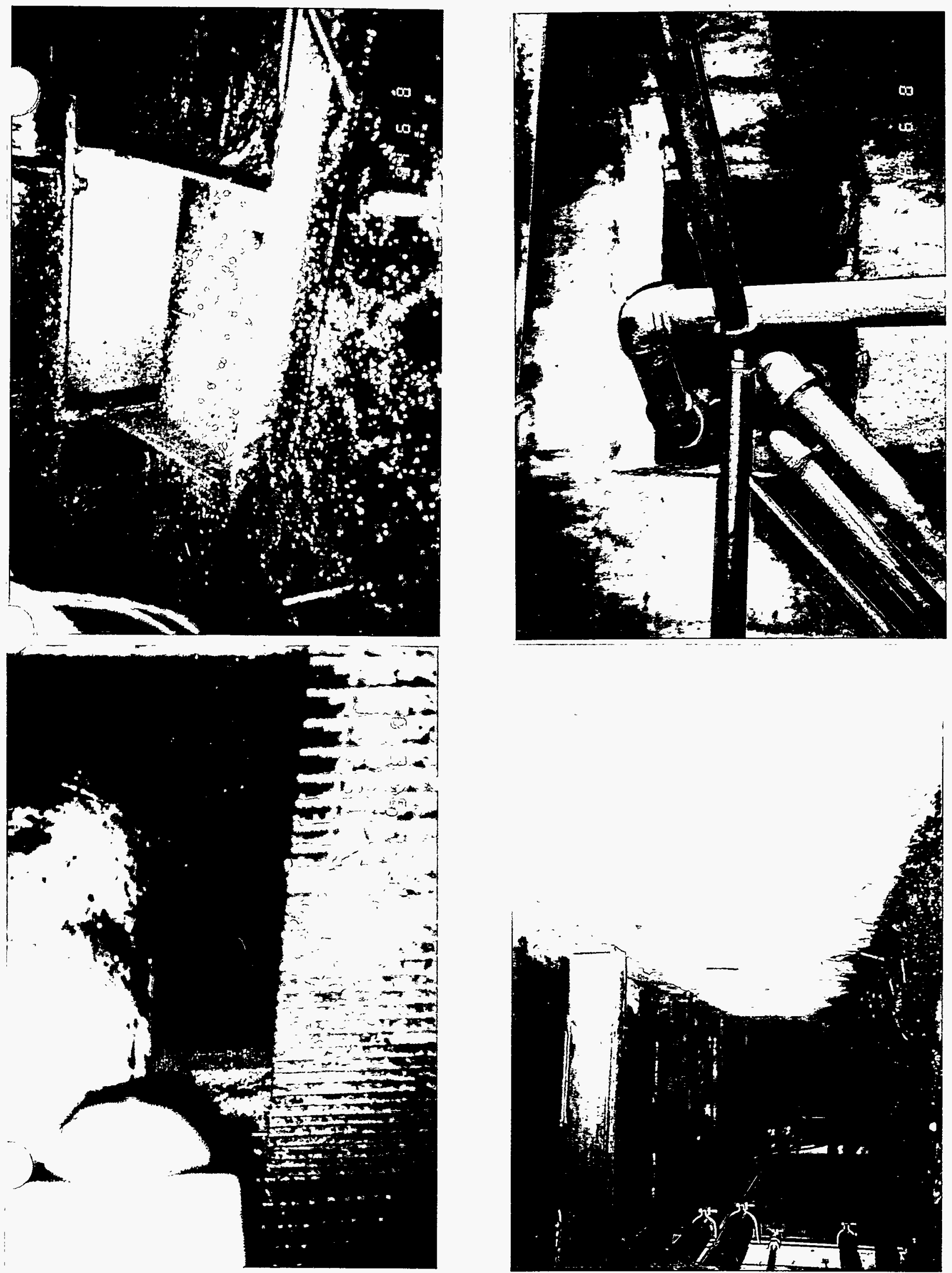
Building \#6

Forging Hammer Trench

Metallic 0ily Water Sludge

Beneath Grating
Building \#6

Forging Hammer Trench

Hammer Dampeners

Sludge Residue

Hydrautic Hosing
Building \#6

Forging Hammer Trench

Oily Metal Shavings
Building \#6

Forging Hammer Trench

Pipe/Conduit Chase 

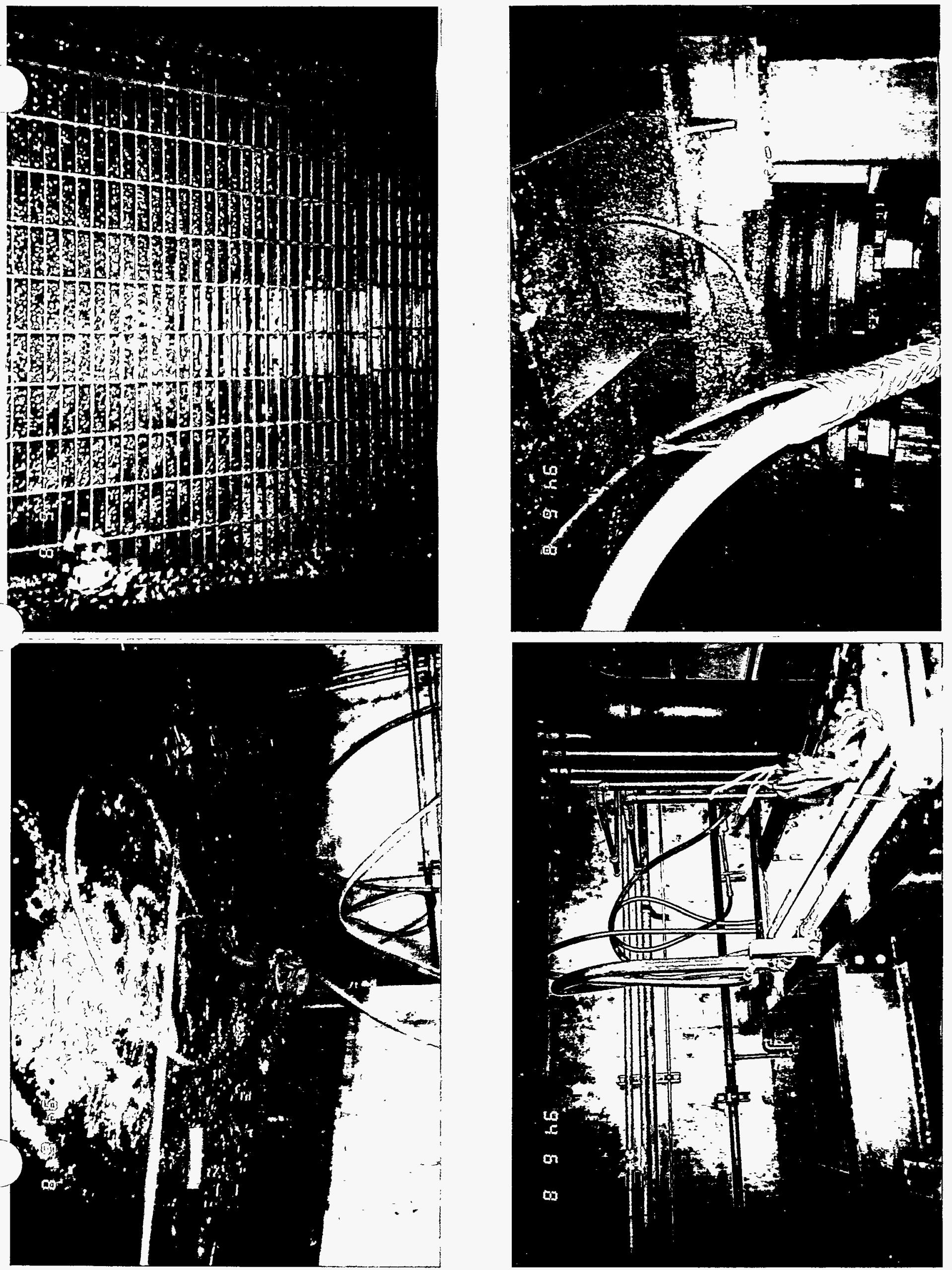


\section{Building \#6}

Forging Hammer Trench Hammer Pedestal Bench 0ily Sheen
Building \#6

Forging Hammer Trench Hammer Pedestal Base

\section{Building \#6}

Forging Hammer Trench South Stairwell Entrance

Fire Extinguisher

Forced Air Ventilation Tube
Building \#6

Forging Hammer Trench

Oily Metal Shavings 

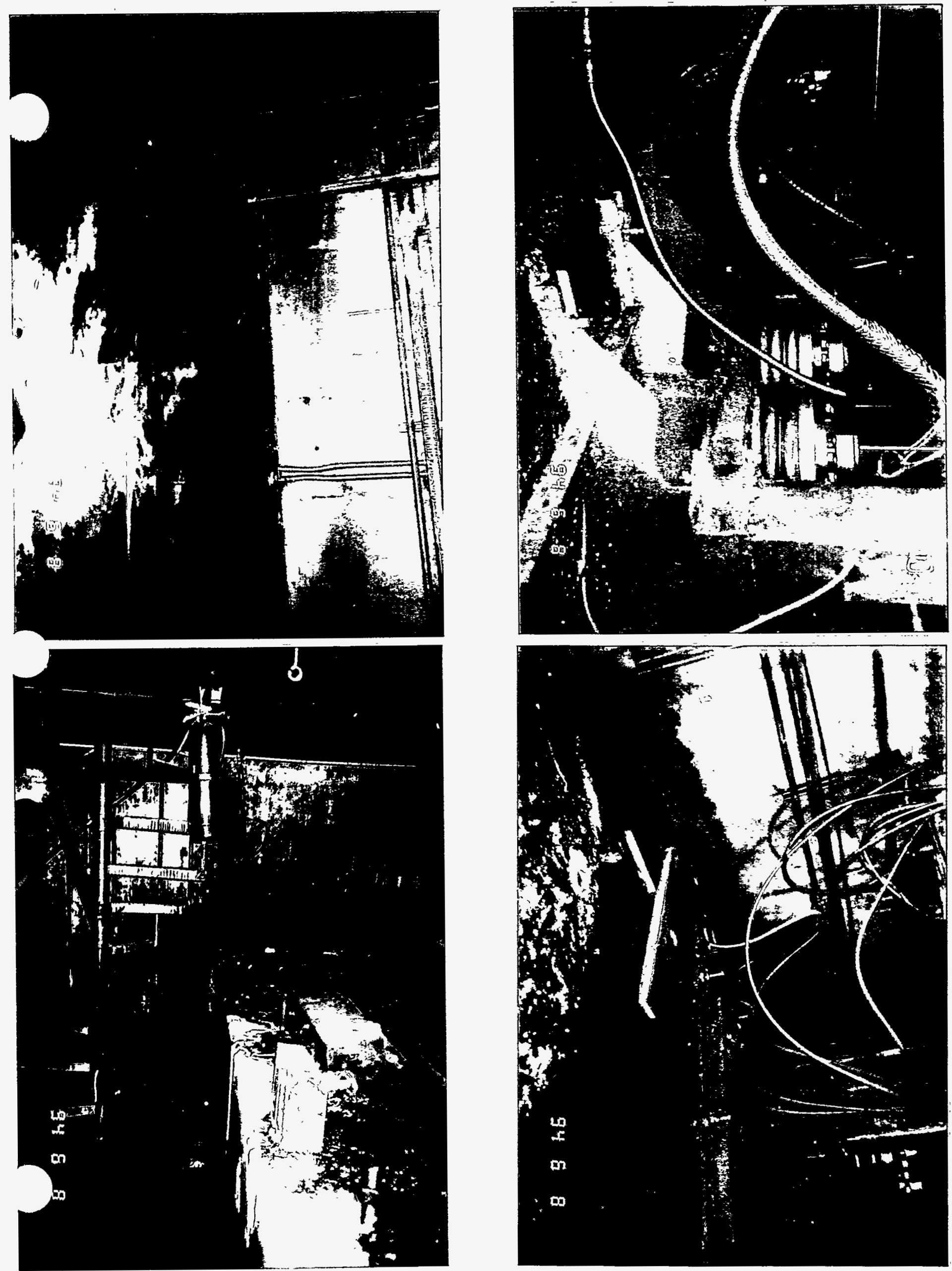
Building \#7

Lubrication Stores

0il/Water Filtration
Boneyard

Used Equipment Storage
Building \#6

Forging Hammer Trench

Forging Hammer Pedestal
Building \#6

Hydraul ic Pump 

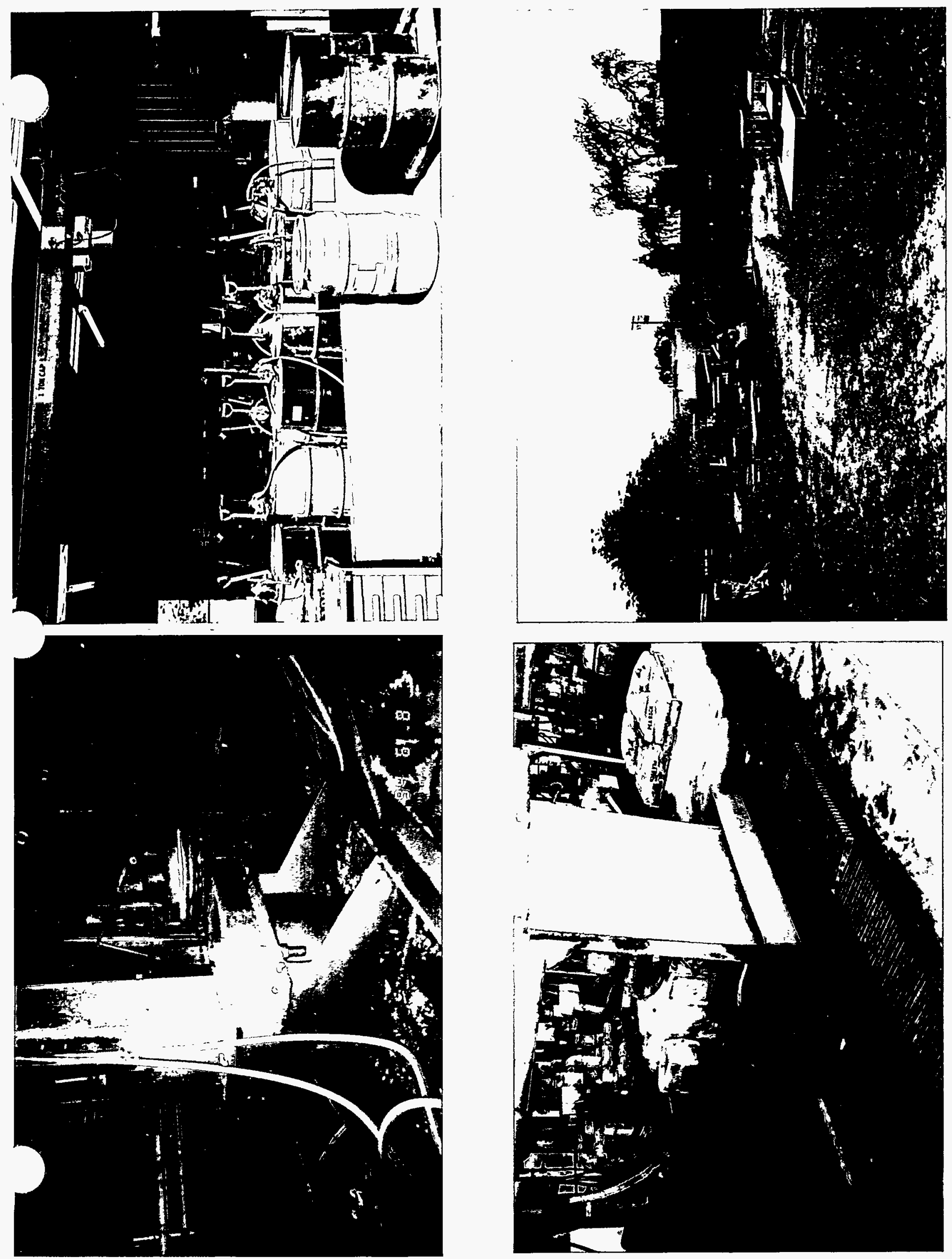
EG\&G Oxnard Rocky Flats

Facility

Administration Building

BLANK

Boneyard

Drum Storage Area
Building \#6

Forging Hammer Trench

View Looking North 

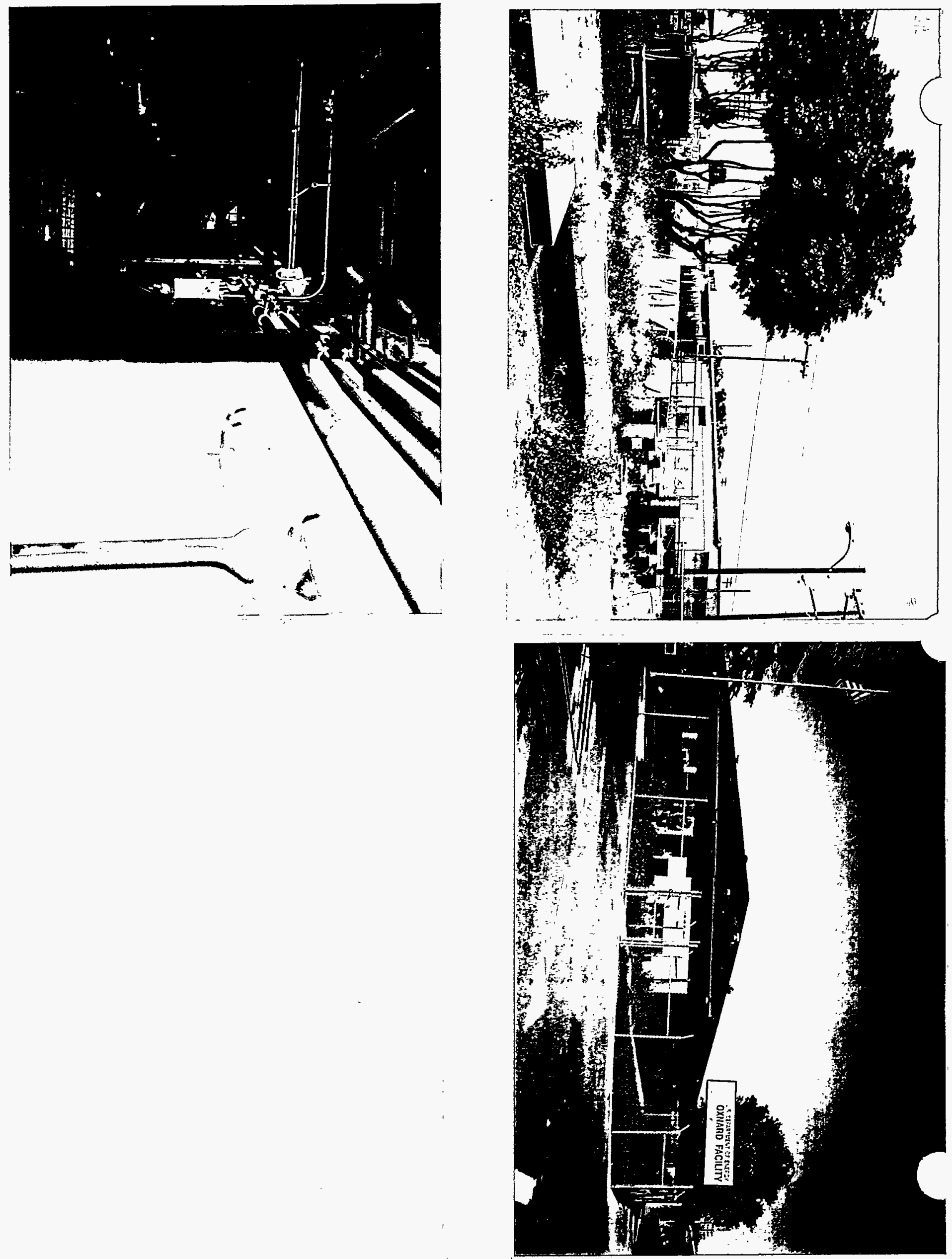
Boneyard

Equipment Storage
Boneyard

Tank/Container Storage
Underground Storage Tank Zyglo Dye Penetrant
Boneyard

Equipment Storage

0ily Residue in Secondary Containment Tray 

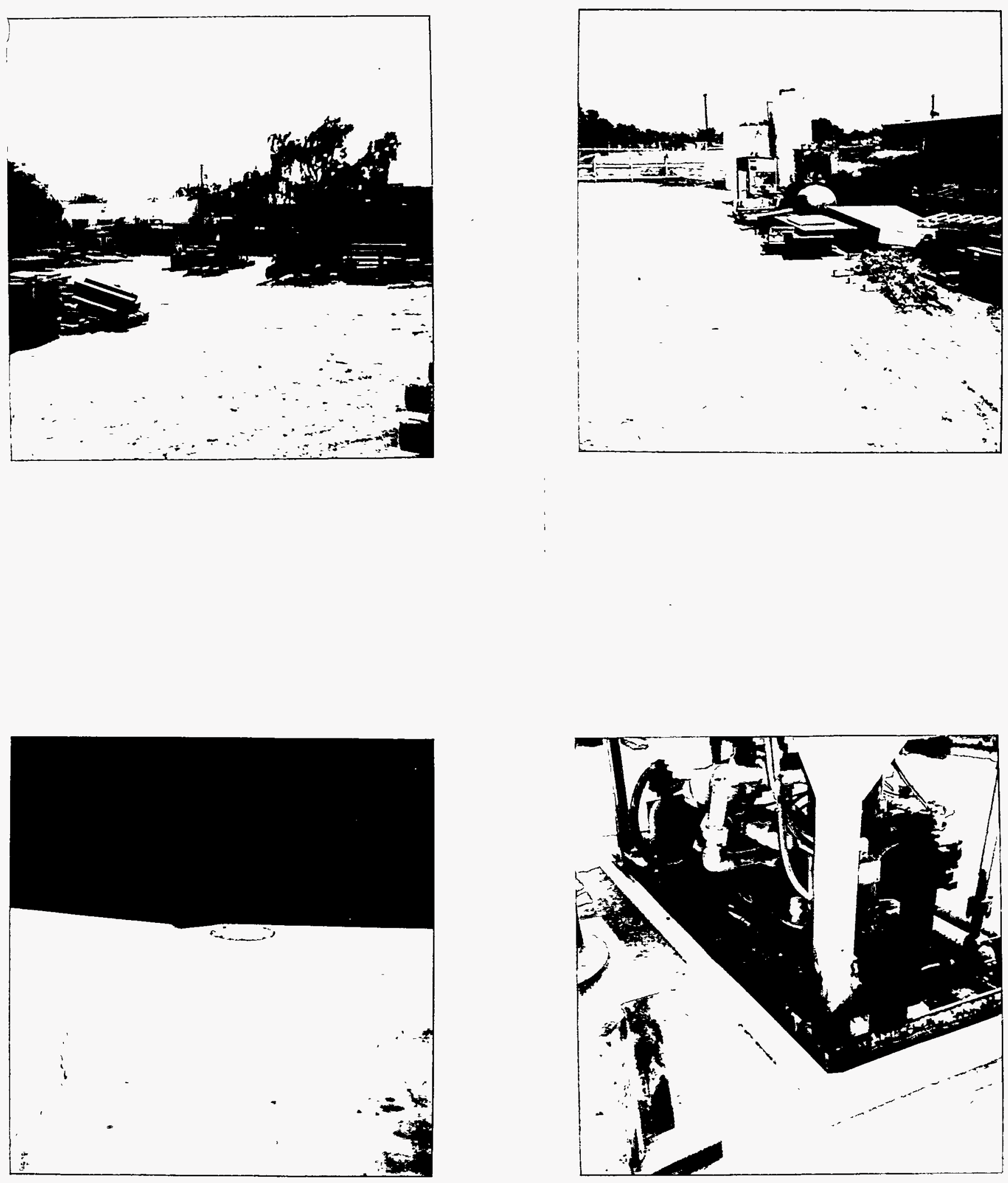
Building \#6

Forging Hammer Trench

Vacuum Suction Line to $0 / W$

Tank from North Trench Sump
Building \#2

West wall - Fabrication BIdg. Used 0i1 Filter Storage
Stockyard

View Looking North
Stockyard

View Looking South 

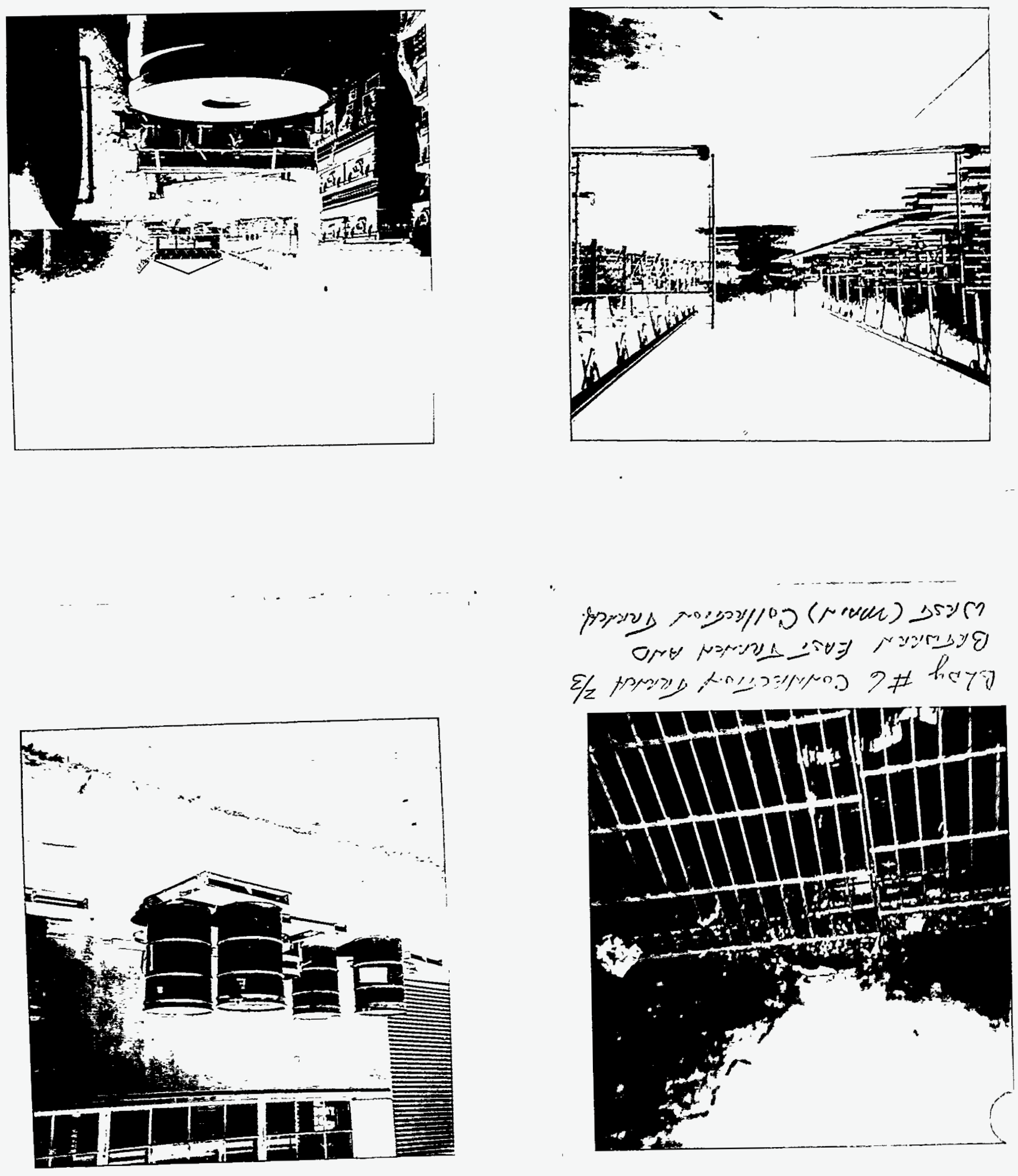
Building \#6

Forging Hammer Trench Vacuum Suction Line to $0 / \mathrm{W}$ Tank from North Trench Sump
Building \#6

Forging Hammer Trench Connection Trench Between

East and West (main) Collection Trench
Building \#6

Forging Hammer Trench

Connection Trench Between

East and West (main)

Collection Trench
Building \#5

3500 Ton Press Pit

Vacuum Suction Line to $0 / W$ Tank 
-200720 cend

rosoese we ?ing m/o ol perpong vannover - Gredid aruft

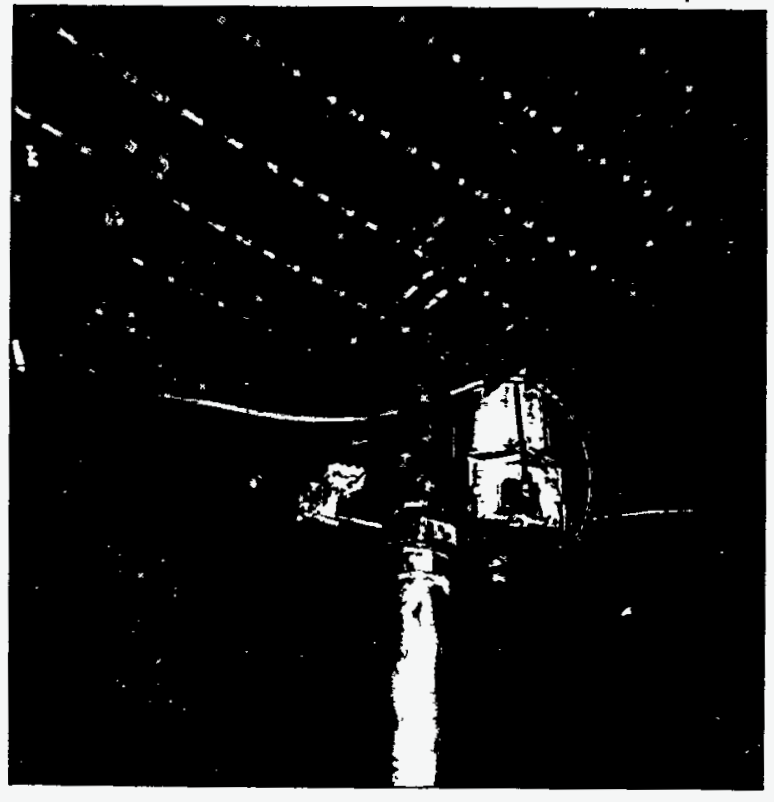

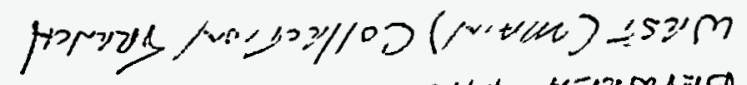

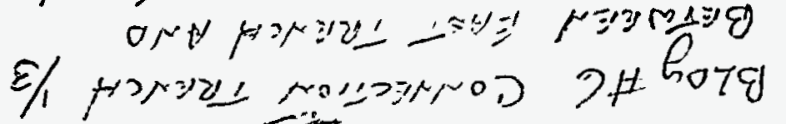

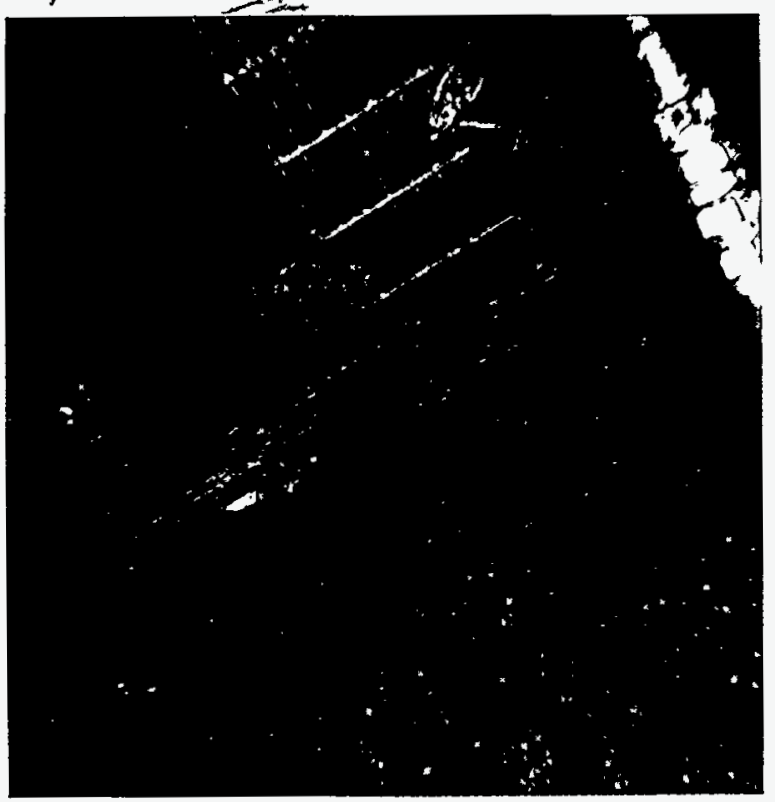

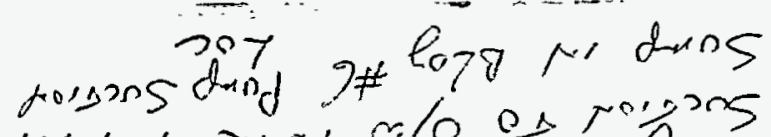

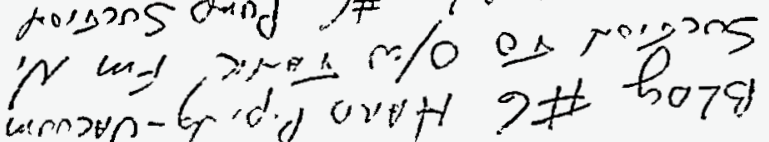

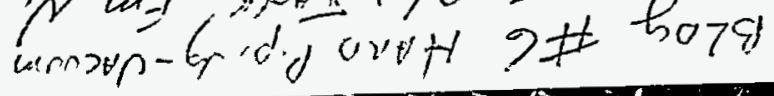

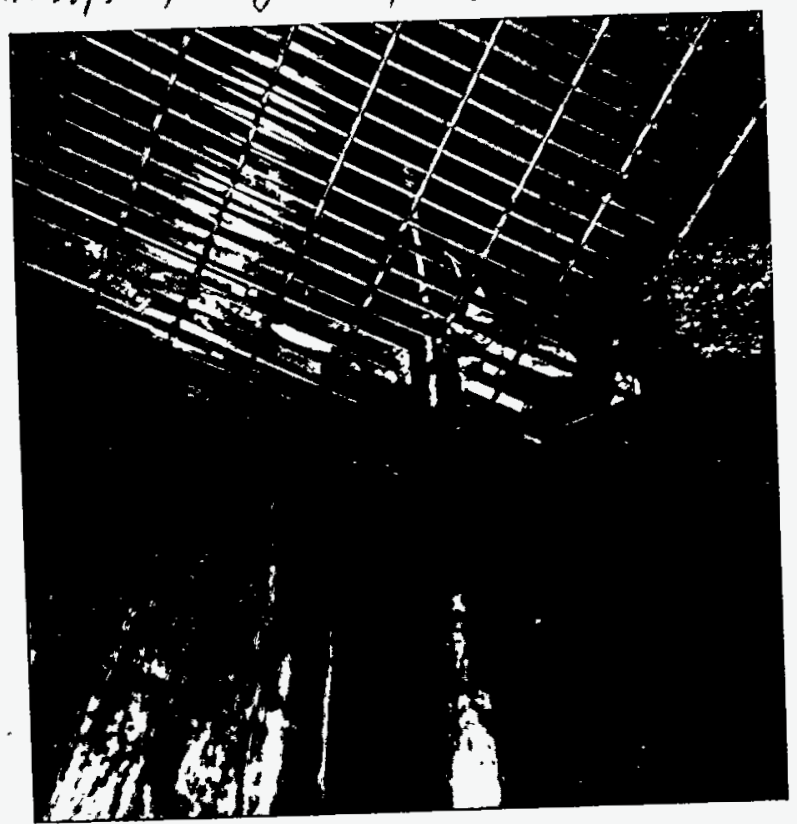

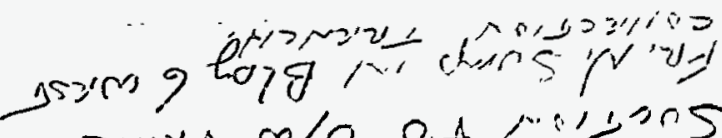

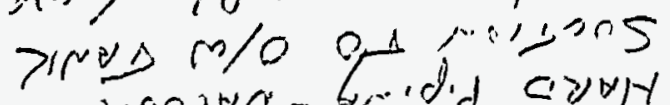
vesisur- ared cony

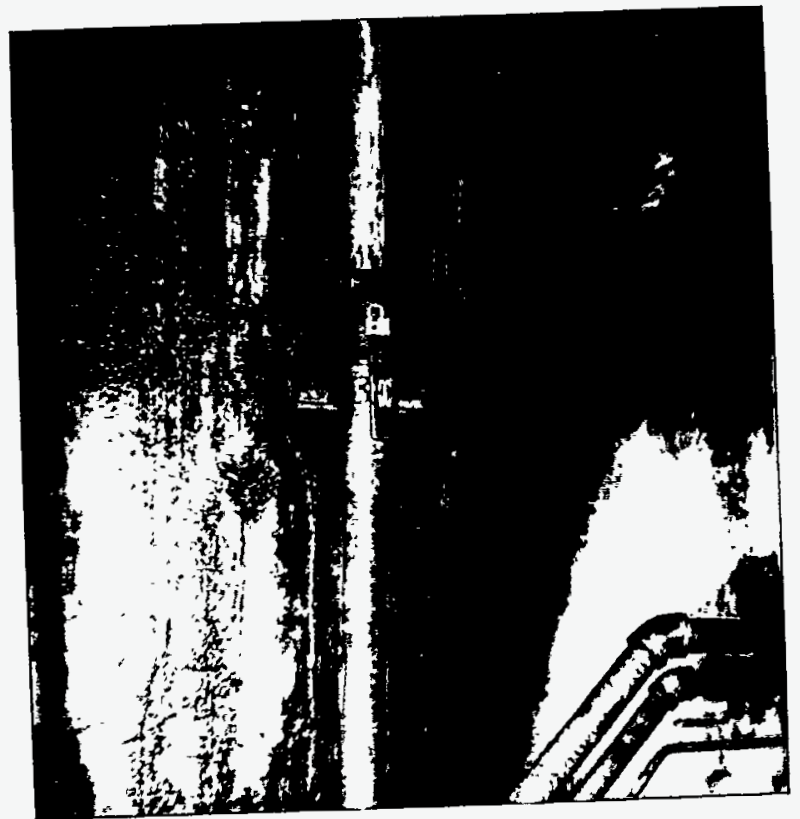


Underground Storage Tank Diesel Tank
BLANK

BLANK 


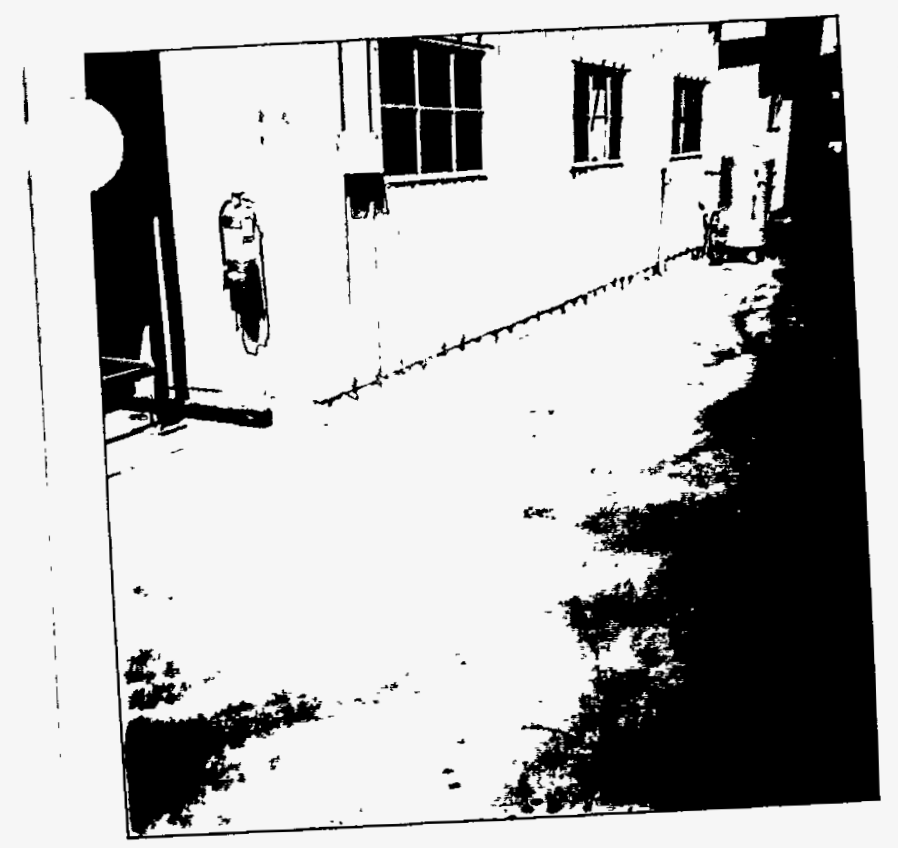




\section{Appendix E}

\section{Design As-Built Drawings \\ Buildings 5 \& 6}




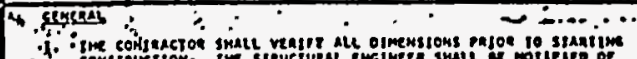

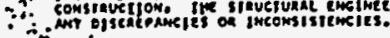

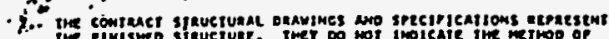

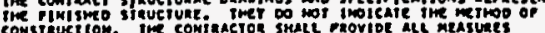

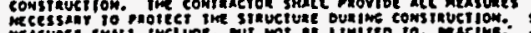

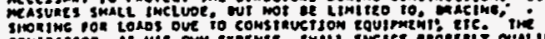

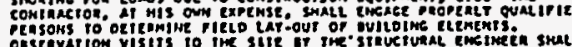

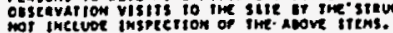

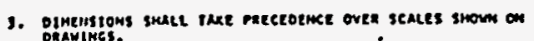

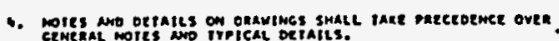

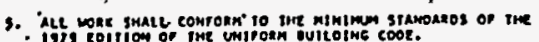

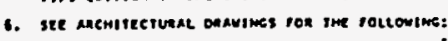

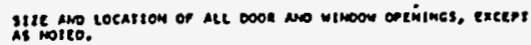

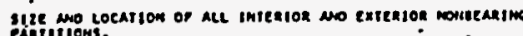

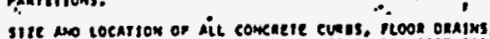

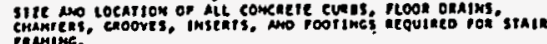

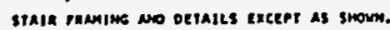
reor rinssurs.

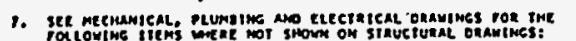

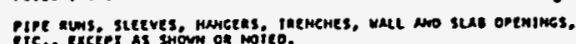

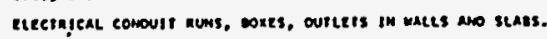

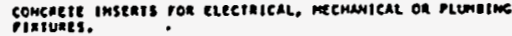

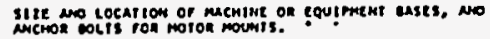

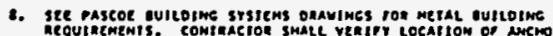

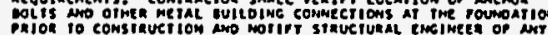

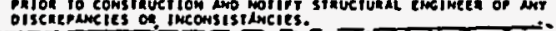

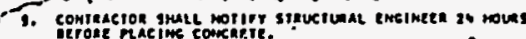

\section{GEN.ERAL}

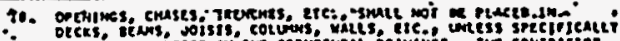

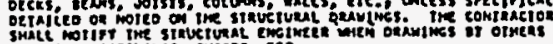

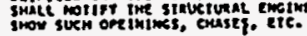

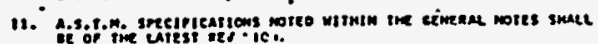

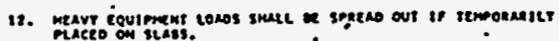

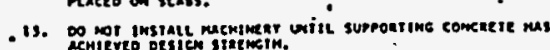

1. evesarion

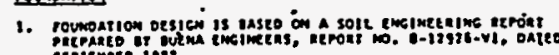

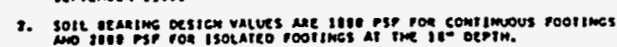

J. sore expansion troex is 0.20 .

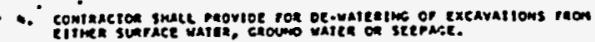

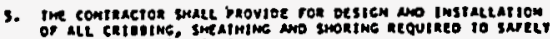

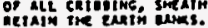

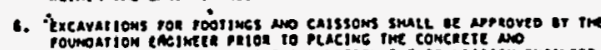

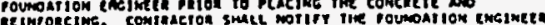

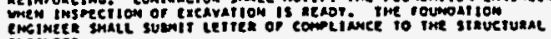

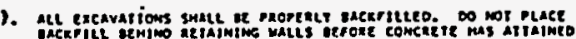

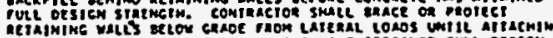

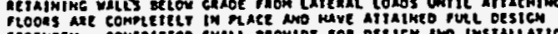

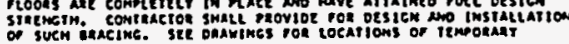

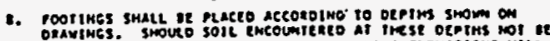

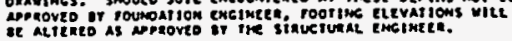

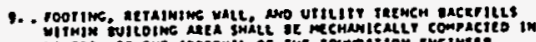

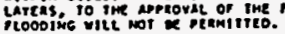

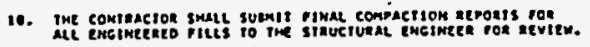

\section{ก} NOTES

sementir

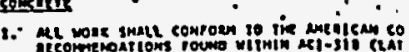

2. Act confezie small oltain the roliovina $n$ precest comentre..........

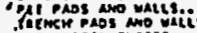

- Merenicat chists.......

J, morlleno

cenzm syau cowroth to a.s.t.

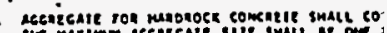

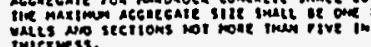

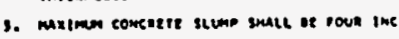

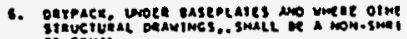

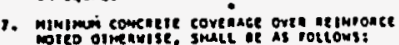

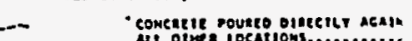

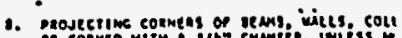

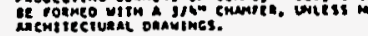

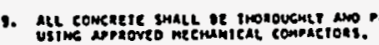

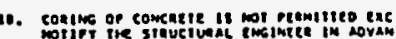

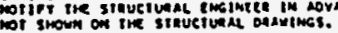

11. Cuelus conpounos usto on concelege imul

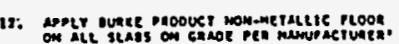

13. concaziz smale ie practo conflimoust a

31. set lespectpons.

- Leinzongting saess

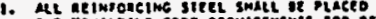

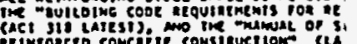

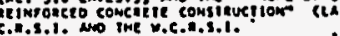

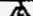
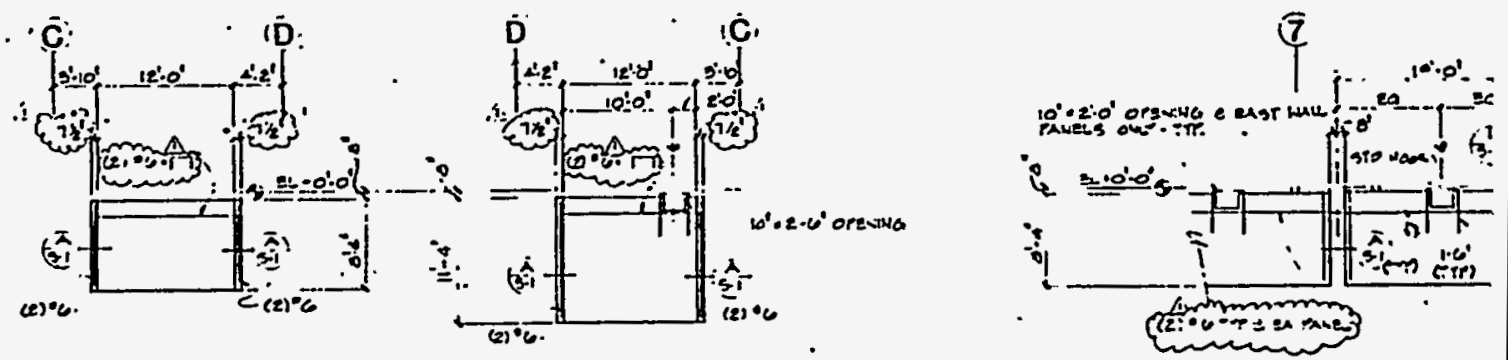

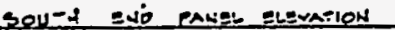

NCRTA SNO RANEL BLEVATION

\section{B TYPICAL PRE-CAST CONC. PANEL ELEVATIONS - CULVERT}

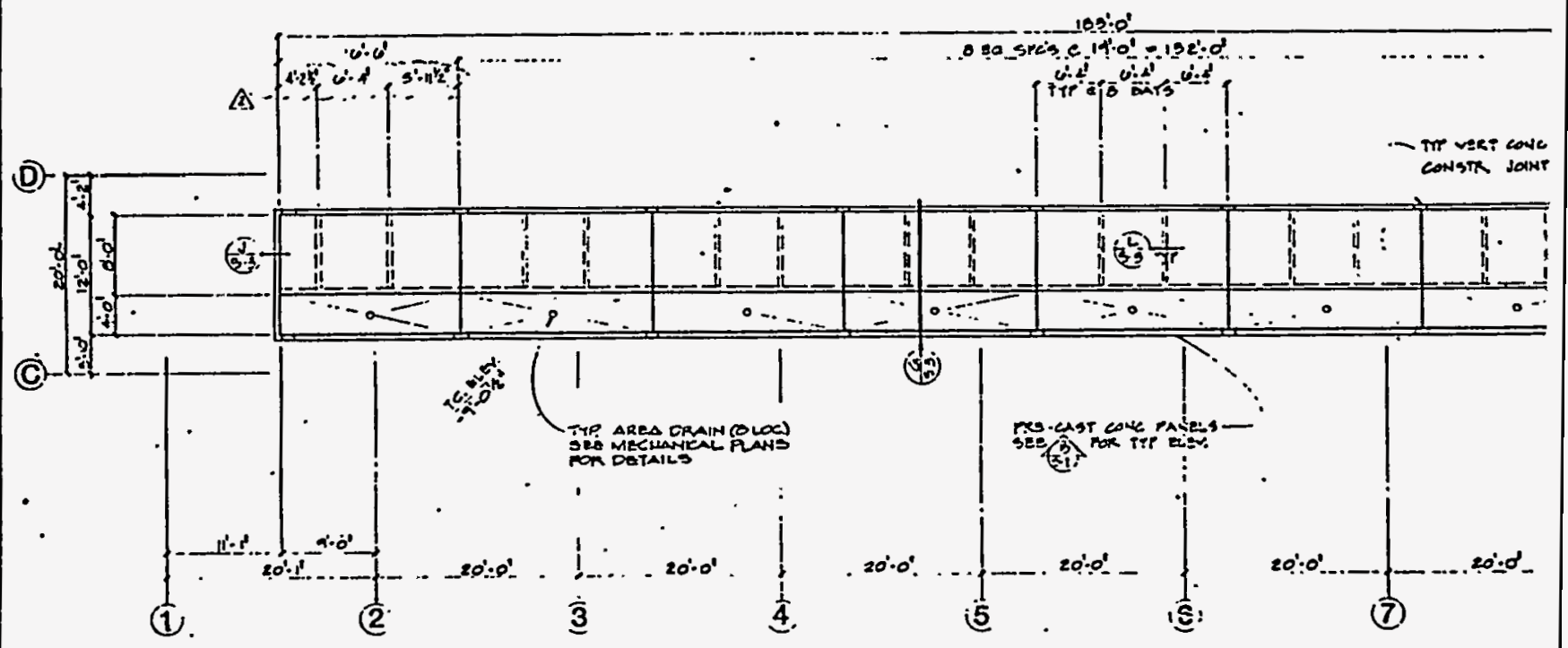

CULVERT FOUNDATION PLAN 


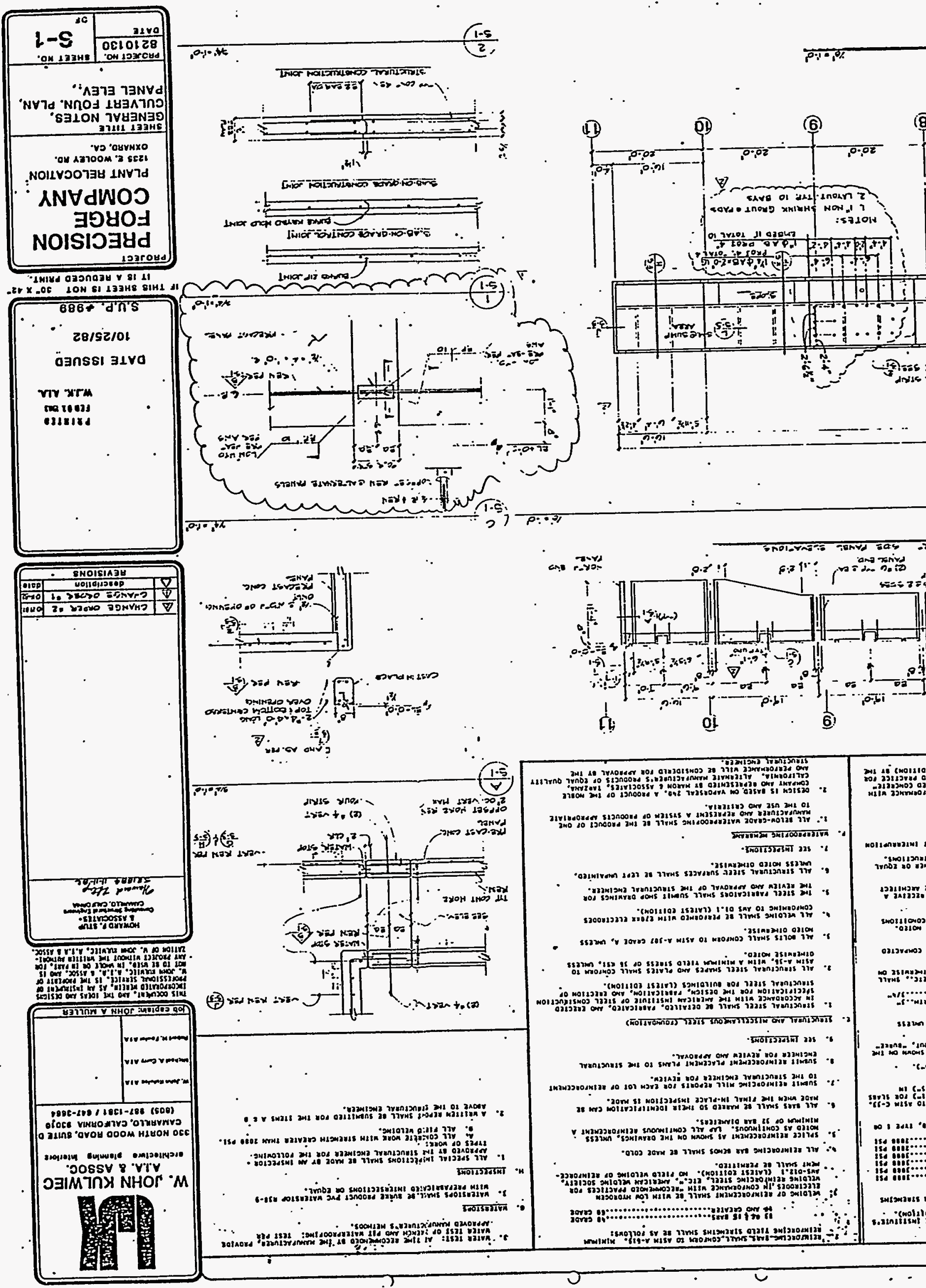



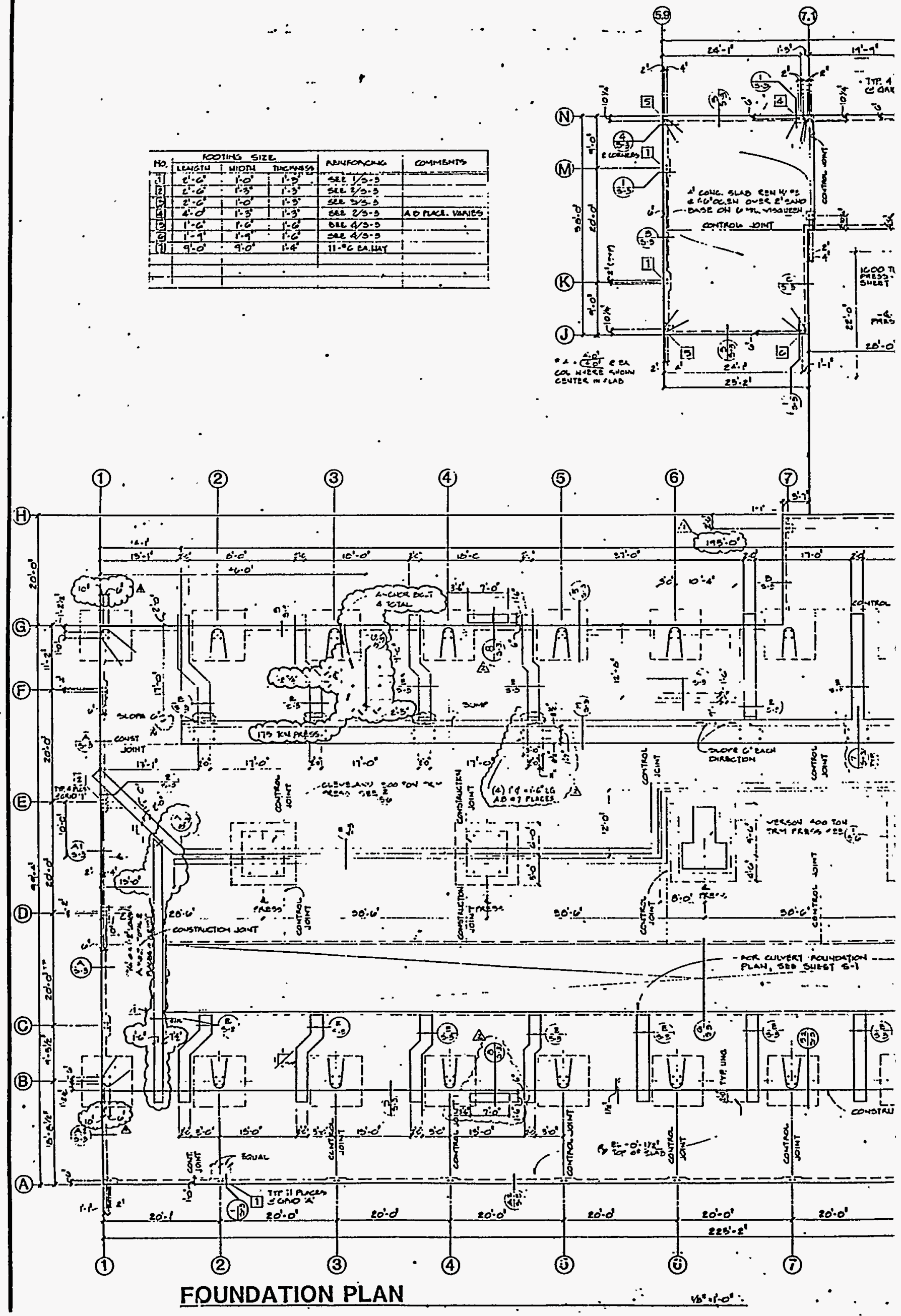


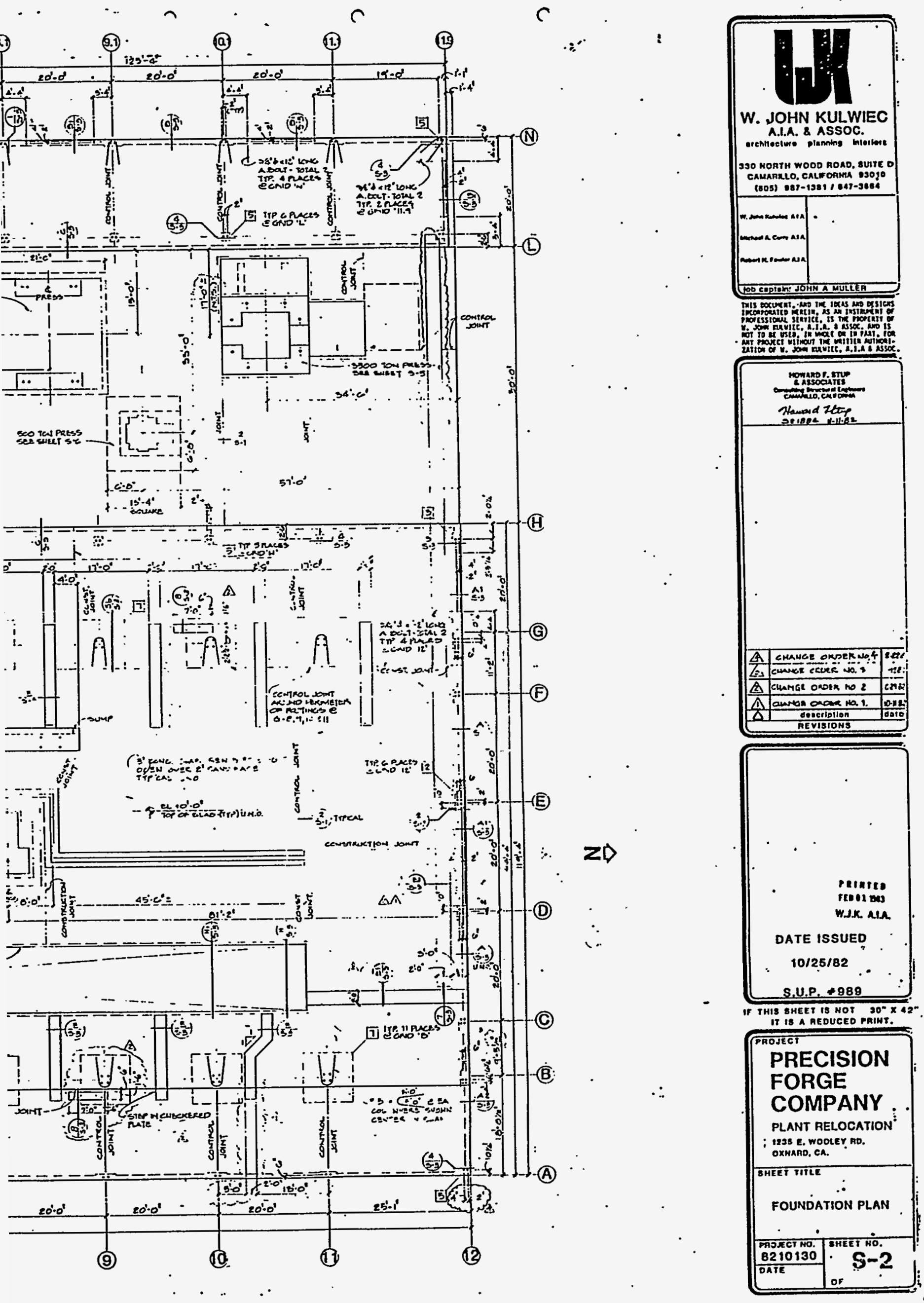




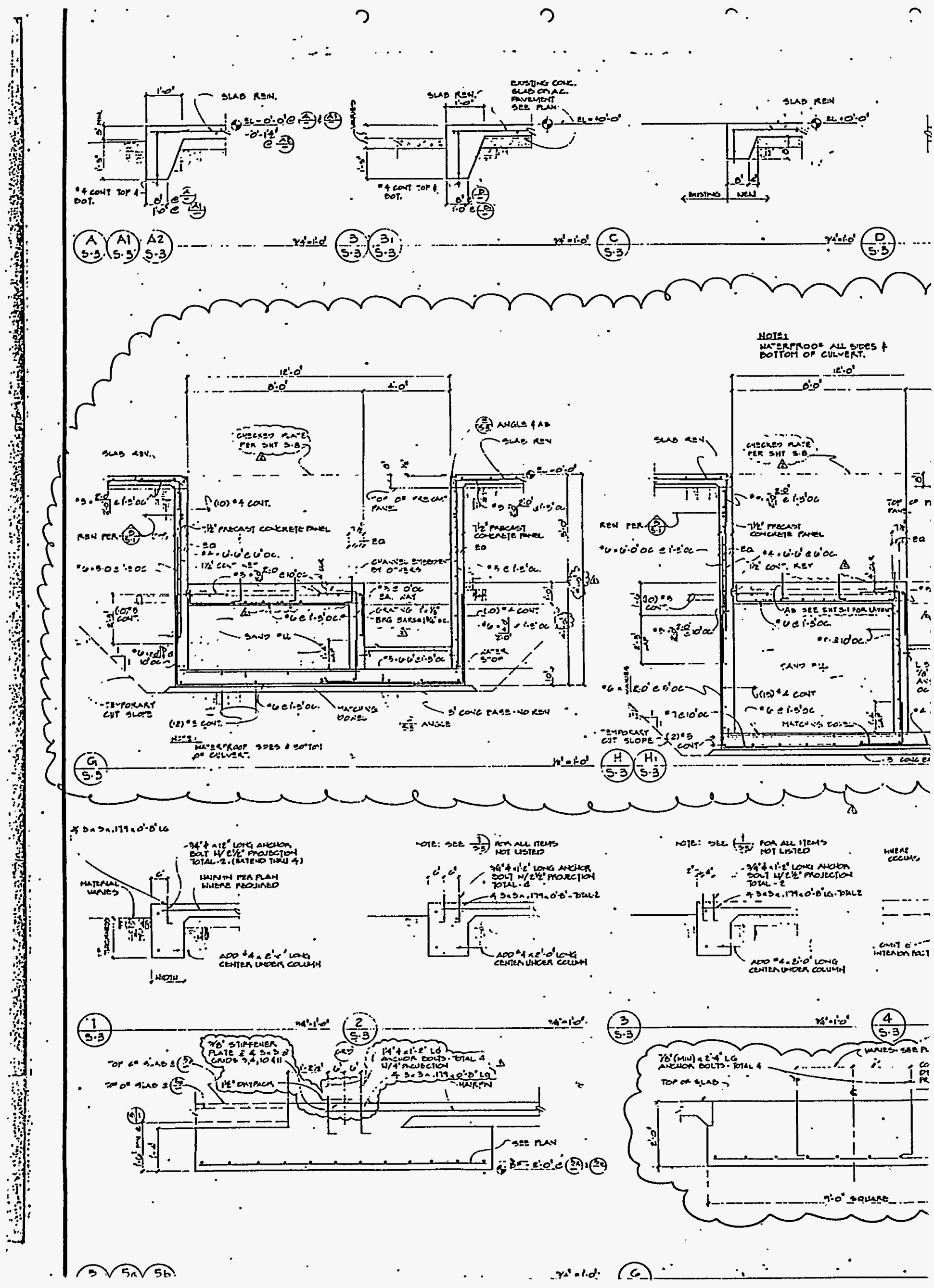




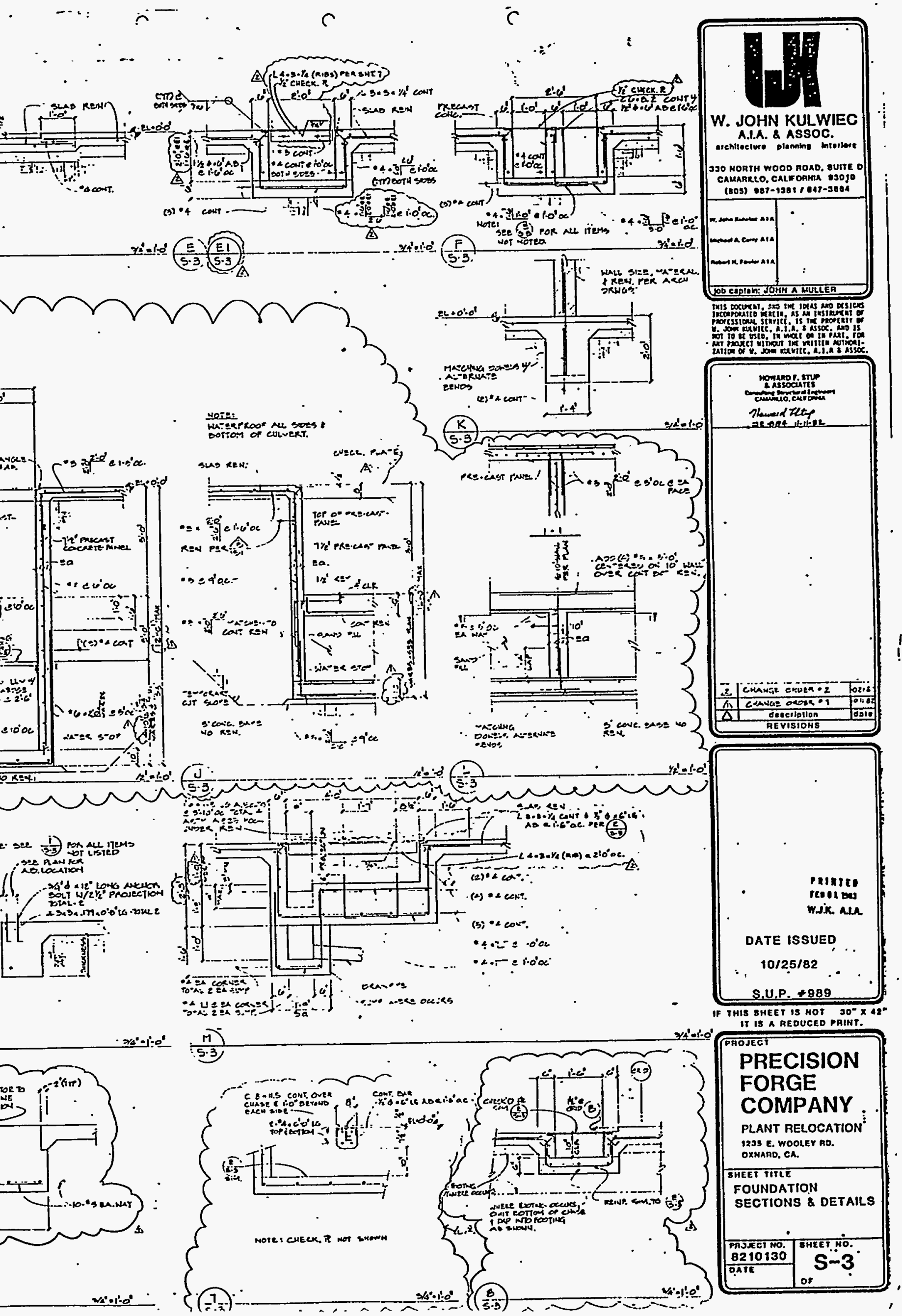




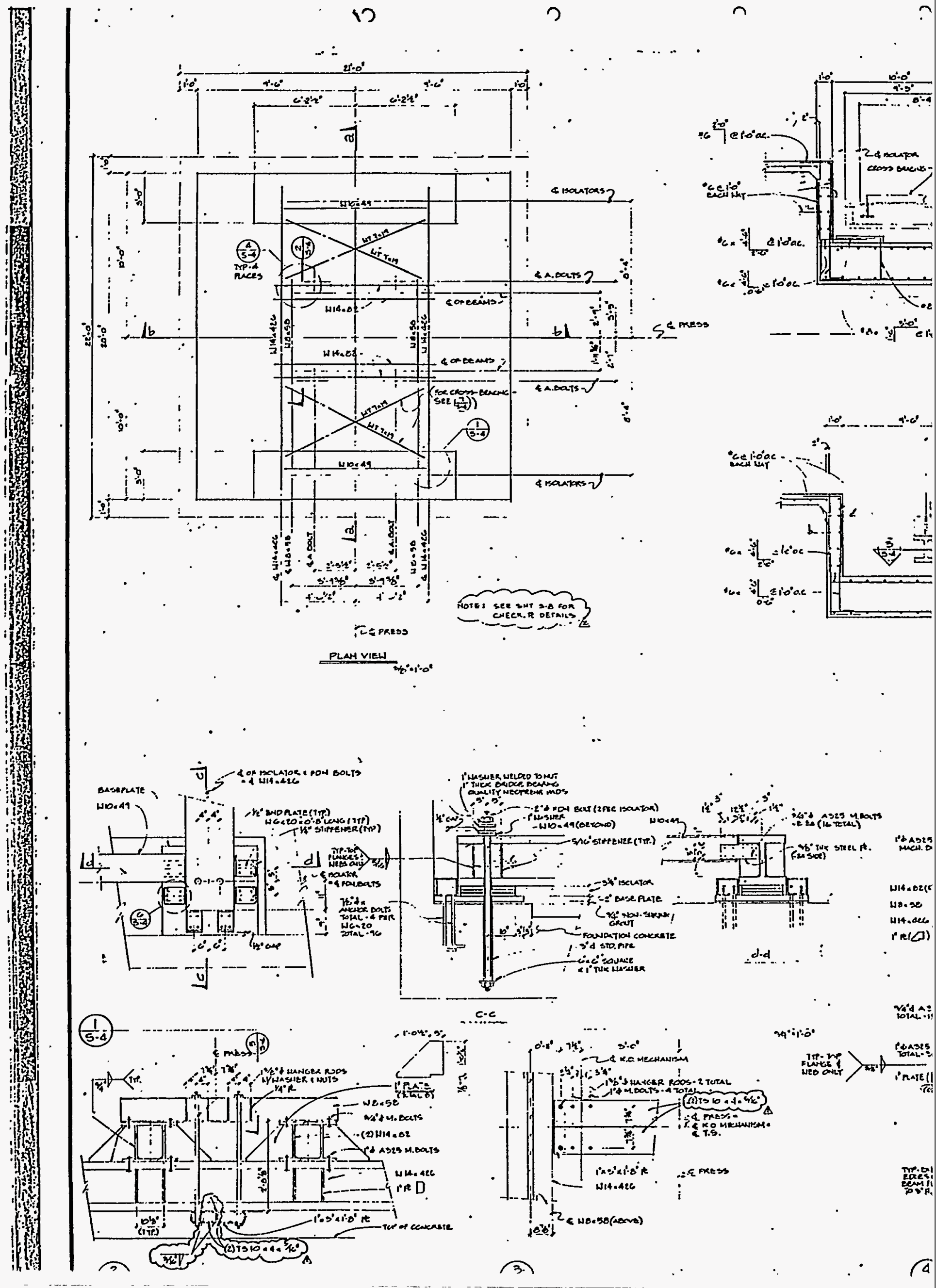




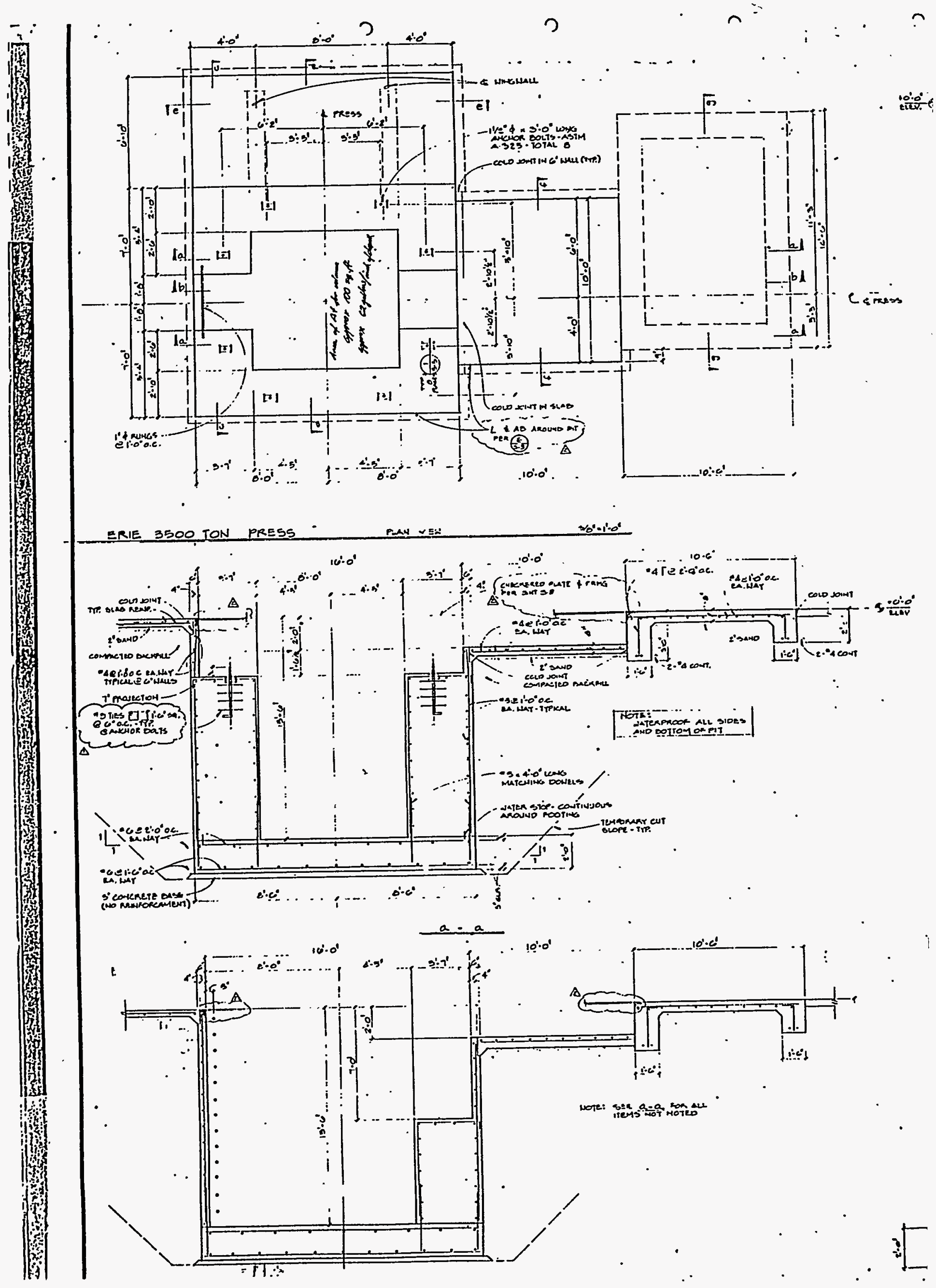




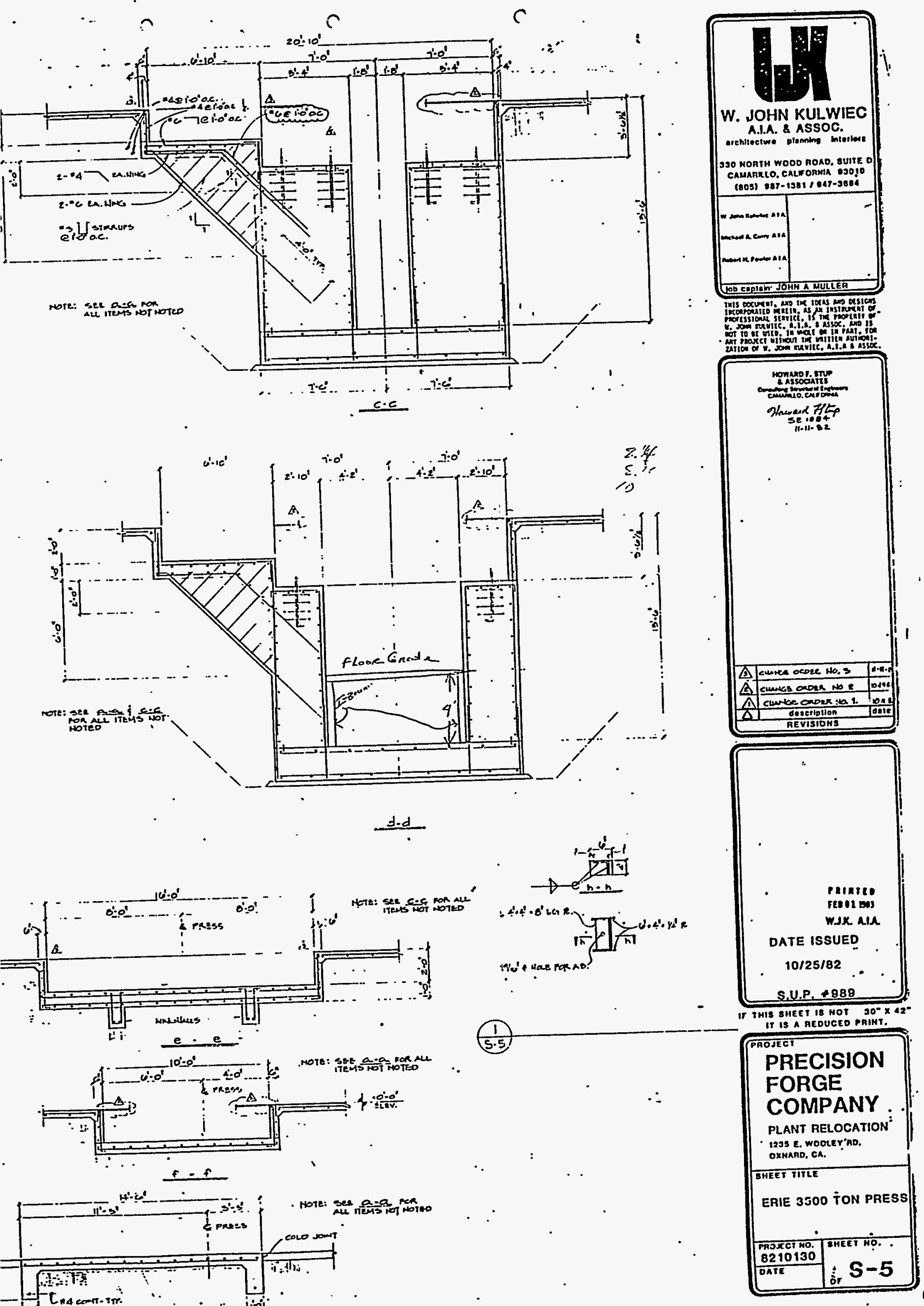




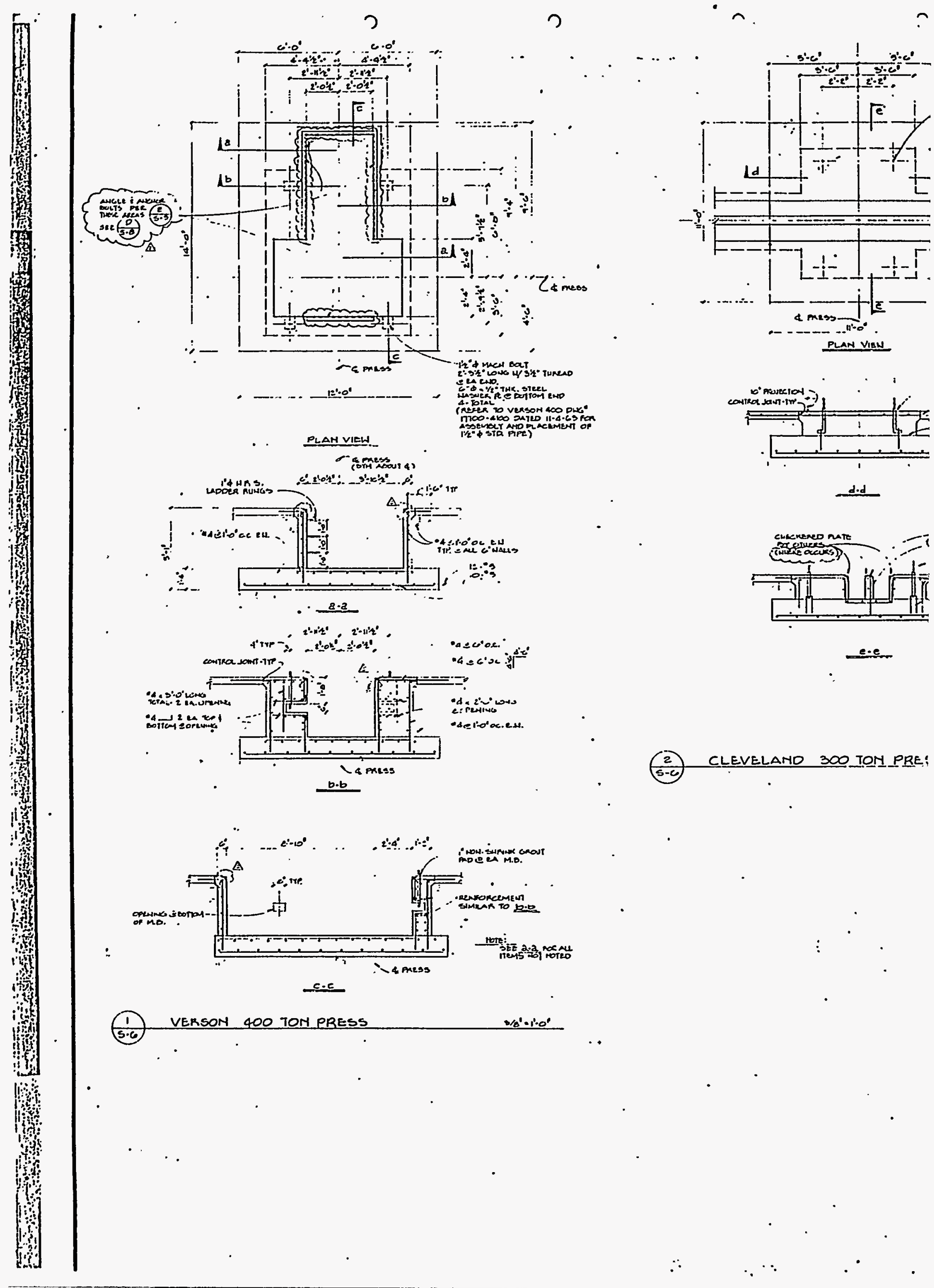




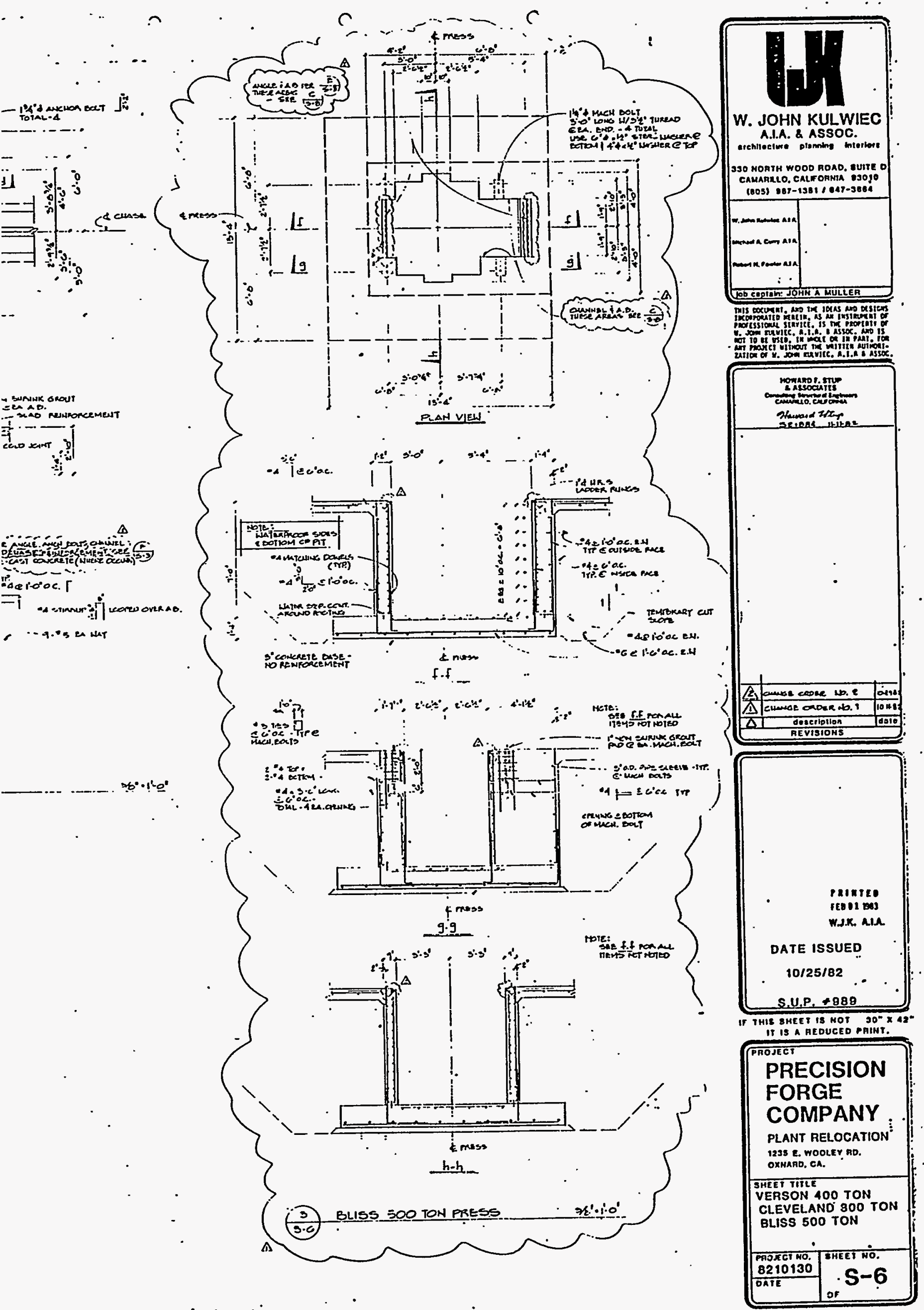




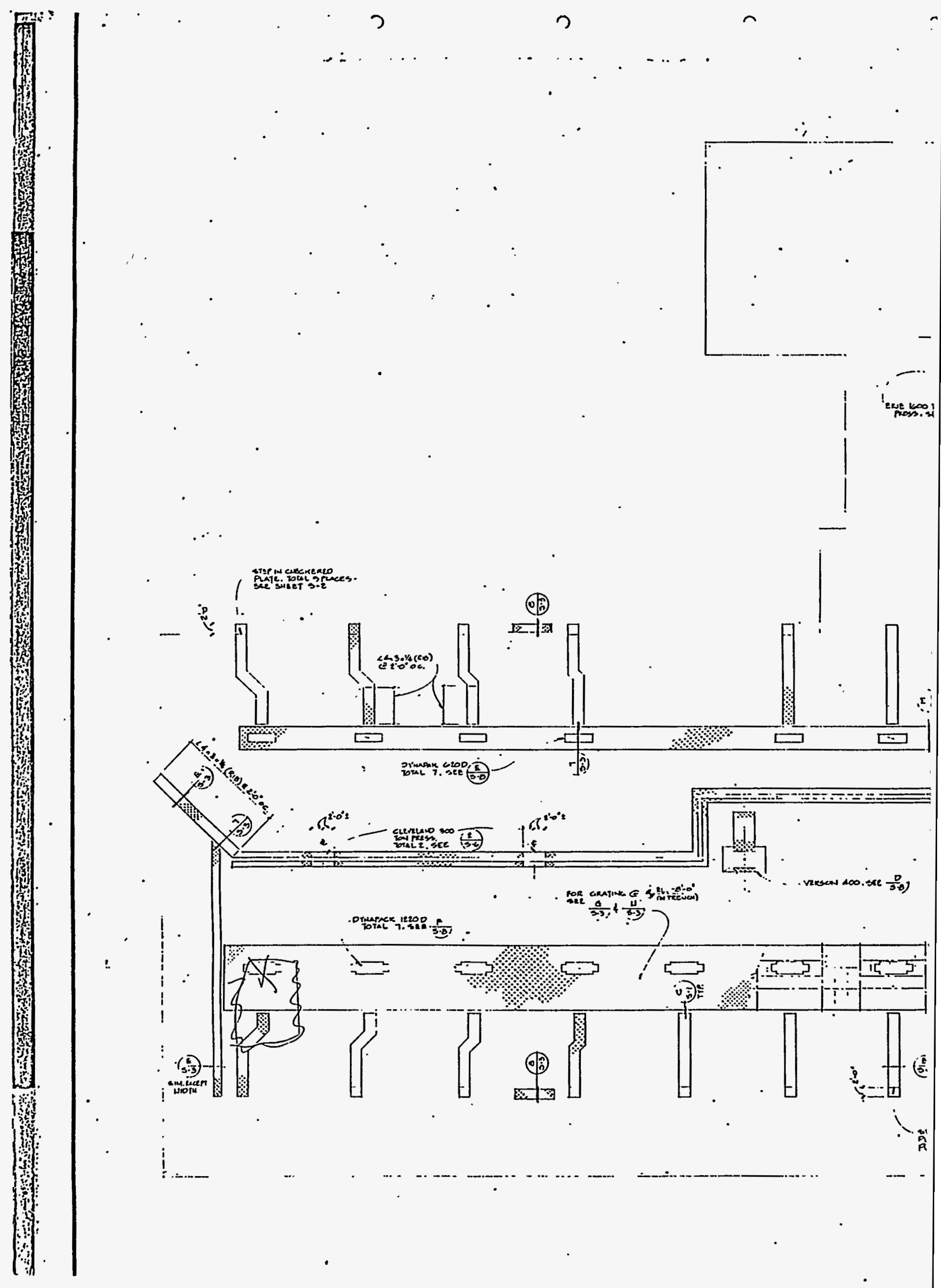




\section{Appendix F}

Hazardous Materials Map

\&

Hazardous Materials Chemical Descriptions 
FOR ADMINSTRATIVE AGENCY USE :

DATE RECEIVED :

FACILITY ID \# :

\section{CHEMICAL DESCRIPTION}

Common Name :OXYGEN

Chemical Name :LLUID OXYGEN

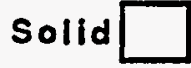

Liquid $\mathrm{X}$ Gas

Trade Secret

Radioactiv $\square$ (If radiocative :

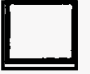

Pure $\mathrm{X}$ Mixture $\square$ Waste

o curries)
CAS \# : 7782-44-7

UN/NA \# : $1072 / 1073$

If waste is checked,

Annual Amount

Generated :

\section{WASTE CLASSIFICATION :}

Toxic $\square$ Ignitable $x$ Corrosive $\square$ Reactive $\square$ Extremely Hazardous $\square$

PHYSICAL AND HEALTH PHYSICAL

HAZARD CATEGORIES : Fire $X$ Reactive $\square$

Sudden Pressure Releası

UNITS OF MEASURE

AMOUNT \& Gals.

TIME AT

FACILITY :<smiles>[13CH3][13CH]1CCC1</smiles>

,Cu. Ft. $\mathrm{X}$
Maximum Average
Daily Amount Daily Amount $\frac{13,500}{9,000}$ \# Days on Site : 9,000 Largest Containe (Amount) :
Immediate Health Delayed Health

(Acute) $x$ (Chronic) $x$

\section{HEALTH}

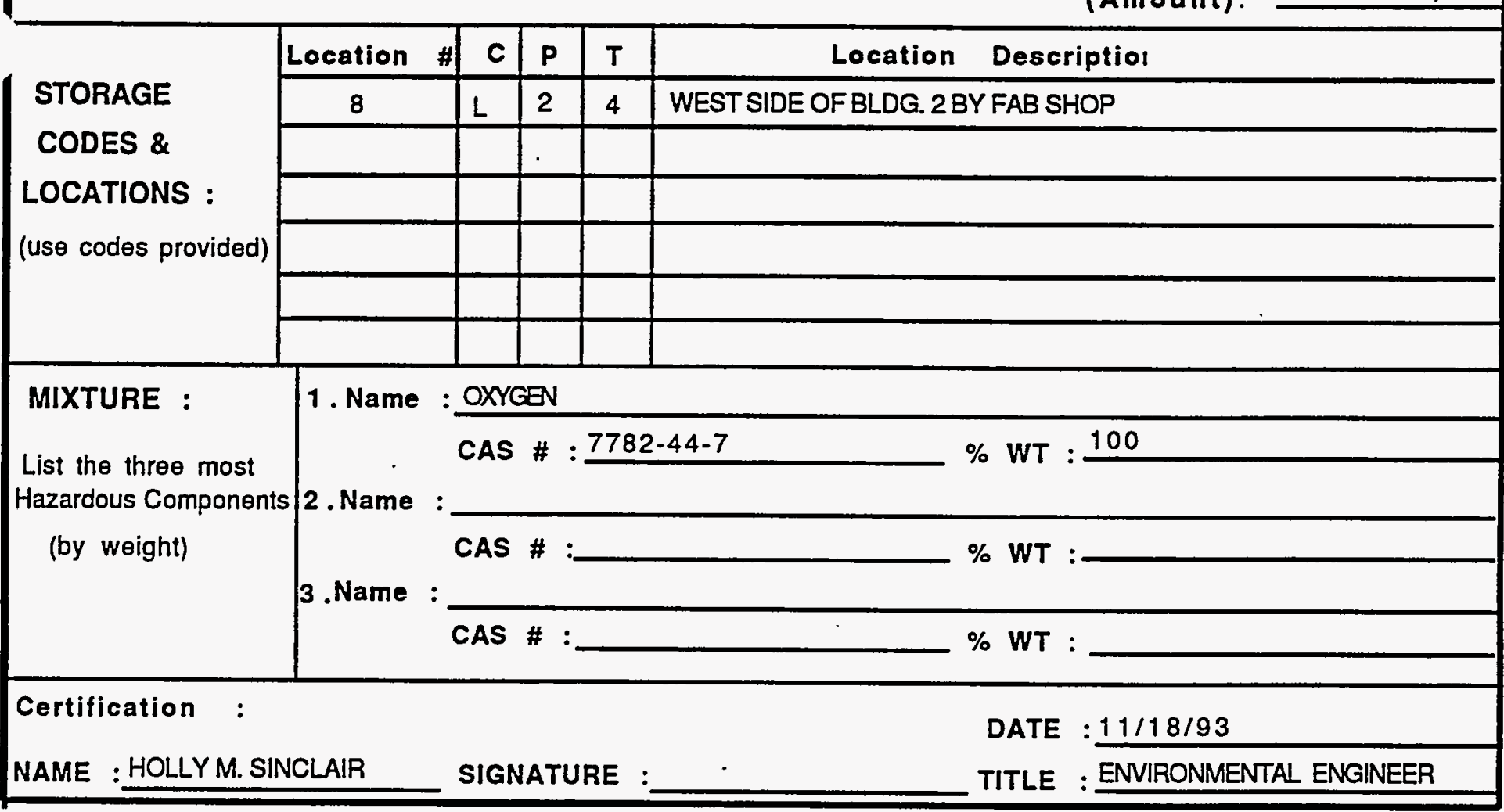




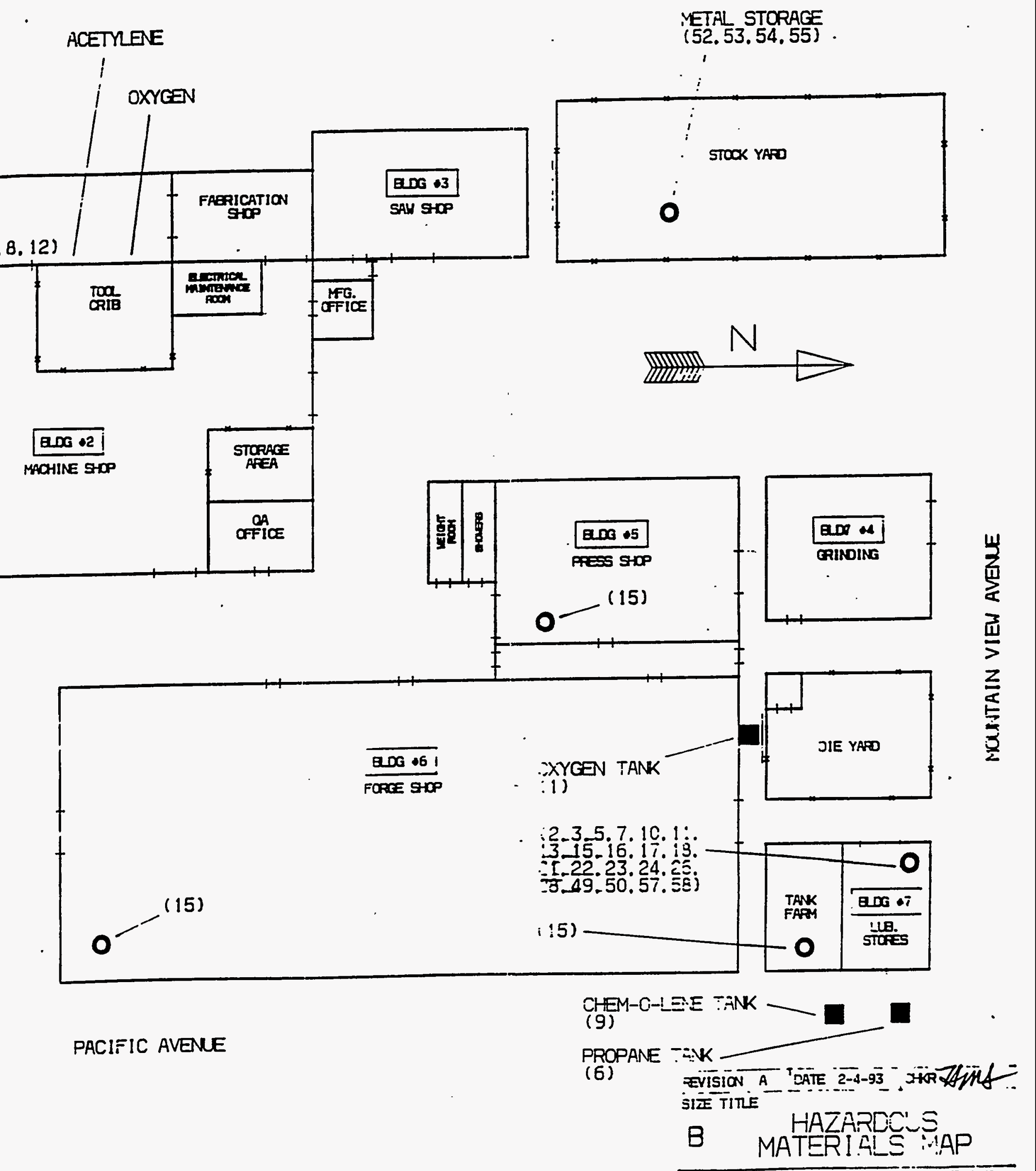




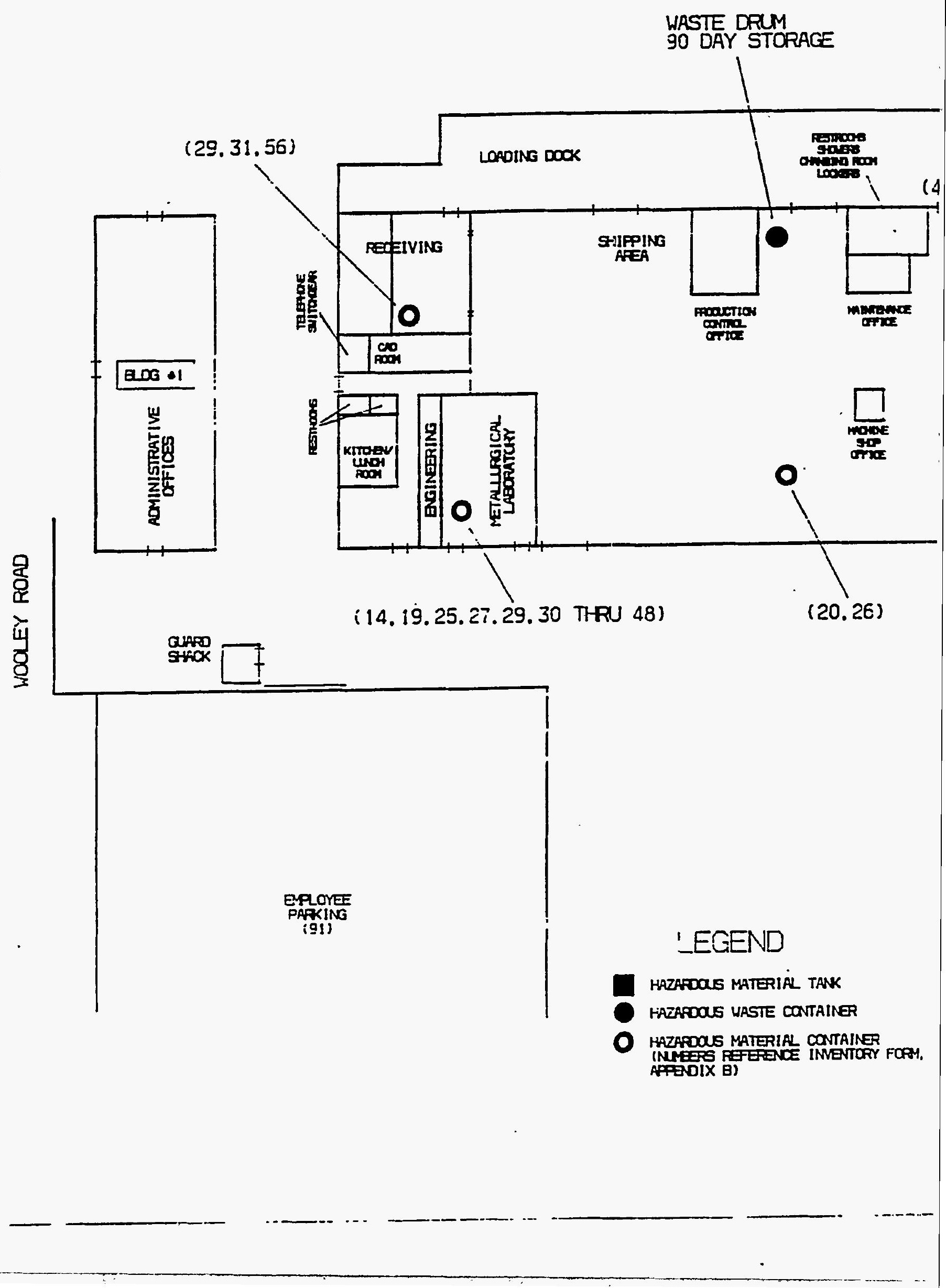




\section{CHEMICAL DESCRIPTION}

Common Name :MOBIL DIESEL FUEL CAS \# : $68334-30-5$

Chemical Name

PETROLEUM DISTTLATE

UN/NA \# : 1268

Solid $\square$

Liquid $X$ Gas

Pure $x$

Mixture $\square$ Waste

Trade Secret Radioactiv $\square$ (If radiocative :

o Curries)

If waste is checked, Annual Amount

WASTE CLASSIFICATION :

Toxic $X$ Ignitable $X$ Corrosive $\square$ Reactive $\square$ Extremely Hazardous $\square$

PHYSICAL AND HEALTH.

HAZARD CATEGORIES : FIre $X$ Reactive

Sudden

Pressure Releas!

\section{PHYSICAL}

UNITS OF MEASURE

AMOUNT \& Gals. $X$

TIME AT

FACILITY :

Cu. Ft.

Lbs.

Maximum

Average
Daily Amount

Daily Amount
55 \# Days on Site : 365

30 Largest Container on-sit (Amount):
Delayed Health

(Chronic)
HEALTH Immediate Health

(Acute)

\begin{tabular}{l}
$\begin{array}{l}\text { STORAGE } \\
\text { CODES \& } \\
\text { LOCATIONS : } \\
\text { (use codes provided) }\end{array}$ \\
\cline { 2 - 8 } \\
\cline { 2 - 7 }
\end{tabular}


FOR ADMINSTRATIVE AGENCY USE :

DATE RECENED:

FACILITY ID \# :

\section{CHEMICAL DESCRIPTION}

Common Name :SHELL TELLUS T OlL 100 CAS \# :

Chemical Name :PETROLEUMHYDROCARBONS UN/NA \# :

Solid $\square$ Liquid $x$ Gas $\square$ Pure $\square$ Mixture $x$ Waste Trade Secret $\square$ Radioactiv $\square$ (If radiocative :

o curries)

If waste is checked, Annual Amount WASTE CLASSIFICATION :

Toxic $X$ Ignitable $\square$ Corrosive $\square$ Reactive $\square$ Extremely Hazardous $\square$

PHYSICAL AND HEALTH HAZARD CATEGORIES
PHYSICAL Fire $\square$ Reactive Sudden Pressure

\section{Releas}

UNITS OF MEASURE

AMOUNT \& Gals. $X$ TIME AT

FACILITY :

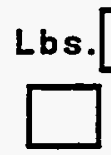

Maximum Average
Daily Amount Daily Amount
440 \# Days on Site : 385 Largest Container on-sit

(Amount):
Delayed Health

(Chronic)
HEALTH

Immediate Health
(Acute) $\square$ (Chronic) $\square$

\begin{tabular}{|c|c|c|c|c|c|}
\hline \multirow{5}{*}{$\begin{array}{l}\text { STORAGE } \\
\text { CODES \& } \\
\text { LOCATIONS : } \\
\text { (use codes provided) }\end{array}$} & Location & C & $\mathbf{P}$ & $T$ & Descriptiol \\
\hline & 7 & $D$ & 1 & 4 & DRUM STORAGE BUILDING \\
\hline & & & & & \\
\hline & & & & & \\
\hline & & & & & \\
\hline & & & & & \\
\hline \multirow{5}{*}{$\begin{array}{l}\text { MIXTURE : } \\
\text { List the three most } \\
\text { Hazardous Components } \\
\text { (by weight) }\end{array}$} & \multicolumn{5}{|c|}{ 1. Name : SOLVENT REFINED HYDROTREATED HEAVY PARAFFINIC DISTILLATE } \\
\hline & 2. Name: & $\begin{array}{l}\text { CAS } \\
\text { SOLI }\end{array}$ & $\#$ & 647 & $\begin{array}{l}\frac{2.54-7}{\text { DHYDROTREATED RESIDUAL OIL }} \text { WT }: 80.85 \\
\end{array}$ \\
\hline & $t^{2}$ & CAS & $\#$ & 247 & $\%$ WT: $: 10-15$ \\
\hline & \multirow[t]{2}{*}{ 3. Name } & ADDI & TIVE & $\mathrm{ACK}$ & \\
\hline & & CAS & \# & & - $\%$ WT $: \leq 5$ \\
\hline \multirow{2}{*}{\multicolumn{2}{|c|}{$\begin{array}{l}\text { Certification : } \\
\text { NAME : HOLLYM.SINCLAIR }\end{array}$}} & & & & DATE : $11 / 18 / 93$ \\
\hline & & SIGI & |AT & & TITLE : ENVIRONMENTAL ENGINEER \\
\hline
\end{tabular}


FOR ADMINSTRATIVE AGENCY USE :

DATERECEIVED :

FACILITY ID \# :

\section{CHEMICAL DESCRIPTION}

Common Name :CARBONDIOXIDE, SOLID

CAS \# : $124-38.9$

Chemical Name :SAME UN/NA \# : 1845

Solid $x$ Liquid $\square$ Gas $\square$ Pure $X$ Mixture $\square$ Waste $\square$

Trade Secret $\square$ Radioactiv $\square$ (lf radiocative :

o curries)

If waste is checked, Annual Amount

WASTE CLASSIFICATION :

Toxic $X$ Ignitable $X$ Corrosive $\square$ Reactive $\square$ Extremely Hazardous $\square$

PHYSICAL AND HEALTH

PHYSICAL

HAZARD CATEGORIES : Fire $X$ Reactive $\square$ Sudden Pressure Releas, $X$

\begin{tabular}{|c|c|}
\multicolumn{3}{c}{ HEALTH } \\
Immediate Health & $\begin{array}{l}\text { Delayed Health } \\
\text { (Acute) } X\end{array}$ \\
\hline
\end{tabular}

UNITS OF MEASURE

AMOUNT \& Gals.

TIME AT

FACILITY :

Lbs. $\square$ Maximum

Cu. Ft. $x$

Average Daily Amount

280 \# Days on Site :

365

FACILITY : (a)

$x$

\begin{tabular}{l}
$\begin{array}{l}\text { STORAGE } \\
\text { CODES \& } \\
\text { LOCATIONS : } \\
\text { (use codes provided) }\end{array}$ \\
\cline { 2 - 8 } \\
\cline { 2 - 7 }
\end{tabular}

TI

(Amount):

280 
FOR ADMINSTRATIVE AGENCY USE :

DATE RECEIVED :

FACILITY ID \# :

\section{CHEMICAL DESCRIPTION}

Common Name :MOBIL DTE OIL HEAVY

CAS \# :

Chemical Name :REFINEDMINERAL OIL UN/NA \# :

Solid $\square$

Liquid $X$ Gas

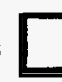

Trade Secret

Radioactiv

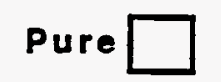

(If radiocative :
Mixture $x$ Waste

o Curries)
If waste is checked,

Annual Amount

Generated :

\section{WASTE CLASSIFICATION :}

Toxic $x$ Ignitable $\square$ Corrosive $\square$ Reactive $\square$ Extremely Hazardous

PHYSICAL AND HEALTH PHYSICAL

HAZARD CATEGORIES : Fire $\square$ Reactive $\square$

Sudden Pressure Releas,
HEALTH

Immediate Health Delayed Health

(Acute) $\square$ (Chronic)

\section{UNITS OF MEASURE}

AMOUNT \& Gals. $X$

TIME AT

FACILITY :

Cu. . Ft.

Lbs.

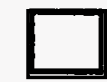

Maximum Daily Amount

Average Daily Amount
55 \# Days on Site :

40 Largest Container on-sit

(Amount):

\section{STORAGE}

CODES \&

LOCATIONS :

(use codes provided)

\begin{tabular}{|c|c|c|c|}
\hline Location \# & $\mathbf{C}$ & $\mathbf{P}$ & $\mathbf{T}$ \\
\hline 7 & $\mathrm{D}$ & 1 & 4 \\
\hline & & & \\
\hline & & & \\
\hline & & & \\
\hline & & & \\
& & & \\
\hline
\end{tabular}

MIXTURE :

1. Name : REFINED MINERAL OILS

CAS \# : SEQ-73-1

$\%$ WT $: \geq 95$

List the three most

Hazardous Components

(by weight)

2. Name : ADDITIVES/OTHERS

CAS \# :

$\% W T: \leq 5$

3. Name :

CAS \# : $\%$ WT :

Certification :

NAME : HOLLYM.SINCLAIR SIGNATURE :

DATE : $11 / 18 / 93$

TITLE : ENVIRONMENTAL ENGINEER 
FOR ADMINSTRATIVE AGENCY USE :

DATE RECEIVED :

FACILITY ID \# :

\section{CHEMICAL DESCRIPTION}

Common Name :PROPANE

Chemical Name :PROPANE

Solld

Liquid $X$ Gas

Trade Secret Radioactiv $\square$ (If radiocative :

Pure $x$ Mixture $\square$ Waste

o curries)
CAS \# : 74-98-6

UN/NA \# : 1075

If waste is checked, Annual Amount

Generated :

\section{WASTE CLASSIFICATION :}

Toxic $x$ Ignitable $x$ Corrosive $\square$ Reactive $\square$ Extremely Hazardous $\square$

\section{PHYSICAL AND HEALTH \\ PHYSICAL}

HAZARD CATEGORIES : Fire $X$ Reactive Sudden Pressure Releas $X$

\section{UNITS OF MEASURE}

AMOUNT \& Gals. $X$

TIME AT

FACILITY :
Lbs.

Cu. Ft.
Maximum

Average Dally Amount
1,125 \# Days on site : 800 Largest Container on-sit

(Amount):
Delayed Health

(Chronic) $x$

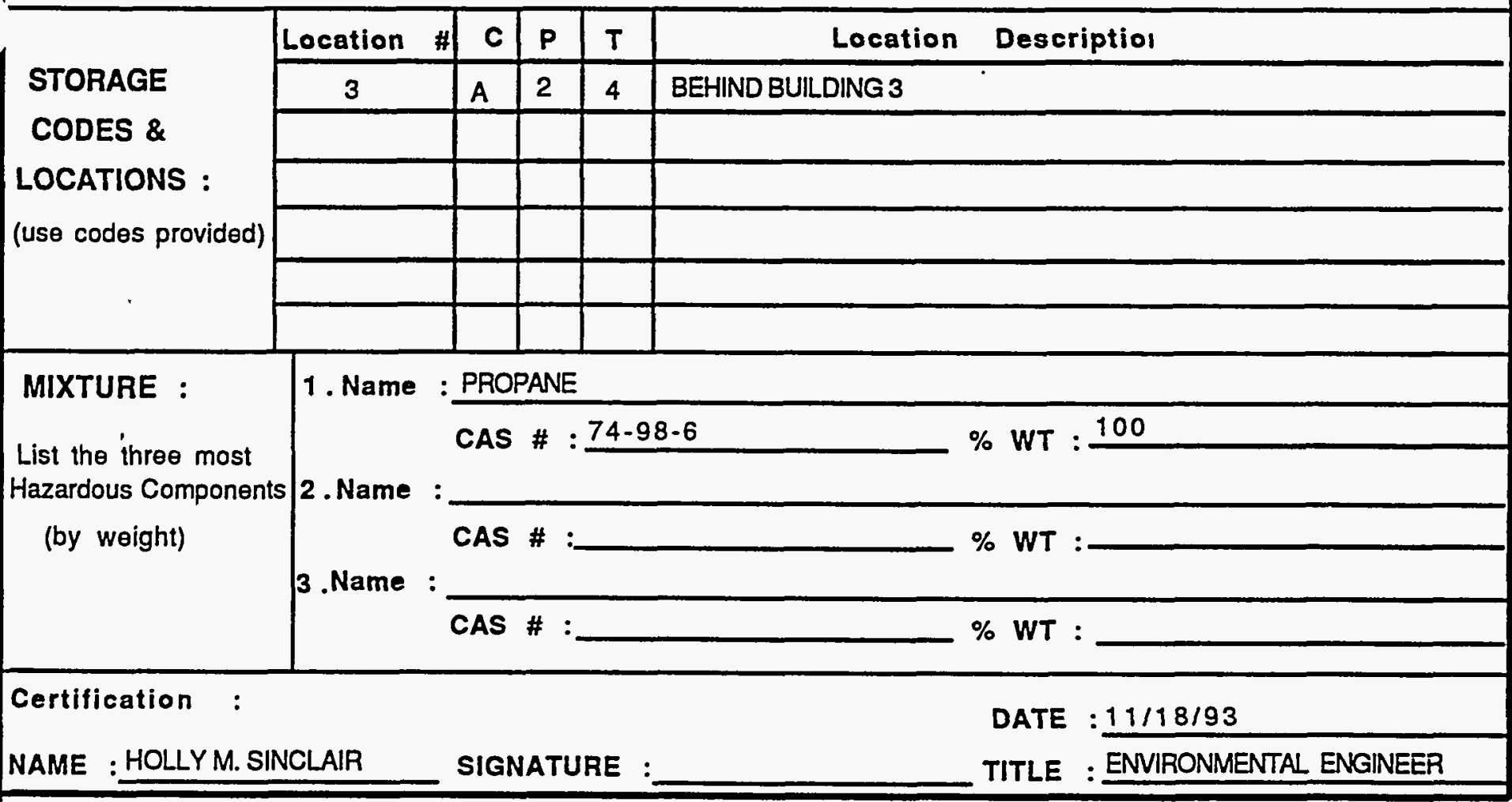




\section{CHEMICAL DESCRIPTION}

Common Name :SHELL OMALA OIL 220 CAS \# :

Chemical Name :PETROLEUMHYDROCARBONS UN/NA \# :

Solid $\square$ Liquid $X$ Gas $\square$ Pure $\square$ Mixture $X$, Waste $\square$

Trade Secret $\square$ Radioactiv $\square$ (lf radiocative :

O curries)
If waste is checked, Annual Amount

Generated :

\section{WASTE CLASSIFICATION :}

Toxic $X$ Ignitable $\square$ Corrosive $\square$ Reactive $\square$ Extremely Hazardous $\square$

\begin{tabular}{|c|c|c|c|c|}
\hline PHYSICAL AND HEALTH & PHYSICAL & \multicolumn{3}{|c|}{ HEALTH } \\
\hline HAZARD CATEGORIES : 'Fire & Reactive & Immediate & Health & Delayed \\
\hline Sudden $P$ & Pressure Releası & (Acute) & & (Chronic) \\
\hline
\end{tabular}

UNITS OF MEASURE

AMOUNT \&

TIME AT

Gais. $X$

FACILITY :

Cu. Ft.

Lbs.

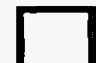

Maximum Daily Amount

55 \# Days on Site :

\begin{tabular}{l}
$\begin{array}{l}\text { STORAGE } \\
\text { CODES \& } \\
\text { LOCATIONS : } \\
\text { (use codes provided) }\end{array}$ \\
\cline { 2 - 7 } \\
\cline { 2 - 7 }
\end{tabular}


FOR ADMINSTRATIVE AGENCY USE :

DATE RECEIVED :

FACILITY ID \# :

\section{CHEMICAL DESCRIPTION}

\section{Common Name :ARGON}

Chemical Name :LIQUIDARGON

Solid $\square$

Trade Secret
Liquid $X$ Gas $\square$ Radioactiv $\square$ (If radiocative :
Pure $x$ Mixture $\square$ Waste

o curries)
CAS \# : 7440-37-1

UN/NA \# : UN 1006

If waste is checked, Annual Amount

Generated : 0

\section{WASTE CLASSIFICATION :}

Toxic $\square$ Ignitable $\square$ Corrosive $\square$ Reactive $\square$ Extremely Hazardous $\square$

\begin{tabular}{|c|c|c|c|c|}
\hline PHYSICAL AND HEALTH & PHYSICAL & \multicolumn{3}{|c|}{ HEALTH } \\
\hline HAZARD CATEGORIES : Fire & Reactive & Immediate & Health & Delayed Health \\
\hline Sudden & Pressure Releası & (Acute) & $\mathbf{x}$ & (Chronic) $x$ \\
\hline
\end{tabular}

UNITS OF MEASURE

AMOUNT \& Gals.

TIME AT

FACILITY :
Lbs. $\square$

Maximum

Average Daily Amount
336 \# Days on site : 336 Largest Container on-sit

(Amount):

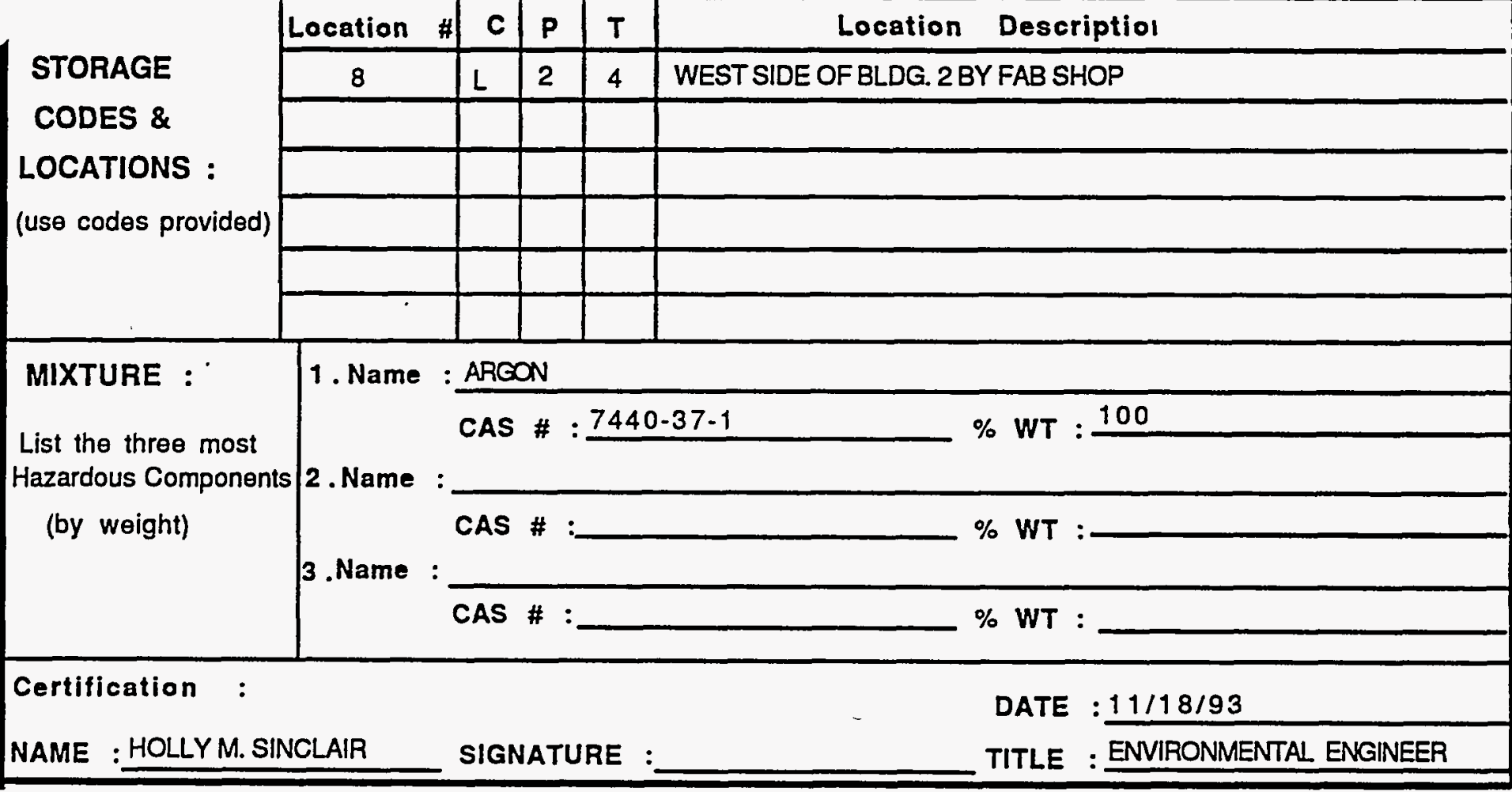


FOR ADMINSTRATIVE AGENCY USE :

DATE RECEIVED :

FACILITY ID \# :

\section{CHEMICAL DESCRIPTION}

Common Name :CHEM-OLENE PLUS FUEL GAS

CAS \# : 74-98-6

Chemical Name :PROPANEMIXTURE

UN/NA \# : 1954

solid $\square$

Trade Secret

\section{Liquid $X$ Gas}

Radioactiv $\square$ (If radiocative :
Mixture $\mathrm{X}$ Waste

o curries)
If waste is checked,

Annual Amount

Generated :

\section{WASTE CLASSIFICATION :}

Toxic $X$ Ignitable $x$ Corrosive $\square$ Reactive $\square$ Extremely Hazardous $\square$

PHYSICAL AND HEALTH PHYSICAL

HAZARD CATEGORIES : Fire $X$ Reactive Sudden Pressure Releas. $\mathrm{X}$

UNITS OF MEASURE

AMOUNT \& Gals. $X$ Lbs.

TIME AT

FACILITY :
Cu. Ft.

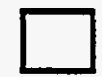

Maximum. Daily Amount Average Daily Amount
1,300 \# Days on Site : 1,150 Largest Container on-sit (Amount):
Delayed Health

(Chronic) $x$
HEALTH

Immediate Health $\begin{aligned} & \text { Delayed Health } \\ & \text { (Acute) } X\end{aligned}$
(Chronic) $X$

\begin{tabular}{|c|c|c|c|c|c|}
\hline \multirow{5}{*}{$\begin{array}{l}\text { STORAGE } \\
\text { CODES \& } \\
\text { LOCATIONS : } \\
\text { (use codes provided) }\end{array}$} & Location & C & $\mathbf{P}$ & $T$ & Descriptiol \\
\hline & 2 & A & 2 & 4 & East side of building 7 \\
\hline & & & & & Behind drum storage bldg. \\
\hline & & & & & \\
\hline & & & & & \\
\hline \\
\hline \multirow{5}{*}{$\begin{array}{l}\text { MIXTURE : } \\
\text { List the three most } \\
\text { Hazardous Components } \\
\text { (by weight) }\end{array}$} & \multicolumn{5}{|c|}{ 1. Name : PROPANE } \\
\hline & \multicolumn{5}{|c|}{$\begin{aligned} \text { CAS \# : } 74-98-6 \\
\text { 2. Name : ETHANE }\end{aligned}$} \\
\hline & \multicolumn{5}{|c|}{ CAS \#: $74-84-0$} \\
\hline & \multirow{2}{*}{ 3. Name : } & \multicolumn{4}{|c|}{ PROPYENE } \\
\hline & & \multicolumn{4}{|c|}{ CÁS \#: $115.07-1$} \\
\hline \multicolumn{6}{|c|}{$\begin{array}{ll}\text { Certification }: & \text { DATE }: \frac{11 / 18 / 93}{} \\
\end{array}$} \\
\hline NAME : HOLLYM.SIN & NCLAIR & \multicolumn{3}{|c|}{ SIGNATURE } & TITLE : ENVIRONMENTAL ENGINEER \\
\hline
\end{tabular}


FOR ADMINSTRATIVE AGENCY USE :

DATE RECEIVED :

FACILITY ID \# :

\section{CHEMICAL DESCRIPTION}

Common Name :MOBIL DTE OIL LIGHT

CAS \# :

Chemical Name :REFINED MINERALOILS

UN/NA \# :

solid $\square$

Liquid $\mathrm{X}$ Gas

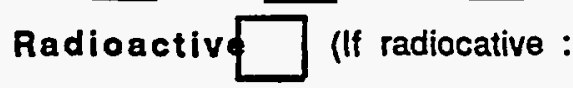

Mixture $x$ Waste

o curries)
If waste is checked, Annual Amount

Generated :

WASTE CLASSIFICATION :

Toxic $X$ Ignitable $\square$ Corrosive $\square$ Reactive $\square$ Extremely Hazardous $\square$

PHYSICAL AND HEALTH

HAZARD CATEGORIES : Fire $\square$ Reactive

Sudden Pressure

Releası

UNITS OF MEASURE

AMOUNT \&

TIME AT

FACILITY :

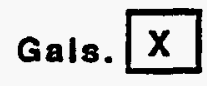

Cu. Ft.

Lbs.

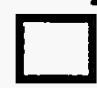

Maximum

Average Daily Amount
55 \# Days on Site :

365

30 Largest Container (Amount) :
Delayed Health

(Chronic)

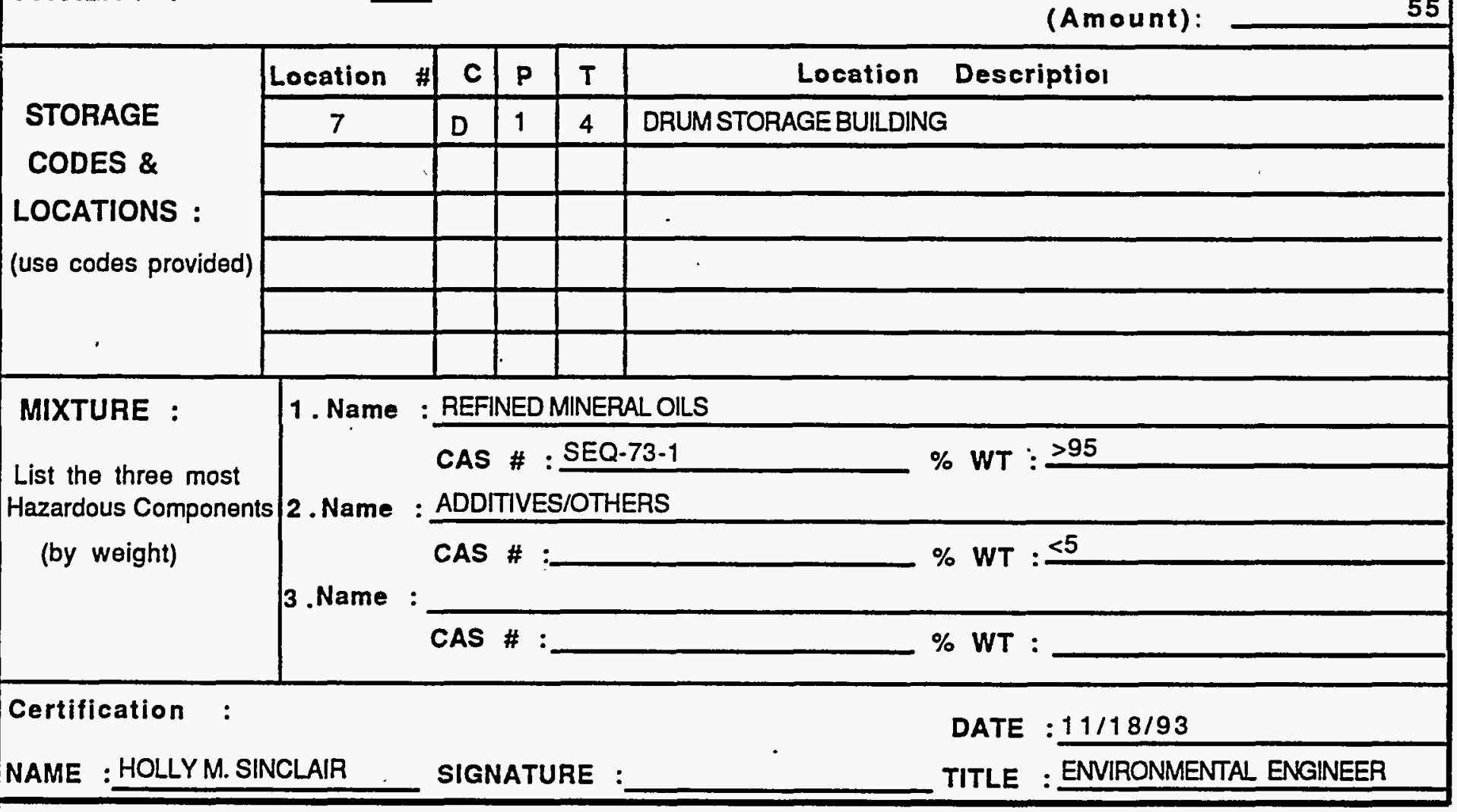


FOR ADMINSTRATIVE AGENCY USE :

DATE RECEIVED :

FACILITY ID \# :

\section{CHEMICAL DESCRIPTION}

Common Name :CUTZOL EDM 30 CAS \# : $8030-30-6$

Chemical Name :MACHINESHOP FUUD UN/NA \# : 1268

Solid $\square$

Trade Secret
Liquid $X$ Gas Radioactiv

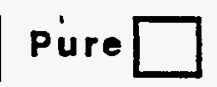

(If radiocative :
Mixture $x$ Waste

o curries)
If waste is checked,

Annual Amount

Generated : 0

\section{WASTE CLASSIFICATION :}

Toxic $x$ Ignitable $x$ Corrosive $\square$ Reactive $\square$ Extremely Hazardous $\square$

\begin{tabular}{|c|c|c|c|c|}
\hline \multirow{3}{*}{$\begin{array}{l}\text { PHYSICAL AND HEALTH } \\
\text { HAZARD CATEGORIES : }\end{array}$} & PHYSICAL & \multicolumn{3}{|c|}{ HEALTH } \\
\hline & Reactive & Immediate & Health & Delayed Health \\
\hline & 'ressure Releas & (Acute) & $\mathbf{x}$ & (Chronic) $x$ \\
\hline
\end{tabular}

\section{UNITS OF MEASURE}

AMOUNT \& Gals. $X$ Lbs. $\square$

TIME AT

Cu. Ft.

Maximum Average Daily Amount

55 \# Days on Site :

FACILITY :

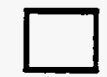

30 Largest Container

(Amount):

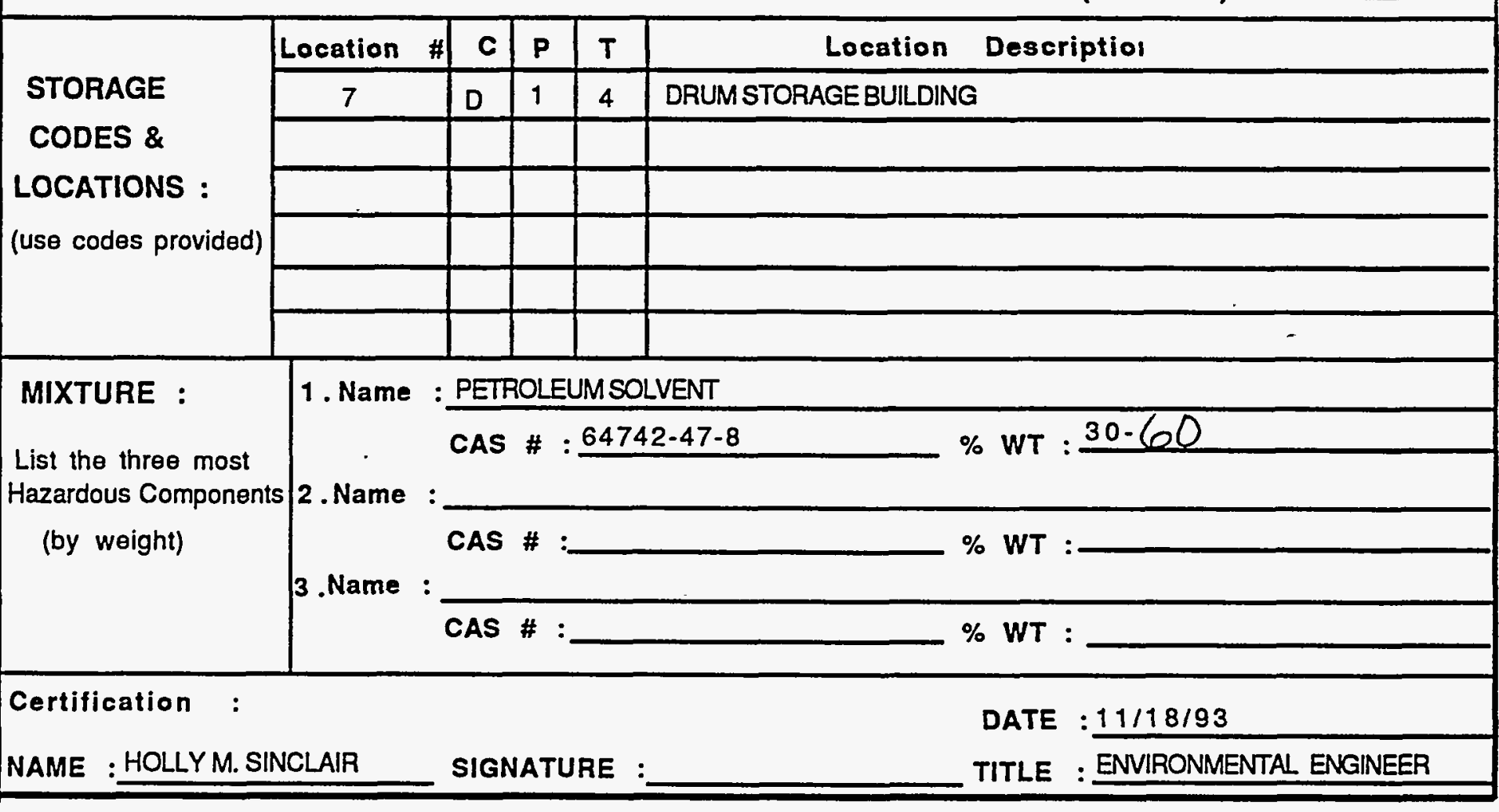


FOR ADMINSTRATIVE AGENCY USE :

DATE RECEIVED :

FACILITY ID \# :

\title{
CHEMICAL DESCRIPTION
}

Common Name :NITROGEN

Chemical Name :NITPOGEN

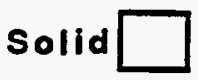

Trade secret
Liquid $x$ Gas

Radioactiv $\square$ (If radiocative :
CAS \# : 7727-37-9

UN/NA \# : 1977

If waste is checked, Annual Amount

Generated :

\section{WASTE CLASSIFICATION :}

Toxic $\square$ Ignitable $\square$ Corrosive $\square$ Reactive $\square$ Extremely Hazardous $\square$

PHYSICAL AND HEALTH

HAZARD CATEGORIES :

\section{PHYSICAL}

Fire $\square$ Reactive

Sudden Pressure Releas

\section{UNITS OF MEASURE}

AMOUNT \&

TIME AT Gals.

FACILITY :

Cu. Ft. $x$

Maximum Daily Amount

Average Daily Amount

O Curries)

\begin{abstract}
Hazardous
\end{abstract}

\section{HEALTH}

Immediate Health Delayed Health

(Acute) $x \quad$ (Chronic) $X$

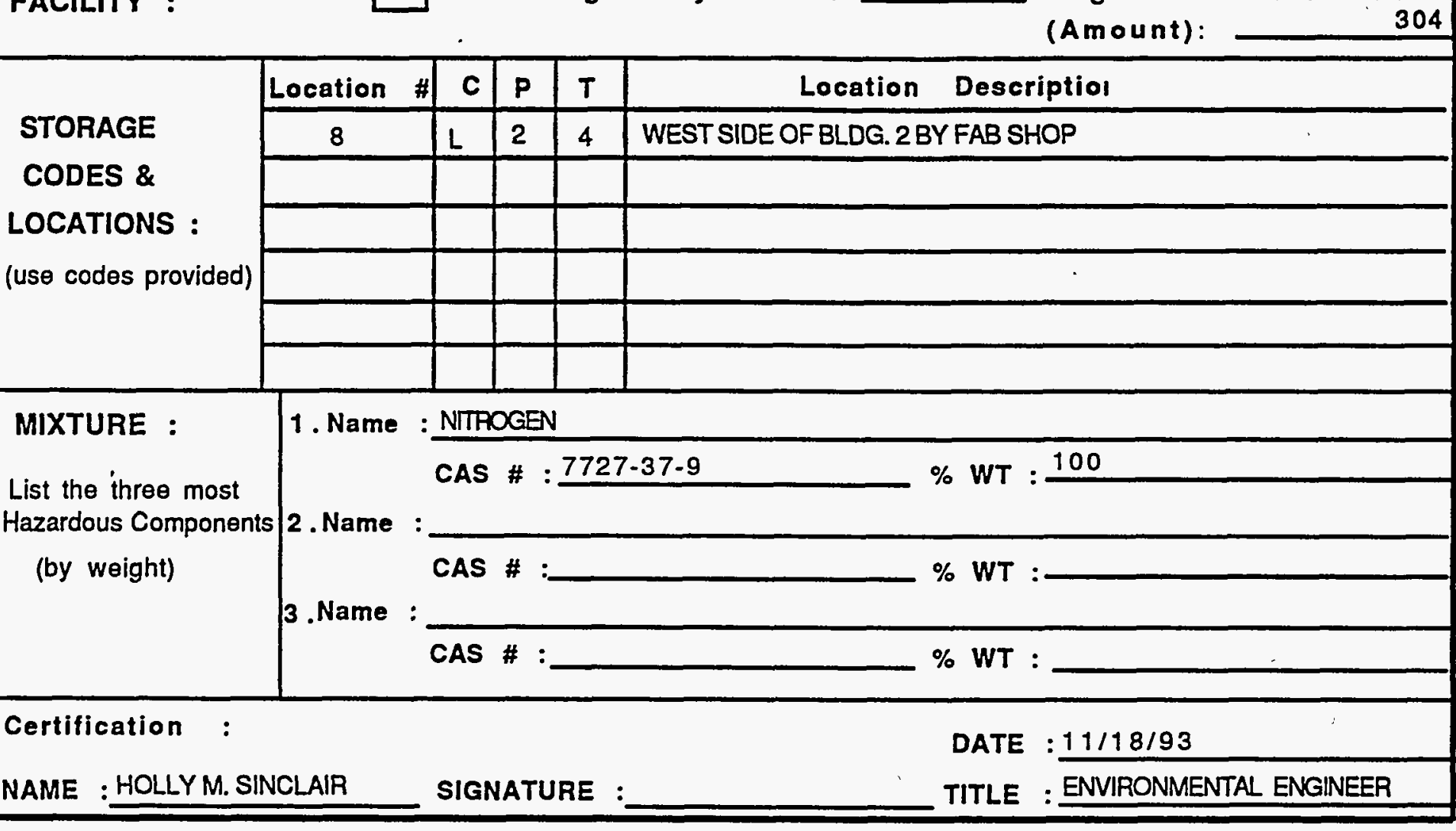


FACILITY ID \# :

\section{CHEMICAL DESCRIPTION}

Common Name :LUBRIZOL 5178

Chemical Name :Antiwear Hydraulic Oil Additive

Solld $\square$ Liquid $x$ Gas $\square$ Pure $\square$ Mixture $x$ Waste $\square$

Trade Secret $\square$ Radioactiv $\square$ (If radiocative :

Ocurries)

CAS \# :

UN/NA \# :

If waste is checked,

Annual Amount

\section{WASTE CLASSIFICATION :}

Toxic $X$ Ignitable $\square$ Corrosive $\square$ Reactive $\square$ Extremely Hazardous $\square$

PHYSICAL AND HEALTH

HAZARD CATEGORIES :
PHYSICAL

Fire $\square$ Reactive $\square$

HEALTH

Immediate Health Delayed Health (Acute) $X \quad$ (Chronic) $X$

\section{UNITS OF MEASURE.}

AMOUNT \& GaIs. $X$ Lbs. $\square$

TIME AT

FACILITY :

Cu. Ft.

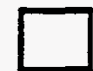

Maximum

Average Daily Amount

55 \# Days on site :

50 Largest Container on-sit

(Amount):

\begin{tabular}{l}
$\begin{array}{l}\text { STORAGE } \\
\text { CODES \& } \\
\text { LOCATIONS : } \\
\text { (use codes provided) }\end{array}$ \\
\cline { 2 - 8 } \\
\cline { 2 - 7 }
\end{tabular}


FOR ADMINSTRATIVE AGENCY USE :

DATE RECEIVED :

FACILITY ID \# :

\section{CHEMICAL DESCRIPTION}

Common Name :AMMONIUM HYDROXIDE

CAS \# : $1336-21-6$

Chemical Name :Ammonium Hydroxide UN/NA \# :

solid

Trade Secret
Liquid $X$ Gas

Radioactiv $\square$ (lf radiocative :
Mixture $\mathrm{X}$ Waste

o curries)
If waste is checked, Annual Amount

Generated :

0

WASTE CLASSIFICATION :

Toxic $\square$ Ignitable Corrosive $x$ Reactive $\square$

Extremely Hazardous $\square$

PHYSICAL AND HEALTH

\section{PHYSICAL}

HAZARD CATEGORIES : Fire $\square$ Reactive Sudden Pressure Releas

\section{UNITS OF MEASURE}

AMOUNT \&

TIME AT

FACILITY :

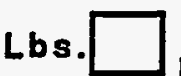

Cu. Ft.
Maximum Daily Amount

Average Daily Amount
HEALTH

Immediate Health Delayed Health

(Acute) $X \quad$ (Chronic) $X$

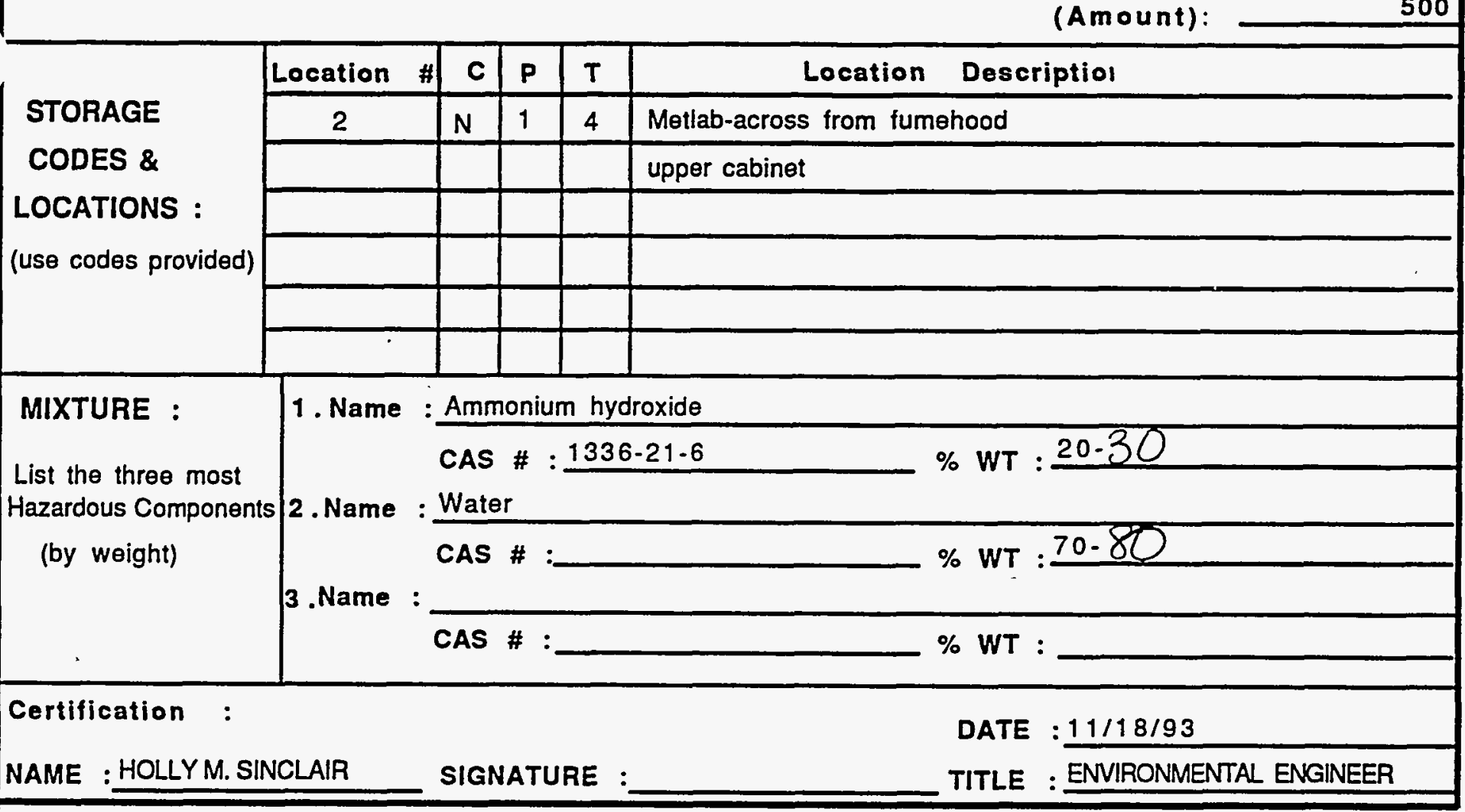


FOR ADMINSTAATIVE AGENCY USE :

DATE RECENED :

FACILITY ID \# :

\section{CHEMICAL DESCRIPTION}

Common Name :SHELL TELLUS TOIL 46

Chemical Name :PETROLEUMHYDROCARBON

Solid $\square$ Liquid $x$ Gas $\square$ Pure $\square$ Mixture $x$ Waste

Trade secret $\square$ Radioactiv $\square$ (If radiocative : CAS \# : UN/NA \# :

If waste is checked, Annual Amount

Generated : 0

WASTE CLASSIFICATION :

Toxic $X$ Ignitable $\square$ Corrosive $\square$ Reactive $\square$ Extremely Hazardous $\square$

PHYSICAL AND HEALTH

PHYSICAL

HAZARD CATEGORIES : Fire $\square$ Reactive

Sudden Pressure Releas

UNITS OF MEASURE

AMOUNT \& Gals. $X$ Lbs. $\square$

TIME AT

FACILITY :

Cu. Ft.

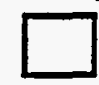

Maximum. Daily Amount

Average Daily Amount
10,000 \# Days on Site : 365 (Amount):

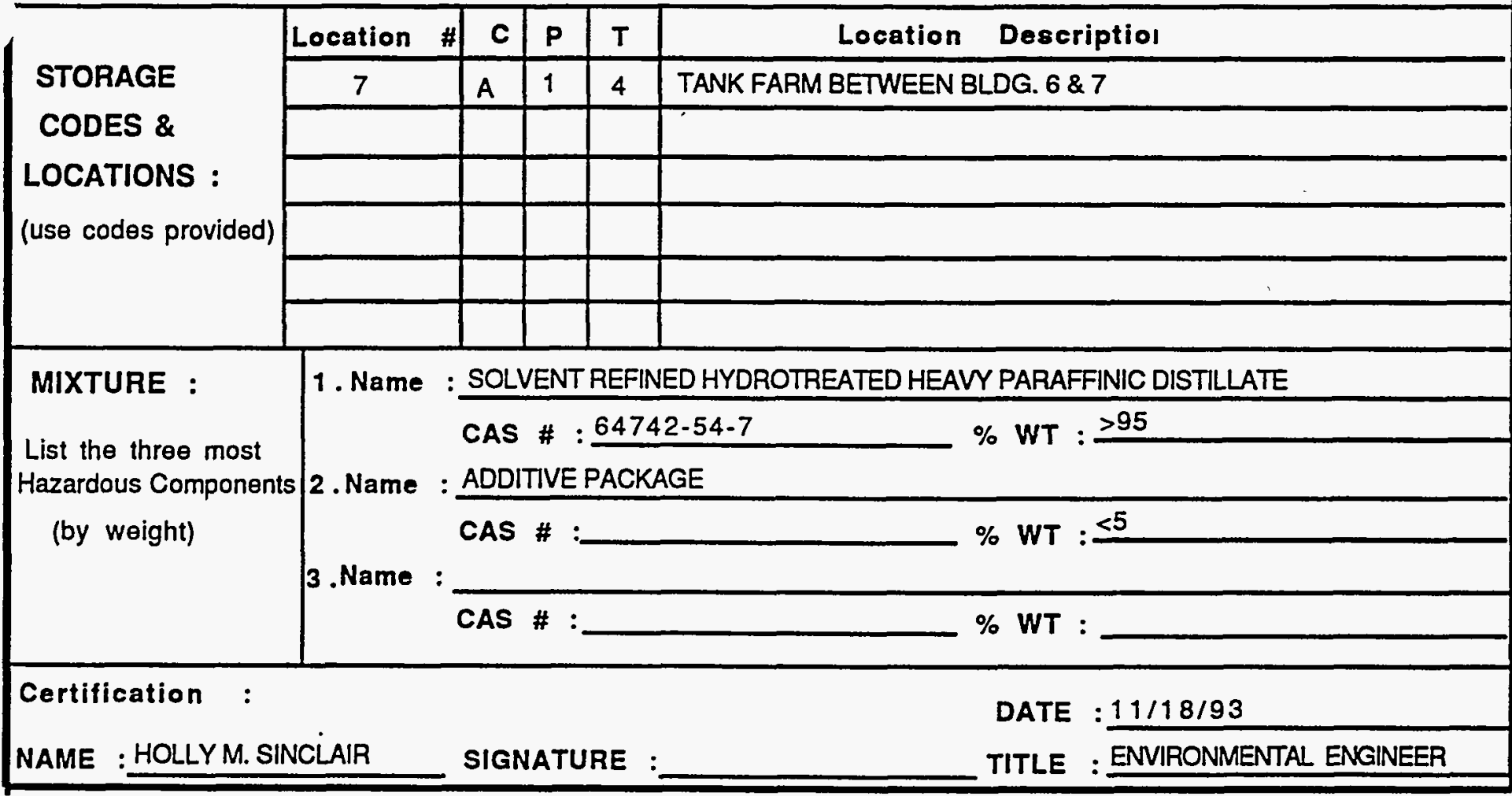


FOR ADMINSTRATIVE AGENCY USE :

DATE RECEIVED:

FACILITY ID \# :

\section{CHEMICAL DESCRIPTION}

Common Name :SHELL TURBOTOIL 68 CAS \# :

Chemical Name :PETROLEUMHYDROCARBONS

Solid $\square$ Liquid $X$ Gas $\square$ Pure $\square$ Mixture $X \quad$ waste $\square$

Trade Secret $\square$ Radioactiv $\square$ (If radiocative : $\quad$ curries) UN/NA \# :

If waste is checked, Annual Amount Generated : 0 WASTE CLASSIFICATION :

Toxic $X$ Ignitable $\square$ Corrosive $\square$ Reactive $\square$ Extremely Hazardous $\square$

PHYSICAL AND HEALTH

PHYSICAL

HAZARD CATEGORIES : Fire $\square$ Reactive

Sudden Pressure Releası

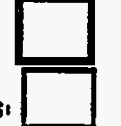

Immediate Health

HEALTH

(Acute)

Delayed Health

(Chronic)

UNITS OF MEASURE

AMOUNT \& Gals. $X$ Lbs.

TIME AT

Cu. Ft.

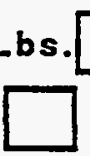

Maximum

Daily Amount

Average Daily Amount

55 \# Days on Site :

FACILITY :

30 Largest Container

(Amount):

\begin{tabular}{l}
$\begin{array}{l}\text { STORAGE } \\
\text { CODES \& } \\
\text { LOCATIONS : } \\
\text { (use codes provided) }\end{array}$ \\
\cline { 2 - 7 } \\
\cline { 2 - 7 }
\end{tabular}


FOR ADMINSTRATIVE AGENCY USE :

DATE RECENED :

FACILITY ID \# :

\section{CHEMICAL DESCRIPTION}

Common Name :BIOBAN TS

CAS \# :

Chemical Name :Substituted Amine

Solld $\square$ Liquid $X$ Gas $\square$ Pure $\square$ Mixture $X$ Waste $\square$ UN/NA \# :

Trade Secret $\square$ Radioactiv $\square$ (If radiocative :

o curries)

If waste is checked,

Annual Amount

Generated :

WASTE CLASSIFICATION :

Toxic $X$ Ignitable $\square$ Corrosive $\square$ Reactive $\square$ Extremely Hazardous $\square$

PHYSICAL AND HEALTH

PHYSICAL

HAZARD CATEGORIES : Fire $\square$ Reactive

Sudden Pressure Releası

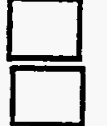

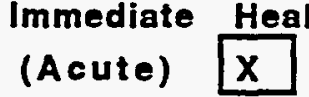

HEALTH

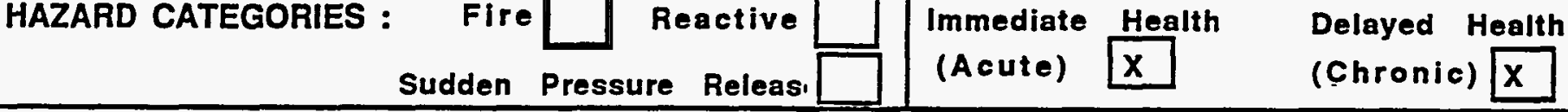

UNITS OF MEASURE

AMOUNT \& Gals. $X$

TIME AT

FACILITY :

Cu. Ft.

Lbs.

Maximum Daily Amount

55 \# Days on Site :

365

STORAGE

CODES \&

LOCATIONS :

(use codes provided)

\begin{tabular}{|l|l|l|l|l}
\hline Location \# & C & P & T & Location Descriptiol \\
\hline
\end{tabular}

(Amount):

Las

MIXTURE :

List the three most

Hazardous Components

(by weight)
Average Daily Amount
50 Largest Container

on-sit

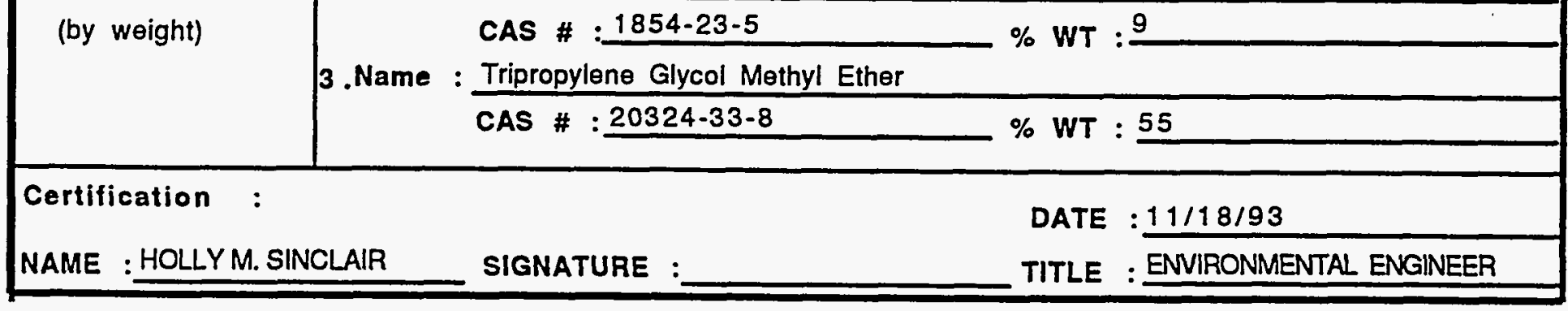


FOR ADMINSTRATIVE AGENCY USE :

DATE RECENED :

FACILITY ID \# :

\section{CHEMICAL DESCRIPTION}

Common Name :AMMONIUM FLUORIDE

CAS \# : $12125-01-8$

Chemical Name :Neutral Ammonium Fluoride

Solid $x$ Liquid $\square$ Gas $\square$ Pure $x$ Mixture $\square$ Waste $\square$

UN/NA \# : UN 2505

\section{Solid $x$}

Trade Secret
Radioactiv $\square$ (If radiocative : o curries)
If waste is checked, Annual Amount

Generated :

\section{WASTE CLASSIFICATION :}

Toxic $X$ Ignitable $\square$ Corrosive $\square$ Reactive $\square$ Extremely Hazardous $\square$

\section{PHYSICAL AND HEALTH \\ PHYSICAL}

HAZARD CATEGORIES :
Fire $\square$ Reactive

Sudden Pressure Releas

UNITS OF MEASURE

AMOUNT \&

TIME AT

FACILITY :

Lbs.

Cu. Ft.
Maximum

Average
Daily Amount
Daily Amount ofand Immediate Health

HEALTH

\begin{tabular}{l}
$\begin{array}{l}\text { STORAGE } \\
\text { CODES \& } \\
\text { LOCATIONS : } \begin{array}{l}\text { Location \# } \\
\text { (use codes provided) }\end{array} \\
$\cline { 2 - 7 }\end{array}$)$ \\
\cline { 2 - 7 }
\end{tabular}


FOR ADMINSTRATIVE AGENCY USE :

DATE RECEIVED :

\section{INVENTORY FORM}

FACILITY ID \# :

\section{CHEMICAL DESCRIPTION}

Common Name :UNOCAL WAY OIL HD 68

Chemical Name :PETROLEUMHYDROCARBONS

Solid $\square$ Liquid $x$ Gas $\square$ Pure $\square$ Mixture $x$ Waste

Trade Secret $\square$ Radioactiv $\square$ (If radiocative :

o curries)

CAS \# : UN/NA \# :

If waste is checked, Annual Amount

Generated : 0

WASTE CLASSIFICATION :

Toxic $x$ Ignitable $\square$ Corrosive $\square$ Reactive $\square$ Extremely Hazardous $\square$

PHYSICAL AND HEALTH PHYSICAL

HAZARD CATEGORIES : Fire $\square$ Reactive $\square$ Sudden Pressure Releası

UNITS OF MEASURE

AMOUNT \&

TIME AT

FACILITY : Gals. $X$

Cu. Ft.

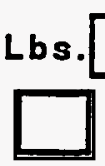

Maximum

Average
Daily Amount

Daily Amount
110 \# Days on Site : 90 Largest Container on-sit (Amount):
Delayed Health

(Chronic)

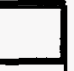

Immediate Health

(Acute)

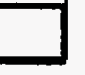

HEALTH

\begin{tabular}{l}
$\begin{array}{l}\text { STORAGE } \\
\text { CODES \& } \\
\text { LOCATIONS : } \\
\text { (use codes provided) }\end{array}$ \\
\cline { 2 - 8 } \\
\cline { 2 - 7 }
\end{tabular}


FOR ADMINSTRATIVE AGENCY USE :

DATE RECEIVED :

FACILITY ID \# :

\section{CHEMICAL DESCRIPTION}

Common Name :MOBIL DTE 24 CAS \# :

Chemical Name :REFINED MINERALOILS UN/NA \# :

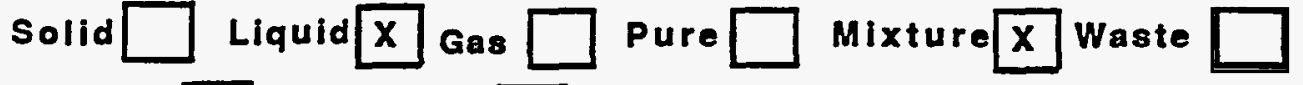

Trade Secret

o curries) Radioactiv $\square$ (If radiocative :

If waste is checked, Annual Amount

Generated : 0

WASTE CLASSIFICATION :

Toxic $X$ Ignitable

\section{Corrosive $\square$ Reactive $\square$}

Extremely Hazardous

PHYSICAL AND HEALTH

PHYSICAL

HAZARD CATEGORIES : FIre $\square$ Reactive

Sudden Pressure

Releas.

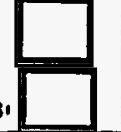

Immediate Health
(Acute)

HEALTH

UNITS OF MEASURE

AMOUNT \& Gals. $X$

TIME AT

Cu. Ft.

Lbs.

Maximum

Average Dally Amount

Daily Amount

110 \# Days on site :

Delayed Health

FACILITY :

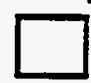

85 Largest Container

(Amount):

55

\begin{tabular}{l}
$\begin{array}{l}\text { STORAGE } \\
\text { CODES \& } \\
\text { LOCATIONS : } \\
\text { (use codes provided) }\end{array}$ \\
\cline { 2 - 8 } \\
\cline { 2 - 7 }
\end{tabular}


FOR ADMINSTRATIVE AGENCY USE :

DATE RECEIVED :

FACILITY ID \# :

\section{CHEMICAL DESCRIPTION}

Common Name :SHELL AUTOMATIC TRANSMISSION FLUID DEXRON II CAS \# :

Chemical Name :PETROLEUMHYDROCARBON UN/NA \# :

solid $\square$

Trade

\section{Liquid $\mathrm{X}$ Gas} Radioactiv $\square$ (If radiocative :
Mixture $\mathrm{X}$ Waste $\square$

o curries)
If waste is checked, Annual Amount

Generated : 0

WASTE CLASSIFICATION :

Toxic $X$ Ignitable $\square$
Reactive

\section{PHYSICAL AND HEALTH HAZARD CATEGORIES :

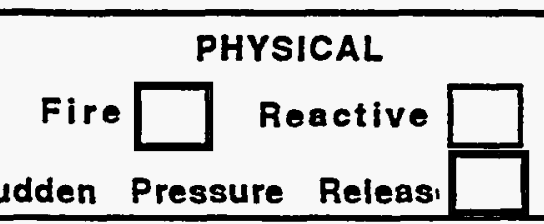 \\ PHYSICAL \\ Sudden Pressure}

\section{UNITS OF MEASURE}

AMOUNT \& Gals. $X$

TIME AT

FACILITY :

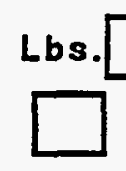

Maximum

Maximum Daily Amount
Average Daily Amount

\begin{tabular}{|c|}
\multicolumn{3}{c}{ HEALTH } \\
Immediate Health \\
(Acute) $\square$ (Chronic) $\square$
\end{tabular}

\section{HEALTH}

(Chronic)

Exiremely Hazardous $\square$

Cu. Ft.

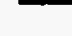

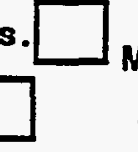

55 \# Days on Site : 40 Largest Container on-sit (Amount):

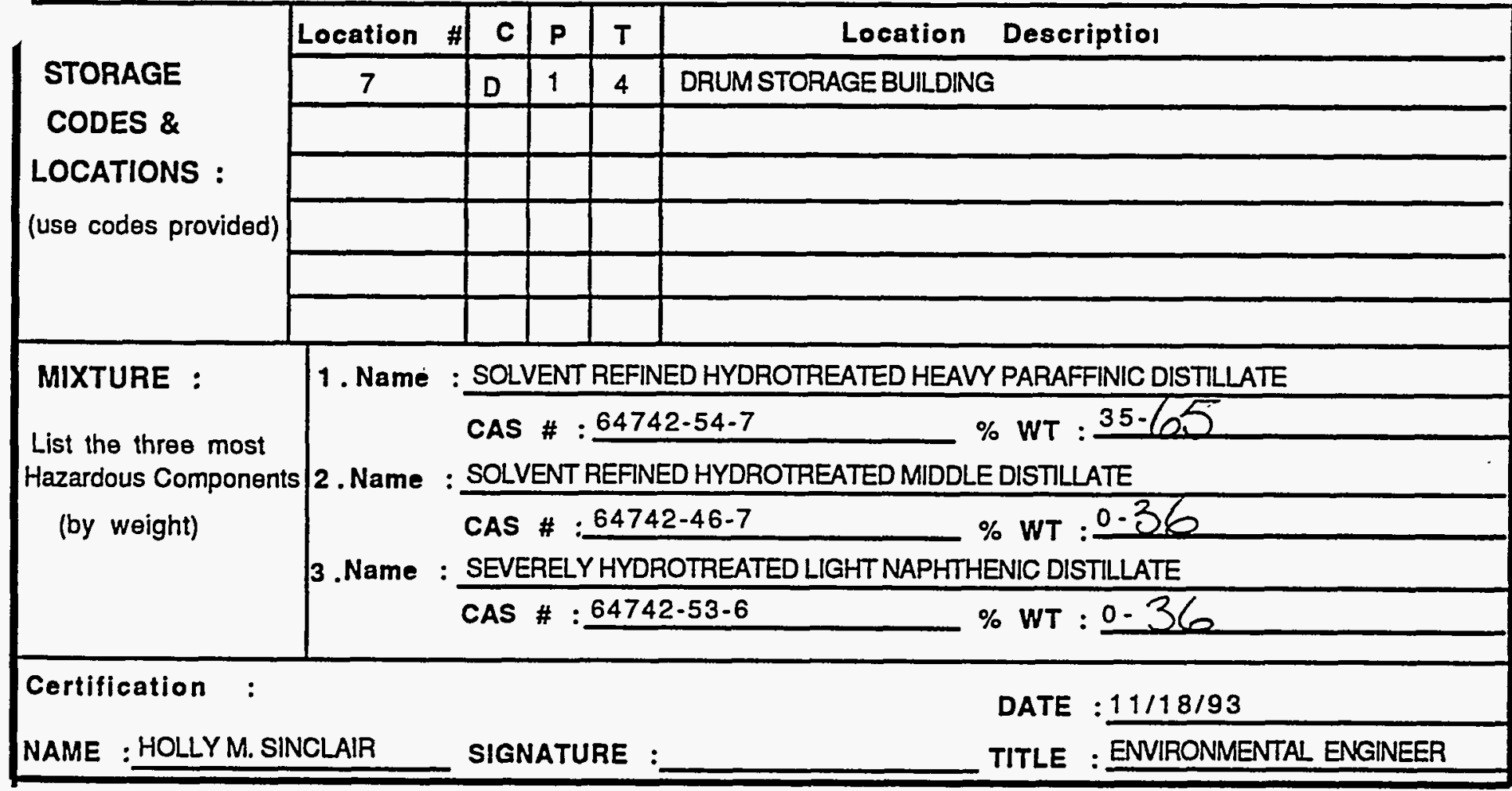


FOR ADMINSTAATIVE AGENCY USE :

DATE RECENED :

FACILITY ID \# :

\section{CHEMICAL DESCRIPTION}

Common Name :MOBIL DELVAC 1230

CAS \# :

Chemical Name :REFINED MINERAL OlLS

UN/NA \# :

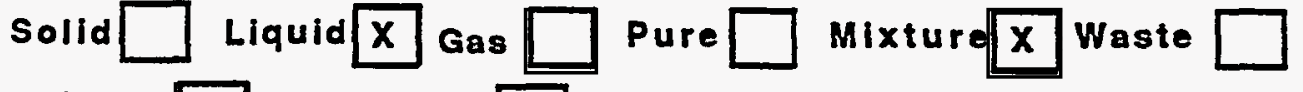

Trade Secret $\square$ Radioactiv $\square$ (If radiocative : $\quad 0$ curries)

If waste is checked, Annual Amount

Generated :

WASTE CLASSIFICATION :

Toxic $x$ Ignitable $\square$ Corrosive $\square$ Reactive $\square$ Extremely Hazardous $\square$

PHYSICAL AND HEALTH

PHYSICAL

HAZARD CATEGORIES : Fire $\square$ Reactive

Sudden Pressure Releas!

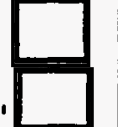

(Acute) Health

HEALTH

\begin{tabular}{c} 
Immediate Health $\begin{array}{l}\text { Delayed Health } \\
\text { (Acute) }\end{array} \quad$ (Chronic) $\square$ \\
\hline
\end{tabular}

UNITS OF MEASURE

AMOUNT \&

TIME AT

FACILITY :

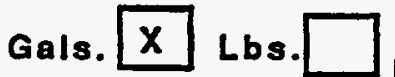

Cu. Ft.

Maximum

Average

Daily Amount

Daily Amount

55 \# Days on site :

30 Largest Container on-sit (Amount):

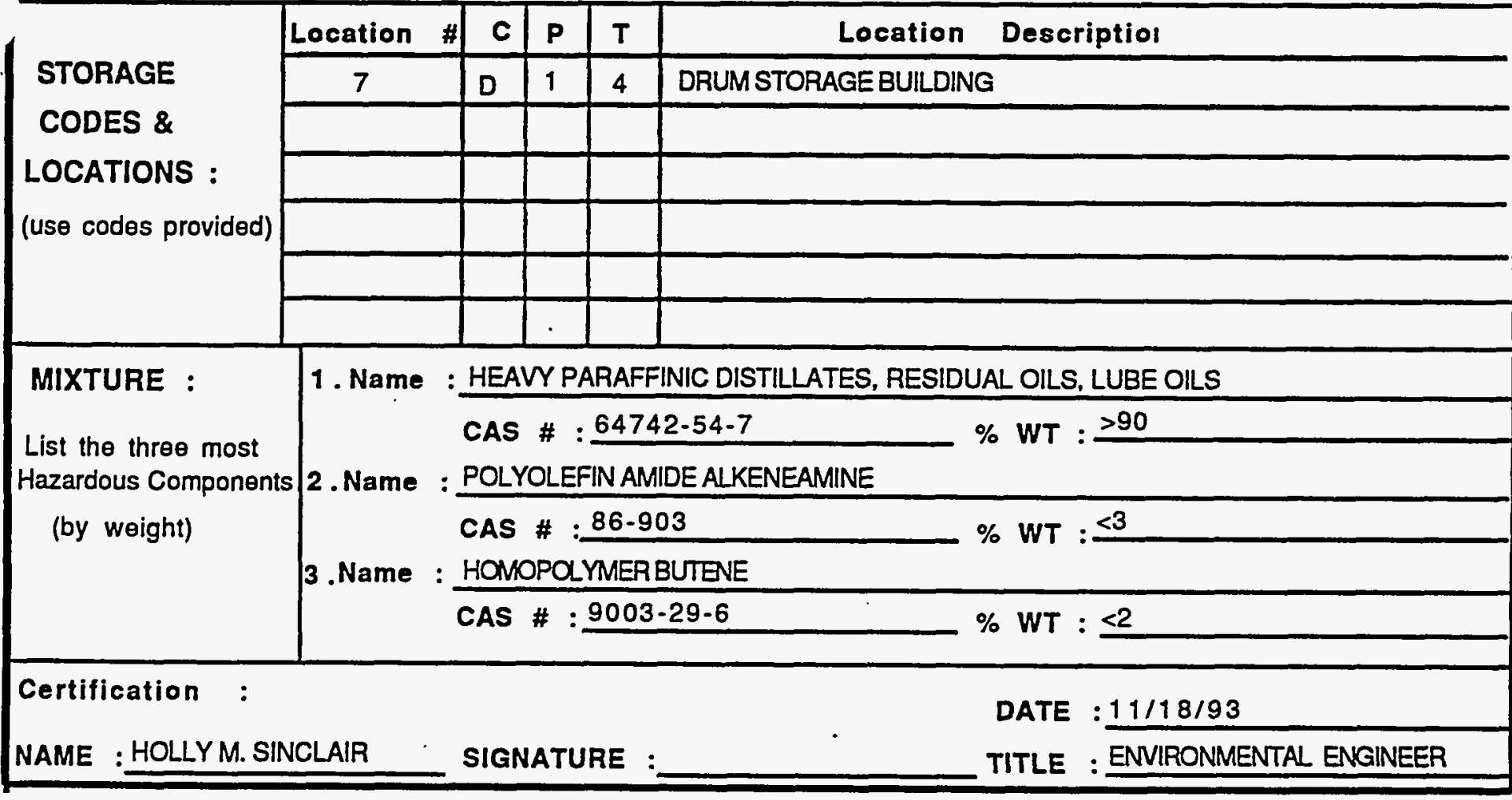


FOR ADMINSTRATIVE AGENCY USE :

DATE RECEIVED :

FACILITY ID \# :

\section{CHEMICAL DESCRIPTION}

Common Name :HYDROGEN PEROXIDE 35\%

CAS \# : 7722-84-1

Chemical Name :Hydrogen Peroxide UN/NA \# :

Solid $\square$ Liquid $X$ Gas
Pure $\square$ Radioactiv $\square$ (If radiocative :
Mixture $x$ Waste

o Curries)
If waste is checked, Annual Amount

Generated :

\section{WASTE CLASSIFICATION :}

Toxic $\square$ Ignitablo $\square$ Corrosive $x$ Reactive $\square$ Extremely Hazardous $\square$

PHYSICAL AND HEALTH PHYSICAL

HAZARD CATEGORIES : Fire $X$ Reactive

Sudden Pressure Releas:

\section{UNITS OF MEASURE}

AMOUNT \&

TIME AT

Gals. $\square$

FACILITY :

Cu. Ft.

Lbs.

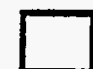

Maximum

Daily Amount

1 \# Days on Site :

Average Daily Amount

1 Largest Container on-sit (Amount):

\section{STORAGE}

CODES \&

LOCATIONS :

(use codes provided)

\section{MIXTURE :}

List the three most

Hazardous Components

(by weight)

\begin{tabular}{|c|c|c|c|l|}
\hline Location $\#$ & C & P & T & Location Descriptios \\
\hline 2 & N & 1 & 4 & Metlab-under fume hood \\
\hline & & & & left cabinet \\
\hline & & & & \\
\hline & & & & \\
\hline & & & & \\
\hline
\end{tabular}

1. Name: Hydrogen Peroxide

$$
\text { CAS \#: }: \underline{7222-84-1} \% \text { WT : } 35
$$

2. Name: Water

CAS \# $\% W T: 65$

3. Name :

CAS \# : \% WT :

Certification : 
FOR ADMINSTRATIVE AGENCY USE :

DATE RECEIVED :

FACILITY ID \# :

\section{CHEMICAL DESCRIPTION}

Common Name :DARACOOL 706LF

CAS \# :

Chemical Name :WATERBASED SYNTHETIC METALWORKING FLUID

Solid $\square$ Liquid $x$ Gas $\square$ Pure $\square$ Mixture $x$ Waste $\square$

Trade Secret $\square$ Radioactiv $\square$ (If radiocative :

o curries) UN/NA \# :

If waste is checked, Annual Amount

Generated :

0

WASTE CLASSIFICATION :

Toxic $\mathrm{X}$ Ignitable $\square$ Corrosive $\square$ Reactive $\square$ Extremely Hazardous $\square$

PHYSICAL AND HEALTH

HAZARD CATEGORIES
PHYSICAL

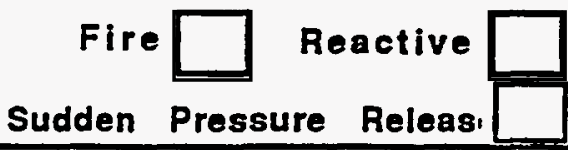

\section{UNITS OF MEASURE}

AMOUNT \& Gals. $X$ Lbs. $\square$

TIME AT

Cu. Ft.

Maximum Daily Amount

165 \# Days on Site :

365

FACILITY :

Average Daily Amount

110 Largest Containe

(Amount):

Delayed Health

(Chronic) $X$

\begin{tabular}{l}
$\begin{array}{l}\text { STORAGE } \\
\text { CODES \& } \\
\text { LOCATIONS : } \\
\text { (use codes provided) }\end{array}$ \\
\cline { 2 - 8 } \\
\cline { 2 - 7 }
\end{tabular}


FOR ADMINSTRATIVE AGENCY USE :

DATE RECEIVED :

\section{INVENTORY FORM}

FACILITY ID \# :

\section{CHEMICAL DESCRIPTION}

Common Name :ACETIC ACID, GLACIAL

Chemical Name :SAME

Solld $\square$ Liquid $x$ Gas $\square$ Pure $x$ Mixture $\square$ Waste Trade Secret $\square$ Radioactiv $\square$ (If radiocative :

WASTE CLASSIFICATION :

Toxic $\square$ Ignitable $x$ Corrosive $x$ Reactive o curries)
CAS \# : $64-19-7$

UN/NA \# : UN 2789

If waste is checked, Annual Amount.

Generated :

HAZARD CATEGORIES : Fire $X$ Reactive $\square$ Sudden Pressure Releas.

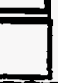
Immediate Health (Acute) $\mathrm{X}$

Delayed Health HEALTH

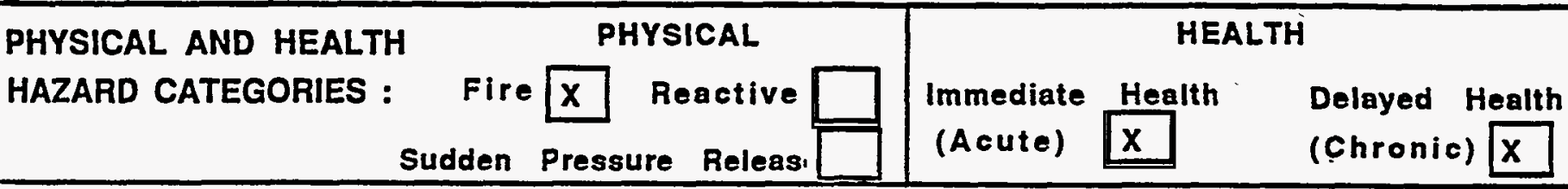

UNITS OF MEASURE

AMOUNT \&

TIME AT

Gals.

FACILITY :

Cu. Ft.

Lb8. $\square$ Maximum

Ditera

Average Daily Amount

5 \# Days on Site :

FACILITY :

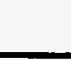

STORAGE
CODES \&
LOCATIONS :

(use codes provided)

MIXTURE :

MIXTURE :

List the three most

Hazardous Components

(by weight)

\begin{tabular}{|c|c|c|c|c|}
\hline Location \# & C & $P$ & $T$ & Location \\
\hline 2 & $M$ & 1 & 4 & Metlab-under fume hood \\
\hline & & & & left cabinet \\
\hline & & & & \\
\hline & & & & \\
\hline & & & & \\
\hline & 1. Name : ACETIC ACID, GLACIAL
\end{tabular}

$$
\text { CAS \# : 64-19-7 }
$$

2. Name :

CAS \# :

\% WT :

3. Name :

CAS \# : $\% W T$ :

Certification :

NAME : HOLLYM.SINCLAIR SIGNATURE :

DATE : $11 / 18 / 93$

TITLE : ENVIRONMENTAL ENGINEER 
FOR ADMINSTRATIVE AGENCY USE :

DATE RECEIVED :

FACILITY ID \# :

\section{CHEMICAL DESCRIPTION}

Common Name :ACETONE

Chemical Name :SAME

solid $\square$

Trade Secret
Liquid $x$ Gas $\square$ Pure $x$ Radioactiv $\square$ (If radiocative :
Mixture $\square$ Waste

o curries)
CAS \# : 67-64-1

UN/NA \# : UN 1090

If waste is checked, Annual Amount

Generated : 0

\section{WASTE CLASSIFICATION :}

Toxic $\square$ Ignitable $x$ Corrosive $\square$ Reactive $\square$ Extremely Hazardous $\square$

PHYSICAL AND HEALTH HAZARD CATEGORIES :

\section{Sudden}

\begin{tabular}{|c|c|c|}
\hline & \multicolumn{2}{|c|}{ PHYSICAL } \\
\hline Fire & $x$ & Reactive \\
\hline
\end{tabular}

PHYSICAL

UNITS OF MEASURE

AMOUNT \&

TIME AT

FACILITY :

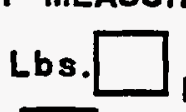

Cu. Ft.

Lbs. $\square$ Maximum

Average Daily Amount
HEALTH

Immediate Health
(Acute) $X x^{\text {Delayed Health }}$
(Chronic) $X$

\begin{tabular}{|c|c|c|c|c|c|}
\hline \multirow{5}{*}{$\begin{array}{l}\text { STORAGE } \\
\text { CODES \& } \\
\text { LOCATIONS : } \\
\text { (use codes provided) }\end{array}$} & Location & C & $\mathbf{P}$ & $T$ & Location Descriptiol \\
\hline & \multirow[t]{2}{*}{2} & \multirow[t]{2}{*}{$M$} & 1 & \multirow[t]{2}{*}{4} & \multirow{2}{*}{$\begin{array}{l}\text { STORED IN FLAMMABLE CABINET OUTSIDE } \\
\text { OF SHIPPINGRECEIVING OFFICE }\end{array}$} \\
\hline & & & & & \\
\hline & & & & & \\
\hline & & & & & \\
\hline & & & & & \\
\hline MIXTURE : & 1. Name: & ACE & ONE & & \\
\hline $\begin{array}{l}\text { List the three most } \\
\text { Hazardous Components }\end{array}$ & 2. Name: & CAS & $\#$ & 67. & $\% W T: 90-100$ \\
\hline (by woight) & Name. & CAS & \# & & \% WT \\
\hline & & CAS & $\#$ & & —WT : \\
\hline Certification : & & & & & DATE : $11 / 18 / 93$. \\
\hline NAME : HOLLYM.SIN & VCLAIR & SIGI & SAT & & TITLE : ENVIRONMENTAL ENGINEER \\
\hline
\end{tabular}


FOR ADMINSTRATIVE AGENCY USE :

DATE RECEIVED :

FACILITY ID \# :

\section{CHEMICAL DESCRIPTION}
Common Name :AMMONIUM CHLORIDE
Chemical Name :SAME
CAS \# : 12125-02-9
UN/NA \# : NA 9085

Solid $x$ Liquid $\square$ Gas $\square$ Pure $x$

Trade secret $\square$ Radioactiv $\square$ (If radiocative

Mixture $\square$ Waste

o Curries)
If waste is checked, Annual Amount

Generated :

\section{WASTE CLASSIFICATION :}

Toxic $X$ Ignitable $\square$ Corrosive $\square$ Reactive $\square$ Extremely Hazardous $\square$

\begin{tabular}{|c|c|c|c|c|}
\hline PHYSICAL AND HEALTH & PHYSICAL & \multicolumn{3}{|c|}{ HEALTH } \\
\hline HAZARD CATEGORIES : & Reactive & Immediate & Health & Delayed Health \\
\hline Sudden & Pressure Releas! & (Acute) & $\mathbf{x}$ & (Chronic) $x$ \\
\hline
\end{tabular}

UNITS OF MEASURE

AMOUNT \& Gals. $\square$ Lbs. $\square$ Ka

TIME AT

Cu. Ft.

Average Daily Amount

10 * Days on Site :

FACILITY :

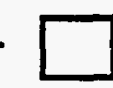

7 Largest Container on-sit (Amount): $500 \mathrm{~g}$.

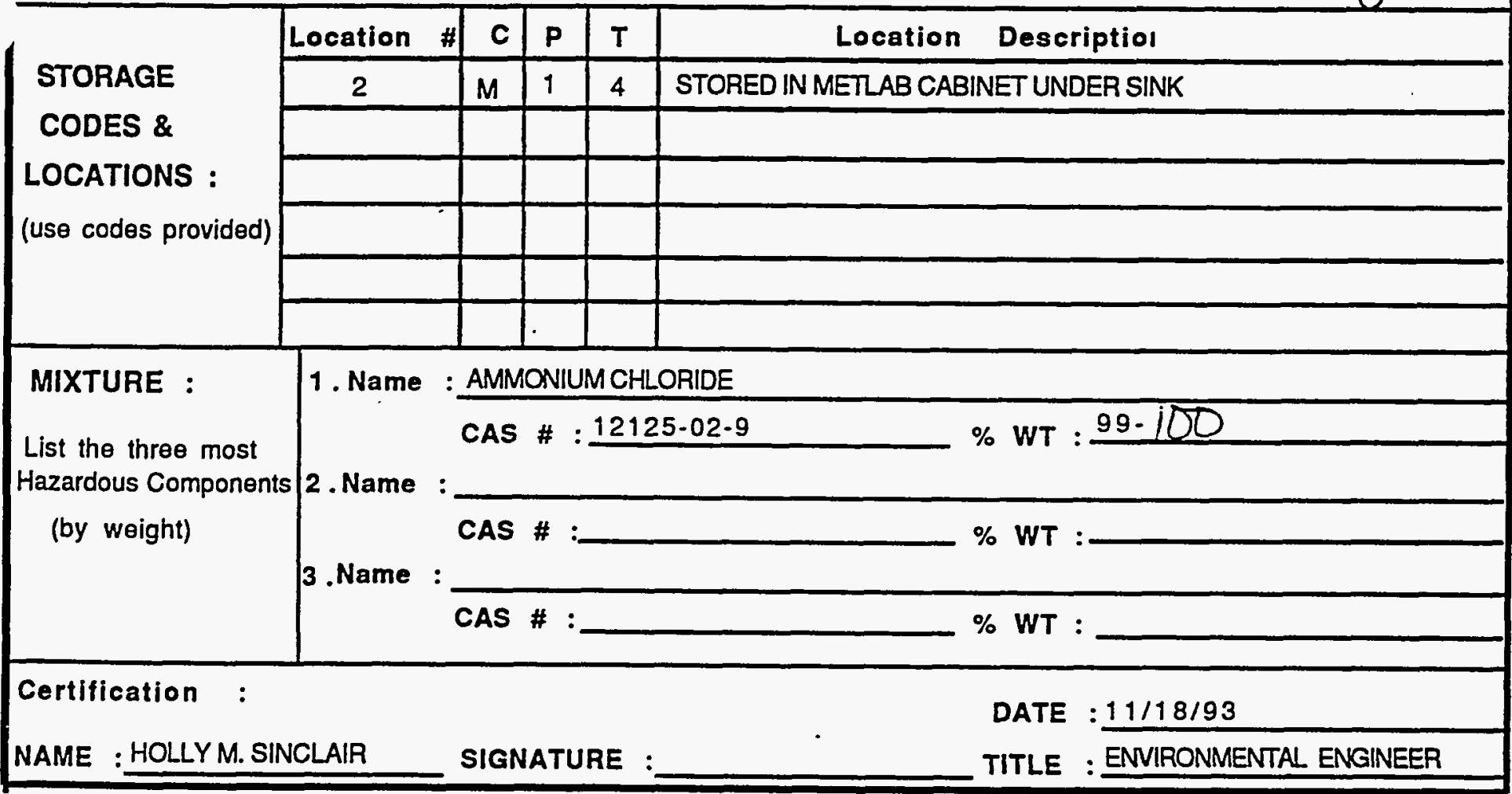


FOR ADMINSTRATIVE AGENCY USE :

DATE RECEIVED :

FACILITY ID \# :

\section{CHEMICAL DESCRIPTION}

Common Name :METHYL ALCOHO

Chemical Name :METHANOL

Solid $\square$ Liquid $X$ Gas $\square$ Pure $X$ Mixture $\square$ Waste $\square$

Trade Secret $\square$ Radioactiv $\square$ (If radiocative :

CAS \# : $67-56-1$

UN/NA \# :

If waste is checked, Annual Amount

Generated :

WASTE CLASSIFICATION :

Toxic $X$ Ignitable $X$ Corrosive $\square$ Reactive $\square$ Extremely Hazardous $\square$

PHYSICAL AND HEALTH

PHYSICAL

HAZARD CATEGORIES : Fire $X$ Reactive

Sudden Pressure Releası

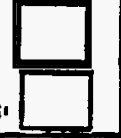

\begin{tabular}{l} 
(Acute) $X$ \\
\hline
\end{tabular}

HEALTH

UNITS OF MEASURE

AMOUNT \& Gais. $x$ entert

TIME AT

Average Daily Amount

3 \# Days on site :

365

FACILITY :

Cu. Ft.

2 Largest Container

on-sit (Amount):

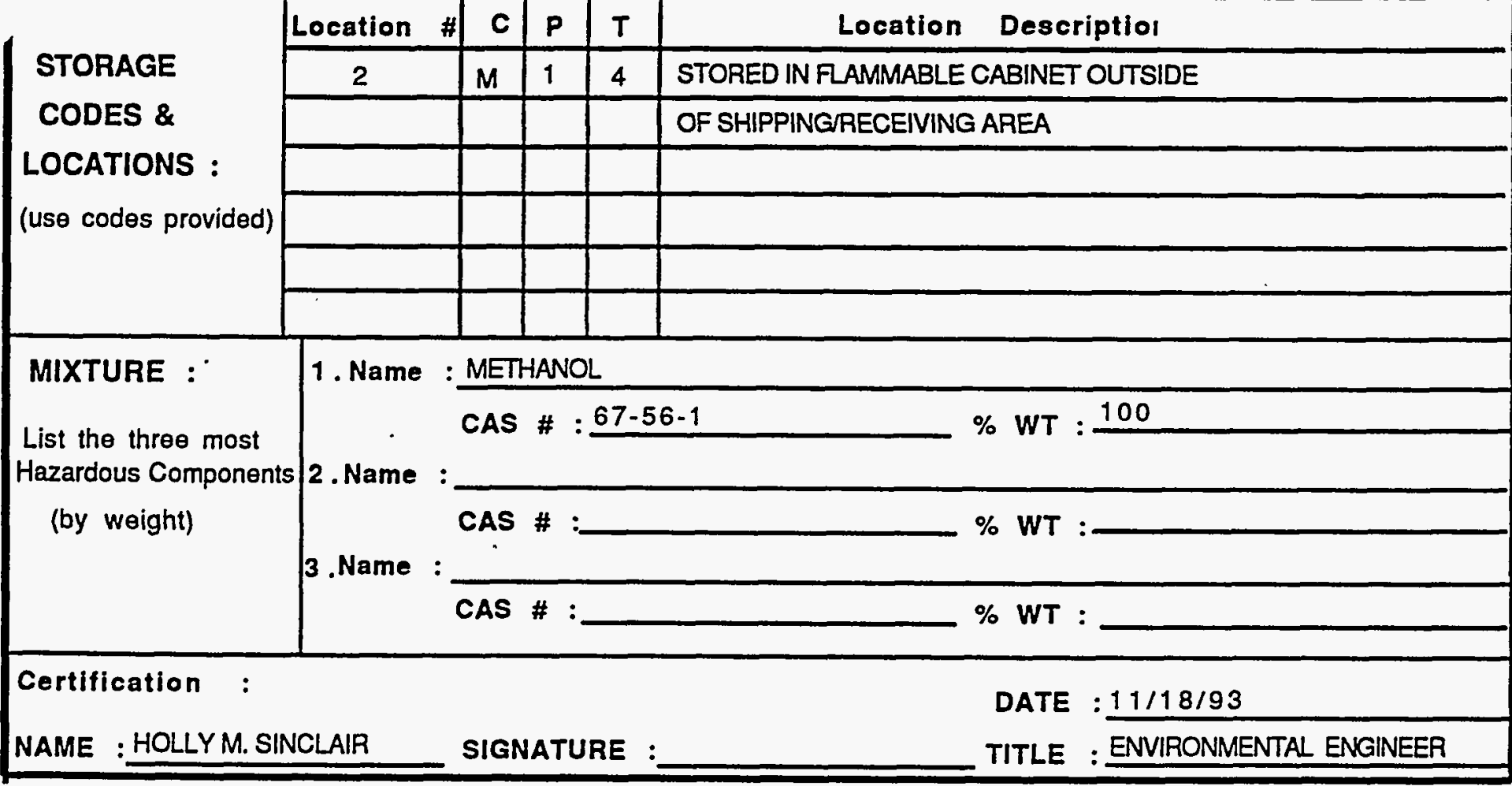


FOR ADMINSTRATIVE AGENCY USE :

DATE RECEIVED :

FACILITY ID \# :

\section{CHEMICAL DESCRIPTION}

Common Name :OXALIC ACID, CRYSTALS CAS \# : $6153 \cdot 56-6$

Chemical Name :ETHANEDIOIC ACID, CRYSTALS UN/NA \# :

solid $x$ Liquid $\square$ Gas Radioactiv $\{$ (If radiocative :
Pure $X$ Mixture $\square$ Waste o curries)
If waste is checked, Annual Amount Generated :

0

\section{WASTE CLASSIFICATION :}

Toxic $X$ Ignitable $\square$ Corrosive $X$ Reactive $\square$ Extremely Hazardous $\square$

PHYSICAL AND HEALTH HAZARD CATEGORIES

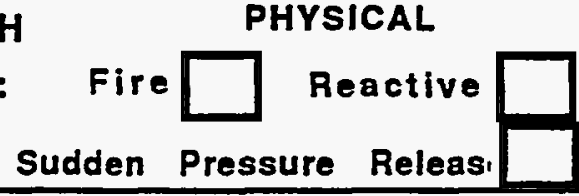

PHYSICAL

\section{AM}

AMOUNT \&

TIME AT

FACILITY :

\section{UNITS OF MEASURE}

Maximum Daily Amount Average Daily Amount

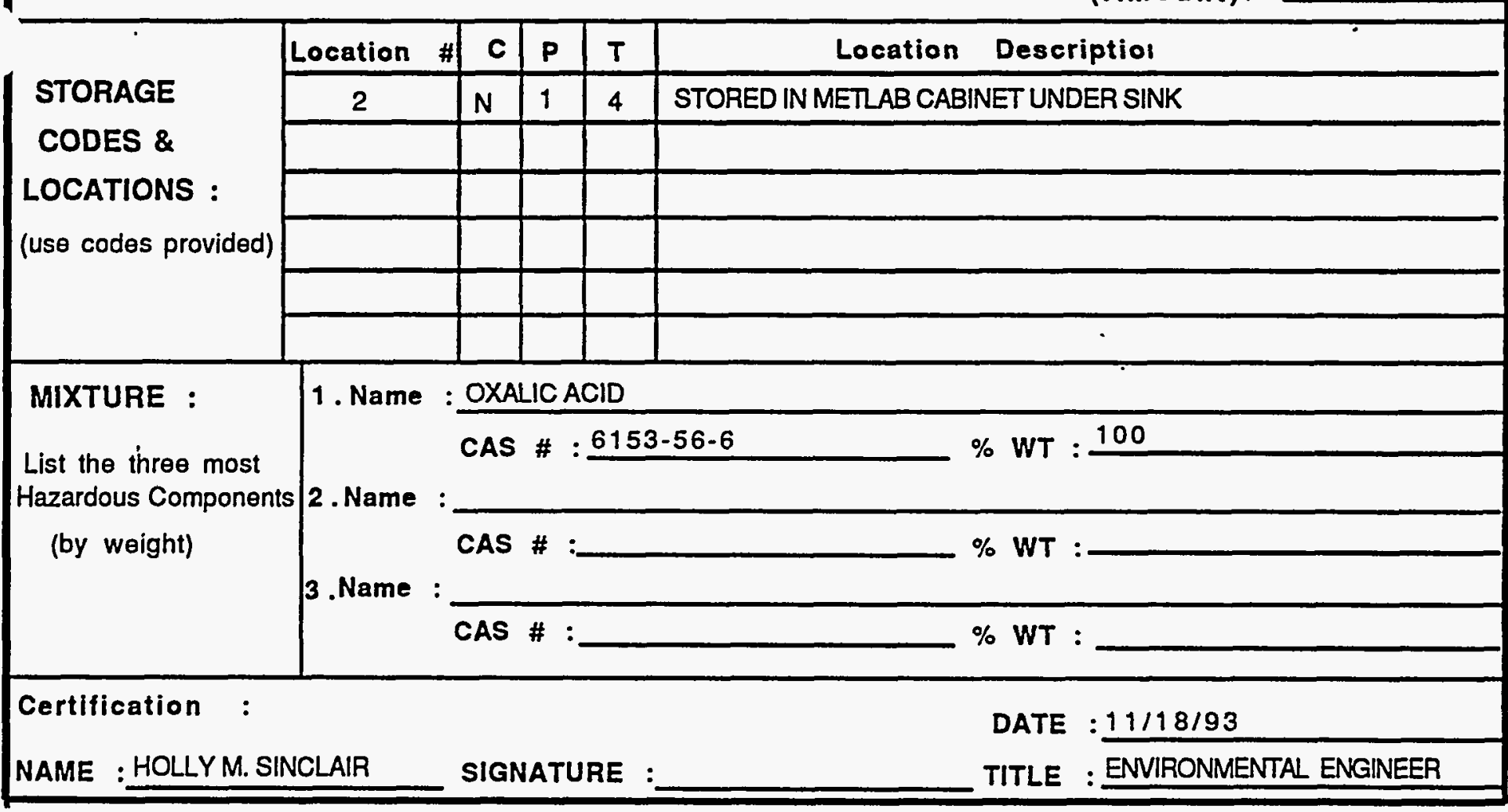

\section{HEALTH}

Immediate Health Delayed Health

(Acute) $X \quad$ (Chronic) $X$ 


\section{CHEMICAL DESCRIPTION}

Common Name :HYDROCHLORIC ACID

CAS \# : 7647-01-1

Chemical Name :Hydrochloric acid

UN/NA \# : UN 1789

solid $\square$

Liquid $x$ Gas
Pure $\square$ Mixture $\mathrm{X}$ Waste

o curries)
If waste is checked, Annual Amount

Generated :

\section{WASTE CLASSIFICATION :}

Toxic $\square$ Ignitable $\square$ Corrosive $x$ Reactive $\square$ Extremely Hazardous $x$

PHYSICAL AND HEALTH PHYSICAL

HAZARD CATEGORIES : Fire $\square$ Reactive

Sudden Pressure Releas!

UNITS OF MEASURE

AMOUNT \& Gals.

TIME AT

FACILITY :

Cu. Ft.

Lbs.

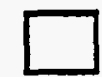

Maximum

Average Daily Amount
HEALTH

Immediate Health Delayed Health

(Acute) $X \quad$ (Chronic) $X$

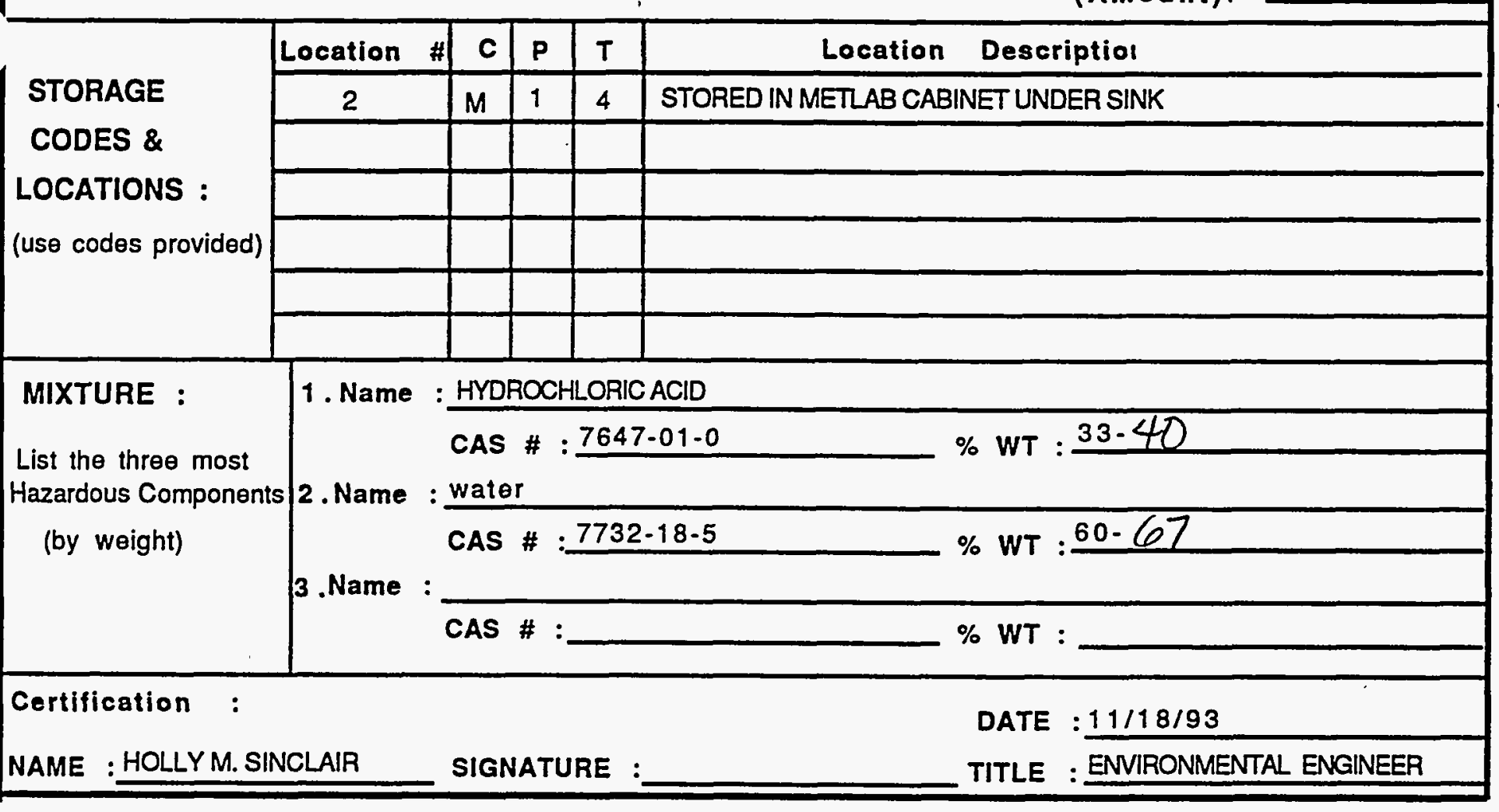


FOR ADMINSTAATIVE AGENCY USE :

DATE RECEIVED :

FACILITY ID \# :

\section{CHEMICAL DESCRIPTION}

Common Name :HYDROFLUORIC ACID

Chemical Name :Hydrofluoric acid

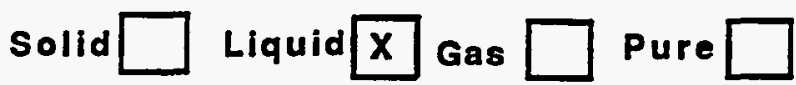

Trade Secret $\square$ Radioactiv $\square$ (If radiocative :
Mixture $x$ Waste

o curries)
CAS \# : $7664-39-3$

UN/NA \# : UN 1790

If waste is checked,

Annual Amount

Generated :

\section{WASTE CLASSIFICATION :}

Toxic $\square$ Ignitable $\square$ Corrosive $x$ Reactive $\square$ Extremely Hazardous $x$

PHYSICAL AND HEALTH PHYSICAL

HAZARD CATEGORIES : Fire $\square$ Reactive

Sudden Pressure Releas,

UNITS OF MEASURE

AMOUNT \& Gals. $\square$

TIME AT

FACILITY :

Cu. Ft.

Lbs. $\square$ Maximum

Average

Daily Amount

Daily Amount

\begin{tabular}{|c|c|}
\hline & HEALTH \\
Immediate Health & Delayed Health \\
(Acute) $X$ & (Chronic) $X$
\end{tabular}

Immediate Health
(Acute) $x$

(Chronic) $x$

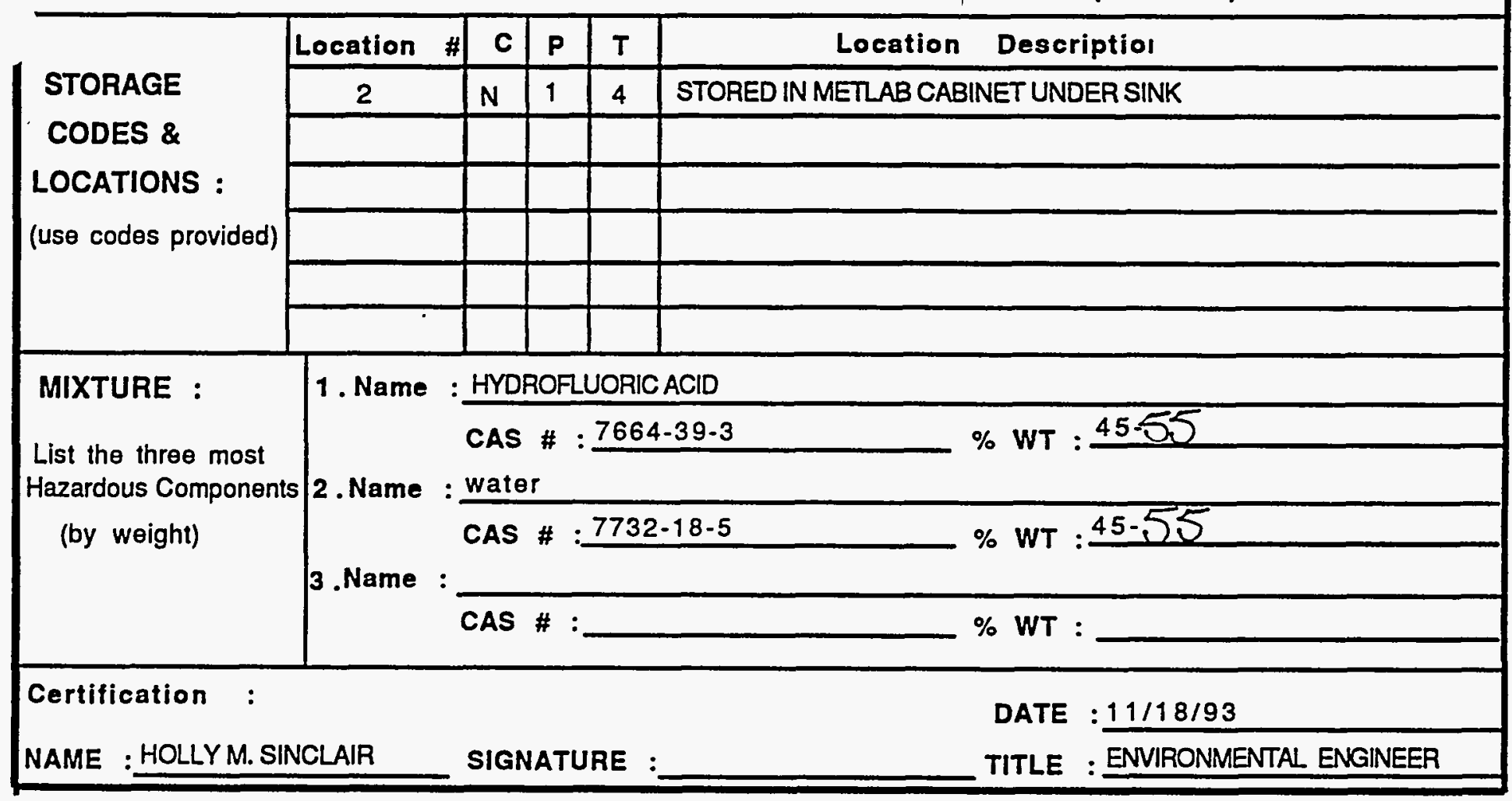


FOR ADMINSTRATIVE AGENCY USE :

DATE RECEIVED :

FACILITY ID \# :

\section{CHEMICAL DESCRIPTION}

Common Name :MIRACLE FORGE 7600 CAS \# :

Chemical Name :Non-Graphite Water Based Lubricant

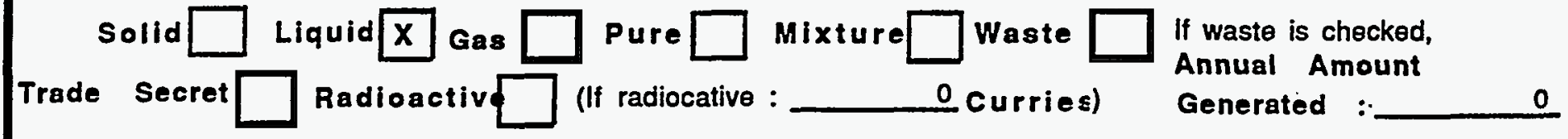
UN/NA \# :

WASTE CLASSIFICATION :

Toxic $\square$ Ignitable $\square$ Corrosive $\square$ Reactive $\square$ Extremely Hazardous $\square$

PHYSICAL AND HEALTH

PHYSICAL

HAZARD CATEGORIES : Fire $\square$ Reactive

Sudden Pressure Releası

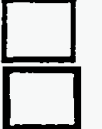
Immediate Health

HEALTH

(Acute) $\square$

Delayed Health

(Chronic)

UNITS OF MEASURE

AMOUNT \& Gals. $X$ Lbs. $\square$ Maximum. Daily Amount
TIME AT

FACILITY : Cu. Ft.

Average Daily Amount

55 \# Days on Site :

365

FACILITY :

45 Largest Containe (Amount):

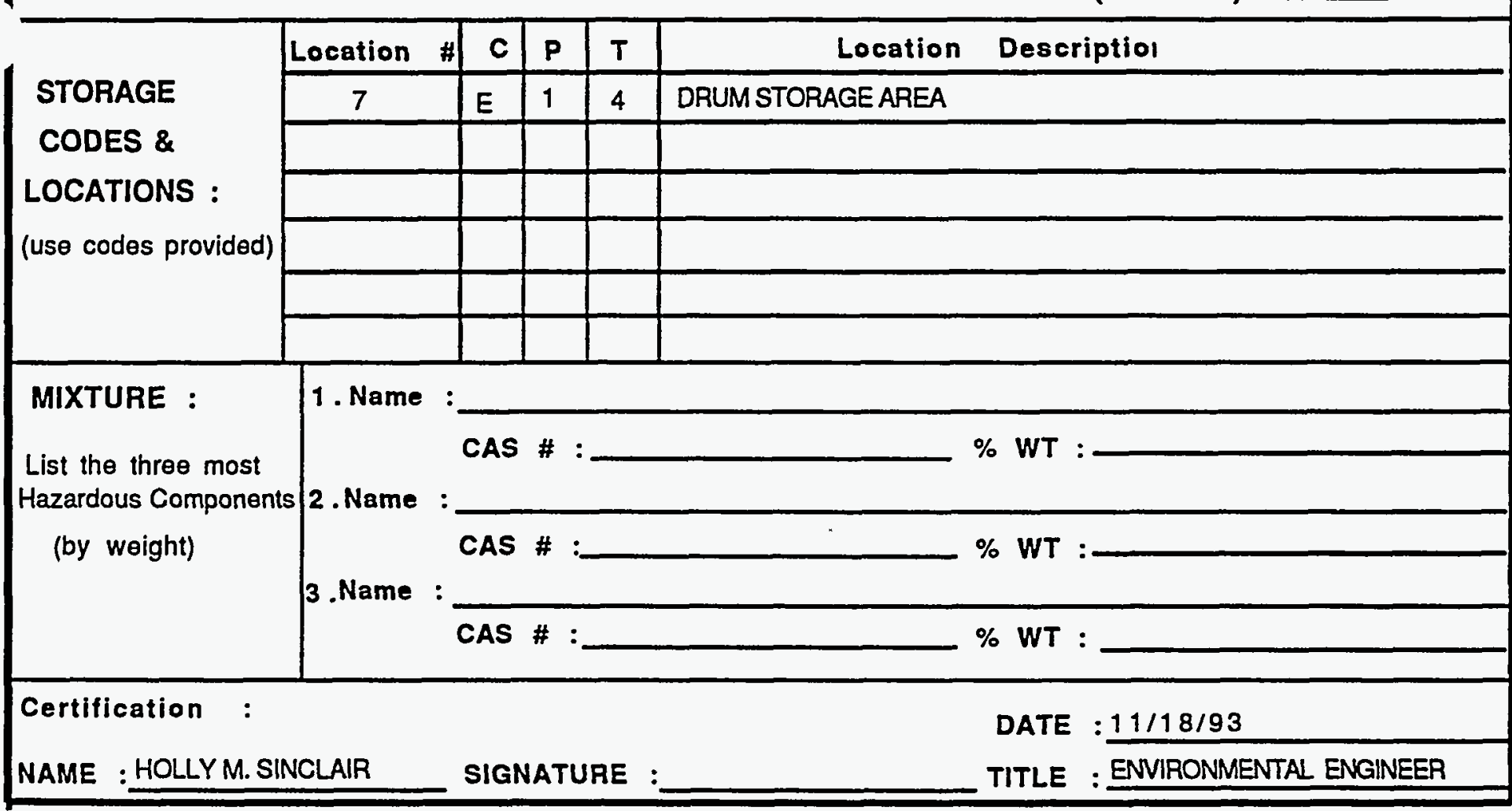


FOR ADMINSTRATIVE AGENCY USE :

DATERECEIVED :

FACILITY ID \# :

\section{CHEMICAL DESCRIPTION}

Common Name :AMMONIUMBIFLUORIDE CAS \# : 1341-49-7

Chemical Name :Ammonium bifluoride UN/NA \# : UN 1727

Solid $x$ Liquid $\square$ Gas Radioactiv
Pure $x$ (If radiocative :
Mixture $\square$ Waste

O curries)
If waste is checked, Annual Amount Generated : 0

\section{WASTE CLASSIFICATION :}

Toxic $\square$ Ignitable $\square$ Corrosive $x$ Reactive $\square$ Extremely Hazardou $\{x$

\section{PHYSICAL AND HEALTH \\ PHYSICAL}

HAZARD CATEGORIES : Fire $\square$ Reactive Sudden Pressure

Releas!
UNITS OF MEASURE

AMOUNT \& Gals. $\square$ Lbs. $\square$ Maximum Dally Amount
TIME AT

FACILITY : Cu. Ft. $\square$ Average Daily Amount

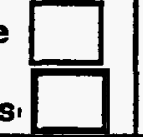

HEALTH
Immediate Health
(Acute) $X x^{\text {Delayed Health }}$
(Chronic) $X$

\section{HEALTH}

soms

\# Days on Site :

1,500 Largest Container on-sit 365 (Amount):

\begin{tabular}{l}
$\begin{array}{l}\text { STORAGE } \\
\text { CODES \& } \\
\text { LOCATIONS : } \\
\text { (use codes provided) }\end{array}$ \\
\cline { 2 - 8 } \\
\cline { 2 - 7 }
\end{tabular}


FOR ADMINSTRATIVE AGENCY USE :

DATE RECEIVED :

FACILITY ID \# :

\section{CHEMICAL DESCRIPTION}

Common Name :NITRIC ACID
Chemical Name :SAME

Solld $\square$ Liquid $x$ Gas $\square$ Pure $\square$

Trade Secret $\square$ Radioactiv $\square$ (If radiocative :
Mixture $\mathrm{X}$ Waste $\square$

o curries)
CAS \# : $7697-37-2$

UN/NA \# : UN 2031

If waste is checked,

Annual Amount

Generated :

WASTE CLASSIFICATION :

Toxic $x$ Ignitable $\square$ Corrosive $x$ Reactive $x$ Extremely Hazardous $\square$

PHYSICAL AND HEALTH

HAZARD CATEGORIES : FIre $\square$ Reactive $X$ Sudden Pressure Releas,

UNITS OF MEASURE

AMOUNT \&

TIME AT

FACILITY :

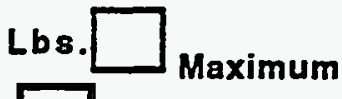

Average

Daily Amount

Daily Amount

\section{HEALTH}

Immediate Health Delayed Health

(Acute) $x \quad$ (Chronic) $x$

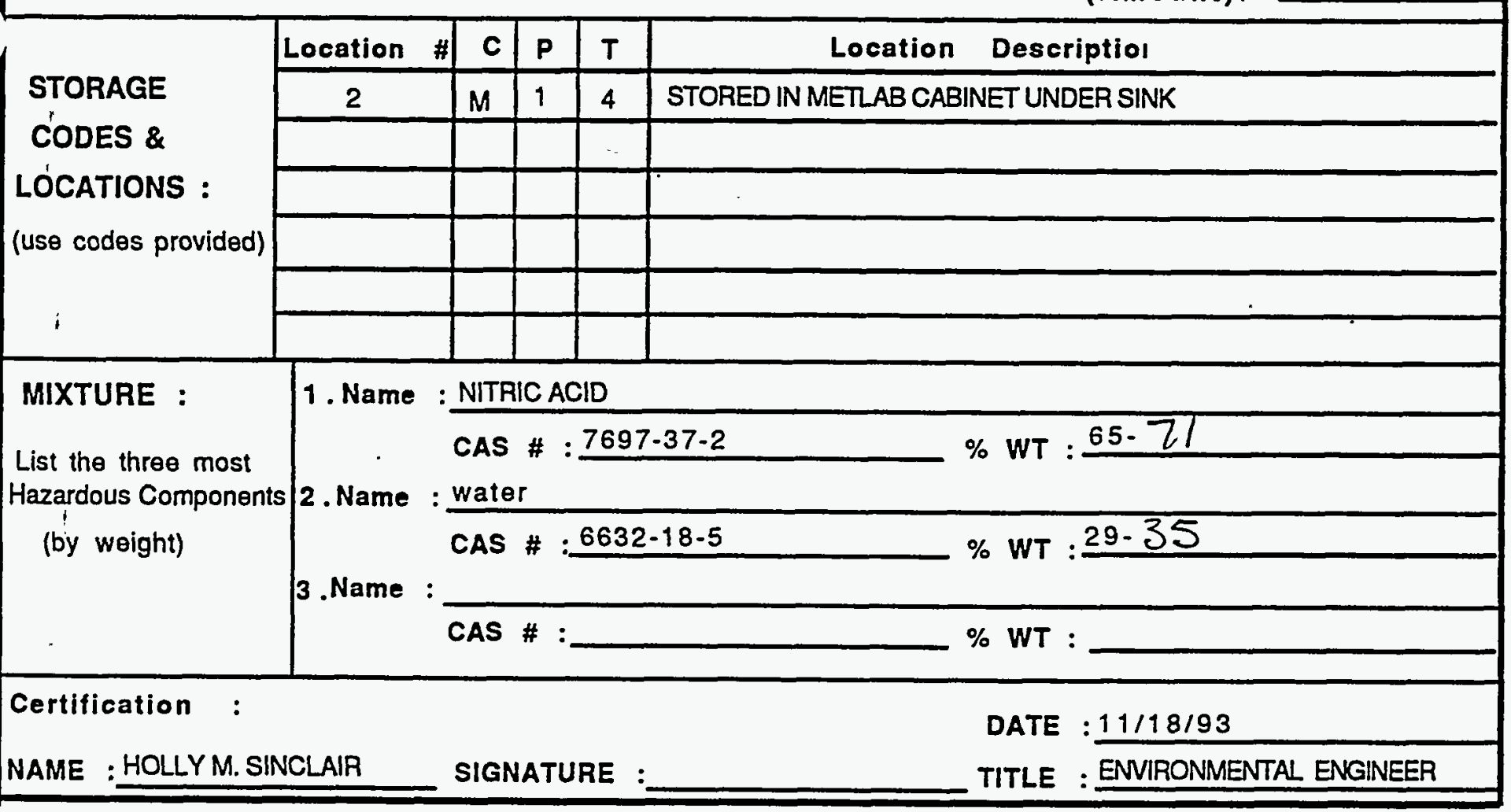


FOR ADMINSTRATIVE AGENCY USE :

DATE RECENED :

FACILITY ID \# :

\section{CHEMICAL DESCRIPTION}

Common Name :POTASSIUM FERRICYANIDE
Chemical Name :SAME

Solid $x$ Liquid $\square$ Gas $\square$ Pure $x$

Trade Secret $\square$ Radioactiv $\square$ (If radiocative :
Mixture $\square$ Waste

o curries)
CAS \# : $13746-66-2$

UN/NA \# :

If waste is checked,

Annual Amount

Generated :

\section{WASTE CLASSIFICATION :}

Toxic $x$ Ignitable $\square$ Corrosive $\square$ Reactive $\square$ Extremely Hazardous $\square$

PHYSICAL AND HEALTH

HAZARD CATEGORIES : Fire $X$ Reactive

Sudden Pressure Releası
PHYSICAL

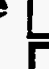

HEALTH

Immediate Health Delayed Health

(Acute) $X$ (Chronic) $X$

\section{UNITS OF MEASURE}

AMOUNT \& Gals. $\square$ Lbs. $X$

TIME AT

FACILITY :

Cu. Ft.

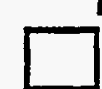

Maximum

Average
Daily Amount

Daily Amount 0.8
1 \# Days on Site :

\begin{tabular}{l}
$\begin{array}{l}\text { STORAGE } \\
\text { CODES \& } \\
\text { LOCATIONS : } \\
\text { (use codes provided) }\end{array}$ \\
\cline { 2 - 8 } \\
\cline { 2 - 7 }
\end{tabular}


FOR ADMINSTAATIVE AGENCY USE :

DATE RECENED :

FACILITY ID \# :

\section{CHEMICAL DESCRIPTION}

Common Name :SODIUMHYDROXDE

Chemical Name :Sodium Hydroxide

Solid $x$ Liquid $\square$ Gas $\square$ Pure $x$

Trade Secret $\square$ Radioactiv $\square$ (If radiocative :
CAS \# : $1310-73-2$

UN/NA \# :

Mixture $\square$ Waste $\square$ If waste is checked,

Annual Amount

Generated :

\section{WASTE CLASSIFICATION :}

Toxic $\square$ Ignitable $\square$ Corrosive $X$ Reactive $\square$ Extremely Hazardous $\square$

\begin{tabular}{l} 
PHYSICAL AND HEALTH $\quad \begin{array}{c}\text { PHYSICAL } \\
\text { HAZARD CATEGORIES : Fire } \square \text { Reactive } \square \\
\text { Sudden Pressure Releas } \square\end{array} \mid \begin{array}{ll}\text { HEALTH } \\
\text { Imediate Health } \\
\text { (Acute) } x\end{array}$ \\
\hline
\end{tabular}

UNITS OF MEASURE

AMOUNT \& Gals. $\square$ Lbs. $\square$ Kaximum

TIME AT Cu. Ft. $\square$ Maximum Daily Amount

FACILITY : Cu. Ft. $\square$ Average Daily Amount

5 \# Days on Site :

365

5 Largest Container on-sit

\begin{tabular}{l}
$\begin{array}{l}\text { STORAGE } \\
\text { CODES \& } \\
\text { LOCATIONS : } \\
\text { (use codes provided) }\end{array}$ \\
\cline { 2 - 8 } \\
\cline { 2 - 7 }
\end{tabular}


FOR ADMINSTRATIVE AGENCY USE :

DATE RECENED :

FACILITY ID \# :

\section{CHEMICAL DESCRIPTION}

Common Name :SODIUM THIOSULFATE

Chemical Name :Sodium Thiosulfate, solutions

Solid $\square$ Liquid $x$ Gas $\square$ Pure $\square$

Trade Secret $\square$ Radioactiv $\square$ (If radiocative :
Mixture $\mathrm{X}$ Waste

o curries)
CAS \# : $7772-98-7$ UN/NA \# :

If waste is checked,

Annual Amount

Generated :

\section{WASTE CLASSIFICATION :}

Toxic $\square$ Ignitable $\square$ Corrosive $\square$ Reactive $\square$ Extremely Hazardous $\square$

$\begin{aligned} \text { PHYSICAL AND HEALTH } & \text { PHYSICAL } \\ \text { HAZARD CATEGORIES : Fire } \square \text { Reactive } \square & \text { Sudden Pressure Releas: }\end{aligned} \mid \begin{aligned} & \text { HEALTH } \\ & \text { Immediate Health } \\ & \text { (Acute) }\end{aligned} \quad \begin{aligned} & \text { Delayed Health } \\ & \text { (Chronic) }\end{aligned}$

\section{UNITS OF MEASURE}

AMOUNT \&

TIME AT

FACILITY :

Lbs. $\square$ Maximum Daily Amount

cu. Ft.

Average

Daily Amount

titers,

1 \# Days on Site

1 Largest Container on-sit (Amount):

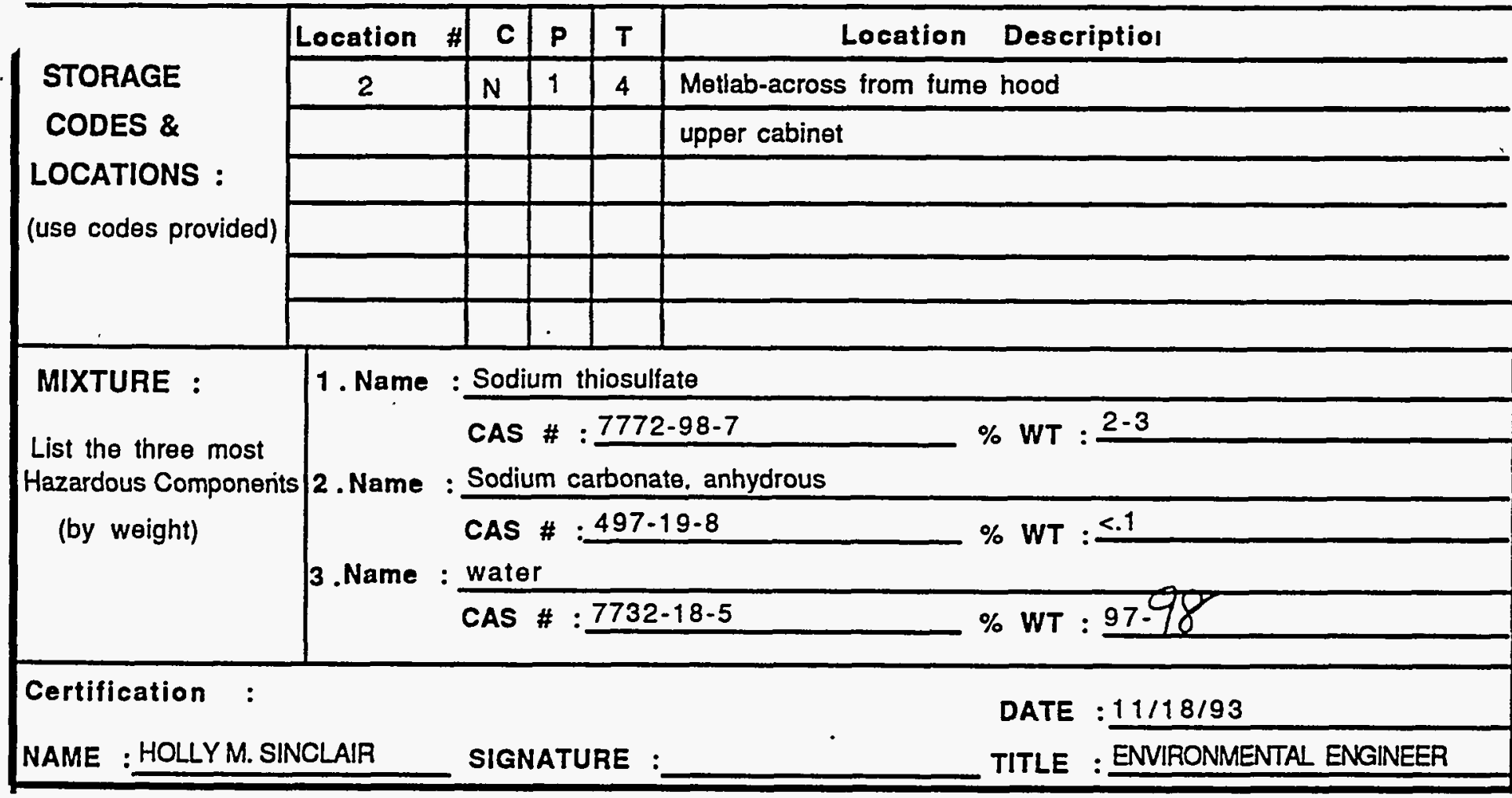


FOR ADMINSTRATIVE AGENCY USE :

DATE RECEIVED :

FACILITY ID \# :

\section{CHEMICAL DESCRIPTION}

Common Name :AMMONIUM SULFATE

Chemical Name :Ammonium sulfate

Solid $x$ Liquid $\square$ Gas $\square$ Pure $x$ Mixture $\square$ Waste

Trade Secret $\square$ Radioactiv $\square$ (If radiocative :
O Curries)
CAS \# : $7783-20-2$ UN/NA \# :

If waste is checked, Annual Amount

Generated : WASTE CLASSIFICATION :

Toxic $X$ Ignitable $\square$ Corrosive $\square$ Reactive $\square$ Extremely Hazardous $\square$

\begin{tabular}{|c|c|c|c|c|}
\hline PHYSICAL AND HEALTH & PHYSICAL & & HE & \\
\hline HAZARD CATEGORIES : Fire & Reactive & Immediate & Health & Delayed \\
\hline Sudden & 'ressure & & & c) \\
\hline
\end{tabular}

\section{UNITS OF MEASURE}

AMOUNT \& Gals.

TIME AT

FACILITY :

Lbs. $X$

Cu. Ft.

Maximum Daily Amount

1 \# Days on site :

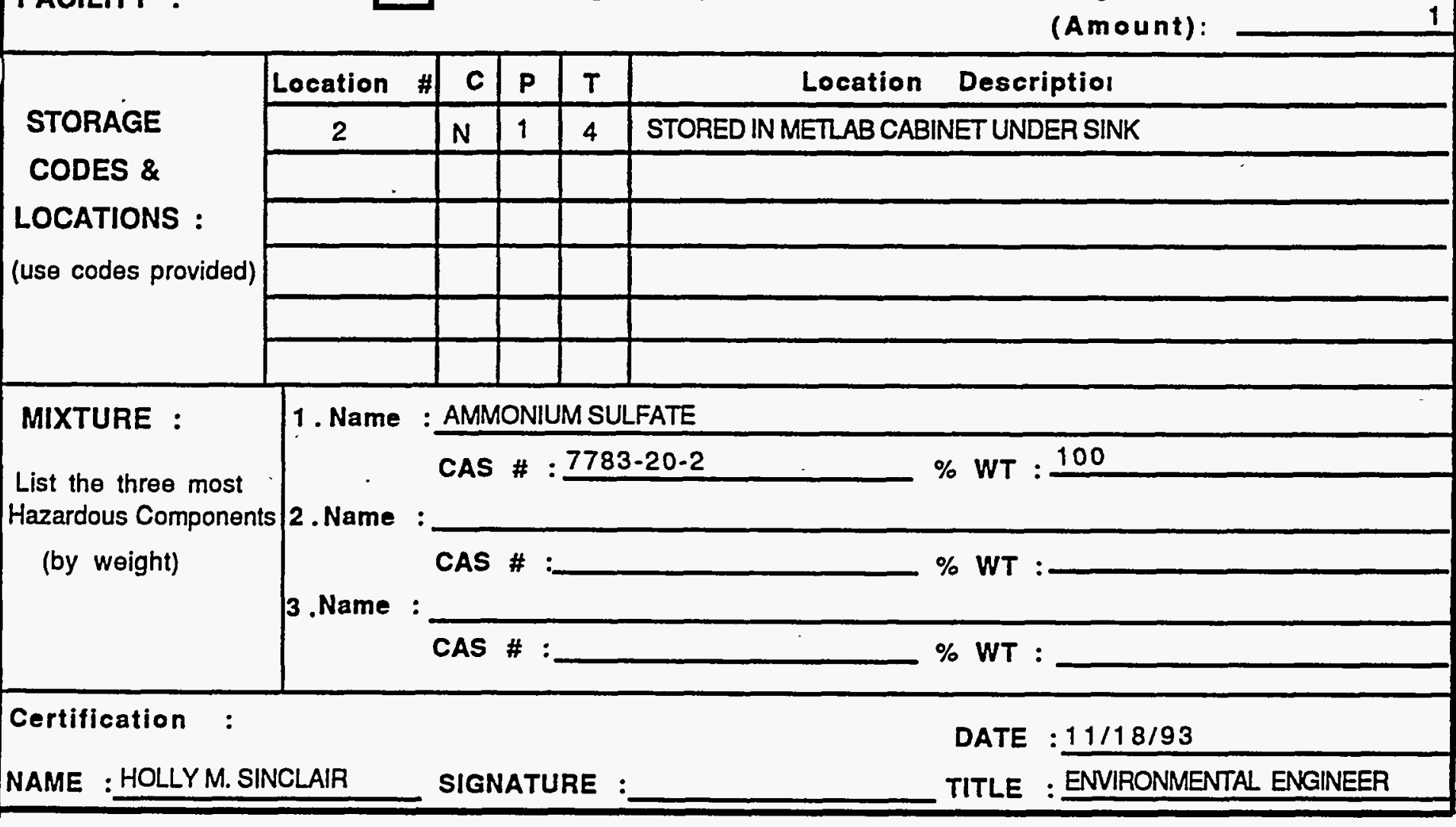


FOR ADMINSTRATIVE AGENCY USE :

DATE RECEIVED :

FACILITY ID \# :

\section{CHEMICAL DESCRIPTION}

Common Name :AMMONIUM PERSULFATE

CAS \# : 7727-54-0

Chemical Name :SAME

Solid $x$ Liquid $\square$ Gas $\square$ Pure $x$ Mixture $\square$ Waste $\square$ UN/NA \# :

If waste is checked, Annual Amount Trade Secret $\square$ Radioactiv $\square$ (If radiocative : _ 0 WASTE CLASSIFICATION :

Toxic $\square$ Ignitable $\square$ Corrosive $x$ Reactive $\square$ Extremely Hazardous $\square$

PHYSICAL AND HEALTH

PHYSICAL

HAZARD CATEGORIES : Fire $X$ Reactive $X$

Sudden Pressure

Releas:

UNITS OF MEASURE

AMOUNT \& Gals.

TIME AT

FACILITY :

Cu. Ft.

Lbs. $X$

t.

Average Daily Amount
HEALTH

$\begin{array}{cll}\text { Immediate } & \text { Health } & \text { Delayed Health } \\ \text { (Acute) } & x & \text { (Chronic) } x\end{array}$

\begin{tabular}{l}
$\begin{array}{l}\text { STORAGE } \\
\text { CODES \& } \\
\text { LOCATIONS : } \\
\text { (use codes provided) }\end{array}$ \\
\cline { 2 - 8 } \\
\cline { 2 - 7 }
\end{tabular}


FOR ADMINSTRATIVE AGENCY USE :

DATE RECEIVED :

\section{INVENTORY FORM}

FACILITY ID \# :

\section{CHEMICAL DESCRIPTION}

Common Name :CUPRIC SULFATE

Chemical Name :SAME

Solid $X$ Liquid $\square$ Gas $\square$ Pure $X$ Mixture $\square$ Waste

Trade Secret $\square$ Radioactiv $\square$ (lf radiocative : $\quad$ curries)

CAS \# : $7758-99-8$

UN/NA \# :

If waste is checked,

Annual Amount

Generated :

0

\section{WASTE CLASSIFICATION :}

Toxic $\square$ Ignitable $\square$ Corrosive $x$ Reactive $\square$ Extremely Hazardous $\square$

PHYSICAL AND HEALTH PHYSICAL

HAZARD CATEGORIES : Fire $\square$ Reactive $\square$

Sudden Pressure Releas,

UNITS OF MEASURE

AMOUNT \& Gals. $\square$ Lbs. $X$

TIME AT

FACILITY :

Cu. Ft.

$\square$

Maximum Dally Amount

Average Daily Amount

Immediate Health

(Acute) $\mathrm{X}$

HEALTH

\begin{tabular}{|c|c|c|c|c|c|}
\hline \multirow{5}{*}{$\begin{array}{l}\text { STORAGE } \\
\text { CODES \& } \\
\text { LOCATIONS : } \\
\text { (Use codes provided) }\end{array}$} & Location \# & C & $\mathbf{P}$ & $T$ & Location Descriptiol \\
\hline & 2 & \multirow[t]{2}{*}{$\mathrm{N}$} & 1 & \multirow[t]{2}{*}{4} & STOREDIN METLAB CABINET UNDER SINK \\
\hline & \multirow{2}{*}{ 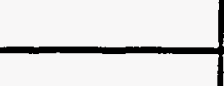 } & & & & \\
\hline & & & & & 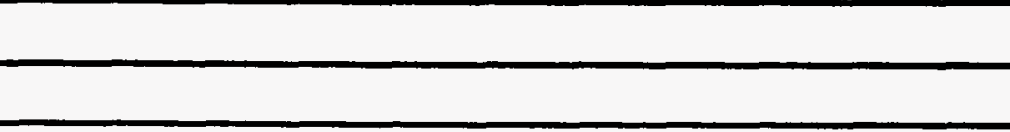 \\
\hline & & & & & \\
\hline & & & & & \\
\hline \multirow{4}{*}{$\begin{array}{l}\text { MIXTURE : } \\
\text { List the three most } \\
\text { Hazardous Components } \\
\text { (by weight) }\end{array}$} & \multirow{4}{*}{$\begin{array}{l}1 . \text { Name : } \\
\text { 2. Name: } \\
3 . \text { Name: }\end{array}$} & \multicolumn{4}{|c|}{ CUPRIC SULFATE } \\
\hline & & \multicolumn{3}{|c|}{ CAS \# : $7758-99-8$} & $\% W T: 100$ \\
\hline & & \multicolumn{3}{|c|}{ CAS \# :- } & — $\%$ WT : \\
\hline & & \multicolumn{3}{|c|}{ CAS \# : } & $\% W T:$ \\
\hline \multicolumn{2}{|l|}{ Certification } & \multirow{2}{*}{\multicolumn{3}{|c|}{ SIGNATURE }} & DATE : $11 / 18 / 93$ \\
\hline NAME : HOLLYM.SIN & VCLAIR & & & & TITLE : ENVIRONMENTAL ENGINEER \\
\hline
\end{tabular}


FOR ADMINSTRATIVE AGENCY USE :

DATE RECENED :

FACILITY ID \# :

\section{CHEMICAL DESCRIPTION}

Common Name :ETHY ALCOHOL

CAS \# : 64-17-5

Chemical Name :ETHANOL

UN/NA \# : UN 1170

solid $\square$

Trade Secret
Liquid $X$ Gas Radioactiv
Pure $\square$

(If radiocative :
Mixture $X$ Waste

o curries)
If waste is checked, Annual Amount

Generated :

\section{WASTE CLASSIFICATION :}

Toxic $\square$ Ignitable $X$ Corrosive $\square$ Reactive $\square$ Extremely Hazardous $\square$

PHYSICAL AND HEALTH PHYSICAL

HAZARD CATEGORIES : Fire $X$ Reactive

Sudden Pressure Releas,

UNITS OF MEASURE

AMOUNT \&

TIME AT

FACILITY :

Lbs.

Cu. Ft.

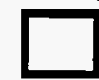

Maximum Daily Amount

Average Daily Amount
HEALTH

Immediate Health Delayed Health

(Acute) $x$

(Chronic) $x$

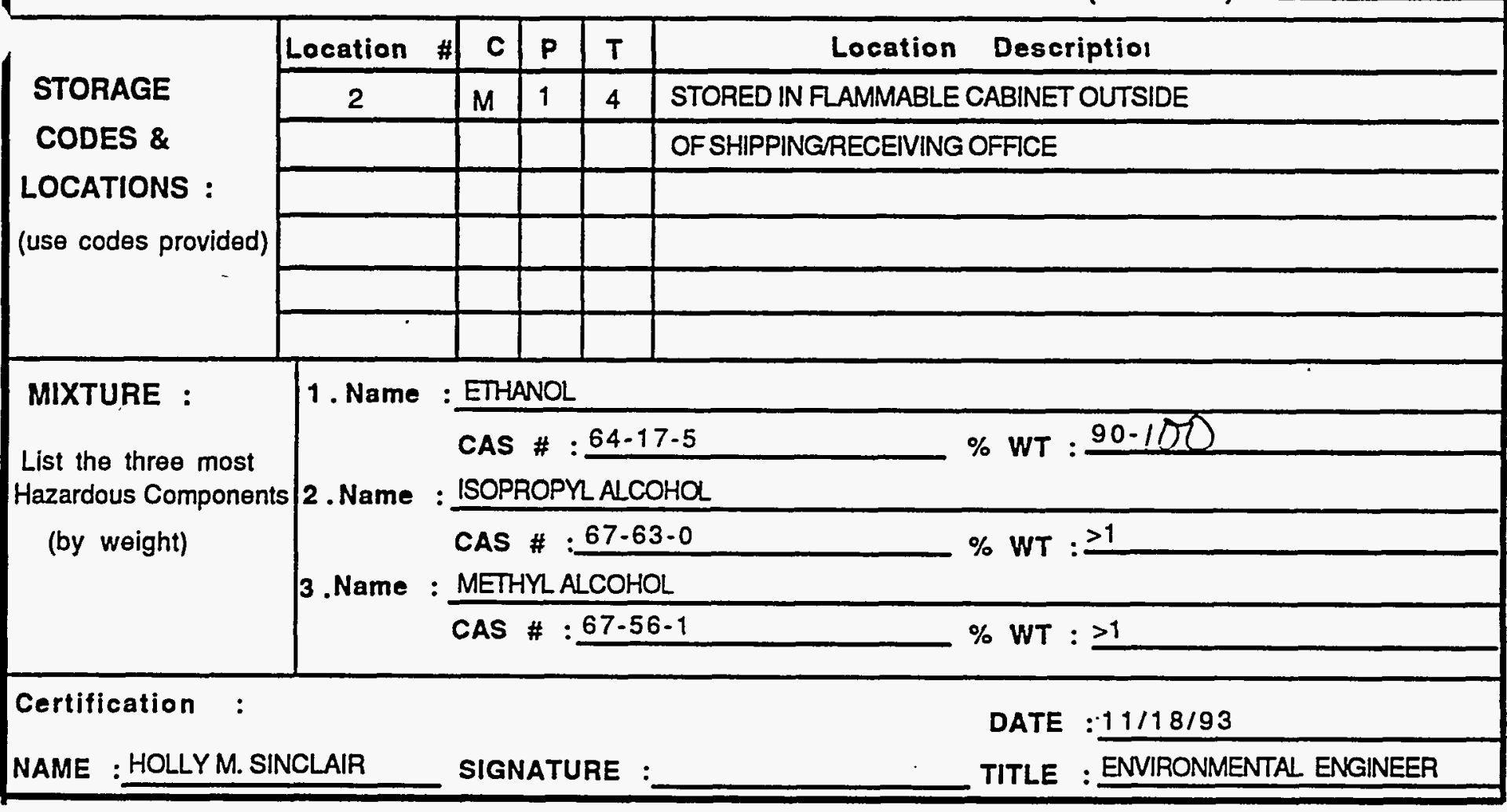


FOR ADMINSTRATIVE AGENCY USE :

DATE RECEIVED :

FACILITY ID \# :

\section{CHEMICAL DESCRIPTION}

Common Name :CUPRIC CHLORIDE, DIHYDRATE CAS \# : $13933-17-0$

Chemical Name :SAME

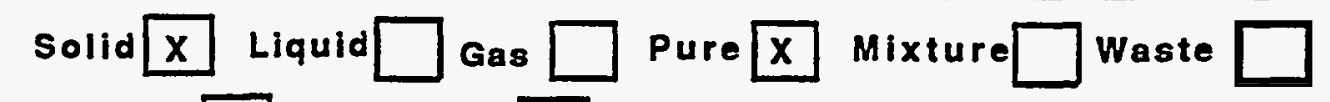

UN/NA \# : UN 2811

Trade secret $\square$ Radioactiv $\square$ (If radiocative : — Curries)

If waste is checked, Annual Amount

Generated :

o

\section{WASTE CLASSIFICATION :}

Toxic $X$ Ignitable $\square$ Corrosive $\square$ Reactive $\square$ Extremely Hazardous $\square$

\begin{tabular}{|c|c|c|c|c|}
\hline \multirow{2}{*}{$\begin{array}{l}\text { PHYSICAL AND HEALTH } \\
\text { HAZARD CATEGORIES : }\end{array}$} & PHYSICAL & \multicolumn{3}{|c|}{ HEALTH } \\
\hline & Reactive & Immediate & Health & Delayed Health \\
\hline Sudden & ressure Releas & (Acute) & $x$ & (Chronic) $x$ \\
\hline
\end{tabular}

\section{UNITS OF MEASURE}

AMOUNT \& Gals. $\square$ Lbs. $X$

TIME AT

FACILITY :

Cu. Ft.

Maximum. Daily Amount

Average Daily Amount

19 \# Days on Site :

365

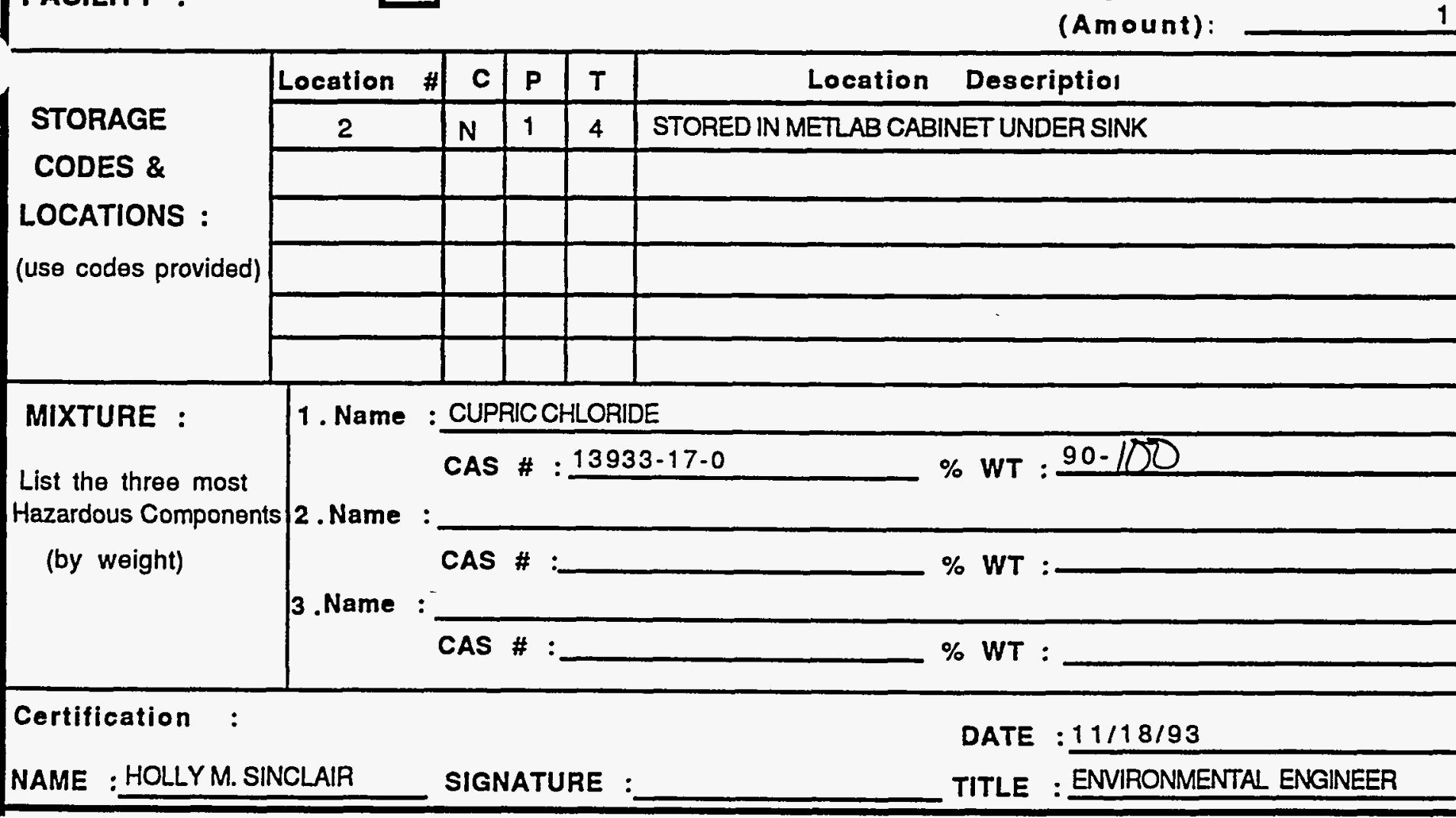


FOR ADMINSTRATIVE AGENCY USE :

DATERECEIVED:

FACILITY ID \# :

\section{CHEMICAL DESCRIPTION}

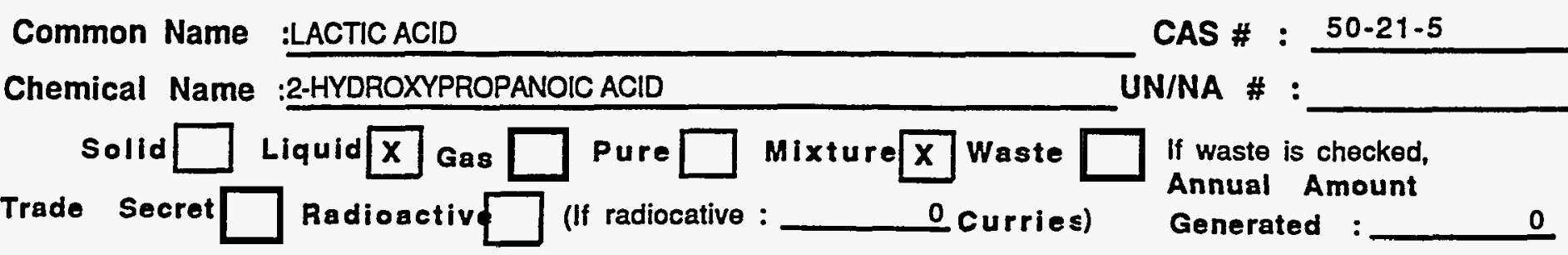

WASTE CLASSIFICATION :

Toxic $\square$ Ignitable $\square$ Corrosive $x$ Reactive $\square$ Extremely Hazardous $\square$

PHYSICAL AND HEALTH PHYSICAL

HAZARD CATEGORIES : Fire $\square$ Reactive

Sudden Pressure Releasi

UNITS OF MEASURE

AMOUNT \&

TIME AT

FACILITY :

Cu. Ft.

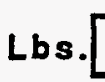

Maximum

Average

Daily Amount

Daily Amount

\begin{tabular}{|c|}
\multicolumn{2}{c}{ HEALTH } \\
Immediate Health \\
(Acute) $x{ }^{\text {Delayed Health }}$ \\
(Chronic) $x$
\end{tabular}

N

500 \# Days on Site 500 Largest Container on-sit (Amount):

\begin{tabular}{l}
$\begin{array}{l}\text { STORAGE } \\
\text { CODES \& } \\
\text { LOCATIONS : } \\
\text { (use codes provided) }\end{array}$ \\
\cline { 2 - 7 }
\end{tabular}


FOR ADMINSTRATIVE AGENCY USE :

DATE RECEIVED :

FACILITY ID \# :

\section{CHEMICAL DESCRIPTION}

Common Name :HYDROGEN PEROXIDE $3 \%$

Chemical Name :SAME

Solid $\square$ Liquid $x$ Gas $\square$ Pure $\square$ Mixture $x$ Waste

Trade secret $\square$ Radioactiv $\square$ (If radiocative : o curries)
CAS \# : 7722-84-1

UN/NA \# :

If waste is checked, Annual Amount

Generated :

은

\section{WASTE CLASSIFICATION :}

Toxic $\square$ Ignitable $\square$ Corrosive $\square$ Reactive $\square$ Extremely Hazardous $\square$

\section{PHYSICAL AND HEALTH \\ PHYSICAL}

HAZARD CATEGORIES
Fire $\square$ Reactive

Sudden Pressure Releas

\section{UNITS OF MEASURE}

AMOUNT \&

TIME AT

FACILITY :

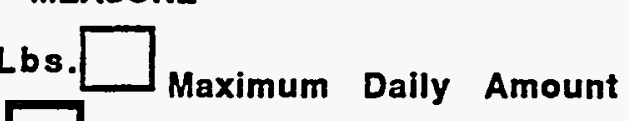

Average Daily Amount

Cu. Ft.

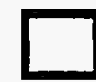

\section{HEALTH}

Immediate Health Delayed Health

(Acute) $x$ (Chronic) $x$

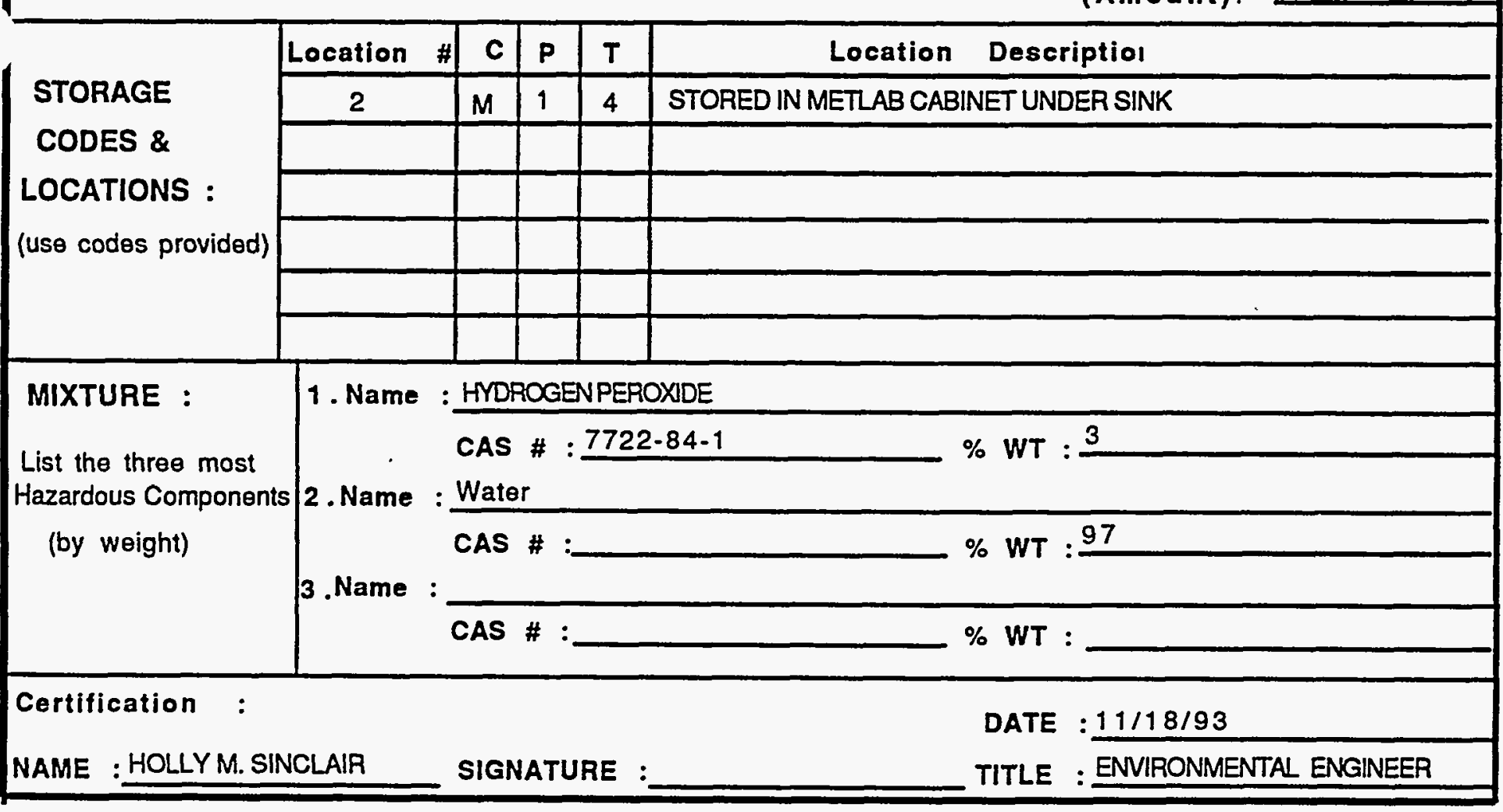


FOR ADMINSTRATIVE AGENCY USE :

DATE RECENED :

FACILITY ID \# :

\section{CHEMICAL DESCRIPTION}

Common Name :DARACLEAN 283 CAS \# :

Chemical Name :AQUEOUS ALKALINE CLEANER

Solid $\square$ Liquid $\mathrm{x}$ Gas $\square$ Pure $\square$ Mixture $\mathrm{X}$ Waste $\square$ UN/NA \# : UN 1824

Trade Secret $\square$ Radioactiv $\square$ (If radiocative : $\quad 0$ curries)

If waste is checked, Annual Amount

Generated :

0

WASTE CLASSIFICATION :

Toxic $x$ Ignitable $\square$ Corrosive $x$ Reactive $\square$ Extremely Hazardous $\square$

PHYSICAL AND HEALTH

HAZARD CATEGORIES :
PHYSICAL

Fire $\square$ Reactive

Sudden Pressure Releas

UNITS OF MEASURE

AMOUNT \&

TIME AT

FACILITY :

Gals. $X$ Lbs. $\square$ Maximum Daily Amount

Cu. Ft.

Average Daily Amount

110 \# Days on Site :

Delayed Health

Immediate Health $\begin{aligned} & \text { Delayed Health } \\ & \text { (Acute) } X\end{aligned}$
(Chronic) $X$

HEALTH

$$
\text { (Amount): }
$$

STORAGE

CODES \&

LOCATIONS :

(use codes provided)

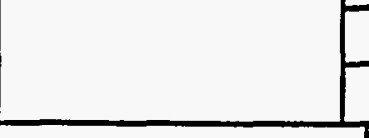

MIXTURE :

List the three most

\begin{tabular}{|c|}
\hline Location \# \\
\hline 7 \\
\hline \\
\hline \\
\hline \\
\hline
\end{tabular}

1. Name : SODIUM HYDROXIDE

CAS \# : 1310-73-2

$\% W T: \leq 2$

Hazardous Components

(by weight)

2. Name: Water

CAS \# : $\%$ WT : $\geq 98$

3 . Name

CAS \# : \% WT :

Certification :

NAME : HOLLYM. SINCLAIR

SIGNATURE :

DATE : $11 / 18 / 93$

TITLE : ENVIRONMENTAL ENGINEER 
FOR ADMINSTAATIVE AGENCY USE :

DATE RECEIVED :

\section{INVENTORY FORM}

FACILITY ID \# :

\section{CHEMICAL DESCRIPTION}

Common Name :ISOGUARD

Chemical Name :Cooling Water Treatment Compound

Solid $\square$ Liquid $x$ Gas $\square$ Pure $\square$ Mixture $x$ Waste

Trade Secret $\square$ Radioactiv $\square$ (If radiocative : o curries)
CAS \# :

UN/NA \# :

If waste is checked,

Annual Amount

Generated :

\section{WASTE CLASSIFICATION :}

Toxic $X$ Ignitable $\square$ Corrosive $\square$ Reactive $\square$ Extremely Hazardous $\square$

\begin{tabular}{|c|c|c|c|c|}
\hline \multirow{2}{*}{$\begin{array}{l}\text { PHYSICAL AND HEALTH } \\
\text { HAZARD CATEGORIES . }\end{array}$} & PHYSICAL & \multicolumn{3}{|c|}{ HEALTH } \\
\hline & Reactive & Immediate & Health & Delayed Health \\
\hline Sudden & Pressure Releas & (Acute) & $x$ & (Chronic) $x$ \\
\hline
\end{tabular}

\section{UNITS OF MEASURE}

AMOUNT \&

TIME AT

Gals. $X$

FACILITY :

Cu. Ft.

Maximum Daily Amount

55 \# Days on Site :

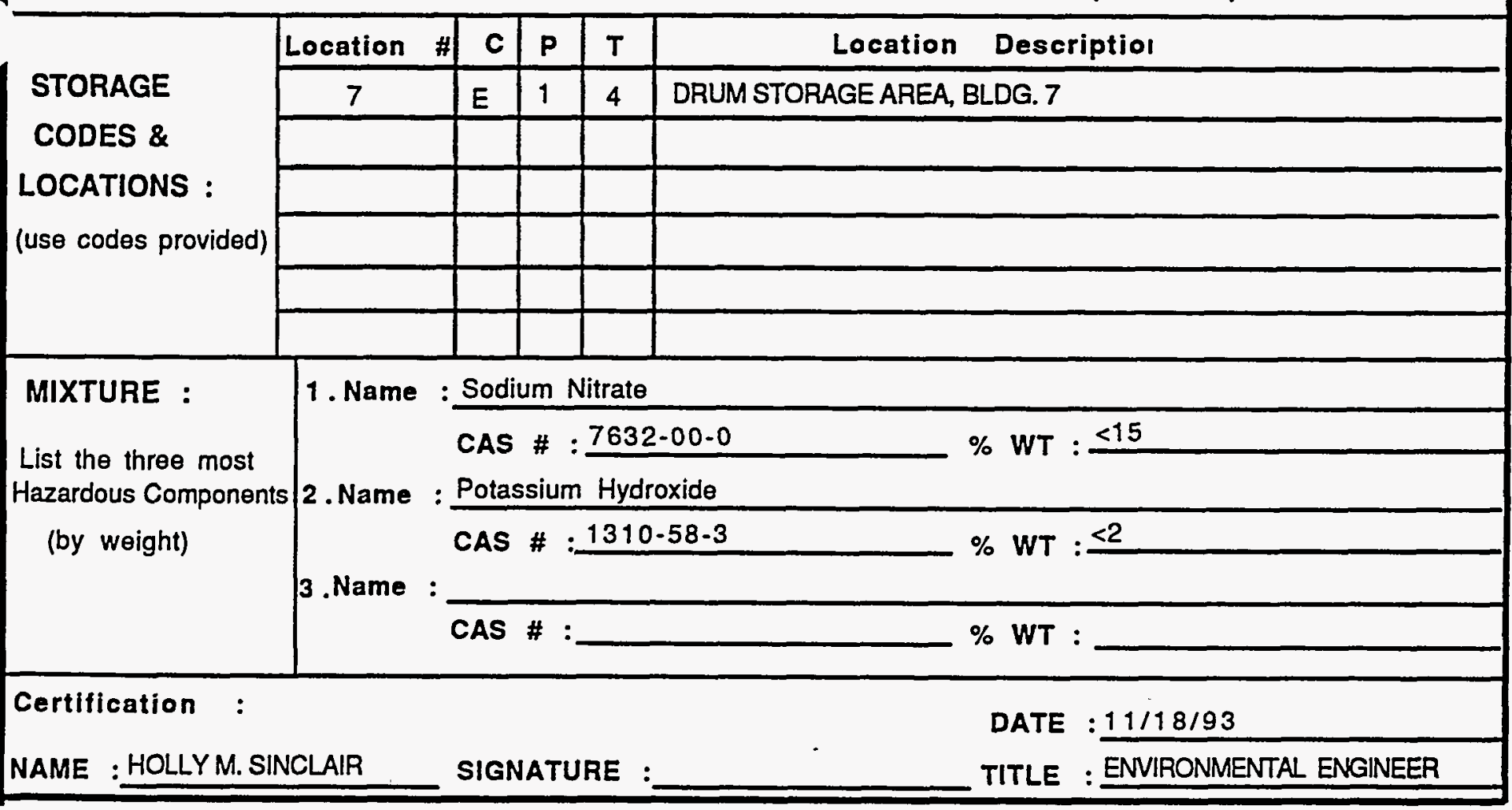


FOR ADMINSTRATIVE AGENCY USE :

DATE RECEIVED :

FACILITY ID \# :

\section{CHEMICAL DESCRIPTION}

Common Name :STAINLESS STEEL

Chemical Name :same

Solid $X]$ Liquid $\square$ Gas $\square$ Pure $\square$ Mixture $X$ Waste $\square$

Trade secret $\square$ Radioactiv $\square$ (If radiocative : CAS \# : UN/NA \#:

If waste is checked, Annual Amount

Generated : 0 WASTE CLASSIFICATION :

Toxic $\square$ Ignitable $\square$ Corrosive $\square$ Reactive $\square$ Extremely Hazardous $\square$

\begin{tabular}{|c|c|c|c|c|c|}
\hline PHYSICAL AND HEALTH & PHYSICAL & \multicolumn{4}{|c|}{ HEALTH } \\
\hline HAZARD CATEGORIES : & Reactive & Immediate & Health & Delayed He & lealth \\
\hline Sudden & 'ressure Releas' & & $x$ & (Chronic) & $\mathbf{x}$ \\
\hline
\end{tabular}

UNITS OF MEASURE

AMOUNT \& Gals. $\square$ Lbs. $X$

TIME AT
FACILITY :

Lbs. $X$ Maximum

Daily Amount

Average Daily Amount

500,000 \# Days on site : 450,000 Largest Container on-sit 365

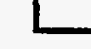

(Amount):

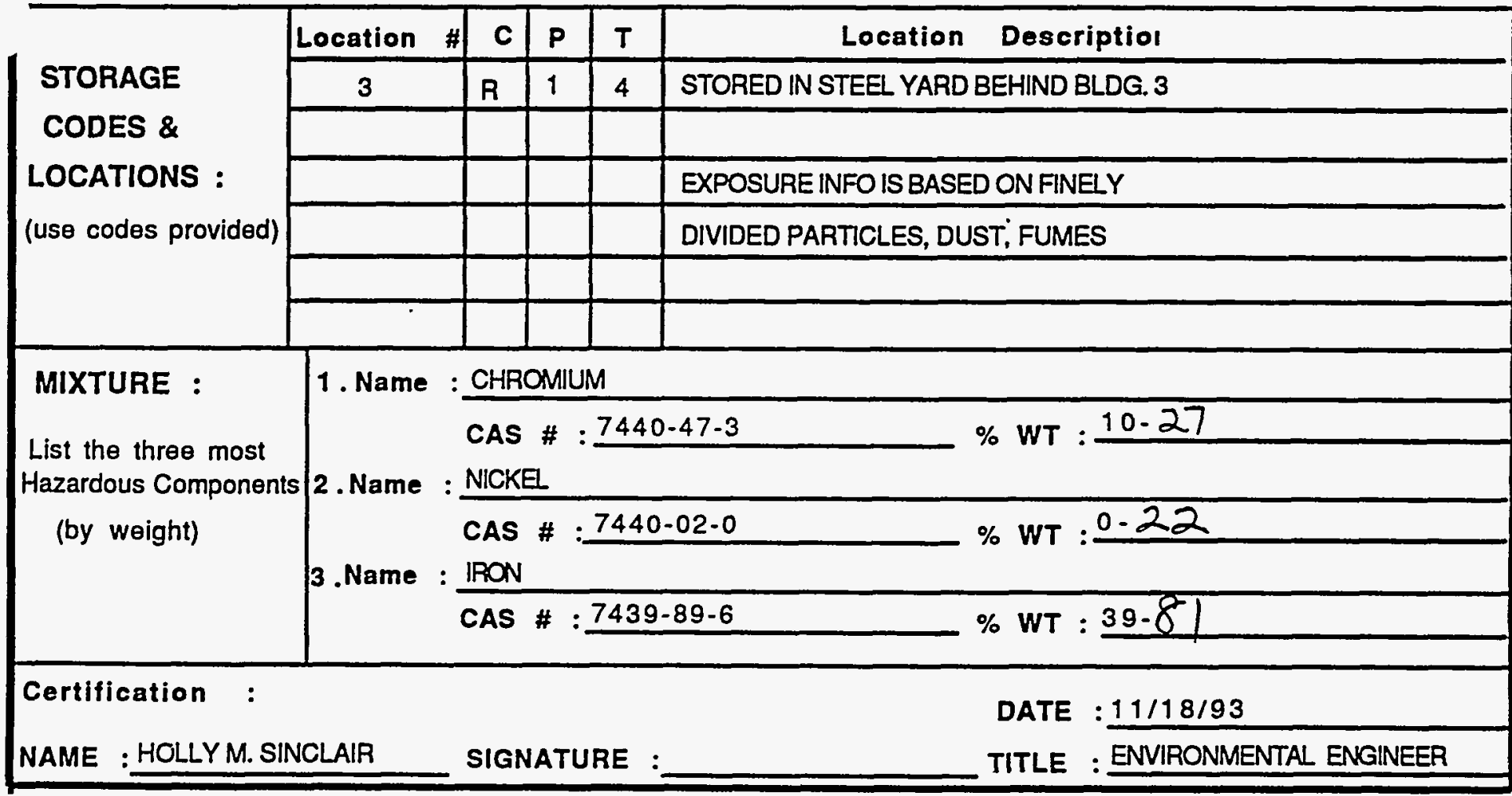


FOR ADMINSTRATIVE AGENCY USE :

DATE RECEIVED:

FACILITY ID \# :

\section{CHEMICAL DESCRIPTION}

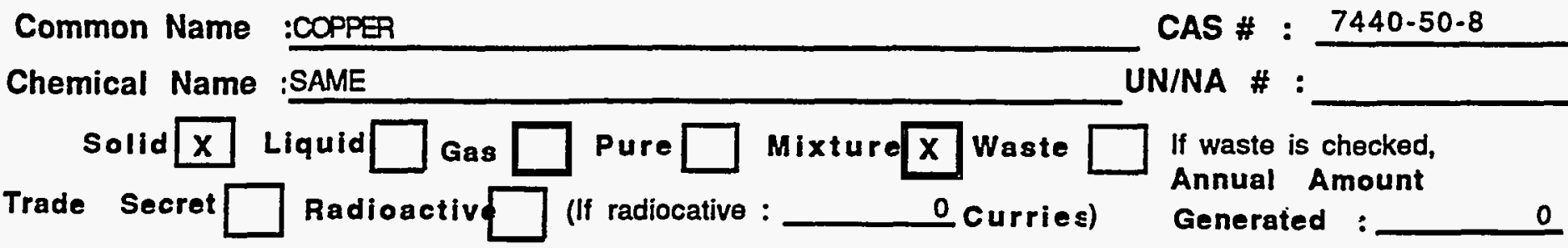

\section{WASTE CLASSIFICATION :}

Toxic $X$ Ignitable $\square$ Corrosive $\square$ Reactive $\square$ Extremely Hazardous $\square$

\begin{tabular}{|c|c|c|c|c|}
\hline \multirow{2}{*}{$\begin{array}{l}\text { PHYSICAL AND HEALTH } \\
\text { HAZARD CATEGORIES : }\end{array}$} & PHYSICAL & \multicolumn{3}{|c|}{ HEALTH } \\
\hline & Reactive & Immediate & Health & Delayed Health \\
\hline Sudden & Pressure & (Acute) & $X$ & (Chronic) $\mid x$ \\
\hline
\end{tabular}

UNITS OF MEASURE

AMOUNT \& Gals.

TIME AT

FACILITY :

Cu. Ft.

Lbs. $X$ Maximum

Average Daily Amount

480 \# Days on Site :

365

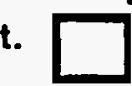

480 Largest Container

(Amount):

\section{STORAGE \\ CODES \& \\ LOCATIONS :}

(use codes provided)

\begin{tabular}{|c|}
\hline Location \# \\
\hline 3 \\
\hline \\
\hline \\
\hline \\
\hline
\end{tabular}

MIXTURE :

1 . Name : COPPER

List the three most

CAS \# : 7440-50-8

$\% W T: \geq 99$

Hazardous Components

(by weight)

2. Name : LEAD (LEADTEX COATING)

$$
\text { CAS \#: } 7439-92-1 \quad \% \text { WT : }: 05
$$

3. Name :

CAS \# :

\% WT :

Certification :

NAME : HOLLYM.SINCLAIR

SIGNATURE :

DATE : $11 / 18 / 93$

TITLE : ENVIRONMENTAL ENGINEER 
FOR ADMINSTRATIVE AGENCY USE :

DATE RECEIVED :

FACILITY ID \# :

\section{CHEMICAL DESCRIPTION}

Common Name :CARBON \& ALLOY STEELS

Chemical Name :steol

Solid $x$ Liquid $\square$ Gas

Trade Secret $\square$ Radioactiv $\square$ (If radiocative :
Mixture $\mathrm{X}$ Waste

O curries)
CAS \# : UN/NA \# :

If waste is checked,

Annual Amount

Generated :

0

\section{WASTE CLASSIFICATION :}

Toxic $\square$ Ignitable $\square$ Corrosive $\square$ Reactive $\square$ Extremely Hazardous $\square$

\begin{tabular}{|c|c|c|c|c|}
\hline PHYSICAL AND HEALTH & PHYSICAL & \multicolumn{3}{|c|}{ HEALTH } \\
\hline HAZARD CATEGORIES : & Reactive & Immediate & Health & Delayed Health \\
\hline Sudden & Pressure Releası & (Acute) & $x$ & (Chronic) $x$ \\
\hline
\end{tabular}

UNITS OF MEASURE

AMOUNT \& Gals. $\square$ Lbs. $X$

TIME AT Cu. Ft.

FACILITY :

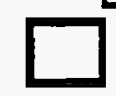

Maximum

Average Dally Amount

Daily Amount

$\frac{110,000}{106,000}$ \# Days on site :

106,000
Largest Container
(Amount):

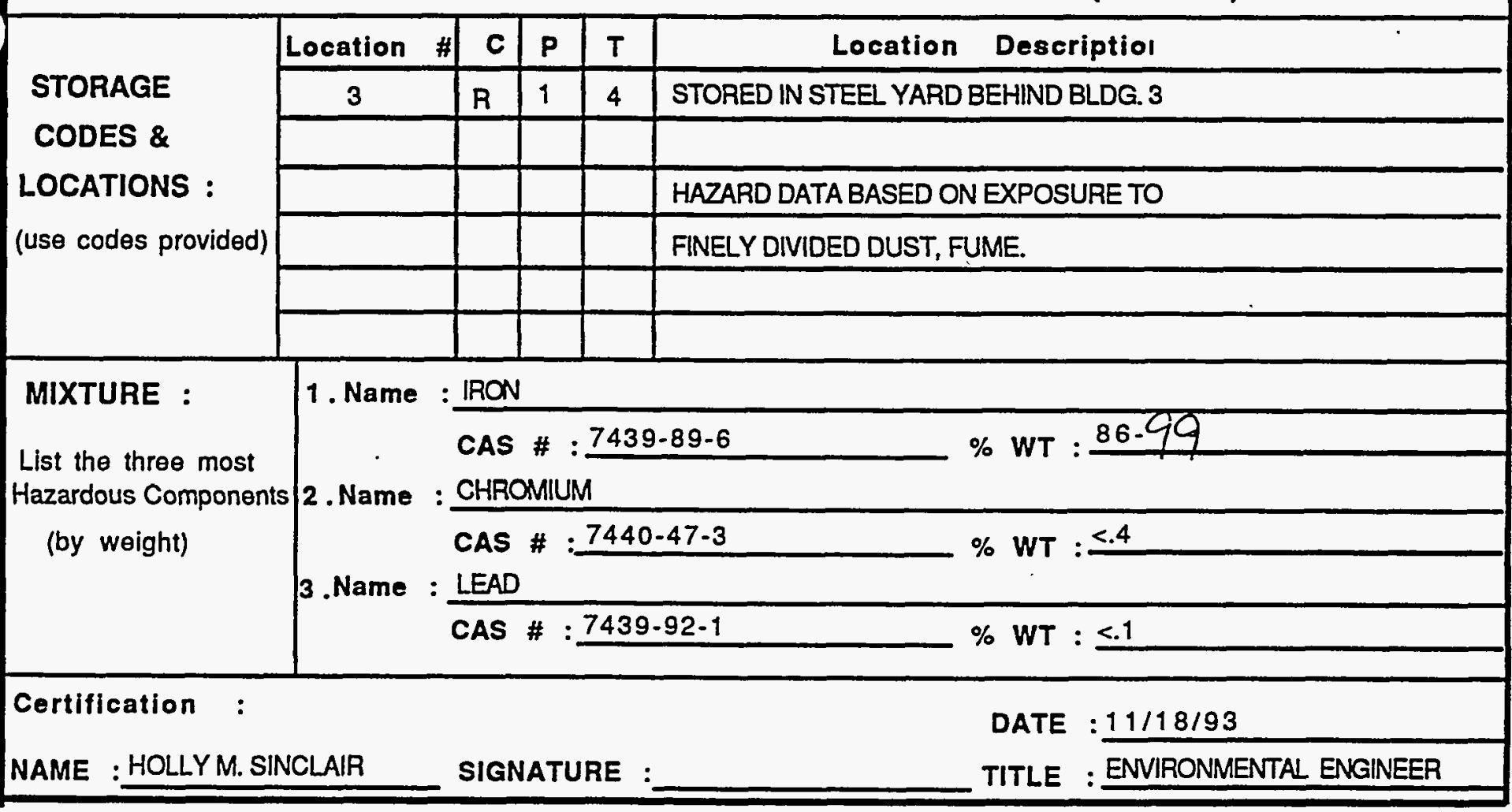


FOR ADMINSTRATIVE AGENCY USE :

DATE RECEIVED :

FACILITY ID \# :

\section{CHEMICAL DESCRIPTION}

Common Name :TITANIUM

Chemical Name :Titanium Alloy

Solid $x$ Liquid $\square$ Gas $\square$ Pure $x$ Mixture $\square$ Waste $\square$

Trade Secret $\square$ Radioactiv $\square$ (If radiocative :
Ocurries)
CAS \# : $13463-64-7$

UN/NA \# :

If waste is checked,

Annual Amount

Generated :

\section{WASTE CLASSIFICATION :}

Toxic $\square$ Ignitable $\square$ Corrosive $\square$ Reactive $\square$ Extremely Hazardous $\square$

\begin{tabular}{|c|c|c|c|c|c|c|c|}
\hline PHYSICAL AND HEALTH & \multicolumn{3}{|c|}{ PHYSICAL } & \multicolumn{4}{|c|}{ HEALTH } \\
\hline HAZARD CATEGORIES : & Fire & $\mathbf{x}$ & Reactive & Immediate & Health & Delayed $H$ & lealth \\
\hline & udden & Pre: & Releası & (Acute). & $x$ & (Chronic) & $x$ \\
\hline
\end{tabular}

\section{UNITS OF MEASURE}

AMOUNT \&

TIME AT

Gals.

Lbs. $X$

FACILITY :

Cu. Ft.

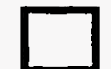

Maximum

Daily Amount

40,000 \# Days on Site :

Average Daily Amount

Largest

(Amount):

\begin{tabular}{|c|c|c|c|c|c|}
\hline \multirow{6}{*}{$\begin{array}{l}\text { STORAGE } \\
\text { CODES \& } \\
\text { LOCATIONS : } \\
\text { (use codes provided) }\end{array}$} & Location \# & C & $\mathbf{P}$ & $\mathbf{T}$ & Descriptiol \\
\hline & 3 & $\mathbf{R}$ & 1 & 4 & STORED IN STEEL YARD BEHIND BLDG. 3 \\
\hline & & & & & HAZARD DESIGNATIONS ARE FOR FINELY \\
\hline & & & & & DIVIDED CHIPS RESULTING FROM \\
\hline & & & & & GRINDING, CUTTING OR POLISHING \\
\hline & & & & & WHILEWET. \\
\hline \multirow{4}{*}{$\begin{array}{l}\text { MIXTURE : } \\
\text { List the three most } \\
\text { Hazardous Components } \\
\text { (by weight) }\end{array}$} & \multicolumn{5}{|c|}{ 1. Name: TITANIUM } \\
\hline & \multirow{3}{*}{$\begin{array}{l}2 . \text { Name: } \\
\text { 3. Name: }\end{array}$} & CAS & $\#$ & 134 & - $\%$ WT : 99 \\
\hline & & CAS & $\#$ & & - $\%$ WT : \\
\hline & & CAS & $\#$ & & $\%$ WT : \\
\hline \multicolumn{2}{|c|}{$\begin{array}{l}\text { Certification : } \\
\text { NAME : HOLLYM.SINCLAIR }\end{array}$} & & & & DATE $: \frac{11 / 18 / 93}{\text { DANOTH }}$ \\
\hline
\end{tabular}




\section{CHEMICAL DESCRIPTION}

Common Name :DENATURED ALCOHOL CAS \# : $64-17-5$

Chemical Name :Ethyl alcohol, anhydrous, denatured UN/NA \# :

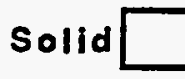

Liquid $X$ Gas

Radioactiv $\square$ (if radiocative :
Mixture $x$ Waste

o curries)
If waste is checked,

Annual Amount

Generated :

0

\section{WASTE CLASSIFICATION :}

Toxic $\square$ Ignitable $X$ Corrosive $\square$ Reactive $\square$ Extremely Hazardous $\square$

PHYSICAL AND HEALTH

HAZARD CATEGORIES :

\section{PHYSICAL}

Fire $X$ Reactive

Sudden Pressure Releas

\section{UNITS OF MEASURE}

AMOUNT \& Gals. $X$ Lbs.

TIME AT

FACILITY :
Cu. Ft.

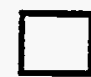

Maximum Average Daily Amount
1 \# Days on Site : Immediate Health Delayed Health (Acute) $x \quad$ (Chronic) $X$

\begin{tabular}{|c|c|c|c|c|c|}
\hline \multirow{6}{*}{$\begin{array}{l}\text { STORAGE } \\
\text { CODES \& } \\
\text { LOCATIONS : } \\
\text { (use codes provided) }\end{array}$} & Location \# & C & $\mathbf{p}$ & $T$ & Location Descriptiol \\
\hline & 2 & $M$ & 1 & 4 & Flammable cabinet outside of \\
\hline & & & & & \\
\hline & & & & & Shipping/Receiving office \\
\hline & & & & & \\
\hline & & & & & \\
\hline \multirow{4}{*}{$\begin{array}{l}\text { MIXTURE : } \\
\text { List the three most } \\
\text { Hazardous Components } \\
\text { (by weight) }\end{array}$} & \multicolumn{5}{|c|}{ 1. Name : methanol } \\
\hline & \multicolumn{5}{|c|}{$\begin{array}{l}\text { CAS \# : } 67-56-1 \\
\text { 2. Name: methyl isobutyl ketone }\end{array}$} \\
\hline & \multicolumn{5}{|c|}{$\begin{array}{l}\text { CAS \#: } 108-10-1 \\
\text { 3. Name: ethanol }\end{array}$} \\
\hline & \multicolumn{5}{|c|}{ CAS \#: $64-17-5$} \\
\hline \multicolumn{6}{|c|}{$\begin{array}{ll}\text { Certification : } & \text { DATE }: 11 / 18 / 93 \\
\end{array}$} \\
\hline NAME : HOLLYM.SIN & NCLAIR & SIG & IATL & & TITLE : ENVIRONMENTAL ENGINEER \\
\hline
\end{tabular}




\section{CHEMICAL DESCRIPTION}

Common Name :Mobil DTE 25 CAS \# :

Chemical Name :Refined mineral oils UN/NA \# : UN 1270

\section{Solid $\square$ \\ Liquid $\mathrm{X}$ \\ Mixture $\mathrm{X}$ Waste}

Radioactiv $\square$ (If radiocative o curries)
If waste is checked, Annual Amount

Generated :

0

\section{WASTE CLASSIFICATION :}

Toxic $X$ Ignitable $\square$ Corrosive $\square$ Reactive $\square$ Extremely Hazardous $\square$

\begin{tabular}{|c|c|c|c|c|}
\hline PHYSICAL AND HEALTH & PHYSICAL & \multicolumn{3}{|c|}{ HEALTH } \\
\hline HAZARD CATEGORIES : & Reactive & Immediate & Health & Delayed \\
\hline Sudden & Dressure & (Acute) & & \\
\hline
\end{tabular}

\section{UNITS OF MEASURE}

AMOUNT \& Gals. $X$ Lbs. $\square$

TIME AT

FACILITY :

Cu. Ft.

Maximum Daily Amount

55 \# Days on Site :

Average Daily Amount

40 Largest Container

(Amount):

\begin{tabular}{|c|c|c|c|c|c|}
\hline \multirow{5}{*}{$\begin{array}{l}\text { STORAGE } \\
\text { CODES \& } \\
\text { LOCATIONS : } \\
\text { (use codes provided) }\end{array}$} & Location : & c & $\mathbf{P}$ & $\mathbf{T}$ & Location Descriptiol \\
\hline & 7 & D & 1 & 4 & Drum storage building \\
\hline & & & & & \\
\hline & & & & & \\
\hline & & & & & \\
\hline & & & & & \\
\hline \multirow{4}{*}{$\begin{array}{l}\text { MIXTURE : } \\
\text { List the three most } \\
\text { Hazardous Components } \\
\text { (by weight) }\end{array}$} & \multicolumn{5}{|c|}{ 1. Name : Refined mineral oil } \\
\hline & \multirow{3}{*}{$\begin{array}{l}2 . \text { Name } \\
3 . \text { Name }\end{array}$} & $\overline{\text { CAS }}$ & \# & - & $\%$ WT $: \geq 95$ \\
\hline & & CAS & $\#$ & & $\%$ WT $: \leq 5$ \\
\hline & & CAS & \# & & — $\mathrm{WT}:$ \\
\hline \multirow{2}{*}{\multicolumn{2}{|c|}{$\begin{array}{l}\text { Certification : } \\
\text { NAME :HOLLYM.SINCLAIR }\end{array}$}} & & & & DATE $: 11 / 18 / 93$ \\
\hline & & SIGI & IATL & & TITLE : ENVIRONMENTAL ENGINEER \\
\hline
\end{tabular}


FOR ADMINSTRATIVE AGENCY USE :

DATE RECENED :

FACILITY ID \# :

\section{CHEMICAL DESCRIPTION}

Common Name :Mobil Vactra \#2 CAS \# :

Chemical Name :Refined mineral oil UN/NA \# : UN 1270 - Solid Liquid $x$ Gas Pure $\square$ Mixture $x$ Waste Trade Secret $\square$ Radioactiv $\square$ (If radiocative : Ocurries) If waste is checked, Annual Amount WASTE CLASSIFICATION :

Toxic $x$ Ignitable $\square$ Corrosive $\square$ Reactive $\square$ Extremely Hazardous $\square$

PHYSICAL AND HEALTH HAZARD CATEGORIES :

\section{PHYSICAL}

Fire $\square$ Reactive $\square$

\section{UNITS OF MEASURE}

AMOUNT \& Gals. $X$ Lbs.

TIME AT

FACILITY :

Cu. Ft.

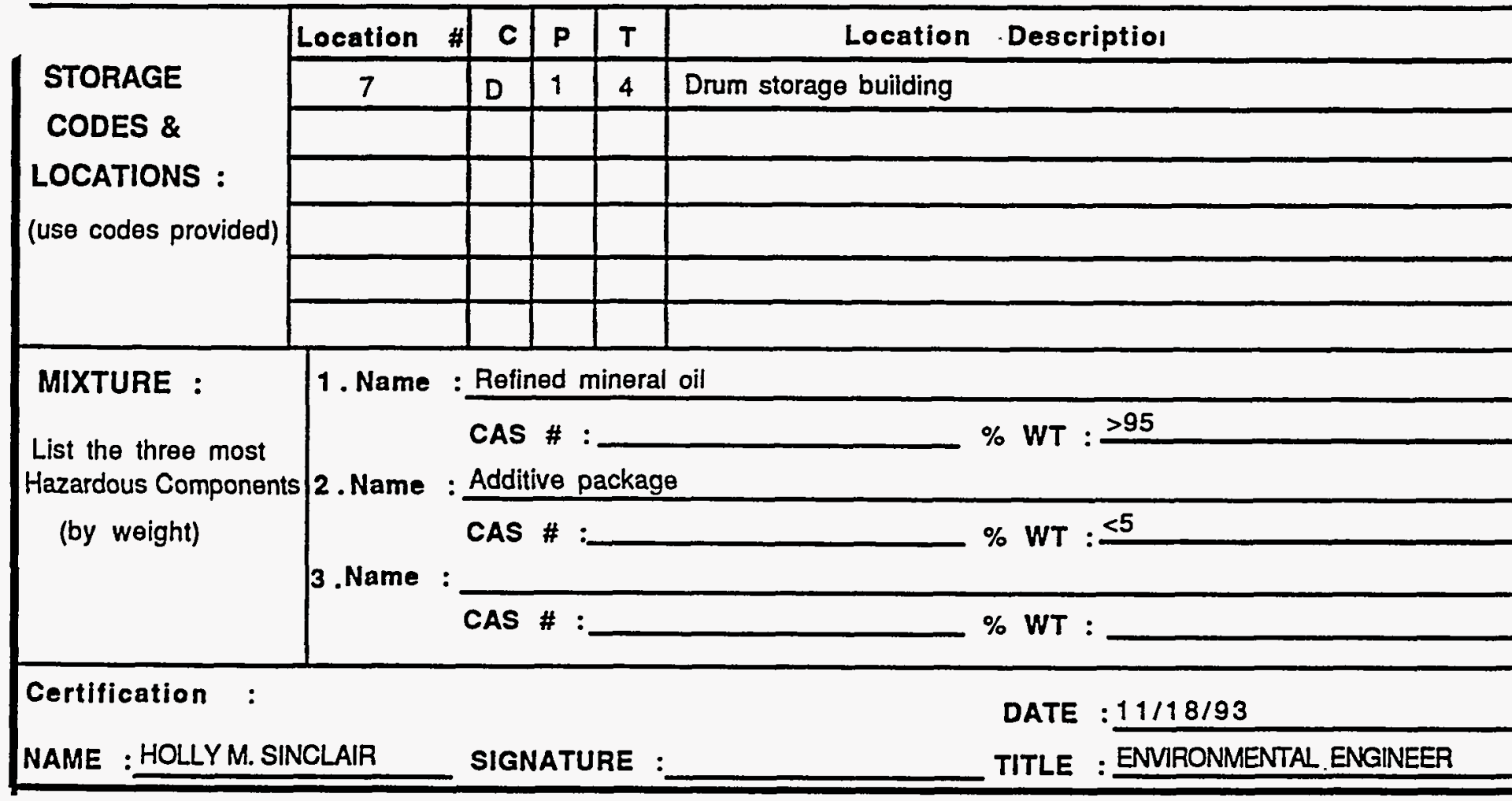




\section{Appendix B}

Phase II Environmental Site Assessment Report for the Kaiser-Hill Company LLC Oxnard Facility Oxnard, California 
This page intentionally blank 
DOEIIDI12584-226

GJPO-OXN-01

\section{Phase II Environmental Site Assessment Report for the Kaiser-Hill Company LLC Oxnard Facility, Oxnard, California}

July 1995

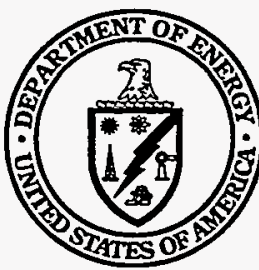

U.S. Department of Energy Grand Junction Projects Office

Approved for public release; distribution is unlimited.

Work Performed Under DOE Contract No. DE-AC04-94AL96907 for the U.S. Department of Energy 
This report was prepared as an account of work sponsored by an agency of the United States Government. Neither the United States Government nor any agency thereof, nor any of their employees, makes any warranty, express or implied, or assumes any legal liability or responsibility for the accuracy, - completeness, or usefulness of any information, apparatus, product, or process disclosed in this report, or represents that its use would not infringe privately owned rights. Reference herein to any specific commercial product, process, or service by trade name, trademark, manufacturer, or otherwise, does not necessarily constitute or imply its endorsement, recommendation, or favoring by the United States Government or any agency thereof. The views and opinions of authors expressed herein do not necessarily state or reflect those of the United States Government or any agency thereof. 


\section{Phase II Environmental Site Assessment Report for the Kaiser-Hill Company LLC Oxnard Facility, Oxnard, California}

July 1995

Work Performed Under Contract No. DE-AC04-94AL96907

Prepared for

U.S. Department of Energy Albuquerque Operations Office Grand Junction Projects Office P.O. Box 2567

Grand Junction, CO 81502-2567

Prepared by Rust Geotech Grand Junction, Colorado 
$: \quad::=\ldots-=$ 


\section{Contents}

Page

1.0 Introduction $\ldots \ldots \ldots \ldots \ldots \ldots \ldots \ldots \ldots \ldots \ldots \ldots$

2.0 Regulatory Framework $\ldots \ldots \ldots \ldots \ldots \ldots \ldots \ldots \ldots \ldots$

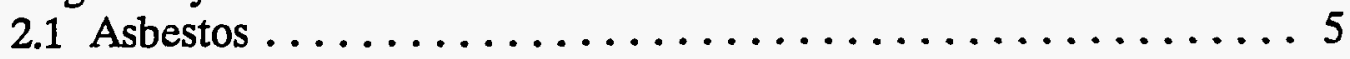

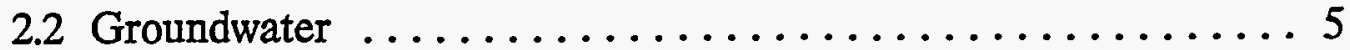

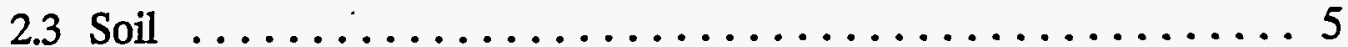

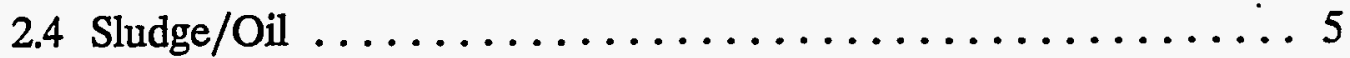

3.0 Field Investigation Results $\ldots \ldots \ldots \ldots \ldots \ldots \ldots \ldots \ldots \ldots$

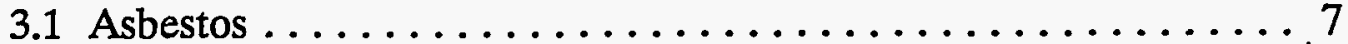

3.1.1 Terminology . .................... 7

3.1.2 Summary of Inspection Activities $\ldots \ldots \ldots \ldots \ldots \ldots .8$

3.1.3 Results ..................... 8

3.1.3.1 Surfacing Materials $\ldots \ldots \ldots \ldots \ldots \ldots \ldots 9$

3.1.3.2 Thermal System Insulation . . . . . . . . . . 9

3.1.3.3 Miscellaneous Materials . . . . . . . . . . . 9

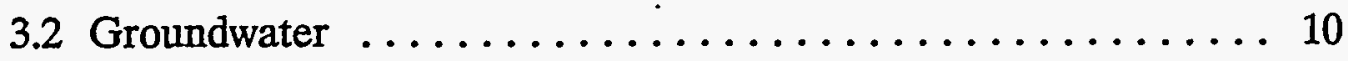

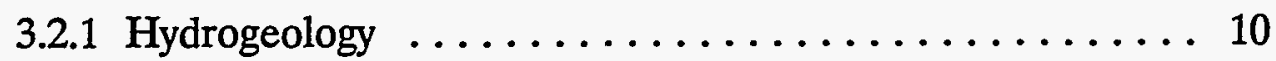

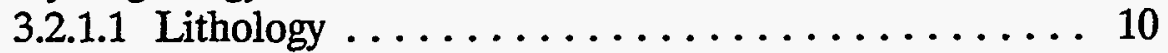

3.2.1.2 Groundwater Flow . . . . . . . . . . 10

3.2 .2 Groundwater Quality ................ 13

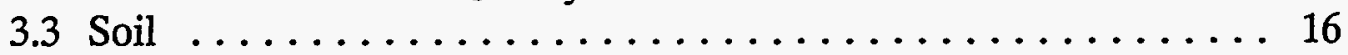

3.4 Radiological Survey $\ldots \ldots \ldots \ldots \ldots \ldots \ldots \ldots \ldots \ldots \ldots \ldots \ldots$

3.5 Waste Disposal . . . . . . . . . . . . . . . . . 18

3.5 .1 Sludge/Oil ....................... 18

3.5.2 Borehole Cuttings .................. 20

3.5.3 Development/Purge Water .............. 20

3.5.4 Personal Protective Equipment ............. 20

3.6 Quality Assurance ...................... 21

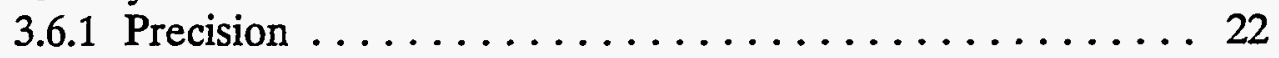

3.6 .2 Accuracy ........................ 22

3.6.3 Completeness .................... 23

$4.0 \quad$ Risk Assessment . . . . . . . . . . . . . . . . . 25

4.1 Preliminary Conceptual Site Model ............. 25

4.2 Data Evaluation ....................... 25

4.3 Exposure Assessment . . . . . . . . . . . . . . . 29

4.4 Toxicity Assessment . . . . . . . . . . . . . . . . 30

4.5 Risk Characterization $\ldots \ldots \ldots \ldots \ldots \ldots \ldots \ldots \ldots \ldots$

5.0 Recommendations ....................... 37 


\section{Contents (continued)}

Page

$6.0 \quad$ References $\ldots \ldots \ldots \ldots \ldots \ldots \ldots \ldots \ldots \ldots \ldots \ldots \ldots \ldots \ldots \ldots . \ldots \ldots$

Appendix A Asbestos Investigation . ................. A-1

Appendix B Borehole Lithologic Logs $\ldots \ldots \ldots \ldots \ldots \ldots \ldots$. . . . . . .

Appendix C Analytes, Laboratory Reporting Limits, and

Analytical Methods ..................... C-1

Appendix D Laboratọry Analytical Data ................. D-1

Appendix E Radiological Survey $\ldots \ldots \ldots \ldots \ldots \ldots \ldots \ldots \ldots$

Appendix F Duplicate-Sample Precision ................F F-1

Figures

Figure 1. Location of the Oxnard Facility in Oxnard, California . . . . . . 2

2. Site Layout of the Oxnard Facility ................ 3

3. Sampling Locations at the Oxnard Facility ............ 11

4. Groundwater Surface Contour Map ................ 12

5. Areas of Concern at the Oxnard Facility . . . . . . . . . . . . 15

6. Risk Assessment Preliminary Conceptual Site Model ...... 26

\section{Tables}

Table 1. Oxnard Facility Areas of Concern ................. 14

2. Risk Assessment Sampling Locations ... . . . . . . . . . . 27

3. Data Summary for Risk Assessment ... . . . . . . . . . . . 28

4. Risk Assessment Scenarios .................... 29

5. Exposure Factors ..................... 30

6. Toxicity Assessment Summary ................ 31

7. Risk Characterization Results .................. 34 


\subsection{Introduction}

The Kaiser-Hill Company LLC Oxnard Facility (Oxnard facility) is a nonferrous metalworking facility occupying 13.75 acres at 1235 East Wooley Road, Oxnard, California (Figure 1). Owned by the U.S. Department of Energy (DOE) and operated by Kaiser-Hill Company LLC, the facility is located in an industrial park within the incorporated city limits of Oxnard and within Ventura County. Seven buildings enclose approximately 86,000 square feet of covered floor space (Figure 2). Bordering the facility are industrial maintenance, manufacturing, and agricultural packaging facilities.

Until Allis-Chalmers (a farm implement manufacturing company) purchased the property in 1949, the site was farmland. Allis-Chalmers built a plant consisting of six buildings and engaged in the activities of founding (foundry casting), forging, machining, welding, cutting, sanding, grinding, painting, and coating. In 1981, DOE helped Precision Forge (a private company that catered to DOE metalworking requirements) move from Santa Monica, California, to the Oxnard facility. By June 1982, Precision Forge completed the transfer to the Oxnard facility. In 1984, DOE acquired Precision Forge, and operated the facility through Rockwell International until 1989 when EG\&G took over operations. Kaiser-Hill Company has operated the facility since the second quarter of 1995. Historically, metalworking at the Oxnard facility involved stainless steel, titanium, aluminum, and copper alloys. Presently, all metal working is with stainless steel, tantalum, molybdenum, and tungsten.

Site investigations conducted by Rust Geotech, the prime contractor for the DOE at the Grand Junction Projects Office (GJPO), started in June 1994 with an Environmental Site Assessment (ESA) Preliminary Evaluation site tour. Results of the Preliminary Evaluation indicated the need for a Phase I and Phase II ESA. A nonintrusive site inspection and data gathering effort in August 1994 resulted in a Phase I ESA report entitled Phase I Environmental Site Assessment for the EG\&G Rocky Flats Oxnard Facility (Phase I ESA) (DOE 1994a). Conclusions and recommendations described in the Phase I ESA report formed the basis for the Phase II intrusive ESA investigation, which was conducted in January and February 1995 and is the focus of this report.

The primary purpose of the Phase II ESA was to investigate areas of the facility considered to be potentially contaminated with regulated substances. These areas required a "due diligence" investigation as described in Site Auditing: Environmental Assessment of Property (Marburg Associates and Parkin 1991) so that contaminated media, if present, could be.identified and accounted for before real estate transfer. Specific objectives of the investigation were:

- To assess the location and type of asbestos and asbestos-containing material (ACM) at the facility. 


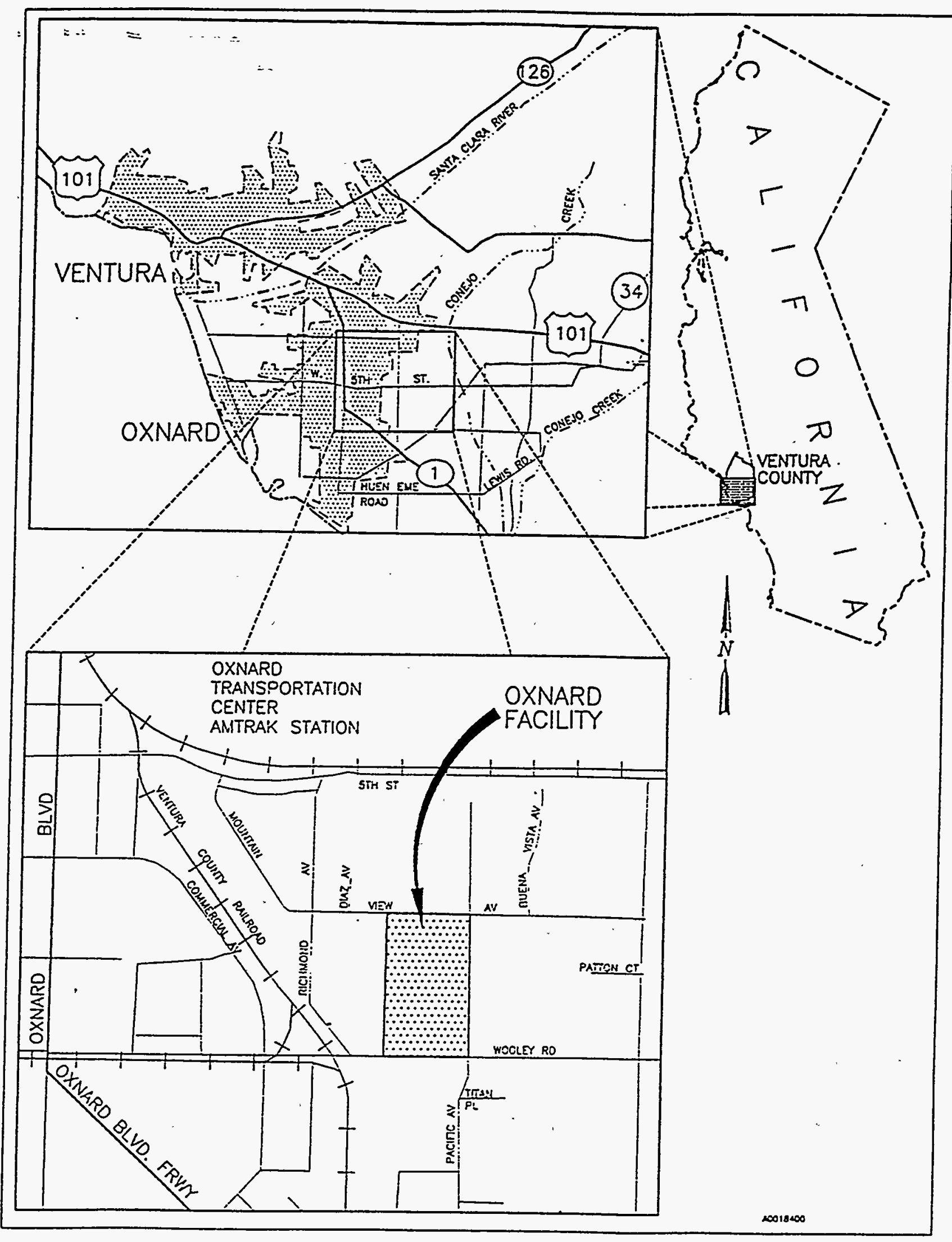

Figure 1. Location of the Oxnard Facility in Oxnard, California 


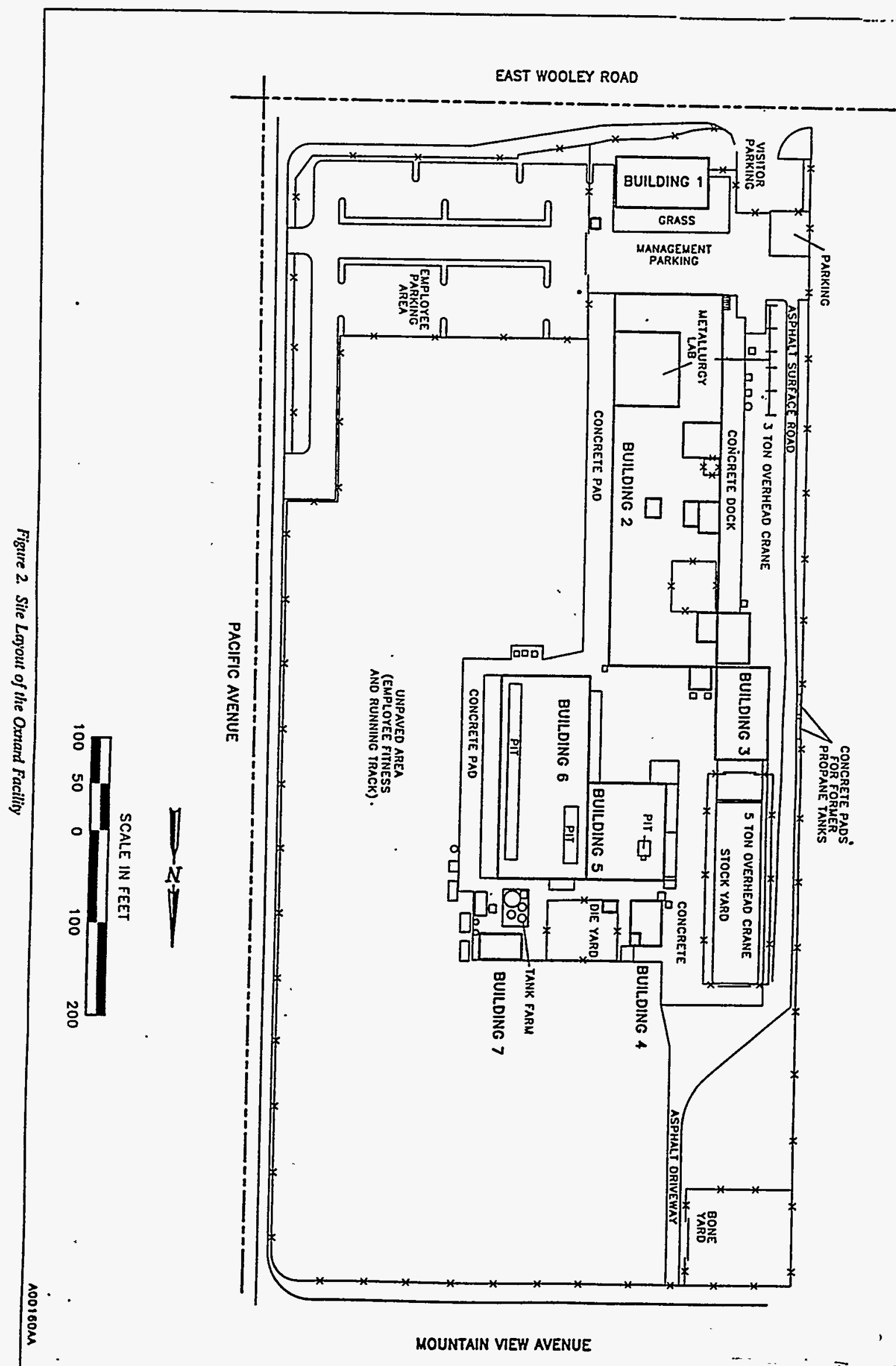


- To assess if groundwater quality at the facility has been affected by on-site and offsite activities.

- To assess if soils at the Oxnard facility have been affected by on-site activities.

- To assess if radiological contamination has occurred in areas where finished products have been returned from contaminated sites.

- To assess the composition of process oil and sludges formed in various collection systems so that waste disposal options and costs associated with facility decommissioning can be identified.

This report is divided into six sections. Section 1.0 describes the types of work performed at the Oxnard facility and the objectives of this Phase II ESA; Section 2.0 provides the regulatory framework used to make decisions from the collected data; Section 3.0 presents the results of the intrusive field investigation and discusses the results in the context of the regulatory framework; Section 4.0 discusses risk assessment; Section 5.0 provides recommendations for addressing health and environmental issues before real estate transfer; and Section 6.0 is a list of references used in this document. 


\subsection{Regulatory Framework}

The National Environmental Policy Act (NEPA) (DOE 1994b) requires consideration be given to environmental factors in the Federal decision-making process. A NEPA checklist was prepared for Phase II ESA activities, which were determined by the DOE Albuquerque Operations Office to be categorically excluded from the requirement to prepare NEPA documentation in the form of either an environmental assessment or an environmental impact statement.

Laboratory analytical work was performed by Truesdail Laboratories, Inc., in Tustin, California. This laboratory is certified by the California Environmental Laboratory Accreditation Program in the methods used to analyze samples collected during the Phase II ESA.

\subsection{Asbestos}

The Toxic Substances Control Act, 40 CFR 763 [U.S. Environmental Protection Agency (EPA) 1994a], establishes requirements concerning the inspection, control, storage, and disposal of asbestos and ACM. The State of California regulates asbestos worker certification, safety, registration, and asbestos emissions through: Title 8, CAL/OSHA, Section 341.15, "Certification of Asbestos Consultants and Site Surveillance Technicians" (Bureau of National Affairs [BNA] 1995a); Title 8, CAL/OSHA, Section 1529, "Asbestos" (BNA 1995b); Title 8, CAL/OSHA, Section 341.6, "Registration Requirements" (BNA 1995c); and Ventura County Air Pollution Control District Rule 62.7, "Asbestos Renovation and Demolition" (BNA 1995d). Asbestos inspection activities were conducted in compliance with these regulations.

\subsection{Groundwater}

Title 22, California Code of Regulations, Division 4.5, Chapter 15, Section 66265.97 (Barclays Law Publishers 1990a), provides guidance for groundwater quality monitoring. Groundwater analytical data were evaluated statistically according to the procedures detailed in this regulation to determine if water quality has been degraded by on-site activities. Title 22 of the California Code of Regulations, Division 4, Chapter 15, Article 4, Section 64431 (Barclays Law Publishers 1990b), provides standards that were used to evaluate groundwater quality.

Groundwater analytical data also were compared to standards set forth in Title 22, California Code of Regulations, Division 4.5, Chapter 11, Article 3, Section 66261.24 (Barclays Law Publishers 1990c), to determine if the groundwater containerized during monitoring well development and purging is considered hazardous waste. 


\subsection{Soil}

Analytical data from composite soil samples also were compared to standards set forth in Title 22, Division 4.5, Chapter 11, Article 3, Section 66261.24 (Barclays Law Publishers 1990c), to determine if borehole cuttings containerized during drilling operations are considered hazardous waste.

An assessment of risk to human health that is due to exposure to contaminated soils was conducted according to the Comprehensive Environmental Response, Compensation, and Liability Act (CERCLA) risk assessment process detailed in Risk Assessment Guidance for Superfund, Volume I, Human Health Evaluation Manual (Part A) (EPA 1989).

\subsection{Sludge/Oil}

Sludge/oil analytical data also were compared to standards set forth in Title 22, Division 4.5, Chapter 11, Article 3, Section 66261.24 (Barclays Law Publishers 1990c), to determine if sludge and oil are considered hazardous waste. An assessment of risk to human health that is due to exposure to sludges and oil was conducted according to the CERCLA risk assessment process detailed in Risk Assessment Guidance for Superfund, Volume I, Human Health Evaluation Manual (Part A) (EPA 1989). 


\subsection{Field Investigation Results}

\subsection{Asbestos}

A preliminary inspection for friable and nonfriable ACM was performed at the Oxnard facility from February 6 through 9, 1995, to determine the presence of ACM. Suspect ACM was sampled for bulk analysis in accordance with the Work Plan/Sampling and . Analysis Plan for the Phase II ESA of the EG\&G Rocky Flats Oxnard Facility, Oxnard, California (Work Plan/SAP) (DOE 1994c) and the regulations listed in Section 2.1 of this report.

\subsubsection{Terminology}

Asbestos is the asbestiform variety of serpentine (chrysotile), riebeckite (crocidolite), cummingtonite-grunerite (amosite), anthophyllite, actinolite, or tremolite. ACM includes both friable and nonfriable material that contains more than 1-percent asbestos as determined by the method specified in Appendix A, Subpart F, 40 CFR Part 763, Section 1, Polarized Light Microscopy (PLM)(EPA 1994a).

A friable ACM can be crumbled, pulverized, or reduced to a powder by hand pressure when dry. A nonfriable ACM cannot be crumbled, pulverized, or reduced to a powder by hand pressure in its dry state. Nonfriable ACM is further described as either Category I or Category II. Category I includes asbestos-containing packings, gaskets, resilient floor covering (floor tile, including asphalt and vinyl floor tile and sheet vinyl floor covering), and asphalt roofing products that are not in poor condition and that contain more than 1-percent asbestos. Category I nonfriable ACM is exempted from the majority of the Air Pollution Control District rules as long as the material is removed, loaded, and transported in a manner that prevents the release of fibers and prevents the material from becoming friable. Category II material includes all nonfriable ACM not considered to be Category I.

Asbestos remediation (also known as abatement) includes the removal of ACM, abatement of asbestos-contaminated facility components, or facility renovation. Facility renovation means altering, removing, or stripping one or more facility components and includes retrofitting for fire protection and installing or removing heating, ventilating, and air conditioning (HVAC) systems. Wrecking or removing load-supporting structures is excluded.

Any waste generated by removal of $\mathrm{ACM}$ associated with demolition or renovation activities is considered asbestos-containing waste material. 


\subsubsection{Summary of Inspection Activities}

Prior to the inspection of the Oxnard facility, a walk-through was conducted with Oxnard staff and management, and floor plans of the buildings to be included in the survey were examined. A sampling plan for each building was then developed. Surface materials, thermal system insulation (TSI), and miscellaneous materials on the exterior and interior of each building were inspected for the presence of suspect asbestos. The location and description of suspect materials assumed to be ACM were recorded, and the suspect materials were grouped into homogeneous sampling areas.

The Work Plan/SAP specifies criteria for determining the number of samples to be taken per homogeneous sampling area and the general locations necessary to obtain a representative sample. It was not within the scope of this inspection to determine the physical condition or potential for disturbance of ACMs or to conduct a hazard assessment.

The Work Plan/SAP also called for a diagram showing the areas to be sampled for friable and nonfriable ACM. Before sampling, a field inspection log was prepared specifying the project name, site name, location (building number), date and time, matrix sampled, inspection and sampler's name, container type, and number of samples collected. Inspection and sampling activities were recorded daily in the field inspection log.

ACM sampling was initiated by spreading a plastic drop cloth at the marked sampling location, labeling a plastic bag with appropriate information (i.e., identification number, sample name, date and time), and recording the sample information in the field inspection log. The sample location was moistened with amended water solution, and the sample was extracted with a clean knife, linoleum cutter, cork borer, or other appropriate tool. Once removed, the sample was placed into the bag and the bag was sealed; the extraction tools were cleaned, and the hole was filled (or cut) with caulk, tar, spackling or duct tape, as appropriate. Tools were decontaminated after each sample was collected. Decontamination material and personal protective equipment were bagged and stored as ACM at the end of the day.

\subsection{3' Results}

Laboratory analysis determined that ACM was present on the Oxnard facility. The Asbestos Summary Sheet (Appendix A) itemizes analytical results for each sample by homogeneous material, functional space, and asbestos content. Bulk asbestos sampling locations are shown in Figures A-1 to A-8 in Appendix A. The following subsections provide details of materials that contain asbestos. If results indicated the absence of ACM, the material and sampling location are not addressed. 


\subsubsection{Surfacing Materials}

The only functional space with surface ACM is the Accounting Offices in Building 1 . The homogeneous ACM is a sprayed-on, textured coating containing 1- to 3-percent chrysotile and 97- to 99-percent minerals, including mica and calcium carbonate. The coating is in excellent condition and is not in a friable or deteriorating state. This coating is on approximately 500 square feet of wall surface.

\subsubsection{Thermal System Insulation}

Six TSI areas exist at the Oxnard facility, specifically in Buildings 1,2, and 6. This insulation is associated with either the HVAC system, located in Building 1, or the air exchangers associated with specific applications at the facility. TSI in Building 1 on the HVAC system piping immediately above the furnace is the only TSI that contains asbestos. This 2-foot-long piping sleeve is a grey, fibrous solid composed of calcium carbonate and silica, and it contains 24- to 28-percent chrysotile and 4- to 6-percent crocidolite asbestos fibers. It is in excellent condition and is not in a friable or deteriorating state.

\subsubsection{Miscellaneous Materials}

Miscellaneous materials sampled for asbestos at the Oxnard facility varied from floor and ceiling tiles to equipment insulation and tank gaskets.

Two functional spaces, the vault and lobby in Building 1, have 9-inch-square ACM vinyl floor tiles. The vault tile is a black, fibrous tile with 50- to 60-percent binder, 40- to 50-percent mineral, and 3- to 5-percent chrysotile asbestos fiber. The lobby tile is a tan, fibrous tile with 50- to 60-percent binder, 40- to 50-percent mineral, and 1- to 2-percent chrysotile asbestos fiber. The combined area of vinyl asbestos tile is approximately 700 square feet.

Storage cabinets in the metallurgical laboratory contain an interior front door fireproof sheathing-a grey, fibrous solid of 70- to 80-percent calcium carbonate and silica and 20- to 30-percent chrysotile asbestos fiber. Given the number of storage cabinets, the estimated area of sheathing is 500 square feet. Two miscellaneous laboratory materials not sampled were the analytical work benches and oven gloves, which are assumed to be ACM throughout the facility.

Several fibrous gaskets were sampled for asbestos. Two sets of gaskets containing asbestos were identified: the emersion heater gaskets in the lubrication stores building and the Lindberg furnace front door gasket in Building 6 . The emersion heater gaskets are small, fibrous gaskets containing 30- to 40-percent binder and 60- to 70-percent chrysotile asbestos fiber. The furnace gasket is a small, green, tubular fibrous gasket containing 5- to 10-percent binder and 90- to 95-percent chrysotile asbestos fiber. 
Window caulk in Building 6 is a calcium carbonate solid with 1- to 3-percent chrysotile asbestos fiber content. Although Building 2 window caulk was not identified as ACM, it is suspected to contain asbestos because Building 2 is 10 to 20 years older than Building 6; future bulk analysis of the Building 2 window caulk may be required.

On the basis of age, all electrical wiring insulation is assumed to be ACM. The brakes on the Cleveland presses also are assumed to be ACM on the basis of discussions with Oxnard facility staff.

\subsection{Groundwater}

\subsubsection{Hydrogeology}

\subsubsection{Lithology}

Lithologic information was obtained by drilling 16 boreholes (Figure 3) at the Oxnard facility during January and February 1995. Split-barrel samples collected from each borehole were used to describe and to document the lithologic sequence. Descriptions of the lithology were prepared for seven soil borings to a depth of 4.5 to 8 feet and for nine monitoring wells to a depth of 12 feet. Although monitoring wells were drilled to a depth of 18 feet, flowing sands encountered at approximately 12 feet prevented splitbarrel sample collection below that point. In general, the shallow lithology beneath the Oxnard facility consists of 2 feet of dark brown, silty-sand topsoil with organic fragments overlying yellowish-brown, medium to coarse-grained, unconsolidated sands intermixed with pebbles and cobbles. Appendix B contains lithologic logs of the boreholes.

\subsubsection{Groundwater Flow}

Water-level elevations were measured in each of the monitoring wells in February 1995 after well development. These water levels were used to prepare the groundwaterelevation contour map in Figure 4. On the basis of this constructed map, groundwater flow in the shallow system is to the southwest, and the lateral hydraulic gradient is 0.002 .

Slug testing data from the nine monitoring wells were analyzed by the method of Bouwer and Rice (1976) and Bouwer (1989). The range of hydraulic conductivities for the tests was 17 to 74 feet per day (ft/d); the arithmetic average was $38 \mathrm{ft} / \mathrm{d}$, and the geometric mean was $33 \mathrm{ft} / \mathrm{d}$. An average linear velocity of the shallow groundwater system was calculated as $0.22 \mathrm{ft} / \mathrm{d}$ by using an estimated effective porosity of 30 percent and the geometric mean of the hydraulic conductivities. 


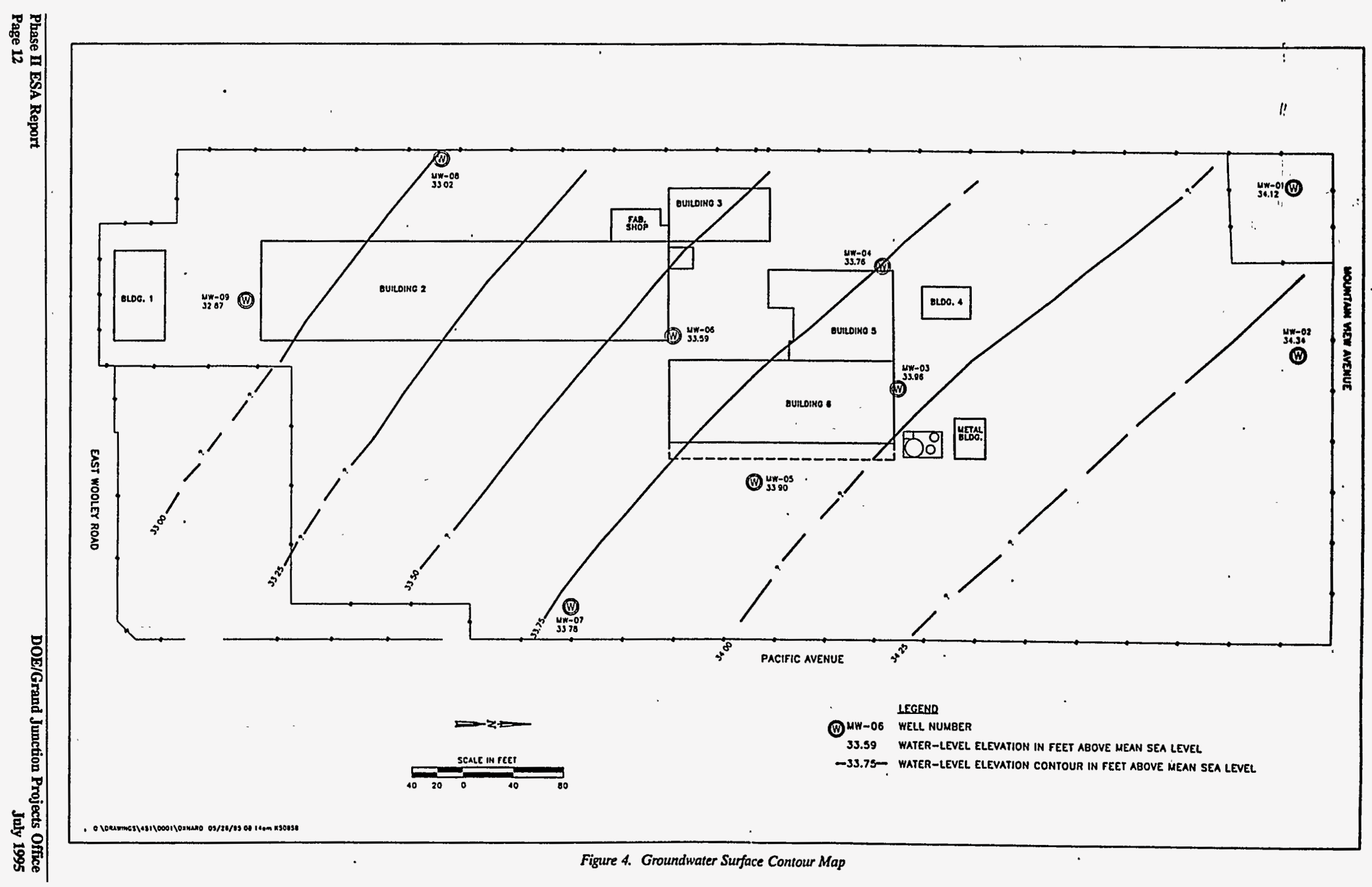




\subsubsection{Groundwater Quality.}

The groundwater monitoring network was designed to investigate "areas of concern" identified in the Phase I ESA. Areas of concern are locations at the facility that have documented historical contamination, suspected historical contamination, or a potential for contaminant release from historical or current operations. Areas of concern are listed in Table 1 and are shown in Figure 5. The primary concerns for on-site groundwater impacts are leaking petroleum products from underground trenches, cellars, and tanks, and unknown contaminants from a historical trash pit under Building 6 . Offsite sources possibly affecting groundwater are leaking petroleum or solvent tanks upgradient of the Oxnard facility.

Water quality samples were collected from nine monitoring wells (Figure 3 ) in February 1995. Groundwater samples were collected for inorganic analyses with a submersible pump or peristaltic pump; groundwater samples were collected for organic analyses with a submersible pump or a Teflon bailer. Sampling procedures are described in the Work Plan/SAP. Groundwater samples were analyzed for metals (Target Compound List [TCL] metals and molybdenum), TCL polychlorinated biphenyls (PCBs), TCL volatile organic compounds, and total petroleum hydrocarbons (TPH). A detailed analyte list is displayed in Appendix C. Analytical results of samples collected from groundwater monitoring wells are listed in Table D-1 in Appendix D.

Analytical results for groundwater samples demonstrate that organic compounds are not affecting the water quality of the shallow aquifer beneath the Oxnard facility. Concentrations of PCBs and TPH were below detection levels in all groundwater samples. With the exception of two organic compounds detected in a sample from MW-2, concentrations of volatile organic compounds also were below detection levels. Because groundwater downgradient of areas of concern did not have detectable concentrations of organic compounds, concerns involving on-site sources (such as leaking trenches, sumps, cellars, tanks, historical pits, and above ground storage spills of organics) have been alleviated.

Monitoring wells MW-2 and MW-7 were located in areas upgradient of the facility to investigate historical soil-gas detections of perchloroethylene (also referred to as tetrachloroethene) and to determine the effects of off-site activities. Perchloroethylene was below the detection level in all groundwater samples. Off-site activities apparently are not affecting the groundwater quality at the Oxnard facility. TPH in samples from the upgradient wells was below the detection level, which alleviates concern about leaking fuel tanks upgradient of the facility. Volatile organic compounds were not detected in the sample from MW-7, and only two volatile organic compounds were detected in the sample from MW-2; concentrations of 1,1,1-trichloroethane and 1,1-dichloroethane were detected at $14.5 \mu \mathrm{g} / \mathrm{L}$ and $4.77 \mu \mathrm{g} / \mathrm{L}$, respectively. Although State of California drinking water standards (Barclays Law Publishers 1990b) are not applicable to the shallow groundwater beneath the Oxnard facility (shallow groundwater 
-Table 1. Oxnard Facility Areas of Concem

\begin{tabular}{|c|c|c|}
\hline Area of Concern & Location & $\begin{array}{c}\text { Potential } \\
\text { Contaminants }\end{array}$ \\
\hline 1. Allis-Chalmers pit & Beneath Building 6 & Metals, unknowns \\
\hline $\begin{array}{l}\text { 2. Trench/sump and oil } \\
\text { collection system }\end{array}$ & Building 6 & $\begin{array}{l}\text { Metals, hydraulic oil, } \\
\text { PCBs }\end{array}$ \\
\hline 3. Bioremediation area & East of Building 6 & Hydraulic oil, PCBs \\
\hline 4. PCB soil area & East of Building 6 & PCBs \\
\hline $\begin{array}{l}\text { 5. Perchloroethylene (PCE) soil- } \\
\text { gas area }\end{array}$ & East side of facility & PCE \\
\hline 6. PCE soil-gas area & North side of facility & PCE \\
\hline 7. Oil collection system & Building 5 & Metals, hydraulic oil, PCBs \\
\hline 8. Tank farm & North of Building 6 & Metals, hydraulic oil, PCBs \\
\hline 9. Abandoned diesel UST & $\begin{array}{l}\text { Outside of south side } \\
\text { of Building } 4\end{array}$ & Diesel fuel \\
\hline 10. PCB soil area & East of the boneyard & PCBs \\
\hline 11. Boneyard & $\begin{array}{l}\text { Northwest corner of the } \\
\text { facility }\end{array}$ & PCBs, metals \\
\hline 12. Asphalt road & $\begin{array}{l}\text { West side of facility and } \\
\text { east of the boneyard }\end{array}$ & Miscellaneous oils, PCBs \\
\hline $\begin{array}{l}\text { 13. Former trichloroethane } \\
\text { aboveground storage tank }\end{array}$ & $\begin{array}{l}\text { Southwest corner of } \\
\text { Building } 3\end{array}$ & TCA \\
\hline $\begin{array}{l}\text { 14. Metal cuttings in recycling } \\
\text { bins }\end{array}$ & West side of Building 2 & Metals, miscellaneous oils \\
\hline 15. Used oil-filter storage area & West side of Building 2 & Metals, miscellaneous oils \\
\hline $\begin{array}{l}\text { 16. Metal slag on ground } \\
\text {, }\end{array}$ & $\begin{array}{l}\text { West of dock next to } \\
\text { Building } 2 \\
\end{array}$ & Metals \\
\hline 17. Loading dock & $\begin{array}{l}\text { Outside of southwest } \\
\text { corner of Building } 2\end{array}$ & $\begin{array}{l}\text { Organics, hydraulic oils, } \\
\text { unknowns }\end{array}$ \\
\hline 18. Metallurgy laboratory & South portion of Building 2 & Metals \\
\hline 19. Cyclone and baghouse & Buildings 4 and 5 & Metals \\
\hline 20. Duct work & Building 1 & Asbestos \\
\hline 21. Vault area & Building 1 & Asbestos \\
\hline
\end{tabular}




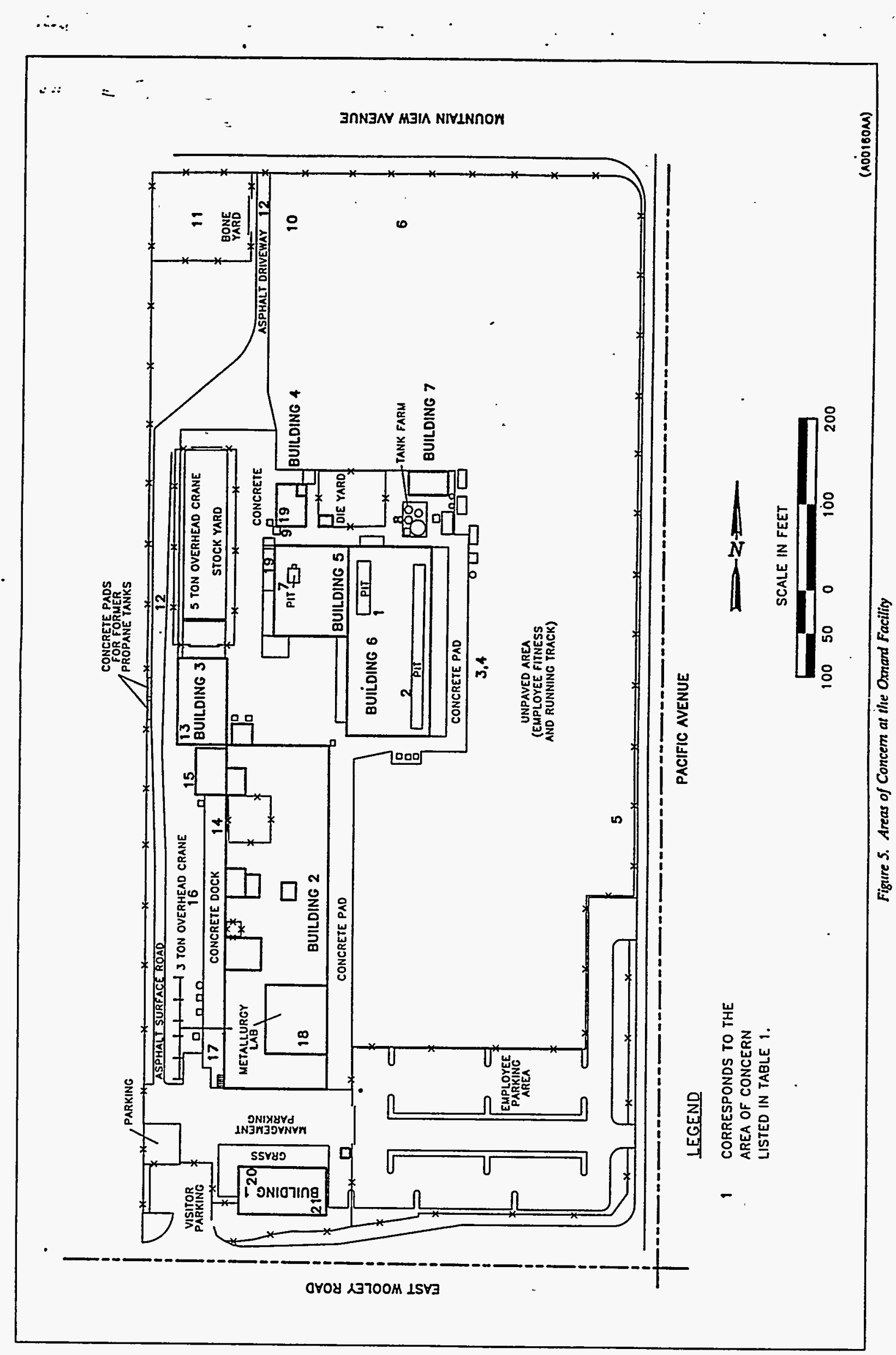


is not used for drinking), they provide a basis for evaluating water quality. The concentrations of the two volatile organic compounds detected are below State drinking water standards and, therefore, do not have a significant effect on water quality.

Analyses of groundwater samples for metals also indicate minimal effect to the shallow aquifer. Silver, arsenic, beryllium, cadmium, cobalt, chromium, copper, mercury, nickel, antimony, selenium, thallium, and vanadium occur in concentrations near or below detection limits and are not considered contaminants in the groundwater. Metals detected in the groundwater (aluminum, lead, iron, zinc) were evaluated statistically using methods described in Section 66265.97 of Title 22 of the California Code of Regulations (Barclays Law Publishers 1990a) to determine if metal concentrations in areas of concern were statistically higher than background metal concentrations. Monitoring wells MW-2 and MW-7 were used as background wells for this analysis. Concentrations of aluminum (MW-1 and MW-6), iron (MW-1 and MW-6), lead (MW-9), and zinc (MW-4 and MW-6) were statistically higher than background concentrations; maximum concentrations of these metals were 2.69 milligrams per liter $(\mathrm{mg} / \mathrm{L})$ of aluminum in MW-1, $4.54 \mathrm{mg} / \mathrm{L}$ of iron in MW-1, $0.082 \mathrm{mg} / \mathrm{L}$ of lead in MW-9, and $0.06 \mathrm{mg} / \mathrm{L}$ of zinc in MW-6.

The California primary drinking water standards for aluminum $(1 \mathrm{mg} / \mathrm{L})$ was exceeded in samples from MW-1 $(2.69 \mathrm{mg} / \mathrm{L})$ and MW-6 $(1.87 \mathrm{mg} / \mathrm{L})$, and the standard for lead $(0.05 \mathrm{mg} / \mathrm{L})$ was exceeded in a sample from MW-9 $(0.082 \mathrm{mg} / \mathrm{L})$. Samples from all wells (except MW-7) in the monitoring network exceeded the California secondary drinking water standard for iron $(0.3 \mathrm{mg} / \mathrm{L})$.

Metal concentrations that are statistically higher than background and above drinking water standards may be elevated because of on-site activities; however, a number of sources of variability (i.e., seasonal variation of groundwater quality and variability of the sampling and analytical process) must be taken into account before these elevated metal concentrations in the groundwater can be definitively attributed to on-site activities. An evaluation of groundwater quality and potential groundwater degradation based on comparison to drinking water standards is a conservative approach. In actuality, the risk to human health from groundwater consumption is insignificant because of an incomplete pathway (see Section 4.0, Risk Assessment).

\subsection{Soil}

Soil samples were collected from 16 boreholes in areas of concern at the Oxnard facility (Figure 3). Soil samples were analyzed for metals, TCL PCBs, TCL volatile organic compounds, and TPH. A detailed analyte list is in Appendix C. Two discrete depthinterval soil samples were collected from each borehole to determine the existence of contamination in soils, and composite soil samples were collected to determine waste disposal options for the borehole cuttings. 
Soil samples were collected with a split-barrel sampler from approximately 0 - to 2- and 4- to 6-foot depths. The Work Plan/SAP required additional soil sample collection in intervals where stained soil was present or elevated organic vapor readings were detected; however, these phenomena did not occur in any soil samples collected at the Oxnard facility. Split-barrel samplers were equipped with stainless steel sleeves so that volatile organic samples could be collected with minimal loss of the volatile compounds. The bottom stainless steel sleeve ( 0.5 foot) in the split-barrel sampler was capped immediately after sampling and prepared for submittal to the laboratory for TCL volatile organic compound analysis. The remaining soil in the split-barrel sampler was removed from the stainless steel sleeves, lithologically described, mixed, and then placed in the samples bottles. Soil sampling procedures are detailed in the Work Plan/SAP.

In general, organic compounds were below detection limits or were detected in low concentrations. Organic analytes below detection included TPH and most of the volatile organic compounds. Volatile organic compounds that were detected included acetone, 2-butanone, toluene, and 1,1,1-trichloroethane. Of the 34 samples analyzed, acetone was detected in 15 samples in concentrations ranging from 16.1 to $845 \mu \mathrm{g} / \mathrm{kg}$;

2-butanone was detected in 15 samples in concentrations ranging from 16.1 to $299 \mu \mathrm{g} / \mathrm{kg}$; and toluene was detected in 19 samples in concentrations ranging from 2.33 to $19.2 \mu \mathrm{g} / \mathrm{kg}$. These compounds are common laboratory contaminants and were found in many of the associated laboratory method blanks. 1,1,1-trichloroethane was detected in 9 samples in concentrations ranging from $2.20 \mu \mathrm{g} / \mathrm{kg}$ to $6.36 \mu \mathrm{g} / \mathrm{kg}$.

PCBs were detected in samples from locations SB-1 (boneyard) and SB-10 (east of the boneyard): aroclor 1254 was detected in a sample from location SB-1 at a concentration of $540 \mu \mathrm{g} / \mathrm{kg}$, and aroclor 1248 was detected in samples from location SB-10 in concentrations ranging from 60 to $3,900 \mu \mathrm{g} / \mathrm{kg}$. This data confirms historical investigation results, which indicated the presence of PCBs in these areas. All other soil samples had PCB concentrations below detection limits.

Concentrations of silver, beryllium, and thallium were below detection limits at all soil sampling locations; all other metal analytes were above detection limits in one or more samples. Metal concentrations detected in soil samples were evaluated statistically using methods described in Section 66265.97 of Title 22 of the California Code of Regulations (Barclays Law Publishers 1990a) to determine if metal concentrations in areas of concern were statistically higher than background metal concentrations. Soil borings SB-2 and SB-7 were used as background locations for this analysis. Two locations, SB-14 and SB-15, had metal concentrations that were statistically higher than background; concentrations of antimony, arsenic, chromium, cobalt, copper, iron, lead, manganese, mercury, and nickel were higher than background in one or both of these soil borings. With the exception of mercury, which was elevated in the 4- to 6-foot depth interval, elevated metal concentrations in these two soil borings were found in the 0 - to 2-foot depth interval. These locations appear to have been affected by on-site activities. Other locations with elevated metal concentrations relative to background included SB-10 (cadmium), SB-3 and SB-13 (lead), SB-11 (lead and zinc), and SB-12 (copper, lead, and 
zinc). All other metal concentrations were not statistically higher than background. Analytical results are in Table D-2, Appendix D.

A risk assessment was conducted to determine if heavy metal, $\mathrm{PCB}$, and volatile organic compound concentrations in exposed soil areas (not under concrete or asphalt) pose a risk to human health. There were no carcinogens in exposed soils that were considered chemicals of potential concern (COPC) when compared to background concentrations; therefore, risk was not calculated. A hazard quotient for exposure to noncarcinogens in exposed soils was calculated at $8.4 \times 10^{-4}$. Risks from noncarcinogens are considered acceptable if the hazard quotient is less than 1 . Risk assessment rationale and calculations are detailed in Section 4.0.

\subsection{Radiological Survey}

The shipping/receiving area in Building 2 was surveyed with a beta-gamma detection instrument to determine if it was radiologically contaminated from products returned from radiologically contaminated sites. The results of the radiological survey demonstrated that the shipping/receiving area is not radiologically contaminated; all direct survey and smear survey readings were at background levels. Results of the survey are in Appendix E.

\subsection{Waste Disposal}

\subsubsection{Sludge/Oil}

Sludge accumulates at the Oxnard facility as a result of gravity drainage of oils and metal fines from the process equipment. Sludge samples were collected from six locations: the trench beneath the forging hammers in Building 6, the cellars beneath the 1,600-ton and 3,500-ton presses in Building 5, the steam cleaning trench east of the tank farm, and the compressor collection trench east of Building 6 (Figure 3). The sewer clarifier/grease trap was not sampled because it did not contain sludge, and the sump at the north end of the collection trench in Building 6 was not sampled because of safety concerns. One oil sample was collected from the tap on the dirty oil tank located in the tank farm (Figure 3). Collection and analysis of sludge and oil samples were conducted to determine options for disposing these wastes should the facility be decommissioned.

Grab samples of sludge were collected using a stainless steel scoop and by following procedures in the Work Plan/SAP. Sludge samples were analyzed for metals and TCL PCBs. The oil sample was analyzed for metals, TCL PCBs, total organic halides, and ignitability. A detailed analyte list is in Appendix C. .

Chromium present at the Oxnard facility is believed to be in the form of chromium metal or trivalent chromium compounds. This assumption is based on process 
knowledge; hexavalent chromium compounds are not presently used at the Oxnard facility, and research of historical records does not indicate use of these compounds. Because hexavalent chromium compounds are not used at the facility, the occurrence of hexavalent chromium is unlikely-chromium metal or trivalent chromium is not oxidized to hexavalent chromium under normal environmental conditions. Therefore, the hazardous waste determination of sludge due to chromium contamination was based on the chromium/trivalent chromium threshold limit value.

Results of sludge and oil sample analysis were compared to threshold limit concentrations listed in Title 22 of the California Code of Regulations (Barclays Law Publishers 1990c) to determine if the oil and sludges are considered hazardous waste. For a waste to be classified as nonhazardous in the State of California, total concentrations must be below the total threshold limit concentration, and soluble concentrations must be below the soluble threshold limit concentration. Total concentrations first were determined for all samples. If analyte concentrations were greater than or "near" the analyte's total threshold limit concentration, the EPA toxicity characteristic leaching procedure (TCLP) and/or the California waste extraction test (WET) procedure were performed to determine soluble concentrations. All sludge and oil analyte concentrations were below their respective total threshold limit concentration. However, sludge and oil samples contained total concentrations of selected metals high enough to require TCLP and/or WET extraction tests.

All TCLP metal concentrations were below their respective soluble threshold limit concentration; however, metal concentrations in samples from three sludge locations exceeded the WET soluble threshold limit concentrations. These excessive concentrations occurred in samples from locations SL-2 (chromium and nickel), SL-5 (lead and zinc), and SL-6 (lead). Therefore, sludge from these locations would be considered hazardous if it were removed from the process. Analytical results of sludge and oil samples collected at the Oxnard facility are in Tables D-3 and D-4 in Appendix D.

The risk (probability of developing cancer) to human health from exposure to carcinogens in the sludge and oil was calculated at $2.7 \times 10^{-6}$, and the hazard quotient for exposure to noncarcinogens in the sludge/oil was calculated at $8.9 \times 10^{-4}$. Under CERCLA, risks from carcinogens greater than $1 \times 10^{-4}$ generally require action to reduce the risks; risks less than $1 \times 10^{-6}$ usually result in no action. When risks are between $1 \times 10^{-6}$ and $1 \times 10^{-4}$, actions are taken on a site-specific basis according to direction from EPA. The calculated risk from carcinogens falls within the range of potential action. However, the risk ranges presented above are for risks to the public, and this risk assessment evaluated risk to the site worker (which was a conservative approach); therefore, the risk due to exposure to carcinogens in the sludge is considered insignificant. Risks from noncarcinogens are considered acceptable if the hazard quotient is less than 1. The calculated hazard quotient of $8.9 \times 10^{-4}$ is well below 1 . Risk assessment assumptions and calculations are detailed in Section 4.0. 


\subsubsection{Borehole Cuttings}

Borehole cuttings generated during drilling operations were containerized and labeled so that proper disposal could be conducted after analytical results were reviewed.

Composite soil samples were collected from each borehole and analyzed for metals, fluoride salts, and TCL volatile organic compounds to determine if the borehole cuttings were hazardous waste.

Analytical results from the composite soil samples were compared to threshold limit concentrations listed in Title 22 of the California Code of Regulations (Barclays Law Publishers 1990c) to determine if the drummed borehole cuttings are hazardous waste. All analyte concentrations were below the respective total threshold limit concentrations; however, two samples had total metal concentrations high enough to require TCLP and/or WET extraction tests. The composite soil sample from SB-13 was analyzed for lead following the TCLP extraction procedure and was analyzed for lead and chromium following the WET extraction procedure. Lead also was analyzed in the composite soil sample from SB-12 following the WET extraction procedure. All TCLP and WET analyte concentrations were below the respective threshold limit concentrations. Therefore, the borehole cuttings, with the exception of those from SB-1 and SB-10, are not hazardous waste. Because PCBs were detected in samples from SB-1 and SB-10, the borehole cuttings from these locations may have to be disposed of at a TSCA-approved facility pending negotiations with California EPA. Analytical results of the composite soil samples are in Tables D-3 and D-5 of Appendix D.

\subsubsection{Development/Purge Water}

Groundwater produced during monitoring well development and groundwater sampling was containerized and labeled so that proper disposal could be conducted after analytical results were reviewed. Groundwater samples were analyzed for metals, TCL PCBs, TCL volatile organic compounds, and TPH to determine if the containerized groundwater was considered hazardous waste.

Analytical results from the groundwater samples were compared to threshold limit concentrations listed in Title 22 of the California Code of Regulations (Barclays Law Publishers 1990c) to determine if the containerized groundwater is considered hazardous waste. All groundwater analyte concentrations were below the respective threshold limit concentrations and, therefore, the containerized groundwater is not hazardous waste. Analytical results of groundwater samples are in Appendix D.

\subsubsection{Personal Protective Equipment (PPE)}

PPE, which was worn during each sampling activity, was containerized and labeled so proper disposal could be conducted after analytical results were reviewed. Analytical results for sludge, oil, soil, groundwater, and asbestos were reviewed to determine if any of these media, and the PPE used to sample them, were hazardous waste. 
Because groundwater is not considered hazardous waste, the PPE used during groundwater sampling is not considered hazardous. PPE used during the sampling of soil borings SB-1 and SB-10 (PCB detections) may have to be disposed of at a TSCAapproved facility pending negotiations with California EPA. Because sludge from locations SL-2, SL-5, and SL-6 was determined to be hazardous, the associated PPE also should be handled and disposed as hazardous waste. PPE was not worn at locations SL-5 because the sample was taken remotely. Because asbestos was detected, PPE worn during the asbestos sampling effort should be treated and disposed of as ACM.

\subsection{Quality Assurance}

Quality assurance measures implemented during the Phase II ESA activities included following procedures established in the Work Plan/SAP, collecting and analyzing field quality control (QC) samples, and analyzing laboratory QC samples. Field QC samples included five trip blanks, four equipment blanks, and five duplicates. Analytical results of field QC samples are in Table D-6 of Appendix D. Laboratory QC consisted of analyzing method blanks, duplicates, blank spikes, blank spike duplicates, matrix spikes, matrix spike duplicates, and laboratory control samples. Volatile organic compounds were detected in many of the method blanks, which indicated laboratory process contamination.

Data quality determinations for the first round of PCB analysis of soil, sludge, and oil samples could not be made because quality indicators did not meet laboratory performance criteria due to matrix interferences. Although PCBs were not detected in any of these samples, a potential for false negatives existed. The laboratory followed the analytical methods detailed in Test Methods for the Evaluation of Solid Waste SW-846 (EPA 1986), which were specified in the Work Plan/SAP. However, SW-846 guidance only suggests methods to clean up samples that have matrix interferences, and no attempt by the laboratory to remedy the matrix interferences was made; therefore, PCB samples from soil, sludge, and oil were reanalyzed in accordance with a detailed set of technical specifications.

All holding times specified in the Work Plan/SAP were met with the exception of the reanalysis of PCBs in soil, sludge, and oil; however, because the samples were stored in the laboratory refrigerator, reanalysis of the samples was acceptable to California EPA despite the expired holding time.

Laboratory analytical data was reviewed, and precision, accuracy, and completeness of the data were calculated for each medium. A general discussion of precision, accuracy, and completeness and a summary of results follows. 


\subsubsection{Precision}

Precision is the agreement between the numerical values of two or more measurements that have been made in an identical manner without any knowledge of the true value. It is determined through the analysis of laboratory and field duplicate samples. Because field duplicate analyses measure both field and laboratory precision, results of the analyses may contain more variability than laboratory duplicate analyses, which measure only laboratory performance.

Precision is expressed as relative percent difference (RPD) according to the following formula:

$$
R P D=\frac{\text { absolute value of (original sample result }- \text { duplicate sample result) }}{\text { (original sample result }+ \text { duplicate sample result) } / 2} \times 100 \%
$$

Field duplicate precision ranged from 0 to 152 percent, and laboratory duplicate precision ranged from 5 to 42 percent. Field and laboratory duplicate RPDs are listed in Appendix F.

\subsubsection{Accuracy}

Accuracy is the closeness of a measurement to the true value. For soil and water samples, it is usually expressed in percent recovery, which is determined through analyses of reference standards and matrix spike samples. The objective of these analyses is to meet the percent recovery defined by the individual analytical methods.

For soil, groundwater, and sludge samples, accuracy is expressed in terms of percent recovery (\%R) during matrix spike sample analysis and RPD of laboratory control . samples:

$$
\% R=\frac{(\text { concentration of spiked sample }- \text { concentration of unspiked sample })}{\text { known concentration of spiked compound }} \times 100 \%
$$

Accuracy for matrix spike recoveries on inorganic samples ranged from 75 to 120 percent. For TPH analyses, matrix spike recoveries ranged from 102 to 110 percent, and blank spikes ranged from 65 to 108 percent. Blank spikes are not an adequate measure of overall accuracy because they do not account for sample matrix interferences. Matrix spikes were not performed on volatile organic compounds; only blank spike analysis was conducted. Blank spike recoveries for volatile organic compounds ranged from 88 to 122 percent. All these recoveries are within the laboratory acceptance criteria. Matrix spike results for the reanalysis of PCBs were not available for review before this report was published. Laboratory control samples also were analyzed for inorganic constituents; recoveries for these analyses ranged from 89 to 110 percent, which is within the laboratory acceptance criteria. 


\subsubsection{Completeness}

The completeness of data collected during this investigation was determined by comparing the number of valid measurements actually obtained to the number of planned measurements and is expressed by the following equation.

$$
\% C=\frac{\text { number of valid measurements }}{\text { total number of planned measurements }} \times 100 \%
$$

The completeness calculation did not include asbestos investigation results because the number of planned asbestos samples was unknown. The calculated completeness for the project was 97 percent. 
$\cdots+\quad-\cdots=$ = 


\subsection{Risk Assessment}

This risk assessment was structured around the CERCLA risk assessment process (EPA 1989), but was simplified considerably over the traditional CERCLA risk assessment. The major differences were a less rigorous data evaluation, a superficial examination of exposure pathways, and the use of only one source for the Toxicity assessment (EPA 1993). The purpose of this risk assessment is to provide a general overview of the potential risks to human health at the Oxnard facility.

\subsection{Preliminary Conceptual Site Model}

Figure 6 presents the conceptual site model for the Oxnard facility. An industrial scenario is assumed for both the current and future. Because the land adjacent to the facility is currently industrial, there is no indication that a residential scenario would apply at this site in the foreseeable future. Major sources at the facility include maintenance/construction disposal, industrial operations, and storage spills. Potentially contaminated media include groundwater, air, and soil/sludges. The site is supplied with drinking water from the city of Oxnard; therefore, exposure from groundwater is not considered a complete pathway. Inhalation of and dermal contact with contaminated soils/sludges are potentially complete pathways, although exposures resulting from these pathways will likely be insignificant compared to exposures from ingestion.

Chromium present at the Oxnard facility is believed to be in the form of chromium metal or trivalent chromium. This assumption is based on process knowledge; hexavalent chromium compounds are not presently used at the Oxnard facility, and historical records do not indicate use of these compounds. The occurrence of hexavalent chromium is unlikely because chromium metal or trivalent chromium is not oxidized to hexavalent chromium under normal environmental conditions.

\subsection{Data Evaluation}

The analytical data collected during the Phase II ESA are presented in Appendix D. Because exposure to groundwater is not considered likely, groundwater data were not used in the risk assessment. No air contaminant concentration data were available, but no violations of State Air quality standards have occurred at the facility. The risks of inhaling contaminated air are expected to be insignificant compared to those of ingesting contaminated soil. 


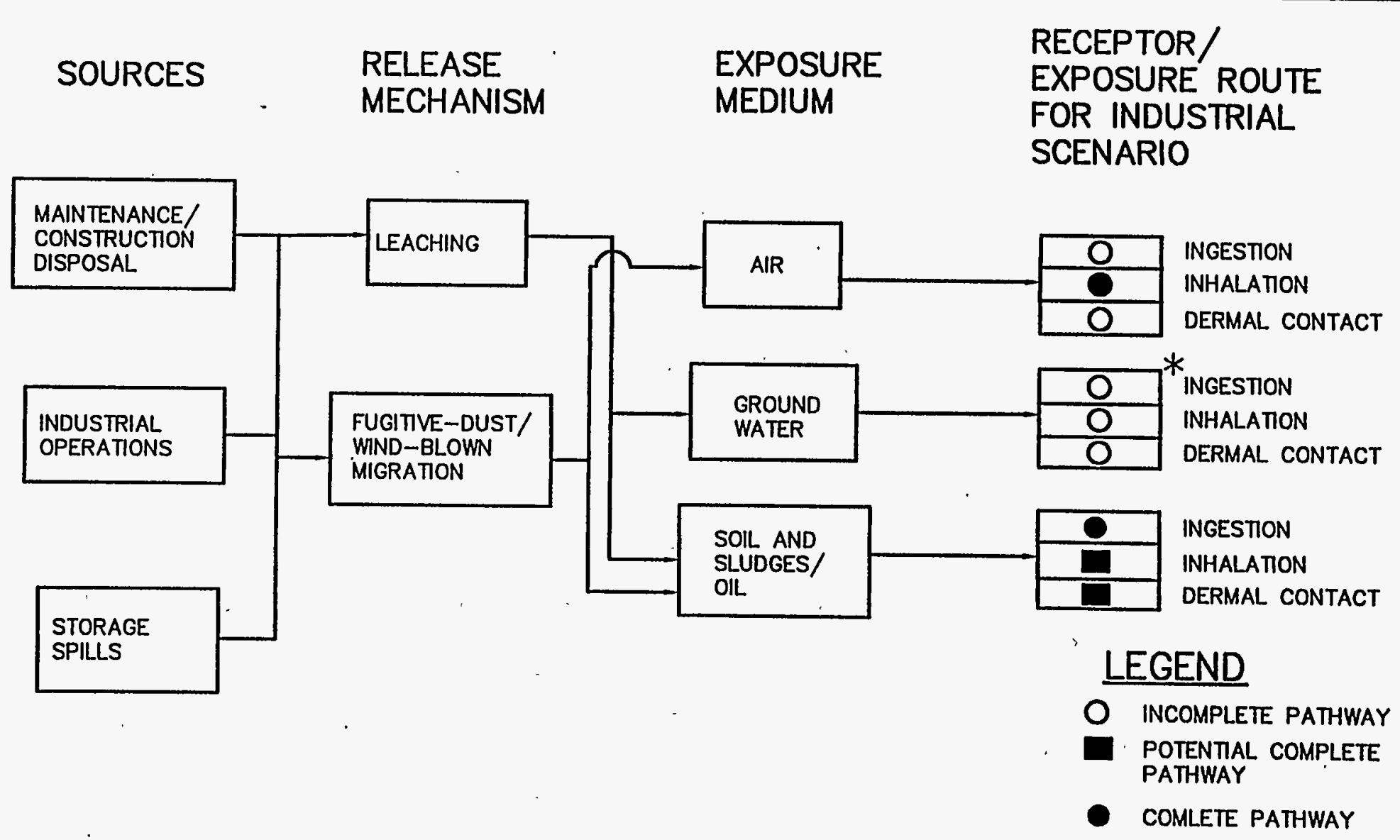

\section{PRELIMINARY CONCEPTUAL SITE MODEL} FOR THE OXNARD FACILITY

* an exposure to contaminated groundwater

IS NOT CURRENTLY OCCURING OR IS EXPECTED

TO OCCUR IN THE FUTURE. THE CITY OF

OXNARD SUPPLIES DRINKING WATER AND THIS

WLL LIKELY CONTNUE. 
Data from the locations listed in Table 2 were used in the risk assessment.

Table 2. Risk Assessment Sampling Locations

\begin{tabular}{||l|l||}
\hline Sample Locations & \multicolumn{1}{|c|}{ Comments } \\
\hline $\begin{array}{l}\text { SB-1, SB-5, SB-8, } \\
\text { SB-9, and SB-10 }\end{array}$ & $\begin{array}{l}\text { Areas where exposure to potentially } \\
\text { contaminated soil is likely. All other } \\
\text { potentially contaminated soil areas are } \\
\text { covered by concrete or asphalt, which } \\
\text { eliminates the potential for exposure. }\end{array}$ \\
\hline SB-2 and SB-7 & Background locations. \\
\hline $\begin{array}{l}\text { Oil-1 and SL-1, } \\
\text { SL-2, SL-3, SL-5, } \\
\text { SL-6, and SL-7 }\end{array}$ & $\begin{array}{l}\text { Data from all of these sample locations were } \\
\text { used because maintenance and cleaning } \\
\text { personnel exposure could occur at all these } \\
\text { points. }\end{array}$ \\
\hline
\end{tabular}

Data from areas of concern were compared to background data as presented in Table 3. When a chemical concentration in a background area was equal to or greater than 90 percent of the concentration of that chemical in the area of concern, that chemical was eliminated as a chemical of potential concern (COPC) in accordance with EPA risk assessment guidance for CERCLA sites. 
-Table 3. Data Summary for Risk Assessment

\begin{tabular}{|c|c|c|c|c|}
\hline Element & $\underset{(\mathrm{mg} / \mathrm{kg})^{2}}{\text { Soil }}$ & $\begin{array}{c}\text { Sludge/Oil } \\
(\mathrm{mg} / \mathrm{kg})^{\mathbf{b}}\end{array}$ & $\begin{array}{c}\text { Background } \\
\text { (mg/kg) }\end{array}$ & $\begin{array}{c}\text { COPC }^{d} \\
\text { Soil/Sludge Oil }\end{array}$ \\
\hline Ag & 0.40 & 0.41 & 0.40 & No \\
\hline As & 3.34 & 2.58 & 4.61 & No \\
\hline $\mathrm{Ba}$ & 104.92 & 37.96 & 93.20 & YES/NO \\
\hline $\mathrm{Be}$ & $0.40^{\prime}$ & 0.41 & 0.40 & No \\
\hline $\mathrm{Cd}$ & 0.58 & 12.29 & 0.40 & YES \\
\hline Co & 5.47 & 39.99 & 6.22 & NO/YES \\
\hline $\mathrm{Cr}$ & 19.36 & 187.07 & 21.45 & NO/YES \\
\hline $\mathrm{Cu}$ & 13.30 & 745.26 & 11.25 & YES \\
\hline $\mathrm{F}$ & 4.86 & - & 2.50 & YES/NO \\
\hline $\mathrm{Hg}$ & 0.034 & 0.031 & 0.016 & YES \\
\hline Mo & 0.52 & 46.06 & 0.40 & YES \\
\hline $\mathrm{Ni}$ & 17.02 & 459.84 & 16.05 & NO/YES \\
\hline $\mathrm{Pb}$ & 14.45 & 171.36 & 12.65 & YES \\
\hline $\mathrm{Sb}$ & 0.40 & 1.54 & $\cdot 0.40$ & NO/YES \\
\hline $\mathrm{Se}$ & 0.17 & 0.05 & 0.22 & NO \\
\hline $\mathrm{Tl}$ & 0.40 & 0.41 & 0.40 & No \\
\hline $\mathrm{V}$ & 31.80 & 24.41 & 41.85 & NO \\
\hline $\mathrm{Zn}$ & 66.06 & 1283.14 & 51.60 & YES \\
\hline $1,1,1$, Trichloroethane & 0.003 & - & 0.002 & YES/NO \\
\hline Acetone & 0.178 & - & 0.008 & YES/NO \\
\hline Toluene & 0.003 & - & 0.002 & YES/NO \\
\hline 2-Butanone & 0.066 & - & 0.008 & YES/NO \\
\hline PCBs & 0.906 & 0.03 & 0.03 & YES/NO \\
\hline
\end{tabular}

These concentrations were calculated by averaging data from sampling locations SB-1, SB-5, SB-8, SB-9, and SB-10. When information on the depth of the sample was given, only data from the $0-2$ foot depth was used. Exposures are not likely to occur from soils greater than 2 feet in depth.

${ }^{b}$ These concentrations were calculated by averaging data from sampling locations Oil-1, SL-1, SL-2, SL-3, SL-5, SL-6, and SL-7.

'According to U.S. EPA Guidance, compounds not detected are assumed to be one-half of the detection limit.

'Based on comparison to background. 


\subsection{Exposure Assessment}

An industrial scenario is the only likely exposure scenario at the facility. Residential use of the facility is not considered likely because of the industrial use of the immediate area. The two possible industrial scenarios are shown in Table 4.

Table 4. Risk Assessment Scenarios

\begin{tabular}{||l|c|l|}
\hline \multicolumn{1}{|c|}{ Scenario } & Exposure Medium & \multicolumn{1}{c|}{ Comments } \\
\hline $\begin{array}{l}\text { Exposure to five } \\
\text { potentially contaminated } \\
\text { soil areas }\end{array}$ & soil & $\begin{array}{l}\text { This assumes regular } \\
\text { exposure to these areas } \\
\text { over the entire work year. }\end{array}$ \\
\hline $\begin{array}{l}\text { Exposure to sludges/oils } \\
\text { through maintenance and } \\
\text { cleaning activities. }\end{array}$ & sludges/oil & $\begin{array}{l}\text { This assumes exposure } \\
\text { will occur infrequently: } \\
\text { one week for } \\
\text { maintenance and one } \\
\text { week for cleaning. }\end{array}$ \\
\hline
\end{tabular}

These scenarios do not include exposure to air or groundwater. Air is not included because data are not available. However, exposures are expected to be limited because no State air violations have been recorded. No contact with groundwater is expected because water is supplied by the City. The only viable exposure route is considered to be ingestion of contaminated soils or sludges/oil. Limited exposure may occur from inhalation or dermal contact with these contaminated media, but this is expected to be insignificant compared to exposure from ingestion.

Exposure to the COPCs was estimated through use of the following equation (from "Risk Assessment Guidance for Superfund")(EPA 1989).

$$
\text { Intake }(m g / k g-d a y)=\frac{C S x I R x C F x F I x E F x E D}{B W x A T} \text {. }
$$

Where:

$\mathrm{CS}=$ Chemical Concentration in Soil $(\mathrm{mg} / \mathrm{kg})$

$\mathrm{IR}=$ Ingestion Rate (mg soil/day)

$\mathrm{CF}=$ Conversion Factor $\left(10^{-6} \mathrm{~kg} / \mathrm{mg}\right)$

$\mathrm{FI}=$ Fraction Ingested from Contaminated Source (unitless)

$\mathrm{EF}=$ Exposure Frequency (days/years)

$\mathrm{ED}=$ Exposure Duration (years)

$\mathrm{BW}=$ Body Weight $(\mathrm{kg})$

$\mathrm{AT}=$ Averaging Time (period over which exposure is averaged - - days) 
Exposure factors used are presented in Table 5.

Table 5. Exposure Factors

\begin{tabular}{|c|c|c|c|c|}
\hline $\begin{array}{c}\text { Exposure } \\
\text { Parameter }\end{array}$ & \multicolumn{2}{|c|}{$\begin{array}{c}\text { Exposure Factor } \\
\text { soil sludge/oil }\end{array}$} & Source & Comment \\
\hline Ingestion Rate & $50 \mathrm{mg} / \mathrm{day}$ & $50 \mathrm{mg} /$ day & USEPA 1991 & $\begin{array}{l}\text { Interim default for adult } \\
\text { ingestion of soil and } \\
\text { dust in the typical work } \\
\text { place. }\end{array}$ \\
\hline Fraction Ingested & 1 & 1 & & Assumes worst case. \\
\hline Exposure Frequency & 250 days & 10 days & : & $\begin{array}{l}\text { Soil: Assumes } 5 \\
\text { days/week } 50 \text { weeks per } \\
\text { yr. } \\
\text { Sludges/Oil: Assumes } \\
\text { one week for cleaning, } \\
\text { one week for } \\
\text { maintenance. A total of } \\
\text { two weeks per year. }\end{array}$ \\
\hline Exposure Duration & 25 yrs. & 25 yrs. & USEPA 1991 & $\begin{array}{l}\text { This is the } 95 \text { percentile } \\
\text { for a worker to stay at } \\
\text { the same location, } \\
\text { according to the Bureau } \\
\text { of Labor statistics. }\end{array}$ \\
\hline Body Weight & $70 \mathrm{~kg}$ & $70 \mathrm{~kg}$ & USEPA 1989 & Standard Values \\
\hline Averaging Time & $\begin{array}{l}70 \text { yrs. for } c \\
25 \text { yrs. for } n\end{array}$ & og. & USEPA 1989 & Standard Values \\
\hline
\end{tabular}

Sources: US EPA 1991. Human Health Evaluation Manual, Supplemental Guidance: "Standard Default Exposure Factors." OSWER Directive 9285.6-03. US EPA 1989. Risk Assessment Guidance for Superfund, EPA/540/1-89/002 (EPA 1991).

\subsection{Toxicity Assessment}

This section presents information on toxicity of the chemicals of potential concern. Table 6 presents carcinogenicity status, slope factor, and reference dose for each contaminant of potential concern. 
Table 6. Toxicity Assessment Summary

\begin{tabular}{|c|c|c|c|}
\hline COPC & Effect ${ }^{2}$ & $\begin{array}{l}\text { Slope Factor } \\
\text { (mg/kg-day) })^{-1}\end{array}$ & $\begin{array}{c}\text { RFD } \\
\text { (mg/kg/day) }\end{array}$ \\
\hline $\mathrm{Ba}$ & $\mathbf{N}$ & - & $7 \times 10^{-2}$ \\
\hline $\mathrm{Cd}$ & & - & -- \\
\hline Co & & - & - \\
\hline $\mathrm{Cr}^{b}$ & $\mathbf{N}$ & $4.1 \times 10^{1}$ & $2 \times 10^{-2}$ \\
\hline $\mathrm{Cu}$ & $\mathbf{N}$ & - & NA \\
\hline F & $\mathbf{N}$ & - & $4 \times 10^{-1}$ \\
\hline $\mathrm{Hg}$ & $\mathbf{N}$ & - & $3 \times 10^{-4}$ \\
\hline Mo & $\mathbf{N}$ & - & $5 \times 10^{-3}$ \\
\hline $\mathrm{Ni}$ & $\mathrm{C} / \mathrm{N}$ & $8.4 \times 10^{-1}$ & $2 \times 10^{-2}$ \\
\hline $\mathrm{Pb}$ & $\mathrm{C} / \mathrm{N}$ & NA & NA \\
\hline $\mathrm{Sb}$ & $N$ & - & $4 \times 10^{-4}$ \\
\hline $\mathrm{Zn}$ & $\mathbf{N}$ & - & NA \\
\hline $\begin{array}{c}1,1,1 \\
\text { Trichloroethane }\end{array}$ & $\mathbf{N}$ & - & NA \\
\hline Acetone & $\mathrm{N}$ & - & $1.0 \times 10^{0}$ \\
\hline Toluene & $\mathrm{N}$ & - & $2.0 \times 10^{0}$ \\
\hline 2-Butanone & & - & NA \\
\hline PCBs & $\mathrm{C}$ & 7.7 & NA \\
\hline
\end{tabular}

${ }^{\circ} \mathrm{C}=$ Carcinogen.

$\mathrm{N}=$ Noncarcinogen.

bAssume chromium and chromium III compounds.

$\mathrm{NA}=$ Not Available.

Source: Health Effects Assessment Summary Tables. EPA 540-R-93-058. 
Toxicity profiles for the chemicals of potential concern are as follows:

- Barium primarily is toxic to the skin, eye, heart, and lung.

- Cadmium is a metal with a level of toxicity similar to that of lead. It is present in most foods and tissues. The element and its compounds are toxic through inhalation, ingestion, and subcutaneous application. Organs most sensitive to cadmium are the kidney and lung.

- Exposure to cobalt can lead to decreased pulmonary function and skin disorders.

- Chromium exists in several valence states, but only trivalent and hexavalent chromium are of biological importance. Trivalent chromium is an essential nutrient. Hexavalent chromium is a human carcinogen through inhalation.

- Copper is an essential trace metal. At high intakes, it is toxic to the brain, liver, and kidneys.

- Fluoride is added to many public water supplies. In high doses, it can cause respiratory, skin, eye, and gastrointestinal irritation. Fluoride is not a carcinogen.

- Mercury primarily is toxic to the skin, respiratory tract, central nervous system, and kidney.

- Molybdenum is an essential trace metal. At high doses, it is toxic to the blood, liver, and eye.

- Nickel and some of its compounds are carcinogenic to humans, a finding based on epidemiological evidence for nickel refinery workers suffering from lung and nose cancer.

- The toxic effects of lead are widespread, and are found in the central nervous system, the peripheral nervous system, the kidneys, and the blood.

- Antimony is similar in toxicity to arsenic. It can damage the reproductive system, skin, heart, lungs, and gastro-intestinal tract.

- Zinc is an essential trace metal in the human diet. Zinc is not an inherently toxic element; however, some of its compounds are toxic and cause dermatitis and intestinal disorders.

- At high levels of exposure, trichloroethane is moderately toxic and depresses the central nervous system. 
- Systemic toxicity in the form of central nervous system depression can occur after ingestion, inhalation, or dermal absorption of acetone.

- At high levels of exposure, toluene is a naricotic and affects the central nervous system, leading to fatigue, weakness, and confusion. In low, chronic exposures, toluene can cause damage to the liver and kidneys.

- Inhalation of 2-butanone can lead to central nervous system depression, narcosis, and cardiorespiratory system failure.

- PCBs are moderately toxic by ingestion and/or skin contact and cause liver and experimental reproductive effects.

In most pathways to human receptors, agents occur in mixtures. When there is a mixture of toxic agents, there exists a potential for interactions among the agents' effects. This potential depends on the components of the mixture, on the carriers along the environmental pathway from source to receptor, and on the way the contaminant enters the body. For carcinogens that lead to the same endpoint (e.g., cancer of the same organ) there is a considerable potential for synergistic or antagonistic interaction. It has been shown, however, that for carcinogens having low cancer probabilities, the interaction generally is antagonistic, which leads to a lower probability of cancer.

\subsection{Risk Characterization}

Risks were estimated as follows:

Carcinogens: $\quad$ Risk $=C D I x S F$

where: Risk $=$ a unitless probability (e.g., $2 \times 10^{-5}$ ) of an individual developing cancer;

$\mathrm{CDI}=$ Chronic daily intake averaged over 70 years $(\mathrm{mg} / \mathrm{kg}$-day); and

$\mathrm{SF} \quad=$ slope factor, expressed in $(\mathrm{mg} / \mathrm{kg} \text {-day })^{-1}$

Noncarcinogens: $\quad$ Noncancer Hazard Quotient $=E / R f D$

where: $\mathrm{E}=$ exposure level (or intake)

$\mathrm{RfD}=$ reference dose; and

$\mathrm{E}$ and $\mathrm{RfD}$ are expressed in the same units and represent the same exposure period (i.e., chronic, subchronic, or shorter-term). 
Risk characterization results are presented in Table 7.

Table 7. Risk Characterization Results

\begin{tabular}{|c|c|c|}
\hline COPC & Soil Scenario & Sludges/Oil \\
\hline & Carcinogens Noncarcinogens & Carcinogens Noncarcinogens \\
\hline $\mathrm{Ba}$ & $7.33 \times 10^{-4}$ & Not a COPC \\
\hline $\mathrm{Cd}$ & \multicolumn{2}{|c|}{ (No EPA slope factor [SF] or reference dose [RFD] available.) } \\
\hline Co & \multicolumn{2}{|c|}{ (No EPA slope factor [SF] or reference dose [RFD] available.) } \\
\hline $\mathrm{Cr}$ & & $1.83 \times 10^{-4}$ \\
\hline $\mathrm{Cu}$ & \multicolumn{2}{|c|}{ (No EPA slope factor [SF] or reference dose [RFD] available.) } \\
\hline $\mathbf{F}$ & $5.9 \times 10^{-6}$ & Not measured \\
\hline $\mathrm{Hg}$ & $5.5 \times 10^{-5}$ & $2.0 \times 10^{-6}$ \\
\hline Mo & $5.1 \times 10^{-5}$ & $1.8 \times 10^{-4}$ \\
\hline $\mathbf{N i}$ & Not a COPC & $2.7 \times 10^{-6}$ \\
\hline $\mathrm{Pb}$ & \multicolumn{2}{|c|}{ (No EPA slope factor [SF] or reference dose [RFD] available.) } \\
\hline $\mathrm{Sb}$ & Not a COPC & $7.5 \times 10^{-5}$ \\
\hline $\mathrm{Zn}$ & \multicolumn{2}{|c|}{ (No EPA slope factor [SF] or reference dose [RFD] available.) } \\
\hline 1,1,1 Trichloroethane & \multicolumn{2}{|c|}{ (No EPA slope factor [SF] or reference dose [RFD] available.) } \\
\hline Acetone & $8.7 \times 10^{-8}$ & Not measured \\
\hline Toluene & $7.3 \times 10^{-10}$ & Not measured \\
\hline 2-Butanone & \multicolumn{2}{|c|}{ (No EPA slope factor [SF] or reference dose [RFD] available.) } \\
\hline PCBs & $5.8 \times 10^{-8}$ & \\
\hline TOTAL & $8.4 \times 10^{-4}$ & $2.7 \times 10^{-6}$ \\
\hline
\end{tabular}

Under CERCLA, risks from carcinogens greater than $1 \times 10^{-4}$ generally require action to reduce the risks; risks less than $1 \times 10^{-6}$ usually result in no action. When risks are between $1 \times 10^{-6}$ and $1 \times 10^{-4}$ actions are taken on a site-specific basis in accordance with direction from EPA. For noncarcinogens, the individual risks (called Hazard Quotients) are summed to become a Hazard Index (HI). Generally, when the HI is less than one, adverse noncarcinogenic effects during a lifetime are not considered to be excessive, within an adequate margin of safety. (For more information on acceptable risk ranges under CERCLA, see "National Oil and Hazardous Substances Pollution Contingency," 40 CFR Part 300, March 8, 1990). 
Risk ranges given are for risks to the public. A CERCLA baseline risk assessment does not include an evaluation of risks to workers because CERCLA focuses on inactive sites. Risks to the general public are expected to be negligible. Therefore, the CERCLA risk interpretation given above provides a worst-case interpretation of the results for this facility.

The risk from carcinogens $\left(2.7 \times 10^{-6}\right)$ is in the $1 \times 10^{-6}$ to $1 \times 10^{-4}$ range but is close to the lower value of the range and is based on a worst-case assumption of risk to site workers. The risk from noncarcinogens is well below the hazard index threshold of 1 . 


\subsection{Recommendations}

All friable asbestos gaskets including the emersion heater and Lindberg furnace gasket should be removed and disposed of in accordance with the Ventura County Air Pollution Control District Rule 62.7, Sections D.2.a, D.3, G, and J. Notification and containment area requirements are not applicable because the total surface area of ACM is less than 100 square feet.

Abatement of the floor tiles (Building 1, Lobby and Vault), window caulk (Building 6), textured wall coating (Building 1, Accounting Offices), laboratory benches and cabinet sheathing, and HVAC sleeve is not necessary because these materials are in excellent condition and are not in a friable state.

In general, ACM floor tiles and mastic are classified as Category I Non-Friable ACM, and the laboratory benches, cabinet sheathing, window caulk, and HVAC sleeve are classified as Category II Non-Friable ACM. As such, these materials are exempt from Rule 62.7 as long as removal procedures prevent the ACM from becoming friable during renovation. The following language is specific to Category I and Category II Non-Friable ACM:

Use removal, loading, and transportation techniques designed to prevent the release of fibers and to prevent nonfriable ACM from becoming friable. Do not use techniques involving sanding, grinding, chipping, drilling, sawing, abrading, dropping, throwing, sliding, or any other technique that may allow the release of fibers or render the material friable. Keep all areas of ACM that are disturbed during cutting or disjoining operations adequately wet. Cover or contain the material so that no ACM is lost during transportation to an appropriate disposal site.

An asbestos management plan for asbestos that will remain in place will need to be prepared because the facility qualifies as a public building in the State of California. If and when renovation or demolition of buildings or building components is planned, a full asbestos inspection of those areas will be required prior to renovation or demolition. The asbestos inspection will allow the asbestos content of materials, such as the electrical wiring insulation, brakes of the Cleveland presses, and window caulk in Building 2 , to be determined.

Significant contamination of the shallow groundwater system beneath the Oxnard facility has not occurred. The absence or low concentrations of organic compounds and most metals in groundwater samples indicates minimal degradation of the shallow aquifer, and groundwater remediation is not warranted. Monitoring wells should be properly abandoned as specified in the well permit issued by the City of Oxnard, which requires wells to be abandoned after the completion of the monitoring program. 
Soils at the Oxnard facility show elevated concentrations of heavy metals and PCBs. Heavy metal concentration in several soil boring samples were statistically higher than those in background samples; however, the risk from exposure to soils in these ares is low, and corrective action is not warranted. PCBs were detected in samples from two soil borings. Negotiations with California EPA should be conducted to determine PCB action levels at the Oxnard facility. Once action levels are established, two courses of action are possible: (1) no action (if PCB concentrations are below established action limits and no further characterization is required); or (2) further characterization of PCBs and appropriate corrective action.

Radiological contamination at the Oxnard facility was not found; no action is required.

Containerized groundwater is not hazardous and can be handled and discarded as wastewater in accordance with local regulations. Sludges in the trench beneath Building 6, the cellar beneath the 1,600-ton press in Building 5, and the collection trench east of Building 6 should be treated as hazardous waste if they are removed for disposal. All containerized soils, with the exception of borehole cuttings from SB-1 and SB-10, are not hazardous and can be returned to the soils on the facility. Borehole cuttings from SB-1 and SB-10 should not be disposed of until negotiations with California EPA concerning PCBs are completed. PPE used to collect asbestos samples should be disposed of in accordance with the Ventura County Air Pollution Control District Rule 62.7. 


\subsection{References}

Barclays Law Publishers, 1990a. Title 22, California Code of Regulations, Division 4.5, Chapter 15, § 66265.97 "General Water Quality Monitoring and System Requirements," South San Francisco, CA.

, 1990b. Title 22, California Code of Regulations, Division 4, Chapter 15, Article 4, § 64431 "Maximum Contaminant Levels - Inorganic Chemicals," South San Francisco, CA.

, 1990c. Title 22, California Code of Regulations, Division 4, Chapter 11, Article 3, § 66261.24 "Characteristic of Toxicity," South San Francisco, CA.

Bouwer, H., and R. C. Rice, 1976. "A slug test for determining hydraulic conductivity of unconfined aquifers with completely or partially penetrating wells," Water Resources Research, v. 12, pp. 423-428.

Bouwer, H., 1989. "The Bouwer and Rice Slug Test - An Update," Ground Water, v 27, pp. 304-309.

Bureau of National Affairs, 1995a. Title 8, California Code of Regulations, Chapter 3.2, California Occupational Safety and Health Regulations (CAL/OSHA) Subchapter 2, Article 2.6, § 341.15 Certification of Asbestos Consultants and Site Surveillance Technicians, Washington, DC.

, 1995b. Title 8, California Code of Regulations, Chapter 3.2, California Occupational Safety and Health Regulations (CAL/OSHA), Construction Safety Orders, Article 4, § 1529 Asbestos, Washington, DC.

, 1995c. Title 8, California Code of Regulations, Chapter 3.2, California Occupational Safety and Health Regulations (CAL/OSHA), Subchapter 2, Article 2.5, § 341.6 Registration Requirements, Washington, DC.

, 1995d. Ventura County Air Pollution Control District Rule 62.7, Asbestos Renovation and Demolition, Washington, DC.

Marburg Associates and William P. Parkin, 1991. Site Auditing: Environmental Assessment of Property, Specialty Technical Publishers, Inc., Vancouver, British Columbia, Canada.

U.S. Department of Energy, 1994a. Phase I Environmental Site Assessment for the EG\&G Rocky Flats Oxnard Facility, Grand Junction Projects Office, Grand Junction, CO 
U.S. Department of Energy, 1994b. 10 CFR 1021.301, "National Environmental Policy Act Implementation Procedures, Subpart C - Implementing Procedures," U.S. Code of Federal Regulations, January 1, 1994.

, 1994c. Work Plan/Sampling and Analysis Plan for the Phase II ESA of the EG\&G Rocky Flats Oxnard Facility, Oxnard, California, Grand Junction Projects Office, Grand Junction, CO.

U.S. Environmental Protection Agency, 1989. Risk Assessment Guidance for Superfund, Volume I: Human Health Evaluation Manual (Part A), EPA/540/1-89/002, Washington, DC. , 1991. Human Health Evaluation Manual, Supplemental Guidance: "Standard Default Exposure Factors", Interim Final," OSWER Directive 9285.6-03, Washington DC. , 1993. Health Effects Assessment Summary Tables, EPA 540-R93-058, March 1993, Washington, DC. , 1994a. 40 CFR Part 763, "Subchapter R-The Toxic Substances Control Act, Asbestos", U.S. Code of Federal Regulations, July 1, 1994. , 1994b. 40 CFR Part 300, "National Oil and Hazardous Substances Pollution Contingency", U.S. Code of Federal Regulations, July 1, 1994.

Ventura County Air Pollution Control District, 1992. Rule 62.7, "Asbestos Renovation and Demolition," Ventura, CA. 


\section{Appendix A \\ Asbestos Investigation}


$\cdots+-\cdots=$

$$
\text { . }
$$


Asbestos : $\quad$ lary Sheet

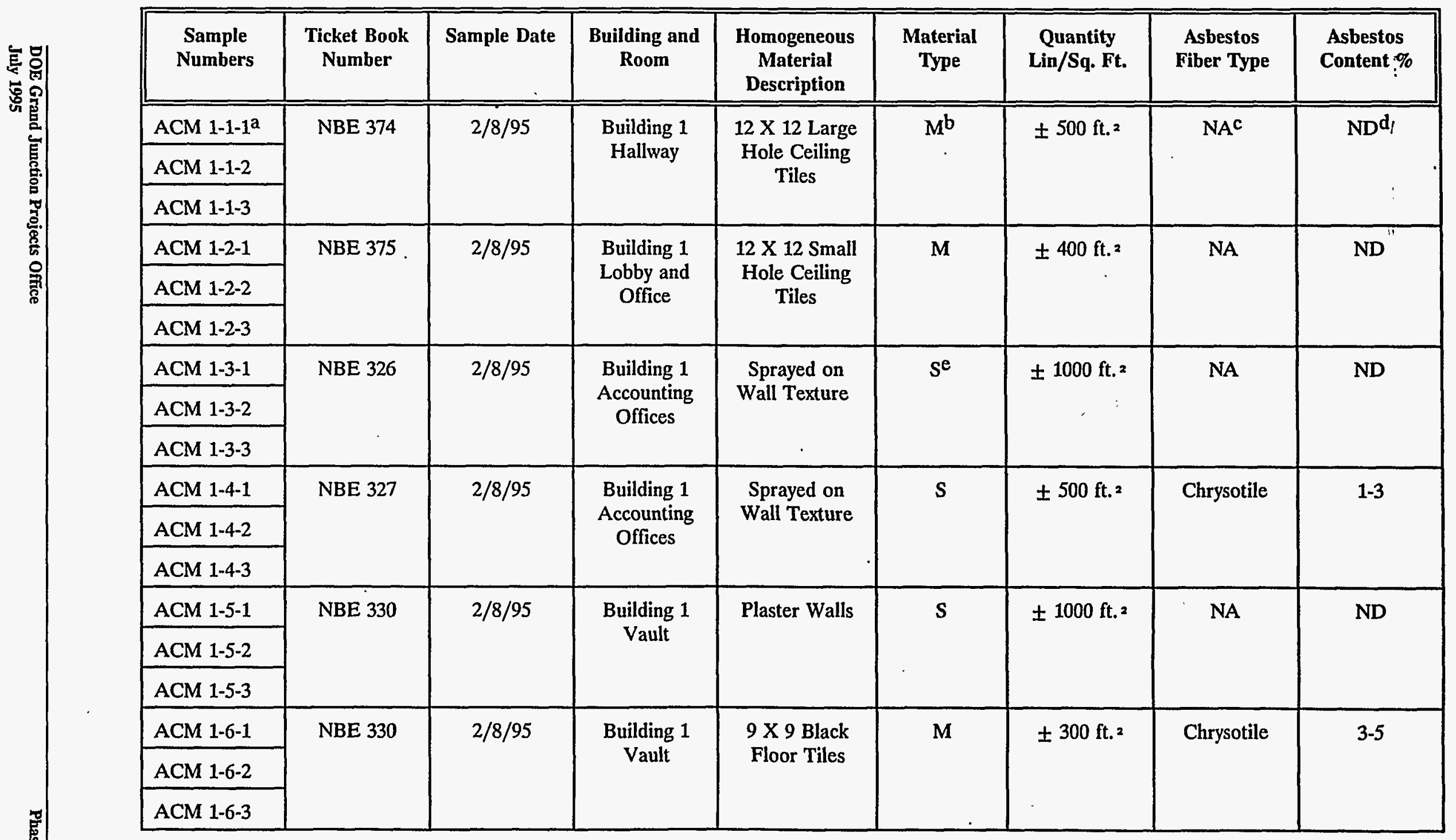

asample designation is as follows: ACM 1-1-1=Asbestos containing material from Building 1, homogeneous material 1, and one sample taken, respectively.

bMiscellaneous.

cNot applicable.

dNot detected.

esurface. 
Asbestos Summary Sheet (continued)

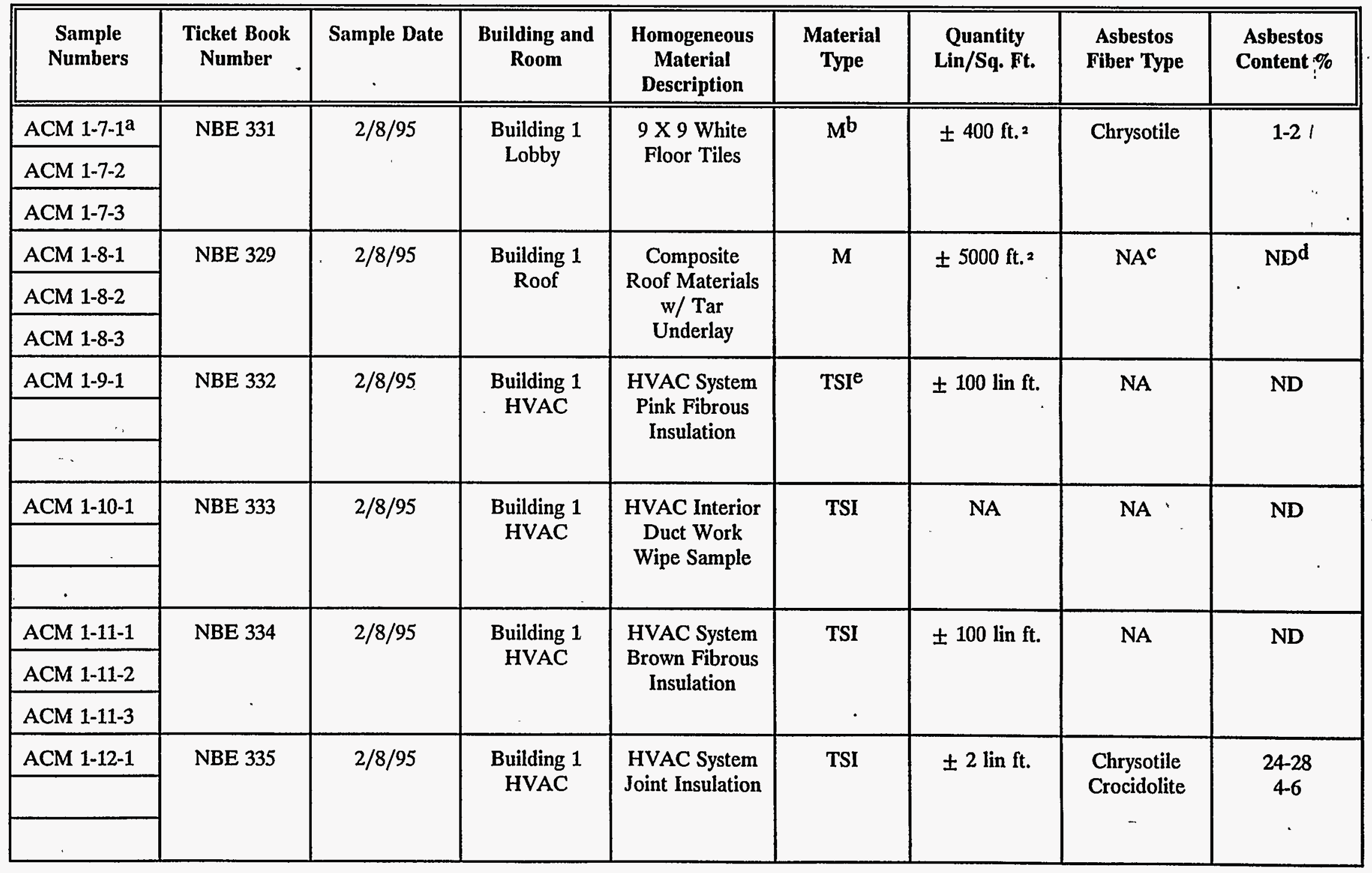

aSample designation is as follows: ACM 1-1-1=Asbestos containing material from Building 1, homogeneous material 1, and one sample taken, respectively.

bMiscellaneous.

cNot applicable.

dNot detected.

eThermal system insulation. 
Asbestos Summi ieet (continued)

\begin{tabular}{|c|c|c|c|c|c|c|c|c|}
\hline $\begin{array}{l}\text { Sample } \\
\text { Numbers }\end{array}$ & $\begin{array}{l}\text { Ticket Book } \\
\text { Number }\end{array}$ & Sample Date & $\begin{array}{l}\text { Building and } \\
\text { Room }\end{array}$ & $\begin{array}{l}\text { Homogeneou } \\
\text { s Material } \\
\text { Description }\end{array}$ & $\begin{array}{c}\text { Material } \\
\text { Type }\end{array}$ & $\begin{array}{l}\text { Quantity } \\
\text { Lin/Sq. Ft. }\end{array}$ & $\begin{array}{l}\text { Asbestos } \\
\text { Fiber Type }\end{array}$ & $\begin{array}{l}\text { Asbestos } \\
\text { Content } \%\end{array}$ \\
\hline ACM 2-1-1 ${ }^{a}$ & \multirow[t]{2}{*}{ NBE 364} & \multirow[t]{2}{*}{$2 / 8 / 95$} & \multirow{2}{*}{$\begin{array}{c}\text { Building } 2 \\
\text { Fabrication } \\
\text { Shop (outside) }\end{array}$} & \multirow{2}{*}{$\begin{array}{c}\text { Air } \\
\text { Exchanger } \\
\text { Insulation } \\
\text { Wrap }\end{array}$} & \multirow[t]{2}{*}{$\mathrm{TSI}^{\mathrm{b}}$} & \multirow[t]{2}{*}{$<4$ lin. ft. } & \multirow[t]{2}{*}{$N A^{c}$} & \multirow{2}{*}{$\mathrm{ND}^{\mathrm{d}}{ }^{\prime}$} \\
\hline & & & & & & & & \\
\hline ACM 2-2-1 & \multirow{3}{*}{ NBE 365} & \multirow{3}{*}{$2 / 7 / 95$} & \multirow{3}{*}{$\begin{array}{l}\text { Building } 2 \\
\text { Engineering } \\
\text { Office } \\
\text { Conference } \\
\text { Room }\end{array}$} & \multirow{3}{*}{$\begin{array}{l}\text { Plaster } \\
\text { Wallboard. } \\
\text { All wallboard } \\
\text { in building } 2 \\
\text { was the same } \\
\text { construction. }\end{array}$} & \multirow{3}{*}{$S^{e}$} & \multirow{3}{*}{ $\pm 1000 \mathrm{ft} .^{2}$} & \multirow{3}{*}{ NA } & \multirow{3}{*}{ ND } \\
\hline ACM 2-2-2 & & & & & & & & \\
\hline ACM 2-2-3 & & & & & & & & \\
\hline ACM 2-3-1 & \multirow{3}{*}{ NBE 366} & \multirow{3}{*}{$2 / 7 / 95$} & \multirow{3}{*}{$\begin{array}{l}\text { Building } 2 \\
\text { Engineering } \\
\text { Office } \\
\text { Conference } \\
\text { Room }\end{array}$} & \multirow{3}{*}{$\begin{array}{c}24 \text { X } 48 \\
\text { Ceiling Tiles }\end{array}$} & \multirow{3}{*}{$\mathrm{M}^{\mathrm{f}}$} & \multirow{3}{*}{ $\pm 1000 \mathrm{ft} .^{2}$} & \multirow{3}{*}{ NA } & \multirow{3}{*}{ ND } \\
\hline ACM 2-3-2 & & & & & & & & \\
\hline ACM 2-3-3 & & & & & & & & \\
\hline ACM 2-4-1 & \multirow{3}{*}{ NBE 367} & \multirow{3}{*}{$2 / 7 / 95$} & \multirow{3}{*}{$\begin{array}{c}\text { Building } 2 \\
\text { Manufacturing } \\
\text { Office }\end{array}$} & \multirow{3}{*}{$\begin{array}{l}9 \times 9 \text { Gray- } \\
\text { brown Floor } \\
\text { Tiles w/ } \\
\text { Black Mastic }\end{array}$} & \multirow{3}{*}{$\mathbf{M}$} & \multirow{3}{*}{ $\pm 100 \mathrm{ft.}{ }^{2}$} & \multirow{3}{*}{ NA } & \multirow{3}{*}{ ND } \\
\hline & & & & & & & & \\
\hline & & & & & & & & \\
\hline ACM 2-5-1 & \multirow{3}{*}{ NBE 368} & \multirow{3}{*}{$2 / 7 / 95$} & \multirow{3}{*}{$\begin{array}{c}\text { Building } 2 \\
\text { Maintenance } \\
\text { Office }\end{array}$} & \multirow{3}{*}{$\begin{array}{l}9 \times 9 \text { Light- } \\
\text { brown Floor } \\
\text { Tiles w/ } \\
\text { Black Mastic }\end{array}$} & \multirow{3}{*}{$\mathbf{M}$} & & & \\
\hline ACM 2-5-2 & & & & & & $\pm 600 \mathrm{ft.}{ }^{2}$ & NA & ND \\
\hline ACM 2-5-3 & & & & & & & & \\
\hline
\end{tabular}

aSample designation is as follows: ACM 1-1-1=Asbestos containing material from Building 1, homogeneous material 1, and one sample taken, respectively.

bThermal system insulation.

cNot applicable.

dNot detected.

eSurface.

$\mathrm{f}_{\text {Miscellaneous. }}$ 
Asbestos Summary Sheet (continued)

\begin{tabular}{|c|c|c|c|c|c|c|c|c|}
\hline $\begin{array}{c}\text { Sample } \\
\text { Numbers }\end{array}$ & $\begin{array}{l}\text { Ticket Book } \\
\text { Number }\end{array}$ & Sample Date & $\begin{array}{l}\text { Building and } \\
\text { Room }\end{array}$ & $\begin{array}{c}\text { Homogeneous } \\
\text { Material } \\
\text { Description }\end{array}$ & $\begin{array}{c}\text { Material } \\
\text { Type }\end{array}$ & $\begin{array}{l}\text { Quantity } \\
\text { Lin/Sq. Ft. }\end{array}$ & $\begin{array}{l}\text { Asbestos } \\
\text { Fiber Type }\end{array}$ & $\begin{array}{c}\text { Asbestos } \\
\text { Content } \%\end{array}$ \\
\hline ACM $2-6-1^{a}$ & \multirow{3}{*}{ NBE 369} & \multirow{3}{*}{$2 / 7 / 95$} & \multirow{3}{*}{$\begin{array}{l}\text { Building } 2 \\
\text { Maintenance } \\
\text { Office }\end{array}$} & \multirow{3}{*}{$\begin{array}{c}24 \times 48 \\
\text { Ceiling Tiles }\end{array}$} & \multirow{3}{*}{$M^{b}$} & \multirow{3}{*}{ $\pm 600 \mathrm{ft}^{2} 2$} & \multirow{3}{*}{$\mathrm{NA}^{\mathrm{c}}$} & \multirow{3}{*}{$\mathrm{ND}^{d^{\prime}}$} \\
\hline ACM 2-6-2 & & & & & & & & \\
\hline ACM 2-6-3 & & & & & & & & \\
\hline ACM 2-7-1 & \multirow{3}{*}{ NBE 370} & \multirow{3}{*}{$2 / 8 / 95$} & \multirow{3}{*}{$\begin{array}{l}\text { Building } 2 \\
\text { Metallurgical } \\
\text { Lab }\end{array}$} & \multirow{3}{*}{$\begin{array}{l}\text { Laboratory } \\
\text { Cabinet } \\
\text { Interior } \\
\text { Fireproof } \\
\text { Sheathing }\end{array}$} & \multirow{3}{*}{$\mathbf{M}$} & \multirow{3}{*}{ $\pm 500 \mathrm{ft}^{2}$} & \multirow{3}{*}{ Chrysotile } & \multirow{3}{*}{$20-30$} \\
\hline & & & & & & & & \\
\hline & & & & & & & & \\
\hline ACM 2-8-1 & \multirow{2}{*}{ NBE 371} & \multirow{2}{*}{$2 / 8 / 95$} & \multirow{2}{*}{$\begin{array}{c}\text { Building } 2 \\
\text { Metallurgical } \\
\text { Lab }\end{array}$} & \multirow{2}{*}{$\begin{array}{l}\text { Tan Furnace } \\
\text { Brick }\end{array}$} & \multirow{2}{*}{$\mathbf{M}$} & \multirow{2}{*}{ $\pm 4 \mathrm{ft} .2$} & \multirow{2}{*}{ NA } & \multirow{2}{*}{ ND } \\
\hline & & & & & & & & \\
\hline ACM 2-9-1 & \multirow[b]{2}{*}{ NBE 372} & \multirow[b]{2}{*}{$2 / 8 / 95$} & \multirow{2}{*}{$\begin{array}{l}\text { Building } 2 \\
\text { Shop Area }\end{array}$} & \multirow{2}{*}{$\begin{array}{c}\text { Window Caulk } \\
\text { Calcium } \\
\text { Carbonate }\end{array}$} & \multirow[b]{2}{*}{$\mathbf{M}$} & \multirow[b]{2}{*}{ $\pm 500 \mathrm{ft.}^{2}$} & \multirow[b]{2}{*}{ NA } & \multirow[b]{2}{*}{ ND } \\
\hline & & & & & & & & \\
\hline ACM 2-10-1 & \multirow[b]{2}{*}{ NBE 373} & \multirow[b]{2}{*}{$2 / 8 / 95$} & \multirow{2}{*}{$\begin{array}{l}\text { Building } 2 \\
\text { Shop Area }\end{array}$} & \multirow{2}{*}{$\begin{array}{l}\text { Drill Press } \\
\text { Deflector } \\
\text { Guard }\end{array}$} & \multirow[b]{2}{*}{$\mathbf{M}$} & & & \\
\hline & & & & & & $\pm 3 \mathrm{ft} .2$ & NA & ND \\
\hline ACM 4-1-1 & & & Building 4 & Asphalt Rolled & & & & \\
\hline ACM 4-1-2 & NBE 351 & $2 / 6 / 95$ & Roof & $\begin{array}{l}\text { Roofing } \\
\text { Material }\end{array}$ & M & $\pm 1000 \mathrm{ft} .{ }^{2}$ & NA & ND \\
\hline ACM 4-1-3 & & & & & & & & \\
\hline
\end{tabular}

- aSample designation is as follows: ACM 1-1-1=Asbestos containing material from Building 1, homogeneous material 1, and one sample taken, respectively.

bMiscellaneous.

cNot applicable.

d Not detected. 
Asbestos Sumır 'reet (continued)

\begin{tabular}{|c|c|c|c|c|c|c|c|c|}
\hline $\begin{array}{l}\text { Sample } \\
\text { Numbers }\end{array}$ & $\begin{array}{l}\text { Ticket Book } \\
\text { Number }\end{array}$ & Sample Date & $\begin{array}{l}\text { Building and } \\
\text { Room }\end{array}$ & $\begin{array}{l}\text { Homogeneous } \\
\text { Material } \\
\text { Description }\end{array}$ & $\begin{array}{l}\text { Material } \\
\text { Type }\end{array}$ & $\begin{array}{l}\text { Quantity } \\
\text { Lin/Sq. Ft. }\end{array}$ & $\begin{array}{l}\text { Asbestos } \\
\text { Fiber Type }\end{array}$ & $\begin{array}{c}\text { Asbestos } \\
\text { Content } \% \\
:\end{array}$ \\
\hline ACM 5-1-1 ${ }^{a}$ & \multirow{3}{*}{ NBE 357} & \multirow{3}{*}{$2 / 7 / 95$} & \multirow{3}{*}{$\begin{array}{l}\text { DP/DG } \\
\text { Room } \\
\text { Equipment } \\
\text { Insulation }\end{array}$} & \multirow{3}{*}{$\begin{array}{c}\text { Yellow Fibrous } \\
\text { Glass }\end{array}$} & \multirow{3}{*}{$M^{b}$} & \multirow{3}{*}{ $\pm 200 \mathrm{ft} .2$} & \multirow{3}{*}{$N A^{c}$} & \multirow{3}{*}{$\mathrm{ND}^{\mathrm{d}^{\prime}}$} \\
\hline & & & & & & & & \\
\hline & & & & & & & & \\
\hline ACM 5-2-1 & \multirow[b]{2}{*}{ NBE 358} & \multirow[b]{2}{*}{$2 / 7 / 95$} & \multirow{2}{*}{$\begin{array}{l}\text { Used Block } \\
\text { Storage Area }\end{array}$} & \multirow{2}{*}{$\begin{array}{c}\text { Brown Silica } \\
\text { Brick (furnace) }\end{array}$} & \multirow[b]{2}{*}{$\mathbf{M}$} & \multirow[b]{2}{*}{ $\pm 300 \mathrm{ft} \cdot{ }^{2}$} & \multirow[b]{2}{*}{ NA } & \multirow[b]{2}{*}{ ND } \\
\hline & & & & & & & & \\
\hline ACM 5-3-1 & \multirow{3}{*}{ NBE 359} & \multirow{3}{*}{$2 / 7 / 95$} & \multirow{3}{*}{$\begin{array}{l}\text { Used Block } \\
\text { Storage Area }\end{array}$} & \multirow{3}{*}{$\begin{array}{c}\text { Tan Silica } \\
\text { Brick and } \\
\text { Mortar } \\
\text { (furnace) }\end{array}$} & \multirow{3}{*}{$\mathbf{M}$} & \multirow{3}{*}{ $\pm 2000 \mathrm{ft}^{2}$} & \multirow{3}{*}{ NA } & \multirow{3}{*}{ ND } \\
\hline ACM 5-3-2 & & & & & & & & \\
\hline ACM 5-3-3 & & & & & & & & \\
\hline ACM 6-1-1 & \multirow[b]{2}{*}{ NBE 360} & \multirow[b]{2}{*}{$2 / 7 / 95$} & \multirow{2}{*}{$\begin{array}{l}\text { Building } 6 \\
\text { Window } \\
\text { Caulking }\end{array}$} & \multirow{2}{*}{$\begin{array}{l}\text { Window Caulk } \\
\text { Calcium } \\
\text { Carbonate }\end{array}$} & \multirow[b]{2}{*}{$\mathbf{M}$} & \multirow[b]{2}{*}{ $\pm 500 \mathrm{ft} .^{2}$} & \multirow[b]{2}{*}{ Chrysotile } & \multirow[b]{2}{*}{$1-3$} \\
\hline & & & & & & & & \\
\hline ACM 6-2-1 & \multirow{3}{*}{ NBE 361} & \multirow{3}{*}{$2 / 7 / 95$} & \multirow{3}{*}{$\begin{array}{l}\text { Lindberg } \\
\text { Furnace } \\
\text { Front Door } \\
\text { Gasket }\end{array}$} & \multirow{3}{*}{$\begin{array}{l}\text { Grey Fibrous } \\
\text { Gasket }\end{array}$} & & & & \\
\hline & & & & & $\mathbf{M}$ & $<5$ lin. $\mathrm{ft}$. & Chrysotile & $90-95$ \\
\hline & & & & & & & & \\
\hline ACM 6-3-1 & & & Lindberg & Brown and & & & & \\
\hline & NBE 363 & $2 / 7 / 95$ & $\begin{array}{l}\text { Furnace } \\
\text { Brick }\end{array}$ & $\begin{array}{c}\text { Tan Silica } \\
\text { Brick (furnace) }\end{array}$ & $\mathbf{M}$ & $<20 \mathrm{ft}^{2}{ }^{2}$ & NA & ND \\
\hline & & & & & & & & \\
\hline
\end{tabular}

aSample designation is as follows: ACM 1-1-1=Asbestos containing material from Building 1, homogeneous material 1, and one sample
. taken, respectively.

bMiscellaneous.

cNot applicable.

dNot detected. 
Asbestos Summary Sheet (continued)

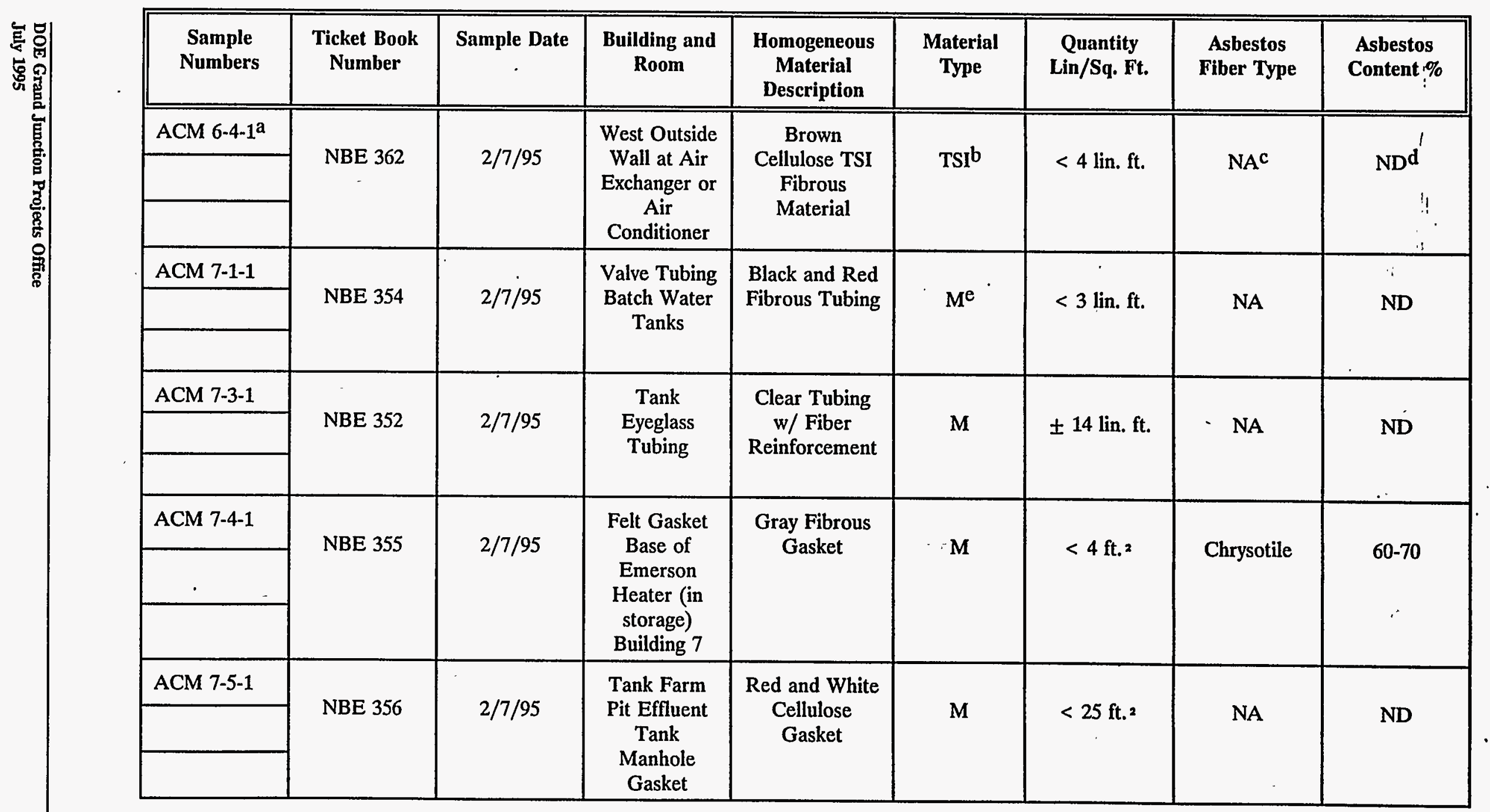

aSample designation is as follows: ACM 1-1-1=Asbestos containing material from Building 1 ; homogeneous material 1, and one sample taken, respectively.

bThermal system insulation.

cNot applicable.

dNot detected.

eMiscellaneous. 


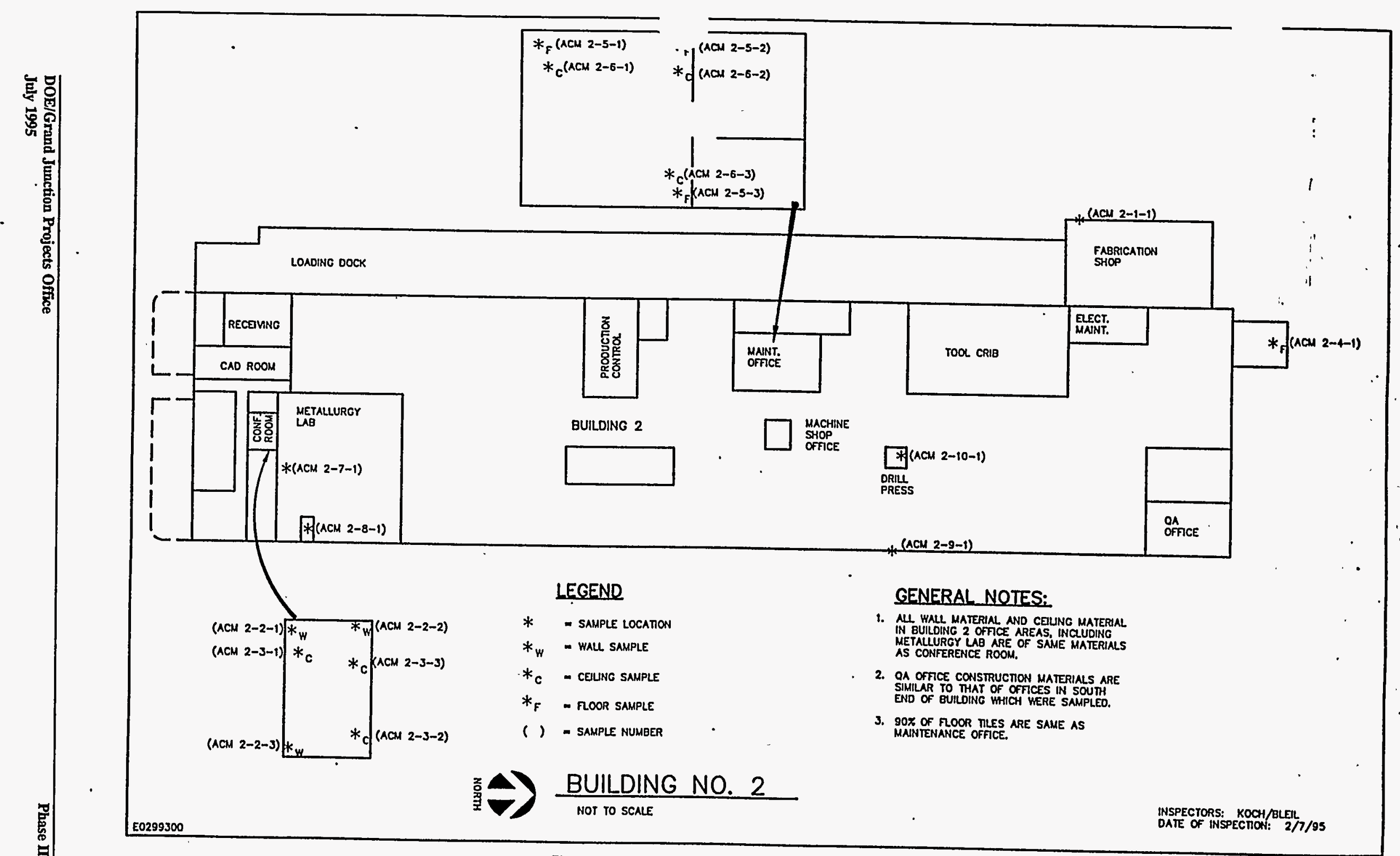

Figure A-1. Ashestos Sampling Locations in Building 2 


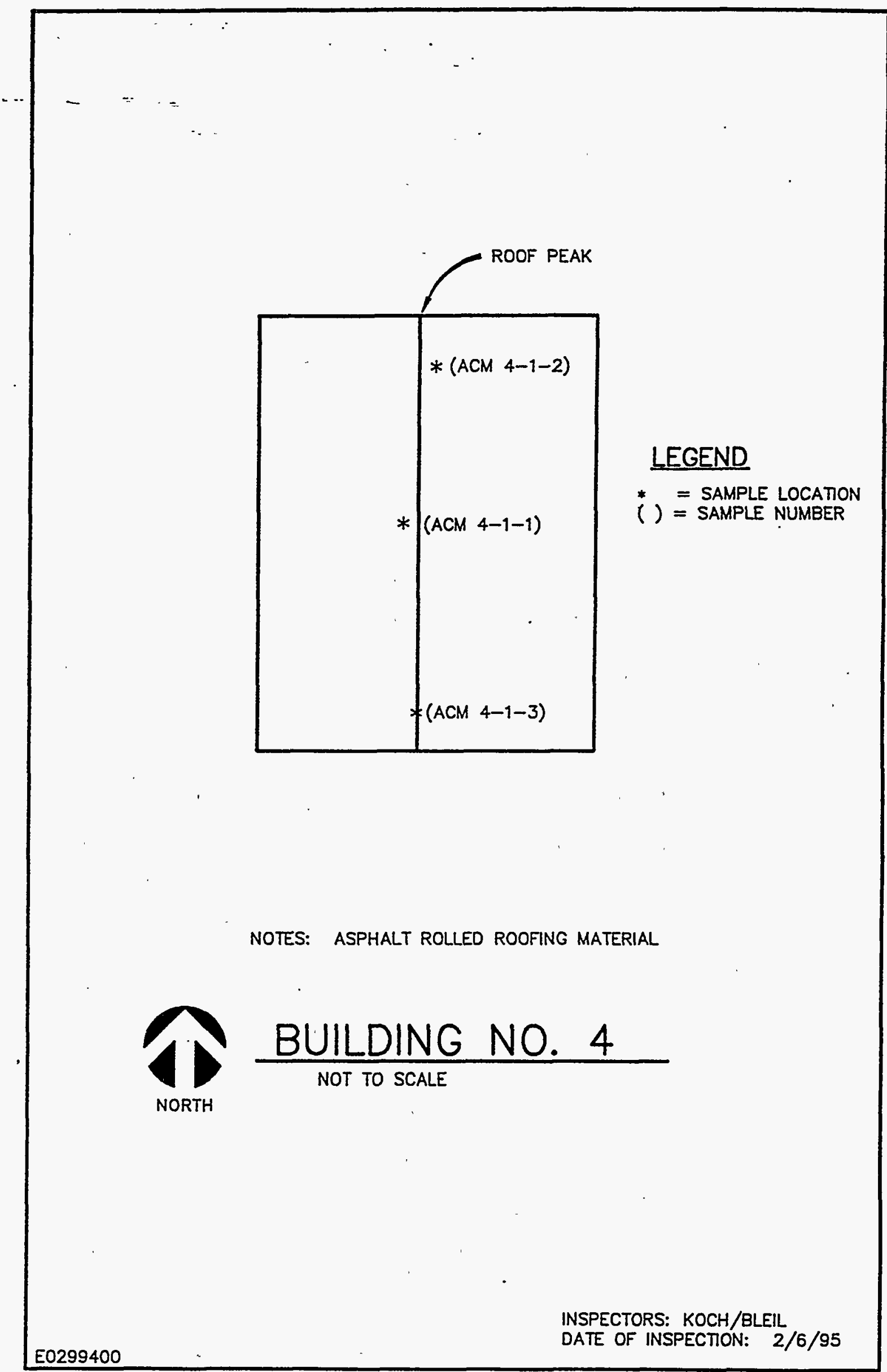

Figure A-2. Asbestos Sampling Locations in Building 4 


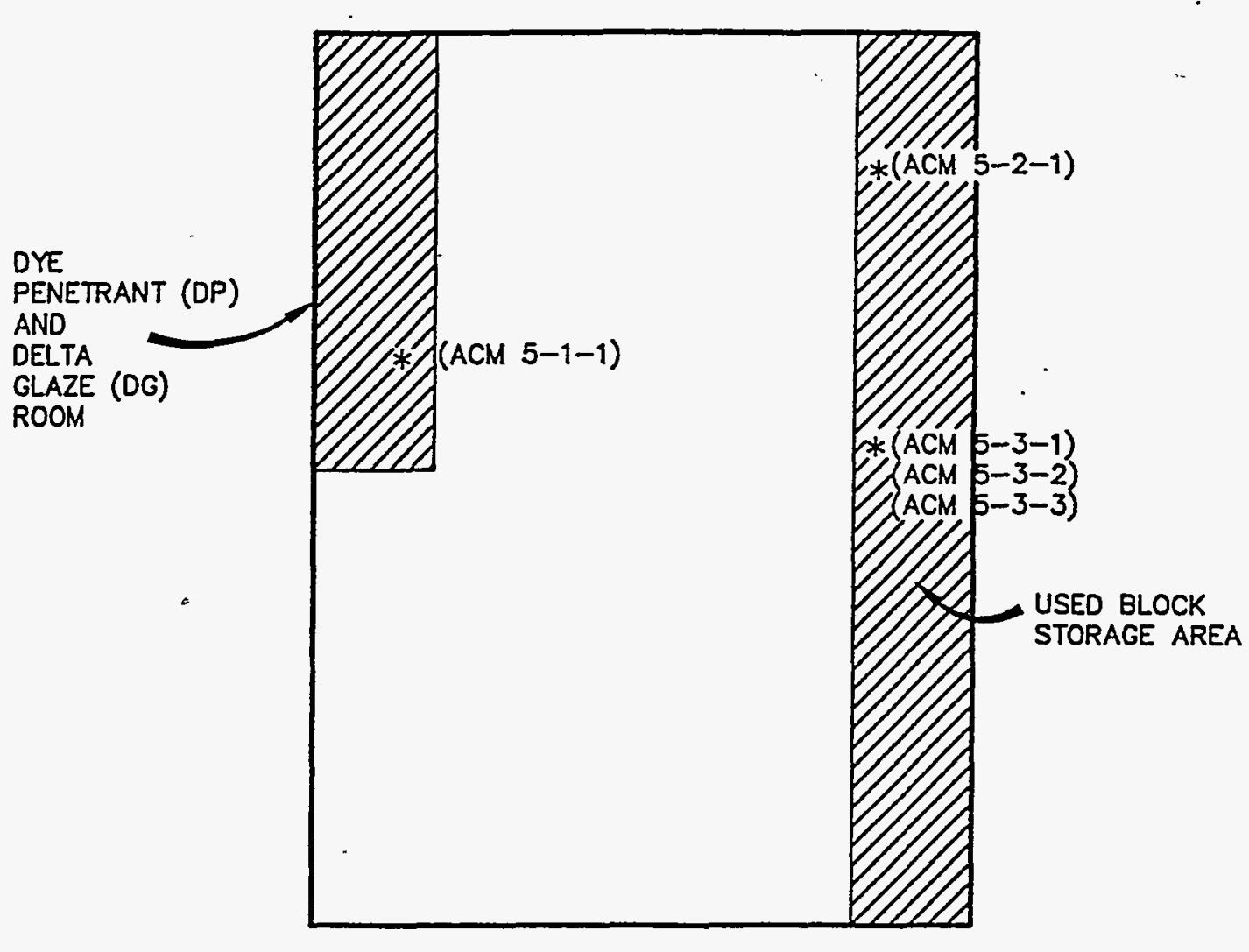

LEGEND

* = SAMPLE LOCATION

() = SAMPLE NUMBER

\section{$7_{\text {NoRTH }} \frac{\text { BUILDING NO. } 5}{\text { NOT TO SCALE }}$}

INSPECTORS: KOCH/BLEIL

DATE OF INSPECTON: $2 / 7 / 95$

Figure A-3. Asbestos Sampling Locations in Building 5 
FOREE SHOP

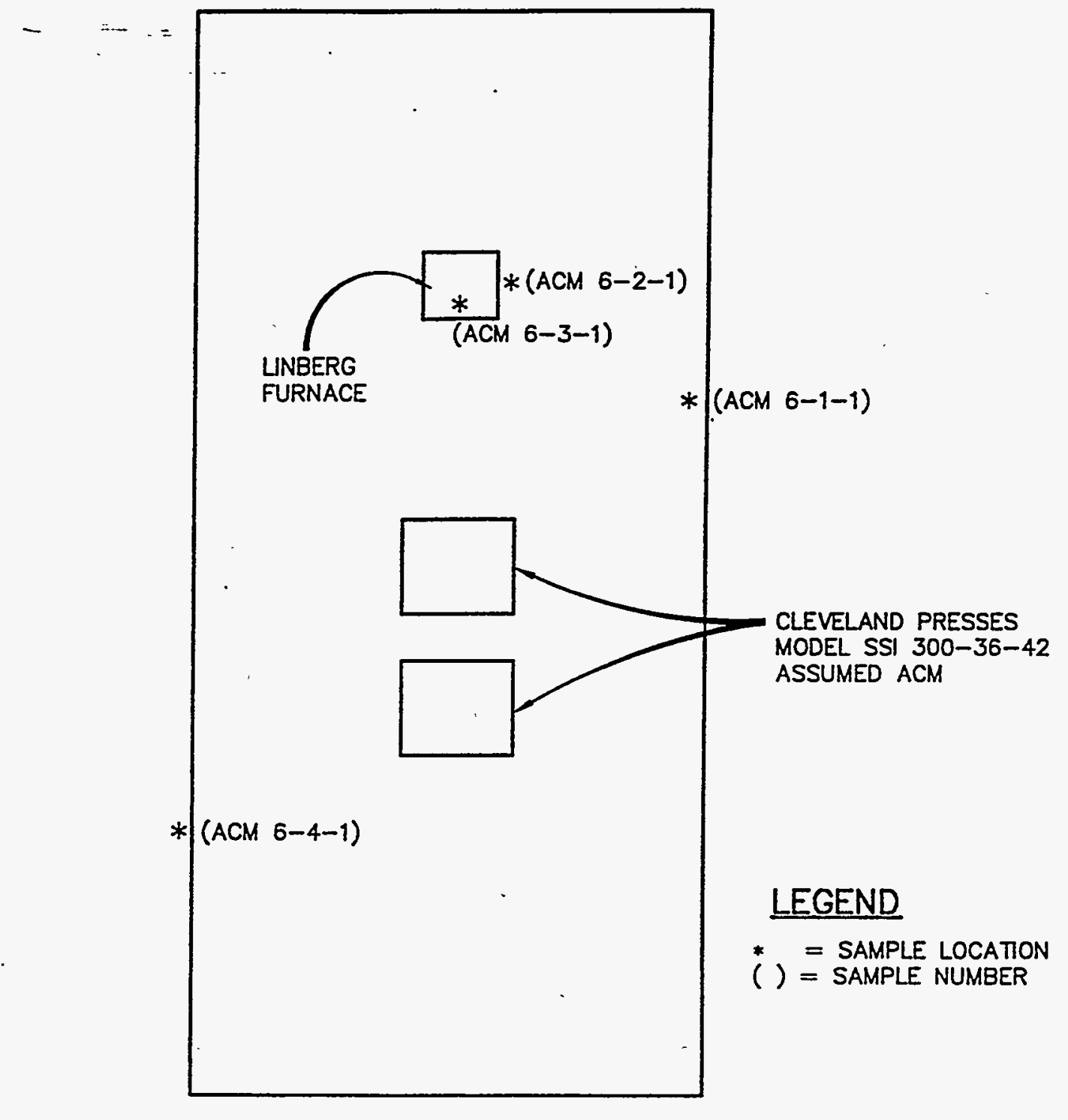

*NOTES: THE BRAKES ON THE CLEVELAND PRESSES ARE

ASSUMED TO BE ACM ON THE BASIS OF AGE AND MAKE.

,

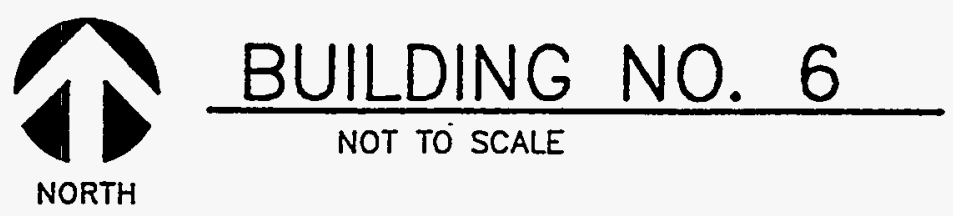

INSPECTORS: $\mathrm{KOCH} / \mathrm{BLEIL}$

DATE OF INSPECTION: $2 / 7 / 95$

Figure A-4. Asbestos Sampling Locations in Building 6 


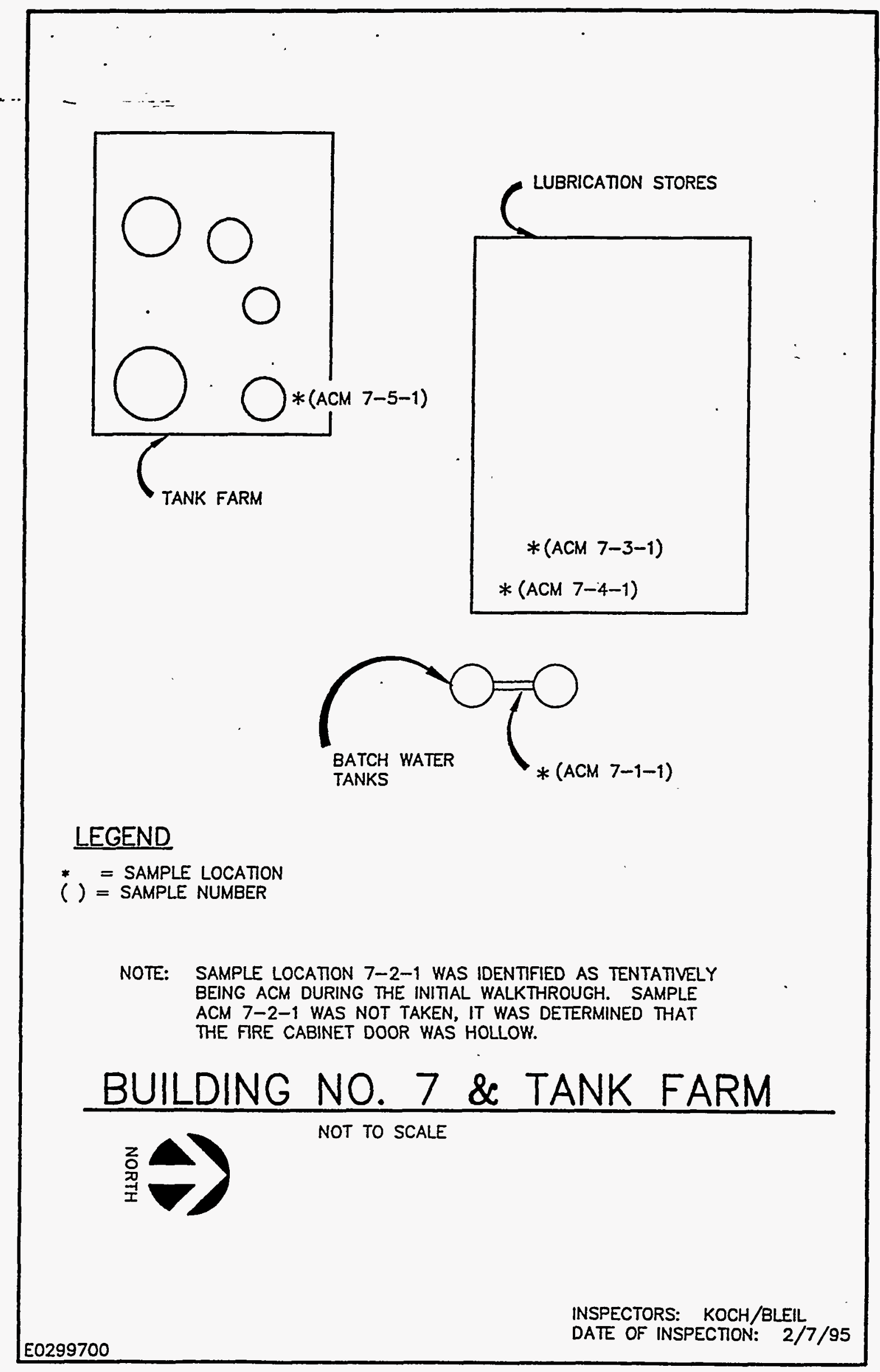

Figure A-5. Asbestos Sampling Locations in Building 7 and Tank Farm 


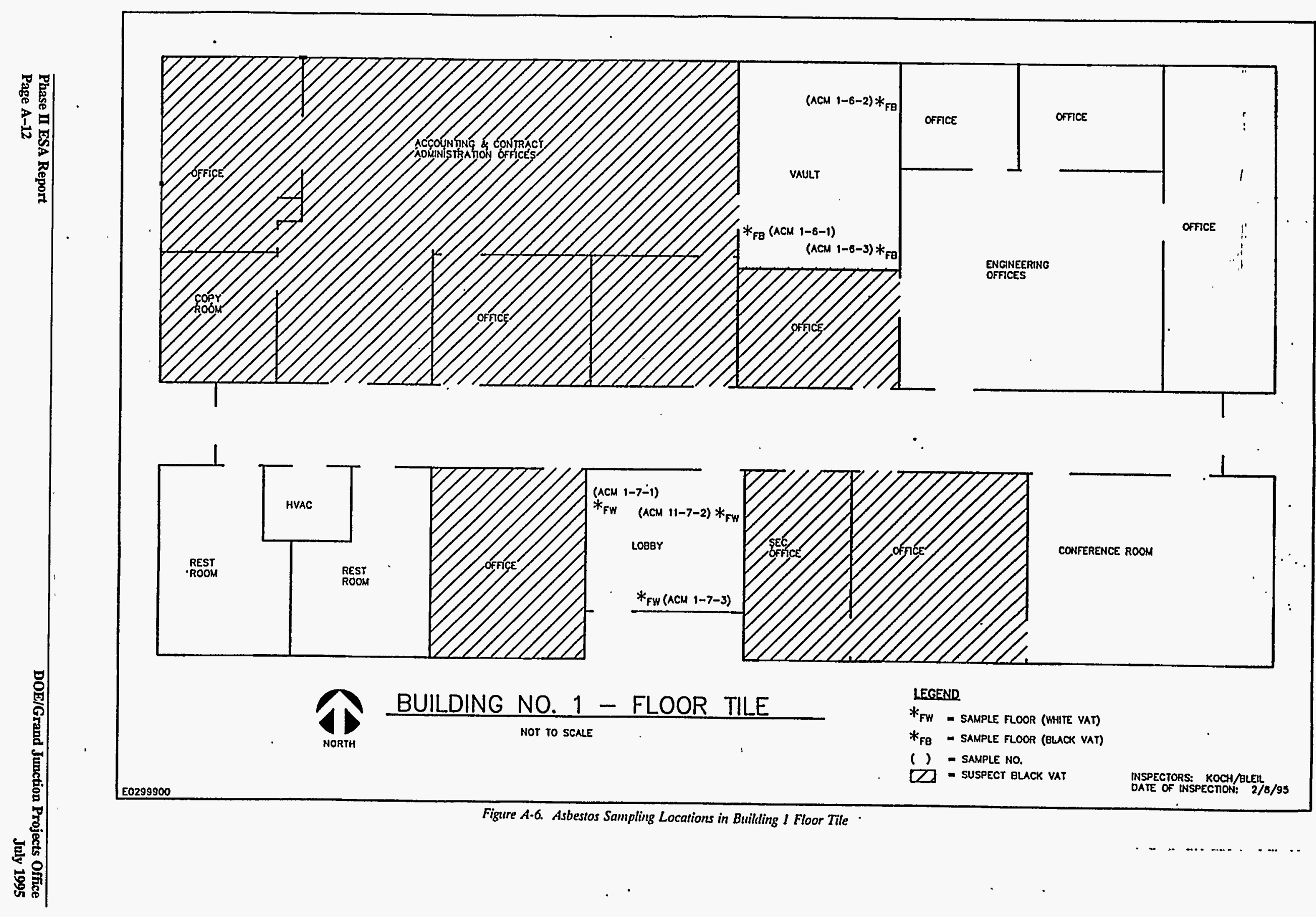




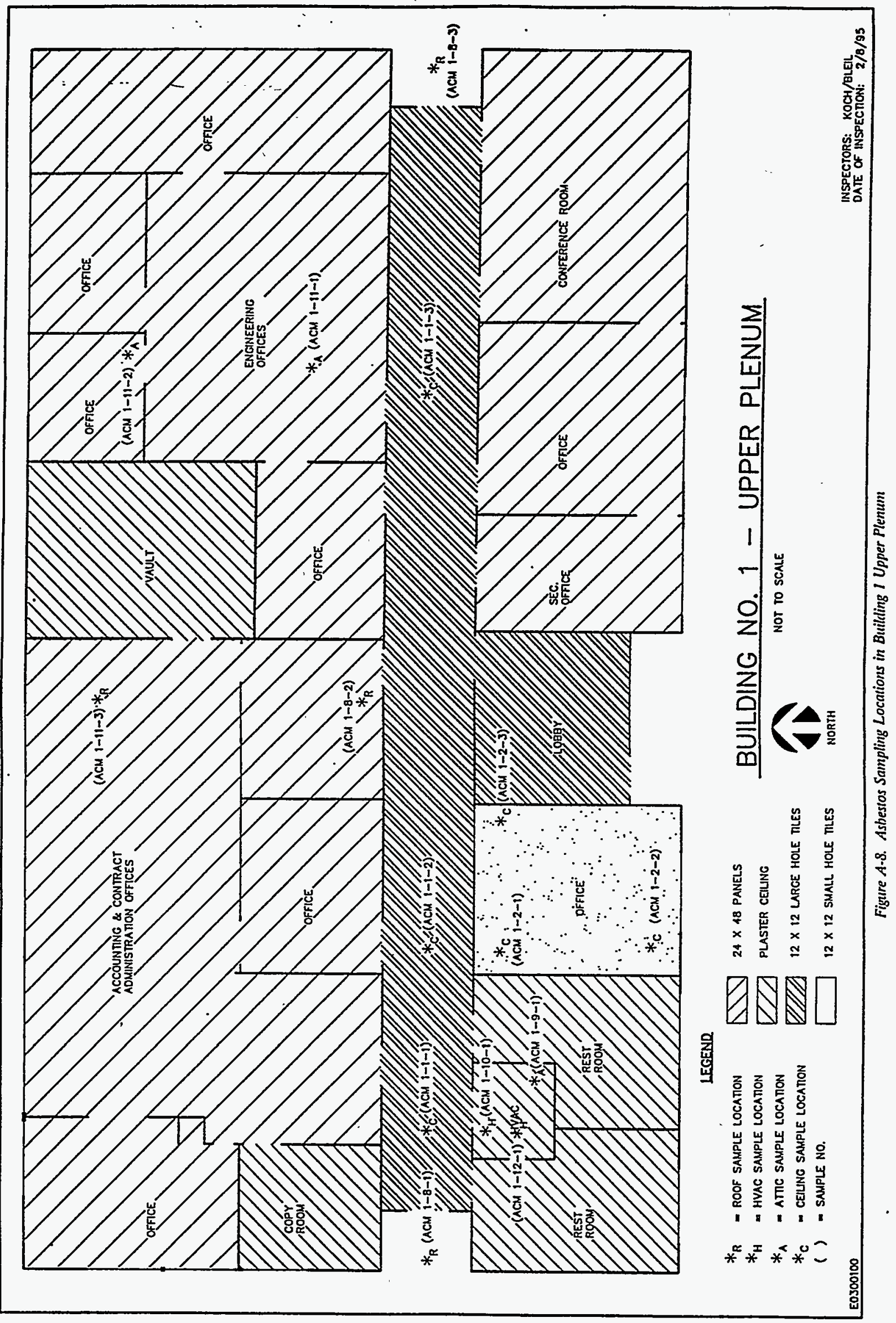




\section{Appendix B}

\section{Borehole Lithologic Logs}


Facility: Orarared DOE FAccotrtito: OxNARD, CA

Boring/Woll No: $M W-O l$ Locotion (rt.) N:

Ground Elev. (It. AMSL): __ Auger/Bit Sizo (in.): $81 / 4$

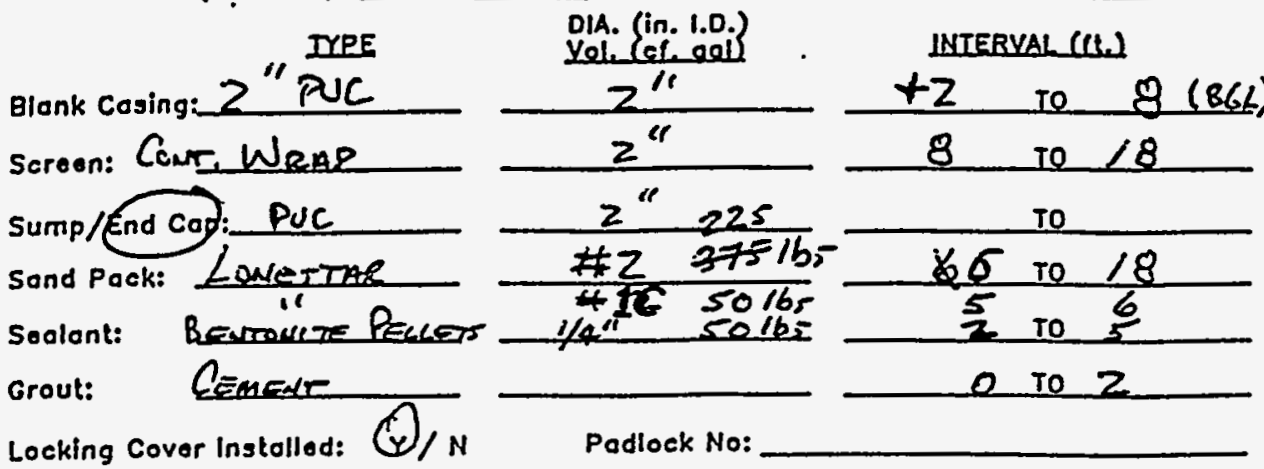

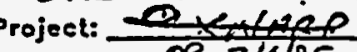
जि: $2 / 4 / 95$

Hole Depth (lt): 18 No. of Complotions: stick-Up Ht. (It.): Z

Slot Sizo: $\quad .0<0$

Localion Sketch:

Drilling Mothad: Aucare

Sompling Method: SPLeT SpooN

Dato Drilled: $\operatorname{fos} 31,95$

Dale Dovelopod:

Fluid Level/Date:

Somplers:_PRice Remorks:

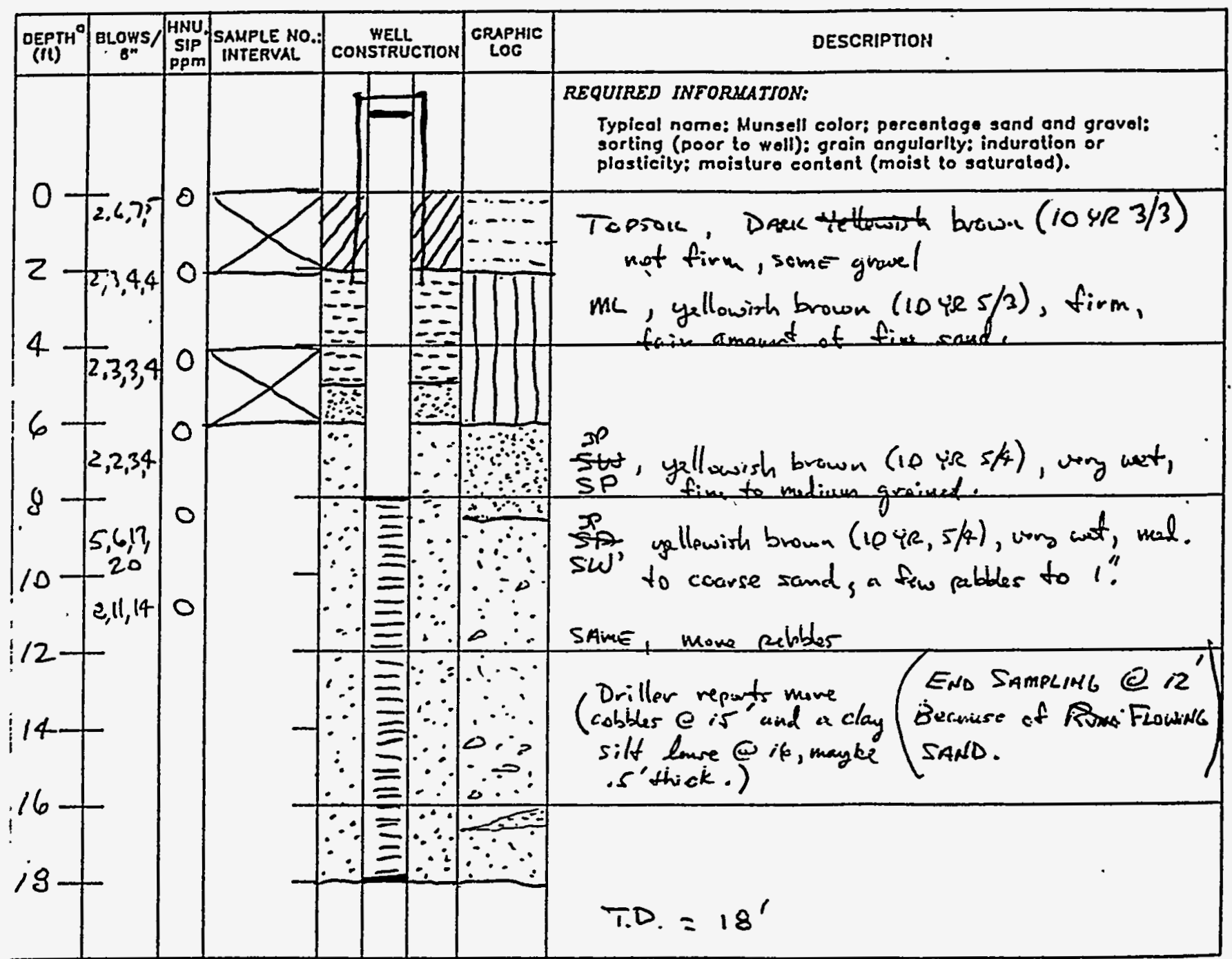

all Dopths From Ground Surfaco. coupleted ay: Jeff Relce VERIFIEO BY: 


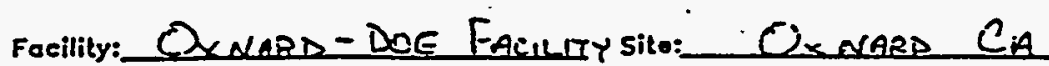

Boring/Wall No: MWU-OZ Ground Elev. (It. AMSL):

IYPE

Blonk Cosing: $2^{\prime \prime} P J C$

screon: Cent, Wrep Púc

Sump/End Cap: PUC

sond Pack: Lwernar ik

Soalont:

isivestar itas

Hentenite Pिचाइ

Grout: - L LenerT

Locking Cover Installod: (y) $N$

Drilling Mothod: Aucer

Dato Drilled: $\tan 31,95$

Somplers: Price
Lacotion (ft.) $\mathrm{N}$ : Auger/Bit Size (in.): $g$ \% $/ 4$ DIA. (in. 1.0.).

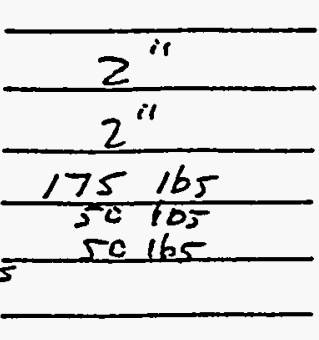

Padlock No:
Dote. Developed:

Remarks:
INTERYAL (U)

\begin{tabular}{rrr}
+2 ro & 8 \\
\hline 8 & ro 18 \\
to & \\
\hline 6 & т0 18 \\
\hline 5 & 10 & 6 \\
\hline 2 & 10 & 5
\end{tabular}

Project: Jite CHetRetTereatre E:

Holo Deplh (ft): $\angle 8$

No. of Complotions: stick-Up Ht. (It.): $\frac{Z}{\pi i}$ Slot Sizo: $.010^{\prime \prime}$

Location Skolch:

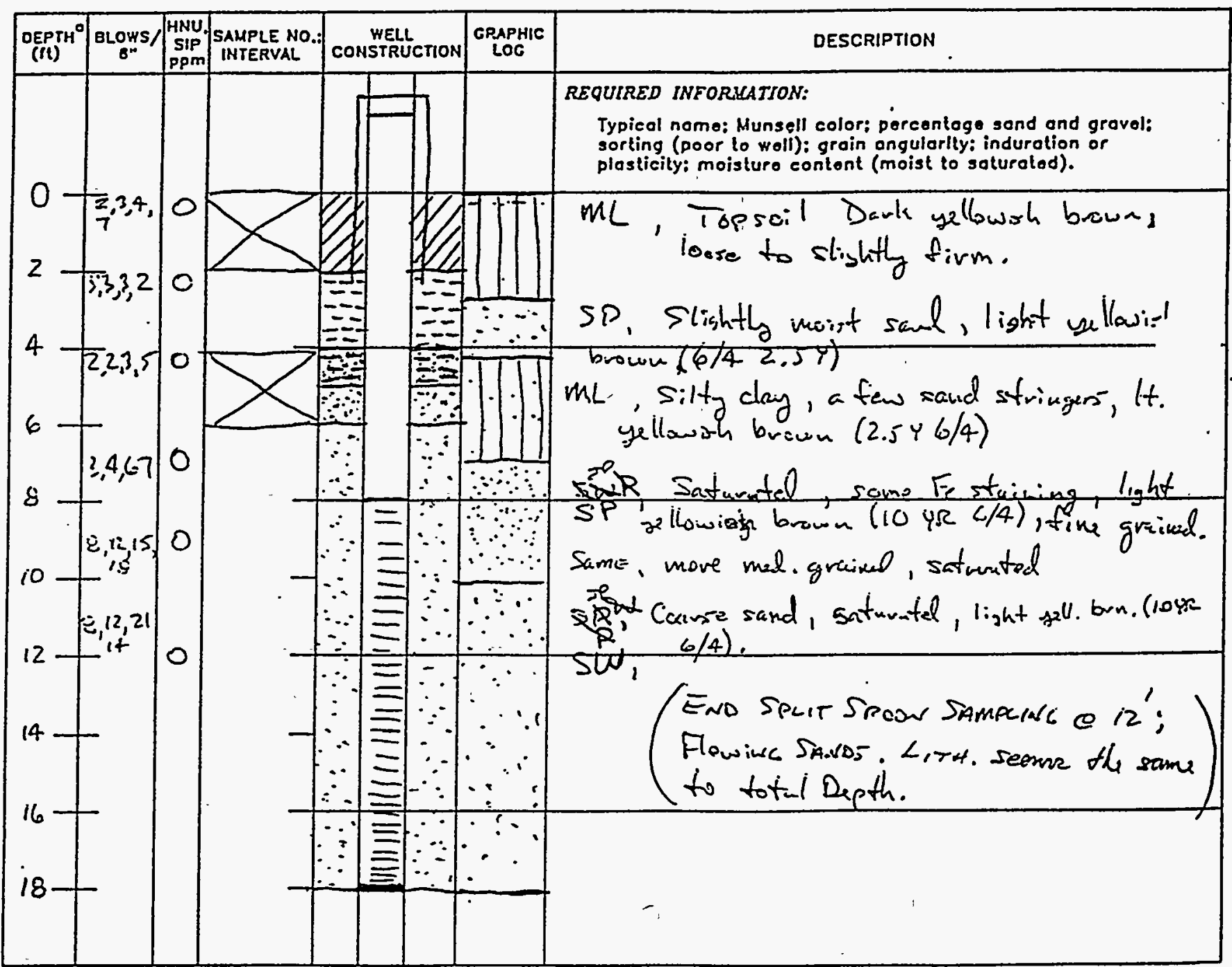

a All Depths From Ground Surfoco.

completeo by: JEFF Rice

Sompling Method: Spur Seane

Fluid Lovel/Dete:

$\angle$ 
Focility: OxNARD Boring/Hall No: ST Q2 MW-O3 sito: OxNaro DQE Aackitu project: STE Ciär. Ground Elev. (It. AMSL): Locotion (ft.) N:

IYPE

Blank Casing: PUC

screen: Cont. Wrap PJC

sump/End Cop PUC

Sond Pook: Lowestar $\$ 2$

Sealont: Bentroutre Peuers

Grout:

Locking Covor Installed: $\quad Y . / N$

Drilling Mothod: Auges

Dato Drillad: 2/1/85

somplers: PrLCE
Auger/Bit Sizo (in.): $91 / 4$ DIA. (in. 1.D.) yol.\{et.ooi\} $z$ il

\begin{tabular}{|c|}
\hline $2^{\prime \prime}$ \\
\hline $2^{\prime \prime}$ \\
\hline $\begin{array}{l}200165 \\
50.165\end{array}$ \\
\hline $50 \mathrm{lbs}$ \\
\hline
\end{tabular}

Padlock No:
INTERYAL (1). $* .25$ 10 8 8 T 18

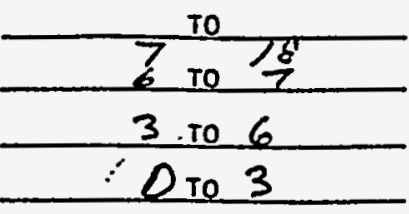
E: Hole Depth (ft): $\angle \&(B G L)$

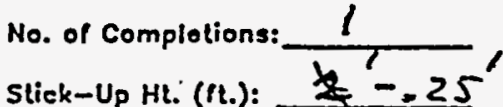
Sloi Sizo: .010 Location Sketch:

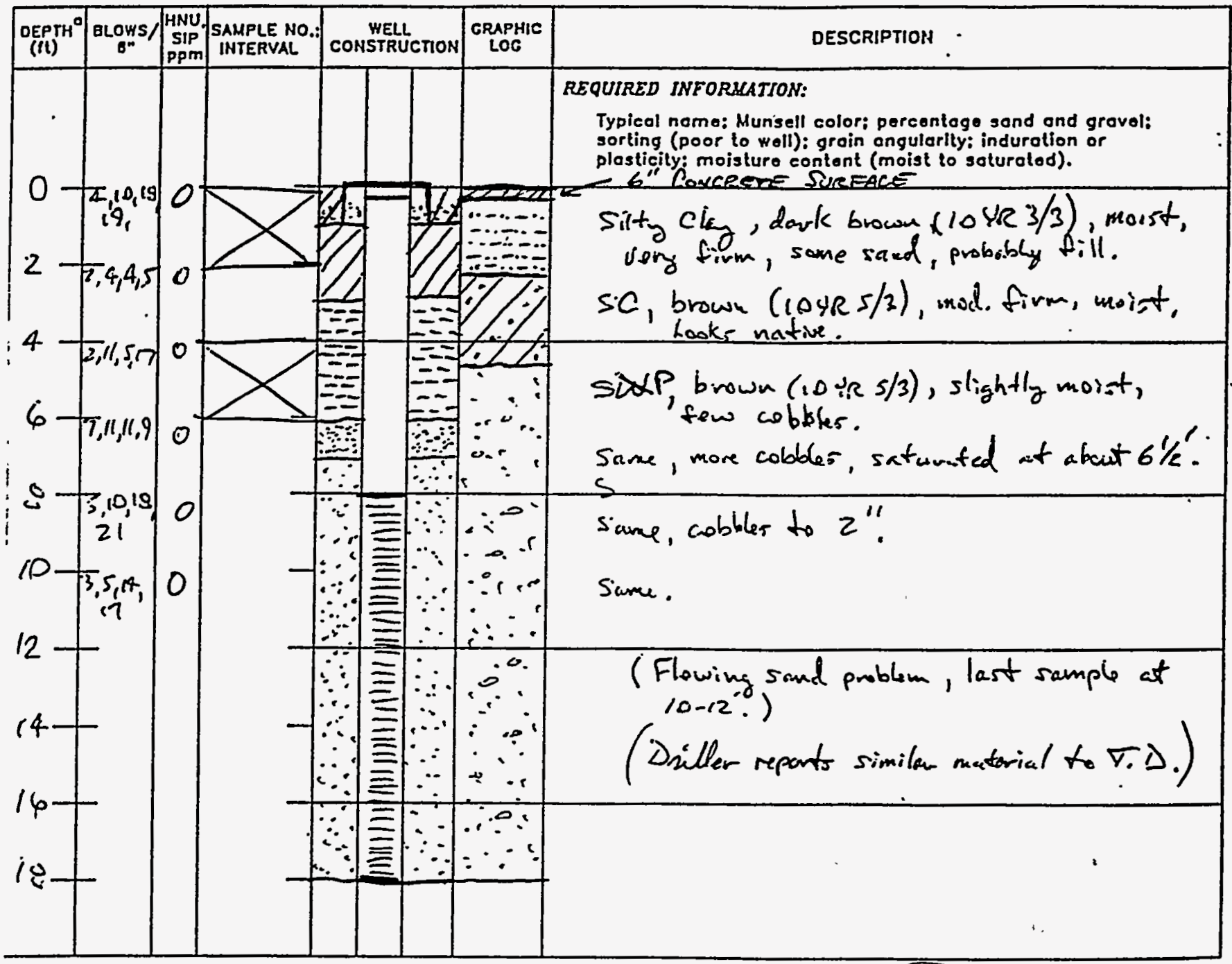

a All Dopths From Ground Surlaco. completed ay: Jefe Price

Sompling Hethod: SpLE SPeod Date Developed: Fluid Level/Oate:

Romarks: 
P.o. Box 11000

Grand Junollon. Colörado 81502

Focility: Oxacare Sito:Oenctics 20 Fe Faccol

Eoring/Holl No: $m w-04$

Augar/Bit Sizo (in.): Location (ft.) N:

Ground Elev. (fl. AMSL):

\section{IYPE}

Blank Casing: PUC

screon: Contiumer Weap Puc

sump/End cop Puc

Sond Pock: Lonentariz

Grout: Cement

Locking Cover Installed: $Y / N$

Drilling Mothod: Auget

Dato Drillad: $2 / 2 / 95$

somplers: RlCÉ
INTERYAL (ft.)

Dla. (in. 1.0.)
Vol. (ci._eail)

$2^{\prime \prime}$

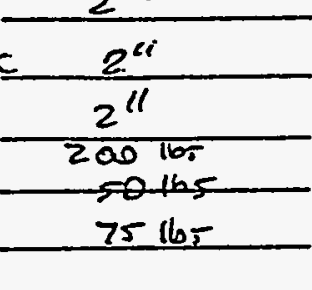

Podlock No: .25 To 8
Date Dovelopad:
SITE CAMR

$\begin{array}{r}8 \text { 10 } / 8 \\ \hline \text { 10 } \\ \hline 7 \text { 10 } 7 \\ \hline 3 \text { т0 } 6 \\ \hline 0 \text { 10 } 3 \\ \hline\end{array}$

E: $2 / 4 / 95$

Holo Depth ( $t$ t): $1 \mathrm{~S}$

No. of Complotions:

Stick-Up Ht. (It.): $=. \dot{2}$

Location Skotch:

Sompling Method: SPLT SPacN

Fluid Levol/Dote:

Slot Sizs: $.010^{11}$

\begin{tabular}{|c|c|c|c|c|c|c|}
\hline $\begin{array}{l}\text { OEPrH } \\
\text { (II) }\end{array}$ & BLows/ & $\begin{array}{l}H N U, \\
\text { SIP } \\
P P M\end{array}$ & $\begin{array}{l}\text { SAMPLE NO.: } \\
\text { INTERVAL }\end{array}$ & $\begin{array}{l}\text { WELL } \\
\text { CONSTRUCTION }\end{array}$ & $\begin{array}{l}\text { GRAPHIC } \\
\text { LOG }\end{array}$ & DESCRIPTION. \\
\hline & & & & & & REQUIRED INFORKCATION: \\
\hline
\end{tabular}

Typical name: Munsell color: porcenlage sond and graval;

sorting (poor to wall): grain angularlty: induration or

$0-13,4,5,5$

\section{2}

$2,3,3,3$

0

4

$\frac{1}{2,3,4,0}$

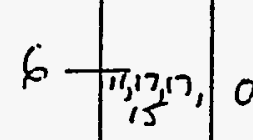

0

@

$\mid \frac{8,12,19}{3 c} 0$

io $-17,14, \pi 0$

23

$1279,14,170$

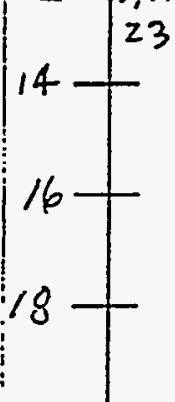

a All Dopths From Ground Surfaco. COMPLETEO BY:

Tere Ruce
(Stop split spoont sampliny, flaving sand troubl.)

(Dille reparte similas restruil to T.D.) 
Focility: OXNARD

Boring/Well No: MW- 05

Ground Elev. (ft. AMSL):

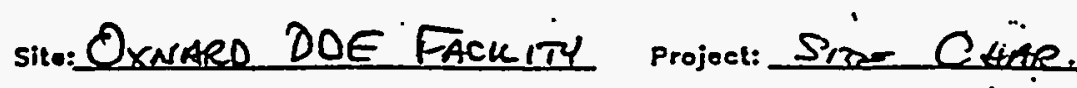

IYPE

Blank Casing: PUC

Sereon: Conr: WraP PUC

Sump/End cad: PUC

Sand Paok: LoNeJTAR \#2

Grout:

CEMEart

Locking Cover Installed: $Q / N$

Drilling Mothod: AUGER

Dato Drilled: $2 / i / 95$

Somplers: Pricé

Remorks:

- All Dopths From Ground Surfoce.

COMPLETED BY: TEFf Rlce

Podlock No:

E:

Hole Depth ( $(t): 19$

No. of Complotions:

stick-Up Ht. (ft.): $Z$

Slot Sizo: . .

Locotion Sketch:

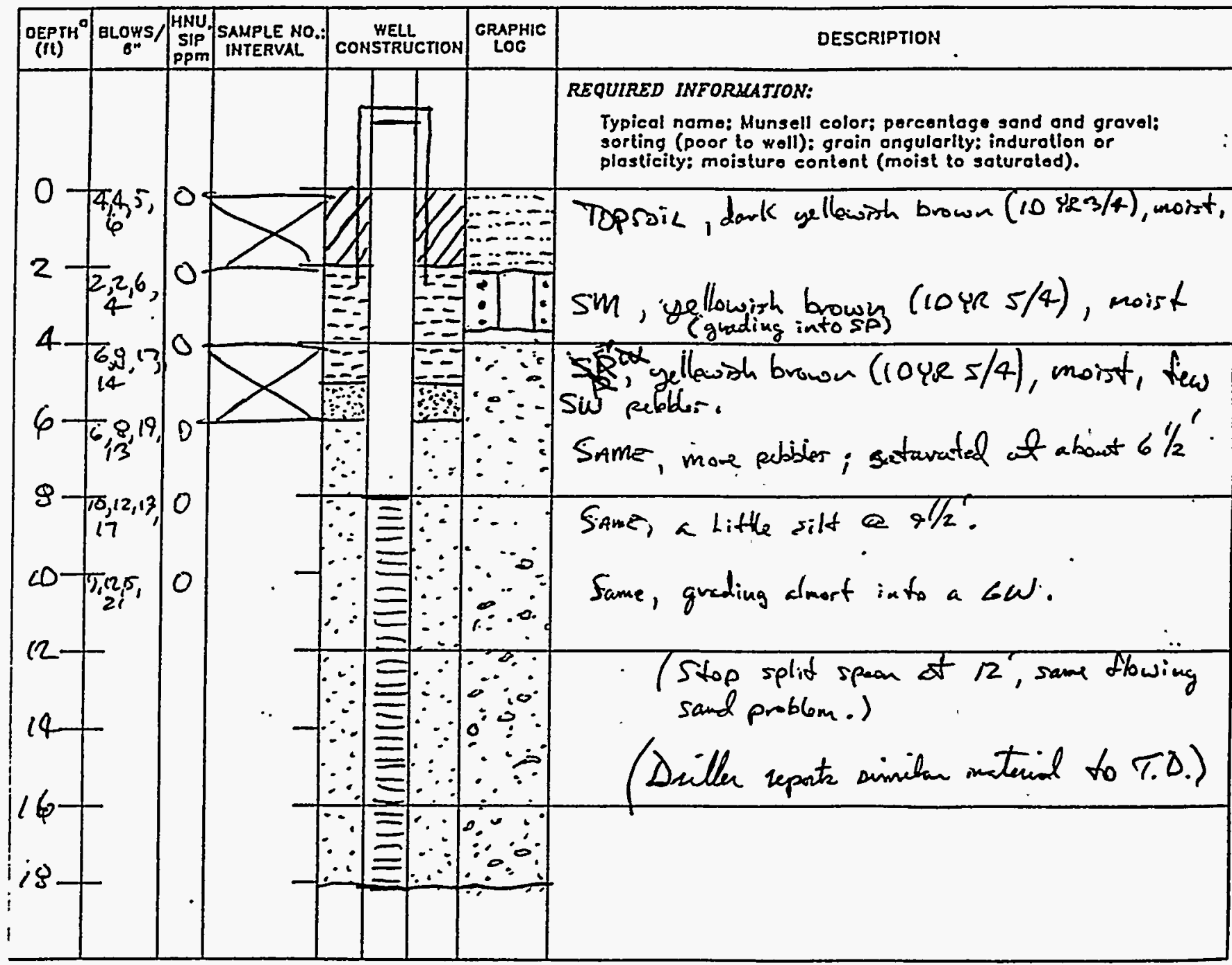

Sompling Method: Secit Seoon

Date Dovelopod: ___ Fluid Lovel/Oate:

INTERVAL (UL)

8 io 18

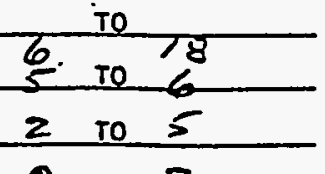

0 To 2 
Facility: Oxncare

\section{,}

-Boring/Hall No: $M W-06$

Ground ETEV. (It: AMSL):

Blank Casing. $z$ "PYPE

sereon: Cont. Wenp PuC

Sump End CaP PUC

Sond Pack LoNestar \#Z

Sond Pack: Lonestitic

Soalont: BENTONITE PELCETS

Grout: CEMENT

Locking Cover Ingtalled: $Y / N$

Drilling Method: AUGER

Dote Drilled: $2 / 2 / 95$

somplers: Pelce
Podlock No:

Sito:OX DARD DDE FAclenty

Locolion (ft.) M:

Auger/Bit Sizo (in.): $\quad 81 / 4 "$

DIA. (in. 1.0.)

Volect eall

$2^{4}$

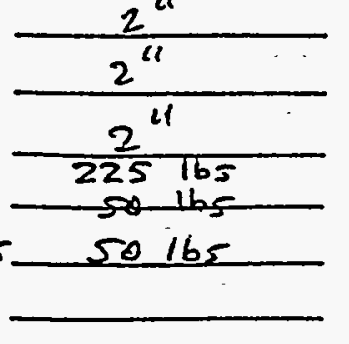

Project: SiTE CHRB

E:

Holo Deplh (tt): $18^{\prime}(B C L)$

No. of Complotions:

stick-Up Ht. (it.): -

Slot Sizs: . . $010^{\prime \prime}$

Locotion Skalch:

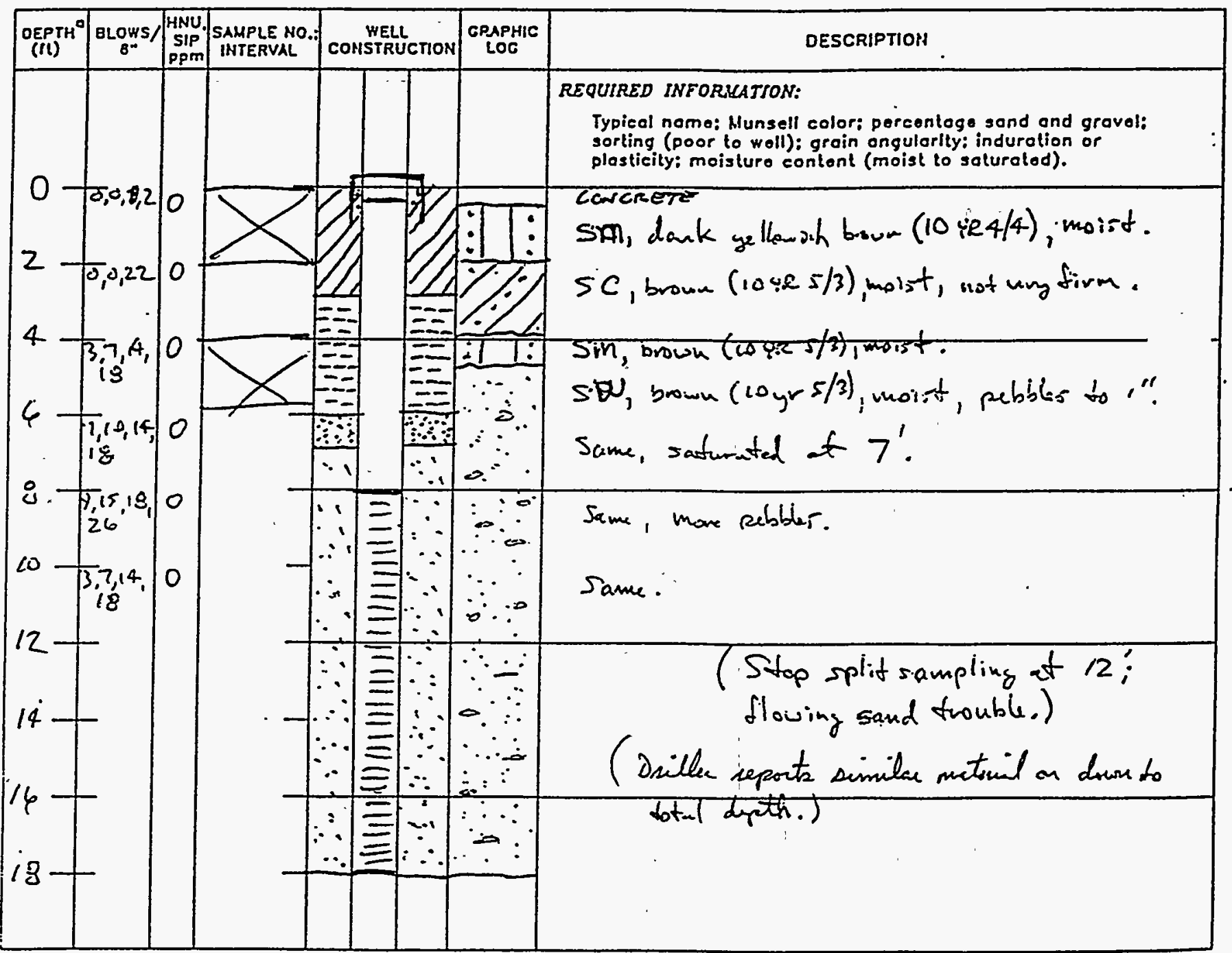

a All Depths From Ground Surfaco.

colifleteo ay: Teff Price

VERIFIEO BY:

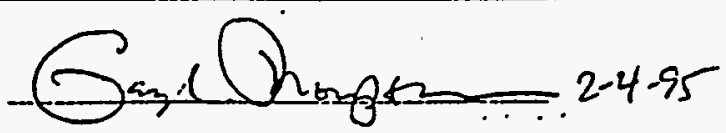


Facility: OxNaes

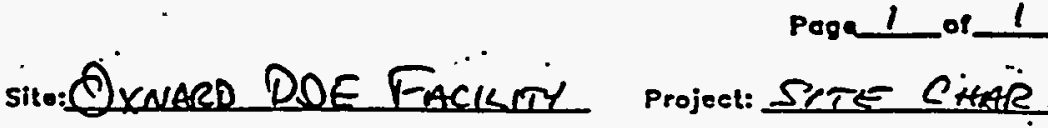

Boring/Hell No: Mul-o 7 Loeation (ft.) N:

Ground Elev. (It. AMSL): Auger/Bit Sizo (in.): $81 / 4^{\prime \prime}$

\section{IYPE}

Blank Cosing: DUC

screon: Court. WRAP RUC

Sump/End Cop: PUC

Sond Pock: $\frac{\text { Lovestar \#2 }}{\text { LOWESTAR IC }}$

Soolont:

Grout:

Boutonite Bilet 1/4

Cement

Loeking Cover installed: $\quad Y / N$

Drilling Mothod: Auger

Dato Drilled: $2 / 1 / 95$

Somplers: Price

\section{INTERYAL (IT)}

+2 To 8 (BLL)

8 io is

$2^{\prime \prime}$

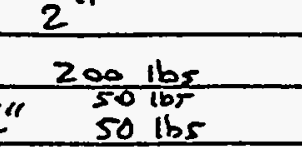

50 lbs

Padlock No:

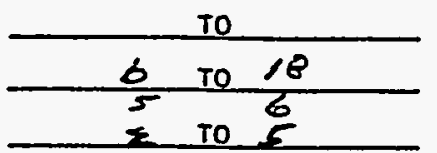

0 To 2
Hole Depth (It): No. of Complotions:

18 stick-Up Ht. (fl.): $2^{\prime}$

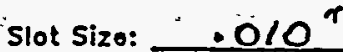
Location Skolch:

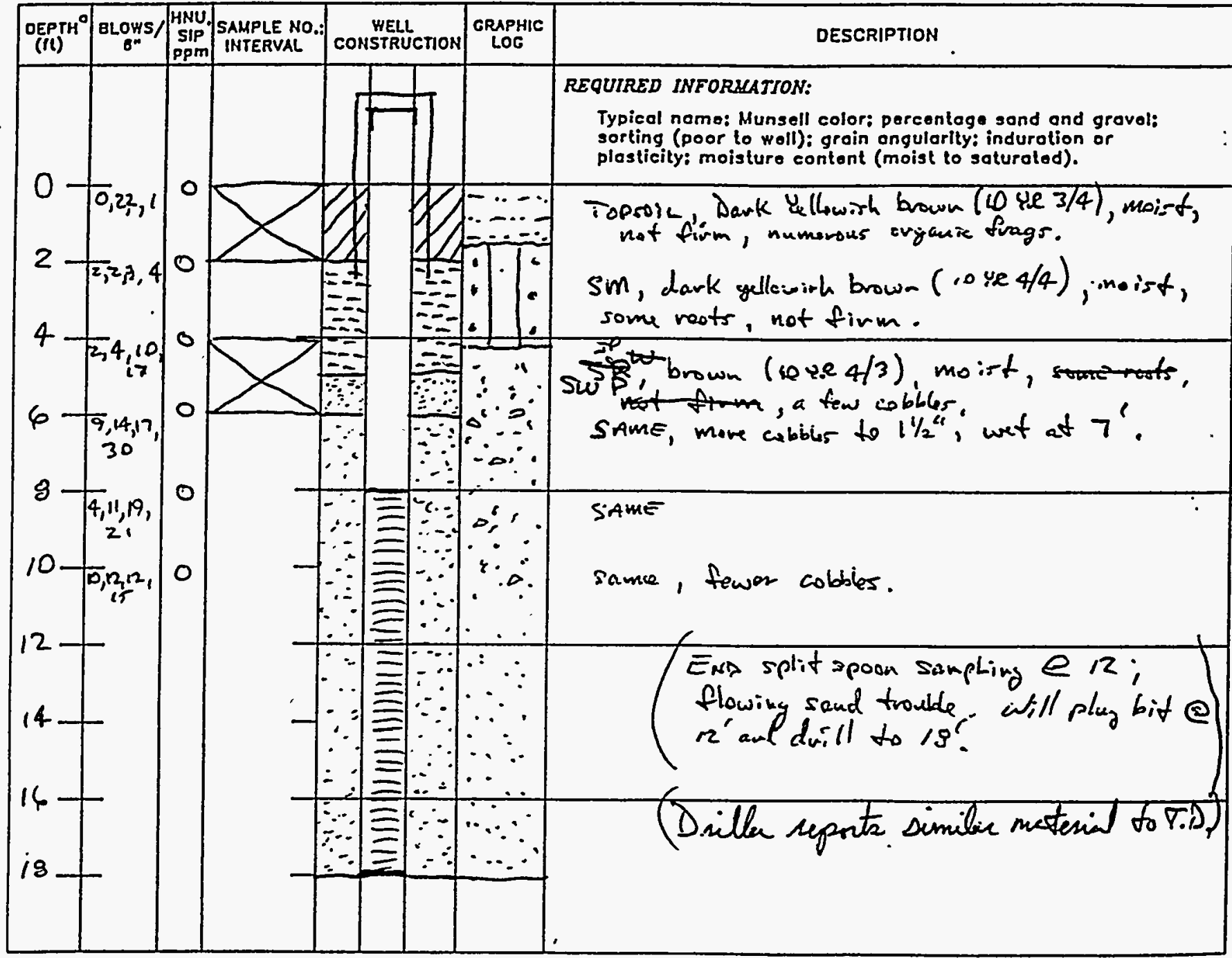

a All Dopths From Ground Surfaco. completeo by: Jeff Rlce Sompling Method: SPLIT SPEaN. Dale Developod: Fluid Level/Date: Remarks:

Typical nome: Munsell color; percentage sand and graval sorting (poor to woll): grain ongularlty: indurotion or not firm, numerous eviganie firags.

Sin, dark yellewirh brown ( 10 ye 4/4), ino ist, some roots, not firm.

brown (se y.e 4/3) mo:-t, sments, fot a fin is ables. same, move cabibis to $11 / 2^{\prime \prime}$, wet af 7 .

SAME

same, fewor cobbles.

\section{Ents split apoon sampling $€ 12$;} flowikg soud troukle, ifill pluy bit e Divile ) VERIFIEO BY:

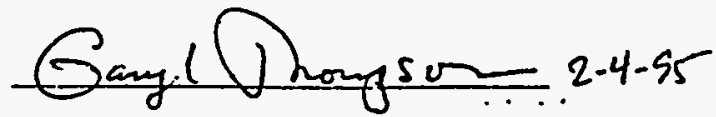


Facility: OXNARP Sito:OEnero DOE FAcicitiY Project: SITE CHAR.

Boring/Hall No: MW - 08

Location (ft.) N:

Ground Elev. (ft. AMSL):

IYPE

glonk Cosing: PUC

Screen: Cont writp PUC

Sumpend Cod: PUC

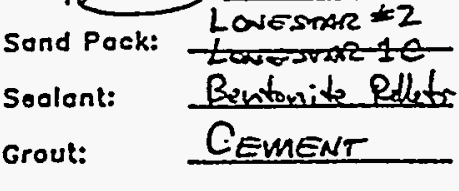

Locking Cover Instolled: $Y / M$

Drilling Mothod: AUGER

Dato Drillad: $2 / 2 / 95$

somplers: Price
Auger/ait Sizo (in.): $\& 1 / 4^{\mu}$

OIA. (in. 1.0.).

Yol. (ch oeil

$2^{\prime \prime}$

$\frac{2^{\prime \prime}}{2^{\prime \prime}}$

225165

solb-

75 lbs

Podlock No:
Dote Doveloped:

Remorks:
E:

Holo Depth (Pt): $1 \xi^{\prime}$ (BCi)

No. of Completions:

stick-Up Ht. (ft.): Z

Slot Size: .010"

Locotion Skoteh:

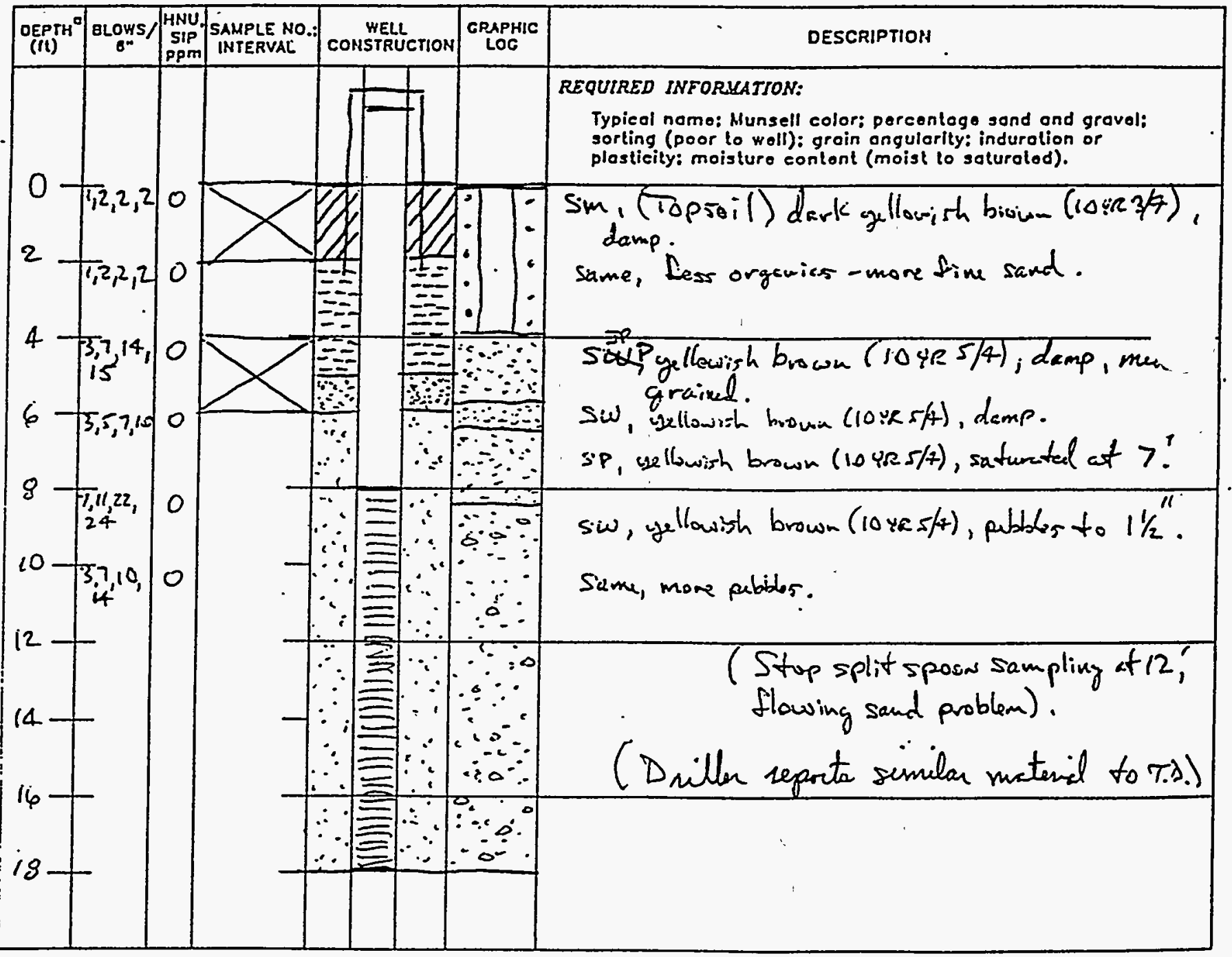

a All Dopths From Ground Surfaco.

completeo ay: JefF Price

Sampling Method: SpLIT SAleN

Fluid Lovel/Dats:

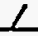

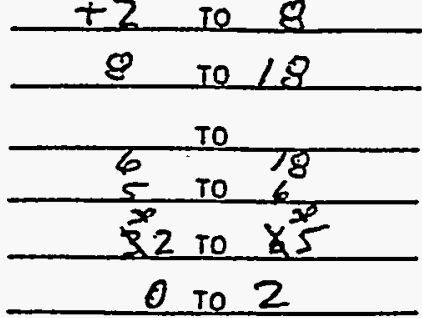

$\cdot$ 
Grand Junollon, Colorado 81802

Poge Lor l 1

Focility: OxNarP

Boring/Well No: mw - OP

Ground Elev. (It. AMSL):

IYPE

.

Sito:OxaraRo DOE EAclc OY Project: SITE CiAR

Blank Casing: PUC

Screen: Canr. Wesp PJC

Sump/Lina Cor: Puc

Sand Pack: Lonerrat 2

soolant: Bavroune Pecats

Grout:

Cenewr

Locking Cover Instolled: $\quad \mathrm{Y} / \mathrm{N}$

Drilling Mothod: AUCER DiA. (in. 1.0.)

Locotion (ft.) M:

Date Drilled: $2 / 3 / 95$

Somplers: Price $2^{\prime \prime}$

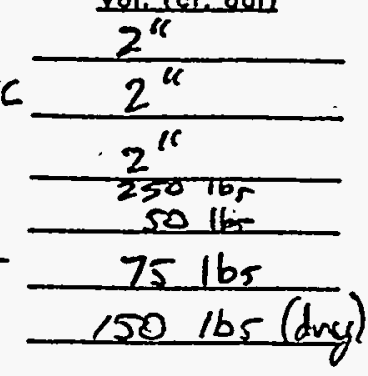

INIERYAL (IL)

Podlock No:

\begin{tabular}{c}
\hline 25 10 8 \\
\hline 9 to 18 \\
\hline to 78 \\
\hline 6 to 7 \\
\hline 3 to 6 \\
\hline 0 to 3 \\
\hline
\end{tabular}

Holo Depth (rt): 18

No. of Complotions:

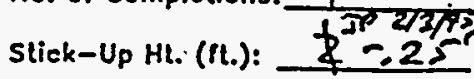

Slot Sizo: $\longrightarrow 0<0$

Locotion Skotch:

Sompling Method: SplT SPONN

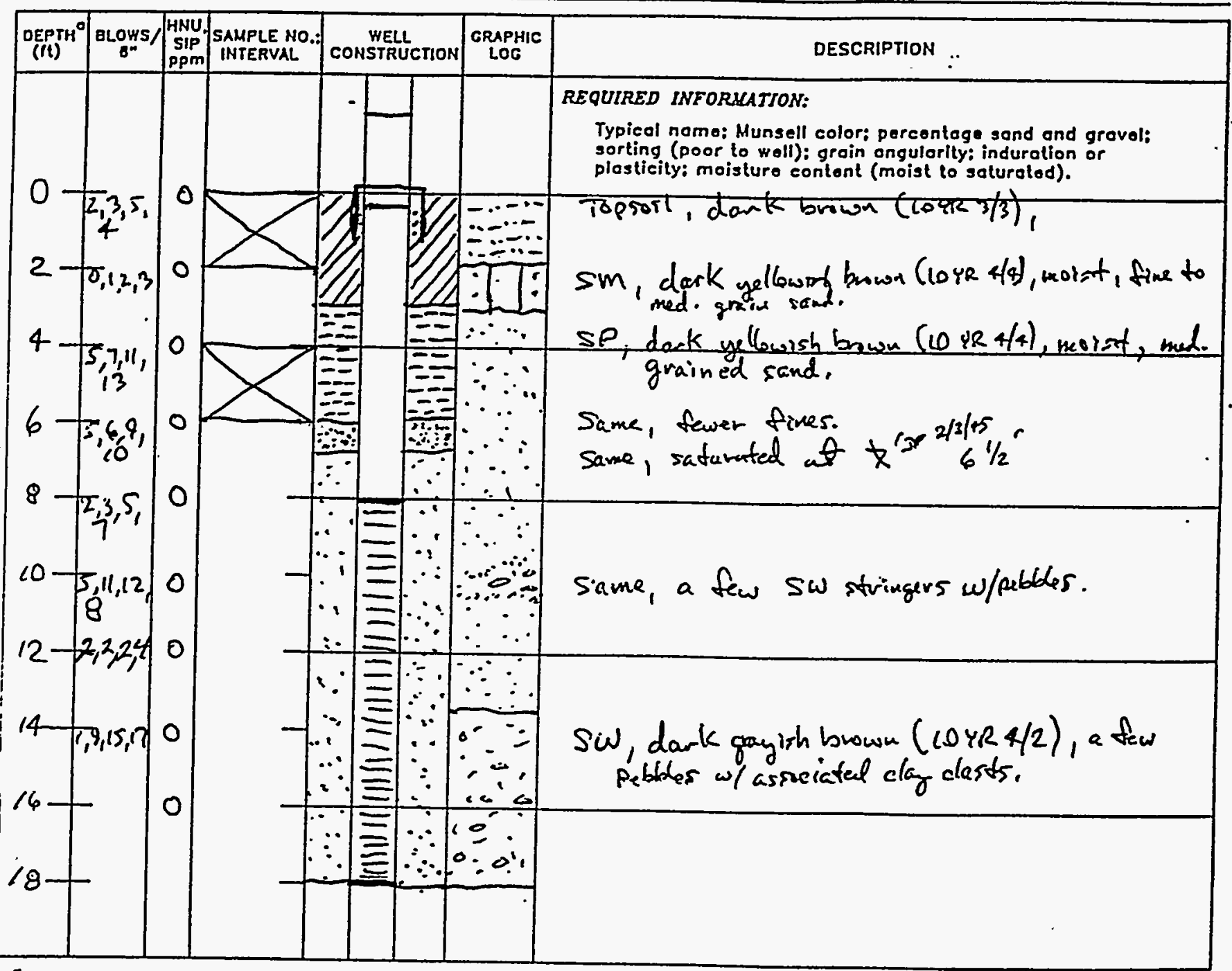

a All Dopths From Ground Surfoco.

completed by: Jeer Rele

VEPIFIED BY:

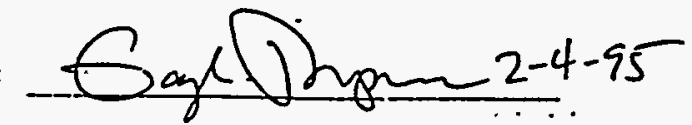


P.O. Box 14000

Grand Junollon, Colorado 81502

Poge 1 or 1

Focility: OXNARD

Boring/Well No: $S B-10$

Ground Elev. (ft. AMSL):

Blank Cosing: $N$ 工YPE

Sereen: $\quad$ L/A

Sump/End Cap: N/A

Sand Pack: NAT DiRT

Soolont: Bartoxite Eecers

Grout: $\quad N / A$

Loeking Cover Installod: $\quad Y / N$

Drilling Mathod: A uzed

sito: Oxararp DDE FAcic ay

Lecation (rt.) M:

Auger/Bit Sizo (in.):

OIA. (in. 1.0.)

yel. cel oeli

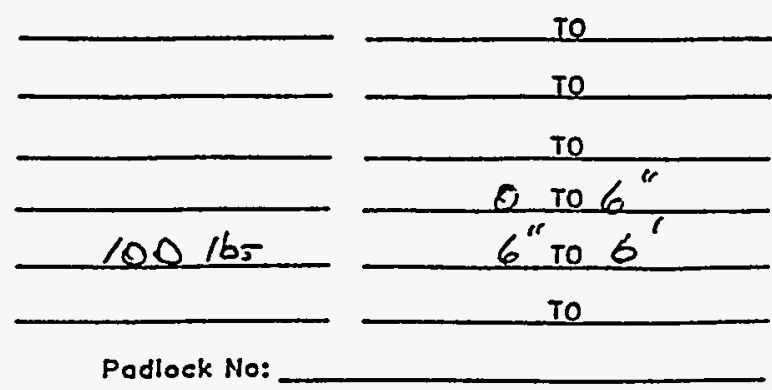

\section{INTERYAL (UL)}

No:

Sompling Method: SPLIT SPaOA

Doto Drilled: $\frac{2 / 4 / 95}{2 / 4}$

samplers: $P R / C E$

Dale Developod:

Fluid Lovol/Doto:

Remorks:

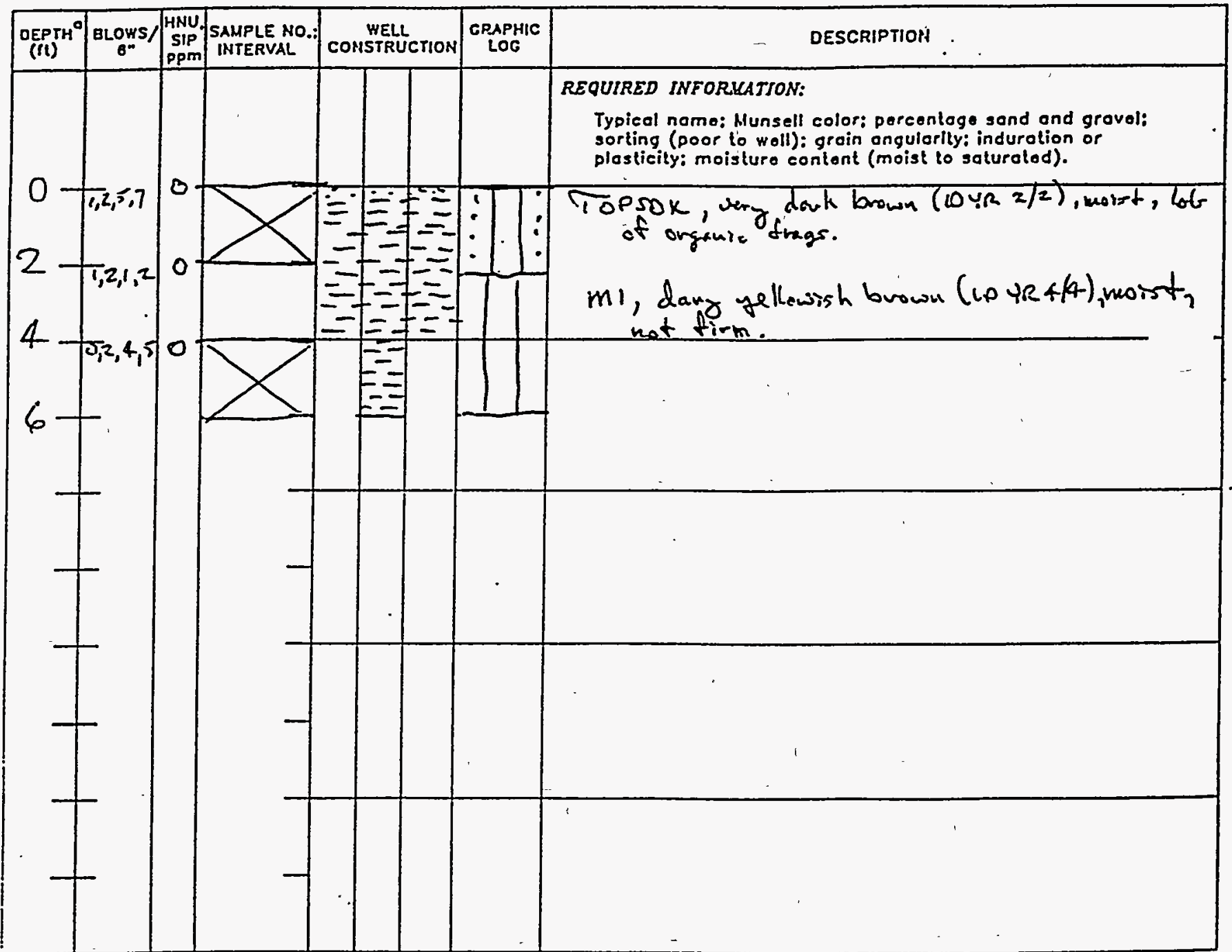

- All Dopths From Ground Surface.

completed ay: Jèfe Rice

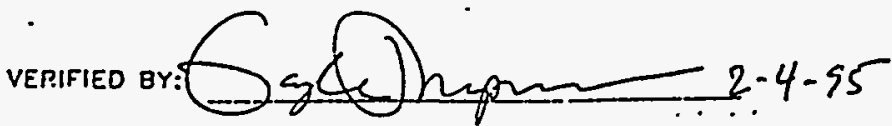


Prand Junollon, Colorado oiso2

Poge 1 of 1

Facility: Oracere

Sito:OxnCARE DOE FACKCTY Boring/Holl No: $S B-11$ (Ne Welt) Locotion (nt.) N:

Ground Elev. (it: AMSL):

Blank Cosing: $\frac{N / A}{N / A}$

Sump/End Cap:_N/A

Sand Pack: LouresTar \#2

$\checkmark$ Seolont: Bentowite Pelcen

Grout:

Locking Cover Ingtolled: $Y / N$

Drilling Mothod: HaNo Aucere

Doto Drilled: $2 / 3 / 95$

Somplers: Rerce
Augar/Bit Sizo (in.):

DIA. (in. 1.0.)

Vol. (ef ool
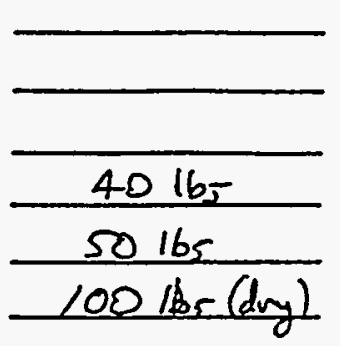

Podlock No:
Date Doveloped: _ N/A Remarks:

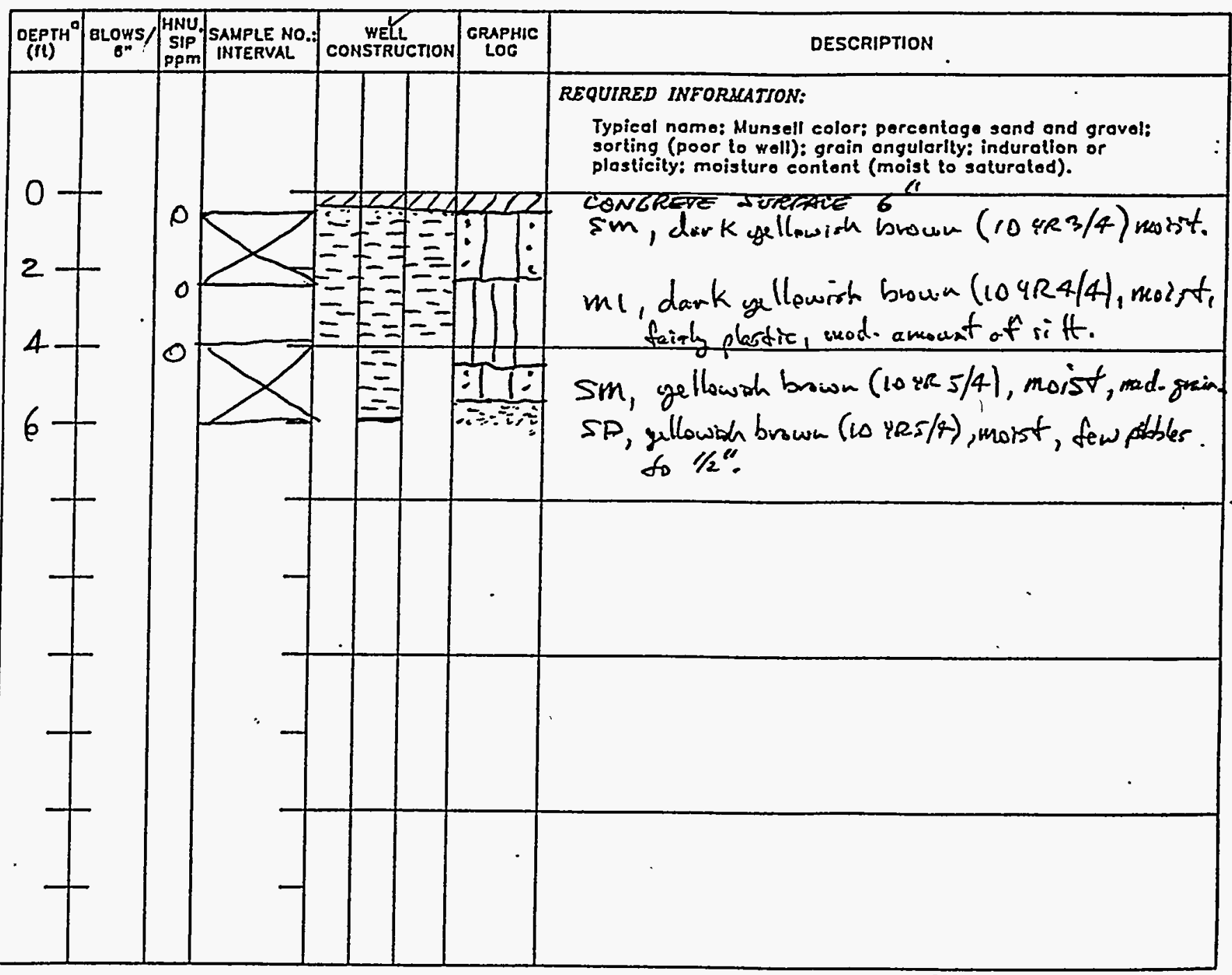

a All Dopths From Ground Surfoco.

coMpleted oy: Seff Rice

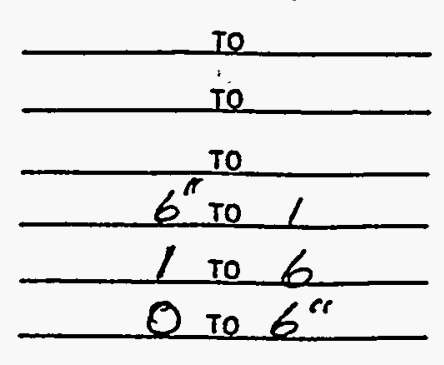

Project: Sits CtHir

E:

Holo Depth (it): 6 No. of Complotions: $N / A$ stick-Up Ht. (It.): $\triangle \mathrm{NC} / A$

Slot Sizo: _ N/A

Location Skotch:

3 "Aoger (third) ,"Drive Tuke Fluid Lovel/Dote: $N / A$ 
Boring/Holl No: $S B-R$ (No Well)

Locotion (ft.) N:

Ground Elev. (ft. AMSL):

Blank Cosing:_ N/A

Screen: N/A

Sump/End Cop: $N / A$

Sond Pack:

Seolont:

BENTONITE PELEDS

Grout:

Locking Cover Installed: $\quad Y / N$

Drilling Method: 2141955

Date Drilled: AJGee $L$

Somplers: PricE
Auger/Bit Sizo (in.): $81 / 4$

DIA. (in. 1.0.)

Yol. \{c. ool. .... INTERVAL (ft.)

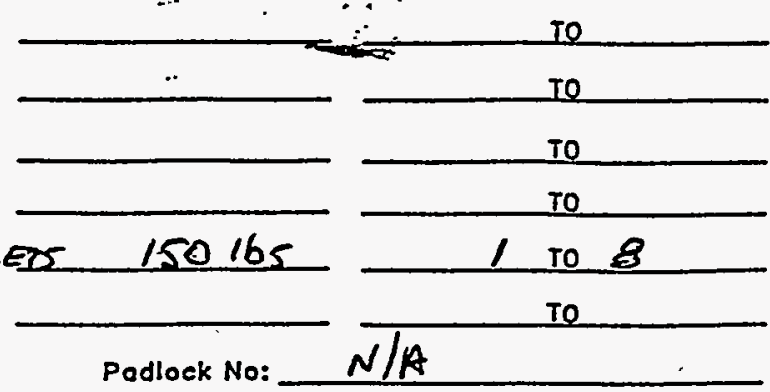

Project: SizE Citäe.

E:

Holo Depth (rt): \&

No. of Complotions: $\Lambda^{\prime}$

stick-Up Ht. (ft.): $-N / A t$

Slot Sizo: N//4

Locotion Skotch:

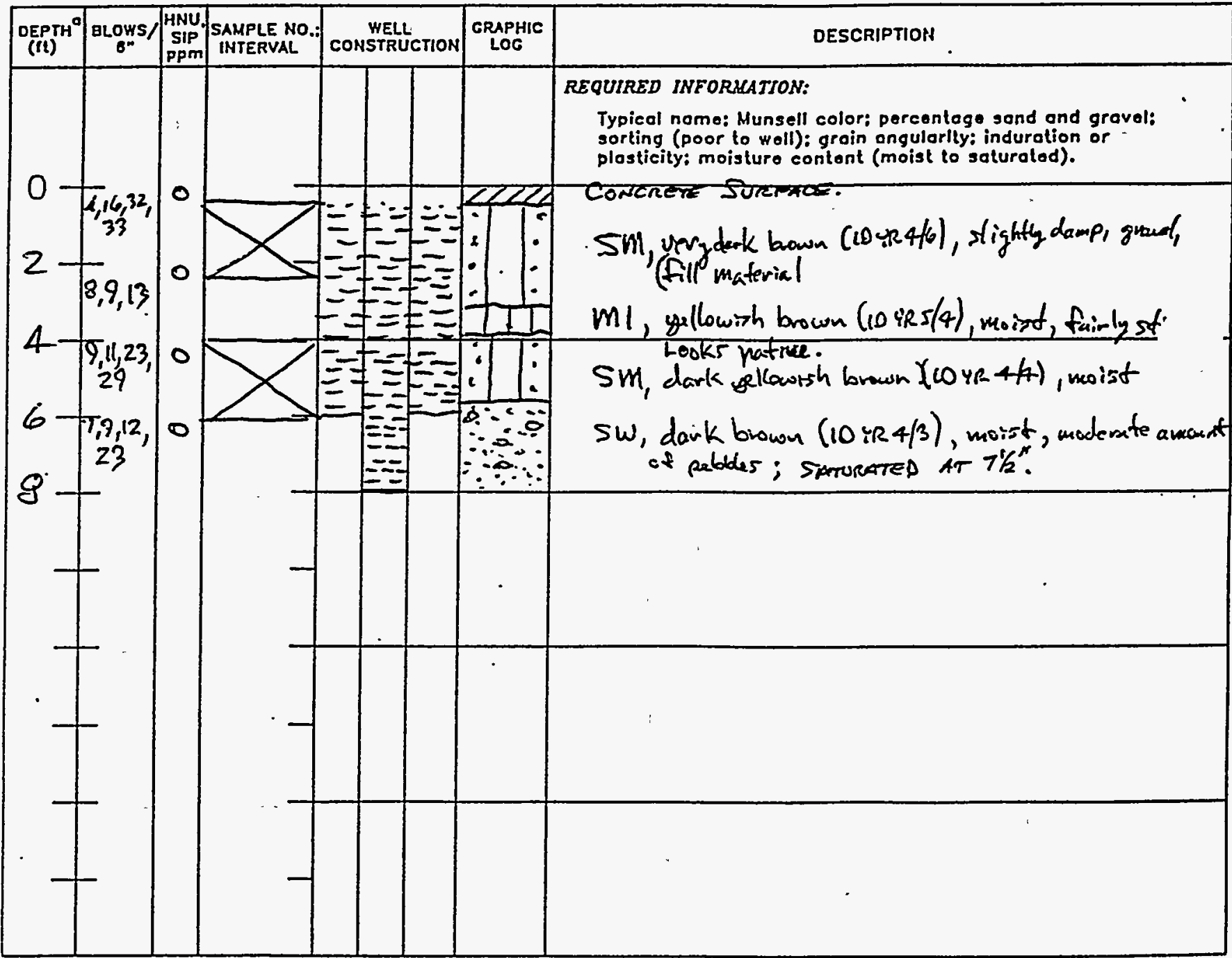

a All Dopths From Ground Surfoco.

completeo by: Jeff Price

Sompling Method: SPLIT SPoor

Date Doveloped: _._N/A Fluid Lovel/Oato: N/A

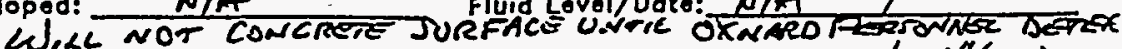

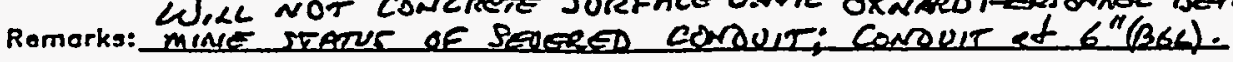

\section{EQUIRED INFORUATION:}

Typical nome: Munsell color: percentege sond and gravel sorting (poor to wall): groin angularlty: induration or

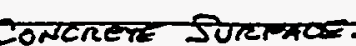

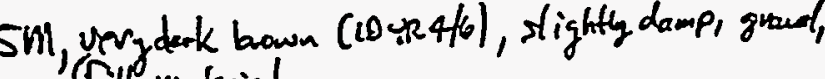
(fill material

SM, dark gllowsh brown ( 10 YR +4$)$, neoist

$S w$, daik biown (10 iR $4 / 3)$, moist, moderate amount of pabbles; sarureated AT 7\%". 
Facillty: QXACARD

Borlng/Wall No: $5 B-13$

Ground Elev. (It. AMSL):

Blonk Cosing: N/A

Sereon: N/A

Sump/End Cap:_N/A

Sand Pack: Lowestar 2

Soolont: Bentokite Peccets

Grout:

Locking Cover Instolled: $Y / M$

Orilling Mothod: Aycer

Date Drillad: $2 / 4 / 95$

somplers: $\mathbb{R} / C E$
Sito:Dxwerd DOE Ficec

Augar/Bit Sizo (in.): $\quad 8^{1 / 4}$

OIA. (in. 1.0.)

Yol. (ch ooll

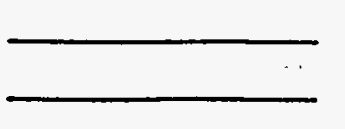

$25 \mathrm{lbr}$.

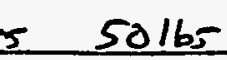

(o) 165

Podlock No:
INTERVAL (Ch)

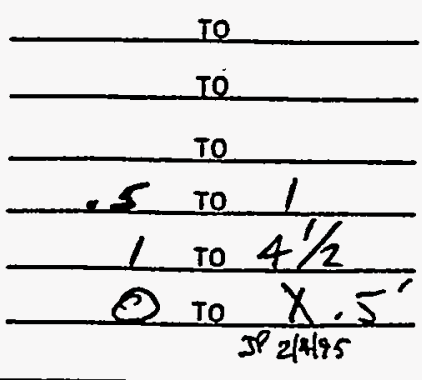

Project: SiQE Crise

E:

Holo Depth (t $t): 41 / 2$

No. of Complotions: N/R

stick-Up Ht. (It.): N/A

Slot Sizo: $N / A$

Location Skotch:

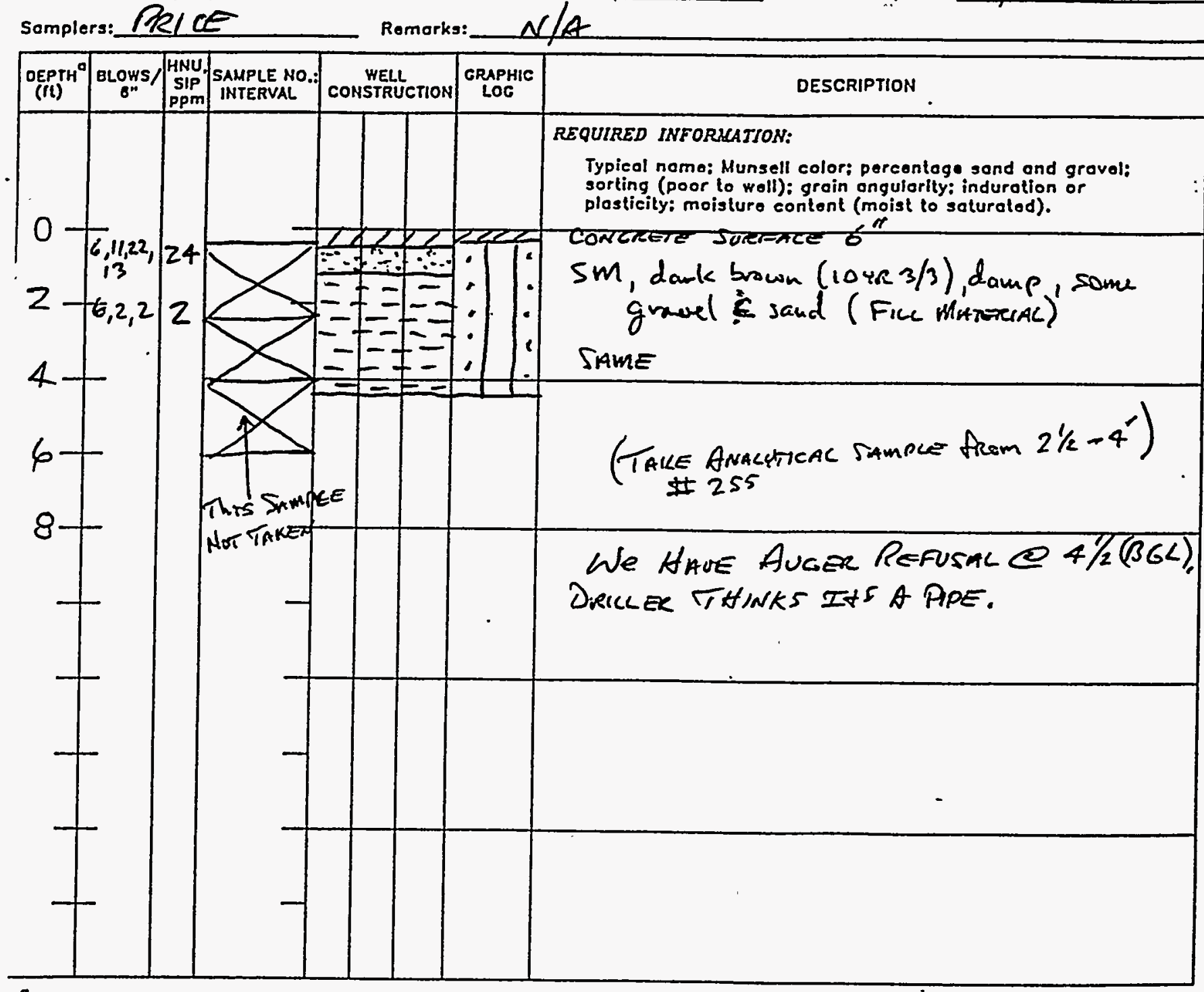

a All Dopths From Ground Surface.

completed ay: Jeff Rice

Sompling Method: SpLIT Spaer

Fluid Lovol/Oato: N/A /

Date Dovelopod: N/A

$+2$

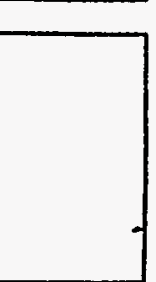


Facility: OXNARD

Sito:Oxama DOE EAcisitY Location (ft.) N: Auger/ait Sizo (in.): $\quad$ g //4 DIA. (in. 1.D.).

Blank Casing:_ $\quad d / 4$

Seroon: $\quad N / A$

Sump/End Capj Mlat

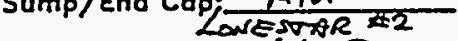

Sond Pock: SLWEINAC SoND

Soulont: Bentonite Pesers

Grout: Coscreors 100 lbs (dyg)

Locking Cover Installed: $Y / N$ Drilling Mothod: Au 6 ER Dato Drilled: $2 / 3 / 95$

Somplers: Pelas Vele. (c) ool

INTERYAL (IV) To

TO

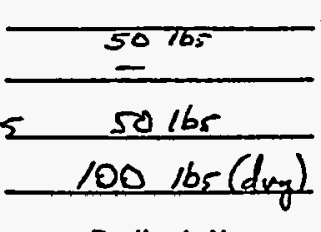

Podlock No: $\mathrm{al} / \mathrm{A}$
Project: STE CHAR

E: $2 / 4 / 45 \sim i 3 p$
Holo Depth (lt): $4^{\prime} Q^{\prime}$

No. of Completions:_ $N$ Stick-Up Ht. (It.): N N/A

Slot Sizo: _ N N/A

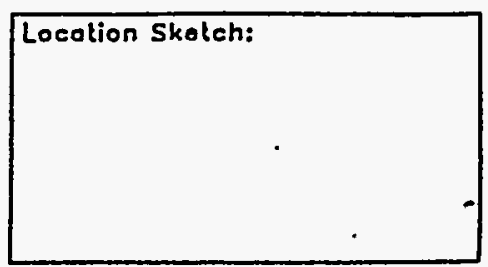

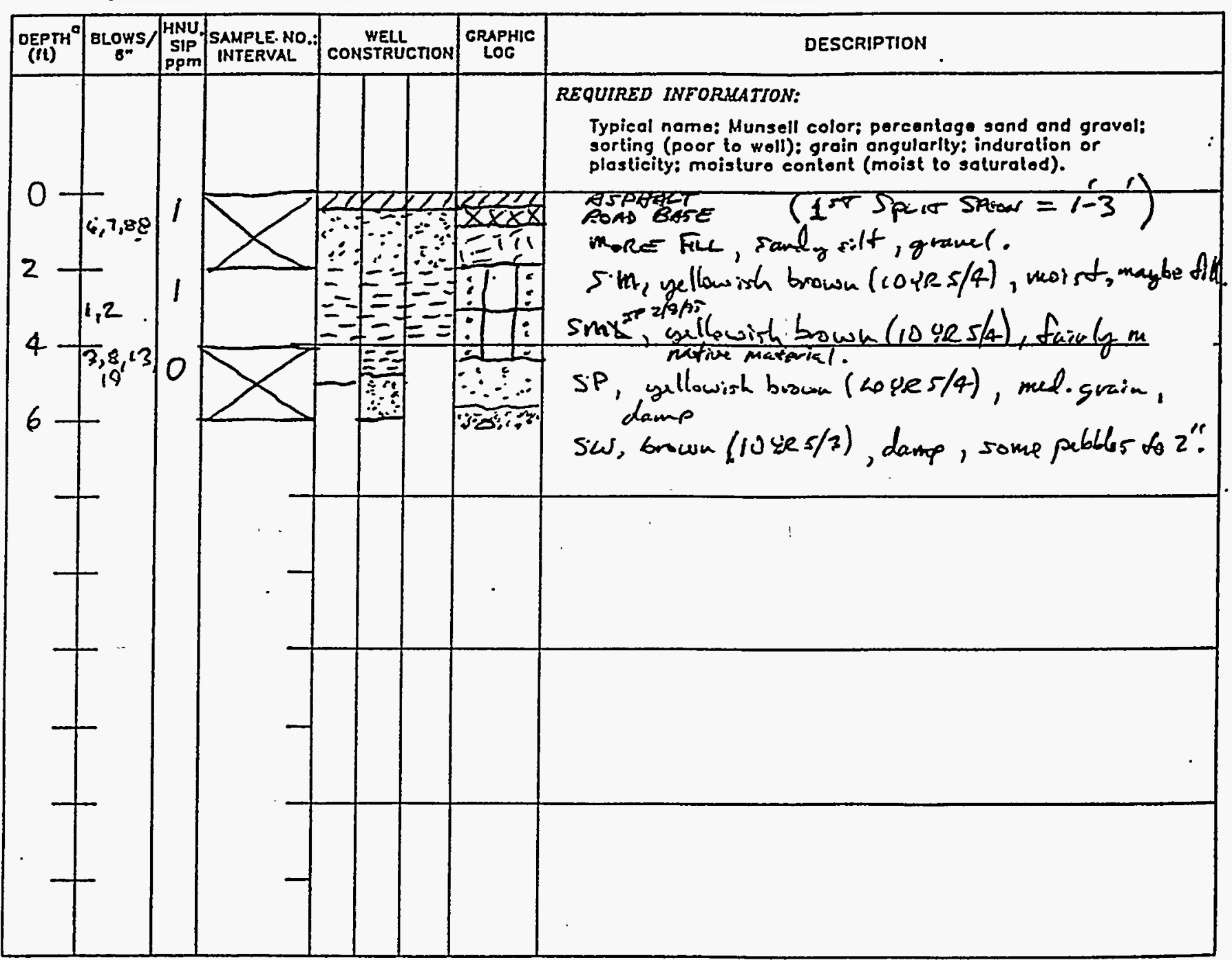

- All Dopths From Ground Surface. completeo ay: Jefe Pese VEPIFIED BY:

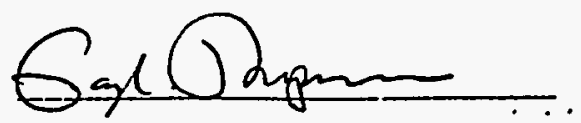


P.o. Box 14000
Grand Junotlon, Colorado 81302 .

Focility: O) $\times$ NARP

goring/Hall No: $S B-15$

Ground Elev. (ft. AMSL):

IYPE

Blank Casing: Norse

Screen: Noste

Sump/End Cop:_Lave

Sond Pack: LW(ESAR "Z (NONE OSEQ)

Soalont: Bevorton To

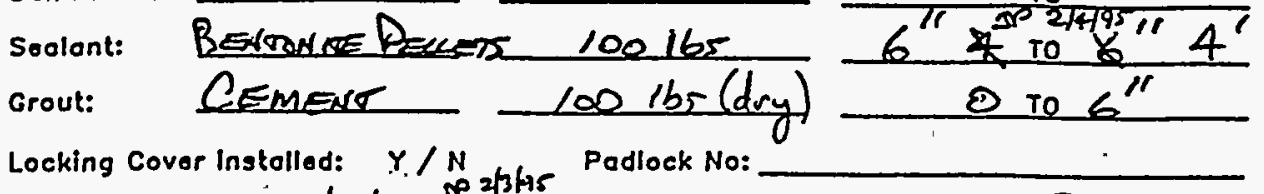
(Ne Well) Location (ft.) $N$ : Auger/Bit Sizo (in.): $\quad 9 / / 4$ DIA. (in. 1.0.)

INTERYAL (UL)

To

To
Poge $i$ or 1

Site: OxNARP DOE Facicity

Aergere Sompling Method:SPht SPore

Date Doveloped: N/A

Fluid Lovol/Doto: $\quad \mu / A /$

Remarks:

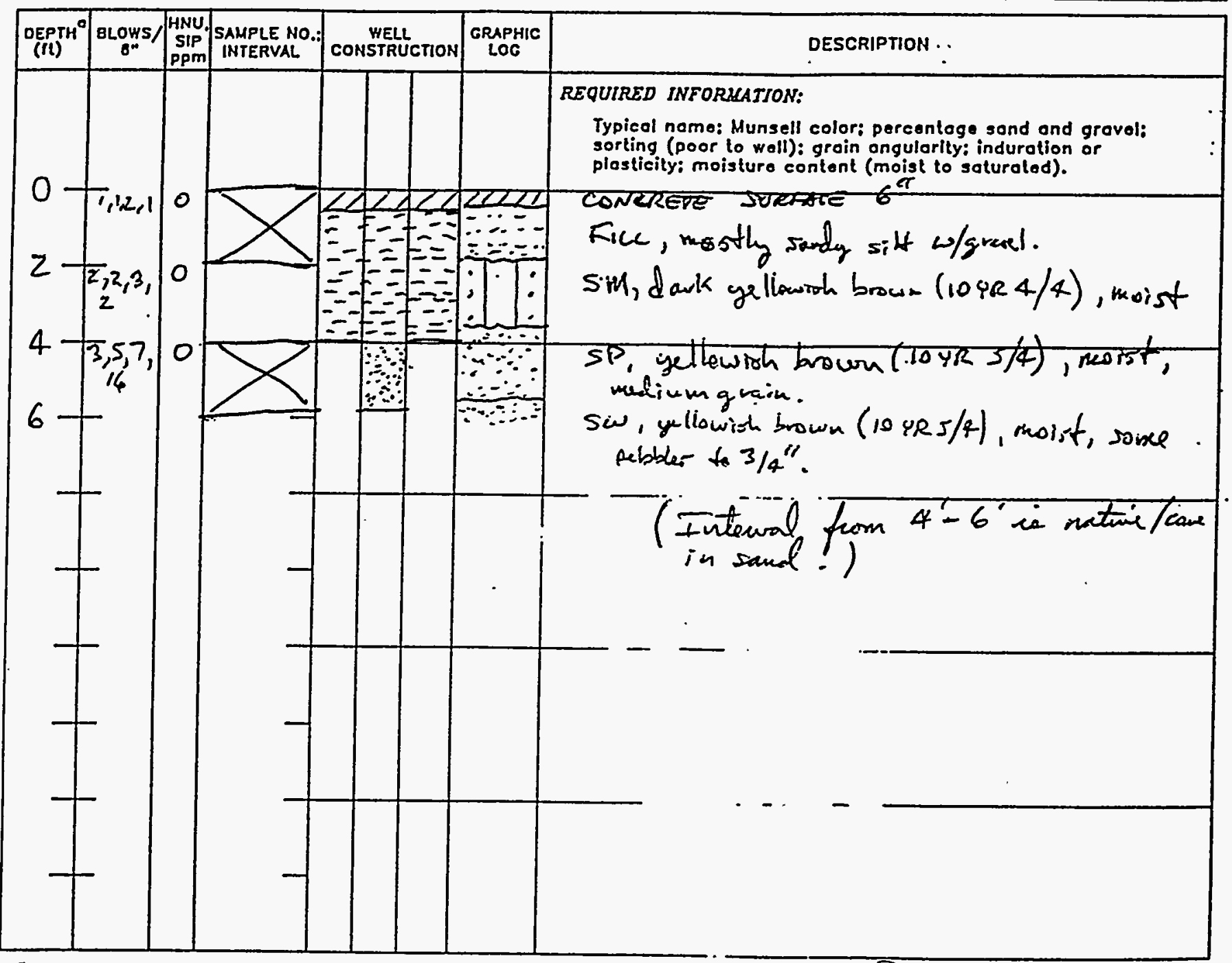

a All Dopths From Ground Surfaco.

completed by: Jefe Rece

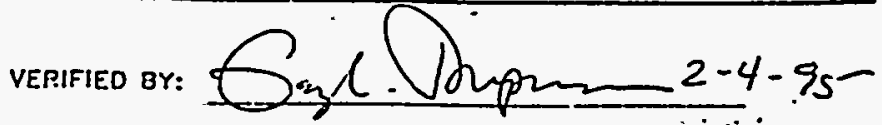

DOE Grand Junction Projects Office

July 1995

Phase II ESA Report

Page B-15 
P.0. Box 14000

Grand Junollon, Colorado atsoz.

Poge 1 of 1

Facility: OXNAPR Boring/Holl No: SB-16 (No Well)

Sito: Oximeo PoE FAcuitu

Project: Site Offige"

Ground Elev. (ft. AMSL):

E: 2/4/95 IP Holo Depth (tt): (4t) $6^{\prime}$

IYPE

DIA. (in. 1.0.)

vol. cei ooli

Blank Casing: Aloke Nooke

Scroon:

Sand Pack: LoNestar \#2

sociont: Bentonige Peacoss

Grout: Aspitimte Covcrate

Nat Sano Ruf

Locking Covor ingtolled: $Y / N$

Drilling Mothod: Auger

Dato Drilled: $2 / 2 / 95$

somplers: Price

Oert oLOHS

\begin{tabular}{|c|c|c|c|c|c|c|}
\hline$\underset{(i t)}{\operatorname{OEPTH}}{ }^{\circ}$ & aLOWS/ & $\begin{array}{l}\text { HNU, } \\
\text { SIP } \\
\text { PPM }\end{array}$ & $\begin{array}{l}\text { SAMPLE NO.: } \\
\text { INTERVAL }\end{array}$ & constrtiefton & LOG & DESCRIPTION . \\
\hline & & & & $\begin{array}{l}\text { Brtekfill } \\
\text { Inforkfurdo }\end{array}$ & & $\begin{array}{l}\text { REQUIRED INFORSATION: } \\
\text { Typical nomo: Munsell color; percentoge sond ond graval: } \\
\text { sorting (poor to woll): grain ongulorlty: indurotion or } \\
\text { plasticity: moisturo conlent (moist to saturotod). }\end{array}$ \\
\hline
\end{tabular}

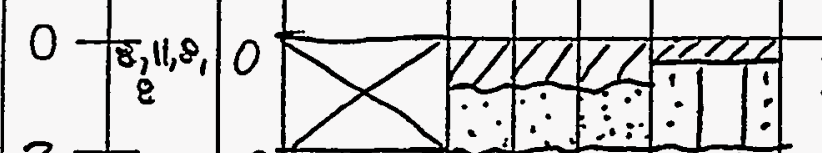

ASPHACTIC CONCRETE SURTALE

5 in, dark brown $(10$ is 4/3), damp, probably fill material.
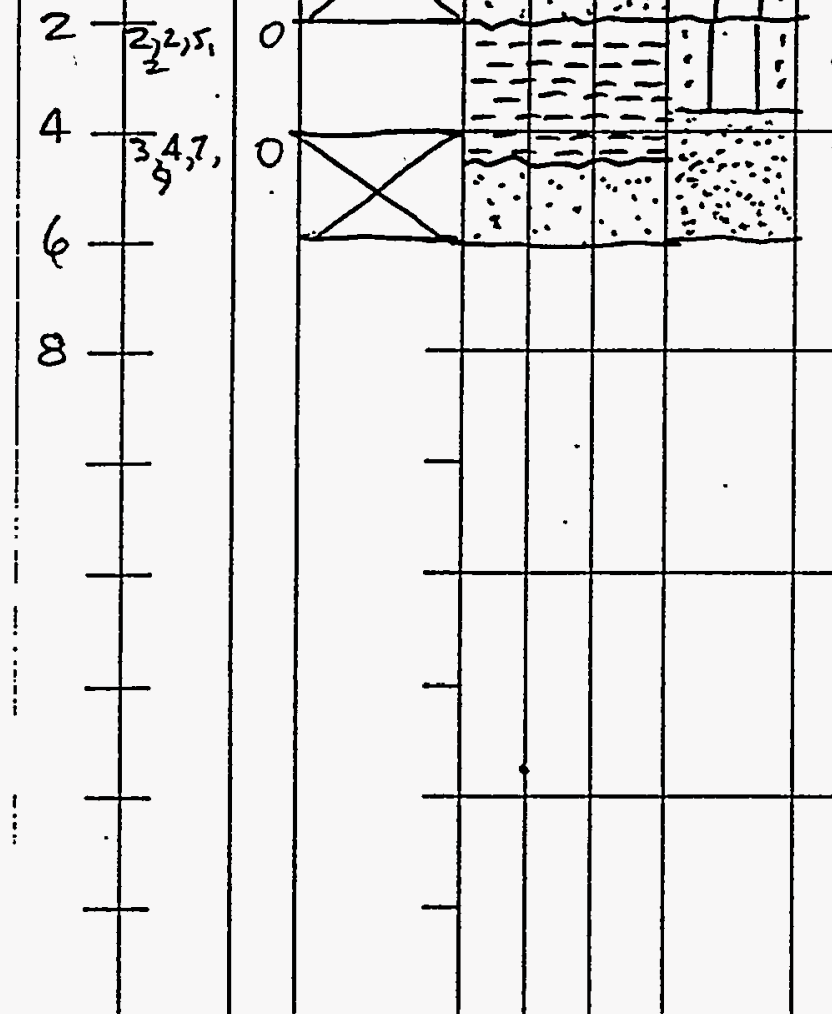

a All Dopths From Ground Surfoco.
completed BY: JEFf RICE
No. of Completions: _ N

Stick-Up Ht. (It.):

Slot Sizo:

N/A

Locotion Skotch: $6^{\prime \prime} \&$ ro $\$ 22$

(3) To

$4 / 2$ to 6
Sompling Method: Split Speon

Fluid Lovol/Dato: $\mathrm{N} / \mathrm{A}$

yellownt brown (10 ys 36$)$, damp; mosthy sand, look like natrue mat.

SP, yellowish brown $(10$ VR5/6), saturatel $5 \%$

(Tomal Deprat of Auger $=4^{\prime}$; SPLIV SPwor PeMETRATIOUT fo b?
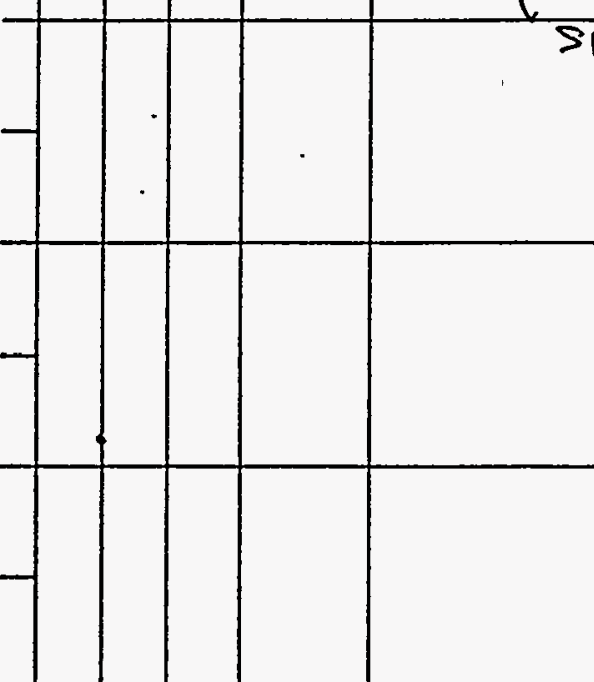
Appendix C

Analytes, Laboratory Reporting Limits, and Analytical Methods 
$\therefore \ldots$ 
Table C-1. Analytes, Laboratory Reporting Limits, and Analytical Methods for Groundwater Sampling

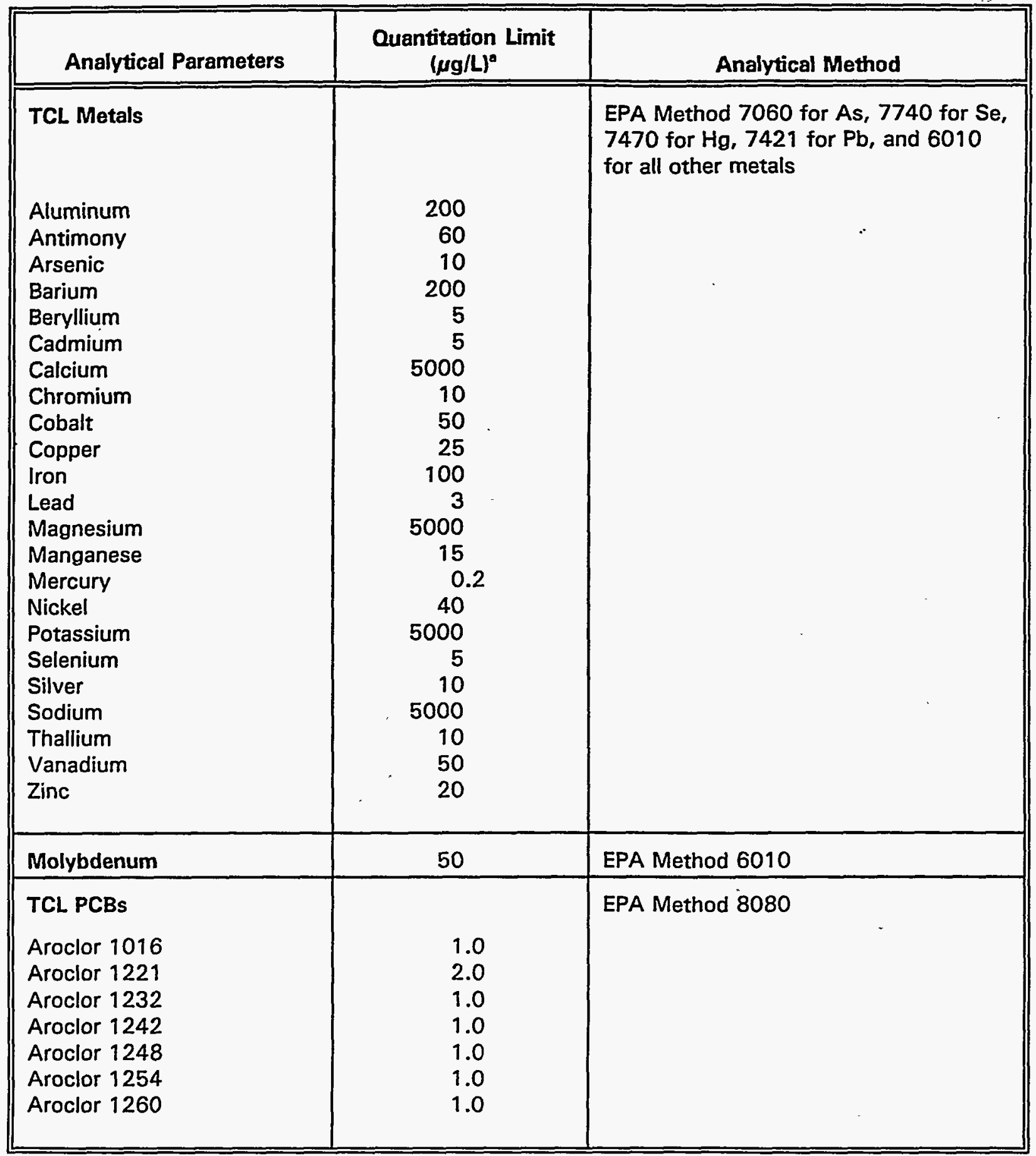

${ }^{\mathrm{a}} \mu \mathrm{g} / \mathrm{L}=$ microgram per liter. 
Table C-1 (continued). Analytes, Laboratory Reporting Limits, and Analytical Methods for Groundwater Sampling

\begin{tabular}{|c|c|c|}
\hline Analytical Parameter & $\begin{array}{l}\text { Quantitation Limit } \\
(\mu \mathrm{g} / \mathrm{L})^{\circ}\end{array}$ & Analytical Method \\
\hline $\begin{array}{l}\text { TCL VOCs } \\
\text { Acetone } \\
\text { Benzene } \\
\text { Bromodichloromethane } \\
\text { Bromoform } \\
\text { Bromomethane } \\
\text { 2-Butanone } \\
\text { Carbon disulfide } \\
\text { Carbon tetrachloride } \\
\text { Chlorobenzene } \\
\text { Chlorodibromomethane } \\
\text { Chloroethane } \\
\text { Chloroform } \\
\text { Chloromethane } \\
\text { 1,1-Dichloroethane } \\
\text { 1,2-Dichloroethane } \\
\text { 1,1-Dichloroethene } \\
\text { trans-1,2-Dichloroethene } \\
\text { 1,2-Dichloropropane } \\
\text { cis-1,3-Dichloropropene } \\
\text { trans-1,3-Dichloropropene } \\
\text { Ethyl benzene } \\
\text { 2-Hexanone } \\
\text { Methylene chloride } \\
\text { 4-Methyl-2-pentanone } \\
\text { Styrene } \\
\text { 1,1,2,2-Tetrachloroethane } \\
\text { Tetrachloroethene } \\
\text { Toluene } \\
\text { 1,1,1-Trichloroethane } \\
\text { 1,1,2-Trichloroethane } \\
\text { Trichloroethene } \\
\text { Vinyl acetate } \\
\text { Vinyl chloride } \\
\text { Xylene }\end{array}$ & $\begin{array}{c}100 \\
5 \\
5 \\
5 \\
10 \\
100 \\
100 \\
5 \\
5 \\
5 \\
10 \\
5 \\
10 \\
5 \\
5 \\
5 \\
5 \\
5 \\
5 \\
5 \\
5 \\
50 \\
5 \\
50 \\
5 \\
5 \\
5 \\
5 \\
5 \\
5 \\
5 \\
50 \\
10 \\
5 \\
\end{array}$ & EPA Method 8240 or 8260 \\
\hline TPH & 500 & $\begin{array}{l}\text { California Modified EPA } \\
\text { Method } 8015\end{array}$ \\
\hline
\end{tabular}

${ }^{\mathrm{a}} \mu \mathrm{g} / \mathrm{L}=$ microgram per liter. 
Table C-2. Analytes, Laboratory Reporting Limits, and Analytical Methods for Soil Sampling

\begin{tabular}{|c|c|c|}
\hline Analytical Parameter & $\begin{array}{l}\text { Quantitation Limit } \\
(\mathbf{m g} / \mathrm{kg})^{\mathrm{n}}\end{array}$ & Analytical Method \\
\hline TCL Metals & & $\begin{array}{l}\text { EPA Method } 7060 \text { for } \mathrm{As}, 7740 \text { for } \\
\mathrm{Se}, 7471 \text { for } \mathrm{Hg}, 7421 \text { for } \mathrm{Pb} \text {, and } \\
6010 \text { for all other metals. }\end{array}$ \\
\hline Aluminum & 40.0 & \\
\hline Antimony & 12.0 & \\
\hline Arsenic & 2.0 & \\
\hline Barium & 40.0 & r \\
\hline Beryllium & 1.0 & \\
\hline Cadmium & 1.0 & $\theta^{\prime}$ \\
\hline Calcium & 1,000 & \\
\hline Chromium & 2.0 & \\
\hline Cobalt & 10.0 & \\
\hline Copper & 5.0 & \\
\hline Iron & 20.0 & \\
\hline Lead & 0.6 & \\
\hline Magnesium & 1,000 . & \\
\hline Manganese & 3.00 & \\
\hline Mercury & 0.04 & . \\
\hline Nickel & 8.0 & \\
\hline Potassium & 1,000 & \\
\hline Selenium & 1.0 & \\
\hline Silver & 2.0 & \\
\hline Sodium. & 1,000 & \\
\hline Thallium & 2.0 & \\
\hline Vanadium & 10.0 & \\
\hline Zinc & 4.0 & \\
\hline Molybdenum & 10.0 & EPA Method 6010 \\
\hline TCL PCBs & $(\mu \mathrm{g} / \mathrm{kg})^{\mathrm{b}}$ & EPA Method 8080 \\
\hline Aroclor 1016 & 33 & \\
\hline Aroclor 1221 & 67 & \\
\hline Aroclor 1232 & 33 & \\
\hline Aroclor 1242 & 33 & \\
\hline Aroclor 1248 & 33 & \\
\hline Aroclor 1254 & 33 & \\
\hline Aroclor 1260 & 33 & \\
\hline
\end{tabular}

"mg/kg = milligram per kilogram.

${ }^{\mathrm{b}} \mu \mathrm{g} / \mathrm{kg}=$ microgram per kilogram. 
Table C-2 (continued). Analytes, Laboratory Reporting Limits, and Analytical Methods for Soil Sampling

\begin{tabular}{|c|c|c|}
\hline Analytical Parameter & $\begin{array}{c}\text { Quantitation Limit } \\
(\mu \mathrm{g} / \mathrm{kg})^{\mathbf{*}}\end{array}$ & Analytical Method \\
\hline $\begin{array}{l}\text { TCL VOCs } \\
\text { Acetone } \\
\text { Benzene } \\
\text { Bromodichloromethane } \\
\text { Bromoform } \\
\text { Bromomethane } \\
\text { 2-Butanone } \\
\text { Carbon disulfide } \\
\text { Carbon tetrachloride } \\
\text { Chlorobenzene } \\
\text { Chlorodibromomethane } \\
\text { Chloroethane } \\
\text { Chloroform } \\
\text { Chloromethane } \\
\text { 1,1-Dichloroethane } \\
\text { 1,2-Dichloroethane } \\
\text { 1,1-Dichloroethene } \\
\text { trans-1,2-Dichloroethene } \\
\text { 1,2-Dichloropropane } \\
\text { cis-1,3-Dichloropropene } \\
\text { trans-1,3-Dichloropropene } \\
\text { Ethyl benzene } \\
\text { 2-Hexanone } \\
\text { Methylene chloride } \\
\text { 4-Methyl-2-pentanone } \\
\text { Styrene } \\
1,1,2,2-\text { Tetrachloroethane } \\
\text { Tetrachloroethene } \\
\text { Toluene } \\
1,1,1-T r i c h l o r o e t h a n e \\
1,1,2-T r i c h l o r o e t h a n e \\
\text { Trichloroethene } \\
\text { Vinyl acetate } \\
\text { Vinyl chloride } \\
\text { Xylene } \\
\end{array}$ & $\begin{array}{c}100 \\
5 \\
5 \\
5 \\
10 \\
100 \\
100 \\
5 \\
5 \\
5 \\
10 \\
5 \\
10 \\
5 \\
5 \\
5 \\
5 \\
5 \\
5 \\
5 \\
5 \\
50 \\
5 \\
50 \\
5 \\
5 \\
5 \\
5 \\
5 \\
5 \\
5 \\
5 \\
5 \\
5 \\
5 \\
5 \\
5\end{array}$ & EPA Method 8240 or 8260 \\
\hline TPH & $10 \mathrm{mg} / \mathrm{kg}^{\mathrm{b}}$ & $\begin{array}{l}\text { California Modified EPA } \\
\text { Method } 8015\end{array}$ \\
\hline
\end{tabular}

${ }^{\mathrm{a}} \mu \mathrm{g} / \mathrm{kg}=$ microgram per kilogram.

$\mathrm{bm} / \mathrm{kg}=$ milligram per kilogram. 
Table C-3. Analytes, Laboratory Reporting Limits, and Analytical Methods for Sludge and Oil Sampling

\begin{tabular}{|c|c|c|}
\hline Analytical Parameter & Quantitation Limit & Analytical Method \\
\hline $\begin{array}{l}\text { TOTAL Metals } \\
\text { Antimony } \\
\text { Arsenic } \\
\text { Barium } \\
\text { Beryllium } \\
\text { Cadmium } \\
\text { Chromium } \\
\text { Cobalt } \\
\text { Copper } \\
\text { Lead } \\
\text { Mercury } \\
\text { Molybdenum } \\
\text { Nickel } \\
\text { Selenium } \\
\text { Silver } \\
\text { Thallium } \\
\text { Vanadium } \\
\text { Zinc }\end{array}$ & $\begin{array}{c}(\mathbf{m g} / \mathbf{K g})^{a} \\
\\
\\
12.0 \\
2.0 \\
40.0 \\
1.0 \\
1.0 \\
2.0 \\
10.0 \\
5.0 \\
0.6 \\
0.04 \\
10.0 \\
8.0 \\
1.0 \\
2.0 \\
2.0 \\
10.0 \\
4.0\end{array}$ & $\begin{array}{l}\text { EPA Method } 7060 \text { for } \mathrm{As}, 7740 \text { for } \\
\mathrm{Se}, 7471 \text { for } \mathrm{Hg}, 7421 \text { for } \mathrm{Pb} \text {, and } \\
6010 \text { for all other metals. }\end{array}$ \\
\hline $\begin{array}{l}\text { TCL PCBs } \\
\text { Aroclor } 1016 \\
\text { Aroclor } 1221 \\
\text { Aroclor } 1232 \\
\text { Aroclor } 1242 \\
\text { Aroclor } 1248 \\
\text { Aroclor } 1254 \\
\text { Aroclor } 1260\end{array}$ & $\begin{array}{c}(\mathbf{m g} / \mathbf{k g})^{\natural} \\
0.05 \\
0.05 \\
0.05 \\
0.05 \\
0.05 \\
1.0 \\
1.0\end{array}$ & EPA-600/481-045 \\
\hline \multicolumn{3}{|c|}{ Following is for oil sample analysis only. } \\
\hline $\begin{array}{l}\text { Total Organic Halides } \\
\text { Ignitability }\end{array}$ & $\begin{array}{c}25 \mathrm{mg} / \mathrm{kg}^{\mathrm{a}} \\
1^{\circ} \mathrm{C}\end{array}$ & $\begin{array}{l}\text { EPA Method } 9076 \\
\text { EPA Method } 1010\end{array}$ \\
\hline
\end{tabular}

"mg/kg = milligram per kilogram. 
Table C-4. Analytes, Laboratory Reporting Limits, and Analytical Methods for Soil Sampling for Waste Disposal Determination

\begin{tabular}{|c|c|c|}
\hline Analytical Parameter & Quantitation Limit & Analytical Method \\
\hline $\begin{array}{l}\text { TOTAL Metals } \\
\text { Antimony } \\
\text { Arsenic } \\
\text { Barium } \\
\text { Beryllium } \\
\text { Cadmium } \\
\text { Chromium } \\
\text { Cobalt } \\
\text { Copper } \\
\text { Lead } \\
\text { Mercury } \\
\text { Molybdenum } \\
\text { Nickel } \\
\text { Selenium } \\
\text { Silver } \\
\text { Thallium } \\
\text { Vanadium } \\
\text { Zinc }\end{array}$ & $\begin{array}{c}(\mathbf{m g} / \mathrm{Kg})^{\mathrm{a}} \\
\\
\\
12.0 \\
2.0 \\
40.0 \\
1.0 \\
1.0 \\
2.0 \\
10.0 \\
5.0 \\
0.6 \\
0.04 \\
10.0 \\
8.0 \\
1.0 \\
2.0 \\
2.0 \\
10.0 \\
4.0\end{array}$ & $\begin{array}{l}\text { EPA Method } 7060 \text { for } \mathrm{As}, 7740 \text { for } \\
\mathrm{Se}, 7471 \text { for } \mathrm{Hg}, 7421 \text { for } \mathrm{Pb} \text {, and } \\
6010 \text { for all other metals. }\end{array}$ \\
\hline Fluoride Salts & 5.0 & $\begin{array}{l}\text { EPA Method } 300.0 \text { modified for } \\
\text { soils }\end{array}$ \\
\hline
\end{tabular}

amg/kg = milligram per kilogram. 
Table C-4 (continued). Analytes, Laboratory Reporting Limits, and Analytical Methods for Soil Sampling for Waste Disposal Determination

\begin{tabular}{|c|c|c|}
\hline Analytical Parameter & $\begin{array}{l}\text { Quantitation Limit } \\
(\mu \mathrm{g} / \mathrm{kg})^{\mathrm{a}}\end{array}$ & Analytical Method \\
\hline $\begin{array}{l}\text { TCL VOCs } \\
\text { Acetone } \\
\text { Benzene } \\
\text { Bromodichloromethane } \\
\text { Bromoform } \\
\text { Bromomethane } \\
\text { 2-Butanone } \\
\text { Carbon disulfide } \\
\text { Carbon tetrachloride } \\
\text { Chlorobenzene } \\
\text { Chlorodibromomethane } \\
\text { Chloroethane } \\
\text { Chloroform } \\
\text { Chloromethane } \\
\text { 1,1-Dichloroethane } \\
\text { 1,2-Dichloroethane } \\
\text { 1,1-Dichloroethene } \\
\text { trans-1,2-Dichloroethene } \\
\text { 1,2-Dichloropropane } \\
\text { cis-1,3-Dichloropropene } \\
\text { trans-1,3-Dichloropropene } \\
\text { Ethyl benzene } \\
\text { 2-Hexanone } \\
\text { Methylene chloride } \\
\text { 4-Methyl-2-pentanone } \\
\text { Styrene } \\
\text { 1,1,2,2-Tetrachloroethane } \\
\text { Tetrachloroethene } \\
\text { Toluene } \\
\text { 1,1,1-Trichloroethane } \\
\text { 1,1,2-Trichloroethane } \\
\text { Trichloroethene } \\
\text { Vinyl acetate } \\
\text { Vinyl chloride } \\
\text { Xylene }\end{array}$ & $\begin{array}{c}100 \\
5 \\
5 \\
5 \\
10 \\
100 \\
100 \\
5 \\
5 \\
5 \\
10 \\
5 \\
10 \\
5 \\
5 \\
5 \\
5 \\
5 \\
5 \\
5 \\
5 \\
5 \\
50\end{array}$ & EPA Method 8240 or 8260 \\
\hline
\end{tabular}

${ }^{\mathrm{a}} \mu \mathrm{g} / \mathrm{kg}=$ microgram per kilogram. 
$=\quad \cdots \quad-\cdots=$

$+\cdots+\cdots$ 


Table D-1. Oxnard Groundwater Data

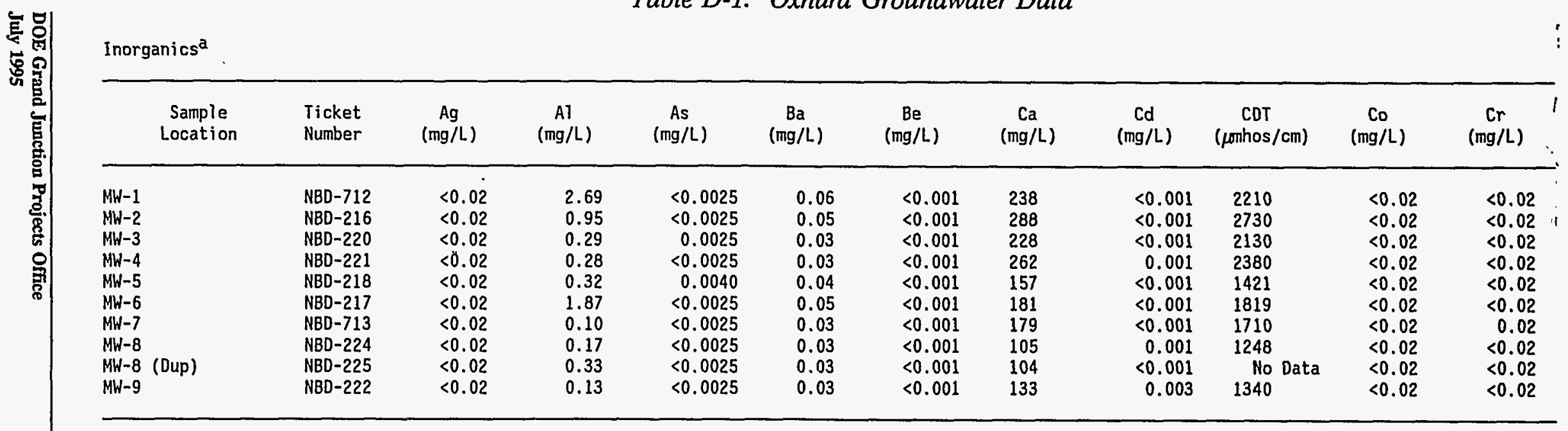

$a_{A}$ " $<"$ indicates that the maximum concentration was below the detection limit (number shown is detection limit). 
Inorganics ${ }^{a}$

\begin{tabular}{|c|c|c|c|c|c|c|c|c|c|c|c|}
\hline $\begin{array}{c}\text { Sample } \\
\text { Location }\end{array}$ & $\begin{array}{l}\text { Ticket } \\
\text { Number }\end{array}$ & $\underset{(\mathrm{mg} / \mathrm{L})}{\mathrm{Cu}}$ & $\begin{array}{c}\mathrm{Fe} \\
(\mathrm{mg} / \mathrm{L})\end{array}$ & $\begin{array}{l}\text { H2O Depth } \\
\text { (feet) }\end{array}$ & $\begin{array}{c}\mathrm{Hg} \\
(\mathrm{mg} / \mathrm{L})\end{array}$ & $\underset{(\mathrm{mg} / \mathrm{L})}{\mathrm{K}}$ & $\underset{(m g / L)}{M g}$ & $\begin{array}{c}M n \\
(m g / L)\end{array}$ & $\begin{array}{c}\mathrm{Na} \\
(\mathrm{mg} / \mathrm{L})\end{array}$ & $\underset{(m g / L)}{N i}$ & $\begin{array}{c}\mathrm{Pb} \\
(\mathrm{mg} / \mathrm{L})\end{array}$ \\
\hline$M W-1$ & NBD -712 & $<0.02$ & 4.54 & 9.91 & $<0.0005$ & 4.5 & 73.5 & 0.12 & 167 & $<0.02$ & 0.003 \\
\hline MH-2 & NBD-216 & $<0.02$ & 1.03 & 9.90 & $<0.0005$ & 6.3 & 91.4 & 1.00 & 228 & $<0.02$ & $<0.001$ \\
\hline$M W-3$ & NBD-220 & $<0.02$ & 0.63 & 7.45 & 0.0008 & 4.8 & 70.8 & 0.66 & 164 & $<0.02$ & 0.001 \\
\hline$M H-4$ & NBD-221 & $<0.02$ & 0.85 & 7.78 & $<0.0005$ & 5.4 & 76.2 & 0.81 & 180 & $<0.02$ & 0.005 \\
\hline$M W-5$ & $\mathrm{NBD}-218$ & $<0.02$ & 1.02 & 9.56 & $<0.0010$ & 3.37 & 49.9 & 0.50 & 88.5 & $<0.02$ & $<0.001$ \\
\hline MH-6 & NBD -217 & $<0.02$ & 2.89 & 7.84 & $<0.0005$ & 5.32 & 56.6 & 0.59 & 132 & $<0.02$ & $<0.001$ \\
\hline$M W-7$ & NBD-713 & $<0.02$ & 0.14 & 9.54 & $<0.0005$ & 3.7 & 64.3 & 0.47 & 115 & $<0.02$ & $<0.001$ \\
\hline MH-8 & NBD -224 & $<0.02$ & 0.27 & 9.34 & $<0.0005$ & 4.2 & 35.8 & 0.33 & 112 & $<0.02$ & 0.011 \\
\hline MH-8 (Dup) & NBD-225 & $<0.02$ & 0.44 & No Data & $<0.0005$ & 4.2 & 35.6 & 0.34 & 111 & $<0.02$ & 0.004 \\
\hline MW-9 & NBD-222 & $<0.02$ & 0.54 & 6.92 & $<0.0005$ & 2.8 & 44.8 & 0.35 & 95.8 & $<0.02$ & 0.082 \\
\hline
\end{tabular}

$\mathrm{a}_{\mathrm{A}}$ "<" indicates that the maximum concentration was below the detection limit (number shown is detection limit). 
Table D-1 (continued). Oxnard Groundwater Data

Inorganics ${ }^{\mathrm{a}}$

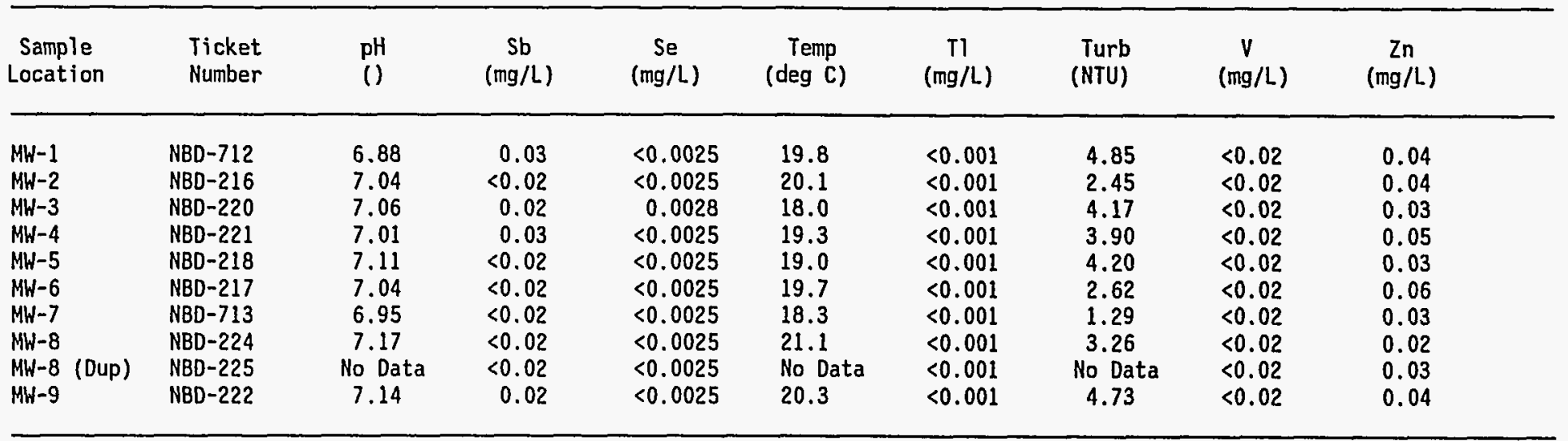

${ }_{\mathrm{A}} \mathrm{C}<"$ indicates that the maximum concentration was below the detection limit (number shown is detection limit).

Detected Volatile Organics

\begin{tabular}{lllll}
\hline $\begin{array}{l}\text { Sample } \\
\text { Location }\end{array}$ & $\begin{array}{l}\text { Ticket } \\
\text { Number }\end{array}$ & Analyte & Result & $\begin{array}{l}\text { Measurement } \\
\text { Unit }\end{array}$ \\
\hline $\begin{array}{llll}M H-2 \\
M H-2\end{array}$ & NBD-216 & $\begin{array}{l}1,1,1-\text { Trichloroethane } \\
1,1-D i c h l o r o e t h a n e\end{array}$ & 14.5 & $\mu \mathrm{g} / \mathrm{L}$ \\
& $N B D-216$ & 4.77 & $\mu \mathrm{L} / \mathrm{L}$ \\
\hline
\end{tabular}

Groundwater Detected PCBs: None.

Groundwater Detected Total Petroleum Hydrocarbons: None. 
Table D-2. Oxnard Soil Data

Inorganics ${ }^{a}$

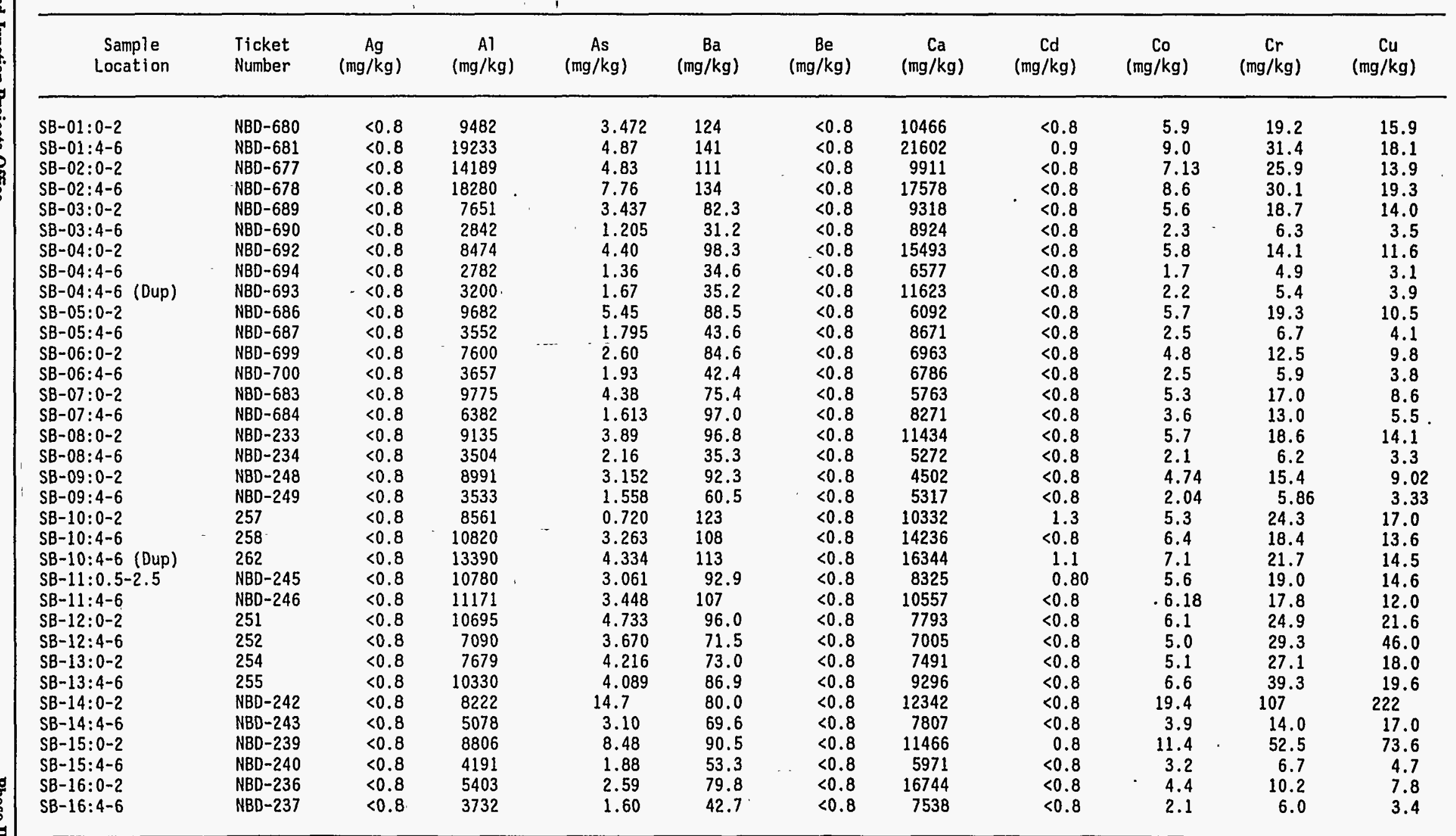

$a_{A}$ "<" indicates that the maximum concentration was below the detection limit (number shown is detection limit). 
Table D-2 (continued). Oxnard Soil Data

Inorganics ${ }^{a}$

\begin{tabular}{|c|c|c|c|c|c|c|c|c|c|c|c|}
\hline $\begin{array}{l}\text { Sample } \\
\text { Location }\end{array}$ & $\begin{array}{l}\text { Ticket } \\
\text { Number }\end{array}$ & $\begin{array}{c}F e \\
(\mathrm{mg} / \mathrm{kg})\end{array}$ & $\begin{array}{c}\mathrm{Hg} \\
(\mathrm{mg} / \mathrm{kg})\end{array}$ & $\begin{array}{c}K \\
(m g / k g)\end{array}$ & $\begin{array}{c}\mathrm{Mg} \\
(\mathrm{mg} / \mathrm{kg})\end{array}$ & $\begin{array}{c}M n \\
(m g / k g)\end{array}$ & $\begin{array}{c}\mathrm{Na} \\
(\mathrm{mg} / \mathrm{kg})\end{array}$ & $\begin{array}{c}N i \\
(m g / k g)\end{array}$ & $\begin{array}{c}\mathrm{Pb} \\
(\mathrm{mg} / \mathrm{kg})\end{array}$ & $\begin{array}{c}S b \\
(m g / k g)\end{array}$ & $\begin{array}{c}\mathrm{Se} \\
(\mathrm{mg} / \mathrm{kg})\end{array}$ \\
\hline $\begin{array}{l}\text { SB-01:0-2 } \\
\text { SB-01:4-6 } \\
\text { SB-02:0-2 } \\
\text { SB-02:4-6 } \\
\text { SB-03:0-2 } \\
\text { SB-03:4-6 } \\
\text { SB-04:0-2 } \\
\text { SB-04:4-6 } \\
\text { SB-04:4-6 (Dup) } \\
\text { SB-05:0-2 } \\
\text { SB-05:4-6 } \\
\text { SB-06:0-2 } \\
\text { SB-06:4-6 } \\
\text { SB-07:0-2 } \\
\text { SB-07:4-6 } \\
\text { SB-08:0-2 } \\
\text { SB-08:4-6 } \\
\text { SB-09:0-2 } \\
\text { SB-09:4-6 } \\
\text { SB-10:0-2 } \\
\text { SB-10:4-6 } \\
\text { SB-10:4-6 (Dup) } \\
\text { SB-11:0.5-2.5 } \\
\text { SB-11:4-6 } \\
\text { SB-12:0-2 } \\
\text { SB-12:4-6 } \\
\text { SB-13:0-2 } \\
\text { SB-13:4-6 } \\
\text { SB-14:0-2 } \\
\text { SB-14:4-6 } \\
\text { SB-15:0-2 } \\
\text { SB-15:4-6 } \\
\text { SB-16:0-2 } \\
\text { SB-16:4-6 }\end{array}$ & 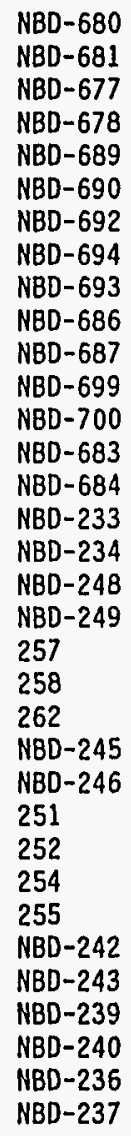 & $\begin{array}{r}20099 \\
27874 \\
20454 \\
28634 \\
18351 \\
6812 \\
16859 \\
5993 \\
7137 \\
17452 \\
8206 \\
14799 \\
7515 \\
15047 \\
11664 \\
21883 \\
6748 \\
13774 \\
6493 \\
17272 \\
19416 \\
21475 \\
17438 \\
18907 \\
20028 \\
20167 \\
25924 \\
19911 \\
142697 \\
16200 \\
52065 \\
8518 \\
11201 \\
6388\end{array}$ & $\begin{array}{c}0.037 \\
0.044 \\
<0.032 \\
0.052 \\
<0.033 \\
0.033 \\
<0.0325 \\
<0.0315 \\
<0.0303 \\
<0.033 \\
<0.033 \\
0.0332 \\
<0.0323 \\
<0.033 \\
<0.030 \\
<0.0323 \\
<0.0275 \\
0.0342 \\
0.0335 \\
0.0669 \\
0.0353 \\
<0.0294 \\
0.0498 \\
0.0558 \\
0.0379 \\
0.0314 \\
0.0459 \\
0.0480 \\
<0.0314 \\
<0.0325 \\
0.0880 \\
<0.0286 \\
0.0421 \\
<0.0292\end{array}$ & $\begin{array}{r}3106 \\
3676 \\
4038 \\
3323 \\
2542 \\
619 \\
2595 \\
587 \\
660 \\
2538 \\
655 \\
2316 \\
716 \\
2672 \\
1273 \\
2419 \\
715 \\
2234 \\
754 \\
3363 \\
2701 \\
3154 \\
2697 \\
2469 \\
2823 \\
1574 \\
1948 \\
2426 \\
2266 \\
1140 \\
2765 \\
1452 \\
1115 \\
755\end{array}$ & $\begin{array}{l}5355 \\
9244 \\
6398 \\
8980 \\
5173 \\
2042 \\
5635 \\
1609 \\
2108 \\
4604 \\
2466 \\
4350 \\
2045 \\
4027 \\
3061 \\
4914 \\
2031 \\
4082 \\
1644 \\
4869 \\
6326 \\
7073 \\
4834 \\
6094 \\
4706 \\
3299 \\
3753 \\
-4842 \\
4488 \\
2638 \\
4349 \\
2439 \\
5915 \\
1820\end{array}$ & $\begin{array}{c}291 \\
371 \\
293 \\
545 \\
256 \\
116 \\
253 \\
108 \\
262 \\
244 \\
141 \\
220 \\
133 \\
208 \\
144 \\
318 \\
90 \\
206 \\
20 \\
93.0 \\
283 \\
262 \\
284 \\
262 \\
260 \\
314 \\
3 \\
239 \\
295 \\
346 \\
1188 \\
172 \\
702 \\
731 \\
131 \\
241 \\
66\end{array}$ & $\begin{array}{l}136 \\
755 \\
206 \\
676 \\
256 \\
173 \\
178 \\
139 \\
110 \\
151 \\
209 \\
116 \\
111 \\
118 \\
148 \\
152 \\
149 \\
179 \\
123 \\
150 \\
288 \\
314 \\
275 \\
519 \\
254 \\
300 \\
198 \\
265 \\
405 \\
269 \\
472 \\
250 \\
235 \\
137\end{array}$ & $\begin{array}{c}18.1 \\
23.8 \\
19.5 \\
24.8 \\
17.9 \\
6.4 \\
16.5 \\
5.6 \\
6.4 \\
15.5 \\
7.4 \\
14.4 \\
6.7 \\
12.6 \\
8.9 \\
17.2 \\
5.3 \\
13.0 \\
5.33 \\
21.3 \\
18.1 \\
19.1 \\
16.5 \\
16.4 \\
17.8 \\
15.1 \\
16.6 \\
18.1 \\
121 \\
13.8 \\
43.2 \\
8.1 \\
10.5 \\
5.3\end{array}$ & $\begin{array}{c}20.5 \\
5.5 \\
14.8 \\
6.0 \\
20.9 \\
2.4 \\
4.6 \\
0.9 \\
1.6 \\
14.3 \\
3.7 \\
4.8 \\
2.0 \\
10.5 \\
3.7 \\
11.1 \\
1.9 \\
6.04 \\
3.01 \\
20.3 \\
3.8 \\
3.9 \\
25.0 \\
5.42 \\
47.6 \\
136 \\
07.5 \\
142 \\
14 \\
51.8 \\
\mid\end{array}$ & $\begin{array}{l}<0.8 \\
<0.8 \\
<0.8 \\
<0.8 \\
<0.8 \\
<0.8 \\
<0.8 \\
<0.8 \\
<0.8 \\
<0.8 \\
<0.8 \\
<0.8 \\
<0.8 \\
<0.8 \\
<0.8 \\
<0.8 \\
<0.8 \\
<0.8 \\
<0.8 \\
<0.8 \\
<0.8 \\
<0.8 \\
<0.8 \\
<0.8 \\
<0.8 \\
<0.8 \\
<0.8 \\
<0.8 \\
<0.8 \\
<0.8 \\
56.4 \\
<0.8 \\
<0.8 \\
<0.8\end{array}$ & $\begin{array}{c}<0.098 \\
0.257 \\
0.319 \\
0.230 \\
0.288 \\
<0.099 \\
0.321 \\
0.295 \\
0.166 \\
0.177 \\
0.106 \\
0.188 \\
0.143 \\
0.119 \\
0.339 \\
0.189 \\
<0.101 \\
0.2473 \\
<0.0996 \\
0.164 \\
0.262 \\
0.232 \\
0.2883 \\
0.1971 \\
0.195 \\
0.113 \\
0.116 \\
0.201 \\
<0.099 \\
<0.098 \\
<0.098 \\
0.110 \\
<0.101 \\
<0.100\end{array}$ \\
\hline
\end{tabular}

$a_{A}$ " $<"$ indicates that the maximum concentration was below the detection 1 imit (number shown is detection limit). 
Table D-2 (continued). Oxnard Soil Data

Inorganics ${ }^{a}$

\begin{tabular}{|c|c|c|c|c|}
\hline $\begin{array}{l}\text { Sample } \\
\text { Location }\end{array}$ & $\begin{array}{l}\text { Ticket } \\
\text { Number }\end{array}$ & $\begin{array}{c}\mathrm{Tl} \\
(\mathrm{mg} / \mathrm{kg})\end{array}$ & $\begin{array}{c}V \\
(\mathrm{mg} / \mathrm{kg})\end{array}$ & $\begin{array}{c}\mathrm{Zn} \\
(\mathrm{mg} / \mathrm{kg})\end{array}$ \\
\hline $\begin{array}{l}\text { SB-01:0-2 } \\
\text { SB-01:4-6 } \\
\text { SB-02:0-2 } \\
\text { SB-02:4-6 } \\
\text { SB-03:0-2 } \\
\text { SB-03:4-6 } \\
\text { SB-04:0-2 } \\
\text { SB-04:4-6 } \\
\text { SB-04:4-6 (Dup) } \\
\text { SB-05:0-2 } \\
\text { SB-05:4-6 } \\
\text { SB-06:0-2 } \\
\text { SB-06:4-6 } \\
\text { SB-07:0-2 } \\
\text { SB-07:4-6 } \\
\text { SB-08:0-2 } \\
\text { SB-08:4-6 } \\
\text { SB-09:0-2 } \\
\text { SB-09:4-6 } \\
\text { SB-10:0-2 } \\
\text { SB-10:4-6 } \\
\text { SB-10:4-6 (Dup) } \\
\text { SB-11:0.5-2.5 } \\
\text { SB-11:4-6 } \\
\text { SB-12:0-2 } \\
\text { SB-12:4-6 } \\
\text { SB-13:0-2 } \\
\text { SB-13:4-6 } \\
\text { SB-14:0-2 } \\
\text { SB-14:4-6 } \\
\text { SB-15:0-2 } \\
\text { SB-15:4-6 } \\
\text { SB-16:0-2 } \\
\text { SB-16:4-6 }\end{array}$ & $\begin{array}{l}\text { NBD-680 } \\
\text { NBD-681 } \\
\text { NBD-677 } \\
\text { NBD-678 } \\
\text { NBD-689 } \\
\text { NBD-690 } \\
\text { NBD-692 } \\
\text { NBD-694 } \\
\text { NBD-693 } \\
\text { NBD-686 } \\
\text { NBD-687 } \\
\text { NBD-699 } \\
\text { NBD-700 } \\
\text { NBD-683 } \\
\text { NBD-684 } \\
\text { NBD-233 } \\
\text { NBD-234 } \\
\text { NBD-248 } \\
\text { NBD-249 } \\
257 \\
258 \\
262 \\
\text { NBD-245 } \\
\text { NBD-246 } \\
251 \\
252 \\
254 \\
255 \\
\text { NBD-242 } \\
\text { NBD-243 } \\
\text { NBD-239 } \\
\text { NBD-240 } \\
\text { NBD }-236 \\
\text { NBD-237 }\end{array}$ & $\begin{array}{l}<0.8 \\
<0.8 \\
<0.8 \\
<0.8 \\
<0.8 \\
<0.8 \\
<0.8 \\
<0.8 \\
<0.8 \\
<0.8 \\
<0.8 \\
<0.8 \\
<0.8 \\
<0.8 \\
<0.8 \\
<0.8 \\
<0.8 \\
<0.8 \\
<0.8 \\
<0.8 \\
<0.8 \\
<0.8 \\
<0.8 \\
<0.8 \\
<0.8 \\
<0.8 \\
<0.8 \\
<0.8 \\
<0.8 \\
<0.8 \\
<0.8 \\
<0.8 \\
<0.8 \\
<0.8\end{array}$ & $\begin{array}{r}33.5 \\
61.6 \\
49.6 \\
57.0 \\
27.2 \\
9.8 \\
26.3 \\
9.7 \\
11.5 \\
35.4 \\
14.5 \\
23.0 \\
7.4 \\
34.1 \\
25.7 \\
32.1 \\
11.6 \\
28.4 \\
13.5 \\
29.6 \\
33.5 \\
39.9 \\
33.4 \\
41.1 \\
34.7 \\
26.7 \\
28.0 \\
33.9 \\
57.9 \\
18.9 \\
45.5 \\
14.9 \\
21.6 \\
12.6\end{array}$ & $\begin{array}{r}67.1 \\
69.7 \\
62.7 \\
66.1 \\
50.9 \\
15.5 \\
48.3 \\
14.9 \\
16.1 \\
52.2 \\
20.7 \\
42.5 \\
18.9 \\
40.5 \\
26.3 \\
78.0 \\
17.7 \\
41.8 \\
16.3 \\
91.2 \\
52.9 \\
56.2 \\
396 \\
50.2 \\
54.1 \\
101 \\
46.6 \\
56.6 \\
69.5 \\
28.1 \\
78.4 \\
21.5 \\
30.7 \\
15.6\end{array}$ \\
\hline
\end{tabular}

detection limit (number shown is detection limit). 
Table D-2 (continued). Oxnard Soil Data

Detected Volatile Organics

\begin{tabular}{|c|c|c|c|c|}
\hline $\begin{array}{l}\text { Sample } \\
\text { Location }\end{array}$ & $\begin{array}{l}\text { Ticket } \\
\text { Number }\end{array}$ & Analyte & Result & $\begin{array}{l}\text { Measurement } \\
\text { Unit }\end{array}$ \\
\hline $\begin{array}{l}\text { SB-10:0-2 } \\
\text { SB-10:0-2 } \\
\text { SB-10:4-6 } \\
\text { SB-11:4-6 } \\
\text { SB-11:4-6 } \\
\text { SB-12:0-2 } \\
\text { SB-12:4-6 } \\
\text { SB-12:4-6 } \\
\text { SB-13:4-6 } \\
\text { SB-13:4-6 } \\
\text { SB-13:4-6 } \\
\text { SB-14:0-2 } \\
\text { SB-14:0-2 } \\
\text { SB-14:0-2 } \\
\text { SB-14:4-6 } \\
\text { SB-14:4-6 } \\
\text { SB-14:4-6 } \\
\text { SB-15:4-6 } \\
\text { SB-15:4-6 } \\
\text { SB-16:0-2 } \\
\text { SB-16:0-2 } \\
\text { SB-16:4-6 }\end{array}$ & $\begin{array}{l}257 \\
257 \\
258 \\
\text { NBD }-246 \\
\text { NBD-246 } \\
251 \\
252 \\
252 \\
255 \\
255 \\
255 \\
\text { NBD-242 } \\
\text { NBD-242 } \\
\text { NBD-242 } \\
\text { NBD-243 } \\
\text { NBD-243 } \\
\text { NBD-243 } \\
\text { NBD-240 } \\
\text { NBD }-240 \\
\text { NBD-236 } \\
\text { NBD }-236 \\
\text { NBD-237 }\end{array}$ & $\begin{array}{l}\text { 2-Butanone } \\
\text { Acetone } \\
\text { Acetone } \\
\text { 2-Butanone } \\
\text { Toluene } \\
\text { Acetone } \\
\text { 2-Butanone } \\
\text { Toluene } \\
\text { 2-Butanone } \\
\text { Acetone } \\
\text { Toluene } \\
\text { 1,1,1-Trichloroethane } \\
\text { 2-Butanone } \\
\text { Toluene } \\
\text { 2-Butanone } \\
\text { Acetone } \\
\text { Toluene } \\
\text { 2-Butanone } \\
\text { Toluene } \\
\text { 2-Butanone } \\
\text { Toluene } \\
\text { Toluene }\end{array}$ & $\begin{array}{c}299 \\
845 . \\
17.1 \\
55.8 \\
9.44 \\
125 . \\
21.3 \\
6.24 \\
35.5 \\
21.9 \\
5.14 \\
4.29 \\
65.7 \\
12.4 \\
51.9 \\
23.2 \\
2.33 \\
23.8 \\
3.03 \\
41.6 \\
6.67 \\
2.67\end{array}$ & $\begin{array}{l}\mu g / \mathrm{kg} \\
\mu g / \mathrm{kg} \\
\mu g / \mathrm{kg} \\
\mu g / \mathrm{kg} \\
\mu g / \mathrm{kg} \\
\mu g / \mathrm{kg} \\
\mu g / \mathrm{kg} \\
\mu g / \mathrm{kg} \\
\mu g / \mathrm{kg} \\
\mu g / \mathrm{kg} \\
\mu g / \mathrm{kg} \\
\mu g / \mathrm{kg} \\
\mu g / \mathrm{kg} \\
\mu g / \mathrm{kg} \\
\mu g / \mathrm{kg} \\
\mu g / \mathrm{kg} \\
\mu g / \mathrm{kg} \\
\mu g / \mathrm{kg} \\
\mu g / \mathrm{kg} \\
\mu g / \mathrm{kg} \\
\mu g / \mathrm{kg} \\
\mu g / \mathrm{kg}\end{array}$ \\
\hline
\end{tabular}

Detected PCBs

\begin{tabular}{lllrr}
\hline $\begin{array}{l}\text { Sample } \\
\text { Location }\end{array}$ & $\begin{array}{l}\text { Ticket } \\
\text { Number }\end{array}$ & Analyte & Result & $\begin{array}{c}\text { Measurement } \\
\text { Unit }\end{array}$ \\
\hline SB-01:0-2 & NBD-680 & Arochlor 1254 & 540 & $\mu \mathrm{g} / \mathrm{kg}$ \\
SB-10:0-2 & 257 & Arochlor 1248 & 3900 & $\mu \mathrm{g} / \mathrm{kg}$ \\
SB-10:4-6 & 258 & Arochlor 1248 & 60 & $\mu \mathrm{g} / \mathrm{kg}$ \\
SB-10:4-6 (Dup) & 262 & Arochlor 1248 & 370 & $\mu \mathrm{g} / \mathrm{kg}$ \\
& & & & \\
\hline
\end{tabular}

Soil Detected Total Petroleum Hydrocarbons: None. 
Table D-2 (continued). Oxnard Soil Data

Detected Volatile Organics

\begin{tabular}{|c|c|c|c|c|c|}
\hline $\begin{array}{l}\text { Sample } \\
\text { Location }\end{array}$ & $\begin{array}{l}\text { Ticket } \\
\text { Number }\end{array}$ & Analyte & & Result & $\begin{array}{l}\text { Measurement } \\
\text { Unit }\end{array}$ \\
\hline $\begin{array}{l}\text { SB-01:0-2 } \\
\text { SB-01:0-2 } \\
\text { SB-01:0-2 } \\
\text { SB-01:4-6 } \\
\text { SB-02:4-6 } \\
\text { SB-02:4-6 } \\
\text { SB-03:0-2 } \\
\text { SB-03:0-2 } \\
\text { SB-03:4-6 } \\
\text { SB-04:0-2 } \\
\text { SB-04:0-2 } \\
\text { SB-04:4-6 } \\
\text { SB-04:4-6 } \\
\text { SB-04:4-6 } \\
\text { SB-04:4-6 } \\
\text { SB-04:4-6 (Dup) } \\
\text { SB-05:0-2 } \\
\text { SB-05:0-2 } \\
\text { SB-05:4-6 } \\
\text { SB-05:4-6 } \\
\text { SB-06:0-2 } \\
\text { SB-06:4-6 } \\
\text { SB-06:4-6 } \\
\text { SB-06:4-6 } \\
\text { SB-07:0-2 } \\
\text { SB-07:0-2 } \\
\text { SB-07:4-6 } \\
\text { SB-07:4-6 } \\
\text { SB-08:4-6 } \\
\text { SB-08:4-6 } \\
\text { SB-08:4-6 } \\
\text { SB-08:4-6 } \\
\text { SB-09:0-2 } \\
\text { SB-09:4-6 } \\
\text { SB-09:4-6 } \\
\text { SB-09:4-6 }\end{array}$ & $\begin{array}{l}\text { NBD-680 } \\
\text { NBD-680 } \\
\text { NBD-680 } \\
\text { NBD-681 } \\
\text { NBD-678 } \\
\text { NBD-678 } \\
\text { NBD-689 } \\
\text { NBD-689 } \\
\text { NBD-690 } \\
\text { NBD-692 } \\
\text { NBD-692 } \\
\text { NBD-694 } \\
\text { NBD-694 } \\
\text { NBD-694 } \\
\text { NBD-694 } \\
\text { NBD-693 } \\
\text { NBD-686 } \\
\text { NBD-686 } \\
\text { NBD-687 } \\
\text { NBD-687 } \\
\text { NBD-699 } \\
\text { NBD-700 } \\
\text { NBD-700 } \\
\text { NBD-700 } \\
\text { NBD-683 } \\
\text { NBD-683 } \\
\text { NBD-684 } \\
\text { NBD-684 } \\
\text { NBD-234 } \\
\text { NBD-234 } \\
\text { NBD-234 } \\
\text { NBD-234 } \\
\text { NBD-248 } \\
\text { NBD-249 } \\
\text { NBD-249 } \\
\text { NBD-249 }\end{array}$ & $\begin{array}{l}\text { 1,1,1-Trichloroethane } \\
\text { Acetone } \\
\text { Toluene } \\
\text { 1,1,1-Trichloroethane } \\
1,1,1-\text { Trichloroethane } \\
\text { Toluene } \\
\text { 2-Butanone } \\
\text { Acetone } \\
\text { Acetone } \\
\text { 2-Butanone } \\
\text { Acetone } \\
\text { 1,1,1-Trichloroethane } \\
\text { 2-Butanone } \\
\text { Acetone } \\
\text { Toluene } \\
\text { Acetone } \\
\text { 1,1,1-Trichloroethane } \\
\text { Toluene } \\
\text { Acetone } \\
\text { Toluene } \\
\text { 2-Butanone } \\
\text { 2-Butanone } \\
\text { Acetone } \\
\text { Toluene } \\
\text { 1,1,1-Trichloroethane } \\
\text { Toluene } \\
\text { 1,1,1-Trichloroethane } \\
\text { Toluene } \\
\text { 1,1,1-Trichloroethane } \\
\text { 2-Butanone } \\
\text { Acetone } \\
\text { Toluene } \\
\text { Toluene } \\
\text { 2-Butanone } \\
\text { Acetone } \\
\text { Toluene }\end{array}$ & . & $\begin{array}{c}6.36 \\
20.6 \\
4.35 \\
5.37 \\
3.70 \\
2.80 \\
31.5 \\
48.3 \\
43.7 \\
16.1 \\
42.9 \\
4.40 \\
35.4 \\
84.2 \\
8.78 \\
57.2 \\
4.97 \\
4.41 \\
16.7 \\
2.34 \\
68.5 \\
42.8 \\
16.1 \\
19.2 \\
3.23 \\
3.28 \\
2.82 \\
2.71 \\
2.20 \\
28.4 \\
30.7 \\
2.97 \\
5.74 \\
29.6 \\
50.8 \\
4.90\end{array}$ & 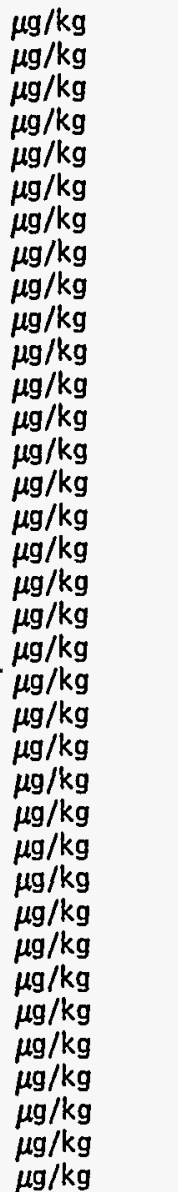 \\
\hline
\end{tabular}


Table D-3. Oxnard WET Test and TCLP Data ${ }^{a}$

\begin{tabular}{|c|c|c|c|c|c|c|c|c|c|c|}
\hline $\begin{array}{l}\text { Sample } \\
\text { Location }\end{array}$ & $\begin{array}{l}\text { Ticket } \\
\text { Number }\end{array}$ & $\underset{(m g / L)}{c d^{b}}$ & $\begin{array}{c}c d^{c} \\
(m g / L)\end{array}$ & $\begin{array}{r}\mathrm{Cr} r^{b} \\
(m g / L)\end{array}$ & $\underset{(m g / L)}{C r^{c}}$ & $\underset{(m g / L)}{C u^{b}}$ & $\underset{(m g / L)}{N i^{b}}$ & $\begin{array}{c}\mathrm{Pb}^{\mathrm{b}} \\
(\mathrm{mg} / \mathrm{L})\end{array}$ & $\begin{array}{c}P b^{C} \\
(m g / L)\end{array}$ & $\begin{array}{l}\mathrm{Zn}^{\mathrm{b}} \\
(\mathrm{mg} / \mathrm{L})\end{array}$ \\
\hline $\begin{array}{l}\text { Oil-1 } \\
\text { SB-12:T.D. } \\
\text { SB-13:T.D. } \\
\text { SL-1 } \\
\text { SL-1 (Dup) } \\
\text { SL-2 } \\
\text { SL-3 } \\
\text { SL-5 } \\
\text { SL-6 } \\
\text { SL-7 }\end{array}$ & $\begin{array}{l}\text { NBD }-711 \\
253 \\
256 \\
\text { NBD }-707 \\
\text { NBD }-708 \\
\text { NBD }-709 \\
\text { NBD }-701 \\
\text { NBD }-702 \\
\text { NBD }-705 \\
\text { NBD }-704\end{array}$ & $\begin{array}{l}\text { No Data } \\
\text { No Data } \\
\text { No Data } \\
\text { No Data } \\
\text { No Data } \\
\text { No Data } \\
\text { No Data } \\
0.1 \\
\text { No Data } \\
0.6\end{array}$ & $\begin{array}{l}\text { No Data } \\
\text { No Data } \\
\text { No Data } \\
\text { No Data } \\
\text { No Data } \\
\text { No Data } \\
\text { No Data } \\
<0.1 \\
\text { No Data } \\
<0.1\end{array}$ & $\begin{array}{l}\text { No Data } \\
\text { No Data } \\
0.2 \\
1.7 \\
1.3 \\
5.7^{d} \\
4.2 \\
\text { No Data } \\
1.3 \\
1.6\end{array}$ & $\begin{array}{l}\text { No Data } \\
\text { No Data } \\
\text { No Data } \\
<0.1 \\
<0.1 \\
<0.1 \\
<0.1 \\
\text { No Data } \\
<0.1 \\
\text { No Data }\end{array}$ & $\begin{array}{l}\text { No Data } \\
\text { No Data } \\
\text { No Data } \\
<0.1 \\
<0.1 \\
<0.1 \\
<0.1 \\
<0.1 \\
3.8 \\
0.6\end{array}$ & $\begin{array}{l}\text { No Data } \\
\text { No Data } \\
\text { No Data } \\
5.0 \\
4.6 \\
21.7 \text { d } \\
\text { No Data } \\
\text { No Data } \\
6.0 \\
7.8\end{array}$ & $\begin{array}{l}<0.16 \\
2.0 \\
3.4 \\
1.4 \\
4.5 \\
0.4 \\
0.2 \\
9.5^{d} \\
6.9 \mathrm{~d} \\
4.8\end{array}$ & $\begin{array}{l}\text { No Data } \\
\text { No Data } \\
<0.1 \\
<0.1 \\
<0.1 \\
<0.1 \\
\text { No Data } \\
0.3 \\
0.1 \\
\text { No Data }\end{array}$ & $\begin{array}{l}\text { No Data } \\
\text { No Data } \\
\text { No Data } \\
\text { No Data } \\
\text { No Data } \\
\text { No Data } \\
\text { No Data } \\
252.1 \text { d } \\
\text { No Data } \\
\text { No Data }\end{array}$ \\
\hline
\end{tabular}

${ }_{A}$ " $<1$ indicates that the maximum concentration was below the detection limit (number shown is detection limit).

b Hazardous Haste evaluation per Title 22 California Administrative Code of Regulations (Waste Extraction (HET) test).

CHazardous Waste evaluation per Title 22 California Administrative Code of Regulations (Toxicity Characteristic Leaching Procedure (TCLP) test).

dithe elements identified with this footnote exceed the limits for hazardous classification. 
Table D-4. Oxnard Sludge and Oil Data

Inorganics ${ }^{a}$

\begin{tabular}{|c|c|c|c|c|c|c|c|c|c|c|c|}
\hline $\begin{array}{l}\text { Sample } \\
\text { Location }\end{array}$ & $\begin{array}{l}\text { Ticket } \\
\text { Number }\end{array}$ & $\begin{array}{c}\mathrm{Ag} \\
(\mathrm{mg} / \mathrm{kg})\end{array}$ & $\begin{array}{c}\mathrm{As} \\
(\mathrm{mg} / \mathrm{kg})\end{array}$ & $\begin{array}{c}\mathrm{Ba} \\
(\mathrm{mg} / \mathrm{kg})\end{array}$ & $\begin{array}{c}\mathrm{Be} \\
(\mathrm{mg} / \mathrm{kg})\end{array}$ & $\begin{array}{c}\mathrm{Cd} \\
(\mathrm{mg} / \mathrm{kg})\end{array}$ & $\begin{array}{c}\text { Co } \\
(\mathrm{mg} / \mathrm{kg})\end{array}$ & $\underset{(\mathrm{mg} / \mathrm{kg})}{\mathrm{Cr}}$ & $\underset{(\mathrm{mg} / \mathrm{kg})}{\mathrm{Cu}}$ & $\begin{array}{c}\mathrm{Hg} \\
(\mathrm{mg} / \mathrm{kg})\end{array}$ & $\begin{array}{c}\text { Ignit } \\
(\operatorname{deg} F)\end{array}$ \\
\hline $\begin{array}{l}0 i 1-1 \\
S L-1 \\
S L-1 \text { (Dup) } \\
\text { SL-2 } \\
\text { SL-3 } \\
\text { SL-5 } \\
S L-6 \\
S L-7\end{array}$ & $\begin{array}{l}\text { NBD-711 } \\
\text { NBD-707 } \\
\text { NBD-708 } \\
\text { NBD-709 } \\
\text { NBD-701 } \\
\text { NBD } 702 \\
\text { NBD-705 } \\
\text { NBD }-704\end{array}$ & $\begin{array}{l}<1.0 \\
<0.8 \\
<0.8 \\
<0.8 \\
<0.8 \\
<0.8 \\
<0.8 \\
<0.8\end{array}$ & $\begin{array}{c}<0.1244 \\
3.249 \\
10.41 \\
\cdot 7.496 \\
1.298 \\
3.971 \\
1.071 \\
0.935\end{array}$ & $\begin{array}{r}2.8 \\
62.0 \\
52.0 \\
51.6 \\
90.6 \\
37.8 \\
13.1 \\
7.8\end{array}$ & $\begin{array}{l}<1.0 \\
<0.8 \\
<0.8 \\
<0.8 \\
<0.8 \\
<0.8 \\
<0.8 \\
<0.8\end{array}$ & $\begin{array}{r}2.8 \\
2.3 \\
3.2 \\
4.8 \\
6.4 \\
40.6 \\
6.7 \\
22.4\end{array}$ & $\begin{array}{c}<1.0 \\
92.6 \\
160 \\
80.2 \\
11.1 \\
12.1 \\
69.4 \\
14.0\end{array}$ & $\begin{array}{c}2.8 \\
381 \\
279 \\
572 \\
131 \\
33.1 \\
129 \\
60.6\end{array}$ & $\begin{array}{c}86.8 \\
1831 \\
892 \\
1411 \\
531 \\
266 \\
765 \\
326\end{array}$ & $\begin{array}{c}<0.0005 \\
0.0314 \\
0.0304 \\
0.061 \\
0.0441 \\
<0.0311 \\
0.0277 \\
0.0389\end{array}$ & $\begin{array}{c}>212 \\
\text { No Data } \\
\text { No Data } \\
\text { No Data } \\
\text { No Data } \\
\text { No Data } \\
\text { No Data } \\
\text { No Data }\end{array}$ \\
\hline
\end{tabular}

$a_{A} "<"$ indicates that the maximum concentration was below the detection limit (number shown is detection limit).

\begin{tabular}{|c|c|c|c|c|c|c|c|c|c|c|}
\hline $\begin{array}{l}\text { Sample } \\
\text { Location }\end{array}$ & $\begin{array}{l}\text { Ticket } \\
\text { Number }\end{array}$ & $\begin{array}{c}\text { Mo } \\
(\mathrm{mg} / \mathrm{kg})\end{array}$ & $\begin{array}{c}\mathrm{Ni} \\
(\mathrm{mg} / \mathrm{kg})\end{array}$ & $\begin{array}{c}\mathrm{Pb} \\
(\mathrm{mg} / \mathrm{kg})\end{array}$ & $\begin{array}{c}S b \\
(m g / k g)\end{array}$ & $\begin{array}{c}\mathrm{Se} \\
(\mathrm{mg} / \mathrm{kg})\end{array}$ & $\begin{array}{c}T 1 \\
(\mathrm{mg} / \mathrm{kg})\end{array}$ & $\begin{array}{c}\text { TOX } \\
(\mathrm{mg} / \mathrm{kg})\end{array}$ & $\begin{array}{c}V \\
(\mathrm{mg} / \mathrm{kg})\end{array}$ & $\begin{array}{c}\mathrm{Zn} \\
(\mathrm{mg} / \mathrm{kg})\end{array}$ \\
\hline $\begin{array}{l}0 i 1-1 \\
\text { SL-1 } \\
\text { SL-1 (Dup) } \\
\text { SL-2 } \\
\text { SL-3 } \\
\text { SL-5 } \\
\text { SL-6 } \\
\text { SL-7 }\end{array}$ & $\begin{array}{l}\text { NBD-711 } \\
\text { NBD-707 } \\
\text { NBD -708 } \\
\text { NBD-709 } \\
\text { NBD -701 } \\
\text { NBD -702 } \\
\text { NBD-705 } \\
\text { NBD-704 }\end{array}$ & $\begin{array}{r}1.3 \\
97.9 \\
122 \\
43.7 \\
73.8 \\
16.4 \\
72.3 \\
17.0\end{array}$ & $\begin{array}{c}14.8 \\
611 \\
476 \\
1543 \\
149 \\
91.1 \\
550 \\
260\end{array}$ & $\begin{array}{l}73.4 \\
226 \\
209 \\
441 \\
76.4 \\
120 \\
201 \\
61.7\end{array}$ & $\begin{array}{r}<1.0 \\
<0.8 \\
<0.8 \\
<0.8 \\
<0.8 \\
8.3 \\
<0.8 \\
<0.8\end{array}$ & $\begin{array}{l}<0.1244 \\
<0.098 \\
<0.098 \\
<0.098 \\
<0.100 \\
<0.098 \\
<0.100 \\
<0.100\end{array}$ & $\begin{array}{l}<1.0 \\
<0.8 \\
<0.8 \\
<0.8 \\
<0.8 \\
<0.8 \\
<0.8 \\
<0.8\end{array}$ & $\begin{array}{l}54 \\
\text { No Data } \\
\text { No Data } \\
\text { No Data } \\
\text { No Data } \\
\text { No Data } \\
\text { No Data } \\
\text { No Data }\end{array}$ & $\begin{array}{r}<1.0 \\
51.7 \\
44.6 \\
42.4 \\
29.9 \\
20.1 \\
20.1 \\
6.2\end{array}$ & $\begin{array}{r}421 \\
999 \\
817 \\
871 \\
998 \\
3735 \\
1497 \\
461\end{array}$ \\
\hline
\end{tabular}

${ }^{a} A$ "<" indicates that the maximum concentration was below the detection limit (number shown is detection 1 imit).

Sludge and 0 il Detected PCBs: None. 


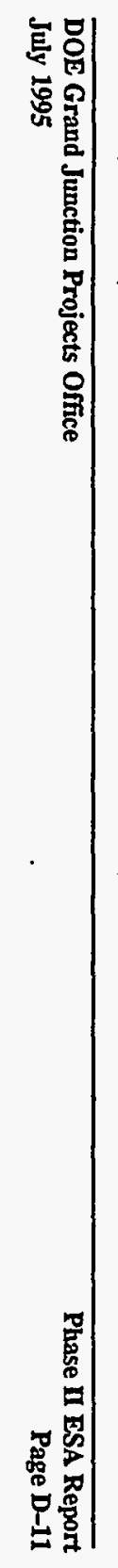

Table D-5. Oxnard Composite Soil Data

Inorganics $a$

\begin{tabular}{|c|c|c|c|c|c|c|c|c|c|c|c|}
\hline $\begin{array}{l}\text { Sample } \\
\text { Location }\end{array}$ & $\begin{array}{l}\text { Ticket } \\
\text { Number }\end{array}$ & $\begin{array}{c}\mathrm{Ag} \\
(\mathrm{mg} / \mathrm{kg})\end{array}$ & $\begin{array}{c}\text { As } \\
(\mathrm{mg} / \mathrm{kg})\end{array}$ & $\begin{array}{c}B a \\
(\mathrm{mg} / \mathrm{kg})\end{array}$ & $\begin{array}{c}B e \\
(\mathrm{mg} / \mathrm{kg})\end{array}$ & $\begin{array}{c}\text { Cd } \\
(\mathrm{mg} / \mathrm{kg})\end{array}$ & $\begin{array}{c}\text { Co } \\
(\mathrm{mg} / \mathrm{kg})\end{array}$ & $\begin{array}{c}\mathrm{Cr} \\
(\mathrm{mg} / \mathrm{kg})\end{array}$ & $\underset{(m g / k g)}{C u}$ & $\underset{(m g / k g)}{F}$ & $\underset{(\mathrm{mg} / \mathrm{kg})}{\mathrm{Hg}}$ \\
\hline $\begin{array}{l}\text { SB-01:T.D. } \\
\text { SB-02:T.D. } \\
\text { SB-03:T.D. } \\
\text { SB-04:T.D. } \\
\text { SB-04:T.D. (Dup) } \\
\text { SB-05:T.D. } \\
\text { SB-06:T.D. } \\
\text { SB-07:T.D. } \\
\text { SB-08:T.D. } \\
\text { SB-09:T.D. } \\
\text { SB-10:T.D. } \\
\text { SB-11:T.D. } \\
\text { SB-12:T.D. } \\
\text { SB-13:T.D. } \\
\text { SB-14:T.D. } \\
\text { SB-15:T.D. } \\
\text { SB-16:T.D. }\end{array}$ & 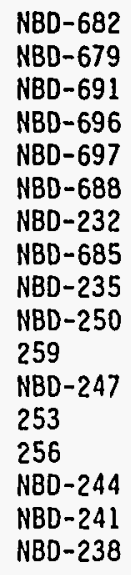 & $\begin{array}{l}<0.8 \\
<0.8 \\
<0.8 \\
<0.8 \\
<0.8 \\
<0.8 \\
<0.8 \\
<0.8 \\
<0.8 \\
<0.8 \\
<0.8 \\
<0.8 \\
<0.8 \\
<0.8 \\
<0.8 \\
<0.8 \\
<0.8\end{array}$ & $\begin{array}{l}1.51 \\
1.658 \\
2.054 \\
4.82 \\
2.18 \\
1.487 \\
1.87 \\
2.222 \\
2.75 \\
0.7633 \\
3.928 \\
2.559 \\
3.163 \\
2.732 \\
9.10 \\
3.59 \\
2.03\end{array}$ & $\begin{array}{r}47.5 \\
54.8 \\
41.2 \\
53.4 \\
54.5 \\
33.4 \\
52.9 \\
44.6 \\
50.5 \\
43.6 \\
115 \\
106 \\
82.6 \\
81.8 \\
86.4 \\
89.0 \\
61.1\end{array}$ & $\begin{array}{l}<0.8 \\
<0.8 \\
<0.8 \\
<0.8 \\
<0.8 \\
<0.8 \\
<0.8 \\
<0.8 \\
<0.8 \\
<0.8 \\
<0.8 \\
<0.8 \\
<0.8 \\
<0.8 \\
<0.8 \\
<0.8 \\
<0.8\end{array}$ & $\begin{array}{l}<0.8 \\
<0.8 \\
<0.8 \\
<0.8 \\
<0.8 \\
<0.8 \\
<0.8 \\
<0.8 \\
<0.8 \\
<0.8 \\
0.9 \\
0.82 \\
<0.8 \\
<0.8 \\
<0.8 \\
<0.8 \\
<0.8\end{array}$ & $\begin{array}{l}3.1 \\
3.4 \\
3.1 \\
1.6 \\
3.0 \\
2.1 \\
3.1 \\
2.9 \\
2.5 \\
2.2 \\
6.4 \\
5.9 \\
4.2 \\
5.6 \\
9.6 \\
5.9 \\
2.9\end{array}$ & $\begin{array}{r}9.3 \\
9.9 \\
7.8 \\
4.8 \\
9.9 \\
6.8 \\
8.8 \\
8.9 \\
7.0 \\
6.5 \\
28.7 \\
20.2 \\
19.3 \\
50.0 \\
40.2 \\
17.8 \\
8.4\end{array}$ & $\begin{array}{r}6.0 \\
5.5 \\
6.5 \\
3.6 \\
6.0 \\
4.3 \\
4.9 \\
4.7 \\
4.1 \\
3.8 \\
21.8 \\
13.0 \\
19.1 \\
21.6 \\
85.2 \\
14.6 \\
4.9\end{array}$ & $\begin{array}{r}9.2 \\
<5.0 \\
5.1 \\
<5.0 \\
5.5 \\
<5.0 \\
5.4 \\
<5.0 \\
<5.0 \\
<5.0 \\
7.6 \\
8.2 \\
11.0 \\
9.8 \\
8.3 \\
10.2 \\
<5.0\end{array}$ & $\begin{array}{c}<0.032 \\
<0.031 \\
<0.032 \\
0.0300 \\
0.0334 \\
<0.031 \\
<0.0296 \\
<0.031 \\
<0.0333 \\
<0.0272 \\
0.0438 \\
0.0415 \\
0.0297 \\
0.0560 \\
<0.0305 \\
0.0341 \\
0.0355\end{array}$ \\
\hline
\end{tabular}

$a_{A}$ "<" indicates that the maximum concentration was below the detection limit (number shown is detection 1 imit). 
Table D-5 (continued). Oxnard Composite Soil Data

Inorganics ${ }^{a}$

\begin{tabular}{|c|c|c|c|c|c|c|c|c|c|}
\hline $\begin{array}{l}\text { Sample } \\
\text { Location }\end{array}$ & $\begin{array}{l}\text { Ticket } \\
\text { Number }\end{array}$ & $\begin{array}{c}\text { Mo } \\
(\mathrm{mg} / \mathrm{kg})\end{array}$ & $\underset{(\mathrm{mg} / \mathrm{kg})}{\mathrm{Ni}}$ & $\begin{array}{c}\mathrm{Pb} \\
(\mathrm{mg} / \mathrm{kg})\end{array}$ & $\begin{array}{c}\mathrm{Sb} \\
(\mathrm{mg} / \mathrm{kg})\end{array}$ & $\begin{array}{c}\mathrm{Se} \\
(\mathrm{mg} / \mathrm{kg})\end{array}$ & $\begin{array}{c}\mathrm{Tl} \\
(\mathrm{mg} / \mathrm{kg})\end{array}$ & $\begin{array}{c}V \\
(m g / k g)\end{array}$ & $\underset{(m g / k g)}{Z n}$ \\
\hline $\begin{array}{l}\text { SB-01:T.D. } \\
\text { SB-02:T.D. } \\
\text { SB-03:T.D. } \\
\text { SB-04:T.D. } \\
\text { SB-04:T.D. (Dup) } \\
\text { SB-05:T.D. } \\
\text { SB-06:T.D. } \\
\text { SB-07:T.D. } \\
\text { SB-08:T.D. } \\
\text { SB-09:T.D. } \\
\text { SB-10:T.D. } \\
\text { SB-11:T.D. } \\
\text { SB-12:T.D. } \\
\text { SB-13:T.D. } \\
\text { SB-14:T.D. } \\
\text { SB-15:T.D. } \\
\text { SB-16:T.D. }\end{array}$ & $\begin{array}{l}\text { NBD-682 } \\
\text { NBD-679 } \\
\text { NBD-691 } \\
\text { NBD-696 } \\
\text { NBD-697 } \\
\text { NBD-688 } \\
\text { NBD-232 } \\
\text { NBD-685 } \\
\text { NBD-235 } \\
\text { NBD-250 } \\
259 \\
\text { NBD-247 } \\
253 \\
256 \\
\text { NBD-244 } \\
\text { NBD-241 } \\
\text { NBD-238 }\end{array}$ & $\begin{array}{r}<0.8 \\
<0.8 \\
<0.8 \\
<0.8 \\
<0.8 \\
<0.8 \\
<0.8 \\
<0.8 \\
<0.8 \\
<0.8 \\
1.0 \\
<0.8 \\
0.8 \\
8.3 \\
2.6 \\
0.8 \\
<0.8\end{array}$ & $\begin{array}{r}8.4 \\
8.1 \\
9.3 \\
5.0 \\
10.2 \\
6.9 \\
7.7 \\
8.0 \\
7.0 \\
7.0 \\
21.4 \\
15.8 \\
12.8 \\
18.0 \\
44.7 \\
16.6 \\
7.7\end{array}$ & $\begin{array}{r}2.3 \\
2.4 \\
6.5 \\
2.0 \\
1.3 \\
2.2 \\
2.7 \\
3.3 \\
1.4 \\
2.3 \\
11.7 \\
19.6 \\
53.3 \\
211 \\
23.7 \\
9.1 \\
2.3\end{array}$ & $\begin{array}{l}<0.8 \\
<0.8 \\
<0.8 \\
<0.8 \\
<0.8 \\
<0.8 \\
<0.8 \\
<0.8 \\
<0.8 \\
<0.8 \\
<0.8 \\
<0.8 \\
<0.8 \\
<0.8 \\
<0.8 \\
<0.8 \\
<0.8\end{array}$ & $\begin{array}{l}0.344 \\
0.172 \\
0.191 \\
1.68 \\
0.231 \\
0.152 \\
0.224 \\
0.237 \\
0.135 \\
0.1496 \\
0.172 \\
0.1844 \\
0.153 \\
0.168 \\
0.116 \\
0.177 \\
0.192\end{array}$ & $\begin{array}{l}<0.8 \\
<0.8 \\
<0.8 \\
<0.8 \\
<0.8 \\
<0.8 \\
<0.8 \\
<0.8 \\
<0.8 \\
<0.8 \\
<0.8 \\
<0.8 \\
<0.8 \\
<0.8 \\
<0.8 \\
<0.8 \\
<0.8\end{array}$ & $\begin{array}{r}18.5 \\
20.3 \\
14.3 \\
8.6 \\
18.1 \\
13.2 \\
17.6 \\
20.1 \\
15.5 \\
14.3 \\
36.8 \\
35.9 \\
24.5 \\
29.6 \\
33.2 \\
27.2 \\
18.7\end{array}$ & $\begin{array}{r}25.1 \\
25.1 \\
26.1 \\
15.5 \\
26.4 \\
18.3 \\
24.4 \\
22.3 \\
19.7 \\
19.0 \\
65.5 \\
131 \\
46.0 \\
59.1 \\
60.4 \\
41.5 \\
21.9\end{array}$ \\
\hline
\end{tabular}

$a_{A}$ "<" Indicates that the maximum concentration was below the detection limit (number shown is detection 1 imit). 
Table D-5 (continued). Oxnard Composite Soil Data

Detected Volatile Organics

\begin{tabular}{lllcc}
\hline $\begin{array}{l}\text { Sample } \\
\text { Location }\end{array}$ & $\begin{array}{l}\text { Ticket } \\
\text { Number }\end{array}$ & Analyte & Result & $\begin{array}{l}\text { Measurement } \\
\text { Unit }\end{array}$ \\
\hline SB-03:T.D. & NBD-691 & Acetone & 15.5 & $\mu \mathrm{g} / \mathrm{kg}$ \\
SB-04:T.D. & NBD-696 & 2-Butanone & 17.0 & $\mu \mathrm{g} / \mathrm{kg}$ \\
SB-04:T.D. & NBD-696 & Acetone & 46.4 & $\mu \mathrm{g} / \mathrm{kg}$ \\
SB-04:T.D. (Dup) & NBD-697 & Acetone & 58.9 & $\mu \mathrm{g} / \mathrm{kg}$ \\
SB-05:T.D. & NBD-688 & Acetone & 17.5 & $\mu \mathrm{g} / \mathrm{kg}$ \\
SB-09:T.D. & NBD-250 & Toluene & 4.59 & $\mu \mathrm{g} / \mathrm{kg}$ \\
SB-10:T.D. & 259 & Acetone & 24.4 & $\mu g / \mathrm{kg}$ \\
SB-12:T.D. & 253 & Acetone & 68.3 & $\mu \mathrm{g} / \mathrm{kg}$ \\
SB-14:T.D. & NBD-244 & 2-8utanone & 28.4 & $\mu \mathrm{g} / \mathrm{kg}$ \\
SB-14:T.D. & NBD-244 & Acetone & 144. & $\mu \mathrm{g} / \mathrm{kg}$ \\
SB-14:T.D. & NBD-244 & Toluene & 2.15 & $\mu \mathrm{g} / \mathrm{kg}$ \\
SB-15:T.D. & NBD-241 & Acetone & 33.9 & $\mu \mathrm{g} / \mathrm{kg}$ \\
SB-16:T.D. & NBD-238 & 2-Butanone & 23.0 & $\mu \mathrm{g} / \mathrm{kg}$ \\
& & & & \\
\hline
\end{tabular}




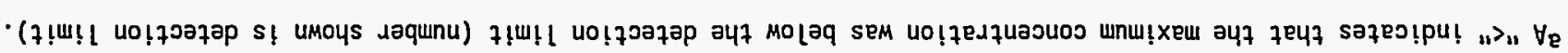

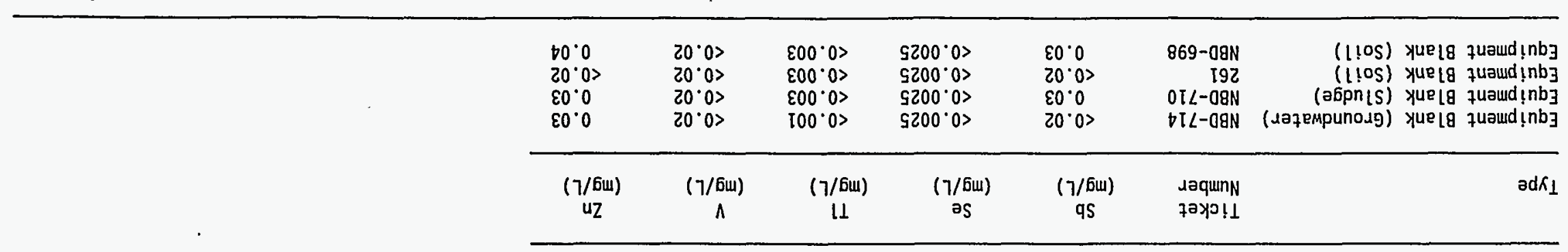

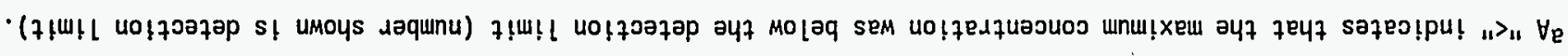

\begin{tabular}{|c|c|c|c|c|c|c|c|c|c|c|c|}
\hline $\begin{array}{l}0\left[00^{\circ} 0\right\rangle \\
\left.0 I 00^{\circ} 0\right\rangle \\
t\left[00^{\circ} 0\right. \\
\varepsilon 00^{\circ} 0\end{array}$ & $\begin{array}{l}20.0> \\
20^{\circ} 0> \\
20^{\circ} 0> \\
20^{\circ} 0>\end{array}$ & $\begin{array}{l}60^{\circ} 0 \\
60^{\circ} \cdot 0> \\
6 T^{\circ} \cdot 2 \\
60^{\circ} 0>\end{array}$ & $\begin{array}{l}20 \cdot 0> \\
20 \cdot 0> \\
20 \cdot 0> \\
20 \cdot 0>\end{array}$ & $\begin{array}{l}60.0> \\
60.0> \\
6 I .0 \\
60.0>\end{array}$ & $\begin{array}{l}60.0> \\
60.0> \\
9 \varepsilon \cdot 0 \\
60.0>\end{array}$ & $\begin{array}{l}9000^{\circ} 0>> \\
9000^{\circ} 0> \\
9000^{\circ} 0> \\
0100^{\circ} 0>\end{array}$ & $\begin{array}{l}\mathrm{ST} \cdot 0 \\
\mathrm{SO} 0^{\circ} 0 \\
\mathrm{SO} 0^{\circ} 0 \\
\angle 0^{\circ} 0\end{array}$ & $\begin{array}{l}60.0 \\
20.07 \\
20.0 \\
20.0>\end{array}$ & $\begin{array}{r}869-09 N \\
\text { I9Z } \\
0 T \angle-09 N \\
\square T \angle-09 N\end{array}$ & $\begin{array}{r}(1 ! \circ s) \\
(\lfloor\div \circ) \\
\text { (aбpnLs) } \\
\text { (土ә7емpunodg) }\end{array}$ & 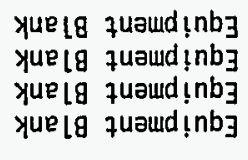 \\
\hline$(7 / 6 \omega)$ & $(7 / 6 \omega)$ & (7/бш) & $(7 / 6 \omega)$ & $(7 / 6 \omega)$ & ( $7 / 6 \omega)$ & $(7 / 6 \omega)$ & (7/6w) & $(7 / 5 w)$ & dequnN & & $\operatorname{ad} \kappa_{1}$ \\
\hline$q_{d}$ & $! N$ & EN & UW & $6 \mathrm{~W}$ & $x$ & 6 & as & nว & zәуฺ!1 & & \\
\hline
\end{tabular}

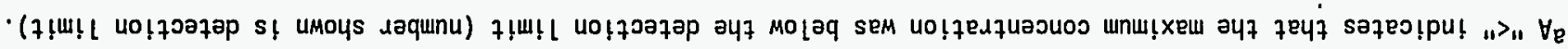

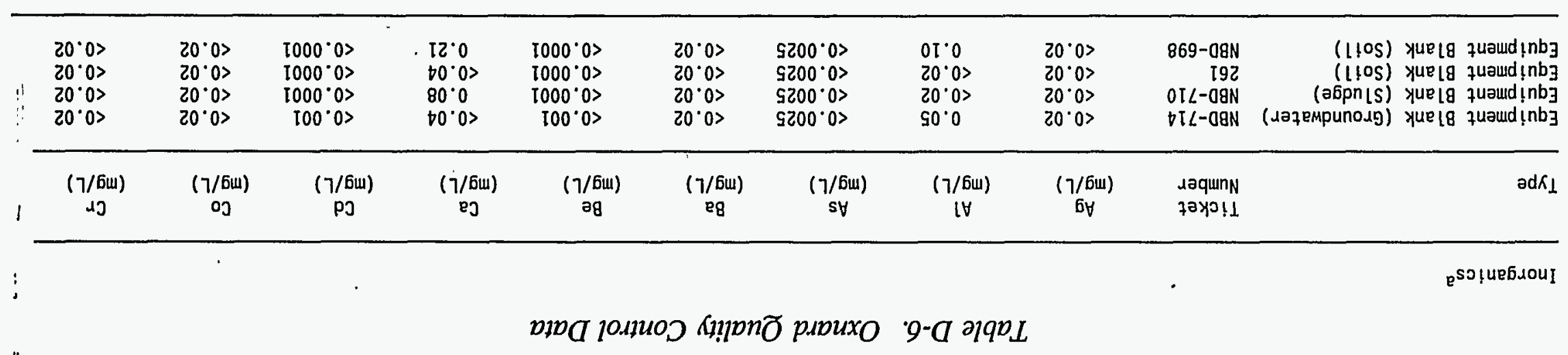


Table D-6 (continued). Oxnard Quality Control Data

Detected Volatile Organics

\begin{tabular}{|c|c|c|c|c|}
\hline Type & $\begin{array}{l}\text { Ticket } \\
\text { Number }\end{array}$ & Analyte & Result & $\begin{array}{l}\text { Measurement } \\
\text { Unit }\end{array}$ \\
\hline $\begin{array}{l}\text { Equipment Blank (Groundwater) } \\
\text { Equipment Blank (Soi1) } \\
\text { Trip Blank } \\
\text { Trip Blank } \\
\text { Trip Blank }\end{array}$ & $\begin{array}{l}\text { NBD-714 } \\
261 \\
\text { NBD-219 } \\
\text { NBD-215 } \\
\text { NBD-219 }\end{array}$ & $\begin{array}{l}\text { Chloroform } \\
\text { Chloroform } \\
\text { 1,2-Dichloroethane } \\
\text { Chloromethane } \\
\text { Chloromethane }\end{array}$ & $\begin{array}{l}3.30 \\
2.01 \\
2.24 \\
6.65 \\
8.27\end{array}$ & $\begin{array}{l}\mu g / L \\
\mu g / L \\
\mu g / L \\
\mu g / L \\
\mu g / L\end{array}$ \\
\hline
\end{tabular}

Equipment Blank Detected PCBs: None.

Equipment Blank Detected Total Petroleum Hydrocarbons: None. 
$\cdots-\cdots=$

.

. 
Appendix E

Radiological Survey 
$\cdots$ 


\section{Radiological Survey Map}

RWP No._N/A_Purpose Contamination Survey OH\&S Technician Candice Burns/CandisuBumes Address/Building No. Building 2-Loading/Beceiving OH\&S Lead Technician Tom Maveal / Toum Maveal DaterTimo 3.06.95/1100 Site ID No. Dxnard Project
Page 1 of 2 Date $02 \cdot 06.95$ Date $3-3 \cdot 95$

\begin{tabular}{|c|c|c|c|c|c|}
\hline No. & Instrument Model & Serlal No. & Probe Model & Probe Serial No. & Calibratlon/Correetlon Faelo \\
\hline 1 & $L \cdot 12$ & 510429 & 44.9 & 512694 & $\begin{array}{l}4.21095 \\
4.24 .95 / E C F \times 10 \text { ACE }\end{array}$ \\
\hline 2 & $L \cdot 12$ & 56836 & 44.9 & 510718 & {$\left[\begin{array}{l}7.3 .95 \\
5010.95 / E C E=10 \text { ACF }=6 .\end{array}\right.$} \\
\hline 3 & $N / A$ & $\mu / A$ & $\alpha / A$ & $N / A$ & $\alpha / A$ \\
\hline
\end{tabular}

] $=$ Tapo pross $\left(4^{\circ} \times 4^{4}\right)$ (no. hisido)

- Smears (no. hisldo)

$\mathrm{O}_{\text {. L Largo area smears }}$

$\Delta=$ Air samples (no. insido)

- Noutron readings in mreminr unless otherwiso noted

Gamma roadings in mrom/hr unloss
otherwiso noted (bota roadings also)

* - Conlact readings (doso rato)

HS : Hol spol

[SOP] = Slop-ofl pad

$K$. Reading at kneo level (when sourcos Irom overhead)

H = Reading al hioad lovel (when sources Irom overtiead)

..... - Contaminalod area

XXXXXX = Radiation area

$\cdot x \cdot x \cdot x \cdot$ - Contaminated/radiation ares

RM - Radloactivo matorial aroa

(2) - Foor drain

ccpm = Correctod or nel cpm (gross back.

nepm . ground) lor direct lirisk, alpha or betal

oamma spocified

\# D Direct lrisk

Highest Dosë Rates

General Area $\frac{N / R}{N / R}$

Highest Conlamination Level
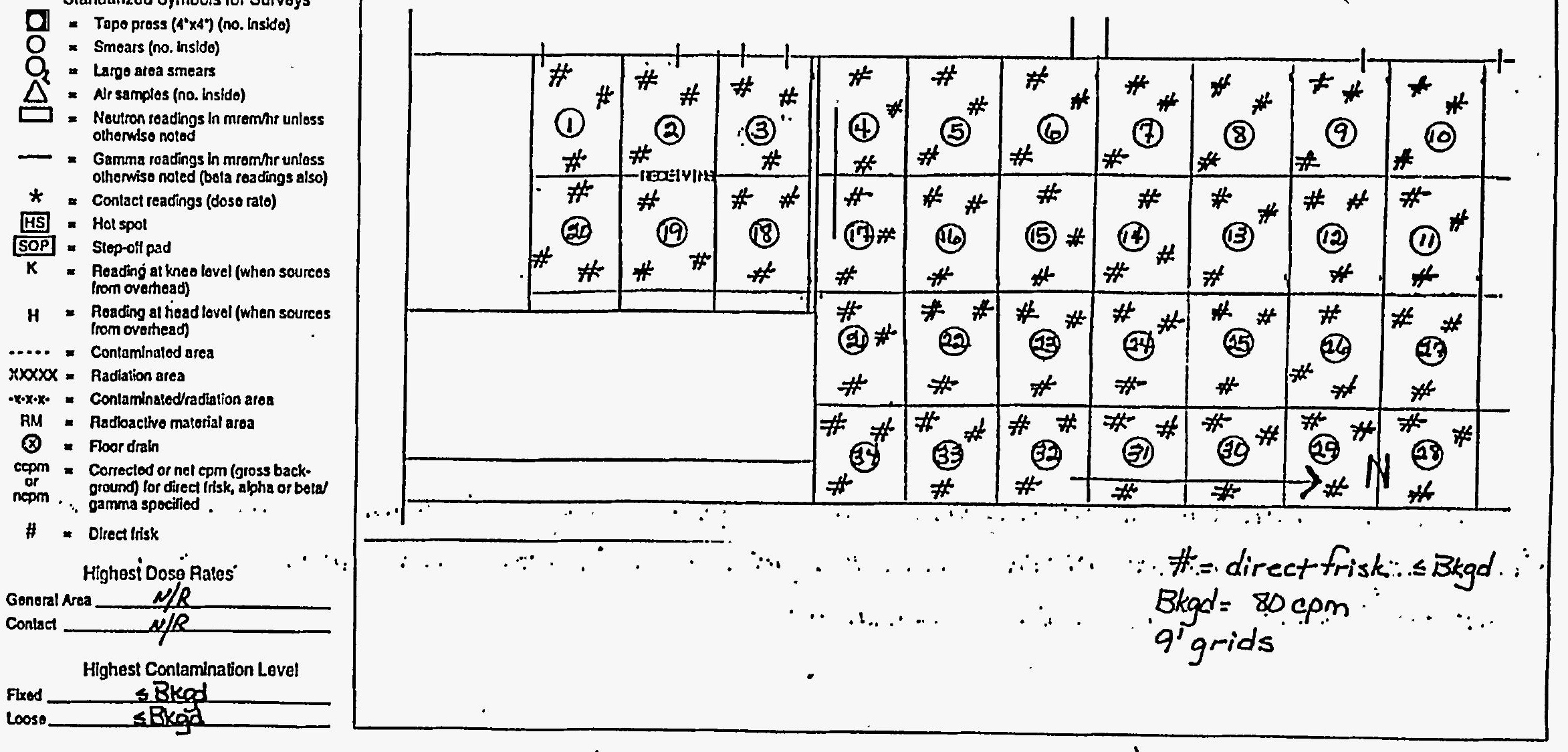


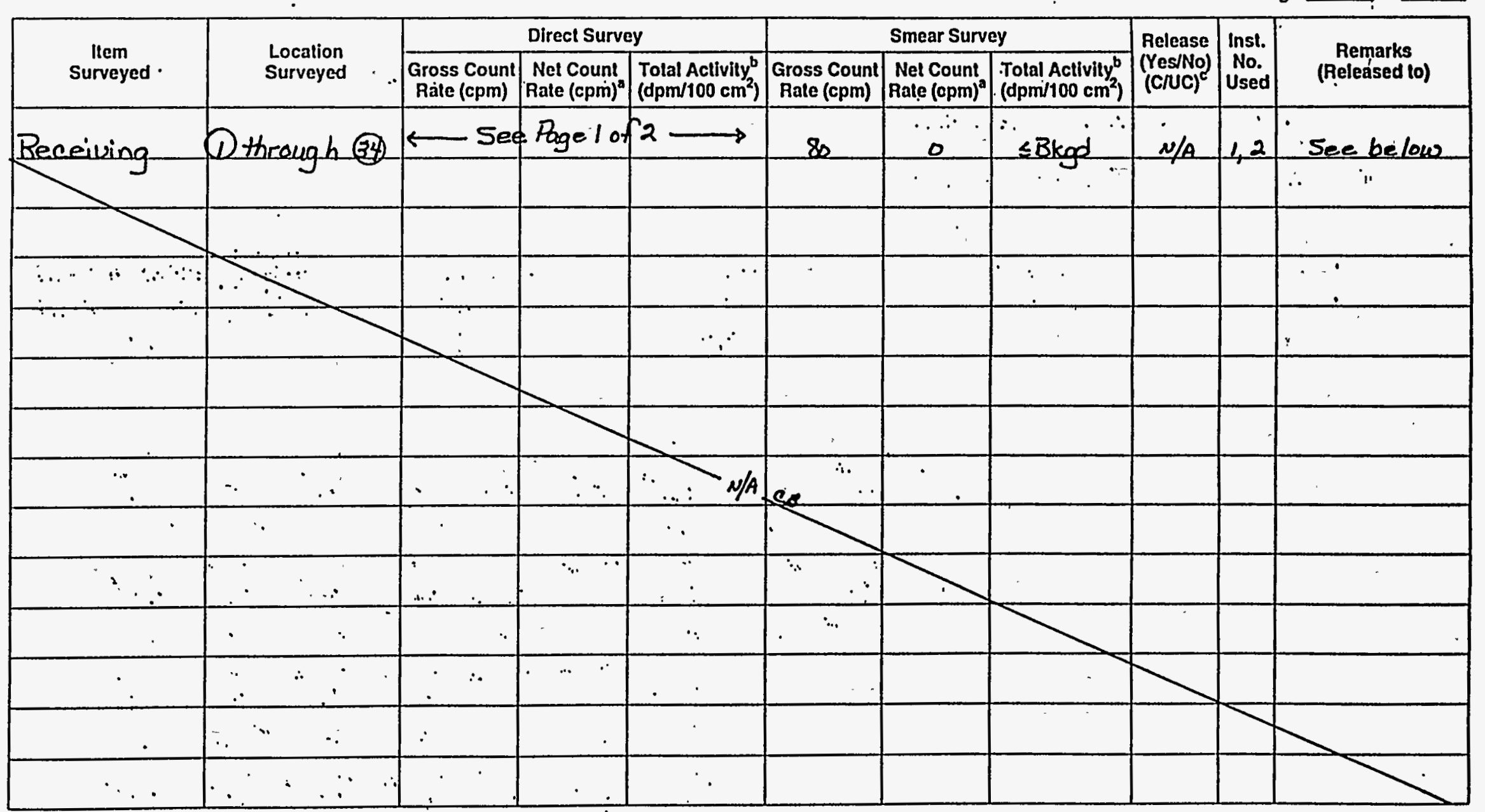

Remarks Coopprehensive "Jurvey of receiving area of Bldg. 2 utilizing 9' grids. Each smear number mepresents. three smears within designated grid. Walls is area were surveyed to a height of 6 fëer with: all readings $\leq$ Bkgd. w/A 


\section{Appendix F \\ Duplicate-Sample Precision}



Table F-1. Field Duplicates Relative Percent Difference Values $a$

\begin{tabular}{|c|c|c|c|c|c|c|}
\hline Analyte & & $M W-8$ & $S B-04: 4-6$ & SB-04:T.D. & SB-10:4-6 & $S L-1$ \\
\hline 1,1,1-Trichloroethane & $\begin{array}{l}\text { Value } \\
\text { Dup } \\
\text { \%RPD }\end{array}$ & & $\begin{array}{l}4.40 \\
<2.0 \\
75\end{array}$ & & & \\
\hline 2-Butanone & $\begin{array}{l}\text { Value } \\
\text { Dup } \\
\text { \%RPD }\end{array}$ & $\because$ & $\begin{array}{l}35.4 \\
<15.0 \\
80.95\end{array}$ & $\begin{array}{l}17.0 \\
<15.0 \\
12.5\end{array}$ & & \\
\hline Acetone & $\begin{array}{l}\text { Vaiue } \\
\text { Dup } \\
\text { \%RPD }\end{array}$ & & $\begin{array}{l}84.2 \\
57.2 \\
38.19\end{array}$ & $\begin{array}{l}46.4 \\
58.9 \\
23.74\end{array}$ & $\begin{array}{l}17.1 \\
<15.0 \\
13.08\end{array}$ & \\
\hline Aluminum & $\begin{array}{l}\text { Value } \\
\text { Dup } \\
\text { \%RPD }\end{array}$ & $\begin{array}{l}0.17 \\
0.33 \\
64\end{array}$ & $\begin{array}{l}2782 \\
3200 \\
13.98\end{array}$ & & $\begin{array}{l}10820 \\
13390 \\
21.23\end{array}$ & \\
\hline Arsenic & $\begin{array}{l}\text { Value } \\
\text { Dup } \\
\text { \%RPD }\end{array}$ & & $\begin{array}{l}1.36 \\
1.67 \\
20.46\end{array}$ & $\begin{array}{l}4.82 \\
2.18 \\
75.43\end{array}$ & $\begin{array}{l}3.263 \\
4.334 \\
28.2\end{array}$ & $\begin{array}{l}3.249 \\
10.41 \\
104.85\end{array}$ \\
\hline Barium & $\begin{array}{l}\text { Value } \\
\text { Dup } \\
\text { \%RPD }\end{array}$ & $\begin{array}{l}0.03 \\
0.03 \\
0\end{array}$ & $\begin{array}{l}34.6 \\
35.2 \\
1.72\end{array}$ & $\begin{array}{l}53.4 \\
54.5 \\
2.04\end{array}$ & $\begin{array}{l}108 \\
113 \\
4.52\end{array}$ & $\begin{array}{l}62.0 \\
52.0 \\
17.54\end{array}$ \\
\hline Cadmium & $\begin{array}{l}\text { Value } \\
\text { Dup } \\
\text { \%RPD }\end{array}$ & $\begin{array}{l}0.001 \\
<0.001 \\
0\end{array}$ & & & $\begin{array}{l}<0.8 \\
1.1 \\
31.58\end{array}$ & $\begin{array}{l}2.3 \\
3.2 \\
32.73\end{array}$ \\
\hline Calcium & $\begin{array}{l}\text { Value } \\
\text { Dup } \\
\text { \%RPD }\end{array}$ & $\begin{array}{l}105 \\
104 \\
.96\end{array}$ & $\begin{array}{l}6577 \\
11623 \\
55.45\end{array}$ & & $\begin{array}{l}14236 \\
16344 \\
13.79\end{array}$ & \\
\hline Chromi um & $\begin{array}{l}\text { Value } \\
\text { Dup } \\
\text { \%RPD }\end{array}$ & & $\begin{array}{l}4.9 \\
5.4 \\
9.71\end{array}$ & $\begin{array}{l}4.8 \\
9.9 \\
69.39\end{array}$ & $\begin{array}{l}18.4 \\
21.7 \\
16.46\end{array}$ & $\begin{array}{l}381 \\
279 \\
30.91\end{array}$ \\
\hline Cobalt & $\begin{array}{l}\text { Value } \\
\text { Dup } \\
\text { \%RPD }\end{array}$ & & $\begin{array}{l}1.7 \\
2.2 \\
25.64\end{array}$ & $\begin{array}{l}1.6 \\
3.0 \\
60.87\end{array}$ & $\begin{array}{l}6.4 \\
7.1 \\
10.37\end{array}$ & $\begin{array}{l}92.6 \\
160 \\
53.37\end{array}$ \\
\hline Copper & $\begin{array}{l}\text { Value } \\
\text { Dup } \\
\text { \%RPD }\end{array}$ & & $\begin{array}{l}3.1 \\
3.9 \\
22.86\end{array}$ & $\begin{array}{l}3.6 \\
6.0 \\
50\end{array}$ & $\begin{array}{l}13.6 \\
14.5 \\
6.41\end{array}$ & $\begin{array}{l}1831 \\
892 \\
68.97\end{array}$ \\
\hline Fluoride & $\begin{array}{l}\text { Value } \\
\text { Dup } \\
\text { \%RPD }\end{array}$ & & & $\begin{array}{l}<5.0 \\
5.5 \\
9.52\end{array}$ & & \\
\hline Iron & $\begin{array}{l}\text { Value } \\
\text { Dup } \\
\text { \%RPD }\end{array}$ & $\begin{array}{l}0.27 \\
0.44 \\
47.89\end{array}$ & $\begin{array}{l}5993 \\
7137 \\
17.43\end{array}$ & & $\begin{array}{l}19416 \\
21475 \\
10.07\end{array}$ & \\
\hline Lead & $\begin{array}{l}\text { Value } \\
\text { Dup } \\
\text { \%RPD }\end{array}$ & $\begin{array}{l}0.011 \\
0.004 \\
93.33\end{array}$ & $\begin{array}{l}0.9 \\
1.6 \\
56\end{array}$ & $\begin{array}{l}2.0 \\
1.3 \\
42.42\end{array}$ & $\begin{array}{l}3.8 \\
3.9 \\
2.6\end{array}$ & $\begin{array}{l}226 \\
209 \\
7.82\end{array}$ \\
\hline
\end{tabular}

Where both the value and the duplicate value were below detection limits, data are not shown. If one value was below detection limits, the detection limit was used in the calculation of RPD. 
Table F-1 (continued). Oxnard Relative Percent Difference Valuesa

\begin{tabular}{|c|c|c|c|c|c|c|}
\hline Analyte & & MW- 8 & SB-04:4-6 & SB-04:T.D. & SB-10:4-6 & $S L-1$ \\
\hline Magnesium & $\begin{array}{l}\text { Value } \\
\text { Dup } \\
\text { \%RPD }\end{array}$ & $\begin{array}{l}35.8 \\
35.6 \\
.56\end{array}$ & $\begin{array}{l}1609 \\
2108 \\
26.85\end{array}$ & & $\begin{array}{l}6326 \\
7073 \\
11.15\end{array}$ & \\
\hline Manganese & $\begin{array}{l}\text { Value } \\
\text { Dup } \\
\text { \%RPD }\end{array}$ & $\begin{array}{l}0.33 \\
0.34 \\
2.99\end{array}$ & $\begin{array}{l}108 \\
262 \\
83.24\end{array}$ & & $\begin{array}{l}262 \\
284 \\
8.06\end{array}$ & \\
\hline Mercury & $\begin{array}{l}\text { Value } \\
\text { Dup } \\
\text { \%RPD }\end{array}$ & & & $\begin{array}{l}0.0300 \\
0.0334 \\
10.73\end{array}$ & $\begin{array}{l}0.0353 \\
<0.0294 \\
18.24\end{array}$ & $\begin{array}{l}0.0314 \\
0.0304 \\
3.24\end{array}$ \\
\hline Molybdenum & $\begin{array}{l}\text { Value } \\
\text { Dup } \\
\text { \%RPD }\end{array}$ & & & & & $\begin{array}{l}97.9 \\
122 \\
21.92\end{array}$ \\
\hline Nickel & $\begin{array}{l}\text { Value } \\
\text { Dup } \\
\text { \%RPD }\end{array}$ & & $\begin{array}{l}5.6 \\
6.4 \\
13.33\end{array}$ & $\begin{array}{l}5.0 \\
10.2 \\
68.42\end{array}$ & $\begin{array}{l}18.1 \\
19.1 \\
5.38\end{array}$ & $\begin{array}{l}611 \\
476 \\
24.84\end{array}$ \\
\hline Potassium & $\begin{array}{l}\text { Value } \\
\text { Dup } \\
\text { \%RPD }\end{array}$ & $\begin{array}{l}4.2 \\
4.2 \\
0\end{array}$ & $\begin{array}{l}587 \\
660 \\
11.71\end{array}$ & $\cdot$ & $\begin{array}{l}2701 \\
3154 \\
15.47\end{array}$ & \\
\hline Selenium & $\begin{array}{l}\text { Value } \\
\text { Dup } \\
\text { \%RPD }\end{array}$ & & $\begin{array}{l}0.295 \\
0.166 \\
55.97\end{array}$ & $\begin{array}{l}1.68 \\
0.231 \\
151.65\end{array}$ & $\begin{array}{l}0.262 \\
0.232 \\
12.15\end{array}$ & \\
\hline Sodium & $\begin{array}{l}\text { Value } \\
\text { Dup } \\
\text { \%RPD }\end{array}$ & $\begin{array}{l}112 \\
111 \\
.9\end{array}$ & $\begin{array}{l}139 \\
110 \\
23.29\end{array}$ & & $\begin{array}{l}288 \\
314 \\
8.64\end{array}$ & \\
\hline Toluene & $\begin{array}{l}\text { Value } \\
\text { Dup } \\
\text { \%RPD }\end{array}$ & & $\begin{array}{l}8.78 \\
<2: 0 \\
125.79\end{array}$ & & & \\
\hline Vanadium & $\begin{array}{l}\text { Value } \\
\text { Dup } \\
\text { \%RPD }\end{array}$ & & $\begin{array}{l}9.7 \\
11.5 \\
16.98\end{array}$ & $\begin{array}{l}8.6 \\
18.1 \\
71.16\end{array}$ & $\begin{array}{l}33.5 \\
39.9 \\
17.44\end{array}$ & $\begin{array}{l}51.7 \\
44.6 \\
14.75\end{array}$ \\
\hline Zinc & $\begin{array}{l}\text { Value } \\
\text { Dup } \\
\text { \%RPD }\end{array}$ & $\begin{array}{l}0.02 \\
0.03 \\
40\end{array}$ & $\begin{array}{l}14.9 \\
16.1 \\
7.74\end{array}$ & $\begin{array}{l}15.5 \\
26.4 \\
52.03\end{array}$ & $\begin{array}{l}52.9 \\
56.2 \\
6.05\end{array}$ & $\begin{array}{l}999 \\
817 \\
20.04\end{array}$ \\
\hline
\end{tabular}

aWhere both the value and the duplicate value are below detection limits, data are not shown. 
Table F-2. Labratory Duplicates Relative Percent Difference Valuesa

\begin{tabular}{|c|c|c|c|c|c|}
\hline Analyte & & 252 & NBD-215 & NBD-238 & NBD-286 \\
\hline 2-Butanone & $\begin{array}{l}\text { VaTue } \\
\text { Dup } \\
\text { \%RPD }\end{array}$ & $\begin{array}{l}21.3 \\
<15.0 \\
34.71\end{array}$ & & $\begin{array}{l}<15.0 \\
23.0 \\
42.11\end{array}$ & $\begin{array}{l}55.8 \\
58.7 \\
5.07\end{array}$ \\
\hline Chloromethane & $\begin{array}{l}\text { Value } \\
\text { Dup } \\
\text { \%RPD }\end{array}$ & & $\begin{array}{l}6.65 \\
7.38 \\
10.41\end{array}$ & & \\
\hline Toluene & $\begin{array}{l}\text { Value } \\
\text { Dup } \\
\text { \%RPD }\end{array}$ & $\begin{array}{l}6.24 \\
5.21 \\
17.99\end{array}$ & & & $\begin{array}{l}9.44 \\
7.66 \\
20.82\end{array}$ \\
\hline
\end{tabular}

aWhere both the value and the duplicate value are below detection limits, data are not shown. 



\section{Appendix C}

Phase III Environmental Site Assessment Report for the U.S. Department of Energy Oxnard Facility Oxnard, California 
This page intentionally blank 


\section{Phase III Environmental Site Assessment Report for the U.S. Department of Energy Oxnard Facility, Oxnard, California}

December 1995

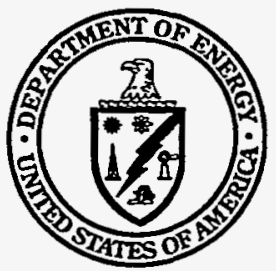

U.S. Department of Energy Grand Junction Projects Office

Approved for public release; distribution is unlimited. 
This report was prepared as an account of work sponsored by an agency of the United States Government. Neither the United States Government nor any agency thereof, nor any of their employees, makes any warranty, express or implied, or assumes any legal liability or responsibility for the accuracy, completeness, or usefulness of any information, apparatus, product, or process disclosed in this report, or represents that its use would not infringe privately owned rights. Reference herein to any specific commercial product, process, or service by trade name, trademark, manufacturer, or otherwise, does not necessarily constitute or imply its endorsement, recommendation, or favoring by the United States Government or any agency thereof. The views and opinions of authors expressed herein do not necessarily state or reflect those of the United States Government or any agency thereof. 


\section{Phase III Environmental Site Assessment Report for the U. S. Department of Energy Oxnard Facility, Oxnard, California}

December 1995

Work Performed Under Contract No. DE-AC04-94AL96907

Prepared for

U.S. Department of Energy

Albuquerque Operations Office

Grand Junction Projects Office

P.O. Box 2567

Grand Junction, CO 81502-2567

Prepared by

Rust Geotech

Grand Junction, Colorado 


\section{Contents}

Page

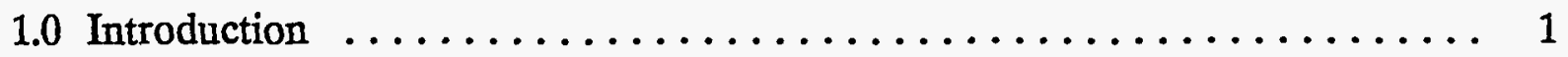

2.0 Regulatory Framework $\ldots \ldots \ldots \ldots \ldots \ldots \ldots \ldots \ldots \ldots \ldots$

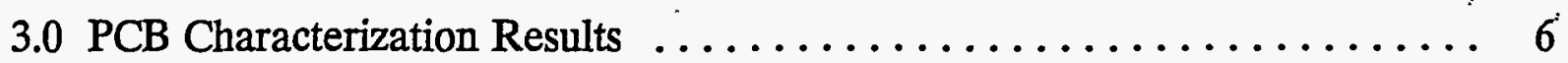

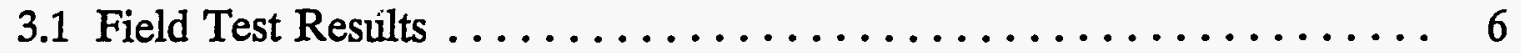

3.2 Laboratory Results $\ldots \ldots \ldots \ldots \ldots \ldots \ldots \ldots \ldots \ldots$

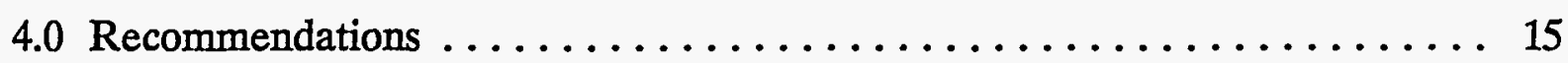

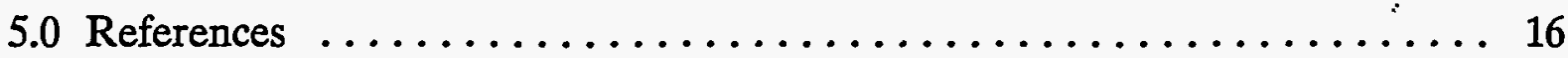

\section{Figures}

Figure 1. Location of the Oxnard Facility in Oxnard, California $\ldots \ldots \ldots \ldots, 2$

2. Site Layout of the Oxnard Facility $\ldots \ldots \ldots \ldots \ldots \ldots \ldots \ldots$

3. PCB Sampling Locations at the Oxnard Facility $\ldots \ldots \ldots \ldots \ldots, 7$

4. PCB Field-Test Concentrations in the $0-1$ Foot Interval . . . . . . . 11

\section{Tables}

Table 1. PCB Field-Test Results $\ldots \ldots \ldots \ldots \ldots \ldots \ldots \ldots \ldots \ldots \ldots \ldots, 8$

2. PCB Laboratory Data ..................... 12

3. Comparison of PCB Field-Test Results with Laboratory Analytical Results . ................... 14 


\subsection{Introduction}

The U.S. Department of Energy (DOE) Oxnard Facility (Oxnard facility) is a nonferrous metalworking facility occupying 13.75 acres at 1235 East Wooley Road, Oxnard, California (Figure 1). Owned by the DOE and operated by Kaiser-Hill Company LLC, the facility is located in an industrial park within the incorporated city limits of Oxnard and within Ventura County. Seven buildings enclose approximately 86,000 square feet of covered floor space (Figure 2). Bordering the facility are industrial maintenance, manufacturing, and agricultural packaging facilities.

Until Allis-Chalmers (a farm implement manufacturing company) purchased the property in 1949, the site was farmland. Allis-Chalmers built a plant consisting of six buildings and engaged in the activities of founding (foundry casting), forging, machining, welding, cutting, sanding, grinding, painting, and coating. In 1981, DOE helped Precision Forge (a private company that catered to DOE metalworking requirements) move from Santa Monica, California, to the Oxnard facility. By June 1982, Precision Forge completed the transfer to the Oxnard facility. In 1984, DOE acquired Precision Forge and operated the facility through Rockwell International until 1989 when EG\&G took over operations. Kaiser-Hill Company has operated the facility since the second quarter of 1995. Historically, metalworking at the Oxnard facility involved stainless steel, titanium, aluminum, and copper alloys. Presently, all metal working utilizes stainless steel, tantalum, molybdenum, and tungsten.

Site investigations conducted by Rust Geotech, the prime contractor for the DOE at the Grand Junction Projects Office (GJPO), started in June 1994 with an Environmental Site Assessment (ESA) Preliminary Evaluation site tour. Results of the Preliminary Evaluation indicated the need for a Phase I and Phase II ESA. A nonintrusive site inspection and data gathering effort in August 1994 culminated in a Phase I ESA report entitled Phase I Environmental Site Assessment for the EG\&G Rocky Flats Oxnard Facility (Phase I ESA) (DOE 1994). Conclusions and recommendations described in the Phase I ESA report formed the basis for the Phase II intrusive ESA investigation, which was conducted in January and February 1995 and culminated in a Phase II report entitled Phase II Environmental Site Assessment Report for the Kaiser-Hill Company LLC Oxnard Facility, Oxnard, California (DOE 1995a). Conclusions and recommendations described in the Phase II report formed the basis for the Phase III investigation, which is the focus of this report.

The primary purpose of the Phase III ESA was to investigate and characterize areas of the facility where polychlorinated biphenyls (PCBs) were detected during the Phase II investigation. These areas required a "due diligence" investigation as described in Site Auditing: Environmental Assessment of Property (Marburg Associates and Parkin 1991) so 


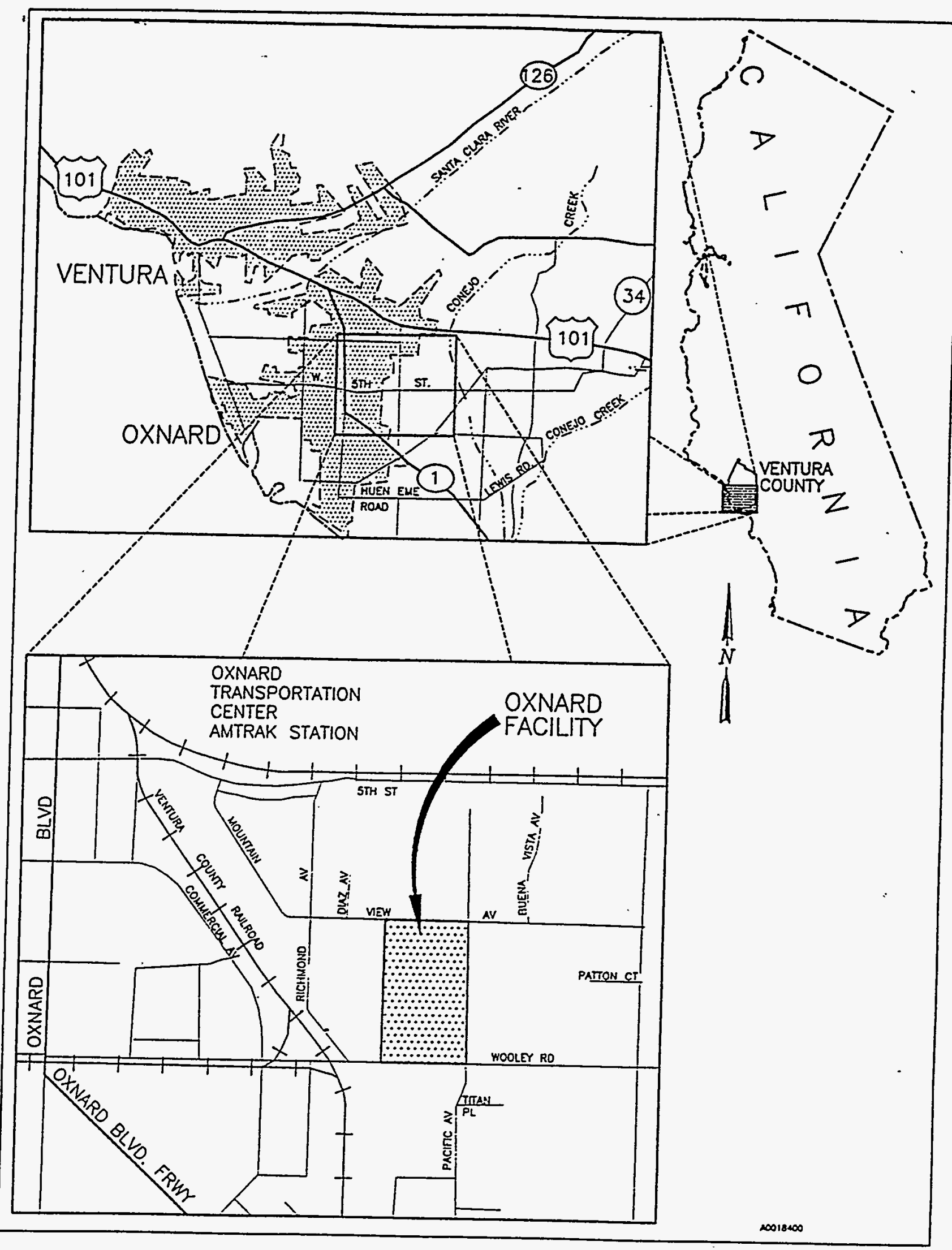

Figure 1. Location of the Oxnard Facility in Oxnard, California 
MOUNTAIN VIEW AVENUE

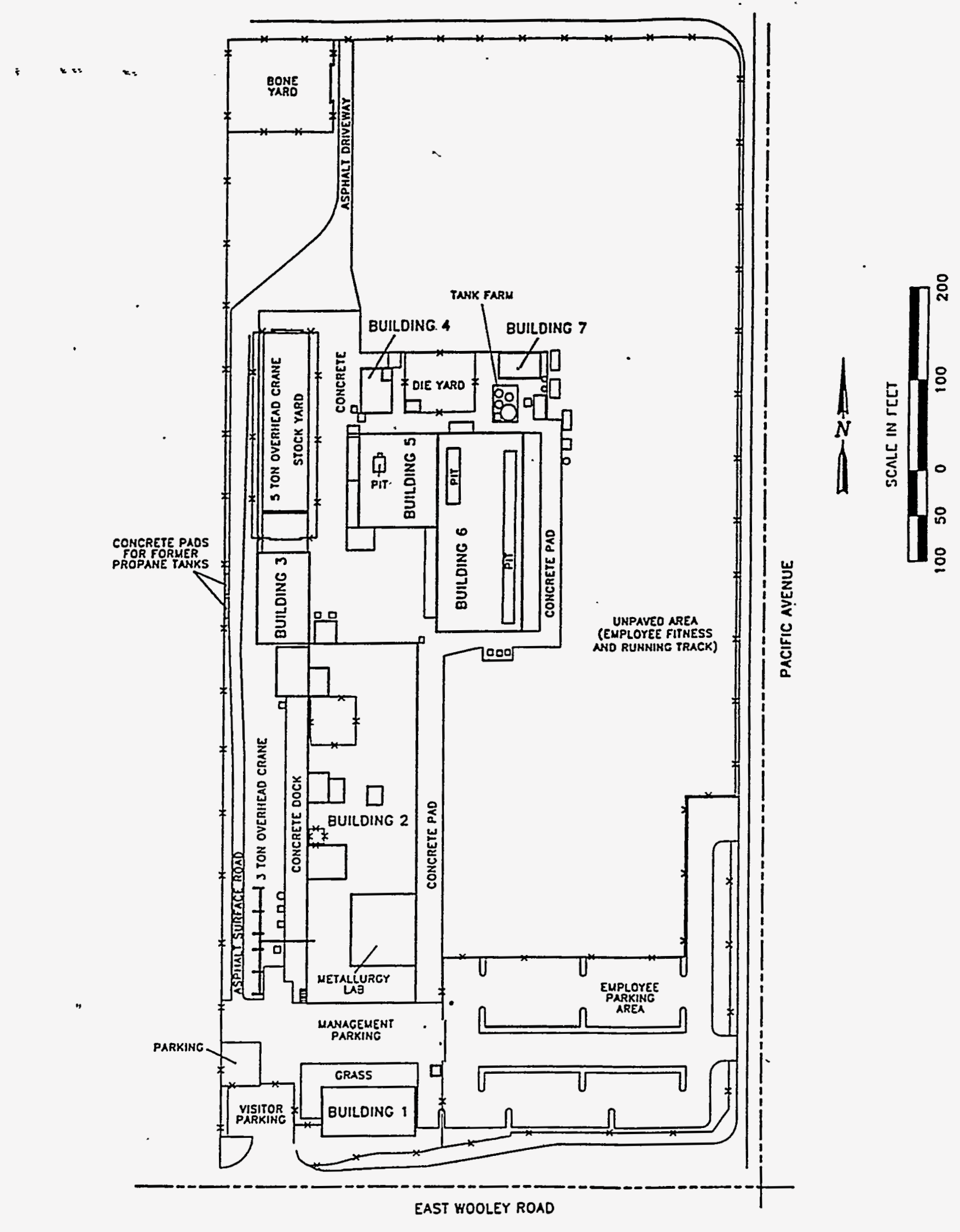

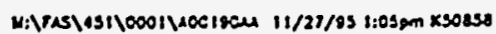

Figure 2. Site Layout of the Oxnard Facility 
that contaminated media, if present, could be identified and accounted for before real estate transfer. Specific objectives of the investigation were:

- To delineate the vertical and horizontal extent of PCBs in the soil.

- To determine if corrective action is required on the basis of a comparison of PCB concentrations in soils to action levels.

- If corrective action is required, to determine the volume of contaminated material so that decisions concerning remediation and disposal options can be made.

This report is divided into five sections. Section 1.0 describes the types of work performed at the Oxnard Facility and the objectives of this Phase III ESA; Section 2.0 provides the regulatory framework used to make decisions from the collected data; Section 3.0 presents the results of the investigation and discusses the results in the context of the regulatory framework; Section 4.0 provides recommendations for adddressing health and environmental issues before real estate transfer; and Section 5.0 lists references used in this document. 


\subsection{Regulatory Framework}

On the basis of the U. S. Environmental Protection Agency (EPA) guidance, $A$ Guide to Remedial Actions at Superfund Sites with PCB Contamination (EPA 1990), which recommends soil action levels of 10-25 ppm for industrial sites, a criterion of $10 \mathrm{ppm}$ of PCBs in soil was established for this characterization.

Use of the 10-ppm criterion is supported by the State of California's Preliminary Endangerment Assessment Guidance Manual (Guidance Marlual)(State of California Environmental Protection Agency 1994), which provides risk-based guidance for determining if a release of a hazardous substance presents a risk to human health or the environment. The Guidance Manual makes use of equations equipped with default values for a residential exposure scenario to determine risk. According to EPA risk assessment guidance (EPA 1991), remedial action is generally not warranted if risks are less than $10^{-4}$, which corresponds to a PCB soil concentration of $8 \mathrm{ppm}$ when using the equations supplied in the Guidance Manual. Although the Oxnard facility will likely remain an industrial site, the Guidance Manual provides a conservative estimate of risk that confirms the 10-ppm criterion suggested by EPA.

Laboratory analytical work was performed by Truesdail Laboratories, Inc. in Tustin, California. This laboratory was certified by the California Environmental Laboratory Accreditation Program in EPA method 8080, which was used to analyze selected samples collected during the Phase III ESA. 


\subsection{PCB Characterization Results}

\subsection{Field Test Results}

To define the extent of PCBs in the shallow soil, samples were collected with an auger and analyzed in the field using EnSys RISc PCB immunoassay field test kits configured for 1- and 10-ppm detection levels. The sampling network consisted of a statistically . based hexagonal design that served two purposes: it allowed delineation of PCB contamination on a small scale, and it allowed a search for PCB-contaminated areas in and adjacent to the Bone Yard. The search for contaminated areas resulted in a certainty of at least 90 percent that PCB-contaminated areas at least 20 feet in diameter in the sampling area were located and subsequently delineated. A complete description of the technical approach used to characterize PCBs for this Phase III ESA is found in the Sampling and Analysis Plan for the Phase III Environmental Site Assessment of the U. S. Department of Energy Oxnard Facility, Oxnard, California (DOE 1995b).

PCB field tests were conducted at 75 sampling locations within and east of the Bone Yard as shown in Figure 3. A total of 121 field tests were performed, consisting of 112 sample tests, 6 field duplicates, and 3 method blanks (see Table 1 for results). The depth interval of the sample is indicated at the end of the sample identification number. For example, sample PCB-46:2-3 was collected from the 2- to 3-foot depth interval.

On the basis of field test results, only two sampling locations, PCB-10 and PCB-12, had PCB concentrations above the 10-ppm criterion established for the project. Sampling location PCB-10 had a PCB concentration greater than 10-ppm in the 0 - to 1-foot interval; PCB-12 had PCB concentrations greater than $10 \mathrm{ppm}$ in the 0 - to 1-, 1- to 2-, and 2- to 3-foot intervals. Results of the PCB field tests in the 0- to 1-foot interval are shown in Figure 4.

\subsection{Laboratory Results}

Twenty-six confirmation samples were submitted to Truesdail Laboratories for PCB analysis using EPA Method 8080. Samples submitted to the laboratory included four samples with PCB field test concentrations over $10 \mathrm{ppm}$, seven samples with PCB field test concentrations greater than $1 \mathrm{ppm}$ but less than $10 \mathrm{ppm}$, seven samples with PCB field test concentrations less than $1 \mathrm{ppm}$, two laboratory duplicates, and six equipment blanks.

All laboratory results were below the 10-ppm criterion established for the project. The highest PCB concentration detected was in sample number PCB-57:0-1, with a concentration of $6.4 \mathrm{ppm}$. Laboratory results are displayed in Table 2. 


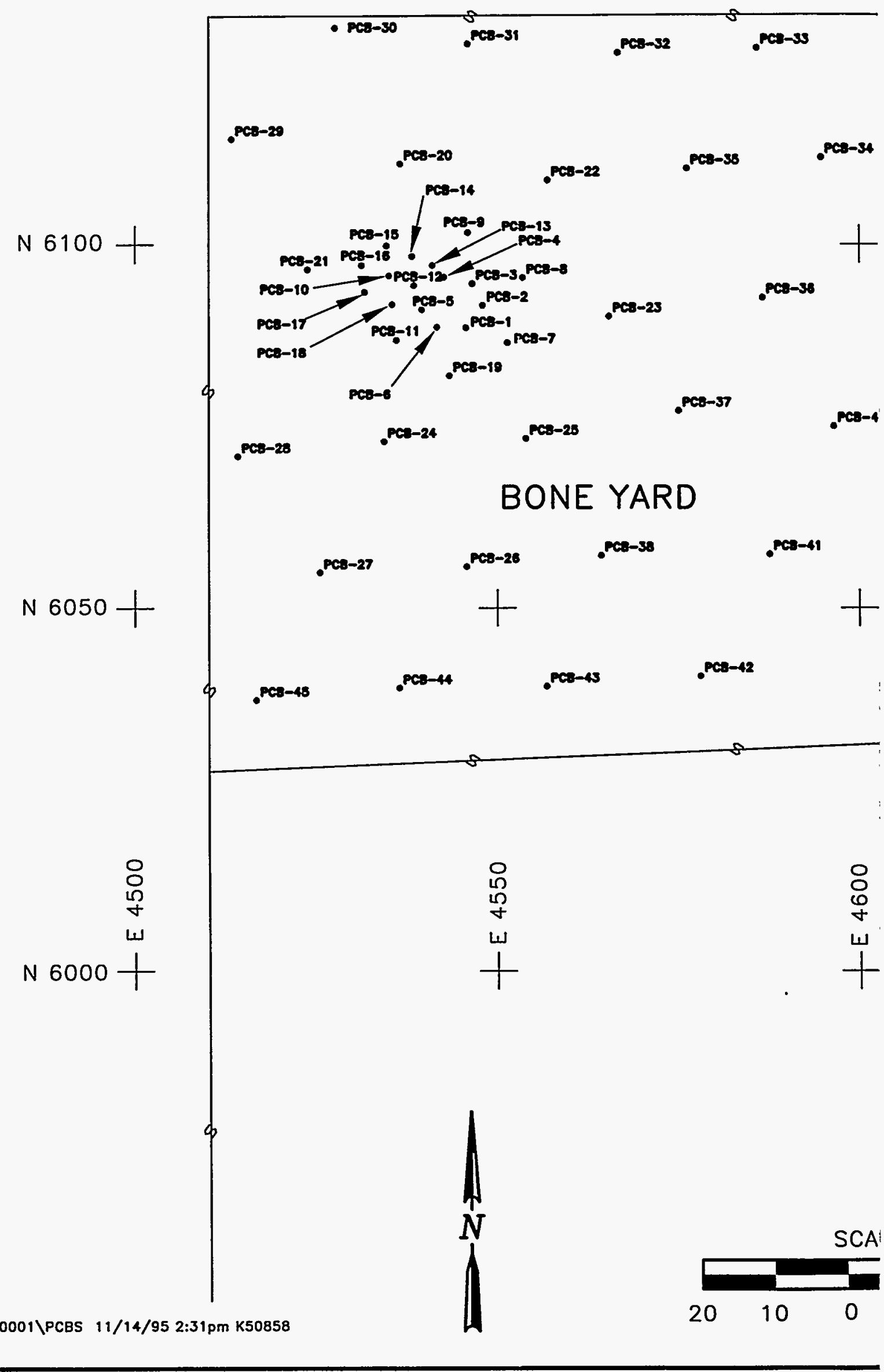


Table 1. PCB Field-Test Results

\begin{tabular}{|c|c|c|c|c|c|}
\hline $\begin{array}{l}\text { Sample } \\
\text { Kumber }\end{array}$ & $\begin{array}{l}\text { Collect } \\
\text { Date }\end{array}$ & Dup & $P C B<1$ & $1<P C B<10$ & PCB>10 \\
\hline 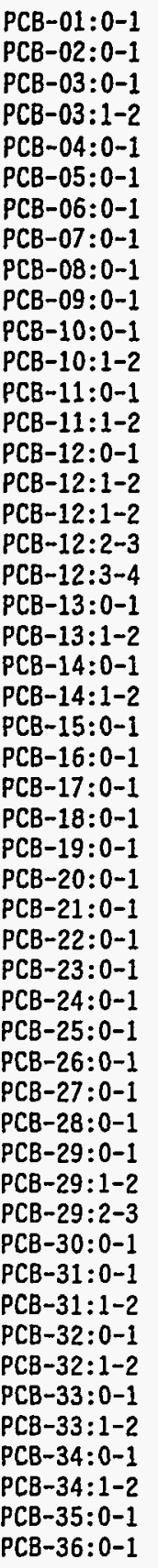 & $\begin{array}{l}1995 / 09 / 11 \\
1995 / 09 / 11 \\
1995 / 09 / 11 \\
1995 / 09 / 11 \\
1995 / 09 / 11 \\
1995 / 09 / 11 \\
1995 / 09 / 12 \\
1995 / 09 / 11 \\
1995 / 09 / 12 \\
1995 / 09 / 12 \\
1995 / 09 / 12 \\
1995 / 09 / 12 \\
1995 / 09 / 12 \\
1995 / 09 / 12 \\
1995 / 09 / 12 \\
1995 / 09 / 12 \\
1995 / 09 / 12 \\
1995 / 09 / 12 \\
1995 / 09 / 12 \\
1995 / 09 / 12 \\
1995 / 09 / 12 \\
1995 / 09 / 12 \\
1995 / 09 / 12 \\
1995 / 09 / 12 \\
1995 / 09 / 12 \\
1995 / 09 / 12 \\
1995 / 09 / 12 \\
1995 / 09 / 12 \\
1995 / 09 / 12 \\
1995 / 09 / 12 \\
1995 / 09 / 12 \\
1995 / 09 / 12 \\
1995 / 09 / 13 \\
1995 / 09 / 13 \\
1995 / 09 / 13 \\
1995 / 09 / 13 \\
1995 / 09 / 13 \\
1995 / 09 / 13 \\
1995 / 09 / 13 \\
1995 / 09 / 13 \\
1995 / 09 / 13 \\
1995 / 09 / 13 \\
1995 / 09 / 13 \\
1995 / 09 / 13 \\
1995 / 09 / 13 \\
1995 / 09 / 13 \\
1995 / 09 / 13 \\
1995 / 09 / 13 \\
1995 / 09 / 13 \\
1995 / 09 / 13 \\
1995 / 09 / 13\end{array}$ & (1) & $\begin{array}{l}x \\
x \\
x \\
x \\
x \\
x \\
x \\
x \\
x \\
x \\
x \\
x \\
x \\
x \\
x \\
x\end{array}$ & $\begin{array}{l} \\
x \\
x \\
x \\
x \\
x \\
x \\
x \\
x\end{array}$ & $\begin{array}{l} \\
x \\
x \\
x \\
x\end{array}$ \\
\hline
\end{tabular}


Table 1 (continued). PCB Field-Test Results

\begin{tabular}{|c|c|c|c|c|c|}
\hline $\begin{array}{l}\text { Sample } \\
\text { Humber }\end{array}$ & $\begin{array}{l}\text { Collect } \\
\text { Date }\end{array}$ & Dup & $P C B<1$ & $1<P C B<10$ & $P C B>10$ \\
\hline 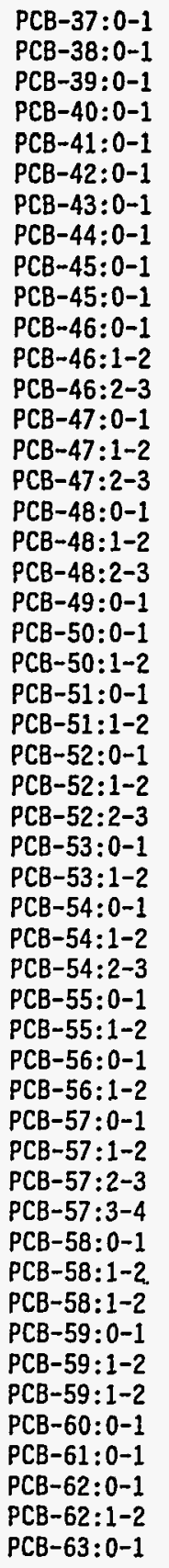 & $\begin{array}{l}1995 / 09 / 13 \\
1995 / 09 / 13 \\
1995 / 09 / 13 \\
1995 / 09 / 13 \\
1995 / 09 / 13 \\
1995 / 09 / 13 \\
1995 / 09 / 13 \\
1995 / 09 / 13 \\
1995 / 09 / 13 \\
1995 / 09 / 13 \\
1995 / 09 / 14 \\
1995 / 09 / 14 \\
1995 / 09 / 14 \\
1995 / 09 / 14 \\
1995 / 09 / 14 \\
1995 / 09 / 14 \\
1995 / 09 / 14 \\
1995 / 09 / 14 \\
1995 / 09 / 14 \\
1995 / 09 / 14 \\
1995 / 09 / 14 \\
1995 / 09 / 14 \\
1995 / 09 / 14 \\
1995 / 09 / 14 \\
1995 / 09 / 14 \\
1995 / 09 / 14 \\
1995 / 09 / 14 \\
1995 / 09 / 14 \\
1995 / 09 / 14 \\
1995 / 09 / 14 \\
1995 / 09 / 14 \\
1995 / 09 / 15 \\
1995 / 09 / 14 \\
1995 / 09 / 15 \\
1995 / 09 / 14 \\
1995 / 09 / 15 \\
1995 / 09 / 14 \\
1995 / 09 / 15 \\
1995 / 09 / 15 \\
1995 / 09 / 15 \\
1995 / 09 / 14 \\
1995 / 09 / 15 \\
1995 / 09 / 15 \\
1995 / 09 / 14 \\
1995 / 09 / 15 \\
1995 / 09 / 15 \\
1995 / 09 / 14 \\
1995 / 09 / 15 \\
1995 / 09 / 15 \\
1995 / 09 / 15 \\
1995 / 09 / 15\end{array}$ & $x$ & $\begin{array}{l}x \\
x \\
x \\
x \\
x \\
x \\
x \\
x\end{array}$ & $\begin{array}{l}x \\
x \\
x \\
x \\
x \\
x \\
x \\
x \\
x \\
x \\
x \\
x \\
x \\
x\end{array}$ & \\
\hline
\end{tabular}


Table 1 (continued). PCB Field-Test Results

\begin{tabular}{|c|c|c|c|c|c|}
\hline $\begin{array}{l}\text { Sample } \\
\text { Humber }\end{array}$ & $\begin{array}{l}\text { Collect } \\
\text { Date }\end{array}$ & Dup & $P C B<1$ & $1<\mathrm{PCB}<10$ & $P C B>10$ \\
\hline $\begin{array}{l}\text { PCB-63:1-2 } \\
\text { PCB-64:0-1 } \\
\text { PCB-65:0-1 } \\
\text { PCB-66:0-1 } \\
\text { PCB-67:0-1 } \\
\text { PCB-68:0-1 } \\
\text { PCB-69:0-1 } \\
\text { PCB-70:0-1 } \\
\text { PCB-71:0-1 } \\
\text { PCB-72:0-1 } \\
\text { PCB-72:0-1 } \\
\text { PCB-72:1-2 } \\
\text { PCB-73:0-1 } \\
\text { PCB-73:0-1 } \\
\text { PCB-74:0-1 } \\
\text { PCB-75:0-1 } \\
\text { PCB-MB } \\
\text { PCB-MB } \\
\text { PCB-MB }\end{array}$ & $\begin{array}{l}1995 / 09 / 15 \\
1995 / 09 / 15 \\
1995 / 09 / 15 \\
1995 / 09 / 15 \\
1995 / 09 / 15 \\
1995 / 09 / 15 \\
1995 / 09 / 15 \\
1995 / 09 / 15 \\
1995 / 09 / 15 \\
1995 / 09 / 15 \\
1995 / 09 / 15 \\
1995 / 09 / 15 \\
1995 / 09 / 15 \\
1995 / 09 / 15 \\
1995 / 09 / 15 \\
1995 / 09 / 15 \\
1995 / 09 / 12 \\
1995 / 09 / 13 \\
1995 / 09 / 15\end{array}$ & $x$ & $\begin{array}{l}x \\
x \\
x \\
x \\
x \\
x \\
x \\
x\end{array}$ & $\begin{array}{l}\mathbf{x} \\
\mathbf{x} \\
.\end{array}$ & \\
\hline
\end{tabular}




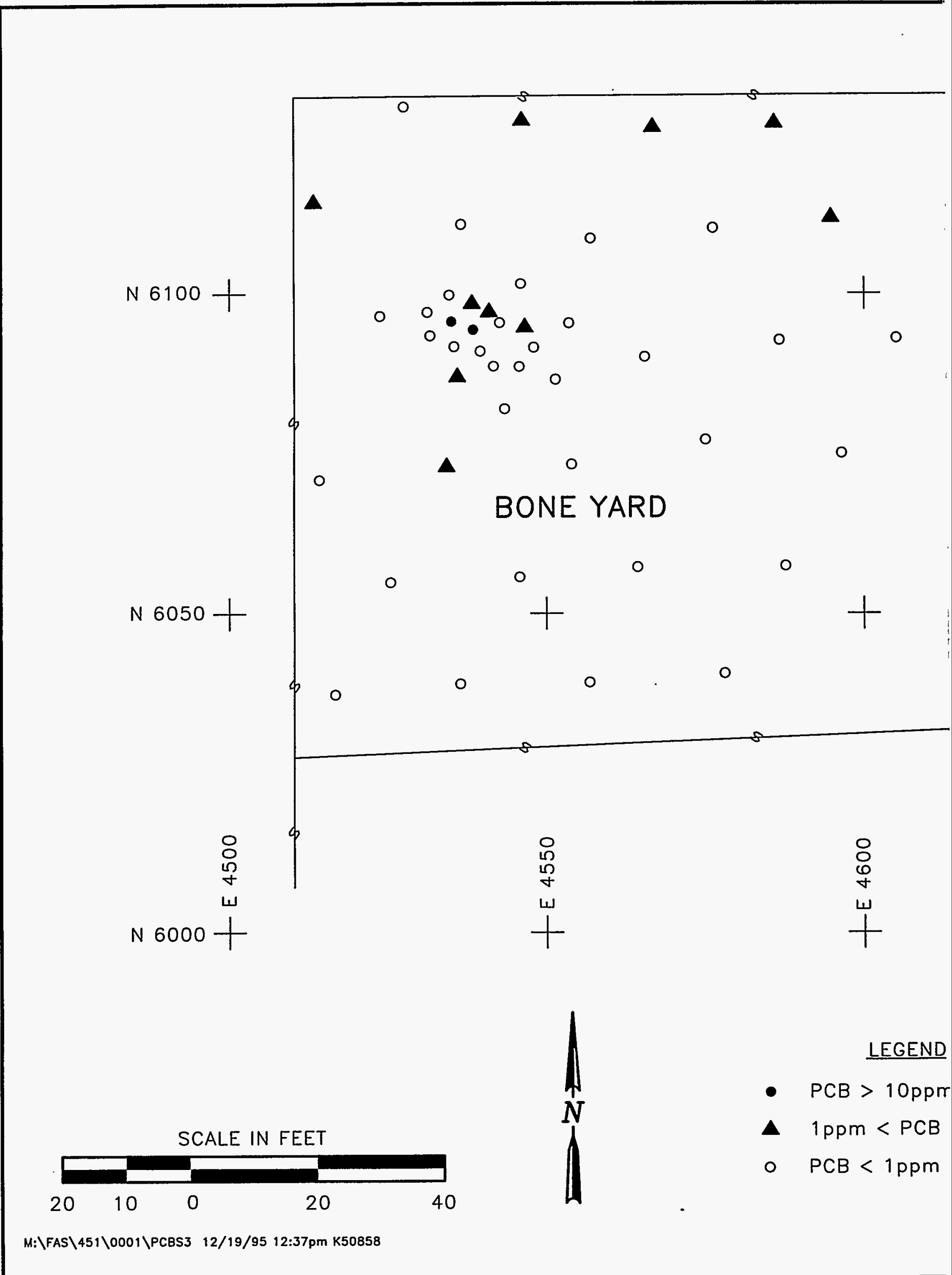


Table 2. PCB Luvoratory Dataa

\begin{tabular}{|c|c|c|c|c|c|c|c|}
\hline $\begin{array}{l}\text { Sample } \\
\text { Kumber }\end{array}$ & $\begin{array}{l}\text { Ticket } \\
\text { Number }\end{array}$ & $\begin{array}{l}\text { Aroclor-1016 } \\
(\mu g / \mathrm{kg})\end{array}$ & $\begin{array}{l}\text { Aroc]or-1221 } \\
(\mu \mathrm{gg} / \mathrm{kg})\end{array}$ & $\begin{array}{l}\text { Arocior-1232 } \\
(\mu \mathrm{g} / \mathrm{kg})\end{array}$ & $\begin{array}{l}\text { Aroc7or-1242 } \\
(\mu \mathrm{g} / \mathrm{kg})\end{array}$ & $\begin{array}{c}\text { Aroclor-1248/1254 } \\
(\mu \mathrm{kg} / \mathrm{kg})\end{array}$ & $\begin{array}{l}\text { Aroclor-1260 } \\
(\mu g / \mathrm{kg})\end{array}$ \\
\hline $\begin{array}{l}\text { PCB-03:0-1 } \\
\text { PCB-10:0-1 } \\
\text { PCB-11:0-1 } \\
\text { PCB-12:0-1 } \\
\text { PCB-12:0-1 (Dup) } \\
\text { PCB-12:1-2 } \\
\text { PCB-12:2-3 } \\
\text { PCB-18:0-1 } \\
\text { PCB-27:0-1 } \\
\text { PCB-33:0-1 } \\
\text { PCB-47:0-1 } \\
\text { PCB-54:0-1 } \\
\text { PCB-54:0-1 (Oup) } \\
\text { PCB-54:2-3 } \\
\text { PCB-57:0-1 } \\
\text { PCB-58:0-1 } \\
\text { PCB-63:1-2 } \\
\text { PCB-66:0-1 } \\
\text { PCB-68:0-1 } \\
\text { PCB-72:1-2 }\end{array}$ & $\begin{array}{l}\text { NBD }-326 \\
\text { NBD }-327 \\
\text { NBD-328 } \\
\text { NBD-329 } \\
\text { NBD-330 } \\
\text { NBD-331 } \\
\text { NBD }-332 \\
\text { NBD }-334 \\
\text { NBD }-335 \\
\text { NBD }-336 \\
\text { NBD }-337 \\
\text { NBD }-340 \\
\text { NBD }-338 \\
\text { NBD }-348 \\
\text { NBD }-341 \\
\text { NBD-342 } \\
\text { NBD-349 } \\
\text { NBD }-344 \\
\text { NBD }-345 \\
\text { NBD }-715\end{array}$ & $\begin{array}{r}<68 \\
<500 \\
<800 \\
<270 \\
<400 \\
<47 \\
<46 \\
<240 \\
<47 \\
<340 \\
<47 \\
<47 \\
<37 \\
<83 \\
<84 \\
<37 \\
<110 \\
<42 \\
<83 \\
<340\end{array}$ & $\begin{array}{r}<68 \\
<500 \\
<800 \\
<270 \\
<400 \\
<47 \\
<46 \\
<240 \\
<47 \\
<340 \\
<47 \\
<47 \\
<37 \\
<83 \\
<84 \\
<37 \\
<110 \\
<42 \\
<83 \\
<340\end{array}$ & $\begin{array}{r}<68 \\
<500 \\
<800 \\
<270 \\
<400 \\
<47 \\
<46 \\
<240 \\
<47 \\
<340 \\
<47 \\
<47 \\
<37 \\
<83 \\
<84 \\
<37 \\
<110 \\
<42 \\
<83 \\
<340 \\
<\quad\end{array}$ & $\begin{array}{r}<68 \\
<500 \\
<800 \\
<270 \\
<400 \\
<47 \\
<46 \\
<240 \\
<47 \\
<340 \\
<47 \\
<47 \\
<37 \\
<83 \\
<84 \\
<37 \\
<110 \\
<42 \\
<83 \\
<340\end{array}$ & $\begin{array}{c}2000 \\
\text { Trace }^{\mathrm{b}} \\
\text { Trace }^{\mathrm{b}} \\
460 \\
\text { Trace }^{\mathrm{b}} \\
<47 \\
<46 \\
\text { Trace }^{\mathrm{b}} \\
700 \\
<340 \\
3200 \\
2800 \\
840 \\
240 \\
6400 \\
2700 \\
340 \\
1700 \\
<83 \\
<340\end{array}$ & $\begin{array}{r}<68 \\
<500 \\
<800 \\
<270 \\
<400 \\
150 \\
150 \\
<240 \\
<47 \\
<340 \\
<47 \\
<47 \\
<37 \\
<83 \\
<84 \\
<37 \\
<110 \\
<42 \\
<83 \\
<340\end{array}$ \\
\hline $\begin{array}{l}\text { Sample } \\
\text { Number }\end{array}$ & $\begin{array}{l}\text { Ticket } \\
\text { Number }\end{array}$ & $\begin{array}{l}\text { Aroclor-1016 } \\
(\mu \mathrm{g} / \mathrm{L})\end{array}$ & $\begin{array}{l}\text { Aroclor-1221 } \\
(\mu \mathrm{g} / \mathrm{L})\end{array}$ & $\begin{array}{l}\text { Aroclor-1232 } \\
(\mu \mathrm{g} / \mathrm{L})\end{array}$ & $\begin{array}{c}\text { Aroctor-1242 } \\
(\mu \mathrm{g} / \mathrm{L})\end{array}$ & $\begin{array}{l}\text { Aroclor-1248/1254 } \\
(\mu \mathrm{m} / \mathrm{L})\end{array}$ & $\begin{array}{l}\text { Aroclor-1260 } \\
(\mu \mathrm{\mu g} / \mathrm{L})\end{array}$ \\
\hline $\begin{array}{l}\text { Equipment Blank } \\
\text { Equipment Blank } \\
\text { Equipment Blank } \\
\text { Equipment Blank } \\
\text { Equipment Blank } \\
\text { Equipment Blank }\end{array}$ & $\begin{array}{l}N B D-333 \\
N B D-339 \\
N B D-343 \\
N B D-346 \\
N B D-347 \\
N B D-350\end{array}$ & $\begin{array}{l}<1.0 \\
<1.0 \\
<1.0 \\
<1.0 \\
<1.0 \\
<1.0\end{array}$ & $\begin{array}{l}<1.0 \\
<1.0 \\
<1.0 \\
<1.0 \\
<1.0 \\
<1.0\end{array}$ & $\begin{array}{l}<1.0 \\
<1.0 \\
<1.0 \\
<1.0 \\
<1.0 \\
<1.0\end{array}$ & $\begin{array}{l}<1.0 \\
<1.0 \\
<1.0 \\
<1.0 \\
<1.0 \\
<1.0\end{array}$ & $\begin{array}{l}<1.0 \\
<1.0 \\
<1.0 \\
<1.0 \\
<1.0 \\
<1.0\end{array}$ & $\begin{array}{l}<1.0 \\
<1.0 \\
<1.0 \\
<1.0 \\
<1.0 \\
<1.0\end{array}$ \\
\hline
\end{tabular}

${ }^{a} A$ " $<"$ symbol indicates that the maximum concentration was below the detection limit (number shown is detection limit); $1000 \mu \mathrm{g} / \mathrm{kg}=1 \mathrm{ppm}$

${ }^{\mathrm{b}}$ Trace $=$ PCBs present in low concentrations but not quantified. 
Comparison of field test data versus laboratory data is shown Table 3. The field test kit predicted the laboratory concentration in 11 out of 18 samples and predicted a higher concentration than the laboratory result (false positive) in 6 of the samples. Only one sample had a test kit result lower than the laboratory result (false negative). However, this false negative had a field result $(<1 \mathrm{ppm})$ and a laboratory result $(1.7 \mathrm{ppm})$ that were similar in magnitude.

Field screening using the PCB test kits was considered effective. In 17 out of 18 cases, the field concentration was equal to or greater than the laboratory concentration (i.e. the test kit results were consistently more conservative than laboratory results).

Although the PCB field test kits performed adequately to meet the goals of the project, the correlation between field and laboratory data was not ideal. The correlation was obscured when the field test predicted concentrations above $10 \mathrm{ppm}$. In each case, the laboratory results were below $1 \mathrm{ppm}$. Possible reasons for the imperfect correlation are

1. The PCB test kits were conservatively configured for aroclor 1248 . This means that the higher aroclors detected by the laboratory (aroclors 1254 and 1260) would cause the detection levels of the test kit to change from $1 \mathrm{ppm}$ and $10 \mathrm{ppm}$ to $0.4 \mathrm{ppm}$ and $4 \mathrm{ppm}$. Therefore, samples having concentrations greater than $10 \mathrm{ppm}$ on the field test actually had concentrations greater than $4 \mathrm{ppm}$.

2. Immunoassay tests are adjusted by EnSys so that the response of the test-kit standard is below the detection level. This adjustment results in a conservative test that rarely gives a false negative result $(<5$ percent); however, some false positives are expected.

3. Laboratory precision for EPA method 8080 can contribute to discrepancies between field-test and laboratory data. Precision of the laboratory duplicate analyzed for this project was 25 percent (relative percent difference).

4) Aliquots taken for field and laboratory analysis may have actually differed in PCB concentration because of heterogeneous soil even though procedures were implemented to homogenize the soil. 
Table 3. Comparison of PCB Field-Test Results with Laboratory Analytical Results

\begin{tabular}{|c|c|c|c|}
\hline Ticket Number & Sample Number & $\begin{array}{c}\text { Field Result } \\
\text { (ppm) }\end{array}$ & $\begin{array}{c}\text { Lab Result } \\
\text { (ppm) }\end{array}$ \\
\hline NBD-337 & PCB-47:0-1 & $1<P C B<10$ & 3.2 \\
\hline NBD -340 & PCB-54:0-1 & $1<P C B<10$ & 2.8 \\
\hline NBD-341 & РСB-57:0-1 & $1<P C B<10$ & 6.4 \\
\hline NBD-342 & PCB-58:0-1 & $1<P C B<10$ & 2.7 \\
\hline NBD -344 & PCB-66:0-1 & PCB $<1$ & 1.7 \\
\hline NBD -345 & PCB-68:0-1 & PCB $<1$ & ND \\
\hline NBD -348 & PCB-54:2-3 & PCB $<1$ & 0.24 \\
\hline NBD-349 & PCB-63:1-2 & $P C B<1$ & 0.34 \\
\hline NBD-715 & PCB-72:1-2 & $P C B<1$ & ND \\
\hline NBD-326 & РСB-03:0-1 & $1<\mathrm{PCB}<10$ & 2.0 \\
\hline NBD -327 & PCB-10:0-1 & $P C B>10$ & Trace \\
\hline NBD -328 & PCB-11:0-1 & $1<\mathrm{PCB}<10$ & Trace \\
\hline NBD -329 & PCB-12:0-1 & $P C B>10$ & 0.46 \\
\hline NBD-331 & PCB-12:1-2 & $P C B>10$ & 0.15 \\
\hline NBD -332 & PCB-12:2-3 & $P C B>10$ & 0.15 \\
\hline NBD-334 & PCB-18:0-1 & $\mathrm{PCB}<1$ & Trace \\
\hline NBD-335 & PCB-27:0-1 & $\mathrm{PCB}<1$ & 0.7 \\
\hline NBD-336 & PCB-33:0-1 & $1<P C B<10$ & ND \\
\hline
\end{tabular}

ND = Not Detected 


\subsection{Recommendations}

Results of this PCB characterization indicate that soils in the vicinity of the Bone Yard at the Oxnard facility are not significantly contaminated with PCBs. The highest confirmed PCB concentration in soil was $6.4 \mathrm{ppm}$, which is below the 10-ppm remediation criterion recommended by the EPA for industrial sites and below the 8-ppm risk-based concentration derived from the State of California guidance. In addition, when risk was assessed using a $6.4 \mathrm{ppm}$ PCB soil concentration and the equations and assumptions from the Phase II ESA report (DOE 1995a), risk from exposure to carcinogens at the Oxnard facility remained essentially unchanged, increasing only slightly from $2.8 \times 10^{-6}$ (Phase II ESA) to $3.2 \times 10^{-6}$. Therefore, remediation of PCBs in soil at the Oxnard facility is not recommended. 


\subsection{References}

Marburg Associates and William P. Parkin, 1991. Site Auditing: Environmental Assessment of Property, Specialty Technical Publishers, Inc., Vancouver, British Columbia, Canada.

State of California Environmental Protection Agency, 1994. Preliminary Endangerment Assessment Guidance Manual, Department of Toxic Substances Control, Sacramento, CA.

U.S. Department of Energy, 1994. Phase I Environmental Site Assessment for the EG\&G Rocky Flats Oxnard Facility, Grand Junction Projects Office, Grand Junction, CO

, 1995a. Phase II Environmental Site Assessment Report for the Kaiser-

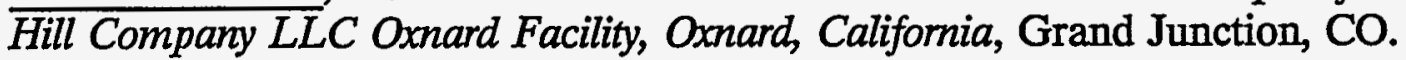

, 1995b. Sampling and Analysis Plan for the Phase III Environmental Site Assessment of the U. S. Department of Energy Oxnard Facility, Oxnard, California, Grand Junction, $\mathrm{CO}$

U. S. Environmental Protection Agency, 1990. A Guide on Remedial Actions at Superfund Sites with PCB Contamination, Directive 9355.4-01FS, Office of Solid Waste and Emergency Response, Washington DC.

, 1991. Role of the Baseline Risk Assessment in Superfund Remedy

Selection Decisions, OSWER Directive 9355.0-30, Washington DC. 


\section{Appendix D}

\section{Asbestos Operation and Maintenance Plan DOE Oxnard Facility}


This page intentionally blank 


\section{ASBESTOS OPERATIONS \& MAINTENANCE PLAN DOE OXNARD FACILITY \\ 1235 EAST WOOLEY ROAD, OXNARD, CA}

Submitted to:

Rust Geotech

USDOE Grand Junction Projects Office POB 14000

Grand Junction, CO 81502-5504

Prepared By:

SCA Environmental, Inc.

2500 Overland Ave.

Suite 100

Los Angeles, CA 90064

TEL: (310) 842-9167

FAX: (310) 842-9168 


\title{
ASBESTOS OPERATIONS \& MAINTENANCE PLAN DOE OXNARD FACILITY \\ 1235 EAST WOOLEY ROAD, OXNARD, CA
}

\author{
Conducted for \\ Rust Geotech \\ USDOE Grand Junction Projects Office \\ POB 14000 \\ Grand Junction, CO 81502-5504
}

January 23, 1996

SCA Project No. L-1421

Prepared by:

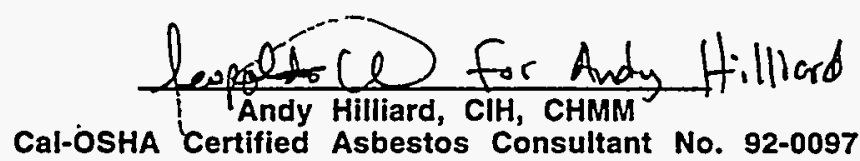

Reviewed by:

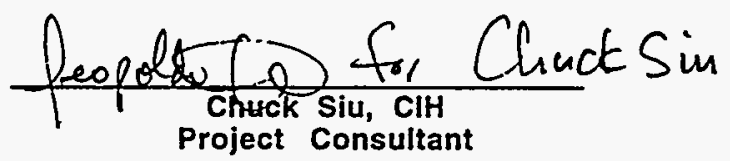

SCA Environmental, Inc. 2500 Overland Ave. Suite 100

Los Angeles, CA 90064

TEL: (310) 842-9167

FAX: (310) 842-9168 



\section{INTRODUCTION and EXECUTIVE SUMMARY}

Throughout the document, each section will have a box like this one which will give a brief summary of the section's contents.

The document you are reading, the Asbestos Operations and Maintenance Plan, contains guidance for controlling asbestos-containing materials located at the DOE Oxnard Facility in Oxnard, CA (hereafter referred to as "the Oxnard Facility"). This document is specifically written to address asbestos occurring in and on the seven structures located at the Oxnard Facility. Understanding and following the guidelines in this document is essential to maintaining a safe workplace and avoiding asbestos exposure to building employees and the public.

This Operations and Maintenance Plan, or "O\&M Plan," includes by reference a survey for asbestos-containing materials which was performed at the site by Rust Geotech in July, 1995. The survey was conducted to estimate the extent of asbestos contamination at the Oxnard facility for purposes of negotiating the transfer or sale of the property. It should not be construed, interpreted or otherwise considered a full asbestos inspection under California statutes and regulations. Because the risk to human health and the environment posed by the type and volume of asbestos identified was negligible, the O\&M Plan was written to minimize the potential need for abatement prior to the transfer or sale. This plan is intended to be transferrable to any new title owner or leaser, provided the transfer does not violate federal, state, or county regulations.

This survey identified asbestos in the following materials:

- $\quad$ Sprayed-on wall texture in the Building 1 Accounting Offices;

- $\quad 9^{11} \times 9^{\prime \prime}$ black floor tiles in the Building 1 Vault;

- $\quad 9 " \times 9$ " white floor tiles in the Building 1 Lobby;

- $\quad$ "HVAC Joint Insulation" in the Building 1 mechanical room (this material is a cement-asbestos flue from the gas heater in the closet);

- Laboratory cabinet interior fireproof sheathing in the Building 2 Metallurgy Lab;

- Window caulk in Building 6;

- $\quad$ Gaskets on the Lindberg Furnace in Building 6;

- Brakes on the Cleveland Presses (assumed asbestos) in Building 6; and

- $\quad$ Gaskets on the Emerson Heater in Building 7.

Since the survey by Rust Geotech was "non-destructive," there is also a potential for concealed asbestos-containing materials to exist at the site. In general, these materials may include vapor barriers, tar papers, window caulking, glues, and mastics. Whenever construction or maintenance activities involve disturbing these materials, then trained personnel should be engaged to sample these materials and determine their asbestos content. 


\section{POLICY STATEMENT}

Building asbestos policy. All employees who may potentially encounter asbestos in their work should be given this policy statement as part of their training.

Policy

Statement:

It is the intent of DOE to have an effective Asbestos Control Program. The purpose of this program is to ensure that employees, visitors and the public are adequately protected from exposure to asbestos fibers in the Oxnard Facility buildings, or as a result of work performed at these buildings by employees or contractors.

This goal will be accomplished by ensuring that all asbestos abatement work, and all maintenance and construction work (including asbestos and demolition) conducted near asbestos, is performed in a safe manner, which minimizes the release of asbestos fibers outside of regulated areas.

An effective Asbestos Control Program requires that individuals at the site fulfill their responsibilities. These responsibilities are summarized in Section 1 of this document.

Asbestos

Impacting

Maintenance

Work:

No building employee may remove, repair, or otherwise work on or with any material containing asbestos. No contractor may remove or repair asbestos materials unless specifically licensed and trained to do so, and approved by the Asbestos Program/ Site Manager. Further, untrained personnel shall not enter areas containing damaged friable asbestos or where an airborne asbestos hazard may exist. All asbestos hazards will be labelled in accordance with federal and state regulations.

As approved by the Asbestos Program/ Site Manager, appropriately trained and equipped contractor personnel are authorized to:

1. Perform small-scale, short-duration removal of asbestos material as necessary for emergency repair work, limited to less than three square feet of material (as defined by OSHA regulations).

2. Perform abatement (removal) of asbestos-containing material following all local, State, and Federal regulations.

\section{Work Evaluation/Permit}

System:

Work which may impact asbestos shall be conducted following the Work Evaluation/Permit System. This system includes determining the presence of asbestos, if any, in areas where construction work is planned to occur. This determination must be performed by a person with EPA Building Inspector training and any required state and county certifications.

Any construction work conducted at the Oxnard Facility should first be reviewed by the Asbestos Program Manager to determine whether the work will impact asbestos-containing materials (ACM). 
Procedures:

Any work which may disturb asbestos shall be performed using specification documents incorporating the latest asbestos regulations as well as state of the art practices, and approved by the Asbestos Program/ Site Manager. Specification documents are typically prepared by an outside consultant with asbestos certification issued by California OSHA.

Whenever asbestos-disturbing work is conducted, clearance inspections and air testing shall be performed as specified in the specification documents listed above.

Whenever asbestos-disturbing work is conducted, an independent industrial hygienist or other trained person approved by the Asbestos Program/ Site Manager shall be present to ensure that work procedures are followed. This industrial hygienist shall have stop work authority if s/he believes that the work operation is creating a hazard for the building personnel or the public.

New Construction

Materials:

As part of this policy, asbestos-containing materials shall not be used when acceptable substitute materials of lower toxicity are available. All construction specifications should include this requirement in writing. The following new materials may contain asbestos:

1) Roofing felts;

2) Vapor barriers, tar paper, and pipe trench liners;

3) Resilient flooring materials;

4) Gaskets;

5) Industrial equipment and vehicle brake pads;

6) Miscellaneous heat shields and protective surfaces;

7) Cement-fiber composite materials (cement-asbestos or "Transite" materials);

8) Other materials incorporating a fibrous binder; and

9) Laboratory tools and equipment.

Construction inspectors and supplies purchasers should examine these new materials in their original packaging to verify that they are labelled "asbestos-free." If the materials are not labelled, the manufacturer should be contacted and asked to provide documentation regarding the composition of the material.

Authority and Enforcement:

Employees found to be operating outside of these policies and procedures will be disciplined. Disciplinary procedures will be consistent with those of other serious offenses. 



\section{RESPONSE TO UNPLANNED ASBESTOS RELEASE}

Actions to take, and people to contact, if there is an accidental disturbance of asbestos materials.

Reporting:

In the event of an actual or suspected unplanned release of asbestos materials, evacuate the area immediately, then obtain the following information before making any phone calls:

- The precise location in the building where the release occurred.

- The exact type of material released (roofing material or some other newlydiscovered material)

- Why/how the material was released (fire, aging, a water leak, etc.)

- How you know the material contains asbestos (i.e., the material was surveyed previously, the material looks like a typical asbestos-containing material).

- The number of people in the area where the release occurred, and the use of the area (office, shop, lobby, etc.).

- Equipment and supplies which may have asbestos material on them, such as desks, books, equipment, etc.

- Potential to evacuate the immediate area of the release.

- Potential to shut off the ventilation system(s) in the area.

Then, contact the following individual:

1. Asbestos Program/ Site Manager

Holly Dumas (805) 486-4881

Procedures:

The following are general procedures to be followed for an asbestos release. They may not apply in all cases. The contacts listed above can give you guidance for your particular situation.

1) Evacuate the immediate area/room where the release occurred. Avoid tracking through asbestos material and spreading it to other areas.

2) Isolate the area, by closing doors, closing windows, setting up barrier tape, or other means.

3) Turn off ventilation systems serving the area.

4) Post warning signs at all possible entrances.

5) Contact appropriate local, state and federal agencies. 

TABLE OF CONTENTS

SECTION 1 - ASBESTOS CONTROL PROGRAM REQUIREMENTS, ROLES, AND

RESPONSIBILITIES.

SECTION 2 - ASBESTOS SURVEY DATABASE AND REPORTS

SECTION 3 - NOTIFICATION AND LABEI IING PROCEDURES FOR OXNARD FACIITY 5

SECTION 4 - MANAGEMENT IN PLACE, PERIODIC ASSESSMENT (SURVEILLANCE) AND ASBESTOS MATERIAL SAMPLING AT THE OXNARD FACILITY................7

SECTION 5 - WORK CONTROL PERMIT SYSTEM................................................ 10 SECTION 6 - WORK PRACTICES FOR OXNARD FACILITY EMPLOYEES PERFORMING MINOR REPAIR OR REMOVAL OF ASBESTOS........................................ 12

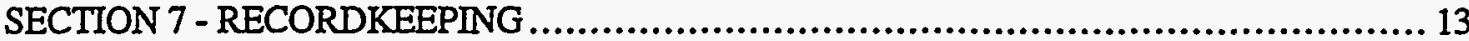

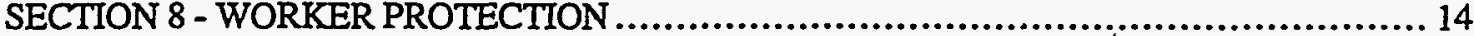

SECTION 9 - TRAINING FOR OXNARD FACILITY EMPLOYEES........................... 15

SECTION 10 - RESPIRATORY PROTECTION FOR OXNARD FACIIITY EMPLOYEES ...... 17

SECTION 11 - MEDICAL SURVEILLANCE FOR OXNARD FACILITY EMPLOYEES........ 18

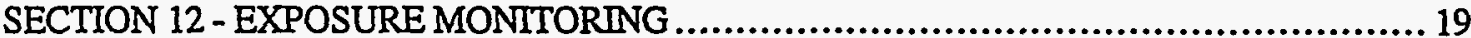

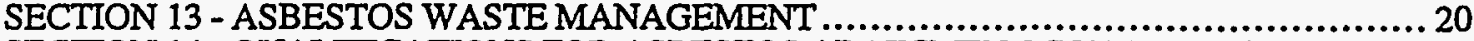

SECTION 14 - QUALIFICATIONS FOR ASBESTOS ABATEMENT CONTRACTORS ........ 21

Attachment A - CONNELLY NOTIFICATION FORMS AND PROCEDURES (Optional Forms - use of these specific forms is not required by regulation; similar forms can be used)

Attachment B - WORK EVALUATION PERMIT FORM (Optional Form - use of this specific form is not required by regulation; similar forms can be used)

Attachment C - WASTE MANIFEST SYSTEM

Attachment D - NOT USED

Attachment E - SUMMARY OF REQUIREMENTS FOR ASBESTOS ABATEMENT CLASSES 


\section{SECTION 1 - ASBESTOS CONTROL PROGRAM REQUIREMENTS, ROLES, AND RESPONSIBILITIES}

This section of the report gives a brief overview of the Asbestos Control Program, including who the players are, and what the elements of the Program are. For more details on responsibilities of individuals, you may wish to read the Management Implementation Plan.

Roles and Responsibilities:

This is a general overview of roles and responsibilities of the Asbestos Control Program participants.

- Asbestos Program/ Site Manager

Note that typically these two roles are separate, with the Asbestos Program Manager being an individual at the Corporate Health and Safety level, who sets overall policy for asbestosrelated work; and the Asbestos Site Manager being at the facility level, and having purchasing authority for small-scale projects.

Because of the nature of the Oxnard Facility, and it's staff (including a full-time Environmental Engineer) the goals of the Asbestos Control Program are best served if these two functions are consolidated.

The Asbestos Program/ Site Manager's responsibilities include:

* maintain contracts with asbestos consultants and contractors;

* approve project-specific asbestos abatement specifications;

* manage the work of asbestos consultants and asbestos abatement contractors; and

* develop an Asbestos Information Notice Program;

* develop a periodic surveillance program;

* develop a response action recordkeeping program;

* develop a work permitting system;

* develop an asbestos awareness training program;

* develop work procedures for Asbestos Operations and Maintenance activities;

* develop and implement a labelling and posting system for ACBM;

* enforce Work Evaluation Permit System for activities which may disturb ACBM;

* $\quad$ supervise Asbestos Operations and Maintenance activities;

* verify that posting and labelling in building is current and complete;

* perform periodic and ongoing surveillance of ACBM;

* maintain on-site records of Asbestos Control Program activities.

- Building Maintenance, Custodial, and Operations Staff

* perform Asbestos Operations and Maintenance activities, where applicable; and

* alert Asbestos Program/ Site Manager to accidental releases or deterioration of ACBM. 
Note that, given the relatively small amount of asbestoscontaining materials identified at the Oxnard Facility, it may not be cost-effective to train Building Maintenance, Custodial, or Operations Staff to perform abatement, even for small scale projects.

- Asbestos Consultant (typically an outside firm)

* prior to construction or other activity potentially disturb ACBM or suspect ACBM, provides Asbestos Inspectors to perform inspection of the work area, including collecting bulk samples for asbestos;

* issues report detailing known ACBM in the work area;

* provides Project Designers to creates specifications and bid documents, assists in selection of qualified contractors to perform abatement work;

* performs construction management services during abatement, including air sampling and quality control review of Abatement Contractor's work activities.

- Asbestos Abatement Contractor (typically an outside firm)

* bids on asbestos abatement tasks;

* performs abatement activities, including small-scale repair of damaged materials, area cleanup and decontamination, and large-scale removal;

* removes asbestos materials in compliance with all applicable regulations and specification requirements;

* disposes of asbestos waste materials and supplies copies of completed waste manifests to building owner.

This is a general overview of roles and responsibilities of the Asbestos Control Program participants. Depending upon the organization of a given facility or building, the roles and responsibilities may be differently distributed.

Elements of the

Asbestos Control

Program:

Several elements must be present for an effective Asbestos Control Program:

1) Trained and equipped personnel:

- Asbestos Program/ Site Manager

- Building Maintenance, Custodial, and Operations Staff

- Asbestos Consultants and Industrial Hygienists

- Accredited Analytical Laboratories

- Asbestos Abatement Contractor

2) Building Surveys

3) Asbestos Operations and Maintenance Plan

4) Asbestos Information Notification System

5) Respiratory Protection Program

6) Medical Surveillance Program

7) Training

- Written Work Procedures

- Emergency Procedures

- Hazard Communication - labels, notices, awareness training;

- Recordkeeping System; and 
- Surveillance/Reinspection

8) Work Evaluation Permit System

9) Equipment and Supplies

10) Cal-OSHA Carcinogen Registration, as applicable

11) Proper Posting and Labelling

12) Ongoing Surveillance Program

These elements of the Asbestos Control Program are explained in detail elsewhere in this document. 


\section{SECTION 2 - ASBESTOS SURVEY DATABASE AND REPORTS}

This section details limitations of the asbestos survey data.

Introduction: The first step in an Asbestos Control Program is the survey of buildings which may contain asbestos. The buildings at Oxnard Facility were surveyed in July, 1995.

While reading this section, you should have a copy of the asbestos survey reports for Oxnard. Facility. If additional asbestos sampling has been performed, you should have that information available also.

Although ACM was generally evaluated for potential risk in the original July, 1995 survey, it should be noted that the condition of friable ACM was not assessed for each and every location where ACM was identified, as assessment was not within the scope of the initial survey. The condition of ACM should be assessed for all friable ACM remaining at the facility (or identified in the future) in order to properly manage it under this plan.

Limitations

of the

Survey:
The survey conducted in the Oxnard Facility was performed as part of a Phase II environmental site assessment (ESA) conducted by Rust Geotech. However, the survey did not include sampling or assessment of concealed materials, for example, vapor barriers inside wall chases. This type of concealed material, and many others, are commonly asbestos-containing in buildings.

The limitations of an asbestos survey can be partially compensated for by vigilance on the part of building maintenance, custodial, and operations staff and construction personnel. Work which disturbs building materials and has the potential to uncover concealed asbestos materials should be performed with the knowledge that these materials may be discovered. All personnel working in this capacity should understand the mechanism for reporting suspect asbestos materials.

Lastly, it cannot be overemphasized that there are limitations to any survey's data. The user should always be skeptical when reviewing the data, and must not accept the data as infallible. Project designers, especially, should accept the data only after they have verified it by means of a site visit and thorough review of the data; satisfaction with the laboratory performing the analysis is critical.

Since Project Designers are wholly responsible for the accuracy of their design, deficiencies in the survey data should not constitute an acceptable excuse for errors and omissions in the designed project. 


\section{SECTION 3 - NOTIFICATION AND LABELLING PROCEDURES FOR OXNARD FACILITY}

This section describes the policy and procedures for notifying employees, contractors, tenants, regarding asbestos in buildings.

A number of regulations require notification of the presence of asbestos. The Connelly Bill (Asbestos Notification Act) requires building owners to notify employees, tenants, and contractors of the presence of asbestos within a building. reference: California Health and Safety Code sections $\$ 25915$ through 25919

Federal OSHA regulations require building owners to notify employees and contractors who may disturb asbestos-containing material. reference: 29 CFR 1926.1101

Federal and California OSHA regulations for Hazard Communication require employers to notify employees of hazardous materials and conditions which may be encountered in the workplace.

reference: 29 CFR 1910.1200

Attachment $A$ of this document contains sample notification forms for building owners/managers to provide to employees, tenants, and contractors.

Note that all asbestos survey and air sampling results are public information. Documents pertaining to asbestos surveys, sampling, and abatement should be made available to the public, however, all inquiries about the status of asbestos in the buildings should be referred to the Asbestos Program/Site Manager so that the latest information is made available. This applies to questions from tenants, contractors, employees, the public, the media, or any other parties.

A variety of strategies exist for labelling asbestos materials in buildings. The intent of the strategies is all the same: to ensure that accidental disturbance of these materials, and asbestos exposure to building occupants, does not occur. For the Oxnard Facility, the following is recommended:

1) Labeling of acoustical sprayed wall material is not recommended; affixing a label to the material would disturb it with potential fiber release, and attaching labels to it would be aesthetically undesirable. Written notices should be used to inform employees and others.

2) Labelling of the the following asbestos materials is practical:

a) fireproof sheathing material in the Building 2 Metallurgy Laboratory cabinets (the cabinet exteriors should be labelled); and

b) cement-asbestos flue in the Building 1 mechanical room.

These materials should be labelled with OSHA-approved warning labels.

3) Labelling of other materials noted, such as floor tiles and gaskets, is not practical. Written notices should be used to inform employees and others. 
California Code of Regulations, Title 8, Section 5208 (n) requires the following information to be placed on asbestos labels, where it is practical to use them: 


\section{SECTION 4 - MANAGEMENT IN PLACE, PERIODIC ASSESSMENT (SURVEILLANCE) AND ASBESTOS MATERIAL SAMPLING AT OXNARD FACILITY}

This section of the report discusses:

1) When it is acceptable to perform "management in place" of asbestos materials, as opposed to abatement of materials.

2) Periodically checking the condition of asbestos materials in the building to identify any new damages or deterioration, known as "ongoing surveillance."

3) When it is advisable to collect more samples for asbestos in a building which has already been surveyed for asbestos.

Management In Place:

This section discusses when it is acceptable to perform management in place of $A C M$ or PACM. Note that this section has fairly generic examples.

Management in place for ACM or PACM is acceptable when the following conditions are met:

1) When a material is nonfriable, or when a material is friable and relatively undamaged;

2) When the material is not in danger of being disturbed by normal operations of the facility;

3) When the material is not in danger of being disturbed by a planned renovation or repair action; and

4) When management in place meets the economic and health and safety goals of the facility.

In order to make an informed decision between abatement of materials and management in place, it is important to understand the costs and risks associated with each option.

Abatement of asbestos involves immediate costs (contract costs, disposal and documentation costs, and costs of "lost use" of portions of a building during abatement). Abatement projects also involve risks of employee exposures, environmental releases, liability associated with waste disposal, etc.

Management of asbestos in place involves costs over time; these costs include record-keeping, training, surveillance, etc. There are also risks associated with management in place, including accidental disturbance or damage. 
The decision between abatement and management in place should be made by personnel familiar with asbestos management, building management, and construction issues.

Performing

Qngoing

Surveillance:

Surveillance means watching the status of asbestos materials in a building over time. Like other parts of a building, asbestos materials are dynamic, and are affected by aging, water damage, accidental disturbance, etc. The goal of surveillance is to catch changes in the asbestos materials early enough so that there is minimal health disturb to employees and the public.

Surveillance involves looking for materials which have been damaged or which may endanger employees, or the public. Building maintenance staff are ideally suited for this task, since they know their buildings very well, and can often observe the condition of asbestos materials as part of a routine of checking other equipment in the building (such as lights, air conditioning system, fire extinguishers, etc.).

The frequency of ongoing surveillance will vary by building. However, in general it is recommended that building-wide surveillance be conducted at least once a year.

Asbestos surveillance involves going through the building and visually assessing each asbestos material, comparing it against the survey report to verify that it has not become more damaged.

Additional Bulk

Sampling:

This section discusses when it is advisable to collect additional bulk samples. Note that this situation has fairly generic examples, which may not apply entirely to the Oxnard Facility buildings.

Additional sampling should be performed in any of the following situations:

1) When there is a potential to disturb "assumed asbestos" or "Presumed Asbestos-Containing Materials" (PACM), a decision should be made whether to collect bulk samples of the material, or to merely treat the material as asbestos. Unless the disturb to the material is very minor, it is usually costeffective to collect samples.

Example: a sheetrock ceiling needs to have several holes drilled into it to hang new lights. No previous sampling has been performed of this material.

The drilling work can either be done using trained personnel under asbestos procedures. Or, samples can be collected of the sheetrock ceiling in the disturbed area; if the samples indicate that the material is non-asbestos, the work can proceed without asbestos procedures. Note that samples should be collected by specially trained personnel (see Section 9).

Warning: because sheetrock, plaster, and other finish materials may appear very similar and yet have different installation dates, it is not usually appropriate to use results from one area of a building to represent the entire building. In the example above, negative results from samples in one location could not be used to state that similar materials in another part of the building were non-asbestos. Determining that sheetrock or plaster has no asbestos throughout an entire building requires a comprehensive sampling strategy, and assumptions based on Building Drawings and history, devised by a person trained in the EPA Building Inspector course, and with experience in this area. 
2) When a suspect material is discovered which is not included in the building's asbestos survey report, the material should either be treated as PACM or sampled.

Example: while cutting into a pipe chase to repair a leak, an employee realizes that there is a vapor barrier material lining the pipe chase. The asbestos survey report does not address the material.

The vapor barrier should be treated as PACM, and the work conducted under asbestos procedures; or, the material should be sampled by a trained individual.

3) When a construction project is being planned which will disturb asbestos materials, PACM, or concealed spaces which may contain asbestos, additional investigation must be performed.

This includes any project which involves moving or replacing a wall (including a non-load-bearing wall). The work control permit system procedures must be used in these cases (see Section 5).

Considerations

for Bulk

Sampling:

The actual act of collecting a bulk sample is fairly simple. However, collecting the sample in accordance with EPA and OSHA requirements, and properly documenting the sample, is fairly complex. Consequently, only individuals who have undergone training to become an EPA accredited Asbestos Inspector should collect bulk samples. This is a requirement of Federal OSHA regulations, 29 CFR 1926.1101 (k) (4) (ii) (B), as well as USEPA and local (Ventura County Air Pollution Control District) regulations .

Bulk sampling and documentation can be conducted fairly rapidly for a small number of samples. However, laboratory analysis of the samples can take a week or longer, depending upon the means used to ship samples to the laboratory, and the turnaround time which the laboratory has agreed upon. Quick sample results ( $<24$ hours) can be achieved through use of couriers to ship the samples, and paying a premium to the laboratory for a "rush" analysis.

In emergency situations, these expenses are well justified. For more routine or foreseeable events, the sampling should be conducted early to allow economical shipping and analysis.

Summary:

To summarize this section:

1) Management in place and abatement are two options for dealing with $\mathrm{ACM}$ or PACM. It is important for the Asbestos Program/ Site Manager, and other decision-makers, to have an understanding of the costs and risks associated with each option.

2) Ongoing surveillance, performed every year or less by building staff, checks for damage to known asbestos materials.

3) Asbestos bulk samples need to be collected in a variety of circumstances, including damaged suspect materials, disturbance to "assumed asbestos" materials, and newly discovered materials. Asbestos bulk sampling requires EPA accreditation as a Building Inspector and specialized training in the use of forms for documentation. For outside (consulting) personnel, accreditation by Cal/OSHA as Certified Site Surveillance Technician or Certified Asbestos Consultant is required as well. 


\section{SECTION 5 - WORK CONTROL PERMIT SYSTEM}

This section lists the "sign-offs" and approvals necessary to perform work which may disturb asbestos. Two types of work permit exist: maintenance work and construction work.

Purpose of the Work Evaluation Permit System:

After a building has been surveyed for asbestos, it is essential that the survey information be used to avoid unplanned disturbances of ACM and PACM. There are many negative consequences of maintenance work or construction work which disturbs ACM or PACM without using asbestos procedures:

1) Most importantly, employees and the public may be exposed to asbestos unknowingly, and thus suffer a risk to their long-term health.

2). Parts of the building may have to be evacuated, resulting in disnuptions to the operations conducted in the building.

3) Money which is intended for building improvement and maintenance may have to be diverted to expensive emergency cleanup work.

How is the Work Evaluation Permit System intended to avoid these problems? The system operates on the principle that any work done in the building must first be compared to the locations of known or presumed ACM.

- For maintenance work, it is essential that all work be approved by the Asbestos Program/ Site Manager prior to beginning. For the Oxnard Facility, this should include any work on the building interior or roof and any work which may disturb concealed materials. In addition, any work on equipment which may have asbestos gaskets or brakes should be approved by the Asbestos Program/ Site Manager.

- For construction work, it is essential that all work be approved by the Asbestos Program/ Site Manager prior to beginning. The Asbestos Program/ Site Manager, needs to determine whether the work has the potential to disturb asbestos. If so, then the Asbestos Program/ Site Manager will engage the services of a qualified consultant to collect additional samples, prepare a scope of work for an abatement contractor; etc.

Construction work as used in this section means non-routine work which may be performed by building maintenance staff, other staff, or outside contractors.

Responsibilities:

For maintenance or construction work, the Asbestos Program/Site Manager has the responsibility to determine whether the work will disturb known or presumed asbestos materials.

Permit Form: 
The Work Evaluation Permit System for construction work should be built into the contract system. No contract for construction work should be approved until the Asbestos Program/ Site Manager completes an Asbestos Work Review form (Attachment B), or similar approved form.

For the Oxnard Facility, this Asbestos Work Review form should be integrated into the other Contractual Forms (such as the existing Work Control Forms and Work Order Packages).

General Elements of Work Evaluations:

The work evaluations conducted for maintenance and construction work involve collecting the following data:

1) the time and location of the work to be performed;

2) description of the work; and

3) any known information about the presence of $\mathrm{ACM}$ (quantity, type, condition).

Once the data has been collected ${ }_{2}$ the steps below are taken:

1) Review data: The reviewer will examine the Asbestos Survey and any other records to determine whether or not any ACM or PACM will be affected by the job.

2) Visit the site: The reviewer will make a visit to the location of the proposed work to verify whether $A C M$ is present and likely to be disturbed. The reviewer will collect additional samples if unrecorded ACM is present, or if PACM is present and the reviewer wants to attempt to rebut its status as PACM. (Note that sample collection may only be performed by an EPA accredited Asbestos Inspector).

3) Record information: Using forms as described above, and as located in Attachment B, record the project's potential disturb to ACM and/or PACM. Maintain a record of these forms along with other asbestos records.

4) Plan work practices: Depending upon the scope and nature of the work, this may include bidding for specialty contractors to perform asbestos work, giving Connelly information to contractors regarding location of asbestos, etc.

5) Visit worksite during work: The reviewer, or reviewer's representative, should visit the location again during the set-up phase of the work, and possibly several times again during the project. The purpose of the visit should be to ensure that the project is proceeding as planned, and that no last minute changes have occurred which might cause an

to $A C M$ or PACM. 


\section{SECTION 6 - WORK PRACTICES FOR OXNARD FACILITY EMPLOYEES} PERFORMING MINOR REPAIR OR REMOVAL OF ASBESTOS

NOTE: The known asbestos materials at the Oxnard Facility are primarily low-maintenance materials. It is not considered practical to have on-site employees trained and equipped to perform minor repair or removal work. Any work which involves cutting, drilling, tearing, or removing asbestos-containing- materials should be performed by an outside contractor with asbestos licensing.

Note that California OSHA regulations require extensive training, medical examinations, and air monitoring for personnel who are assigned to work with asbestos. In addition, a one-time facility permit as a carcinogen user must be obtained through Cal/OSHA. 


\section{SECTION 7 - RECORDKEEPING}

All documentation regarding asbestos should be maintained by the Asbestos Program/ Site Manager. This includes Connelly notifications, sampling results, waste manifests, etc. It is preferable to create a single place to store all asbestosrelated data, rather than combining it with other project or tenant records.

Note that forms related to air sampling and employee training are required to be stored for 30 years by OSHA regulations. It is generally good practice to store all asbestos-related documentation for this same time period. For liability control reasons, it is recommended that the records be stored beyond the 30 year period (i.e, stored indefinitely).

Since these forms are legal documents which verify compliance with regulations and state of the art practices, it is essential that they are completed legibly, and that the personnel completing them are trained to do so properly. 


\section{SECTION 8 -. WORKER PROTECTION}

NOTE: Because the only known asbestos materials at the Oxnard Facility are low-maintenance materials, it is not considered practical to have employees trained and equipped to perform minor repair or removal work. Any work which involves cutting, drilling, tearing, or removing asbestos-containing materials should be performed by an outside contractor with asbestos licensing.

Consequently, the typical worker protection elements for building employees (training, protective equipment, medical surveillance, engineering controls, and administrative controls) are not entirely applicable.

Outside contractors who are hired to perform work should be required to show proof of adequate worker protection practices (see Section 14 for more details). 


\section{SECTION 9 - TRAINING FOR OXNARD FACILITY EMPLOYEES}

This section details asbestos training requirements.

NOTE: Because Oxnard Facility employee contact with asbestos is expected to be minimal, the majority of this section does not apply. However, it is presented for informational purposes, as well as an assistance when hiring outside contractors and consultants.

Note that Oxnard Facility employees would be required to receive hazard communication training for asbestos materials, per 29 CFR 1910.1200 .

The following table lists recommended training protocols for different staff who take part in the Asbestos Control Program. The training programs abbreviated in this table are explained in more detail later in this section.

\begin{tabular}{|c|c|c|c|c|c|c|c|c|}
\hline \multirow[b]{2}{*}{ Staff } & \multicolumn{8}{|c|}{$\begin{array}{l}\text { Recommended Training Requirements for Oxnard Facility } \\
\text { (as applicable) }\end{array}$} \\
\hline & $\begin{array}{l}1 . \\
\mathrm{CN}\end{array}$ & $\begin{array}{r}2 . \\
\text { AAT }\end{array}$ & $\begin{array}{l}3 . \\
\text { M }\end{array}$ & $\begin{array}{l}4 . \\
\text { AAW }\end{array}$ & $\begin{array}{l}5 \\
\mathrm{c} / \mathrm{S}\end{array}$ & $\begin{array}{l}6 . \\
\text { WP }\end{array}$ & $\begin{array}{l}7 . \\
\text { RP }\end{array}$ & $\begin{array}{l}8 \\
\text { AI }\end{array}$ \\
\hline $\begin{array}{l}\text { Custodial, Maintenance, and } \\
\text { Facility Staff with potential for } \\
\text { accidental asbestos contact }\end{array}$ & $\sqrt{ }$ & $\sqrt{ }$ & & & & & & \\
\hline Asbestos Program/ Site Mgr & & $\sqrt{ }$ & & & & & & \\
\hline $\begin{array}{l}\text { Maintenance Staff performing } \\
\text { Class II work* }\end{array}$ & $\checkmark$ & & & $\sqrt{ }$ & & $\sqrt{ }$ & $\sqrt{ }$ & \\
\hline $\begin{array}{l}\text { Maintenance Supervisors for } \\
\text { Class II work* }\end{array}$ & $\sqrt{ }$ & & & & $\sqrt{ }$ & $\sqrt{ }$ & $\sqrt{ }$ & \\
\hline $\begin{array}{l}\text { Maintenance Staff performing } \\
\text { Class III work* }\end{array}$ & $\sqrt{ }$ & & $\sqrt{ }$ & & & $\sqrt{ }$ & $\sqrt{ }$ & \\
\hline $\begin{array}{l}\text { Maintenance Staff performing } \\
\text { Class IV work* }\end{array}$ & $\sqrt{ }$ & $\sqrt{ }$ & & & & $\sqrt{ }$ & $\checkmark$ & \\
\hline $\begin{array}{l}\text { Asbestos Consultant Staff } \\
\text { performing asbestos inspections } \\
\text { and monitoring monitoring }\end{array}$ & $\sqrt{ }$ & & & & $\sqrt{ }$ & $\sqrt{ }$ & $\checkmark$ & $\sqrt{ }$ \\
\hline $\begin{array}{l}\text { All building tenants and } \\
\text { others not listed above }\end{array}$ & $\sqrt{ }$ & & & & & & & \\
\hline
\end{tabular}

*Note that Federal OSHA has divided asbestos work into four categories. Each category requires a different level of training for employees. The categories are described below. A detailed description of each category is contained in Appendix E: 


\begin{tabular}{|c|l|}
\hline OSHA Work Category & Description \\
\hline $\begin{array}{c}\text { (typically only performed by } \\
\text { outside contractors) }\end{array}$ & $\begin{array}{l}\text { Activities involving the removal of Surfacing and } \\
\text { TSI materials which are asbestos or presumed } \\
\text { asbestos (ACM or PACM). This involves primarily } \\
\text { friable materials. }\end{array}$ \\
\hline $\begin{array}{c}\text { II } \\
\text { (typically only performed by } \\
\text { outside contractors) }\end{array}$ & $\begin{array}{l}\text { Activities involving the removal of asbestos or } \\
\text { presumed asbestos (ACM or PACM) materials } \\
\text { which are not surfacing or TSI. This involves } \\
\text { primarily nonfriable materials. }\end{array}$ \\
\hline III & $\begin{array}{l}\text { Repair and maintenance activities which may disturb } \\
\text { ACM or PACM (including small-scale removal of } \\
\text { less than one glovebag or waste bag of friable } \\
\text { materials). }\end{array}$ \\
\hline IV & $\begin{array}{l}\text { Maintenance and custodial activities involving } \\
\text { contact with ACM or PACM; as well as cleanup of } \\
\text { waste and debris containing ACM or PACM. }\end{array}$ \\
\hline
\end{tabular}

Following is a summary table of the training programs referenced above. Note that the more general Federal OSHA and EPA regulations are cited, instead of the state regulations (Ventura County Air Pollution Control District regulations are also cited).

\begin{tabular}{|c|c|c|c|c|}
\hline Code & Training Title & Length & Taught By & $\begin{array}{l}\text { Description \& Regulatory } \\
\text { Reference }\end{array}$ \\
\hline 1. $\mathrm{CN}$ & $\begin{array}{l}\text { Connelly } \\
\text { Notification }\end{array}$ & varies & $\begin{array}{l}\text { N/A; } \\
\text { Written notice } \\
\text { only }\end{array}$ & $\begin{array}{l}\text { California Health and Safety Code } 9 \\
25915 \text { through } 25919\end{array}$ \\
\hline AिT & $\begin{array}{l}\text { Asbestos Awareness } \\
\text { Training }\end{array}$ & $\begin{array}{l}2 \\
\text { hours }\end{array}$ & $\begin{array}{l}\text { staff, approved } \\
\text { staff, or } \\
\text { qualified } \\
\text { consultant }\end{array}$ & $\begin{array}{l}\text { Health effects of asbestos; locations in } \\
\text { building; work practices for Class IV } \\
\text { work; housekeeping requirements and } \\
\text { response to fiber release episodes. } \\
\text { Federal: } 29 \text { CFR } 1926.1101 \text { (k) (8), } \\
\text { 1910.1001 (j) (7). 1910.1200 }\end{array}$ \\
\hline OM & $\begin{array}{l}\text { Operations and } \\
\text { Maintenance Training } \\
\text { (per EPA Model } \\
\text { Accreditation Plan, or } \\
\text { "MAP") }\end{array}$ & $\begin{array}{l}16 \\
\text { hours }\end{array}$ & $\begin{array}{l}\text { staff, approved } \\
\text { staff, or } \\
\text { qualified } \\
\text { consultant }\end{array}$ & $\begin{array}{l}\text { Same as AAT + work practices for Class } \\
\text { III activities } \\
\text { Federal: } 29 \text { CFR } 1926.1101 \text { (k) (8), } \\
\text { 1910.1001 (j) (7), 1910.1200, Sec. } 15 \text {, } \\
\text { PL 101-637 }\end{array}$ \\
\hline AAW & $\begin{array}{l}\text { Asbestos Abatement } \\
\text { Worker Training (per } \\
\text { EPA Model } \\
\text { Accreditation Plan) }\end{array}$ & $\begin{array}{l}32 \\
\text { hours }\end{array}$ & $\begin{array}{l}\text { EPA-approved } \\
\text { AHERA } \\
\text { training facility }\end{array}$ & $\begin{array}{l}\text { Same as OM + work practices for Class } \\
\text { II activities } \\
\text { Federal: } 29 \text { CFR 1926.1101 (k) (8), } \\
\text { 1910.1001 (j) (7), 1910.1200, Sec. 15, } \\
\text { PL 101-637 }\end{array}$ \\
\hline $\begin{array}{l}5 \\
\mathrm{C} / \mathrm{S}\end{array}$ & $\begin{array}{l}\text { Asbestos Abatement } \\
\text { Supervisor Training } \\
\text { (per EPA MAP), also } \\
\text { called } \\
\text { "Competent Person" } \\
\text { Training }\end{array}$ & $\begin{array}{l}40 \\
\text { hours }\end{array}$ & $\begin{array}{l}\text { EPA-approved } \\
\text { AHERA } \\
\text { training facility }\end{array}$ & $\begin{array}{l}\text { Same as AAW + additional detail } \\
\text { regarding safety inspections } \\
\text { Federal: } 29 \text { CFR 1926.1101 (k) (8), } \\
\text { 1910.1001 (j) (7), 1910.1200, Sec. 15, } \\
\text { PL 101-637 }\end{array}$ \\
\hline 6. WP & $\begin{array}{l}\text { Work practice- } \\
\text { specific training }\end{array}$ & varies & $\begin{array}{l}\text { staff, approved } \\
\text { staff, or } \\
\text { qualified } \\
\text { consultant }\end{array}$ & $\begin{array}{l}\text { Detailed training regarding procedures for } \\
\text { performing specific Class II, III, or IV } \\
\text { activities. } \\
\text { No regulatory requirement }\end{array}$ \\
\hline 7. $\mathrm{RP}$ & $\begin{array}{l}\text { Respiratory } \\
\text { Protection Training }\end{array}$ & $\begin{array}{l}\text { approx } \\
2 \\
\text { hours }\end{array}$ & $\begin{array}{l}\text { staff, approved } \\
\text { staff, or } \\
\text { qualified } \\
\text { consultant }\end{array}$ & $\begin{array}{l}\text { Respirator selection, terminology, fit } \\
\text { testing, use, maintenance, and limits. } \\
\text { Federal: } 29 \text { CFR } 1910.134 \text { (d) }\end{array}$ \\
\hline 8. $\mathrm{AI}$ & $\begin{array}{l}\text { Asbestos Inspector } \\
\text { Training (per EPA } \\
\text { MAP) }\end{array}$ & $\begin{array}{l}24 \\
\text { hours }\end{array}$ & $\begin{array}{l}\text { EPA-approved } \\
\text { AHERA } \\
\text { training facility }\end{array}$ & $\begin{array}{l}\text { Federal: } 29 \text { CFR 1926.1101 (k) (4), } \\
\text { 1910.1001 (j) (8), Sec. 15, PL 101-637 } \\
\text { Regional: Ventura County Air } \\
\text { Pollution Control District, rule } 62.7\end{array}$ \\
\hline
\end{tabular}




\section{SECTION 10 - RESPIRATORY PROTECTION FOR OXNARD FACILITY EMPLOYEES}

NOTE: Because Oxnard Facility employee contact with asbestos is expected to be minimal, the majority of this section does not apply.

OSHA regulations stipulate that respiratory protection is required if personal exposures are in excess of 0.1 fiber of asbestos per cubic centimeter of air $(0.1 \mathrm{f} / \mathrm{cc})$. This level is not expected to be exceeded by Oxnard Facility employees under foreseeable conditions.

If it is determined that persons employed at Oxnard Facility should have respiratory protection, OSHA regulations will require the employees to have the following typical tests and documentation:

- respirator fit test, provided by a competent person, every 6 months

- medical examination provided by occupational health physician, every 12 months

- training in use of respirator, every 12 months

- if asbestos will be disturbed, a minimum 2-hour asbestos awareness training is required every 12 months. This training is typically provided by an outside asbestos consultant.

Note that asbestos exposures require a specific type of respirator cartridge.

Reference: 29 CFR 1926.1101 


\section{SECTION 11 - MEDICAL SURVEILLANCE FOR OXNARD FACILITY}

\section{EMPLOYEES}

NOTE: Because work which disturbs asbestos will be performed by outside specialty contractors, no medical surveillance for asbestos will be required for Oxnard Facility employees.

OSHA regulations stipulate that medical surveillance is required if personal exposures are in excess of $0.1 \mathrm{f} / \mathrm{cc}$, and/or if respirators are used to control asbestos exposure. This level is not expected to be exceeded by Oxnard Facility employees under foreseeable conditions.

Reference: 29 CFR 1926.1101 (m) 
SECTION 12 - EXPOSURE MONITORING

NOTE: Because work which disturbs asbestos will be performed by outside specialty contractors, no exposure monitoring for asbestos will be required for Oxnard Facility employees.

OSHA regulations stipulate that exposure monitoring is required if personal exposures are likely to be in excess of $0.1 \mathrm{f} / \mathrm{cc}$, and/or if respirators are used to control asbestos exposure. This level is not expected to be exceeded by Oxnard Facility employees under foreseeable conditions.

In the case of accidental damage to asbestos material, and resultant fiber release, qualified industrial hygiene personnel should perform air monitoring to assess the seriousness of the release, and to formulate response strategies.

Reference: 29 CFR 1926.1101 


\section{SECTION 13 - ASBESTOS WASTE MANAGEMENT}

\section{Asbestos waste is generated by outside contractors performing abatement work in} the building. Specific procedures must be followed.

Definition:

Asbestos waste is regulated by a variety of agencies, including California and Federal EPA, California and Federal OSHA, and the Ventura County Air Pollution Control District (VCAPCD).

\section{General}

Requirements:

In general, asbestos waste must be stored in sealed, impermeable containers. It must be labelled according to the requirements of the agencies listed above.

For most asbestos waste, a manifest is required. This is a document with several copies. The copies are used to track the waste's transportation and disposal, in order to verify that it winds up in an appropriate landfill and not by the side of the road somewhere.

See Attachment $C$ for a diagram of the various waste manifest parts, and the respective parties whom they are sent to.

Generator ID

Numbers:

California EPA assigns a unique number to every facility which generates asbestos waste. To obtain this ID number, the Asbestos Program/ Site Manager should contact the local office of the California EPA. In addition, the Asbestos Program/ Site Manager will need to obtain a tax ID number from the local office of the Board of Equalization.

Note that these numbers are not required until waste is generated, i.e. until an abatement project takes place.

Disposal of

Asbestos Waste

from Abatement

Activities:

When abatement activities generate asbestos waste, the abatement contractor typically handles the storage and disposal requirements. The contractor will need to receive the Generator ID number (as well as the Tax ID number) from the Asbestos Program/ Site Manager.

Manifest

Handling:

Although the asbestos abatement contractor disposes of the asbestos waste from abatement, the manifest must be signed by a representative of the "generator," i.e. the building where the waste is from. Manifests are fairly complex documents, with precise requirements. Fines for not filling them out correctly can be very high.

It is strongly recommended that the manifests be signed only by the Asbestos Program/ Site Manager, or by an outside consultant approved by these programs. 


\section{SECTION 14 - QUALIFICATIONS FOR ASBESTOS ABATEMENT CONTRACTORS}

Minimal qualifications for contracting asbestos abatement work with outside firms.

In the absence of similar company or federal procurement practices, the documentation and submittals outlined in this section are recommended when asbestos work is being performed by outside contractors:

- The prospective Abatement Contractor should provide documentation of current and valid licensing in the State of California in accordance with the provisions of Chapter 9, Division 3 (as amended) of the Business and Professions Code.

- The prospective Abatement Contractor should provide documentation of current and valid certification in the State of California to perform asbestos-related work by the Contractor's State License Board.

- The prospective Abatement Contractor shall provide documentation of current and valid registration with the California Department of Industrial Relations - Division of Occupational Safety and Health to perform asbestos-related work as well as registration for carcinogen usage.

- The prospective Abatement Contractor should submit documentation of successful completion of asbestos work involving at least three jobs similar in work and extent. Documentation should include the following:

1. Agency or Company for which the work was done.

2. Type of work.

3. Name of the agency or company representative responsible for performance inspections.

4. Results of any air monitoring, including laboratory analytical reports.

5. Results of any inspections by other agencies (e.g., Environmental Protection Agency (EPA), Federal Occupational Safety and Health Administration (OSHA), Califormia Department of Industrial Relations - Division of Occupational Safety and Health (DOSH), Air Pollution Control Agency, etc.)

- Prospective Abatement Contractors should submit a statement, signed by an officer of the Company, containing the following information:

1. A record of any citations issued by federal, state, or regulatory agencies related to asbestos abatement activity. Such records shall include identification of the project, dates and resolutions or other information.

2. A list of penalties incurred through non-compliance with asbestos abatement project specifications, including liquidated damages, overruns in scheduled time limitations, and resolutions.

3. A description of the situations in which an asbestos-related contract has been terminated, including projects, dates, and reasons for terminations. 
4. A list of asbestos-related legal proceedings/claims in which the Abatement Contractor (or employees scheduled to participate in this project) has participated or is currently involved.

- The prospective Abatement Contractor should also submit the following information:

1. Evidence of adequate insurance coverage, specifically insurance with pollution coverage.

2. Names and resumes of the project manager(s) and job superintendent(s) for the abatement project.

3. A proposed schedule for completion of the work, including work hours and number of days required to complete the work.

4. Examples of the "Certificate of Completion" the Abatement Contractor intends to provide at the completion of asbestos abatement described in these specifications.

5. Where the asbestos will be disposed and certified that the facility is authorized to accept asbestos.

NOTE: Abatement, maintenance, and repair of asbestos should be accomplished during off-peak work hours whenever possible. 
- 
Attachment A

Connelly Notification Forms and Procedures

(Optional Forms - use of these specific forms is not required by regulation; similar forms can be used) 
1.

Appoint Asbestos Site Manager and Asbestos Program Manager (see Operations and Maintenance Plan for description of duties)

2. Compile information about asbestos in building.

3. Prepare notices for employees, lessees, and contractors.

4. Distribute notices to each employees or tenants listed above. Obtain written verification of receipt from each employee or tenants.

5. Distribute notices to lessees, agents, and contractors. Remind them that they have to forward the notices to their own employees, agents, lessees, and contractors.

6.

Distribute supplemental notices on the following occasions:

a.

b.

Asbestos information known at this time (original notification).

Asbestos information which becomes known during a 90-day period (quarterly notification); supplemental notice withtin 15 days of the end of the 90 day period.

c. All asbestos information to new employees within 15 days of the date

they begin work.

d. All asbestos information to new lessees, agents, and contractors within 15 days of the date they begin the new relationship.

e. All asbestos information to all employees, lessees, and agents on an annual basis. 
NOTICE TO EMPLOYEES - ASBESTOS IN BUILDINGS (INITIAL NOTICE) FOR PERIOD BEGINNING:

(SUPPLEMENTAL NOTICE) FOR QUARTER ENDING:

FOR BUILDING LOCATED AT:

In January of 1989, Assembly Bill 3713 was signed into law and added to the California Health and Safety Code. This bill provides for written notice to employees concerning specific matters related to working in a building with asbestos-conttaining construction materials. It applies to buildings built before 1979 where the owner knows that the building contains asbestos-containing materials; it does not require that a building be surveyed to determine the presence of asbestos.

\section{WHAT IS ASBESTOS?}

Asbestos is a naturally-occurring group of fibrous minerals which have been used extensively in public buildings, apartments, and homes. Asbestos was incorporated into pipe insulation, acoustic plaster, acoustic tile, duct and furnace insulation, floor tiles; textiles, roofing, and hundreds of other building materials.

\section{WHERE DOES ASBESTOS OCCUR IN MY BUILDING?}

See the attached excerpt from the asbestos survey report for your building for information about which materials contain asbestos.

\section{WHY IS ASBESTOS HAZARDOUS?}

Asbestos is a concern because of the potential health risks associated with breathing asbestos fibers. It is important for you to know that most people with asbestos-related diseases were asbestos workers before 1972 . These workers were repeatedly exposed to high levels of asbestos each working day with little or no protection. Asbestos workers today are required to follow specific work procedures and wear appropriate protection to minimize exposure.

Significant exposure to asbestos fibers can lead to asbestosis and certain forms of cancer. Asbestosis is one of the many dust-related lung diseases. It is associated with chronic exposure to relatively high levels of asbestos and is characterized by the permanent deposition of asbestos fibers in the respiratory tract. The earliest and most prominent clinical finding, breathlessness upon exertion, rarely becomes apparent until after at least a decade of exposure.

In addition to asbestosis, the association of asbestos and lung cancer has been well established over the past two decades. Scientists have studied insulation and shipyard workers who were exposed to HIGH AIRBORNE LEVELS of asbestos. These studies indicate that asbestos workers were about five times as likely to get lung cancer as non-asbestos workers who did not smoke. Asbestos workers who also smoke were found to be at much greater risk (about 50 times) of dying of lung cancer than nonsmoking, non-asbestos workers. Mesothelioma, a rare form of cancer of the chest or abdominal cavity, occurs among occupational groups exposed to certain types of asbestos.

\section{ASBESTOS SAMPLING RESULTS}

A summary of the results of recent bulk sampling or air sampling is attached to this notice (if no new sampling has been performed beyond the original asbestos survey, then the asbestos survey executive summary will be attached). 


\section{REGULATIONS}

A number of standards exist for allowable airbome levels of asbestos in the workplace. Most of these standards require medical monitoring, respirators, and specialized training if airborne levels exceed a certain concentration in air.

\begin{tabular}{|c|c|c|c|}
\hline Source & Level & Nature & Comments \\
\hline Cal/OSHA ${ }^{1}$ & $1.0 \mathrm{f} / \mathrm{cc}$ & $\begin{array}{l}\text { Occupational } \\
\text { \& mandatory }\end{array}$ & $\begin{array}{l}\text { 8-hour Time Weighted Average (TWA) Permissible } \\
\text { Exposure Level (PEL) } \\
\text { Excursion Limit (EL) for } 30 \text { minutes period }\end{array}$ \\
\hline $\mathrm{NIOSH}^{2}$ & $0.1 \mathrm{f} / \mathrm{cc}$ & Recommended & Occupational PEL \\
\hline ACGIH $^{3}$ & $0.2 \mathrm{f} / \mathrm{cc}$ & Recommended & $\begin{array}{l}\text { Occupational Threshold Limit Value (TLV) for } \\
\text { chrysotile asbestos }\end{array}$ \\
\hline $\begin{array}{l}\text { Calif. Prop } \\
65^{4}\end{array}$ & vague & Mandatory & $\begin{array}{l}\text { Standard and monitoring method are unclear, but } \\
\text { generally interpreted as comparable to outside ambient } \\
\text { air or } 100 \text { fibers per day, whichever is greater }\end{array}$ \\
\hline $\begin{array}{l}\text { Industry } \\
\text { Standard, } \\
\text { adopted } \\
\text { from } \\
\text { AHERA } \\
\text { regulations }\end{array}$ & $\begin{array}{l}0.01 \mathrm{f} / \mathrm{cc} \\
\text { (PCM) } \\
70 \mathrm{str} / \mathrm{mm}^{2} \\
(\mathrm{TEM})\end{array}$ & $\begin{array}{l}\text { Primary } \\
\text { Clearance } \\
\text { Criteria } \\
\text { Secondary } \\
\text { Clearance } \\
\text { Criteria } \\
\end{array}$ & $\begin{array}{l}\text { Perimeter action level. } \\
\text { - Clearance standard for small scale, short duration } \\
\text { abatement zones. } \\
\text { TEM clearance criterion; if failed, then compare against } \\
\text { ambient level outside of work area. Originating from } \\
\text { AHERA } 5 \text { regulations }\end{array}$ \\
\hline
\end{tabular}

1 California Department of Industrial Relations, Division of Occupational Safety and Health, 1995

2 National Institute of Occupational Safety and Health

${ }^{3}$ American Conference of Governmental Industrial Hygienists, 1994-5

${ }^{4}$ California Proposition 65

5 Asbestos Hazard Emergency Response Act (AFERA); 40 CFR Part 763, applicable to schools but adopted for larger scale projects or where warranted

\section{GENERAL PROCEDURES AND HANDLING RESTRICTIONS}

As you can see, the concern is with asbestos fibers in the air. When asbestos materials are in good condition, it is unlikely that fibers will be released into the air. Do not cut into, drill into, nail, or pin anything onto, sand, move, bump, rub against, or otherwise disturb any asbestos-containing materials. If you should discover any damaged asbestos-containing material, do not touch it; do not attempt to clean it up. Contact your supervisor or building representative/manager immediately and report the situation.

Work that requires disturbance of asbestos materials is performed by outside contractors working under specifications which include work practice procedures, removal techniques, clean up, and clearance air sampling.

If any construction, maintenance, or remodeling is conducted in an area of the building where there is the potential for employees to come in contact with, or release and disturb asbestos-containing building materials, it is required that the area be posted with a clear and conspicuous warning sign. The waming sign must read:

-CAUTION, ASBEstos

CANCER AND LUNG DISEASE HAZARD

DO NOT DISTURB WITHOUT TRAINING AND EQUIPMENT* 
Much of this information may be new to you. If you have questions, or if you wish to read or photocopy the full text of the asbestos survey report for the building, you may contact the designated contact person at your site:

Name:

Phone Number:

Between the hours of and

This written announcement fulfills the asbestos notification requirement of Division 20, Chapter 10.4, Section 25915 of the California Health and Safety Code (Assembly Bill 3713). 


\section{NOTICE TO OWNERS; LESSEES, SUBLESSEES, AGENTS, AND CONTRACTORS - ASBESTOS IN BUILDINGS}

OR

(INITIAL NOTICE) FOR PERIOD BEGINNING:

(SUPPLEMENTAL NOTICE) FOR QUARTER ENDING;

FOR BUILDING LOCATED AT:

In January of 1989, Assembly Bill 3713 was signed into law and added to the California Health and Safety Code. This bill provides for written notice to employees concerning specific matters related to working in a building with asbestos-conttaining construction materials. It applies to buildings built before 1979 where the owner knows that the building contains asbestos-containing materials; it does not require that a building be surveyed to determine the presence of asbestos.

\section{WHAT IS ASBESTOS?}

Asbestos is a naturally-occurring group of fibrous minerals which have been used extensively in public buildings, apartments, and homes. Asbestos was incorporated into pipe insulation, acoustic plaster, acoustic tile, duct and furnace insulation, floor tiles, textiles, roofing, and hundreds of other building materials.

\section{WHERE DOES ASBESTOS OCCUR IN MY BUILDING?}

See the attached excerpt from the asbestos survey report for your building for information about which materials contain asbestos.

\section{WHY IS ASBESTOS HAZARDOUS?}

Asbestos is a concem because of the potential health risks associated with breathing asbestos fibers. It is important for you to know that most people with asbestos-related diseases were asbestos workers before 1972. These workers were repeatedly exposed to high levels of asbestos each working day with little or no protection. Asbestos workers today are required to follow specific work procedures and wear appropriate protection to minimize exposure.

Significant exposure to asbestos fibers can lead to asbestosis and certain forms of cancer. Asbestosis is one of the many dust-related lung diseases. It is associated with chronic exposure to relatively high levels of asbestos and is characterized by the permanent deposition of asbestos fibers in the respiratory tract. The earliest and most prominent clinical finding, breathlessness upon exertion, rarely becomes apparent until after at least a decade of exposure.

In addition to asbestosis, the association of asbestos and lung cancer has been well established over the past two decades. Scientists have studied insulation and shipyard workers who were exposed to HIGH AIRBORNE LEVELS of asbestos. These studies indicate that asbestos workers were about five times as likely to get lung cancer as non-asbestos workers who did not smoke. Asbestos workers who also smoke were found to be at much greater risk (about 50 times) of dying of lung cancer than nonsmoking, non-asbestos workers. Mesothelioma, a rare form of cancer of the chest or abdominal cavity, occurs among occupational groups exposed to certain types of asbestos.

\section{ASBESTOS SAMPLING RESULTS}

A summary of the results of recent bulk sampling or air sampling is attached to this notice (if no new sampling has been performed beyond the original asbestos survey, then the asbestos survey executive summary will be attached). 
A number of standards exist for allowable airborne levels of asbestos in the workplace. Most of these standards require medical monitoring, respirators, and specialized training if airborne levels exceed a certain concentration in air.

\begin{tabular}{|c|c|c|c|}
\hline Source & Level & Nature & Comments \\
\hline Cal/OSHA ${ }^{1}$ & $\begin{array}{l}0.1 \mathrm{f} / \mathrm{cc} \\
1.0 \mathrm{f} / \mathrm{cc}\end{array}$ & $\begin{array}{l}\text { Occupational } \\
\text { \& mandatory }\end{array}$ & $\begin{array}{l}\text { 8-hour Time Weighted Average (TWA) Permissible } \\
\text { Exposure Level (PEL) } \\
\text { Excursion Limit (EL) for } 30 \text { minutes sampling duration }\end{array}$ \\
\hline $\mathrm{NIOSH}^{2}$ & $0.1 \mathrm{f} / \mathrm{cc}$ & Recommended & Occupational PEL \\
\hline ACGIH ${ }^{3}$ & $0.2 \mathrm{f} / \mathrm{cc}$ & Recommended & $\begin{array}{l}\text { Occupational Threshold Limit Value (TLV) for } \\
\text { chrysotile asbestos }\end{array}$ \\
\hline $\begin{array}{l}\text { Calif. Prop } \\
65^{4}\end{array}$ & vague & Mandatory & $\begin{array}{l}\text { Standard and monitoring method are unclear, but } \\
\text { generally interpreted as comparable to outside ambient } \\
\text { air or } 100 \text { fibers per day, whichever is greater }\end{array}$ \\
\hline $\begin{array}{l}\text { Industry } \\
\text { Standard, } \\
\text { adopted } \\
\text { from } \\
\text { AHERA } \\
\text { regulations }\end{array}$ & $\begin{array}{l}0.01 \mathrm{f} / \mathrm{cc} \\
(\mathrm{PCM}) \\
70 \mathrm{str} / \mathrm{mm}^{2} \\
(\mathrm{TEM})\end{array}$ & $\begin{array}{l}\text { Primary } \\
\text { Clearance } \\
\text { Criteria } \\
\text { Secondary } \\
\text { Clearance } \\
\text { Criteria }\end{array}$ & $\begin{array}{l}\text { Perimeter action level. } \\
\text { - Clearance standard for small scale, short duration } \\
\text { abatement zones. } \\
\text { TEM clearance criterion; if failed, then compare against } \\
\text { ambient level outside of work area. Originating from } \\
\text { AHERA }{ }^{5} \text { regulations }\end{array}$ \\
\hline
\end{tabular}

1 California Department of Industrial Relations, Division of Occupational Safety and Health

${ }^{2}$ National Institute of Occupational Safety and Health

3 American Conference of Govermmental Industrial Hygienists, 1994-5

4 Califormia Proposition 65

5 Asbestos Hazard Emergency Response Act (AFERA); 40 CFR Part 763, applicable to schools but adopted for larger scale projects or where warranted

\section{GENERAL PROCEDURES AND HANDLING RESTRICTIONS}

As you can see, the concem is with asbestos fibers in the air. When asbestos materials are in good condition, it is unlikely that fibers will be released into the air. Do not cut into, drill into, nail, or pin anything onto, sand, move, bump, rub against, or otherwise disturb any asbestos-containing materials. If you should discover any damaged asbestos-containing material, do not touch it; do not attempt to clean it up. Contact your supervisor or building representative/manager immediately and report the situation.

Work that requires disturbance of asbestos materials is performed by outside contractors working under specifications which include work practice procedures, removal techniques, clean up, and clearance air sampling.

If any construction, maintenance, or remodeling is conducted in an area of the building where there is the potential for employees to come in contact with, or release and disturb asbestos-containing building materials, it is required that the area be posted with a clear and conspicuous warning sign. The warning sign must read:

"CAUTION, ASBESTOS

CANCER AND LUNG DISEASE HAZARD

DO NOT DISTURB WITHOUT TRAINING AND EQUIPMENT* 
Much of this information may be new to you. If you have questions, or if you wish to read or photocopy the full text of the asbestos survey report for the building, you may contact the designated contact person at your site:

Name: Phone Number:

Between the hours of and

This written announcement fulfills the asbestos notification requirement of Division 20, Chapter 10.4, Section 25915 of the California Health and Safety Code (Assembly Bill 3713).

\section{IMPORTANT NOTE:}

Lessees, sublessees, agents, and contractors are responsible for providing copies of this notification to their own sublessees, agents, contractors, and employees. 
Attachment B

Work Evaluation Form

(Optional Form - use of this specific form is not required by regulation; a similar form can be used) 
BUILDING:

BUILDING \#:

EVALUATION PERFORMED

BY

IDATE: .

EVALUATION REQUESTED

BY

IJOB ORDER \#:

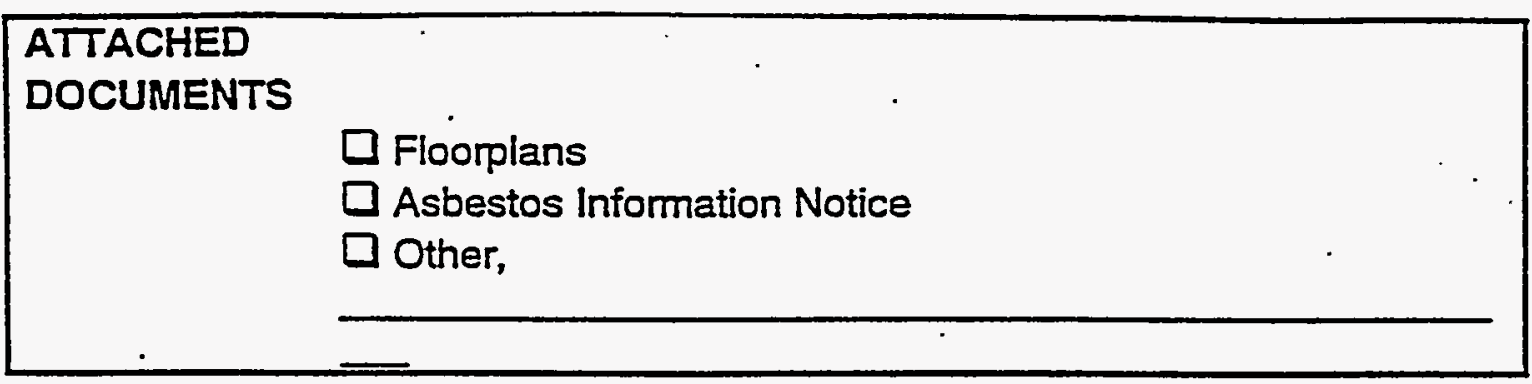

\section{DESCRIPTION OF WORK:}

FINDINGS:

Asbestos is not present in the vicinity of the work.

Asbestos.is present in the vicinity of the work, but disturbance is not expected. If the scope of the work changes, further evaluation is needed and asbestos abatement may be required.

Asbestos is present in the vicinity of the work and may be disturbed. 


\section{Attachment C}

Waste Manifest System 


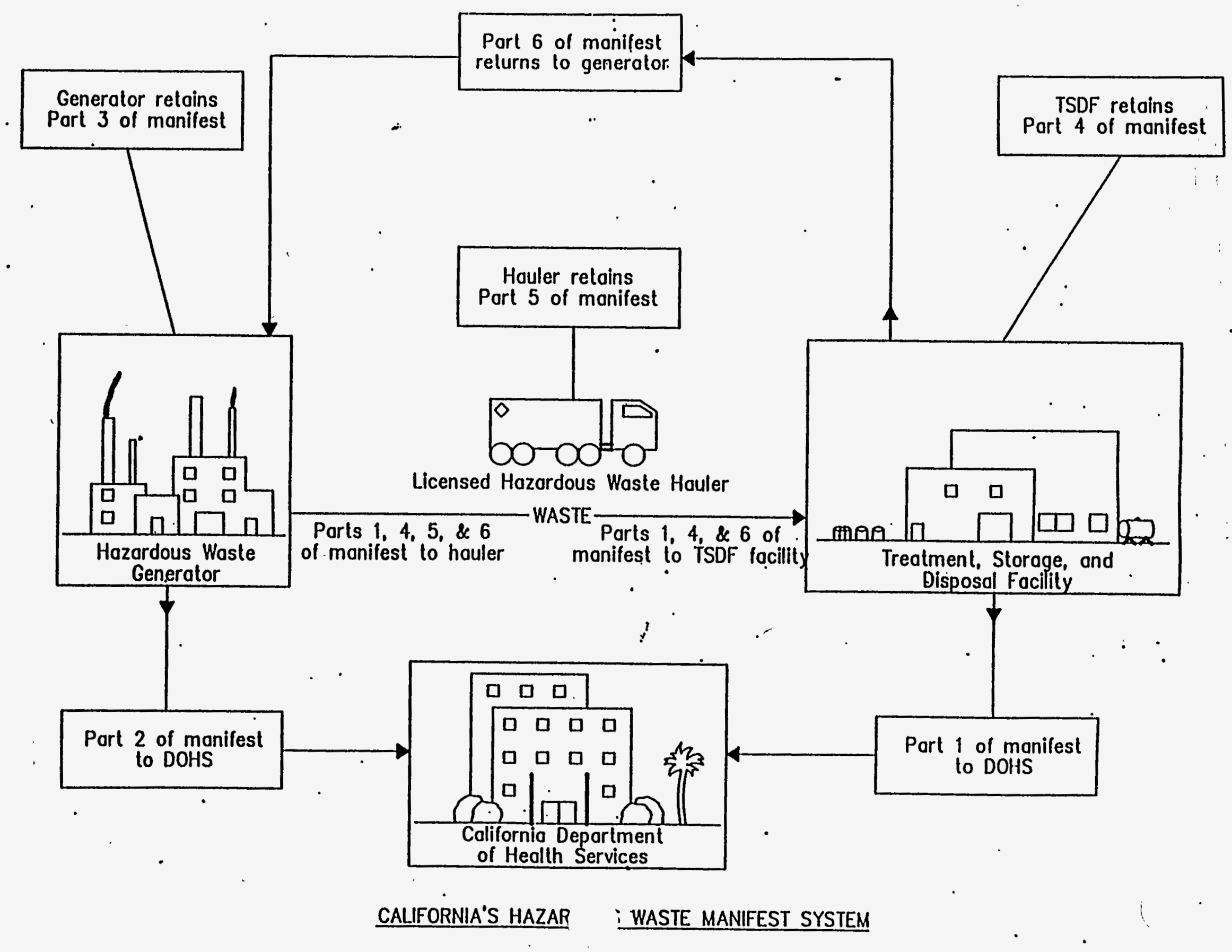




\section{Attachment D}

Not Used 

Asbestos Operations and Maintenance Plan - January, 1996

SCA Environmental, line 1235 East Wooled Road, Oxnard, CA

Attachment E

Summary of Requirements for Asbestos Abatement Classes 1 - 4

$4: 18$ PM $-1 / 24 / 96$ 
Summary of Asbestos Abatement Procedures for Abatement Class Activities

\begin{tabular}{|c|c|c|c|c|}
\hline & Abatement Class I & Abatement Class II & Abatement Class III & Abatement Class IV \\
\hline Activities: & $\begin{array}{l}\text { Removal of TSI or surfacing } \\
\text { matcrial that is ACM or } \\
\text { PACM }\end{array}$ & $\begin{array}{l}\text { Removal of ACM, not } \\
\text { including TSI or surfacing } \\
\text { material (c.g. wallboard, } \\
\text { flooring, roofing, siding, } \\
\text { gaskets and mastic) }\end{array}$ & $\begin{array}{l}\text { Repair and maintenance of } \\
\text { small amounts of TSI or } \\
\text { surfacing ACM or PACM (not } \\
\text { exceeding one } 60 " \text { glovebag or } \\
\text { waste bag) }\end{array}$ & $\begin{array}{l}\text { Maintenance and custodial } \\
\text { activities contacting ACM or } \\
\text { PACM (including clean-up of } \\
\text { ACM or PACM dust and } \\
\text { dcbris) } \\
\end{array}$ \\
\hline Regulated Area: & Yes & Yes & Yes & No (unless PEL exceeded) \\
\hline $\begin{array}{l}\text { Personnel Air } \\
\text { Sampling } \\
\text { Requirements: }\end{array}$ & $\begin{array}{l}\text { Daily for each shift and } \\
\text { activity; } 8 \mathrm{hr} \text { TWA and } 30 \mathrm{~min} \\
\text { STEL }\end{array}$ & $\begin{array}{l}\text { Daily for cach shift and } \\
\text { activity; } 8 \mathrm{hr} \text { TWA and } 30 \mathrm{~min} \\
\text { STEL }\end{array}$ & \begin{tabular}{|l|} 
Sufficient to document expected \\
exposure
\end{tabular} & $\begin{array}{l}\text { Sufficient to document expected } \\
\text { exposure }\end{array}$ \\
\hline \multicolumn{5}{|l|}{$\begin{array}{l}\text { Procedural } \\
\text { Requirements: }\end{array}$} \\
\hline a. wet methods & Yes & Yes & Yes & Yes \\
\hline b. HEPA vacuum & Yes & Yes & Yes & Yes \\
\hline c. prompt clean-up & Yes & Yes & Yes & Yes \\
\hline $\begin{array}{l}\text { d. negative } \\
\text { pressure } \\
\text { enclosure }\end{array}$ & $\begin{array}{l}\text { Disturbances of }>25 \mathrm{LF} \text { or } \\
>10 S \mathrm{ACM} \text { or PACM } \\
\text { requires }>0.02 " \text { negative } \\
\text { pressure differential; }>4 \text { air } \\
\text { changes/hr; air movement away } \\
\text { from workers towards HEPA } \\
\text { unit; and clectrical circuits off } \\
\text { unless using GFI }\end{array}$ & $\begin{array}{l}\text { Indoors when ACM or PACM } \\
\text { is not removed substantially } \\
\text { intact or when PEL may be } \\
\text { exceeded }\end{array}$ & $\begin{array}{l}\text { If PEL may be exceeded; isolate } \\
\text { area when feasible or use } \\
\text { glovebags and/or mini- } \\
\text { enclosures with vacuum }\end{array}$ & No (unless PEL exceeded) \\
\hline e. dropcloths & Yes & Yes & Yes & Yes \\
\hline Control Methods: & $\begin{array}{l}\text { Negative pressure enclosure; } \\
\text { glovebag system; negative } \\
\text { pressure glovebag; glove box; } \\
\text { water spray system ; and/or } \\
\text { mini-enclosures }\end{array}$ & Specific to material removed & $\begin{array}{l}\text { Glovebag, and/or mini- } \\
\text { enclosure }\end{array}$ & N/A \\
\hline Decontamination: & $\begin{array}{l}\text { 3-chamber personal decon with } \\
\text { shower }\end{array}$ & $\begin{array}{l}\text { Minimum of dropcloth, HEPA- } \\
\text { vacuuming coveralls, and } \\
\text { bucket or Hudson sprayer for } \\
\text { hands, face, and equipment }\end{array}$ & $\begin{array}{l}\text { Minimum of dropcloth, HEPA- } \\
\text { vacuuming coveralls, and } \\
\text { bucket or Hudson sprayer for } \\
\text { hands, face, and equipment }\end{array}$ & $\begin{array}{l}\text { Minimum of dropcloth, HEPA- } \\
\text { vacuuming coveralls, and } \\
\text { bucket or Hudson sprayer for } \\
\text { hands, face, and cquipment }\end{array}$ \\
\hline
\end{tabular}


Appendix E

\author{
Condition Assessment Survey \\ for the \\ Kaiser-Hill LLC Oxnard Facility
}


This page intentionally blank 


\section{Condition Assessment Survey for the Kaiser-Hill Company LLC Oxnard Facility}

September 1995

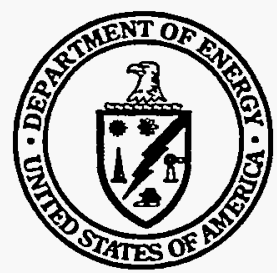

U.S. Department of Energy Grand Junction Projects Office 
This report was prepared as an account of work sponsored by an agency of the United States Government. Neither the United States Government nor any agency thereof, nor any of their employees, makes any warranty, express or implied, or assumes any legal liability or responsibility for the accuracy, completeness, or usefulness of any information, apparatus, product, or process disclosed in this report, or represents that its use would not infringe privately owned rights. Reference herein to any specific commercial product, process, or service by trade name, trademark, manufacturer, or otherwise, does not necessarily constitute or imply its endorsement, recommendation, or favoring by the United States Government or any agency thereof. The views and opinions of authors expressed herein do not necessarily state or reflect those of the United States Government or any agency thereof. 


\section{Condition Assessment Survey for the Kaiser-Hill Company LLC Oxnard Facility}

September 1995

Work performed under DOE Contract No. DE-AC04-94AL96907

Prepared for U.S. Department of Energy Albuquerque Operations Office Grand Junction Projects Office

Prepared by Rust Geotech Grand Junction, Colorado 

TABLE OF CONTENTS

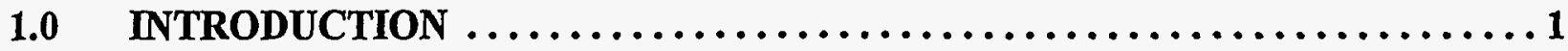

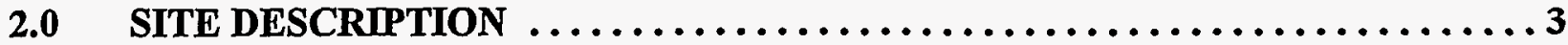

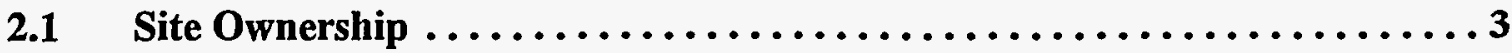

2.2 Physical Location/Setting $\ldots \ldots \ldots \ldots \ldots \ldots \ldots \ldots \ldots \ldots \ldots \ldots \ldots$

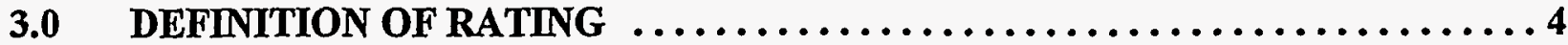

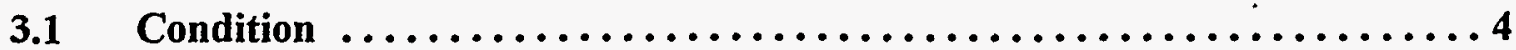

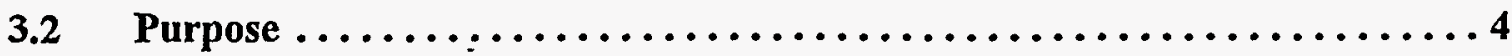

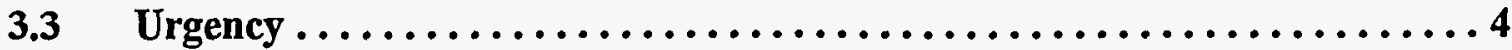

4.0 CONDITION ASSESSMENT SURVEY SUMMARY $\ldots \ldots \ldots \ldots \ldots \ldots \ldots \ldots 6$

Building 1 CAS Summary $\ldots \ldots \ldots \ldots \ldots \ldots \ldots \ldots \ldots \ldots \ldots \ldots \ldots \ldots, \ldots \ldots$

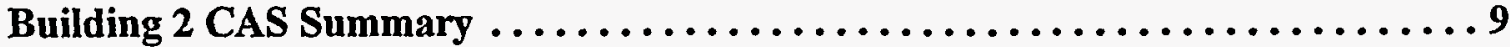

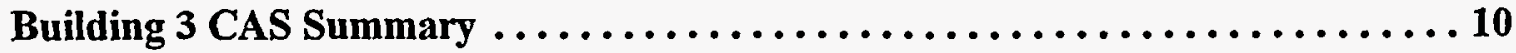

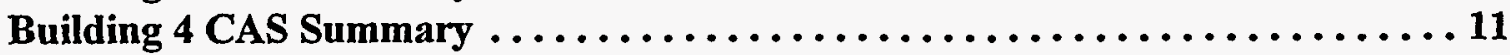

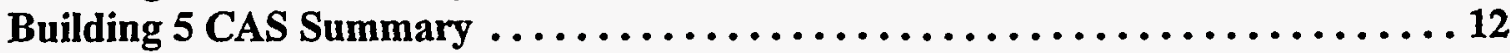

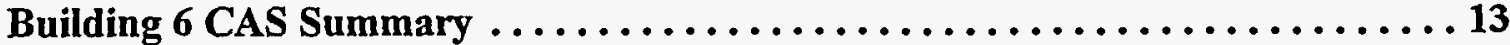

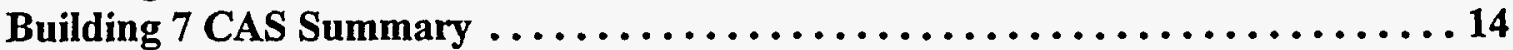





\section{CONDITION ASSESSMENT SURVEY \\ OXNARD FACILITY}

\subsection{INTRODUCTION}

The Condition Assessment Survey (CAS) process consisted of a thorough non-intrusive . assessment of the Kaiser-Hill Company LLC-operated Oxnard Facility and its seven buildings' systems, structures and components. The buildings were assessed for physical condition of plumbing, electrical, architectural and structural by a U.S. Department of Energy (DOE)-approved and certified CAS inspection team from Rust Geotech, Grand Junction Projects Office (under DOE Contract No. DE-AC04-94AL96907), Grand Junction, Colorado. The CAS process began on September 12, 1995, and was completed on September 20, 1995. Uniform Building Codes (UBC), National Fire Protection Association (NFPA) standards, and Occupational Safety and Health Administration (OSHA) standards are the principal references used in this assessment and referenced by DOE CAS Program Deficiency Standards and Inspections Methods Manuals (Volumes 1 through 12). Environmental assessment is not a part of the scope of this CAS process; however, obvious contamination or presence of asbestos is noted as suspected.

The Data Collection Devices were not used in this assessment as the Project Manager selected the option of a documented process versus the automated/documented process; however, the documentation as such includes the details and deficiency codes in accordance with the DOE CAS Program Deficiency Standards and Inspections Methods Manuals necessary to upload this information into the Condition Assessment Information System (CAIS), if desired, at a later date.

The report furnished with this assessment includes copies of the field notes taken by inspectors, a summary of the overall condition for each building and/or major system, and an overall condition of the facility in general, emphasizing the facility's immediate code/safety/ maintenance and repair concerns.

The Oxnard site description, addressing the site ownership, physical location/setting, building size, utilities, geology, seismology, demography, history, current use, etc., is available in numerous other documents including the Phase 1 Environmental Site Assessment document, dated September 30, 1994, and therefore, will not be duplicated in this report. 
The DOE-certified CAS Inspectors participating in this assessment are as follows:

Kyle Brannon

Ronald Hall

Thomas Masias

Joel Swetnam
CAS Administrator/Lead Inspector \#GJ50714

Electrical Inspector \#GJ50573

Architectural/Structural Inspector \#GJ50417

(970) $248-6610$

(970) $248-6610$

(970) $248-6610$

(970) $248-6605$

Mechanical Inspector \#GJ50687 


\subsection{SITE DESCRIPTION}

\subsection{Site Ownership}

The Oxnard facility is owned by the DOE and is operated by Kaiser-Hill Company LLC.

\subsection{Physical Location/Setting}

The Oxnard facility sits on 13.75 acres located in an industrial park within the city limits of Oxnard, county of Ventura. Various industrial facilities surround the Oxnard facility such as Deardorff-Jackson Inc. and Boskovich Corp.

(vegetable/fruit packing house); Kingstone Wheel Corp. (metal wheel fabrication); Gold Coast Steel (machining); and the City of Oxnard, which operates a vehicle maintenance garage immediately to the east of this facility.

The facility consists of seven buildings with approximately 86,000 square feet of covered floor space and approximately 89,500 square feet of roof area. The seven buildings include:

\begin{tabular}{|c|c|}
\hline Building 1 & Administrative Offices \\
\hline Building 2 & $\begin{array}{l}\text { Machine Shop, Engineering Office, Quality Control Office, } \\
\text { Metallurgical Laboratory, Production Control Office, } \\
\text { Maintenance Offices, and Lunch Room }\end{array}$ \\
\hline Building 3 & Saw Shop \\
\hline Building 4 & Grinding Shop \\
\hline Building 5 & Press Shop, Wheelabrator Room, Dye Penetrant Room \\
\hline Building 6 & Forge Shop \\
\hline Building 7 & $\begin{array}{l}\text { Tank Farm and Lubrication Storage/Waste Water Filtration } \\
\text { and Separator }\end{array}$ \\
\hline
\end{tabular}




\subsection{DEFINITION OF RATING}

\subsection{Condition}

Condition ratings used are as follows:

- Excellent: No defects or deficiencies

- Fair: Minor deficiencies/overall condition of the inspection unit (IU)

- Good: Minor defects or deficiencies but better than poor condition

- Poor: Numerous minor deficiencies and/or major deficiencies normally requiring attention within 1 year or immediately

- Fail: - Major safety and/or code violations that have immediate potential of injury. This should be immediately identified as "DO NOT OPERATE."

\subsection{Purpose}

Purpose indicators are used as follows:

- Maintenance: The condition is a maintenance issue and does not necessarily violate code or affect the operation of the unit yet.

- Functional: The condition is determined to have or will soon have an affect on the performance and/or function of this IU.

- Code: This condition is in violation of the UBC, National Building Code, OSHA, NFPA, or known directive/regulation usually requiring action within 1 year.

- Safety: This condition directly affects or will soon affect the safety of personnel and should be corrected immediately in most cases.

\subsection{Urgency}

Urgency codes are recommendations for response to the identified deficiencies based on the inspectors knowledge and experience with identical/similar conditions. Safety deficiencies should be resolved immediately, and maintenance issues should be evaluated and prioritized using a graded approach. In some cases, the inspector cannot make an accurate assessment during a non-intrusive type assessment but believes that the potential exists for an underlying condition. In this case, they may use a condition rating of FAIR and an urgency of $<1$ Year. 


\subsection{CONDITION ASSESSMENT SURVEY SUMMARY OF THE OXNARD FACILITY}

In general, the Oxnard facility is in fair condition considering the age, environment, and history of the structures and their components and systems. Some of the electrical systems . are outdated and do not comply with new construction codes but, in most cases, are adequate for their current use. Wall penetrations that serve utility lines, piping, water lines, etc. are not sealed or escutcheons in place throughout the facility. Piping has been removed from service but exists in various locations, as noted in the CAS Field Reports. Backflow protection for domestic water systems is lacking throughout most of the facility, which offers the potential for water contamination and human ingestion of such contamination. Electrical equipment is typically outdated throughout the facility. In most cases, labeling of electrical equipment/systems, piping, and equipment is not compliant with OSHA or DOE Order 5480.19, Conduct of Operations, requirements. Other than the conditions noted, the buildings are functionally adequate for their intended use. It is also noted that the facility has been well maintained and, considering the age and environment, the buildings are cosmetically appealing.

Due to the age, environment, and numerous deteriorating conditions found on the facility, it is recommended that a CAS be performed annually of this facility at least for the next 3 years. It is also recommended that engineering studies be performed immediately on the deteriorating concrete columns. 


\section{Building 1 CAS Summary}

Administration Office: Technical and Administrative staff, Accounting, Purchasing, Human Resources, etc.

Size: 4,500 square feet

Year Built: 1950

The general condition of this building is good. On the exterior of the building, paint was found peeling, chipping and cracking, and the stucco cracking in some locations. Window units displayed cracked and weathered glazing, and lead-based paint is suspected to be present on fascia and other trim.

The interior of the building is also in good condition with some dropped ceiling panels displaying water stains indicating leaking at the roof. Asbestos containing material is suspected in various locations throughout the building's interior including flooring and vinyl asbestos tile. While in the attic, it was discovered that the interior roofing member supports are not sufficient, and collar ties are missing. Two leaks in the roof were found at northwest center section.

Mechanically, this building is in good condition. Backflow protection is lacking in four outside hose bibs and on the janitor's closet faucet. A $2 \frac{1}{2}$-inch gate valve is leaking on the supply side of the double-check backflow prevention device. The water heater relief valve is improperly plumbed.

Electrically, the assessed condition of this building is fair. In some cases, outdoor electrical raceways are installed using fittings designed for indoor applications. A questionable condition exists as to the heat load in the attic. Three $10 \mathrm{KVA}$ transformers reside in the attic with minimal ventilation. This condition should be evaluated and modifications made, as necessary. In some cases, GFCI protection is not adequate according to OSHA and UBC codes.

See attached CAS Field Reports for additional deficiencies. 


\section{Building 2 CAS Summary}

Machine Shop: Shipping and Receiving, Production, Planning and Control office, Maintenance office, electrical maintenance room, stock room, Quality Assurance office, machining floor, Metallurgical Laboratory, and Engineering/Computer Assisted Drafting office

Size: 42,016 square feet

Year Built: 1950

The general condition of this building is fair with some major deficiencies as follows:

- Column E-8 supports the east end of the 5-ton Gantry crane and is the load-bearing wall support for this side of the building. The concrete column is disintegrating and may be deteriorating from the inside, which makes it difficult to know the extent of the degradation. This is a major concern and should be evaluated immediately.

- Concrete support columns bearing the weight of the external Gantry crane, rail, and supports have significant impact damage. Anchors are broken, structural rebar is exposed, and corrosion is evident. These columns are numbered G1W, G2W, G4W, and G5W.

- Concrete Column E-7 also has exposed rebar and displays spalling.

Mechanically, the assessed condition of this building is good with one exception:

- Backflow prevention is lacking in this building, which presents a potential for domestic water contamination.

Electrically, the assessed condition of this building is good with the following exceptions:

- There are exposed wires visible in the locker room at Column W11.

- The Fabrication Shop has several 110-volt outlets that require GFCI protection.

- A battery-powered floodlight, located between Columns A10 and A11, is inoperative. Corrosion is noted in various locations.

See attached CAS Field Reports for additional deficiencies. 


\section{Building 3 CAS Summary}

Saw Shop: Various saws, 5-ton overhead bridge crane

Size: 5,200 square feet

Year Built: 1949

The general condition of this building is poor. The concrete poured-in-place south wall is cracking and displays efflorescence on exterior. The crane rail support pad, located at the west wall Column 3 W20, has been cosmetically repaired after the original concrete disintegrated. The other concrete support pads are showing the same type of disintegration. An engineering study is recommended.

Electrical assessment indicates fair condition. Minor code violations, corrosion, and outdated electrical equipment exist.

Mechanical condition is assessed as fair. Vacuum breakers are missing in various locations, as noted on the attached field reports.

See attached CAS Field Reports for additional deficiencies. 


\section{Building 4 CAS Summary}

Grinding Shop: Numerous grinding stations, air-abatement particle separator Size: 1,485 square feet

Year Built: 1950

The general condition of this building is poor. The building requires a major facelift and would probably be more practical to tear down and rebuild. Structural supports have been cut out and modified for a monorail system that is no longer used. The metal siding is damaged, pieced together, and requires full replacement.

Electrically, minor code violations were found, but overall, the assessed condition of the building is good. Outdated electrical equipment is also noted.

Mechanically, the assessed condition of this building is fair. Labeling is either non-existent or non-compliant.

See attached CAS Field Reports for additional deficiencies. 


\section{Building 5 CAS Summary}

Press Shop: 1,600-ton mechanical press, 3,500-ton hydraulic press, trim press, natural gas-fired furnaces

Size: 7,950 square feet

Year Built: 1950

The general condition of this building is assessed as fair. Some of the structural supports (swaybraces), located on the interior of the building, have been removed for the convenience of installing a large piece of manufacturing equipment. The missing supports are located at Columns 5E26 through 5E24 and should be reinstalled immediately.

Mechanically, the building is assessed as fair. Hanging piping is not properly suspended and supported with required hardware. Piping labeling is non-compliant. The natural gas odor appears to be excessive. Leak tests should be performed and the ventilation modified accordingly.

Electrically, the condition is assessed as fair. Rubber raceway used, in various applications, is not adequate and requires proper hard-wiring.

See attached CAS Field Reports for additional deficiencies. 


\section{Building 6 CAS Summary}

Forge Shop: 16 High Energy-Rated Forging (HERF) hammers of various energy ratings, gas-fired furnaces, mechanical trim presses, a pit area, tank farm

Size: 24,544 square feet

Year Built: Mid-1980's

The general condition of the building is assessed as good with a few maintenance and code issues as follows:

- Numerous 110-volt outlets are located in the pit area and are not GFCI protected.

- East side exterior under awning has five 110-volt outlets that are not GFCI protected.

Labeling is non-existent/non-compliant.

See attached CAS Field Reports for additional deficiencies. 


\section{Building 7 CAS Summary}

Oil/Waste Water Filtration System: Tanks, drums, 3-wall structure

Size: 1,380 square feet

Year Built: 1950

The general condition of this building is good. A concern about the tank containment area exists. It appears that the tank containment area received some demolition work but was left unfinished, thereby affecting the integrity of the containment area.

- All 110-volt outlets should be GFCI protected.

- Backflow protection is inadequate or non-existent.

See attached CAS Field Reports for additional deficiencies. 


\title{
Oxnard CAS Field Notes
}

\author{
Building 1
}





\section{Oxnard Condition Assessment Survey Field Report}

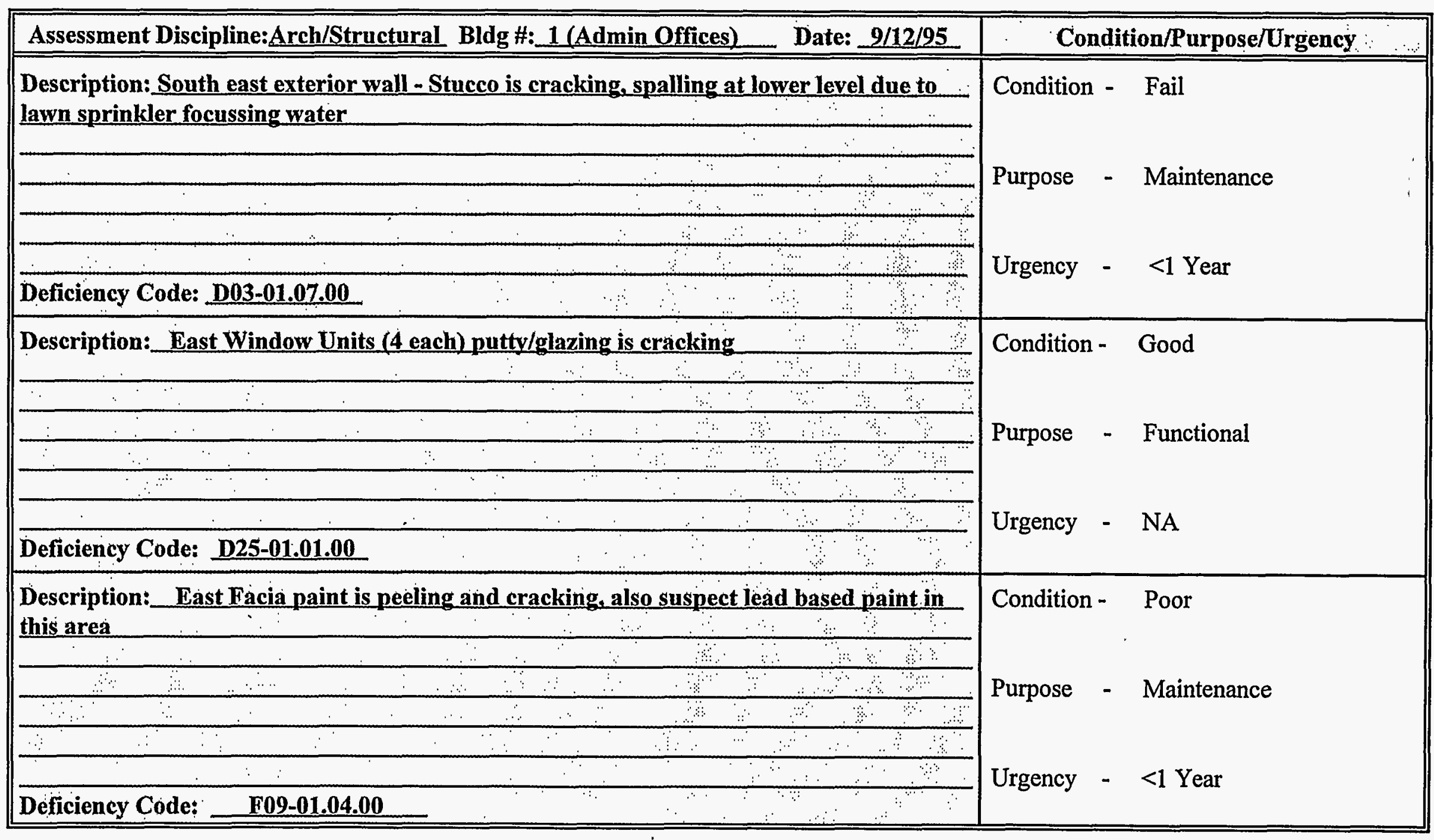




\section{Oxnard Condition Assessment Survey Field Report}

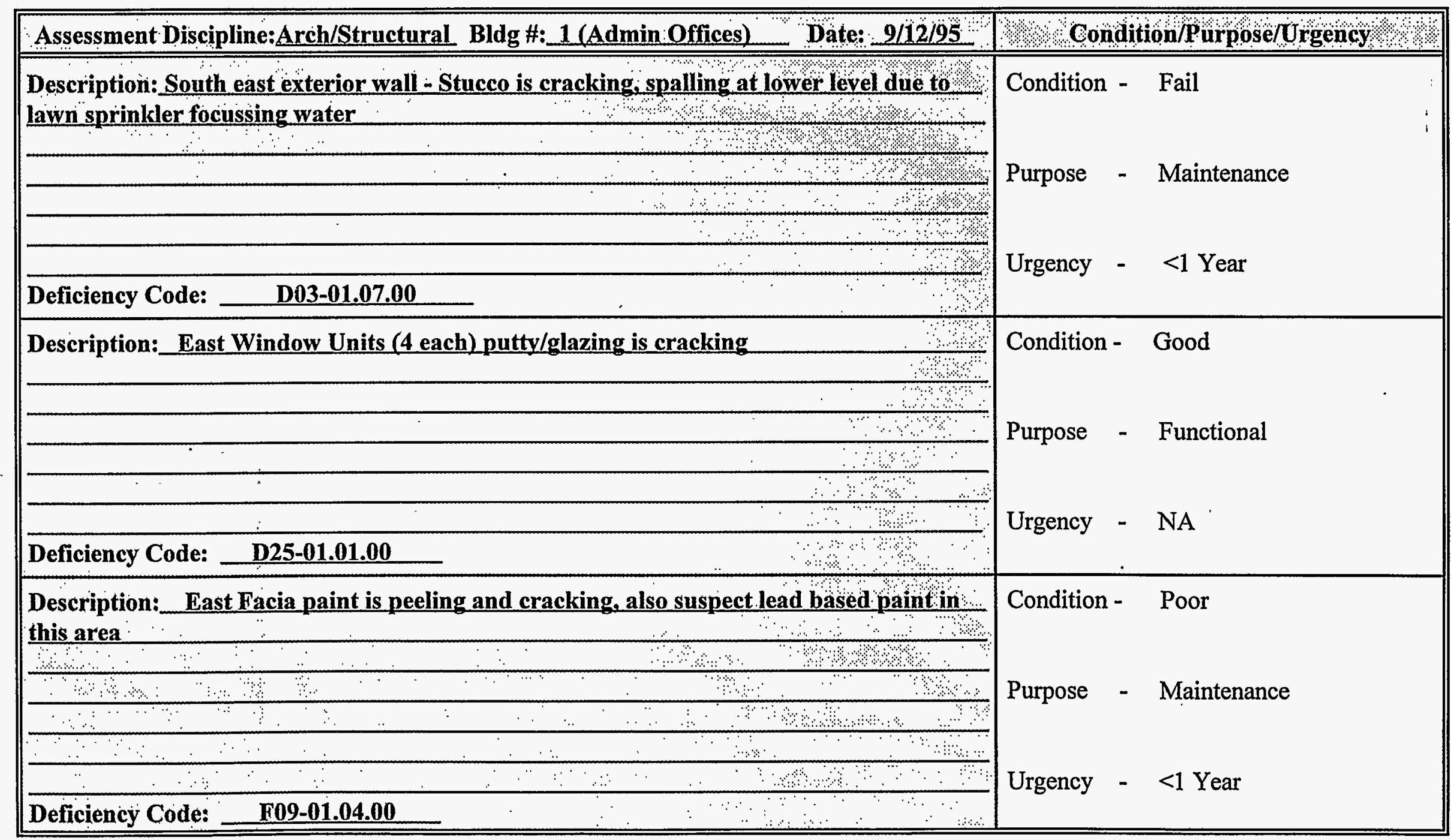




\section{Oxnard Condition Assessment Survey Field Report}

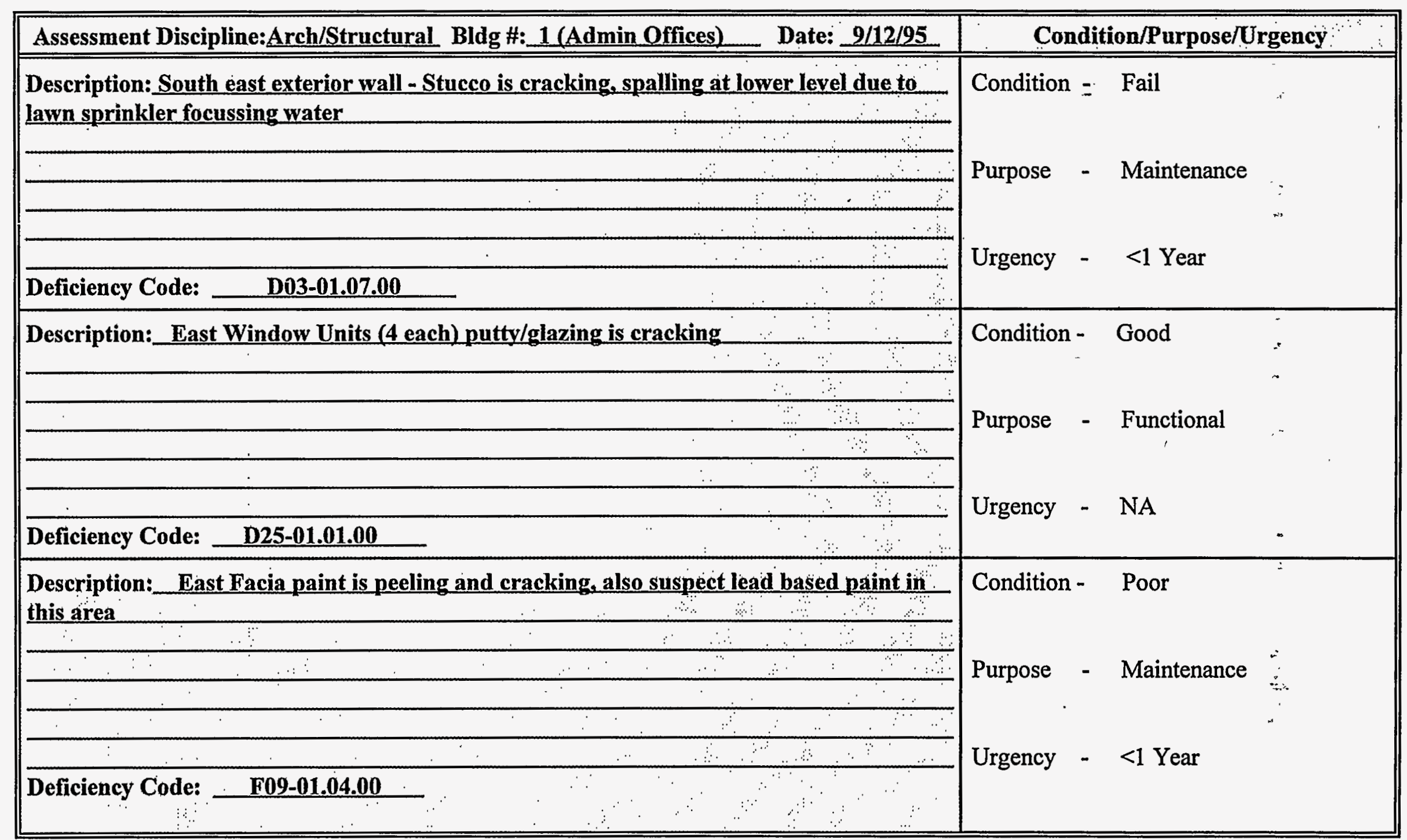




\section{Oxnard Condition Assessment Survey Field Report}

\begin{tabular}{|c|c|c|}
\hline Assessment Discipline:Arch/Structural Bldg \#: 1 (Admin Offices) I Date: 9/12/95. & $\therefore$ Con & dition/Purpose/Urgency \\
\hline Description: Interior hallway suspect (ACM) $\quad$ I & Condition - & Good \\
\hline 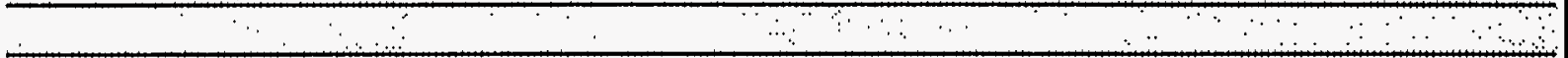 & \multirow{5}{*}{$\begin{array}{l}\text { Purpose } \\
\text { Urgency }\end{array}$} & \multirow{5}{*}{$\begin{array}{l}\text { - Functional } \\
\text { - NA }\end{array}$} \\
\hline$\therefore \quad \therefore \quad \therefore \quad \div \quad \div \quad \div \quad$ & & \\
\hline 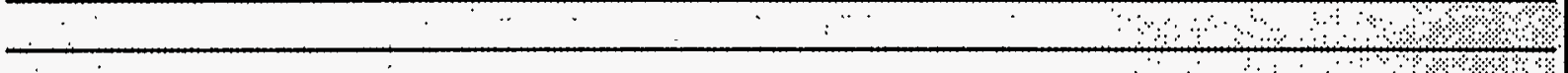 & & \\
\hline$\therefore \quad+\quad \div \quad+\quad \div$ & & \\
\hline Deficiency Code: F15-01.11.00 & & \\
\hline Description: Lobby area, numerous loose ceiling tiles (VAT Flooring Suspected) & \multicolumn{2}{|c|}{ Condition - Good } \\
\hline : : : : : & \multirow{2}{*}{\multicolumn{2}{|c|}{ Purpose - Maintenance }} \\
\hline$\because \quad \because \cdots \because \because$ & & \\
\hline 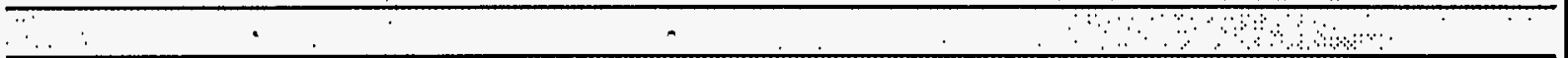 & \multirow{4}{*}{\multicolumn{2}{|c|}{ Urgency - NA }} \\
\hline$\therefore \therefore \therefore \therefore$ & & \\
\hline$\because \ldots \ldots$ & & \\
\hline Deficiency Code: $\ldots$ F15-01.11.00 & & \\
\hline Description Engineering/Purchasing/Payroll offices (VAT flooring Suspected) & \multirow{2}{*}{\multicolumn{2}{|c|}{ Condition - Good }} \\
\hline$\because \cdots \quad+\cdots$ & & \\
\hline 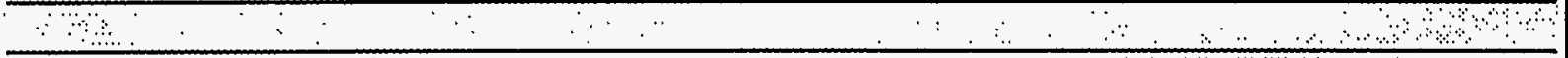 & \multirow[t]{3}{*}{ Purpose } & \multirow[t]{3}{*}{ - Maintenance } \\
\hline 10 & & \\
\hline$\ldots \ldots$ & & \\
\hline$\therefore \cdots$ & \multirow[t]{2}{*}{ Urgency } & \multirow[t]{2}{*}{$-\quad \mathrm{NA}$} \\
\hline Deficiency Code: F15-01.11.00 & & \\
\hline
\end{tabular}




\section{Oxnard Condition Assessment Survey Field Report}

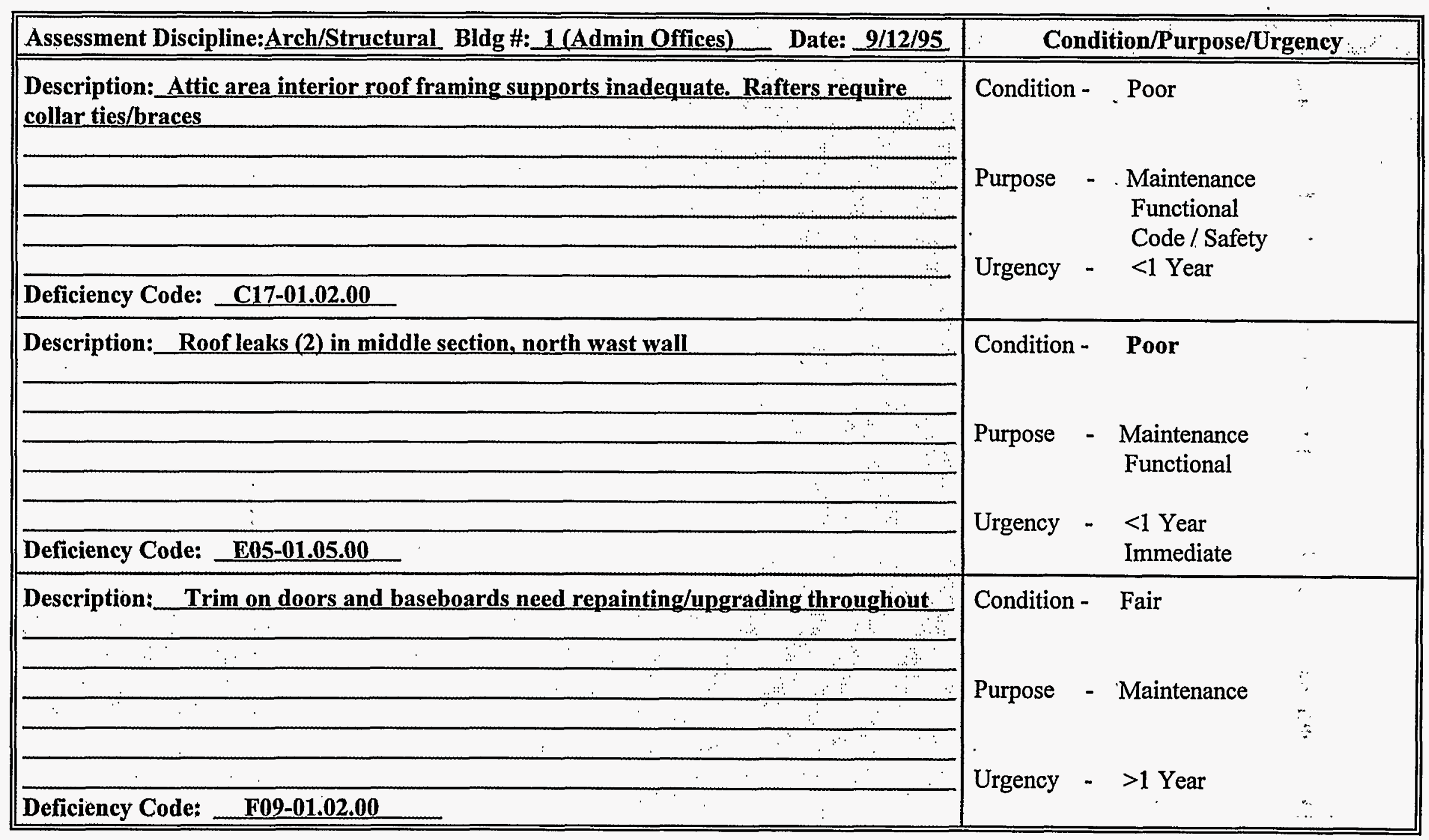




\section{Oxnard Condition Assessment Survey Field Report}

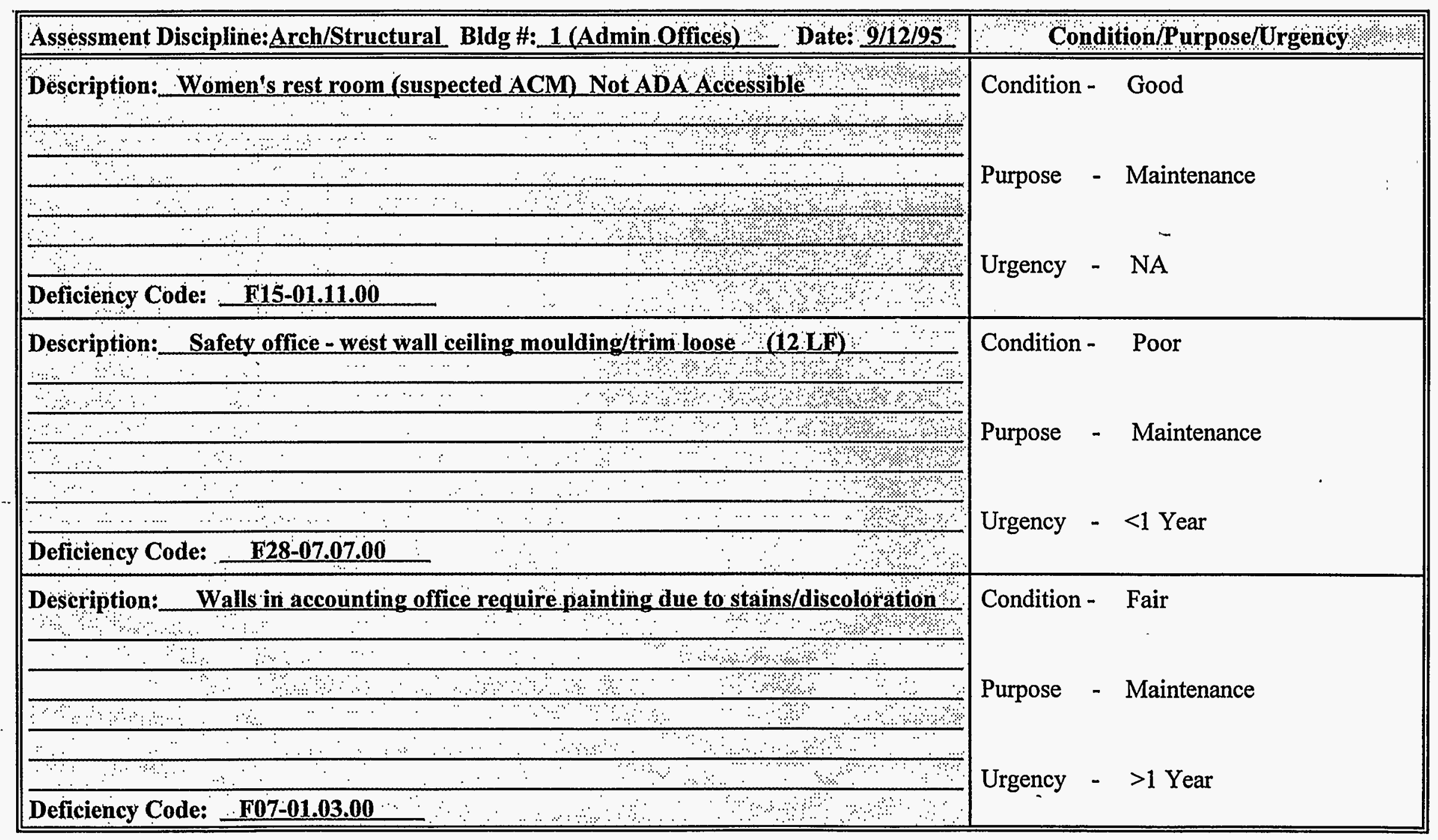




\section{Oxnard Condition Assessment Survey Field Report}

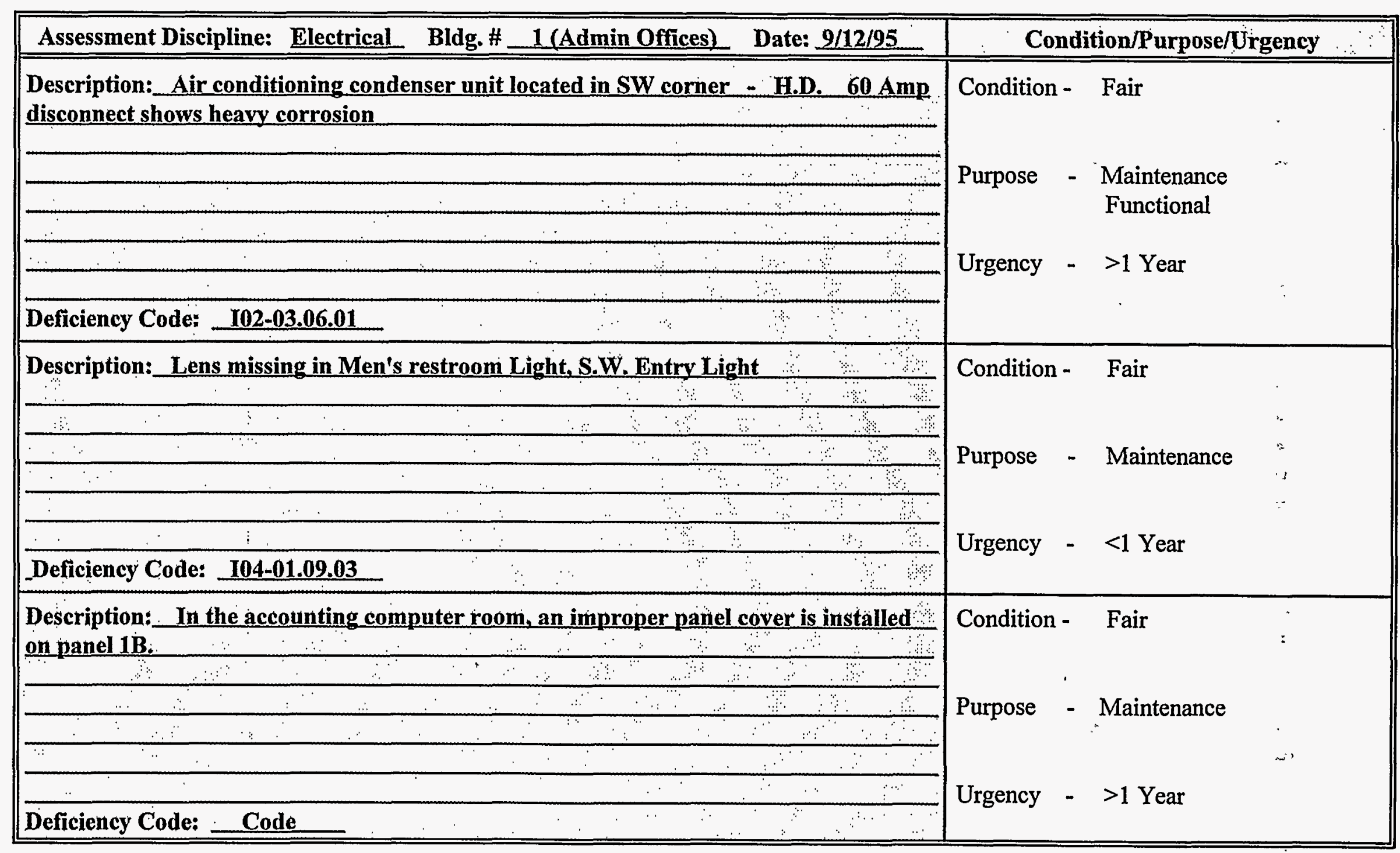




\section{Oxnard Condition Assessment Survey Field Report}

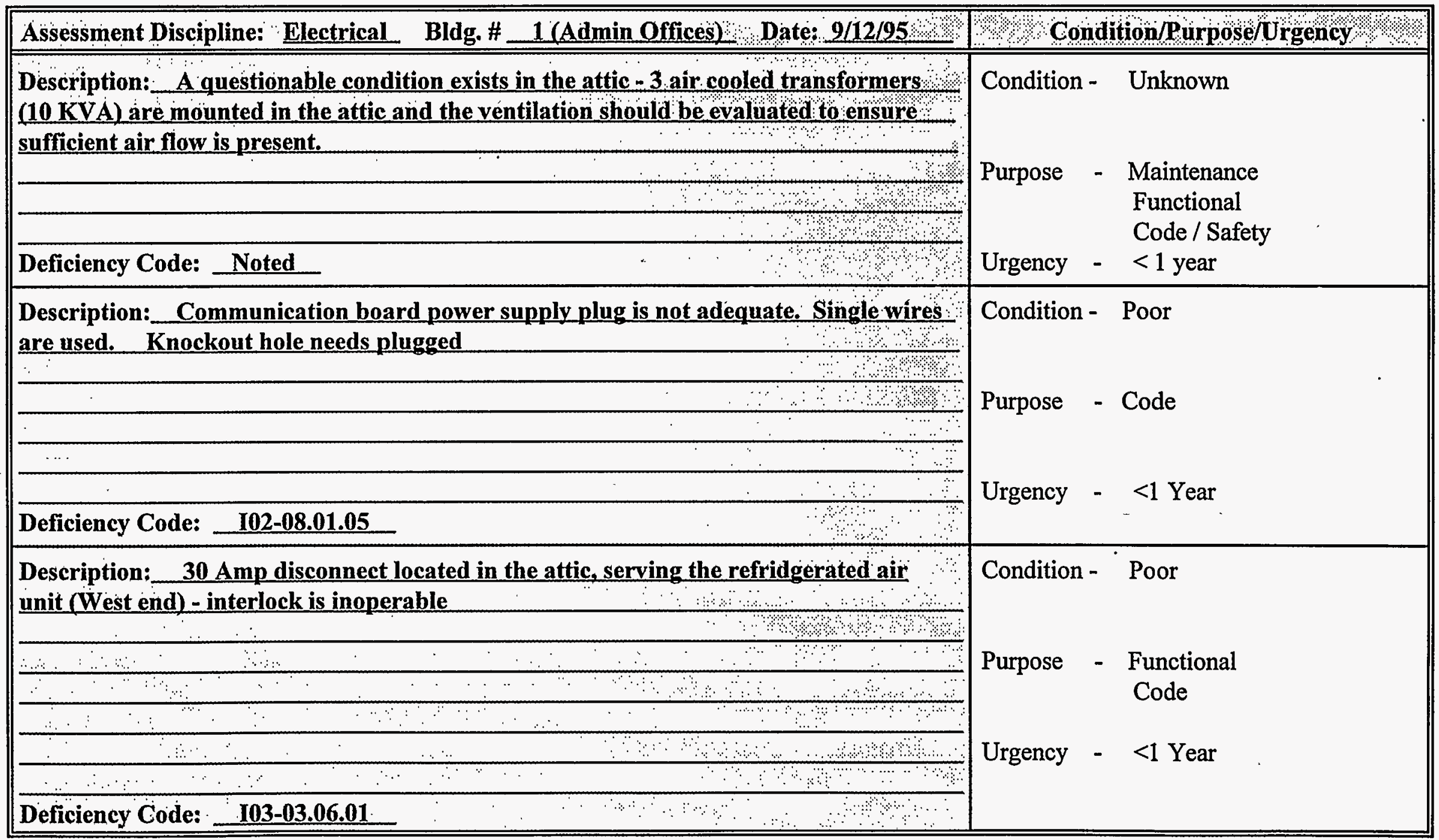




\section{Oxnard Condition Assessment Survey Field Report}

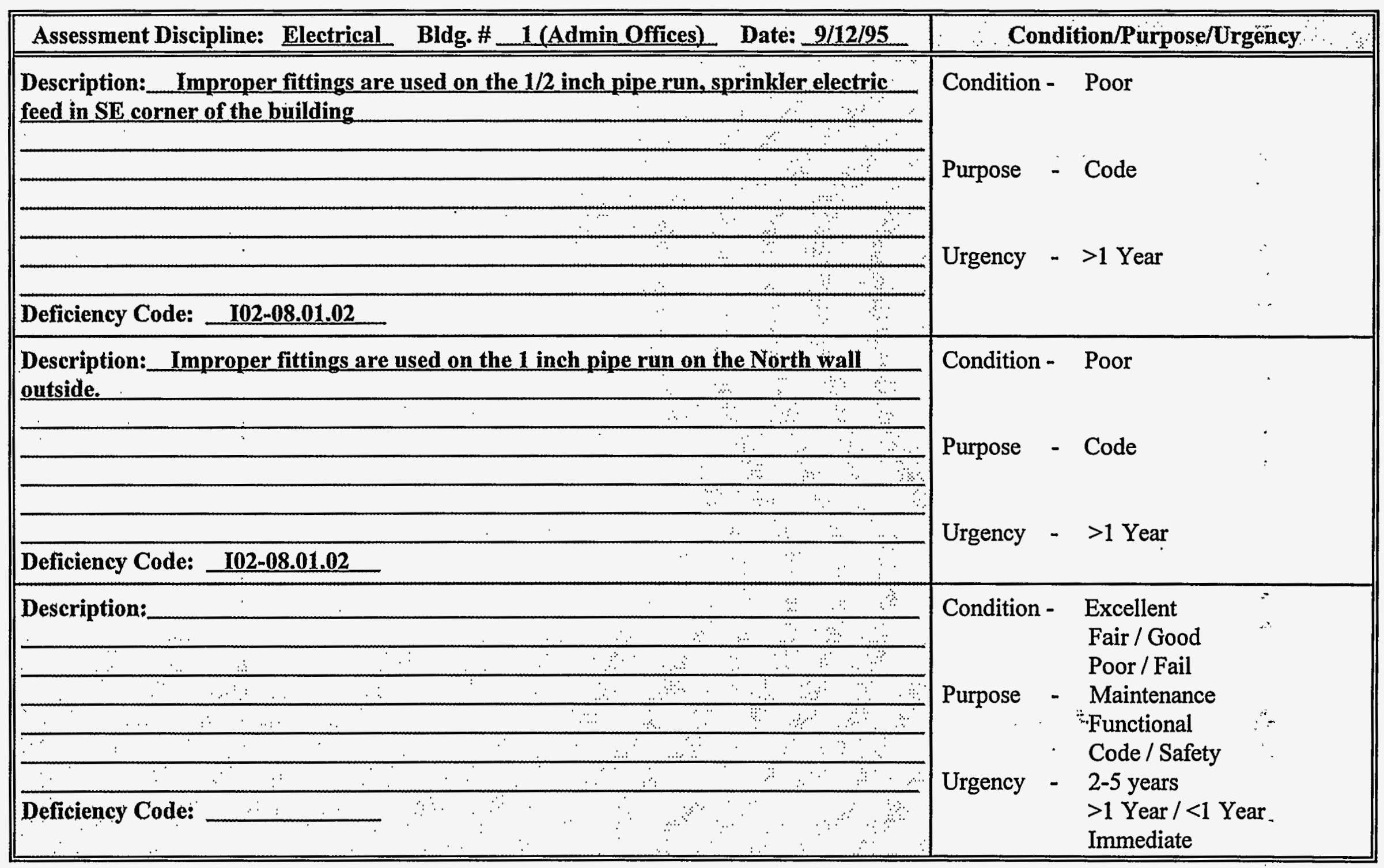




\section{Oxnard Condition Assessment Survey Field Report}

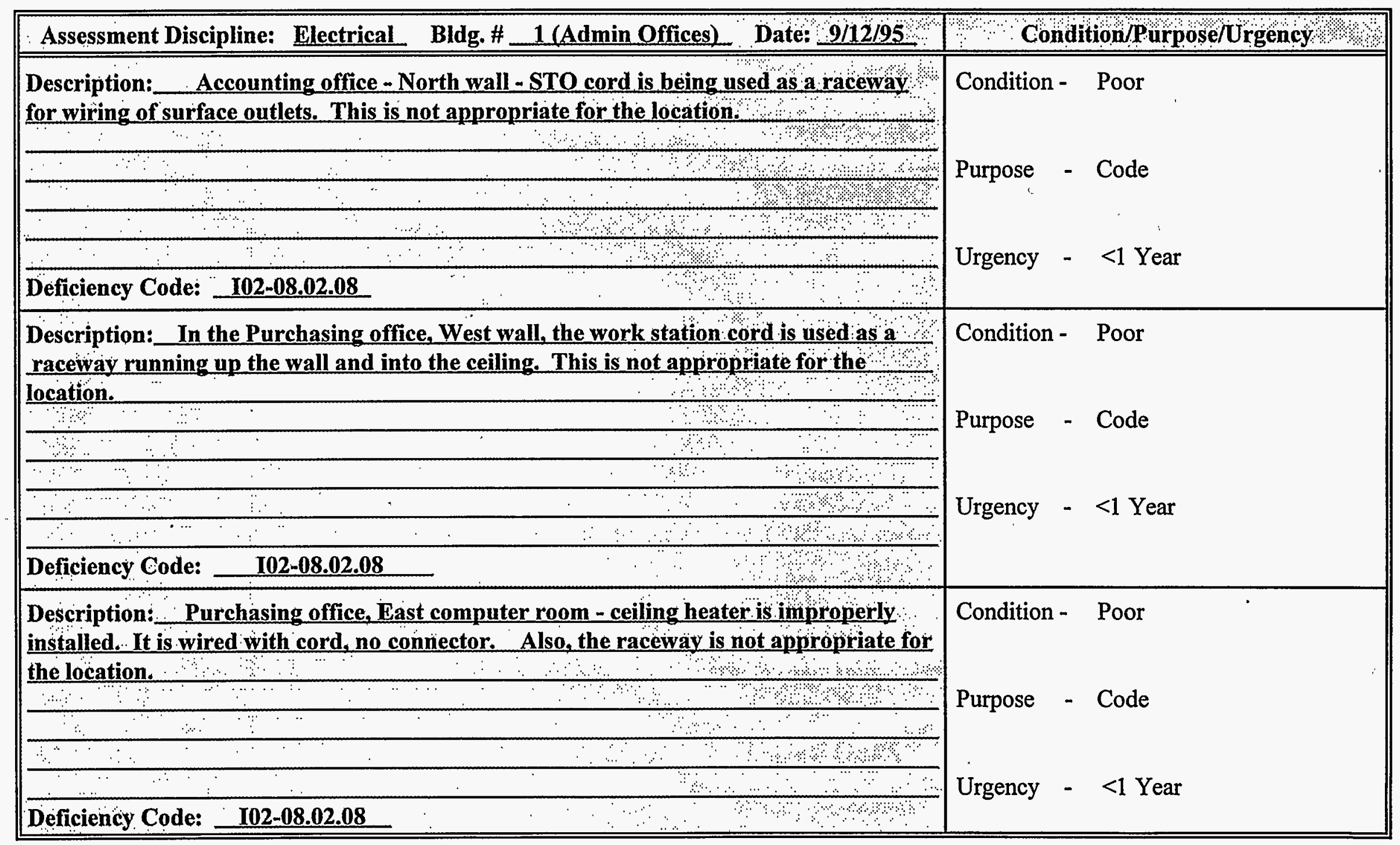




\section{Oxnard Condition Assessment Survey Field Report}

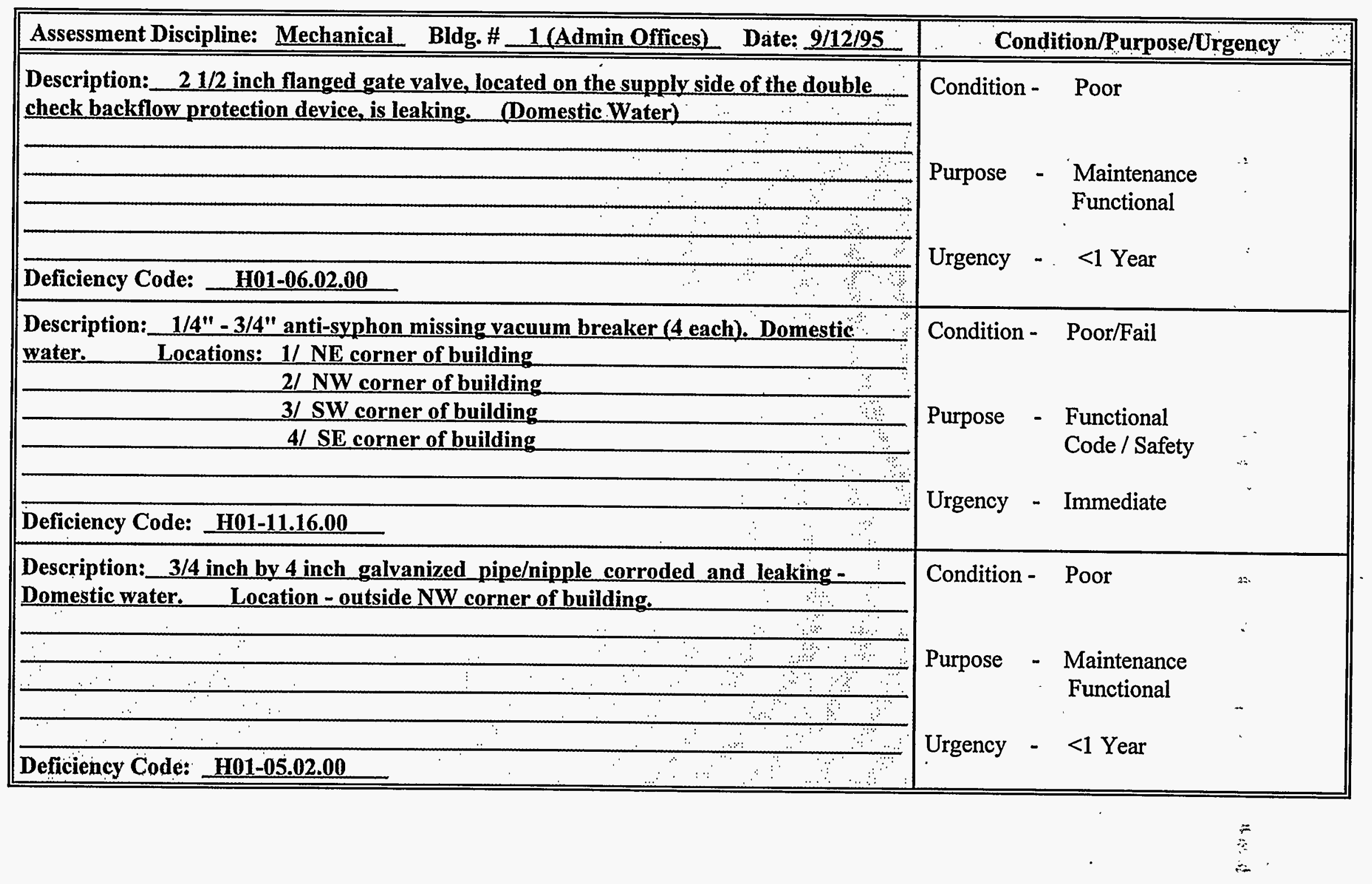




\section{Oxnard Condition Assessment Survey Field Report}

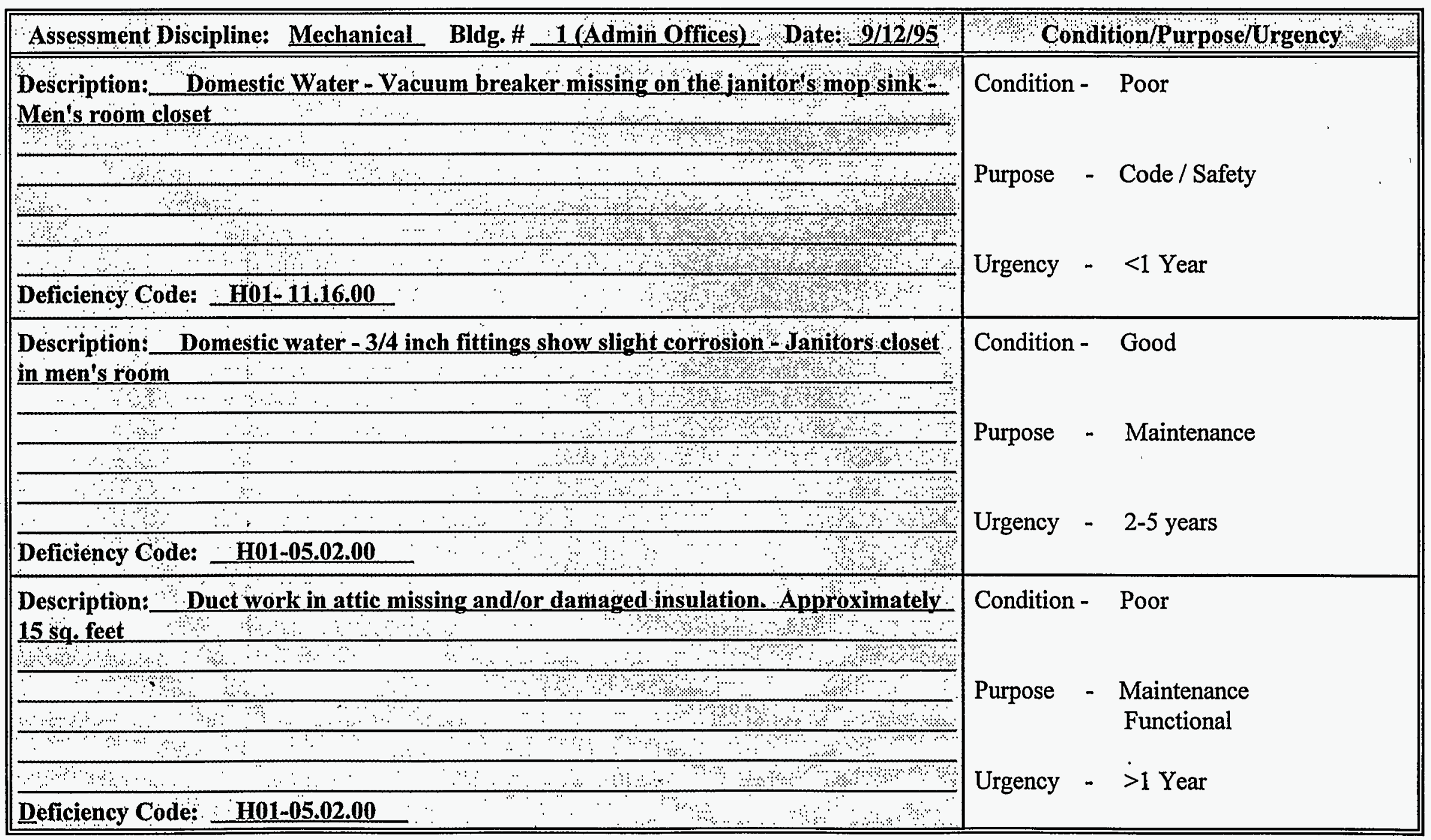




\section{Oxnard Condition Assessment Survey Field Report}

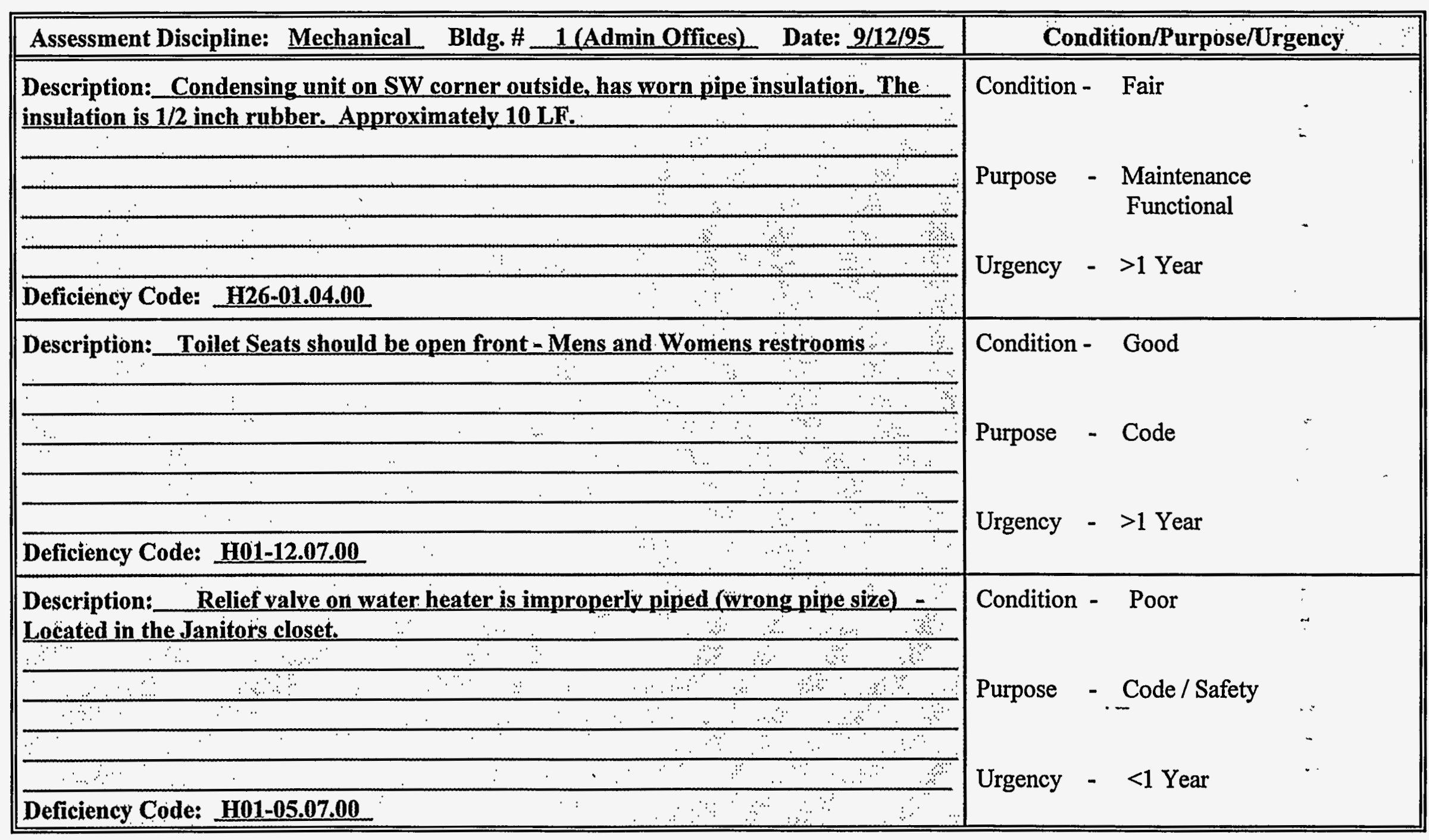




\section{-..}

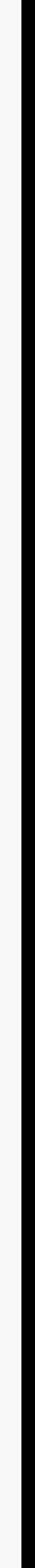




\title{
Oxnard CAS Field Notes
}

\author{
Building 2
}




\section{Oxnard Condition Assessment Survey Field Report}

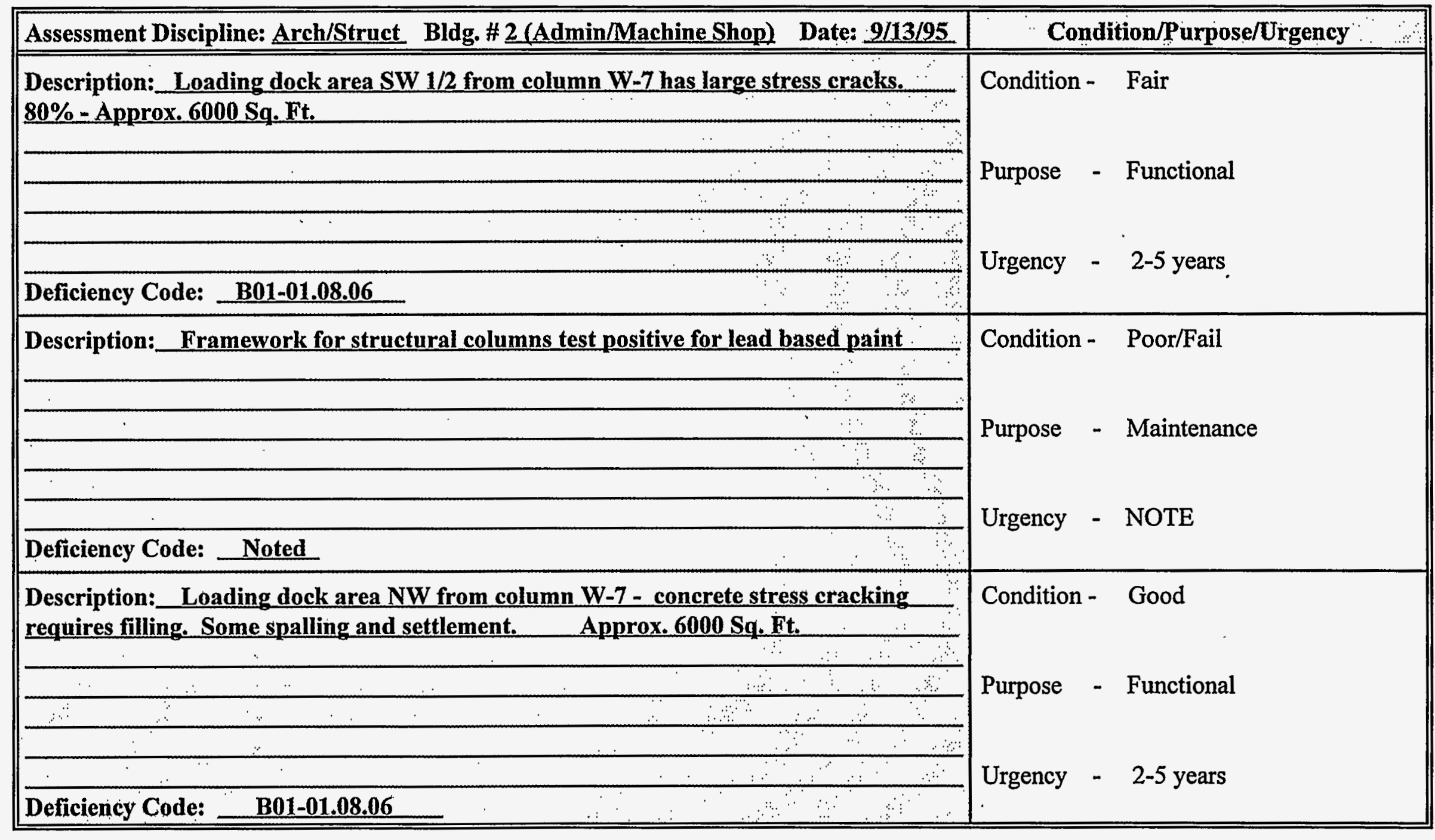




\section{Oxnard Condition Assessment Survey Field Report}

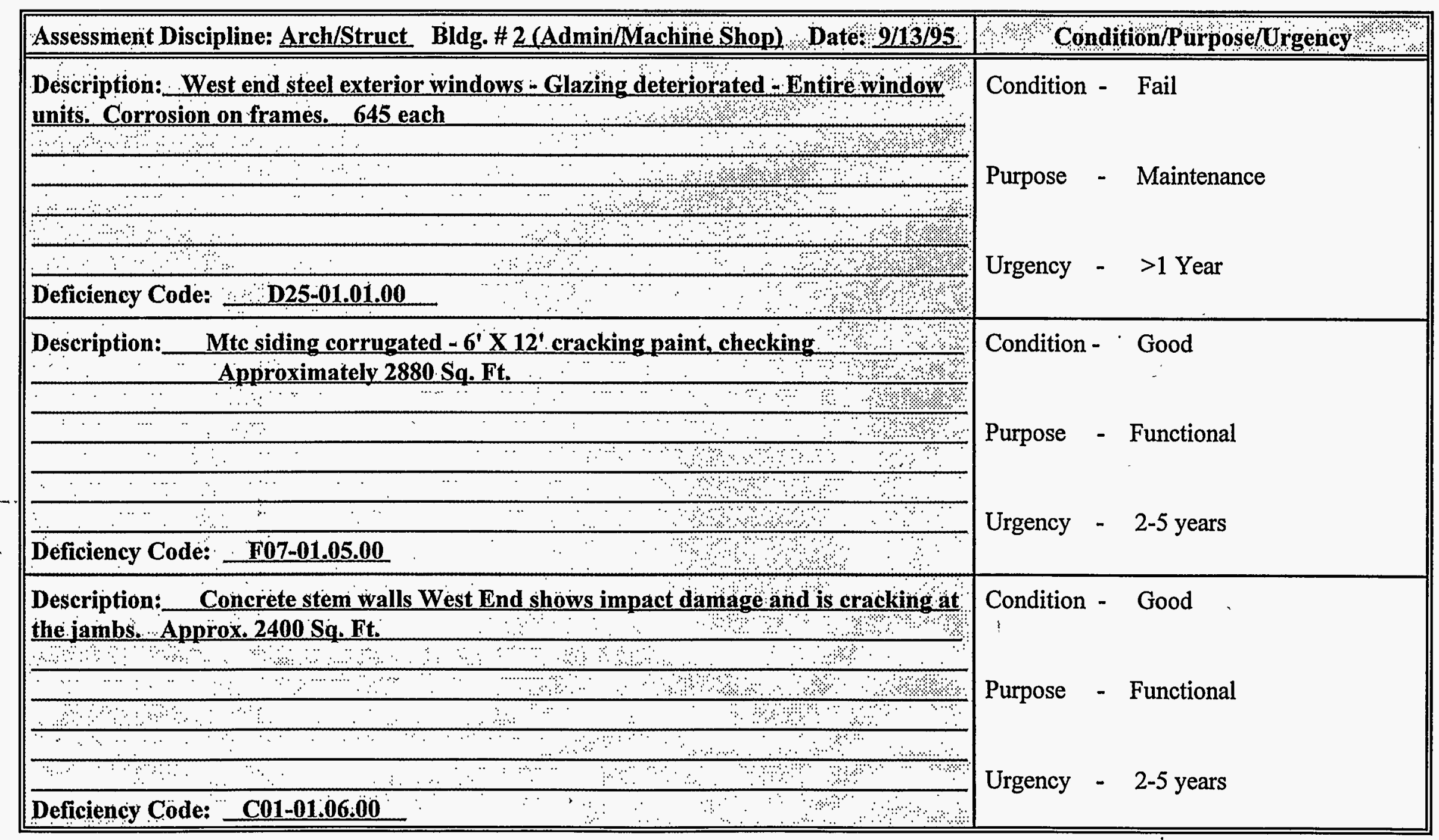




\section{Oxnard Condition Assessment Survey Field Report}

\begin{tabular}{|c|c|c|c|}
\hline Assessment Discipline: Arch/Struct Bldg. \# 2 (Admin/Machine Shop) Date: 9/13/95 & \multicolumn{3}{|c|}{ Condition/Purpose/Urgency } \\
\hline $\begin{array}{l}\text { Description: Overhead doors on West side of building show impact damage and paint } \\
\text { peeling. } 5 \text { each @ } 10^{\prime} \mathrm{W} \times 12^{\prime} \mathrm{H} \text { and } 1 \text { each } 16^{\prime} \mathrm{W} \text {. Protective coating/paint }\end{array}$ & \multirow{4}{*}{$\begin{array}{l}\text { Condition - } \\
\text { Purpose - }\end{array}$} & - Good & \\
\hline 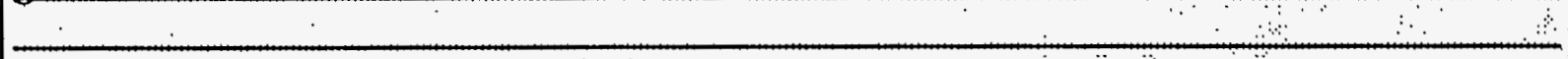 & & - Functional & \\
\hline$\therefore$ & & & \\
\hline$\therefore$ & & \multirow{2}{*}{$-2-5$ years } & \\
\hline Deficiency Code: D16-03.01.01 & Urgency & & \\
\hline Description: North end partial CMU addition. NW corner holes and soiling $W-17$ & \multirow{3}{*}{ Condition - } & \multirow{3}{*}{- Good } & \multirow{3}{*}{$\therefore$} \\
\hline 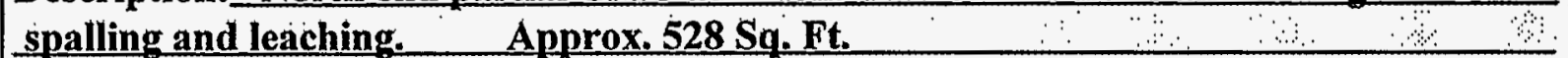 & & & \\
\hline 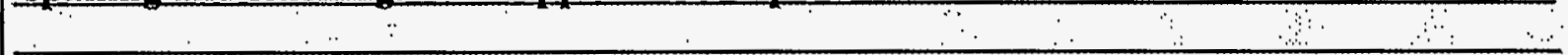 & & & \\
\hline$\therefore$ & \multirow[t]{3}{*}{ Purpose } & \multirow[t]{3}{*}{ - Maintenance } & . \\
\hline$\because \quad \vdots$ & & & \\
\hline$\because \quad \because \quad \cdots \quad \because \cdots$ & & & \\
\hline$\because$ & \multirow[t]{2}{*}{ Urgency } & \multirow[t]{2}{*}{ - $>1$ Year } & \\
\hline Deficiency Code: D02-01.08.00 & & & \\
\hline Description: North end from W-17 window units grazing deterioration & \multirow[t]{2}{*}{ Condition - } & Fail & \\
\hline 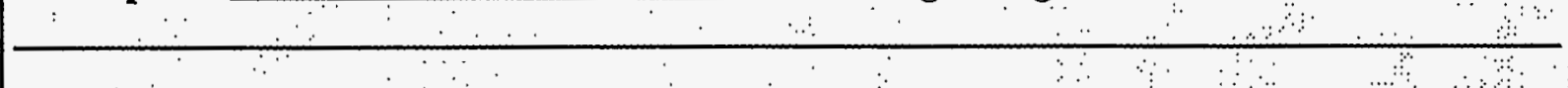 & & & \\
\hline$\therefore \quad \vdots \quad \cdots \quad \therefore \quad \vdots$ & \multirow[t]{3}{*}{ Purpose } & \multirow[t]{3}{*}{ - Maintenance } & \\
\hline$\ldots \quad \ldots$ & & & \\
\hline$\because \because:$ & & & \\
\hline$\therefore \quad \div \quad \div$ & \multirow[t]{2}{*}{ Urgency } & \multirow[t]{2}{*}{$->1$ Year } & \\
\hline Deficiency Code: $\quad$ D25-01.01.00 & & & \\
\hline
\end{tabular}




\section{Oxnard Condition Assessment Survey Field Report}

\begin{tabular}{|c|c|c|}
\hline Assessment Discipline: Arch/Struct Bldg. \#2 (Admin/Machine Shop) Date: $9 / 13 / 95$ & \multicolumn{2}{|c|}{ \% Condition/Purpose/Urgency $\%$} \\
\hline Description, North end overhead doors (2) $10^{\dagger} \times 12^{\prime}$ (1) fixed Paint coating peeling & \multirow{4}{*}{\multicolumn{2}{|c|}{$\begin{array}{l}\text { Condition - Fair } \\
\text { Purpose - Maintenance }\end{array}$}} \\
\hline 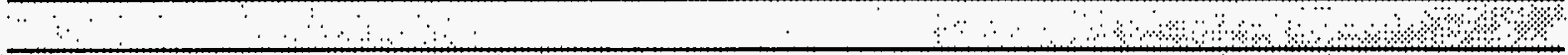 & & \\
\hline & & \\
\hline ४०० \%०० & & \\
\hline $\mathrm{B}$ & \multirow{2}{*}{\multicolumn{2}{|c|}{ Urgency - >1 Year }} \\
\hline Deficiency Code: D16-03.01.01 & & \\
\hline $\begin{array}{c}\text { Description: North exterior stem wall shows impact damage, holes and anchors. } \\
\text { Approx. 125 Sq. Ft. }\end{array}$ & \multicolumn{2}{|c|}{ Condition - Good } \\
\hline & \multirow{3}{*}{\multicolumn{2}{|c|}{ Purpose - Functional }} \\
\hline अथ. & & \\
\hline अa: & & \\
\hline अें & \multirow{2}{*}{ Urgency } & \multirow{2}{*}{ - $2-5$ years } \\
\hline Deficiency Code: $\quad$ C01-01.06.00 & & \\
\hline Description: $\quad$ NE corner corrugated metal - Paint is peeling - Approx. 100 Sq. Ft. & \multirow{5}{*}{\multicolumn{2}{|c|}{$\begin{array}{l}\text { Condition - . Poor } \\
\text { Purpose - Maintenance }\end{array}$}} \\
\hline (2) & & \\
\hline 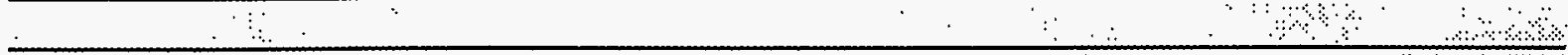 & & \\
\hline$\therefore \quad \therefore \quad 3$ & & \\
\hline 20 & & \\
\hline$\therefore \quad \because \quad \because$ & \multirow{2}{*}{\multicolumn{2}{|c|}{ Urgency $->1$ Year }} \\
\hline Deficiency Code: $\quad$ F07-01.05.00 & & \\
\hline
\end{tabular}




\section{Oxnard Condition Assessment Survey Field Report}

\begin{tabular}{|c|c|c|c|}
\hline Assessment Discipline: Arch/Struct Bldg. \# 2 (Admin/Machine Shop) Date: 9/13/95 & \multicolumn{3}{|c|}{ Condition/Purpose/Urgency. } \\
\hline Description:__East stem wall shows impact damage, spalling & \multicolumn{3}{|c|}{ Condition - Poor } \\
\hline$\ldots$ & \multirow{4}{*}{$\begin{array}{l}\text { Purpose } \\
\text { Urgency }\end{array}$} & \multirow[b]{2}{*}{ - Functional } & \multirow{4}{*}{ - } \\
\hline$\because$ & & & \\
\hline & & \multirow[t]{2}{*}{$-\quad>1$ Year } & \\
\hline Deficiency Code: C01-01.06.00 & & & \\
\hline Description: East window wall section -580 each windows - spackling deterioration & Condition & Poor & \multirow{5}{*}{$\begin{array}{l}\cdots \\
\cdots \\
\cdots \\
\cdots\end{array}$} \\
\hline$\therefore$ & \multirow{4}{*}{$\begin{array}{l}\text { Purpose } \\
\text { Urgency }\end{array}$} & - Maintenance & \\
\hline & & & \\
\hline$\therefore$ & & \multirow{2}{*}{$-\quad>1$ Year } & \\
\hline Deficiency Code: $\quad$ D25-01.01.00 & & & \\
\hline Description: Overhead doors (6 each) on East side of building $-10^{\prime} \times 12$ ' - Paint & Condition - & \multirow[t]{2}{*}{ Fair } & $\because-$ \\
\hline peeling. & & & \\
\hline$\because \quad \therefore \quad \therefore \quad \therefore$ & Purpose & - Maintenance & \multirow{4}{*}{ 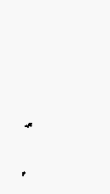 } \\
\hline$+\ldots$ & \multirow{4}{*}{ Urgency } & & \\
\hline \begin{tabular}{c|c|c}
$\cdots \cdots$ & $\vdots$ \\
\end{tabular} & & & \\
\hline (2) & & \multirow[t]{2}{*}{$-2-5$ years } & \\
\hline Deficiency Code: $\quad$ D16-03.01.01 & & & \\
\hline
\end{tabular}




\section{Oxnard Condition Assessment Survey Field Report}

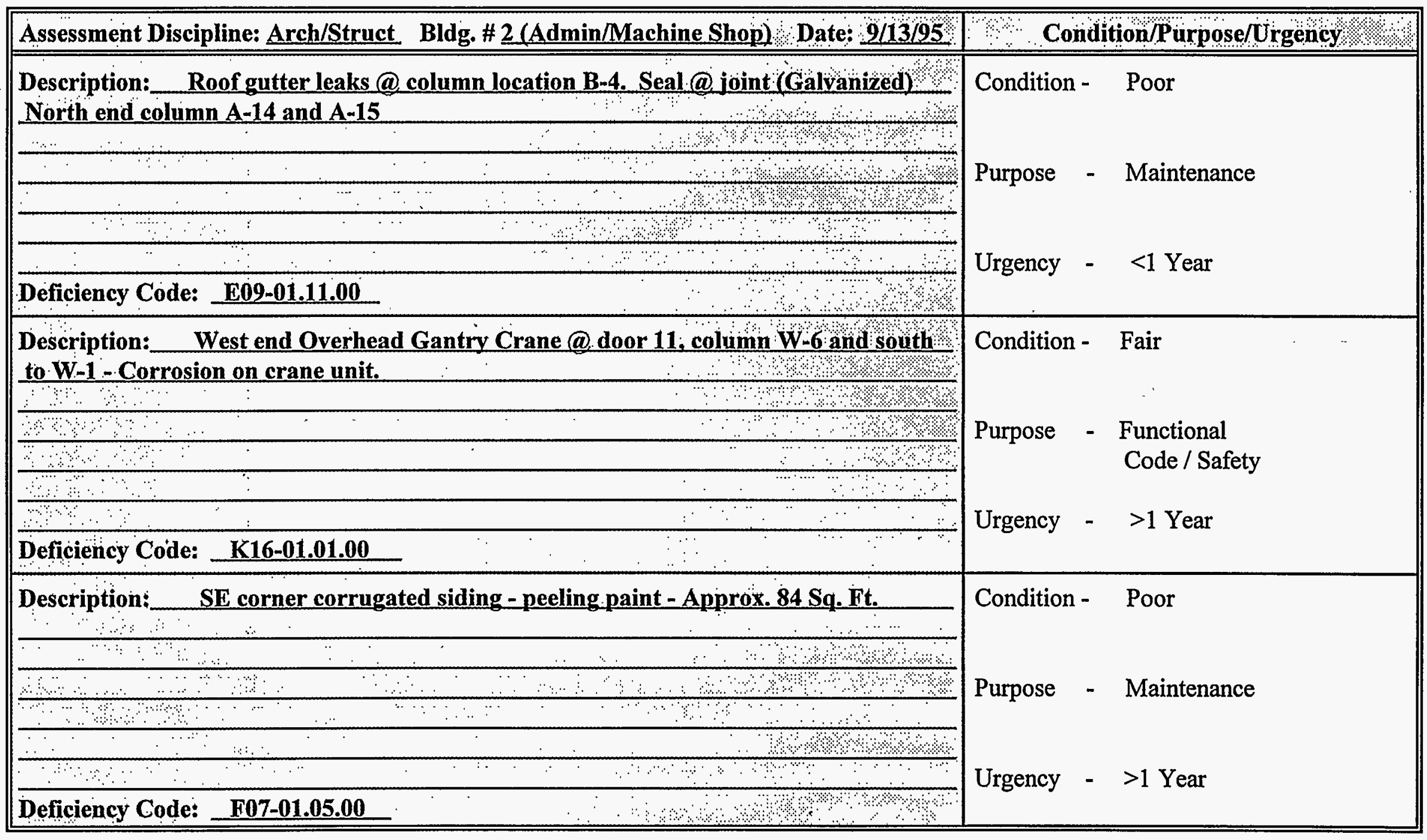




\section{Oxnard Condition Assessment Survey Field Report}

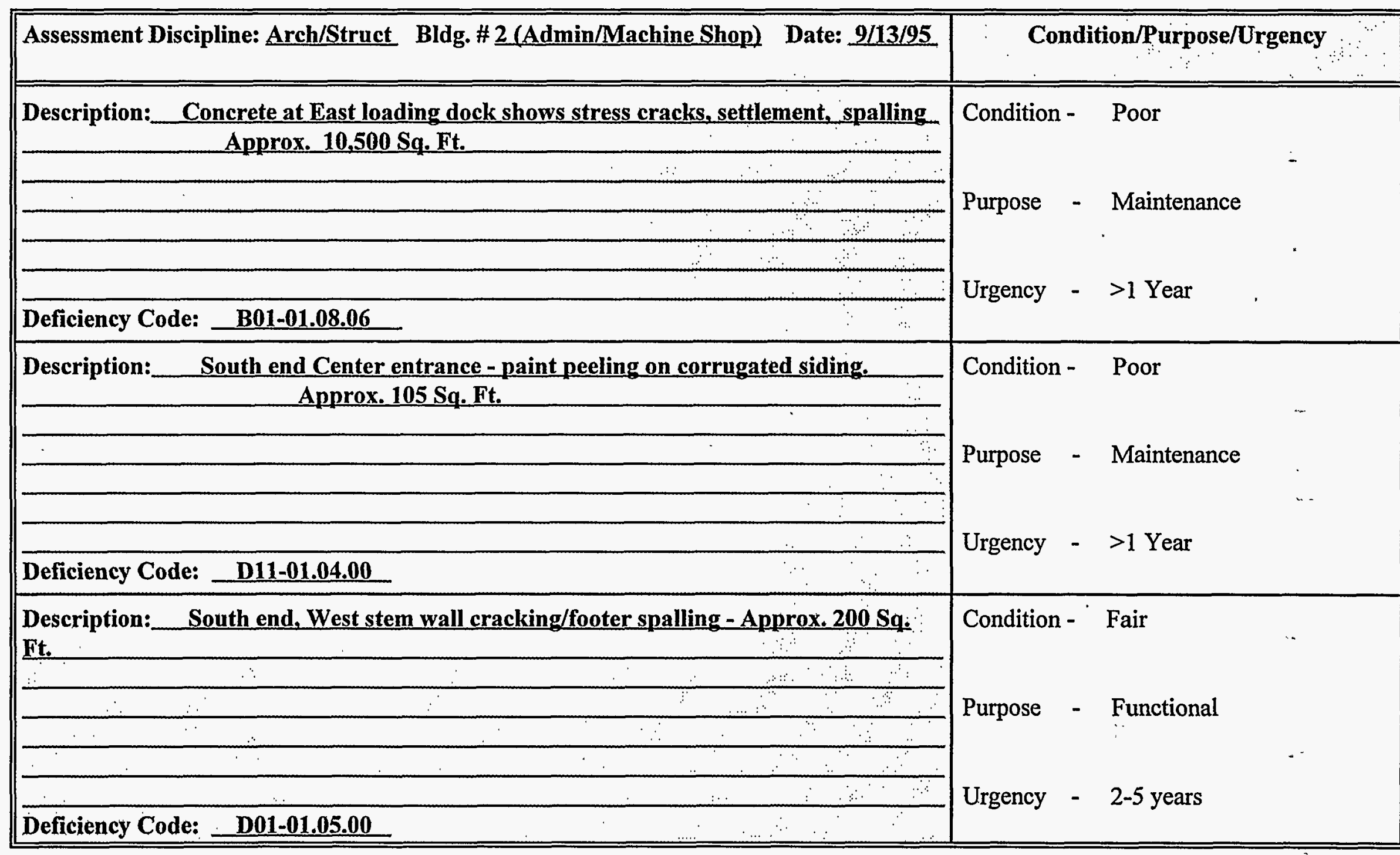




\section{Oxnard Condition Assessment Survey Field Report}

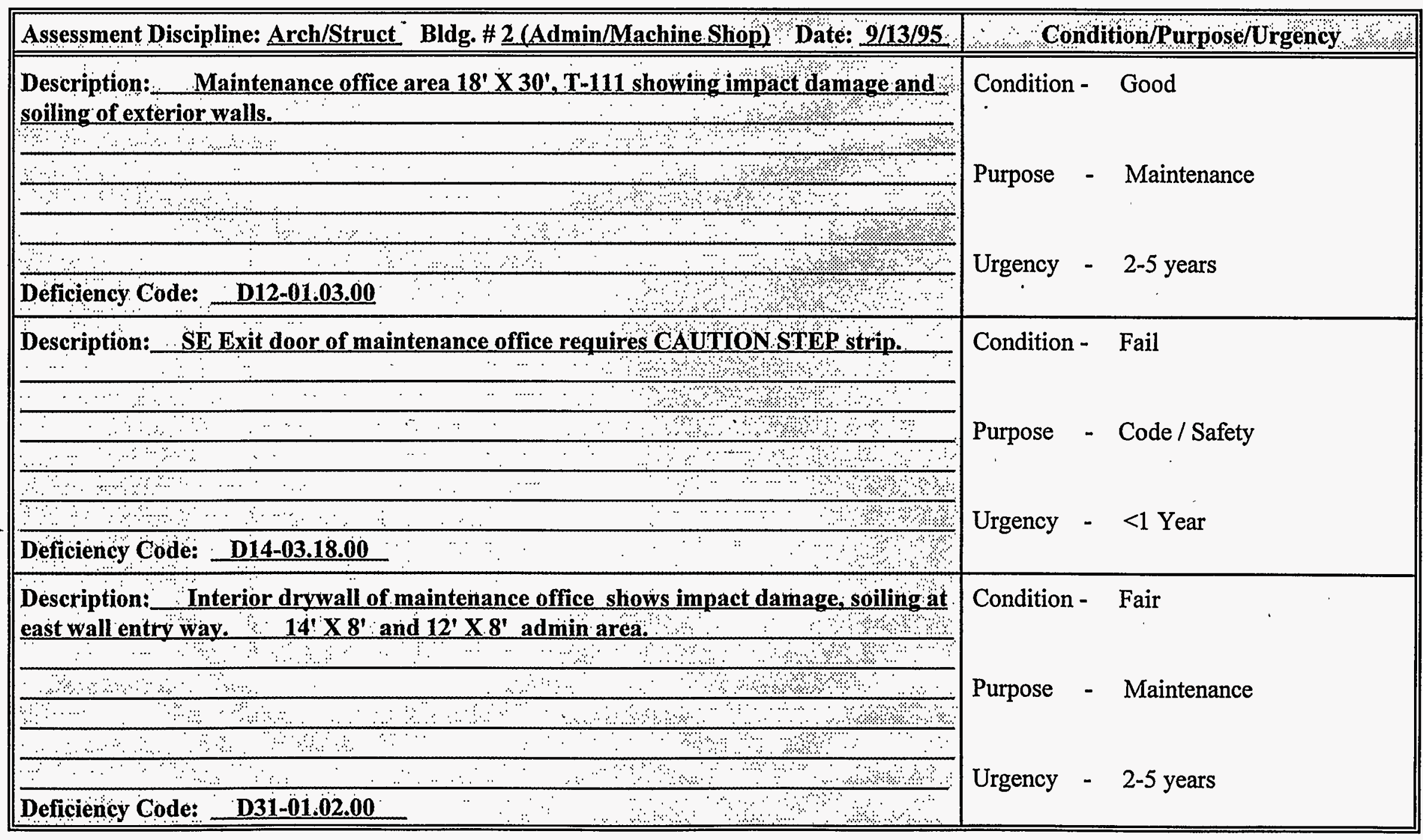




\section{Oxnard Condition Assessment Survey Field Report}

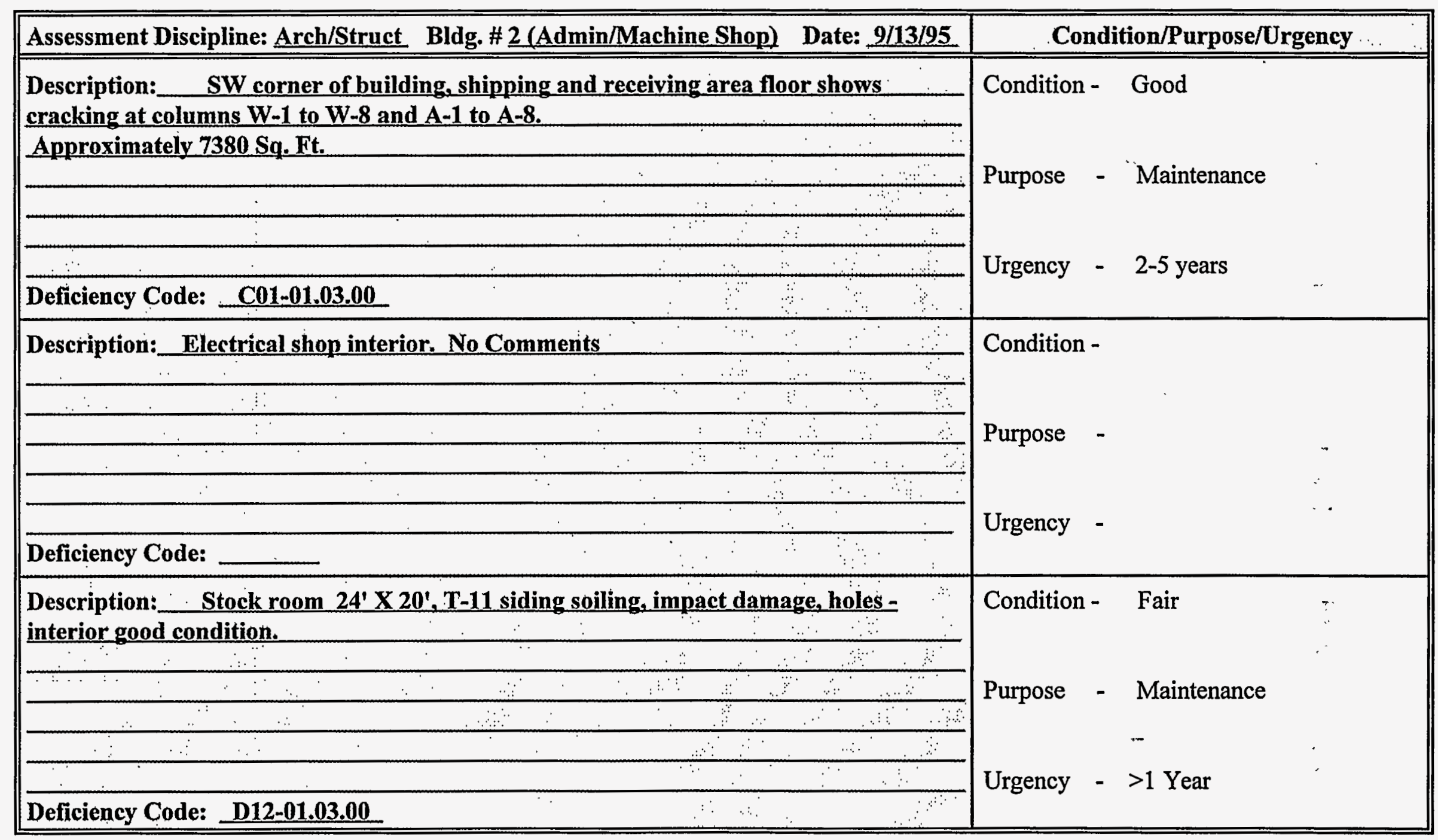




\section{Oxnard Condition Assessment Survey Field Report}

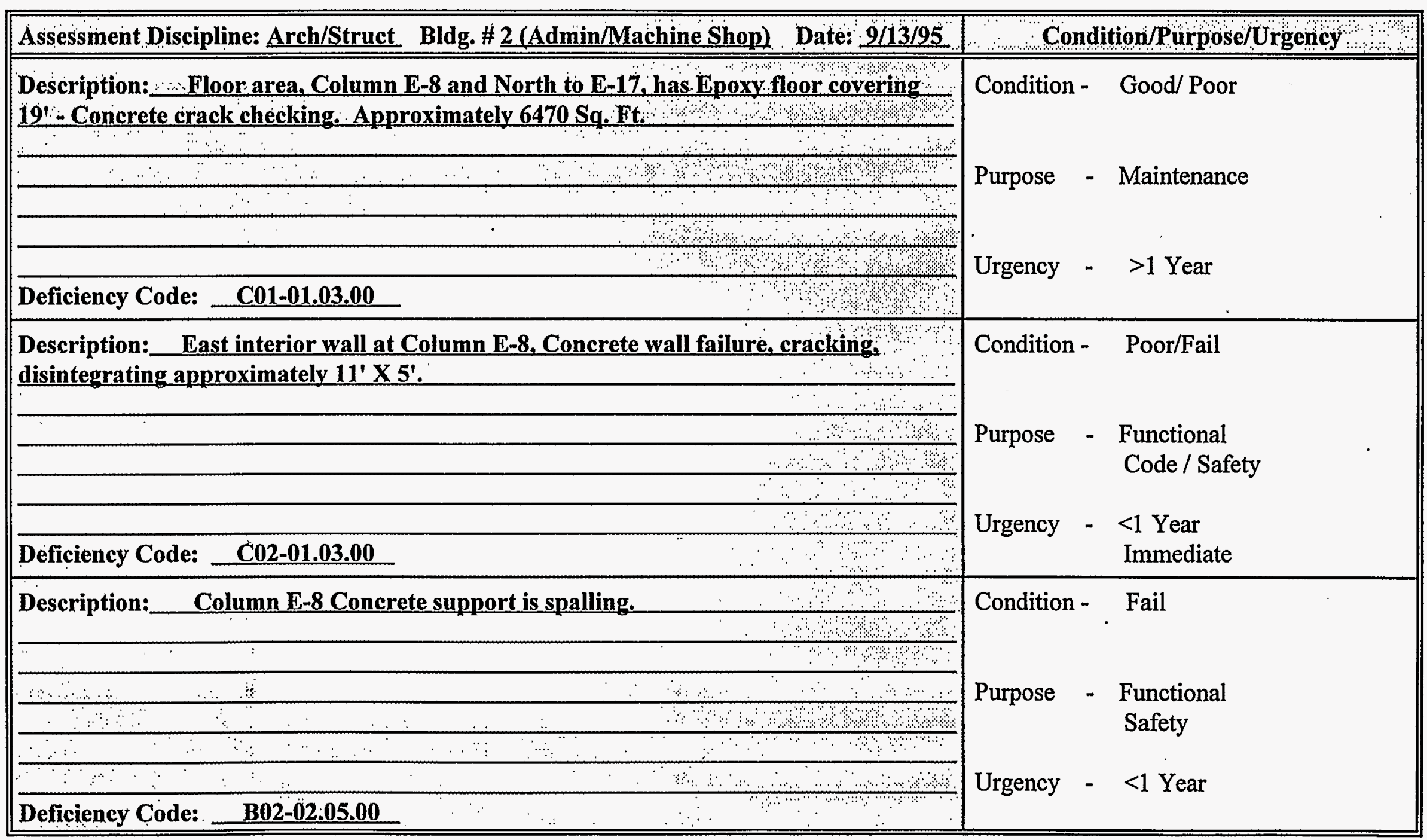




\section{Oxnard Condition Assessment Survey Field Report}

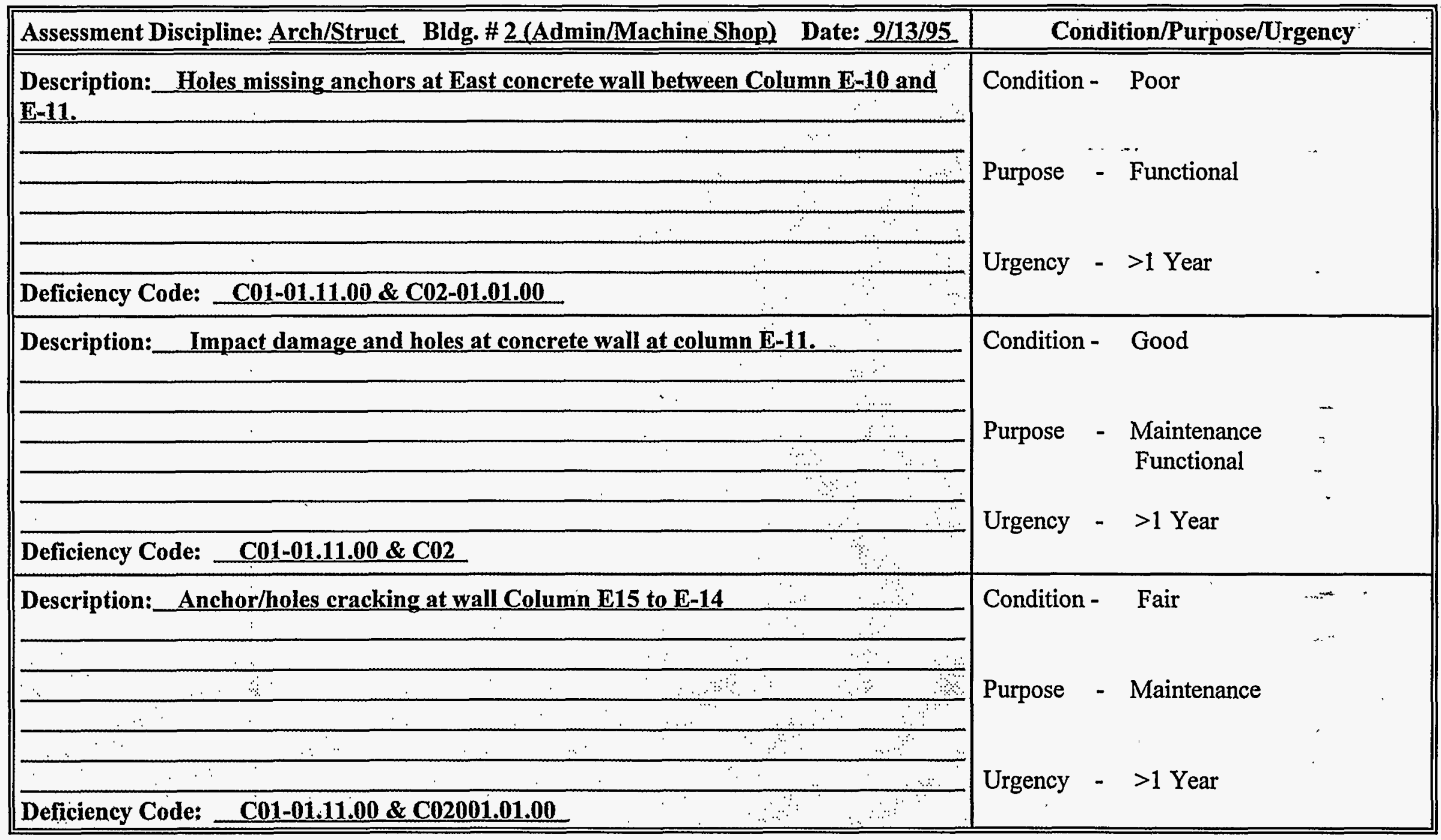




\section{Oxnard Condition Assessment Survey Field Report}

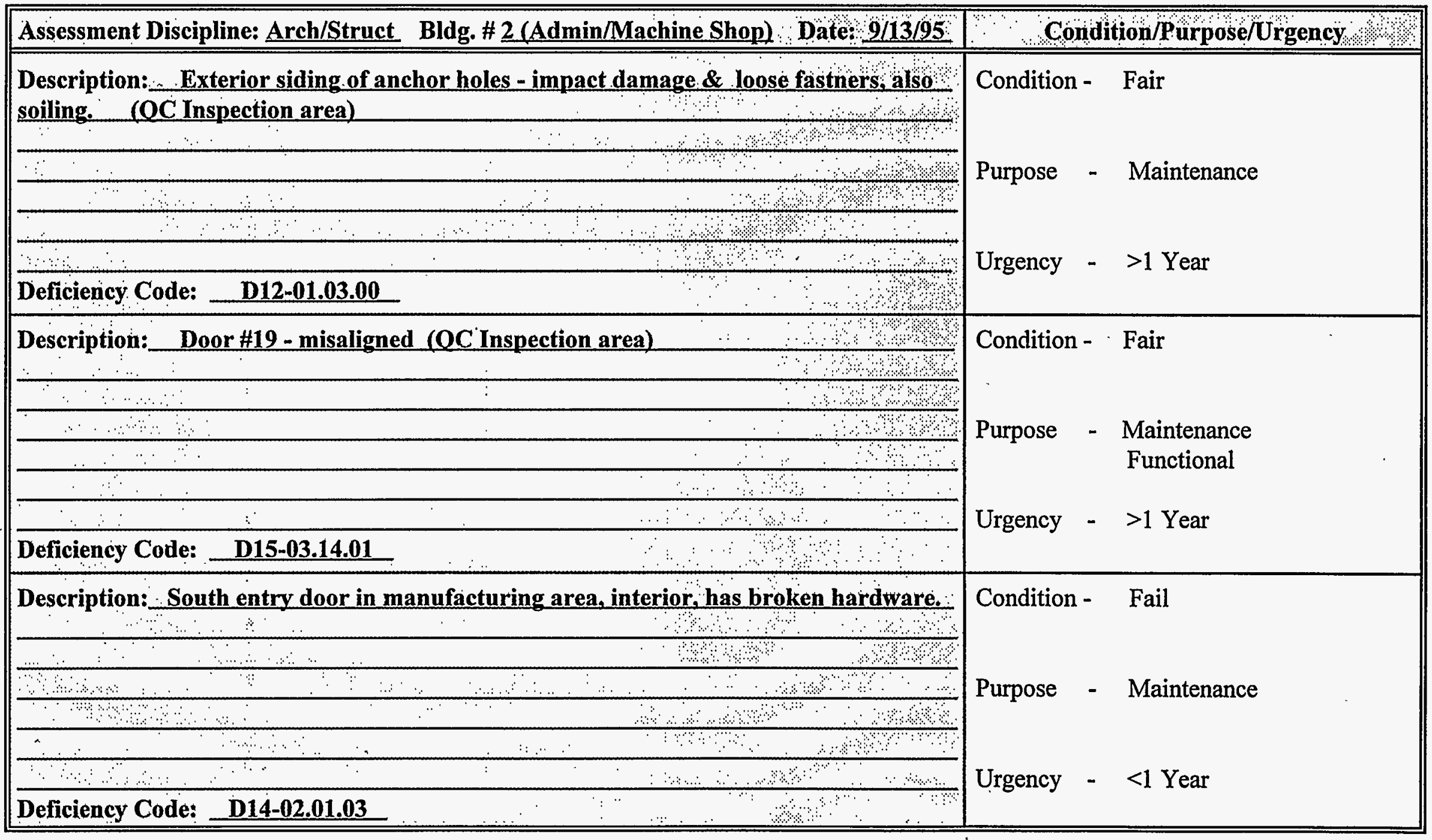




\section{Oxnard Condition Assessment Survey Field Report}

\begin{tabular}{|c|c|c|c|}
\hline Assessment Discipline: Arch/Struct Bldg. \# 2(Admin/Machine Shop) Date: $9 / 13 / 95$ & \multicolumn{3}{|c|}{ Condition/Purpose/Urgency } \\
\hline Description: Manufacturing office interior staining of walls. & \multirow{2}{*}{\multicolumn{2}{|c|}{$\begin{array}{l}\text { Condition - Good } \\
\text { Purpose - Maintenance }\end{array}$}} & \multirow{4}{*}{$\cdot$} \\
\hline 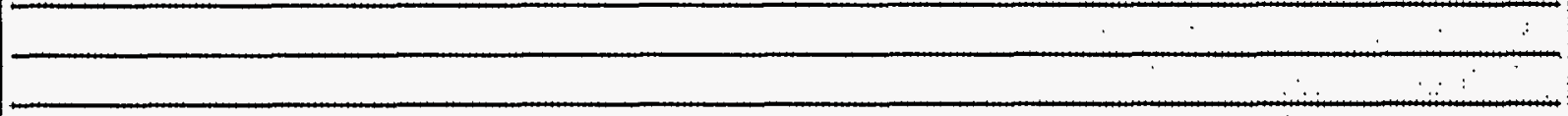 & & & \\
\hline$\therefore$ & \multirow{3}{*}{ Urgency } & & \\
\hline 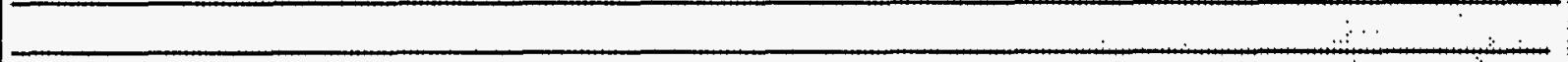 & & \multirow{2}{*}{$->1$ Year } & \\
\hline Deficiency Code: $\quad$ D29-01.03.00 & & & \\
\hline Description: NW corner ceiling area, Manufacturing office - leak damage & Condition - & Poor & \multirow{5}{*}{$\begin{array}{l}\cdots \\
\cdots \\
\cdots \\
\cdots\end{array}$} \\
\hline$\therefore$ & \multirow{4}{*}{$\begin{array}{l}\text { Purpose } \\
\text { Urgency }\end{array}$} & \multirow{2}{*}{ - Maintenance } & \\
\hline ... & & & \\
\hline$\therefore$ & & \multirow[t]{2}{*}{$-\quad>1$ Year } & \\
\hline Deficiency Code: & & & \\
\hline Description: ${ }_{-} \quad$ North office door (Manufacturing office) interior - Passage set missing. & \multirow[t]{2}{*}{ Condition - } & Fail & \multirow{2}{*}{$\ldots$} \\
\hline $\begin{array}{lllll} & & & \vdots \\
\end{array}$ & & & \\
\hline$\therefore \therefore \cdot$ & \multirow{4}{*}{$\begin{array}{l}\text { Purpose } \\
\text { Urgency }\end{array}$} & \multirow{2}{*}{$\begin{array}{l}\text { - Maintenance } \\
\text { Functional }\end{array}$} & \multirow{3}{*}{. } \\
\hline$\frac{\cdots}{3}$ & & & \\
\hline 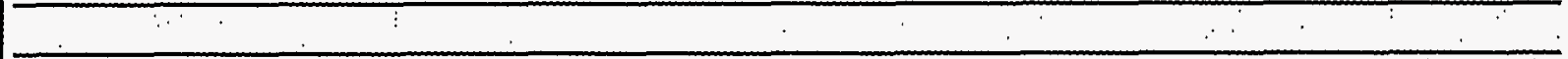 & & \multirow{2}{*}{$-\quad<1$ Year } & \\
\hline Deficiency Code: $\quad$ D14-01.01.03 & & & * \\
\hline
\end{tabular}




\section{Oxnard Condition Assessment Survey Field Report}

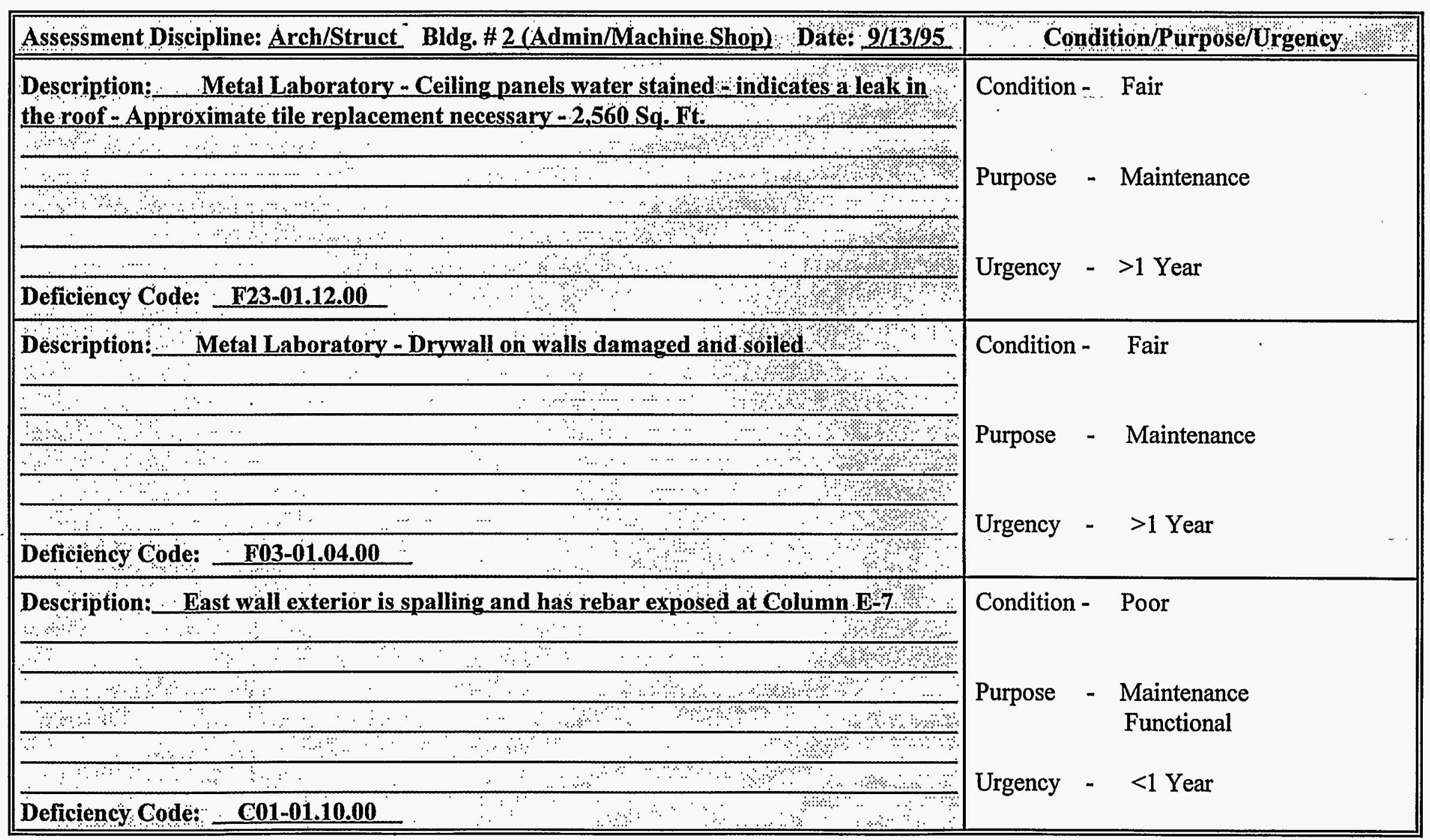




\section{Oxnard Condition Assessment Survey Field Report}

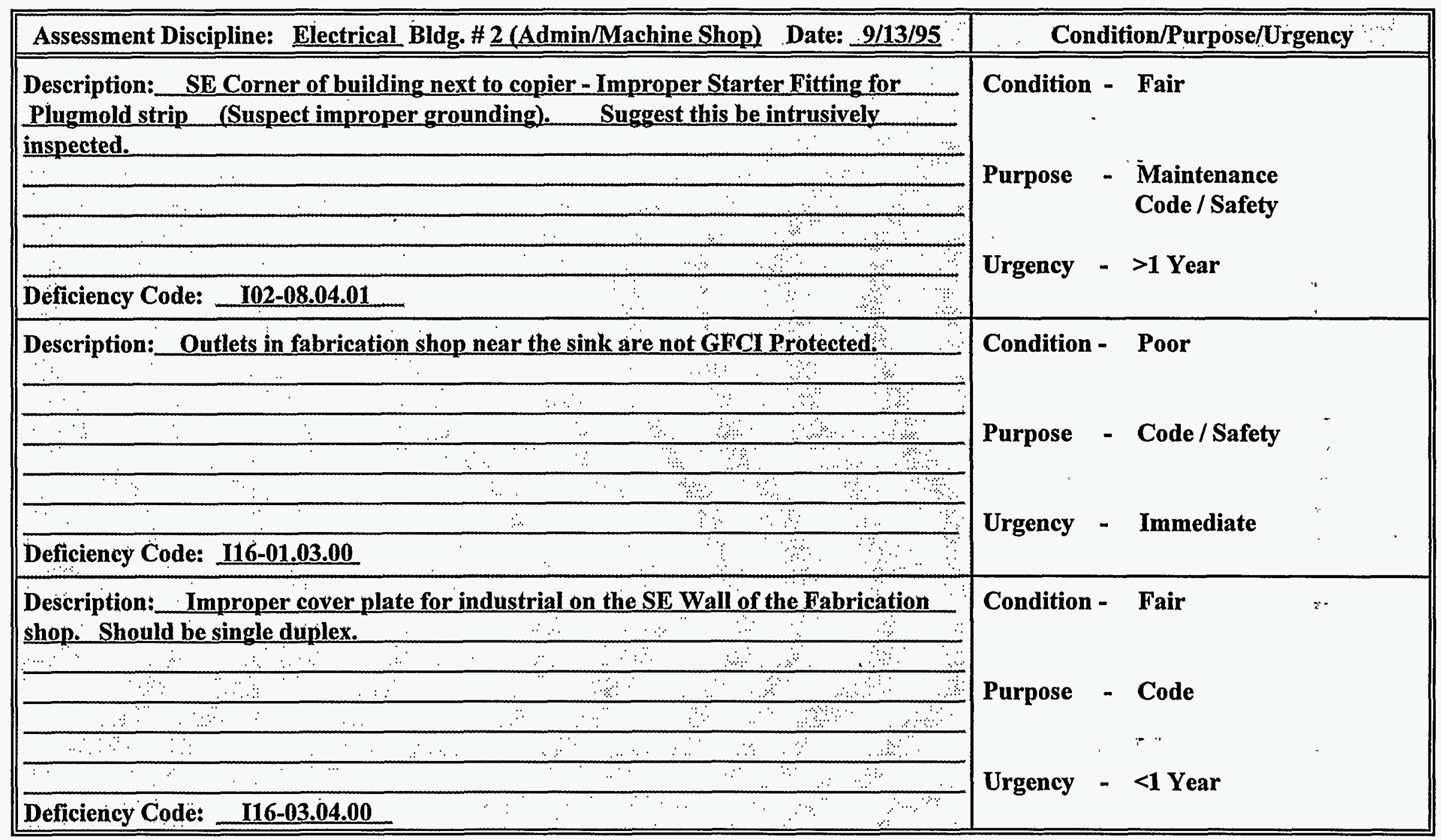




\section{Oxnard Condition Assessment Survey Field Report}

\begin{tabular}{|c|c|c|}
\hline Assesssment Discipline: Electrical Bldg. \# 2 (Admin/Machine Shop) Date: 9/13/95. & Con & dition/Purpose/Urgency \\
\hline Description: Mop closet in men's locker room - Outlet near sink is not GFCI & \multirow{7}{*}{\multicolumn{2}{|c|}{$\begin{array}{ll}\text { Purpose } & \text { - Code / Safety } \\
& \cdot \\
\text { Urgency - Immediate }\end{array}$}} \\
\hline Protected & & \\
\hline $\begin{array}{l}3 \\
-\end{array}$ & & \\
\hline$\therefore \quad \therefore \quad \cdots \quad \cdots \quad \cdots$ & & \\
\hline$\therefore \quad-\quad 38 \div \quad 3$ & & \\
\hline$\therefore \because \cdots$ & & \\
\hline Deficiency Code: I16-01.03.00 & & \\
\hline Description: On beam in shower area - electrical pipe with exposed wires hanging & \multirow{4}{*}{\multicolumn{2}{|c|}{$\begin{array}{l}\text { Condition - Poor } \\
\text { Purpose - Code / Safety }\end{array}$}} \\
\hline$\because \because \cdots$ & & \\
\hline$\because ;: \therefore$ & & \\
\hline ?. & & \\
\hline 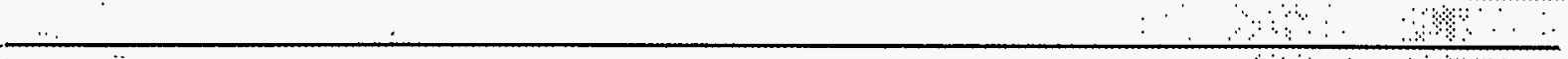 & \multirow[t]{2}{*}{ Urgency - } & \multirow[t]{2}{*}{ Immediate } \\
\hline Deficiency Code: 102-02.02.01 & & \\
\hline $\begin{array}{l}\text { Description: A-8/11 between Columns A-10 and A-11 offices - Emergency battery } \\
\text { pack for flood light is not working. }\end{array}$ & \multirow{2}{*}{\multicolumn{2}{|c|}{ Condition - . Poor }} \\
\hline (n) $100 \%$ & & \\
\hline$\because \quad \because \quad \%$ & \multirow{5}{*}{$\begin{array}{l}\text { Purpose } \\
\text { Urgency }\end{array}$} & \multirow{3}{*}{ - Maintenance } \\
\hline in & & \\
\hline 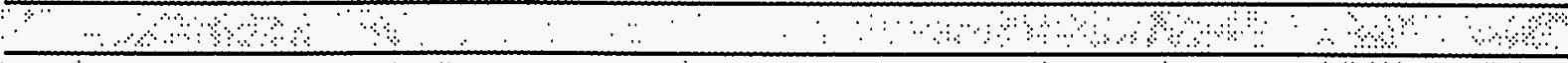 & & \\
\hline \% $: \quad n:$ & & Immediate \\
\hline Deficiency Code: I04-01.03.05 & & \\
\hline
\end{tabular}




\section{Oxnard Condition Assessment Survey Field Report}

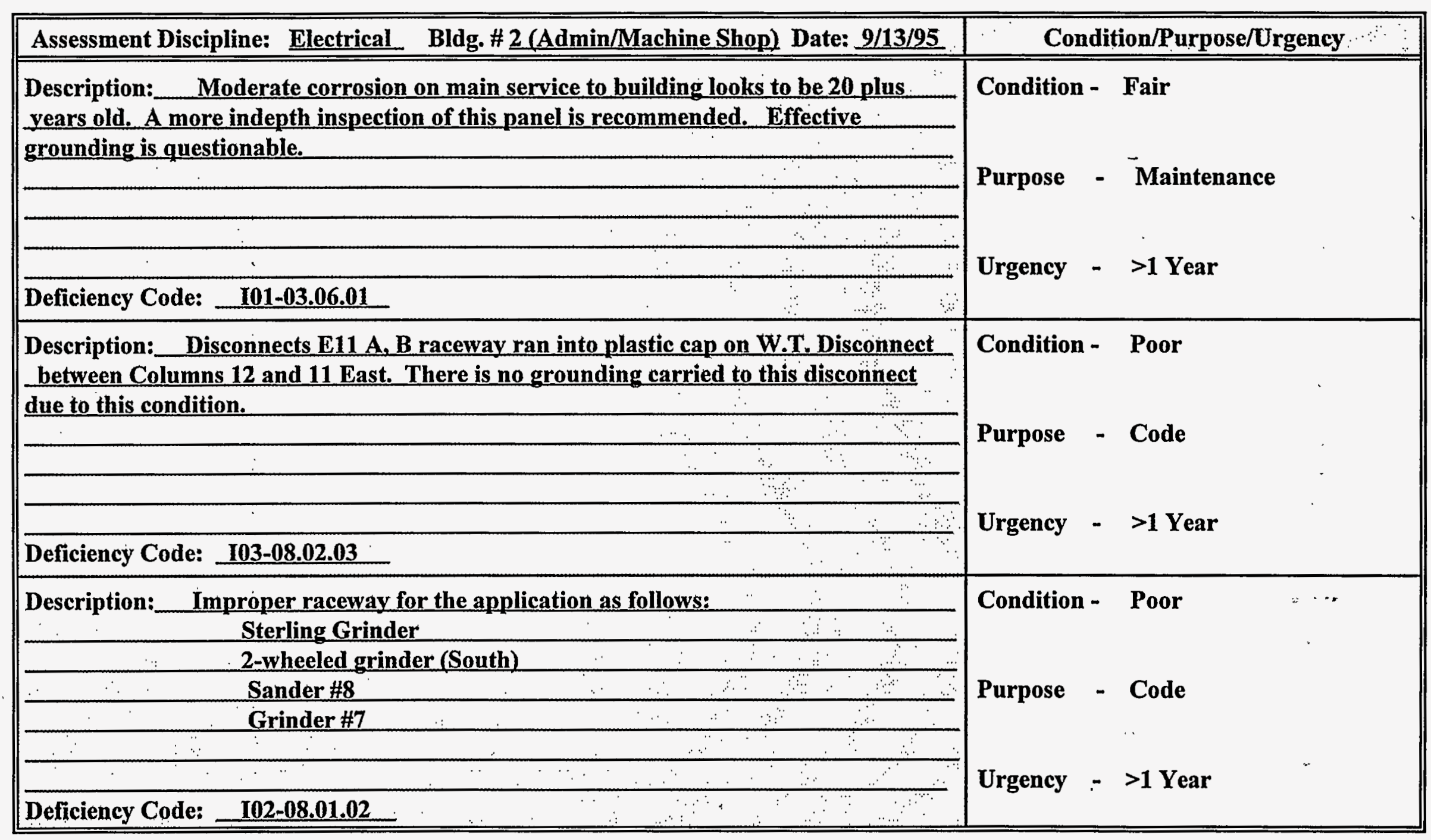




\section{Oxnard Condition Assessment Survey Field Report}

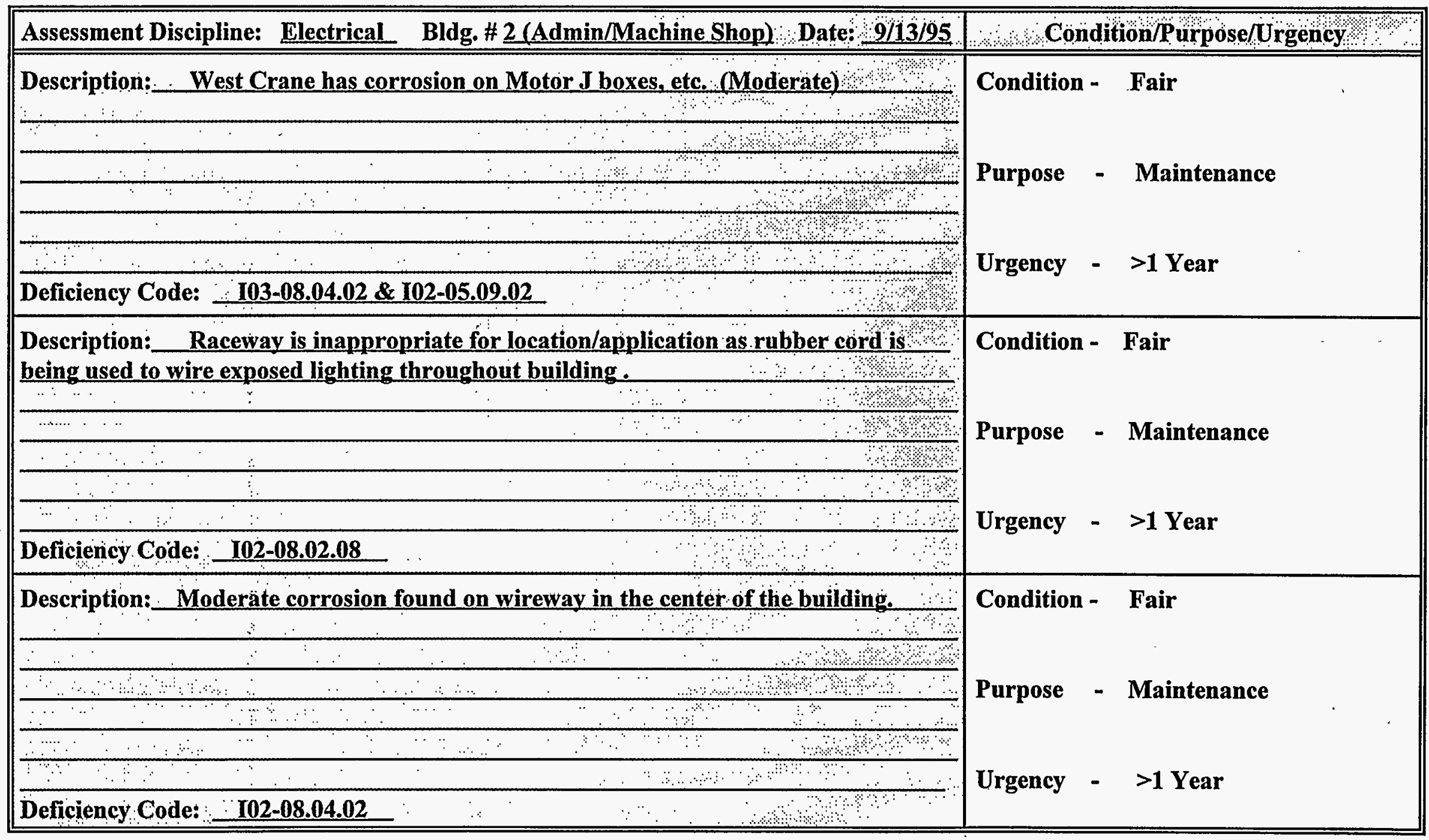




\section{Oxnard Condition Assessment Survey Field Report}

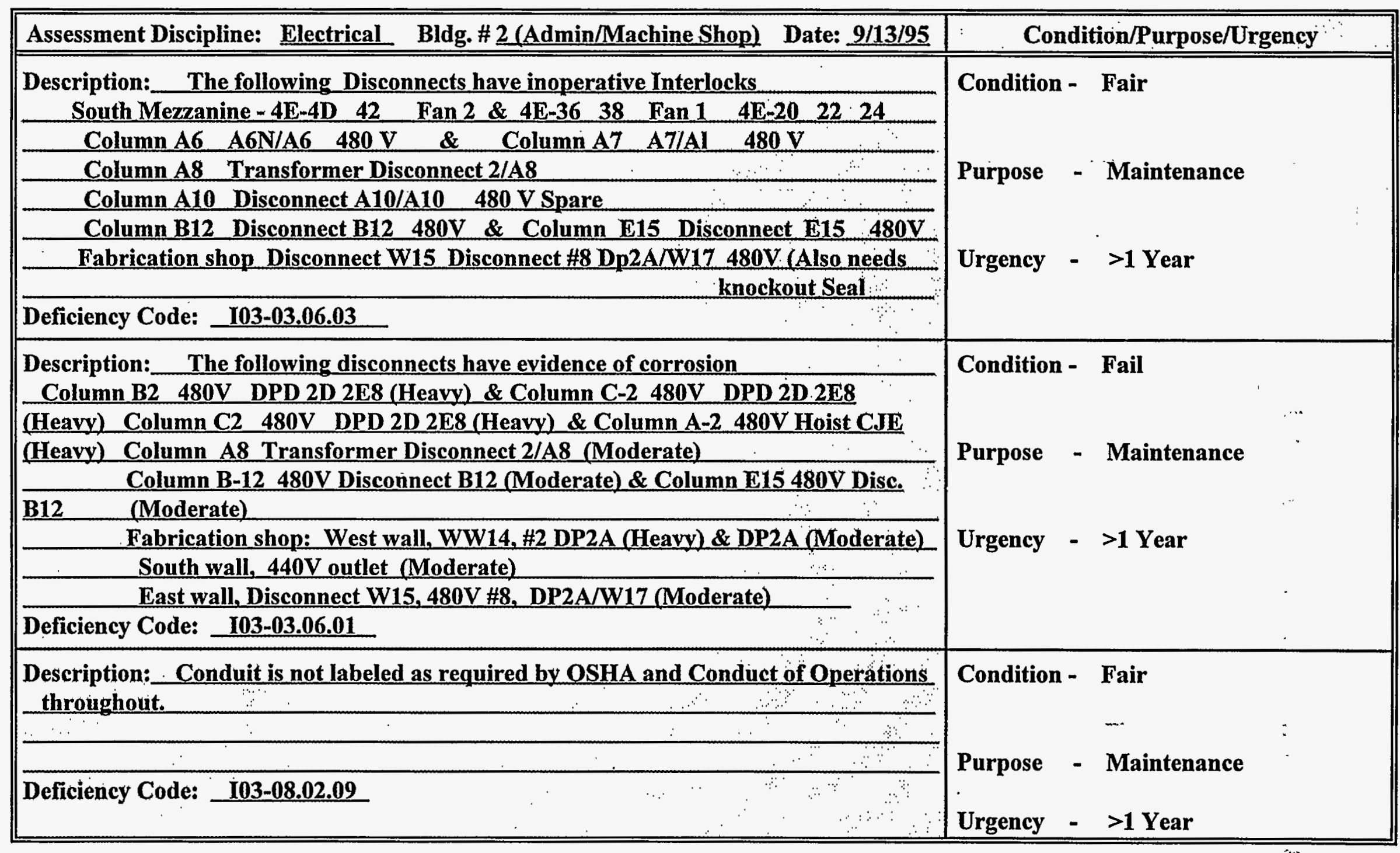




\section{Oxnard Condition Assessment Survey Field Report}

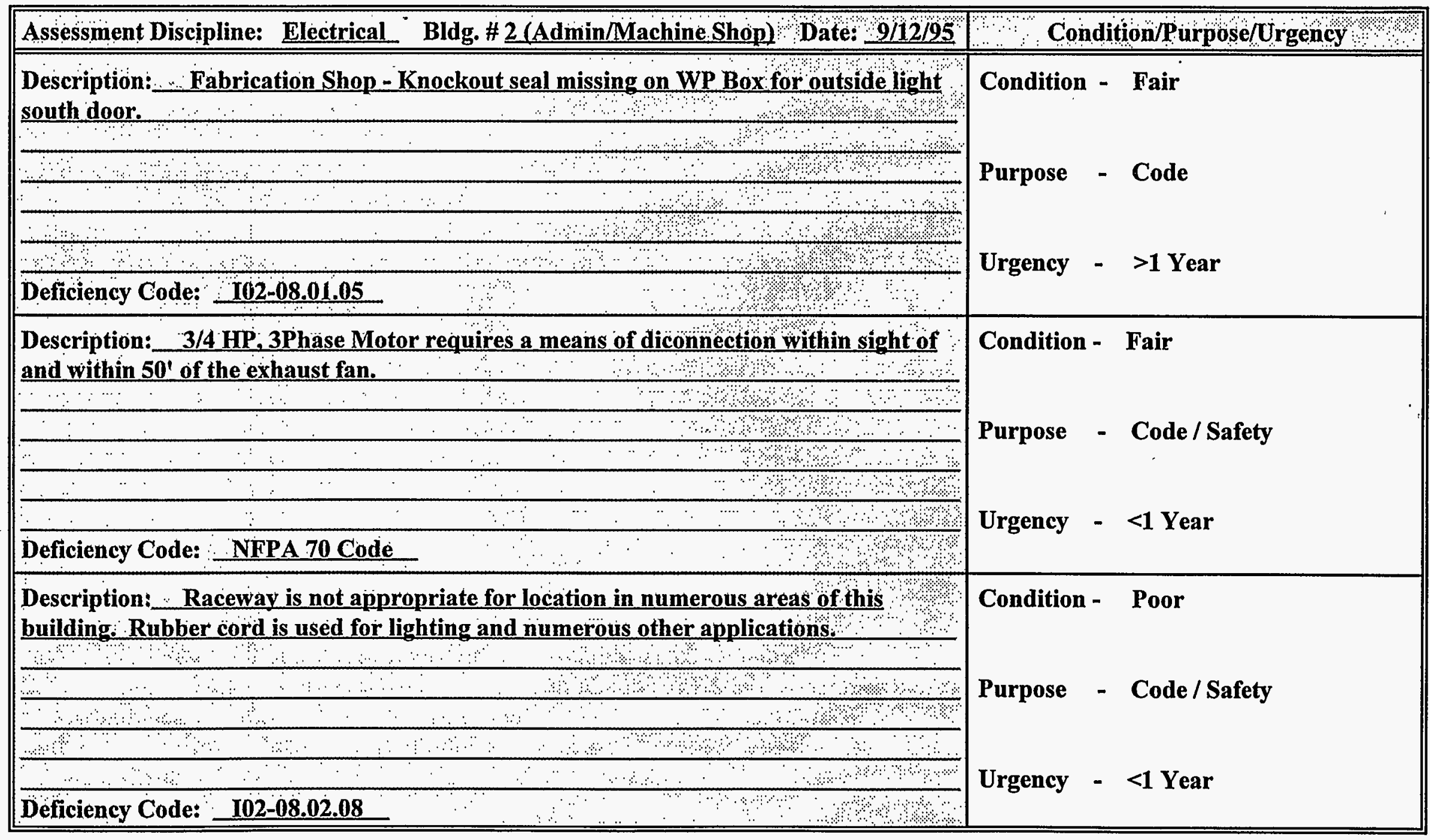




\section{Oxnard Condition Assessment Survey Field Report}

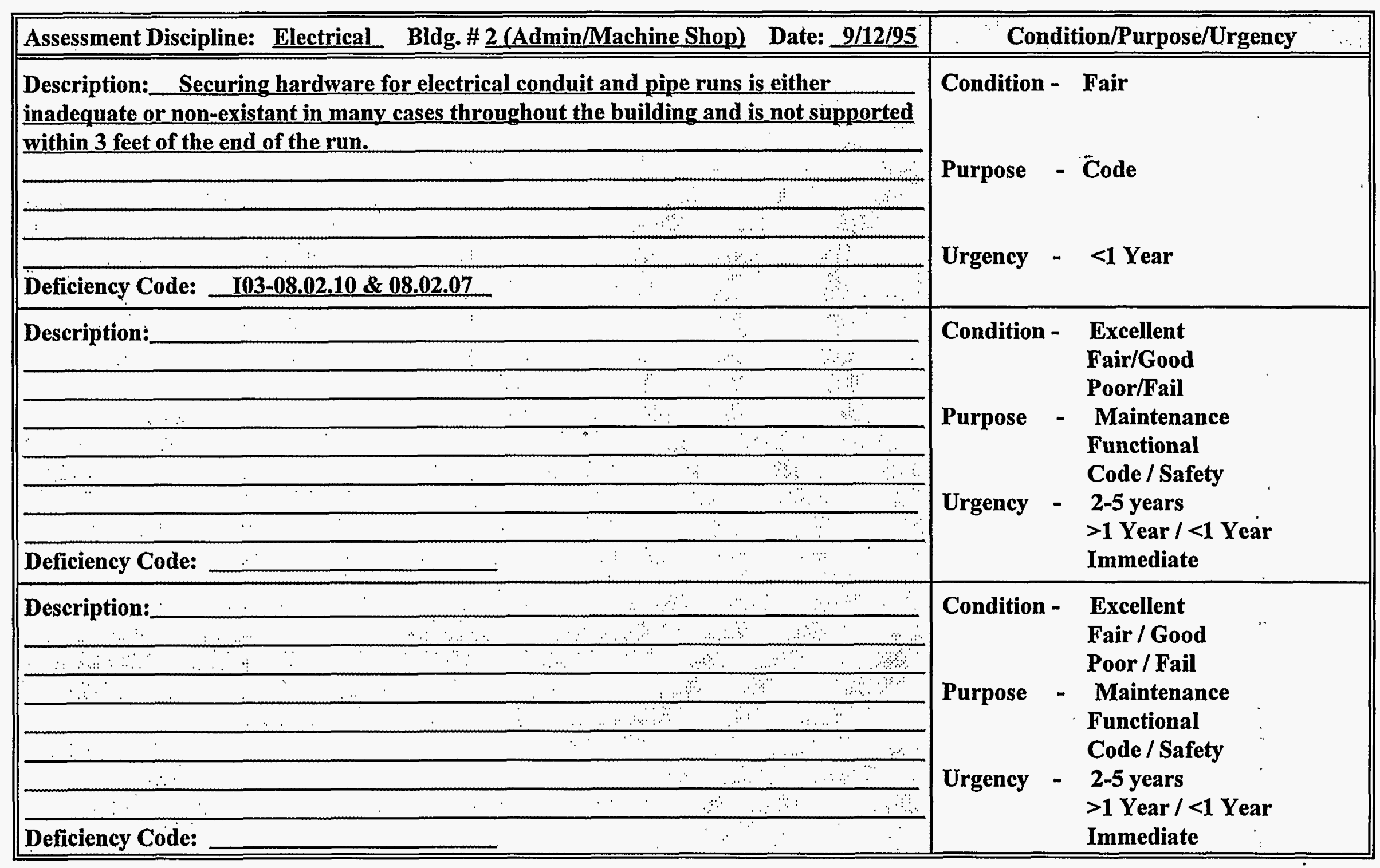




\section{Oxnard Condition Assessment Survey Field Report}

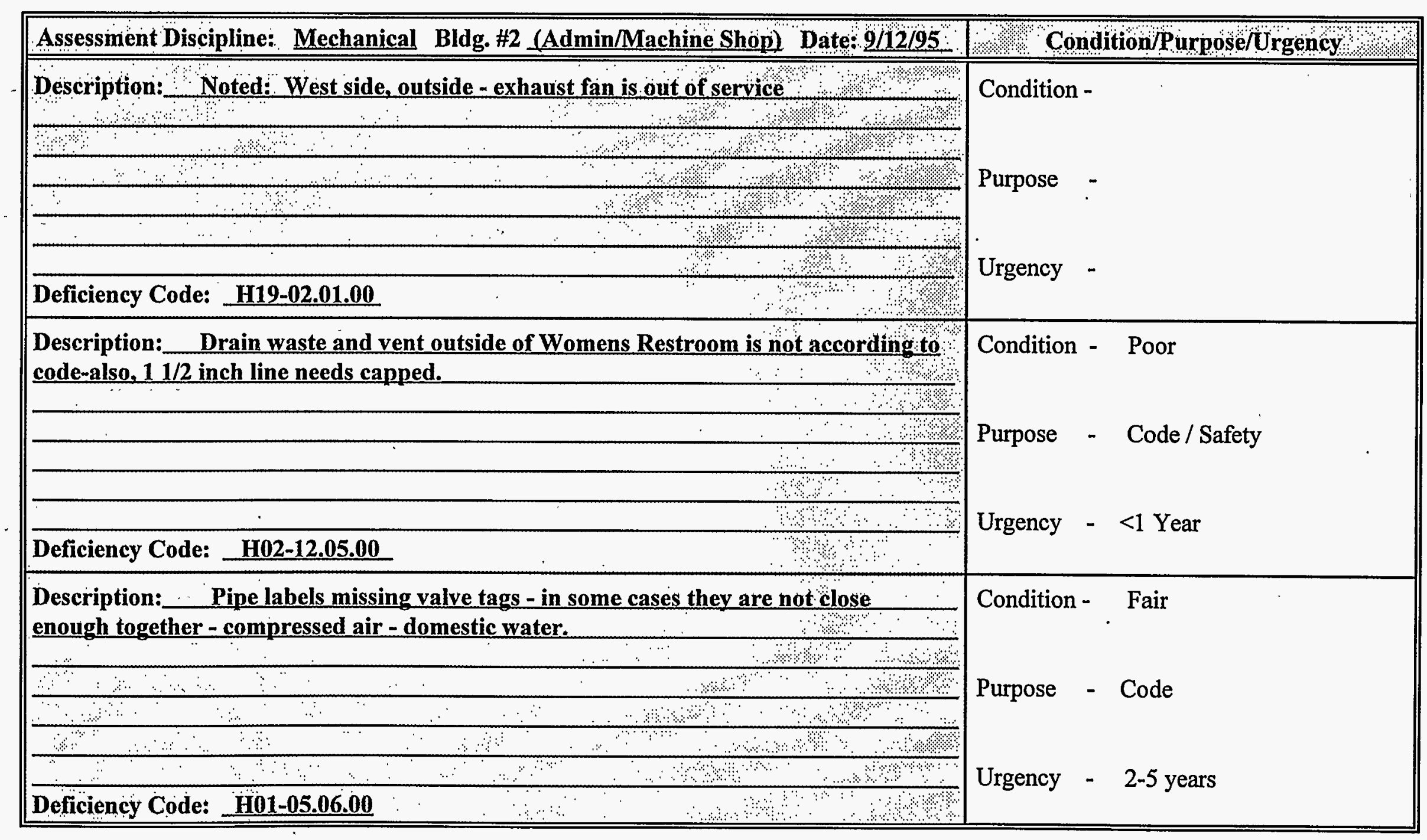




\section{Oxnard Condition Assessment Survey Field Report}

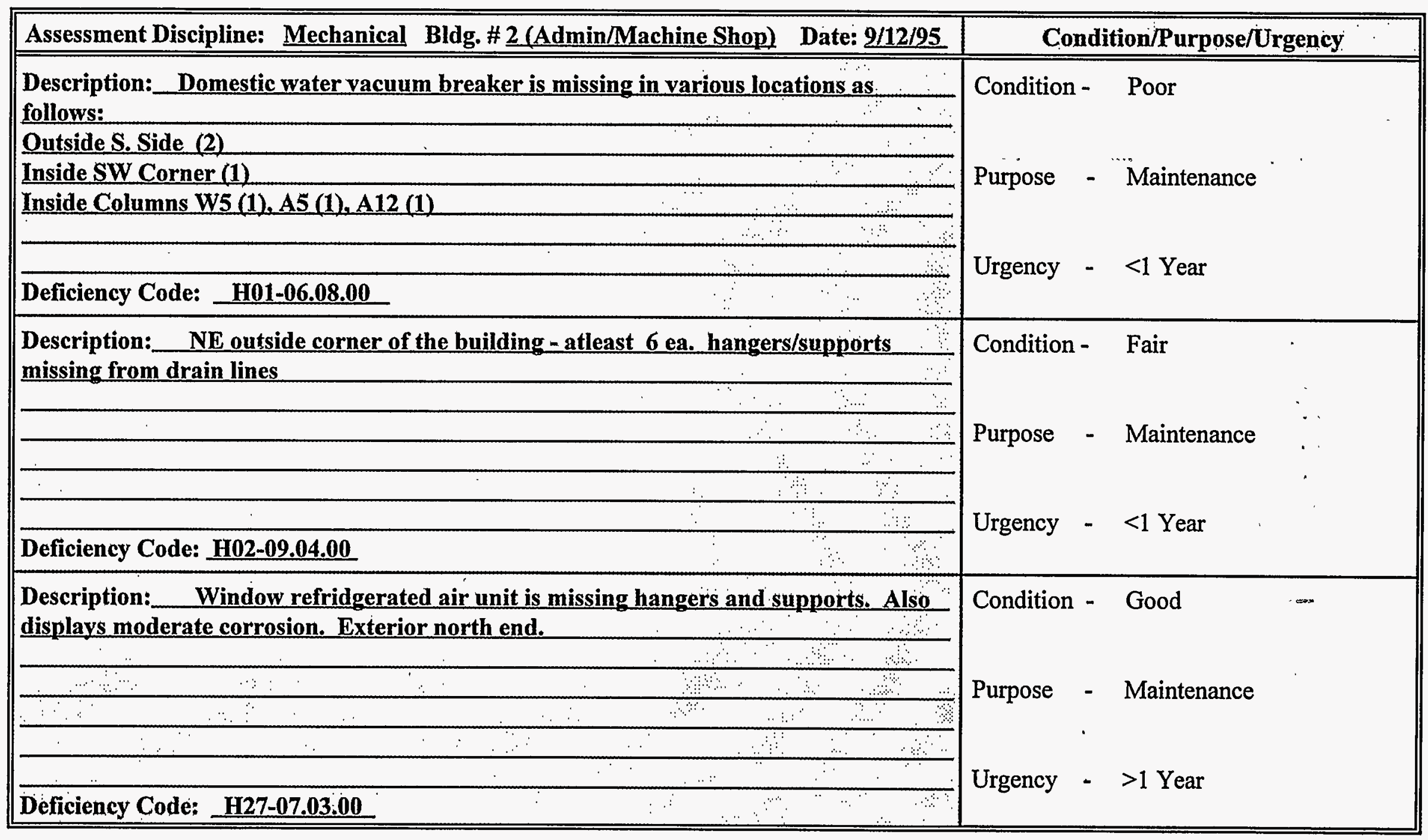




\section{Oxnard Condition Assessment Survey Field Report}

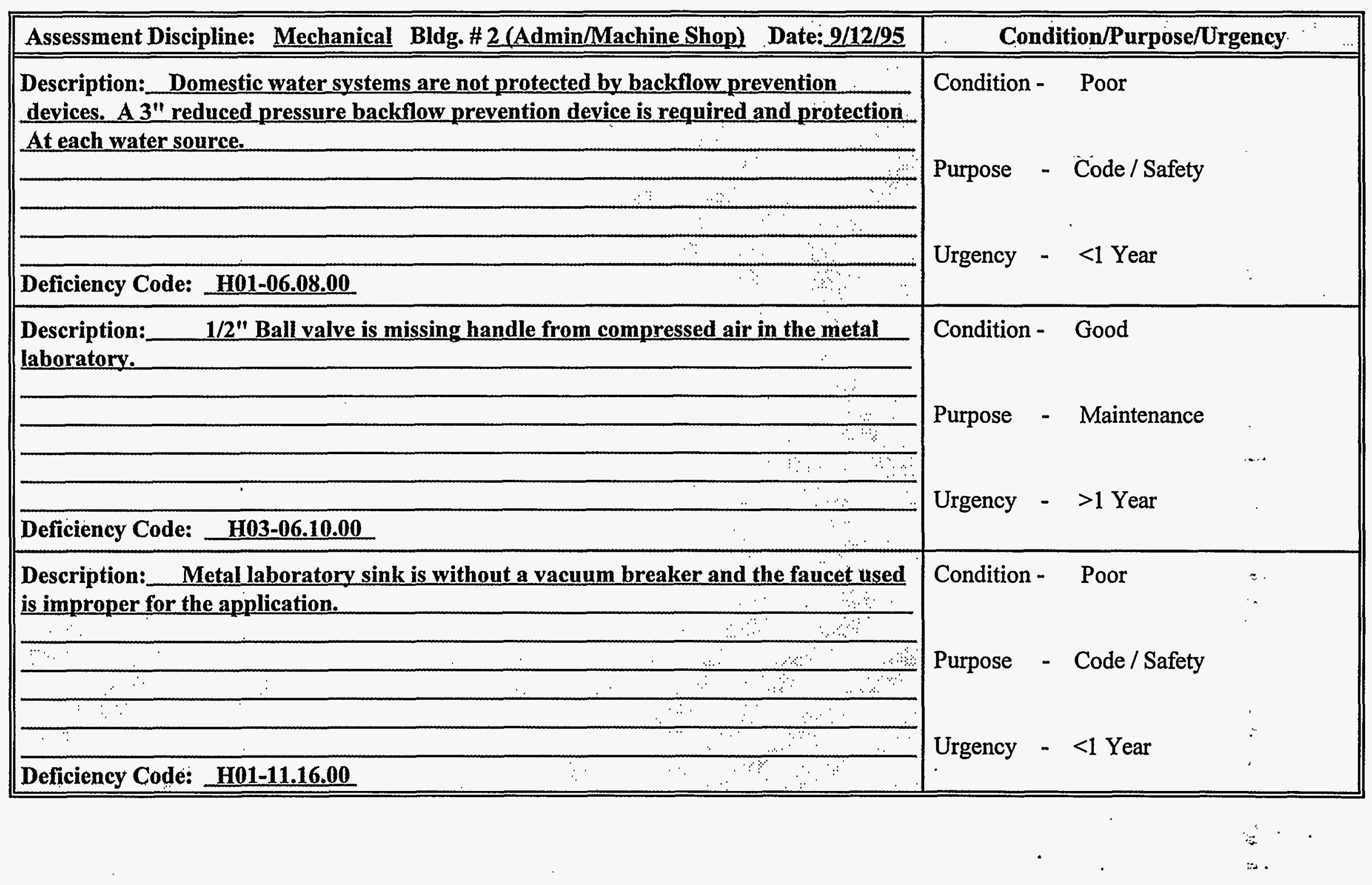




\section{Oxnard Condition Assessment Survey Field Report}

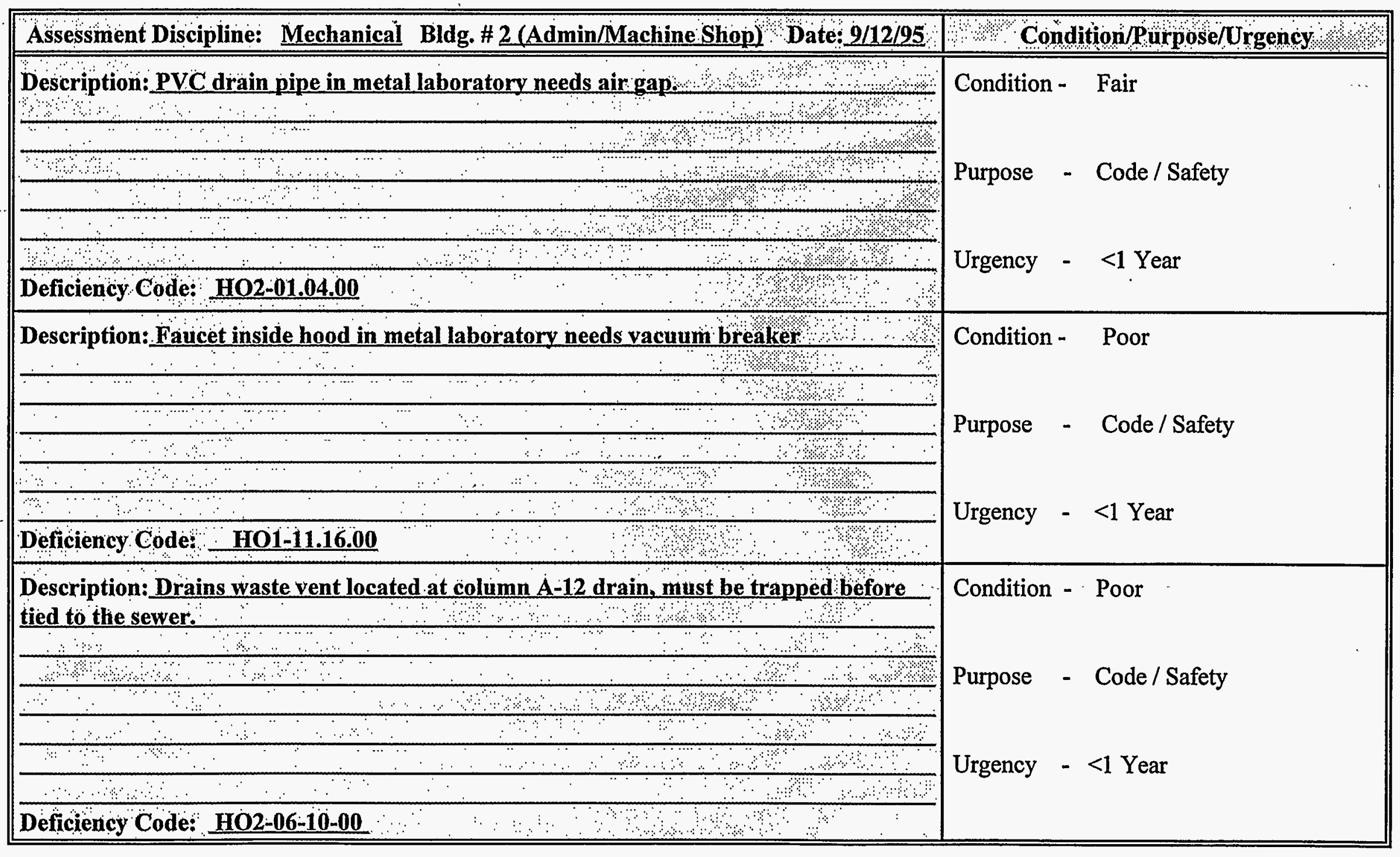




\section{Oxnard Condition Assessment Survey Field Report}

\begin{tabular}{|c|c|c|c|}
\hline Assessment Discipline: Mechanical Bldg. \# 2 (Admin/Machine Shop) & \multicolumn{3}{|c|}{ Condition/Purpose/Urgency } \\
\hline Description: Handle on domestic water $1 / 2$ " ball valve is missing in Column A-12 & \multirow{2}{*}{\multicolumn{2}{|c|}{$\begin{array}{l}\text { Condition - Fair } \\
\text { Purpose - Maintenance }\end{array}$}} & \multirow{4}{*}{$\therefore$} \\
\hline & & & \\
\hline 5 & \multirow{2}{*}{\multicolumn{2}{|c|}{ Urgency - $<1$ Year }} & \\
\hline Deficiency Code: H01-06.11.00 & & & \\
\hline $\begin{array}{l}\text { Description: } 11 / 2 \text { " black natural gas pipe in the mid east side, is missing the } \\
\text { escutcheon at the wall penetration. }\end{array}$ & \multicolumn{3}{|c|}{ Condition - Fair } \\
\hline$\because \therefore \quad-\quad$ & \multirow{2}{*}{\multicolumn{2}{|c|}{ Purpose - Maintenance }} & . \\
\hline$\because \quad \because \quad$ क्ष & & & \\
\hline Deficiency Code: HO1-04.05.00 & \multicolumn{2}{|c|}{ Urgency $-<1$ Year } & \\
\hline Description: $3 / 4^{\prime \prime}$ brass valve handle is broken on compressed air unit. & \multirow{2}{*}{\multicolumn{2}{|c|}{ Condition - Fair }} & $\cdots$ \\
\hline & & & $\because$ \\
\hline $3 \quad-\quad+\quad-\quad$ & \multicolumn{2}{|c|}{ Purpose - Maintenance } & \\
\hline$\therefore \quad$ & \multirow{3}{*}{ Urgency } & & $\because$ \\
\hline 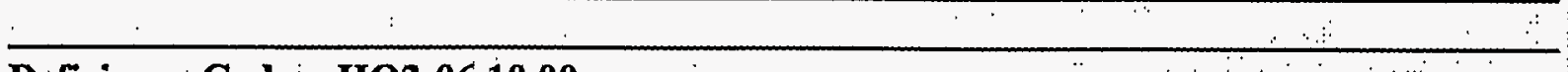 & & - $>1$ Year & \\
\hline Deficiency Code: HO3-06.10.00 & & & \\
\hline
\end{tabular}




\section{Oxnard Condition Assessment Survey Field Report}

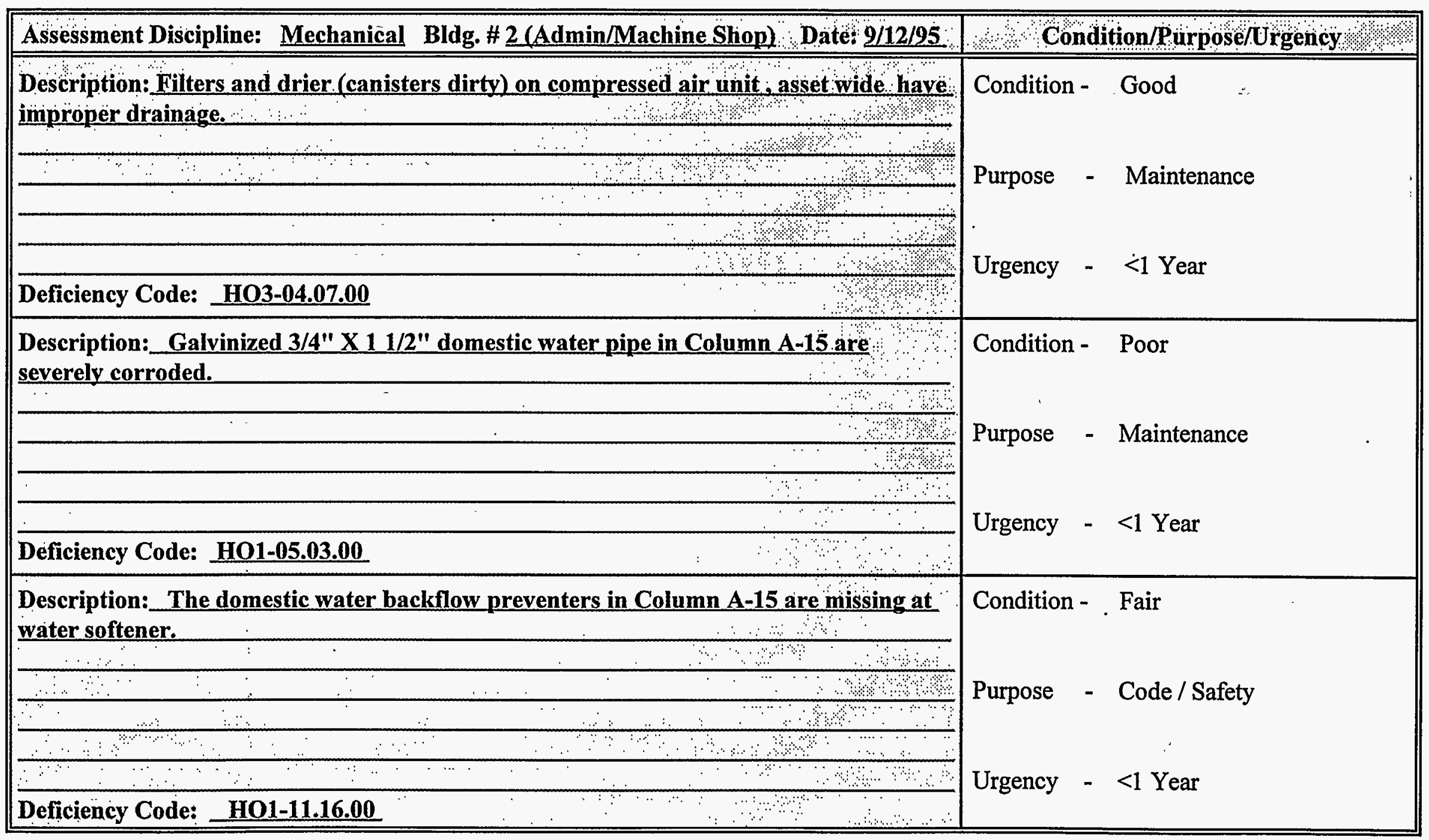




\section{Oxnard Condition Assessment Survey Field Report}

\begin{tabular}{|c|c|c|c|}
\hline Assessment Discipline: Mechanical Bldg. \# 2 (Admin/Machine Shop) Date: 9/13/95 & \multicolumn{3}{|c|}{ Condition/Purpose/Urgency } \\
\hline Description: Window refrigeration frame and fin damage near break room. & \multirow{2}{*}{\multicolumn{2}{|c|}{$\begin{array}{l}\text { Condition - Fair } \\
\text { Purpose - Maintenance }\end{array}$}} & \\
\hline & & & \\
\hline & \multirow{2}{*}{ Urgency } & \multirow{2}{*}{ - $>1$ Year } & \multirow{2}{*}{ 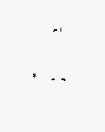 } \\
\hline Deficiency Code: H25/H26-0104.00 & & & \\
\hline Description: Back siphon possible in fabrication shop's domestic water/mop sink. & \multirow{4}{*}{\multicolumn{2}{|c|}{$\begin{array}{l}\text { Condition - Poor } \\
\text { Purpose - Code / Safety }\end{array}$}} & \multirow{6}{*}{$\begin{array}{l}. . \\
. . \\
. \\
-\end{array}$} \\
\hline$\therefore \cdots$ & & & \\
\hline$\because$ & & & \\
\hline ? & & & \\
\hline & \multirow{2}{*}{ Urgency } & \multirow{2}{*}{$-<1$ Year } & \\
\hline Deficiency Code: HO1-11.16.00 & & & \\
\hline Description: No escutcheon or p-trap (code) in mop sink in fabrication shop. & \multirow{2}{*}{\multicolumn{2}{|c|}{ Condition - Poor }} & $\cdots$ \\
\hline$\therefore$ & & & \multirow{5}{*}{$\therefore$} \\
\hline : & \multirow{4}{*}{$\begin{array}{l}\text { Purpose } \\
\text { Urgency }\end{array}$} & \multirow{2}{*}{ - Code / Safety } & \\
\hline 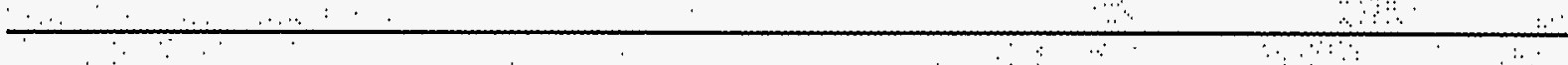 & & & \\
\hline & & : $<1$ Year & \\
\hline Deficiency Code: HO2-06.10.00 & & & \\
\hline
\end{tabular}




\section{Oxnard Condition Assessment Survey Field Report}

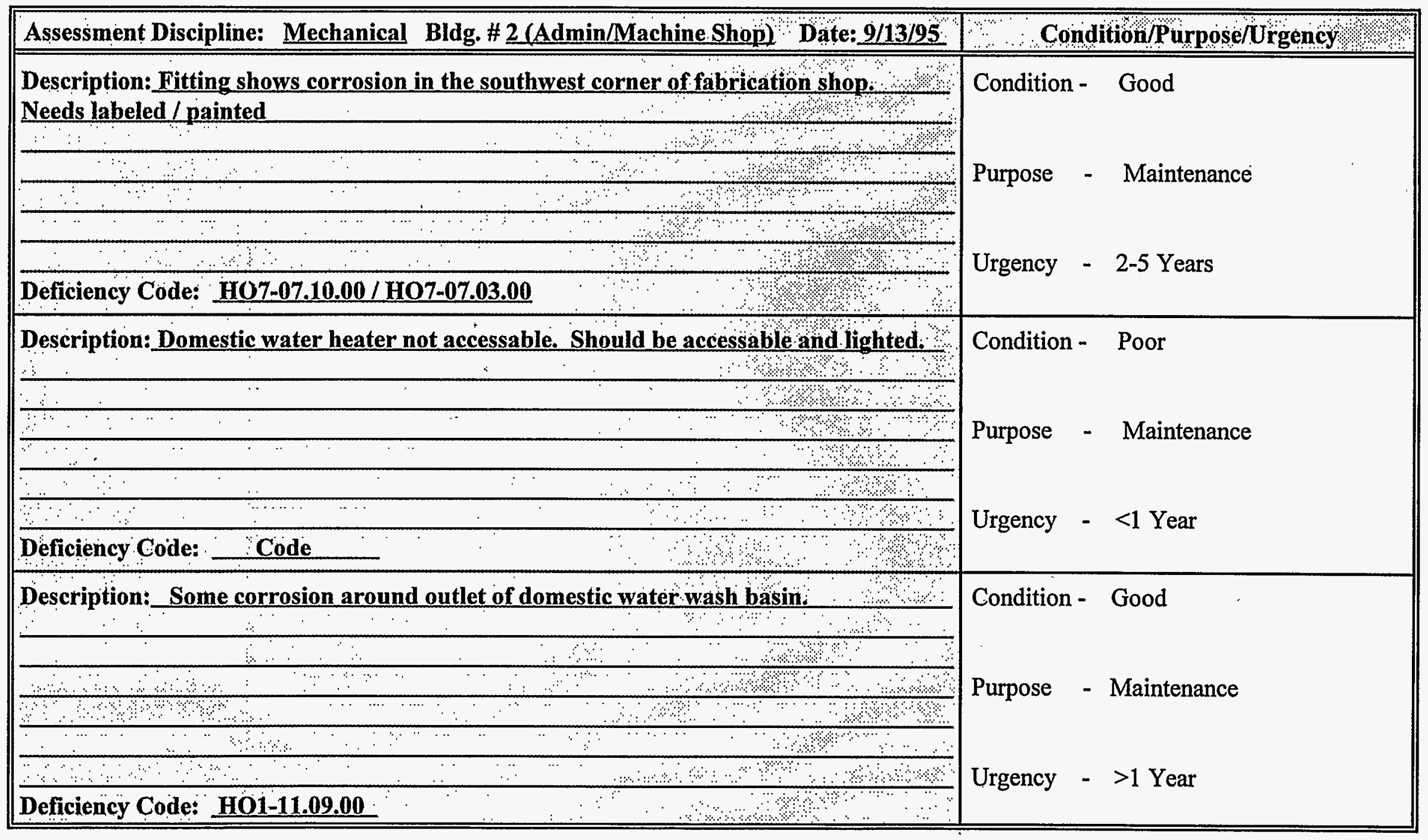




\section{Oxnard Condition Assessment Survey Field Report}

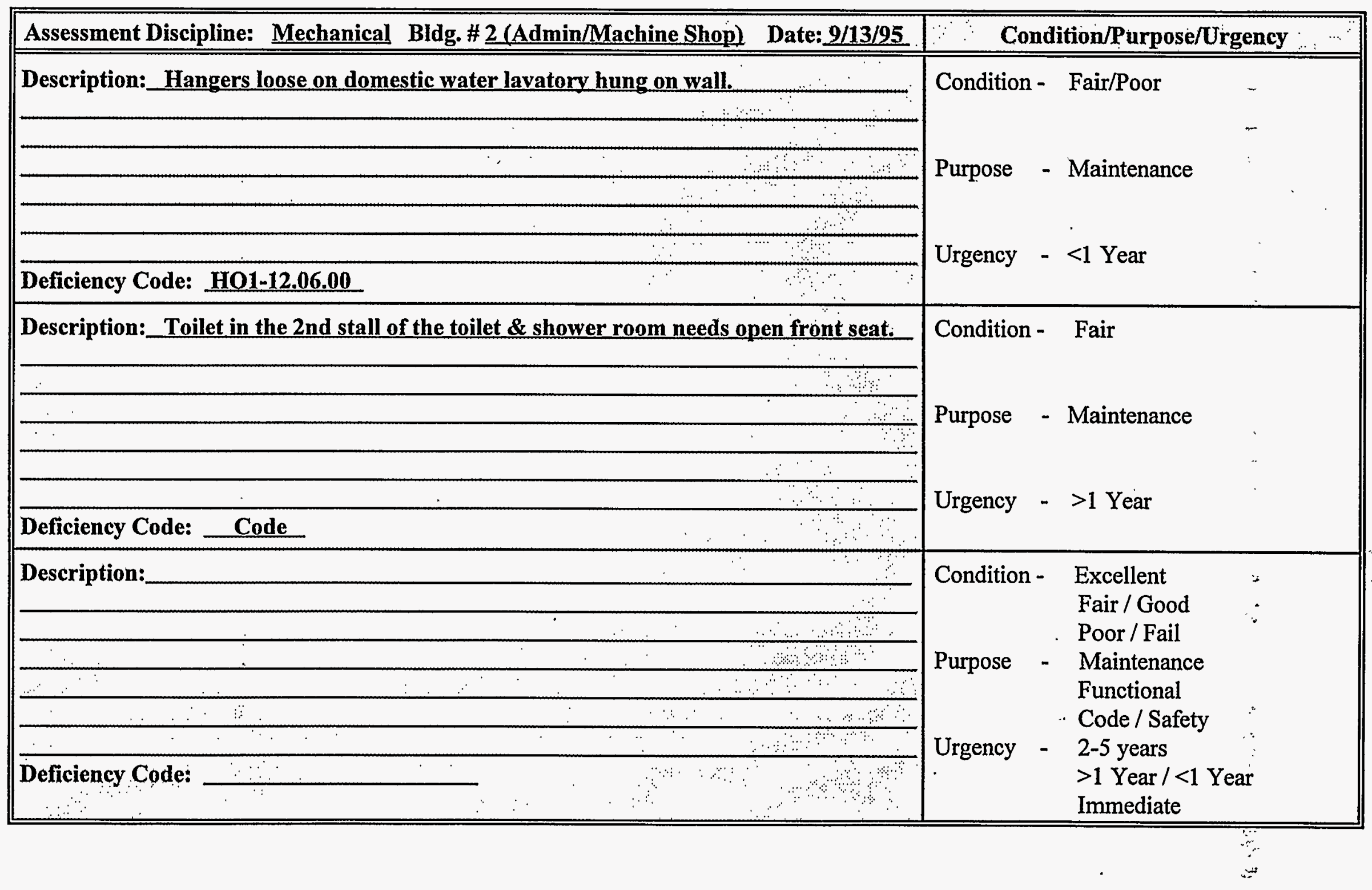




\section{Oxnard Condition Assessment Survey Field Report}

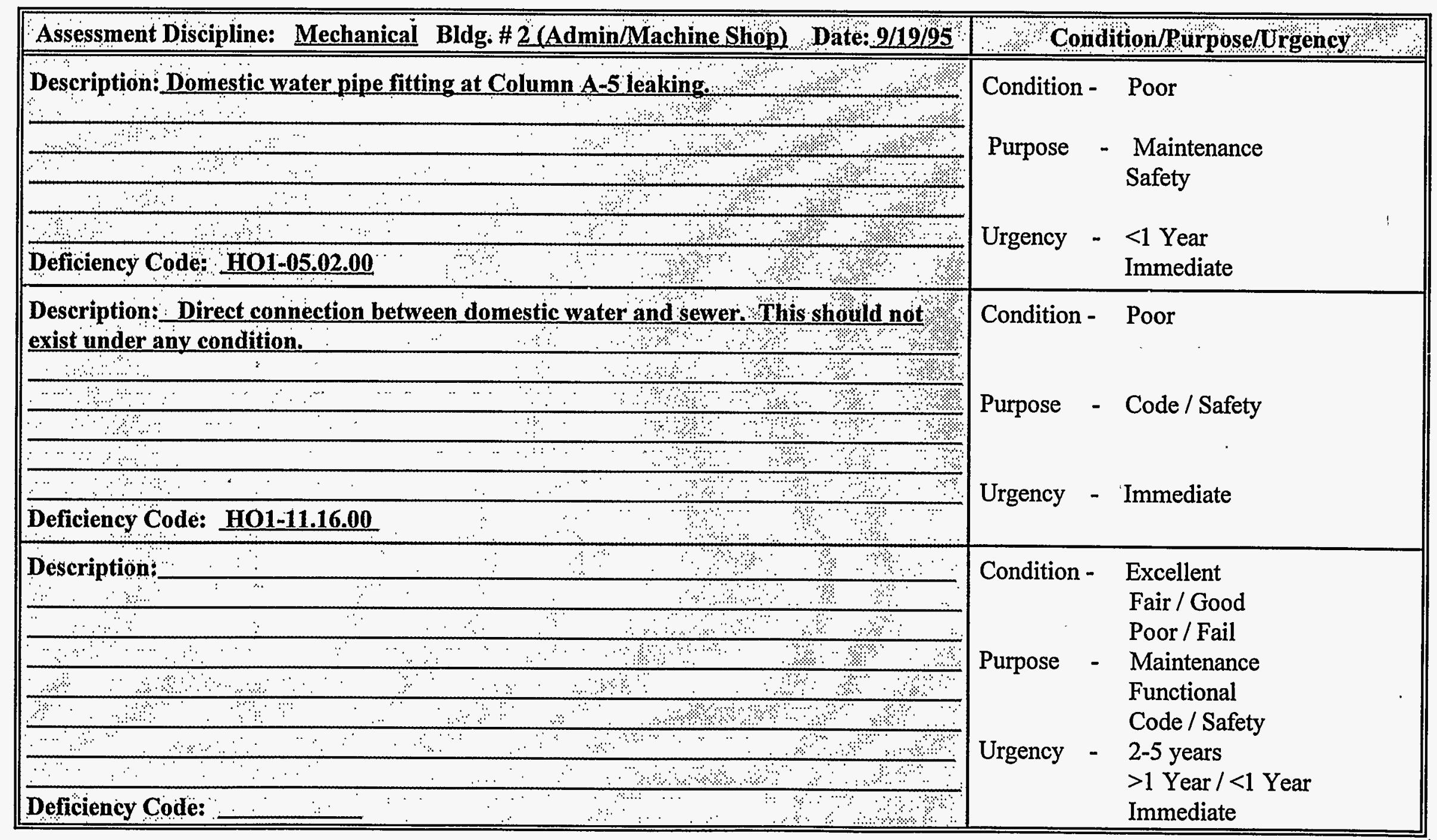




\title{
Oxnard CAS Field Notes
}

\author{
Building 3
}




\section{Oxnard Condition Assessment Survey Field Report}

\begin{tabular}{|c|c|c|}
\hline Assessment Discipline: Arch/Struct & \multicolumn{2}{|c|}{ Condition/Purpose/Urgency } \\
\hline $\begin{array}{l}\text { Description: Upper SE Corner of concrete structure displays stress cracks. } \\
\text { Movement will cause broken concrete to fall. (Potential safety hazard) }\end{array}$ & \multirow{4}{*}{$\begin{array}{l}\text { Condition } \\
\text { Purpose } \\
\text { Urgency }\end{array}$} & \multirow{4}{*}{$\begin{array}{l}\text { Poor / Fail } \\
\text { Functional } \\
\text { Code / Safety } \\
<1 \text { Year }\end{array}$} \\
\hline & & \\
\hline 2 & & \\
\hline Deficiency Code: $\quad$ C01-01.02.05 & & \\
\hline Description: Exterior East wall area of structural concrete is cracking and crazing & Condition - & Fair \\
\hline$\therefore$ & \multirow{4}{*}{$\begin{array}{l}\text { Purpose } \\
\text { Urgency }\end{array}$} & \multirow[b]{2}{*}{ - Functional } \\
\hline$\frac{16}{16}$ & & \\
\hline & & \multirow{2}{*}{ - $>1$ Year } \\
\hline Deficiency Code: CO1.01.02.06 & & \\
\hline $\begin{array}{l}\text { Description: Window caulking/glazing cracked and deteriorating and metal frames } \\
\text { are showing surface corrosion. (360 each) }\end{array}$ & \multicolumn{2}{|c|}{ Condition - Poor } \\
\hline$\ldots \quad \therefore \quad \cdots$ & \multirow{3}{*}{$\begin{array}{l}\text { Purpose } \\
\text { Urgency }\end{array}$} & \multirow{2}{*}{$\begin{array}{l}\text { Maintenance } \\
\text { Functional }\end{array}$} \\
\hline$\therefore$ & & \\
\hline Deficiency Code: D24-01.10.00\& D24-01.15.00 & & \\
\hline
\end{tabular}




\section{Oxnard Condition Assessment Survey Field Report}

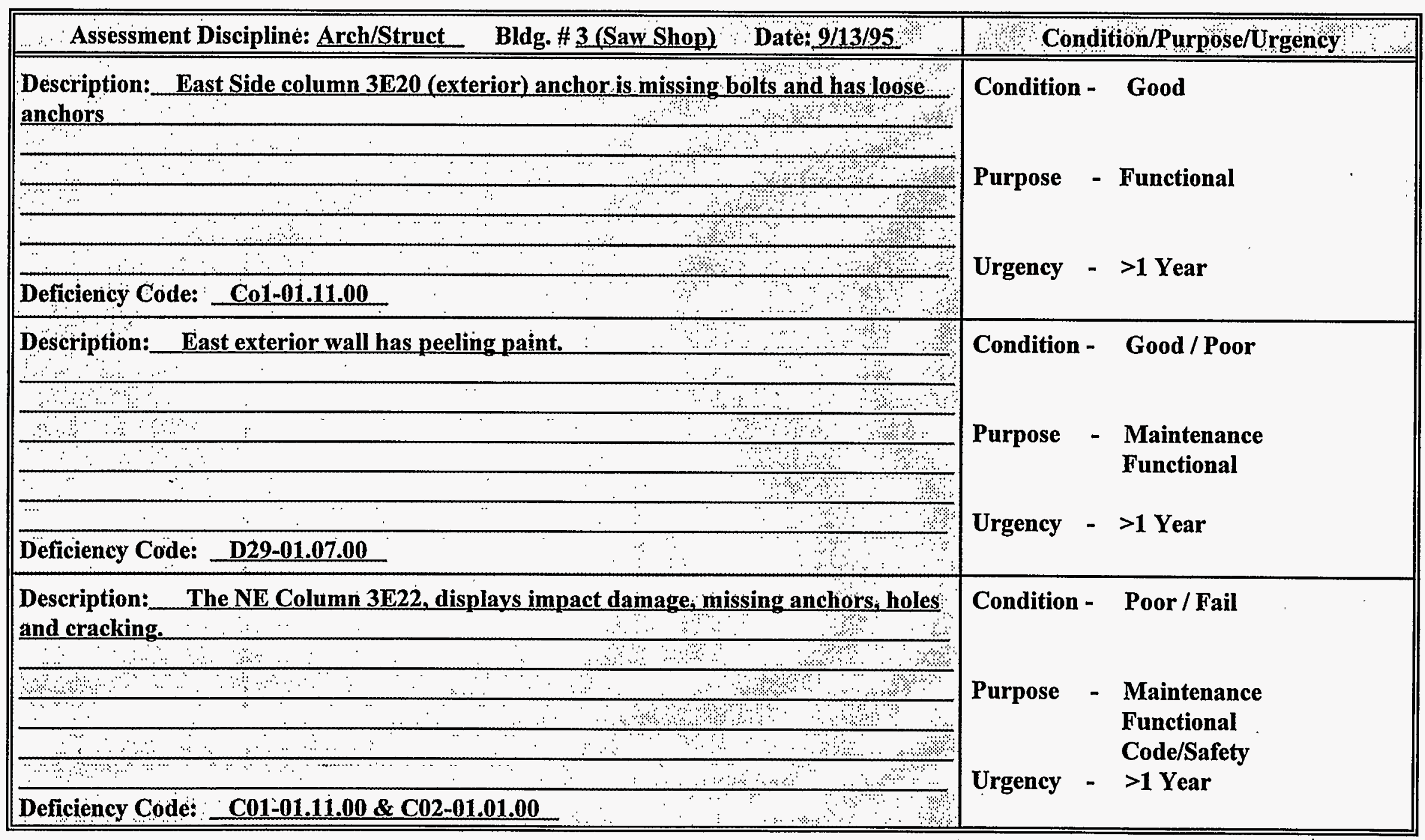




\section{Oxnard Condition Assessment Survey Field Report}

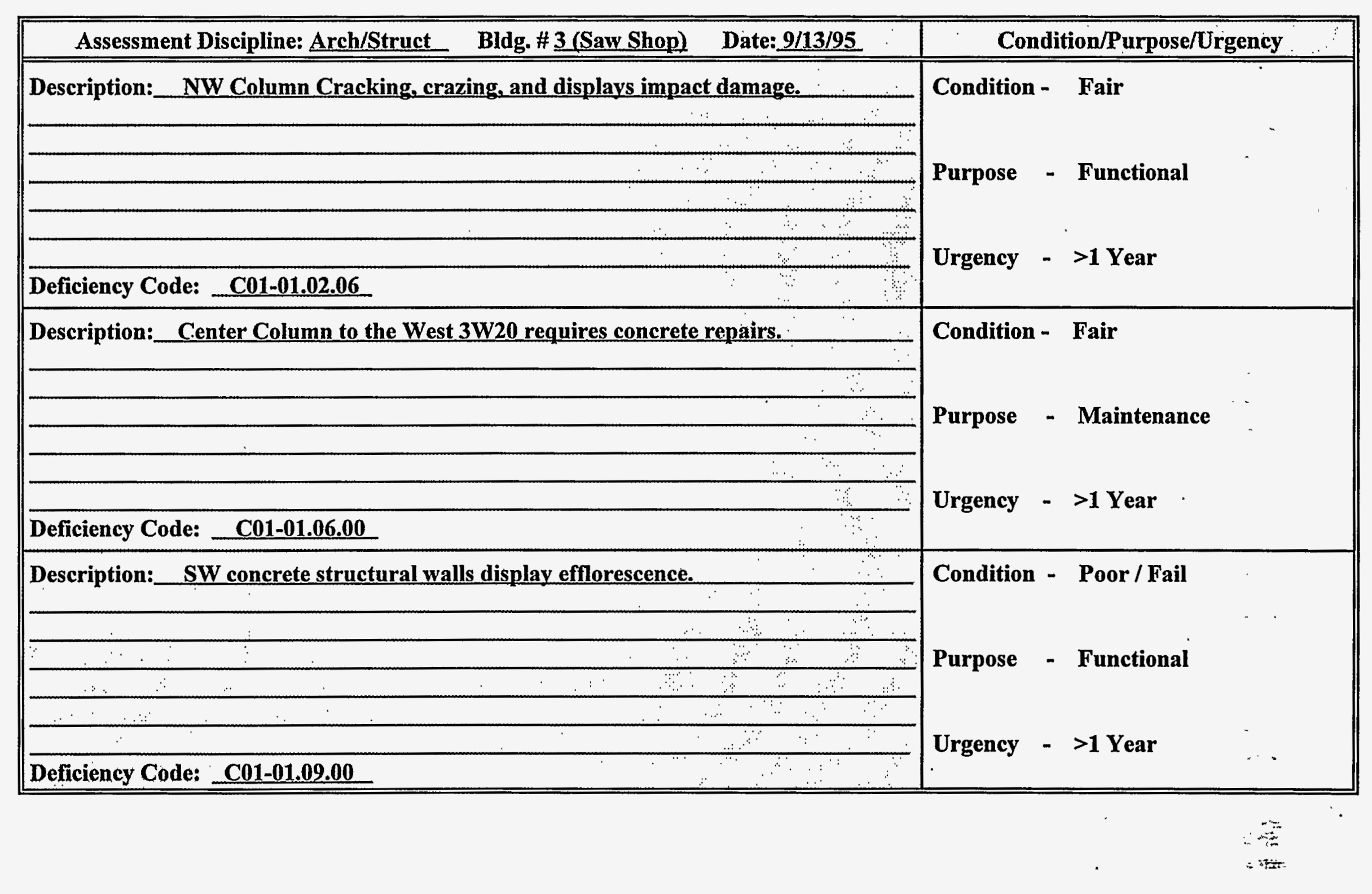




\section{Oxnard Condition Assessment Survey Field Report}

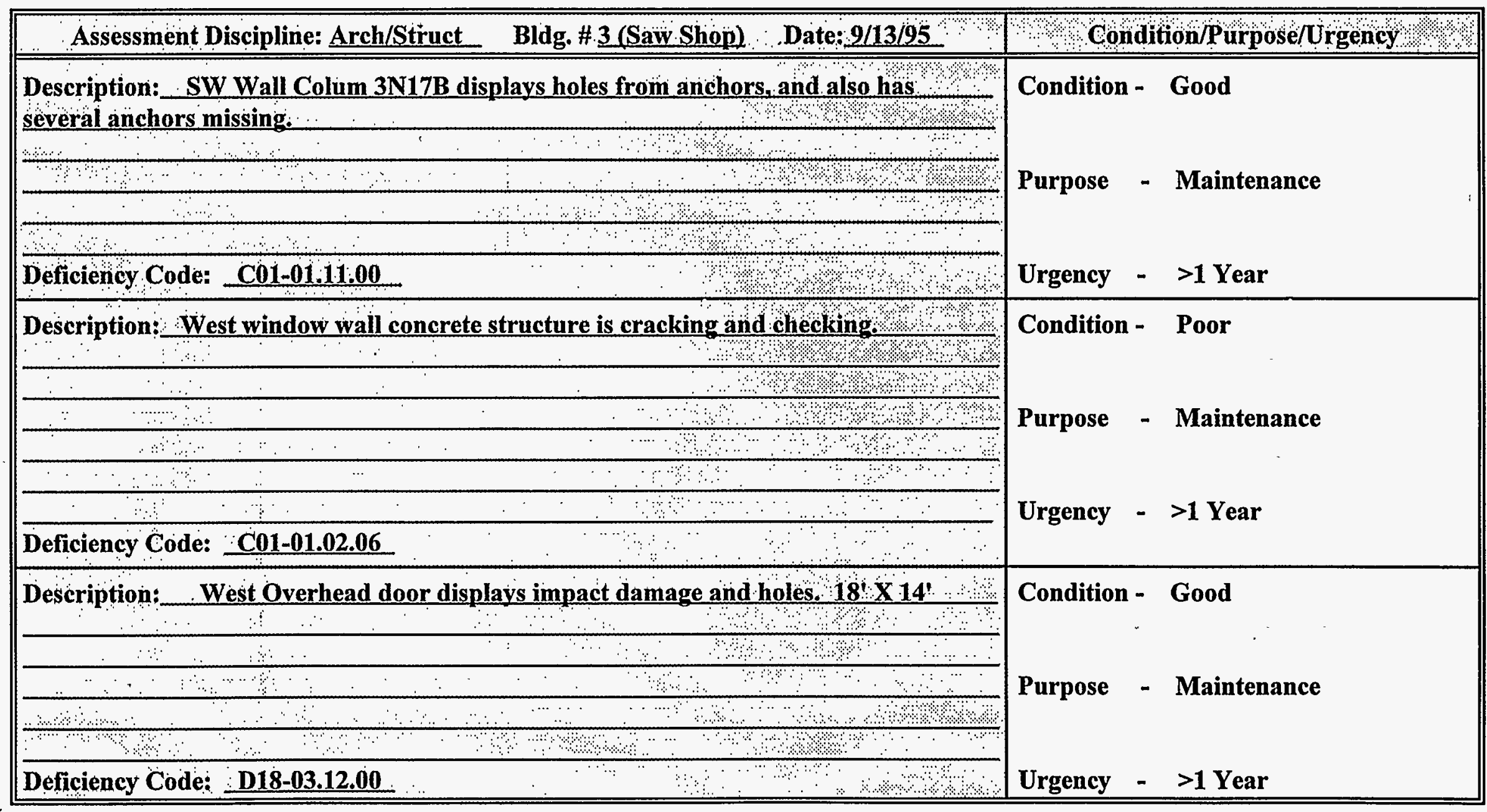




\section{Oxnard Condition Assessment Survey Field Report}

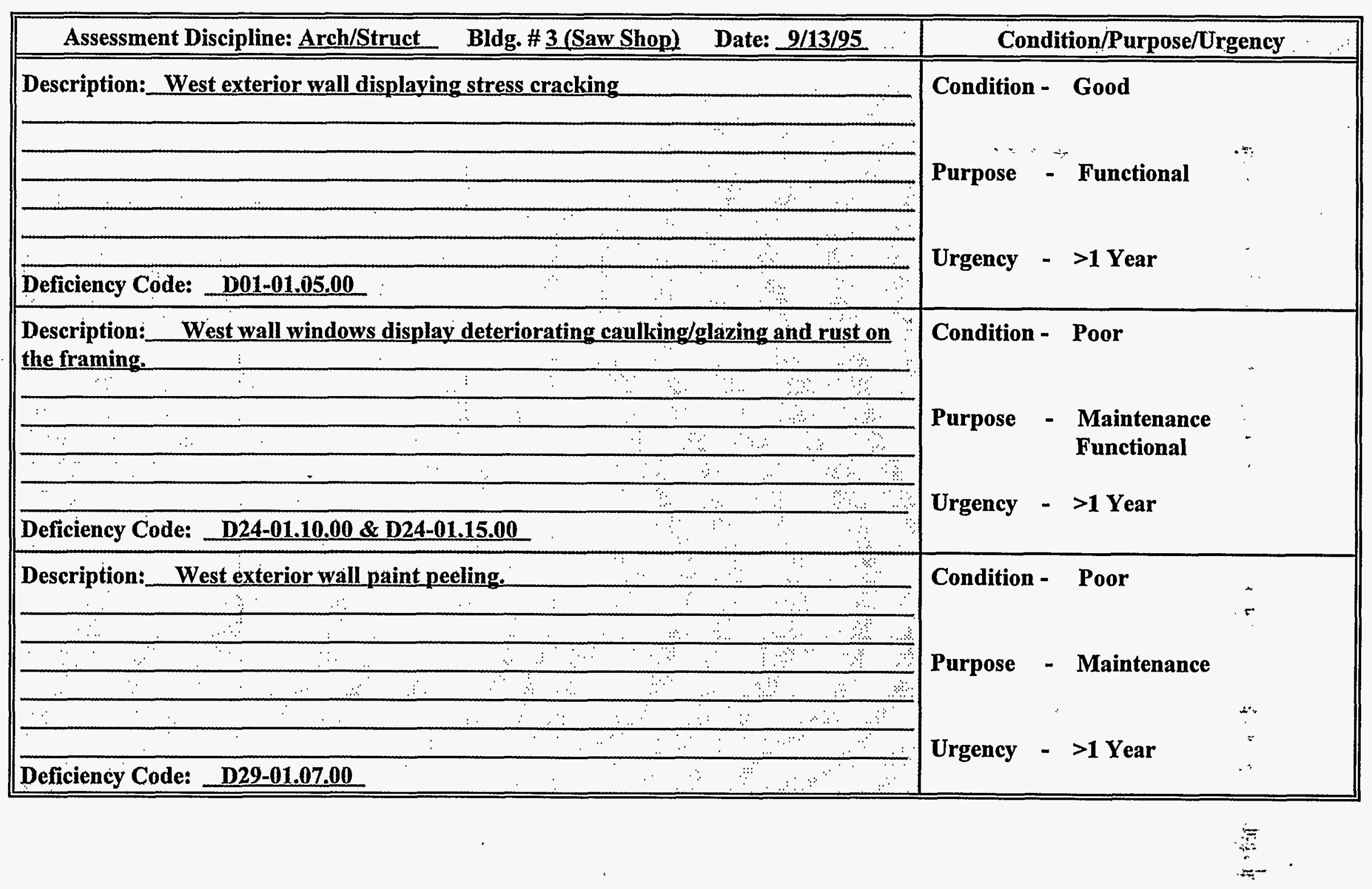




\section{Oxnard Condition Assessment Survey Field Report}

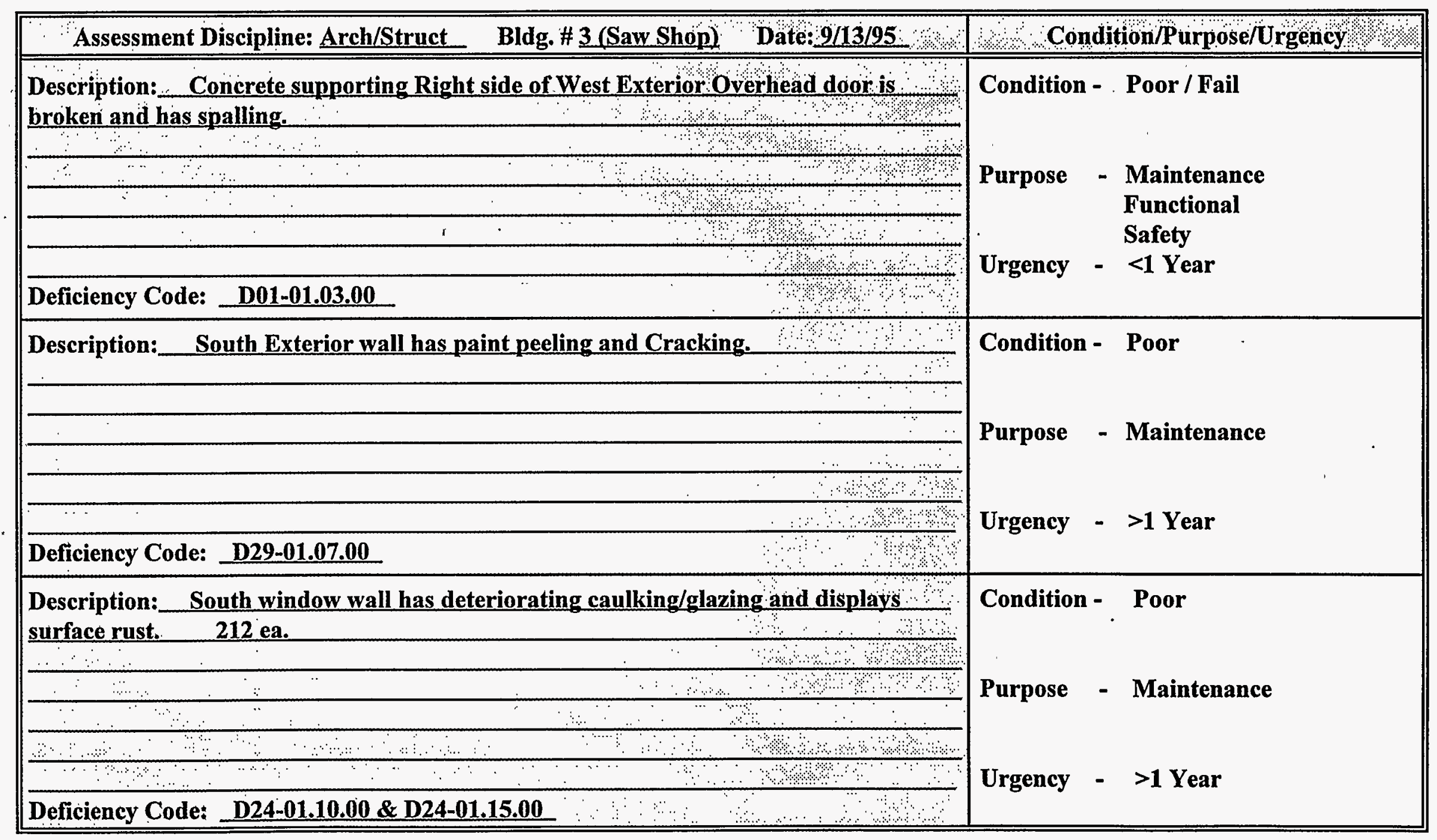




\section{Oxnard Condition Assessment Survey Field Report}

\begin{tabular}{|c|c|c|}
\hline Assessment Discipline: Arch/Struct Bldg. \# 3 (Saw Shop) & Condition/Purpose/Ur & gency \\
\hline Description: Overhead gantry crane rails show signs of corrosion. & \multirow{2}{*}{$\begin{array}{l}\text { Condition - Good } \\
\text { Purpose - - Maintenance }\end{array}$} & \multirow{4}{*}{ i. } \\
\hline & & \\
\hline (a) & \multirow{2}{*}{ Urgency - >1 Year } & \\
\hline Deficiency Code: C03-01.04.00 & & \\
\hline $\begin{array}{l}\text { Description: North of Bldg. } 3 \text { - Overhead gantry crane rail system ( } 5 \text { ton) displays } \\
\text { corrosion along the entire length of the system. }\end{array}$ & \multirow{3}{*}{$\begin{array}{l}\text { Condition - Poor } \\
\text { Purpose - Maintenance }\end{array}$} & \\
\hline$\therefore \quad \%$ & & \\
\hline$\because \quad \because \quad \vdots$ & & \\
\hline$\therefore \quad \therefore$ & \multirow{2}{*}{ Urgency - >1 Year } & \\
\hline Deficiency Code: C03-01.01.00 & & \\
\hline $\begin{array}{l}\text { Description: East Overhead door displays impact damage and peeling paint. } \\
11^{\prime} \times 14^{\prime}\end{array}$ & \multirow{3}{*}{$\begin{array}{l}\text { Condition - Fair } \\
\text { Purpose - Maintenance }\end{array}$} & \multirow{2}{*}{ - } \\
\hline 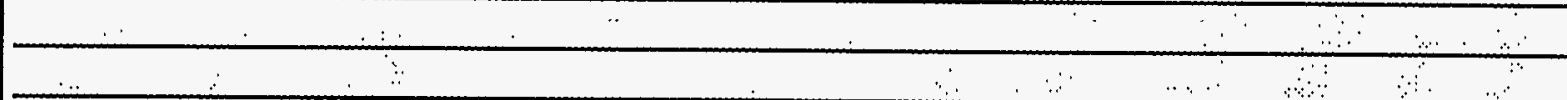 & & \\
\hline $\begin{array}{lll}3 & \vdots \\
2 & \vdots\end{array}$ & & \\
\hline 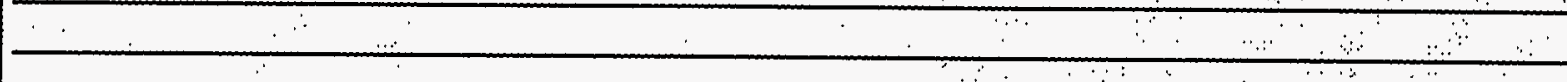 & \multirow[b]{2}{*}{ Urgency - >1 Year } & $\therefore$ \\
\hline Deficiency Code: D16-03.12.00 & & $\therefore$ \\
\hline
\end{tabular}




\section{Oxnard Condition Assessment Survey Field Report}

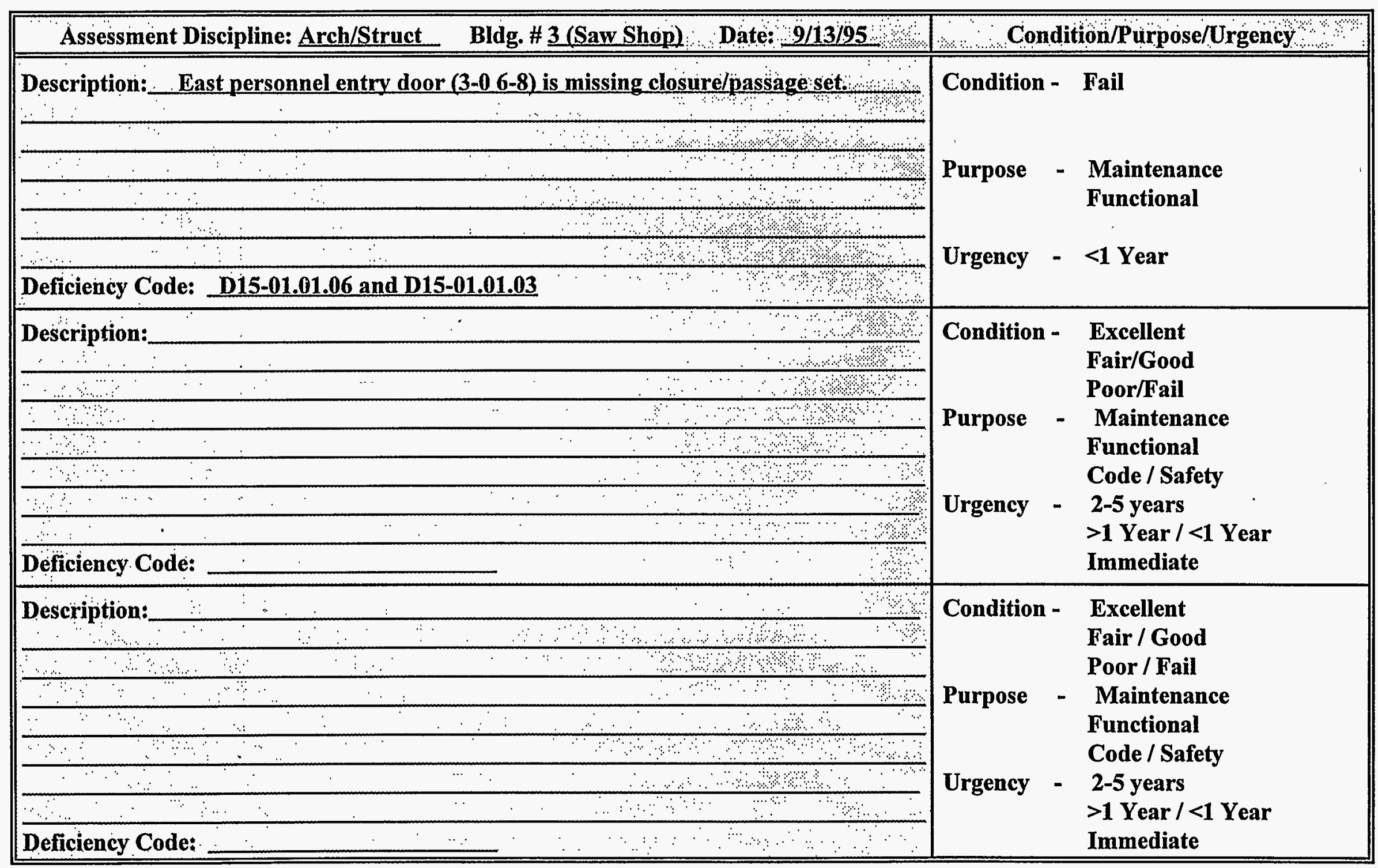




\section{Oxnard Condition Assessment Survey Field Report}

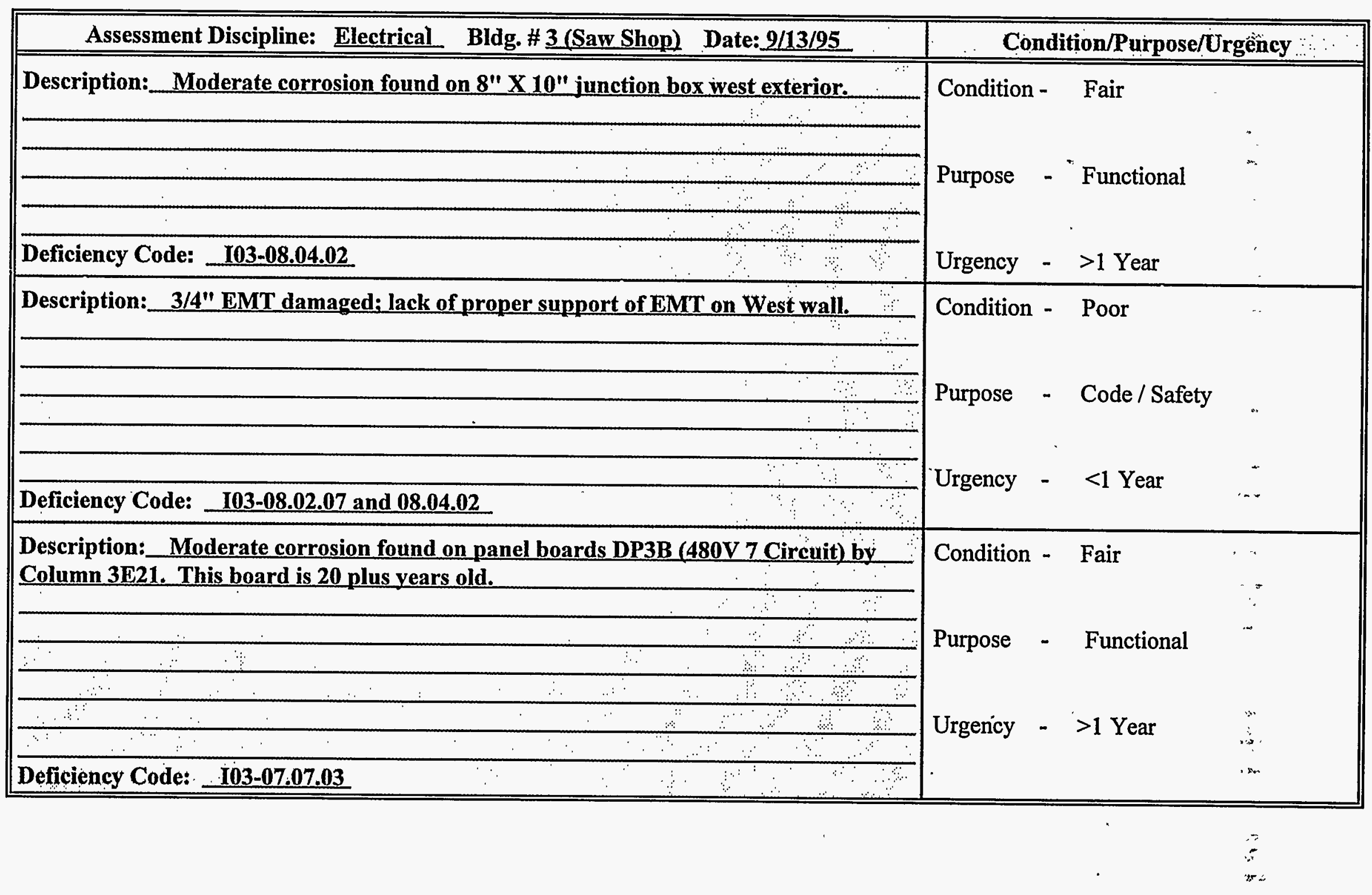




\section{Oxnard Condition Assessment Survey Field Report}

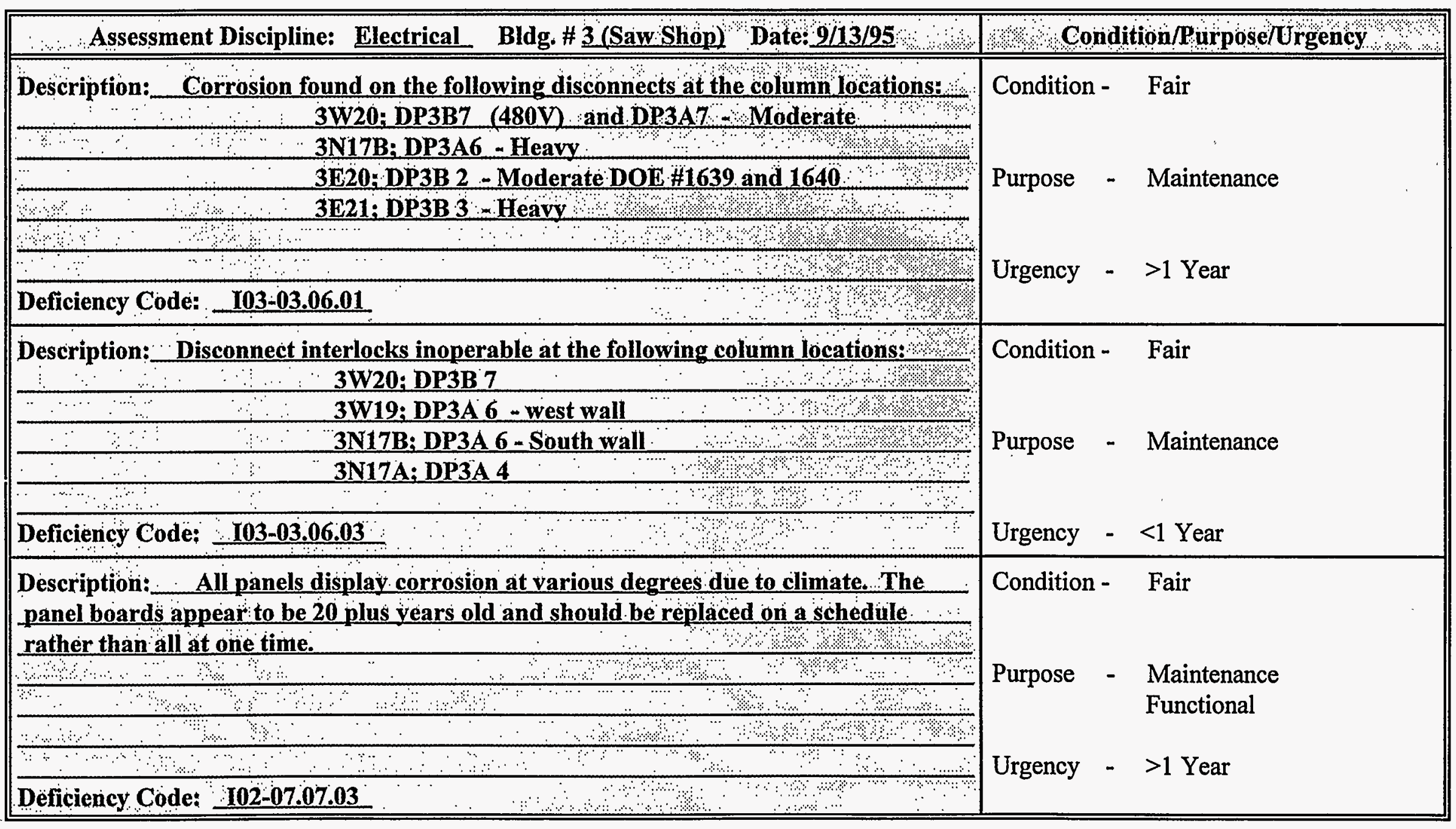




\section{Oxnard Condition Assessment Survey Field Report}

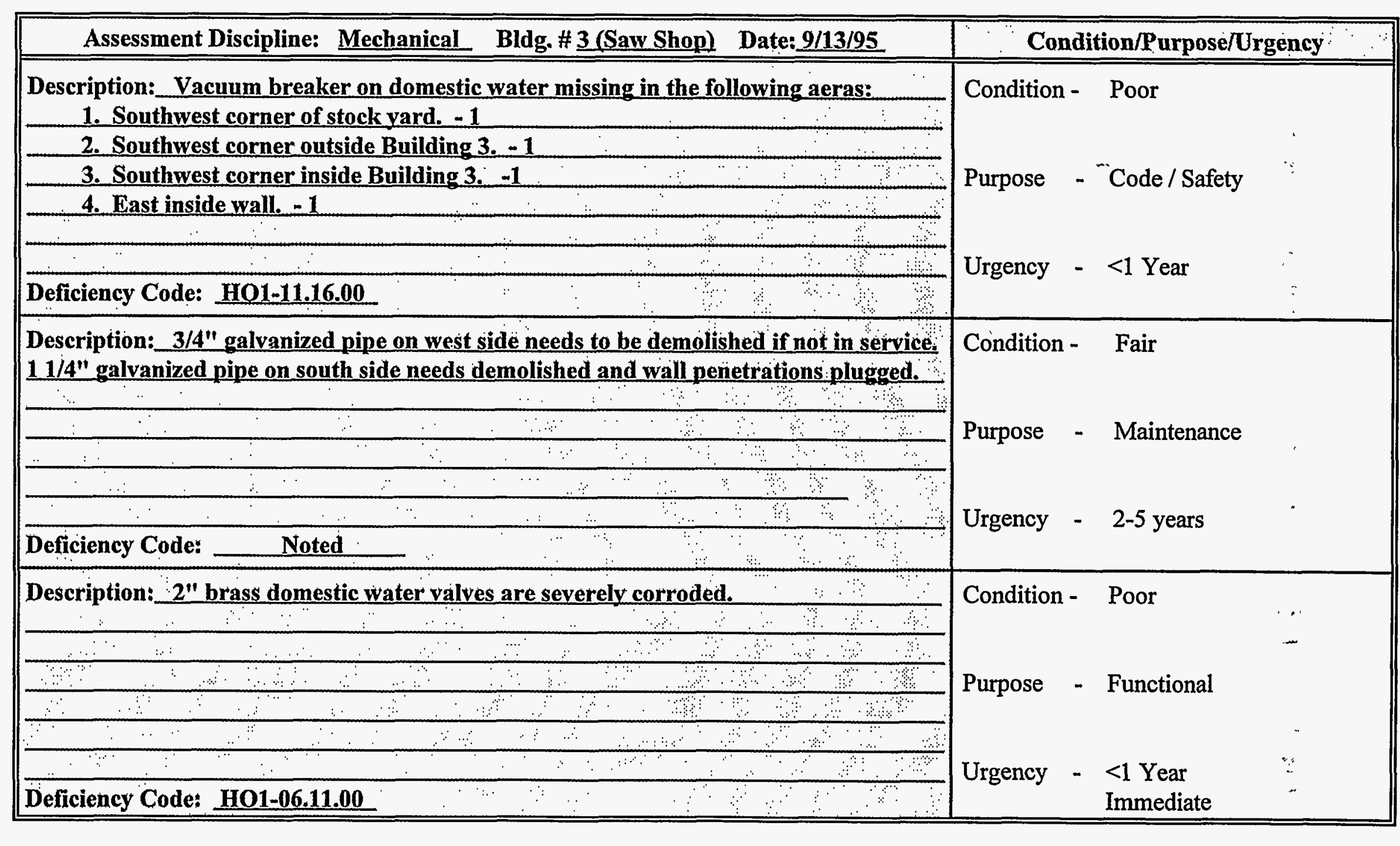




\section{Oxnard Condition Assessment Survey Field Report}

\begin{tabular}{|c|c|}
\hline Assessment Discipline: Mechanical Bldg. \# 3(Saw Shop) Date: 9/13/95 .... & Condition/Rurpose/Urgency \\
\hline Description: Compressed air filters \& driers drain to floor asset wide. & \multirow{3}{*}{$\begin{array}{l}\text { Purpose - Safety } \\
\text { Urgency - >1 Year }\end{array}$} \\
\hline 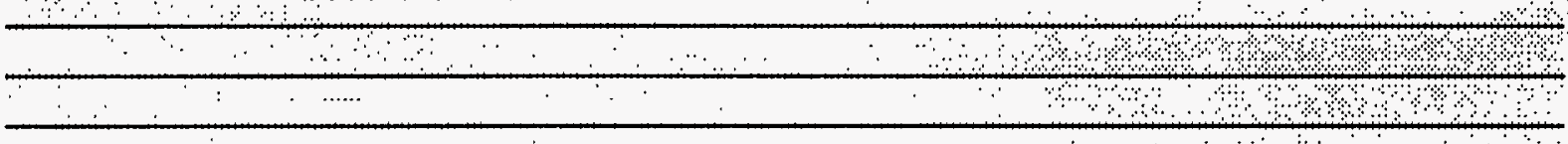 & \\
\hline Deficiency Code: $\mathrm{HO3-04.07.00}$ & \\
\hline $\begin{array}{l}\text { Description: Domestic water pipes not labeled. Drain, waste \& vent pipes not labeled: } \\
\text { Compressed air line pipes not labeled in stock yard. }\end{array}$ & \multirow{2}{*}{$\begin{array}{l}\text { Condition - Good } \\
\text { Purpose - Maintenance }\end{array}$} \\
\hline$\frac{1 \%}{4 \% ?}$ & \\
\hline $\begin{array}{lllll}\text { Deficiency Code: HO1-05.06.00/HO2-01.05.00/HO3-05.06.00 } & & \\
\end{array}$ & Urgency - $2-5$ years \\
\hline Description: 2 " ball valve trd handle missing on compressed air unit. & \multirow{3}{*}{$\begin{array}{l}\text { Condition - - Fair } \\
\text { Purpose - Maintenance }\end{array}$} \\
\hline 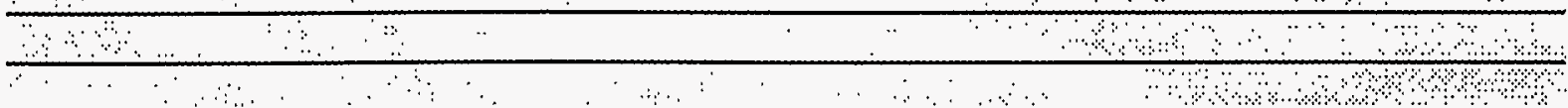 & \\
\hline 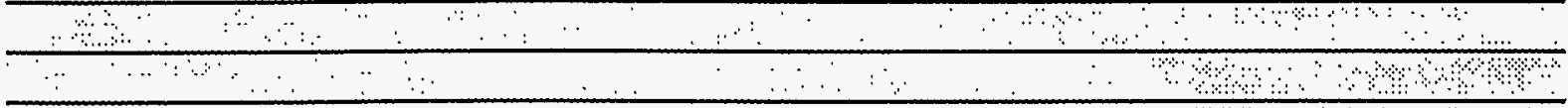 & \\
\hline Deficiëncy Code: HO3-06.10.00 & Urgency $-<1$ Year \\
\hline
\end{tabular}




\section{Oxnard Condition Assessment Survey Field Report}

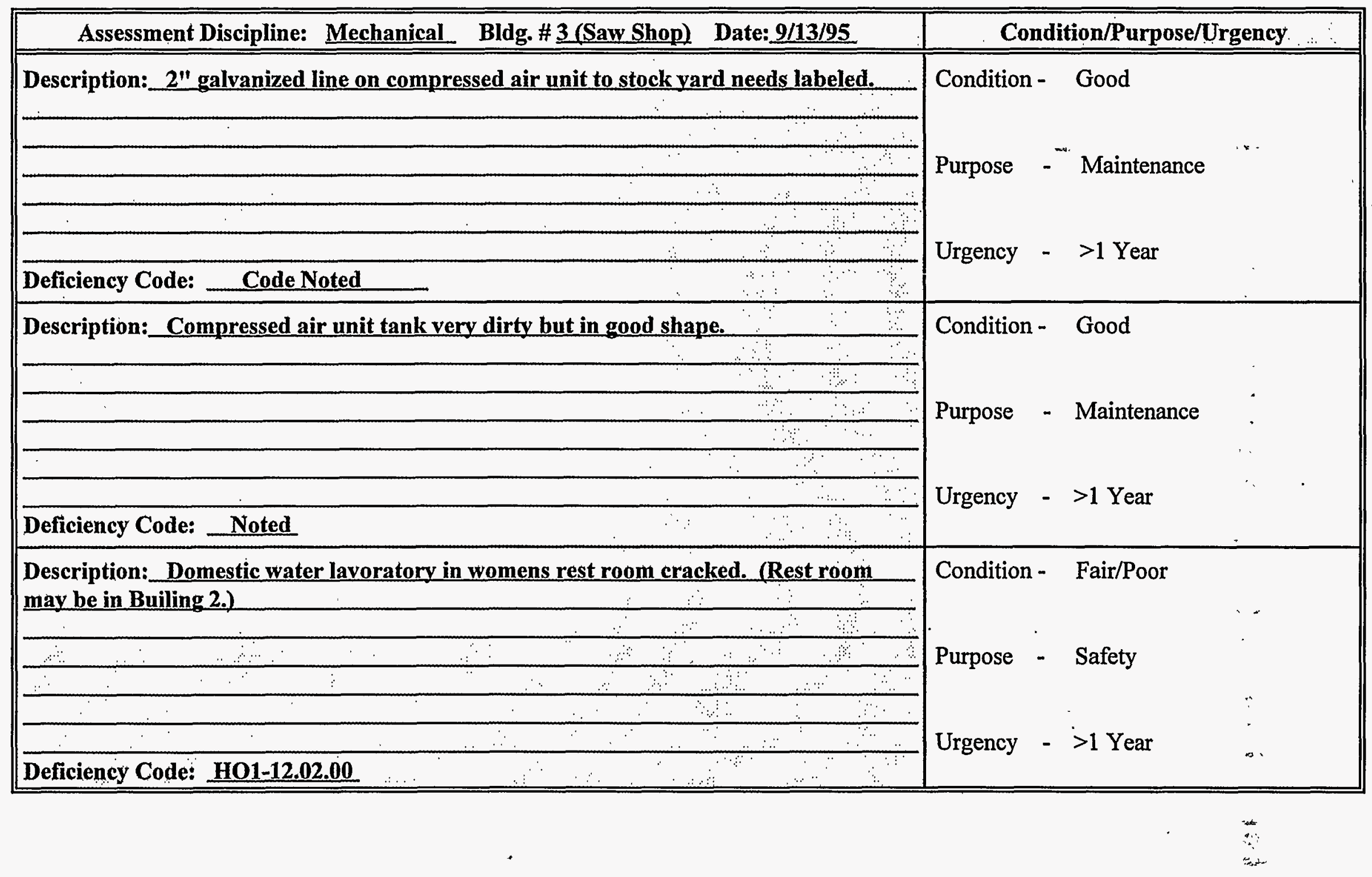




\section{Oxnard Condition Assessment Survey Field Report}

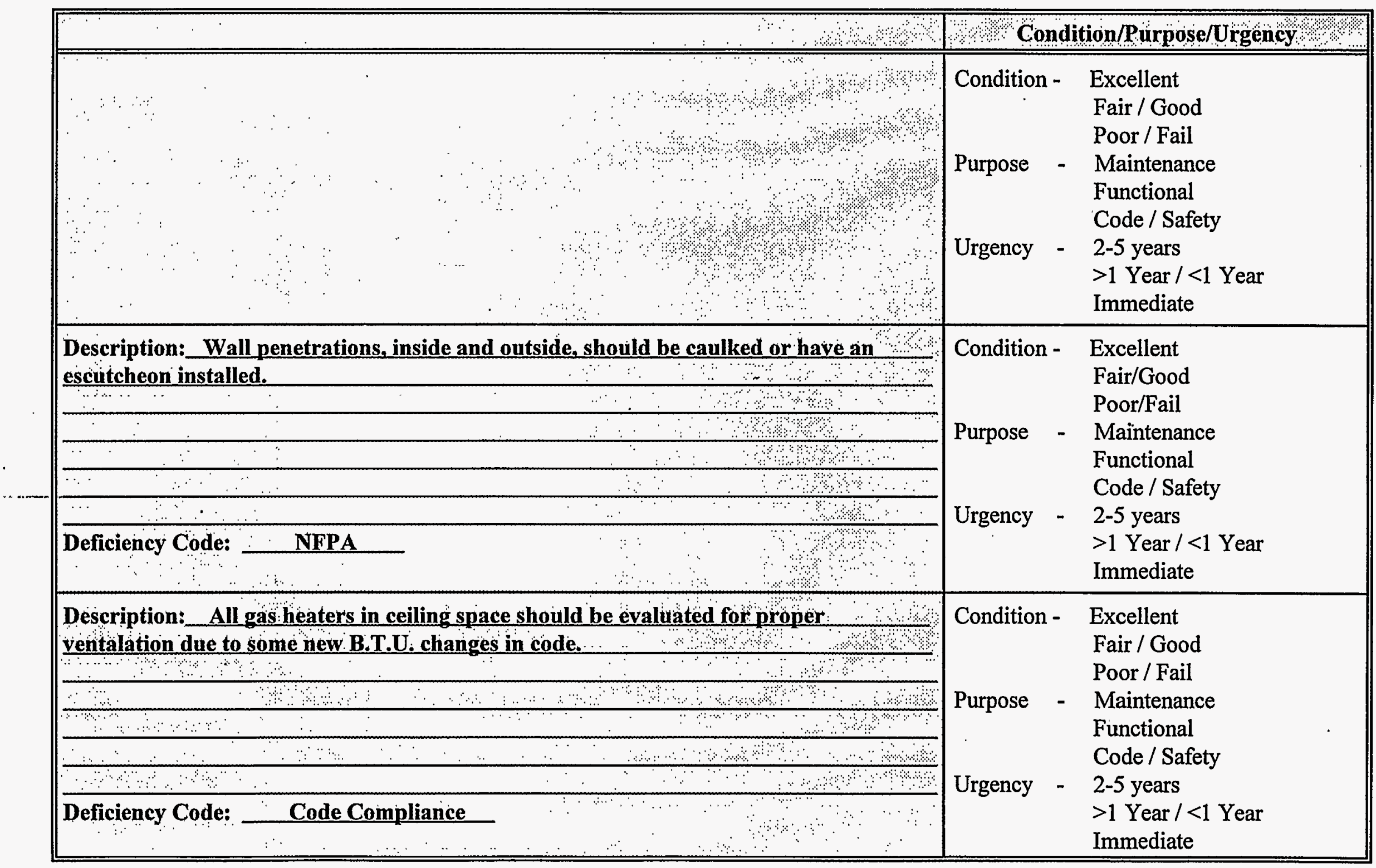




\section{Oxnard CAS Field Notes}

Building 4 



\section{Oxnard Condition Assessment Survey Field Report}

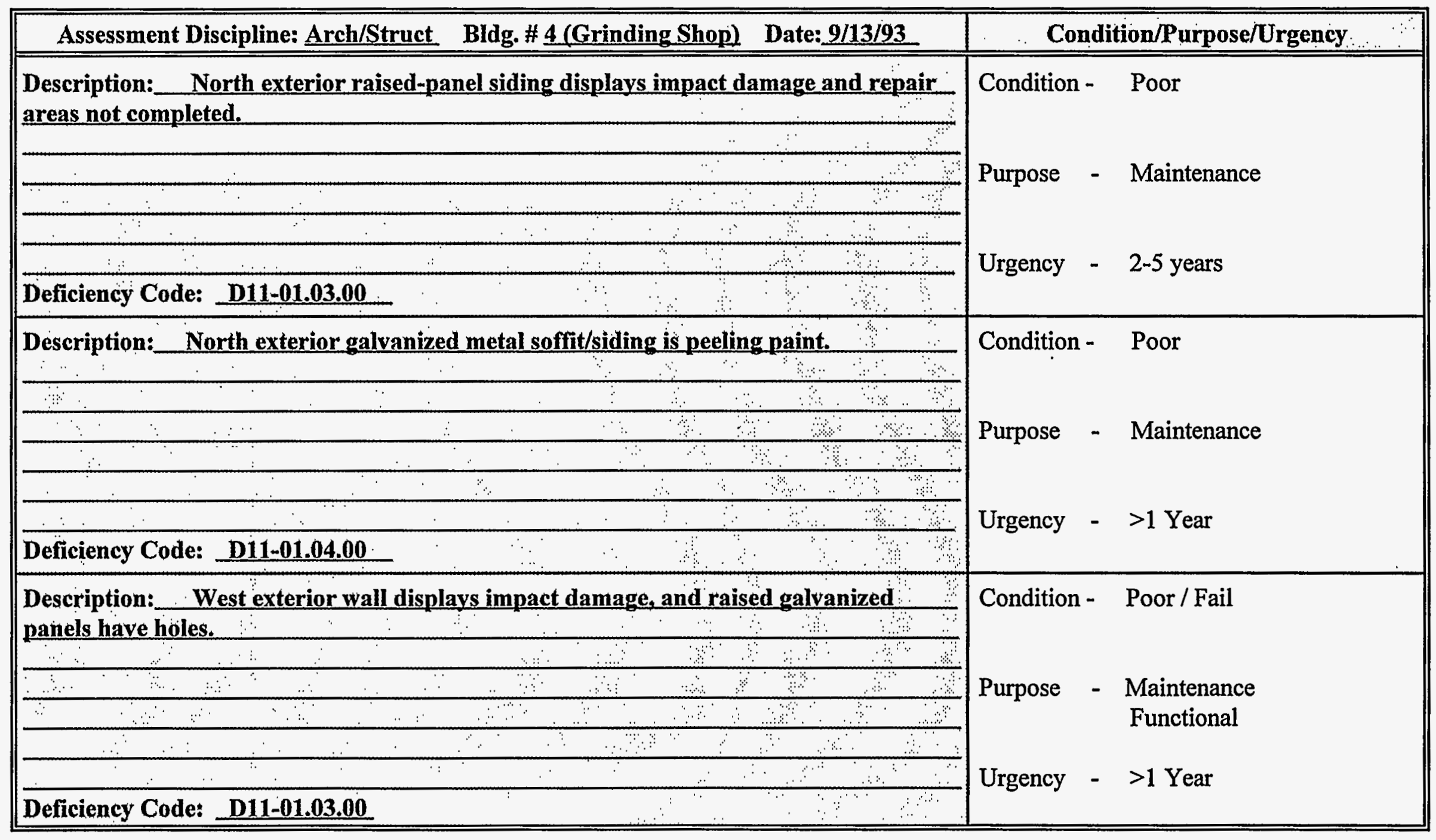




\section{Oxnard Condition Assessment Survey Field Report}

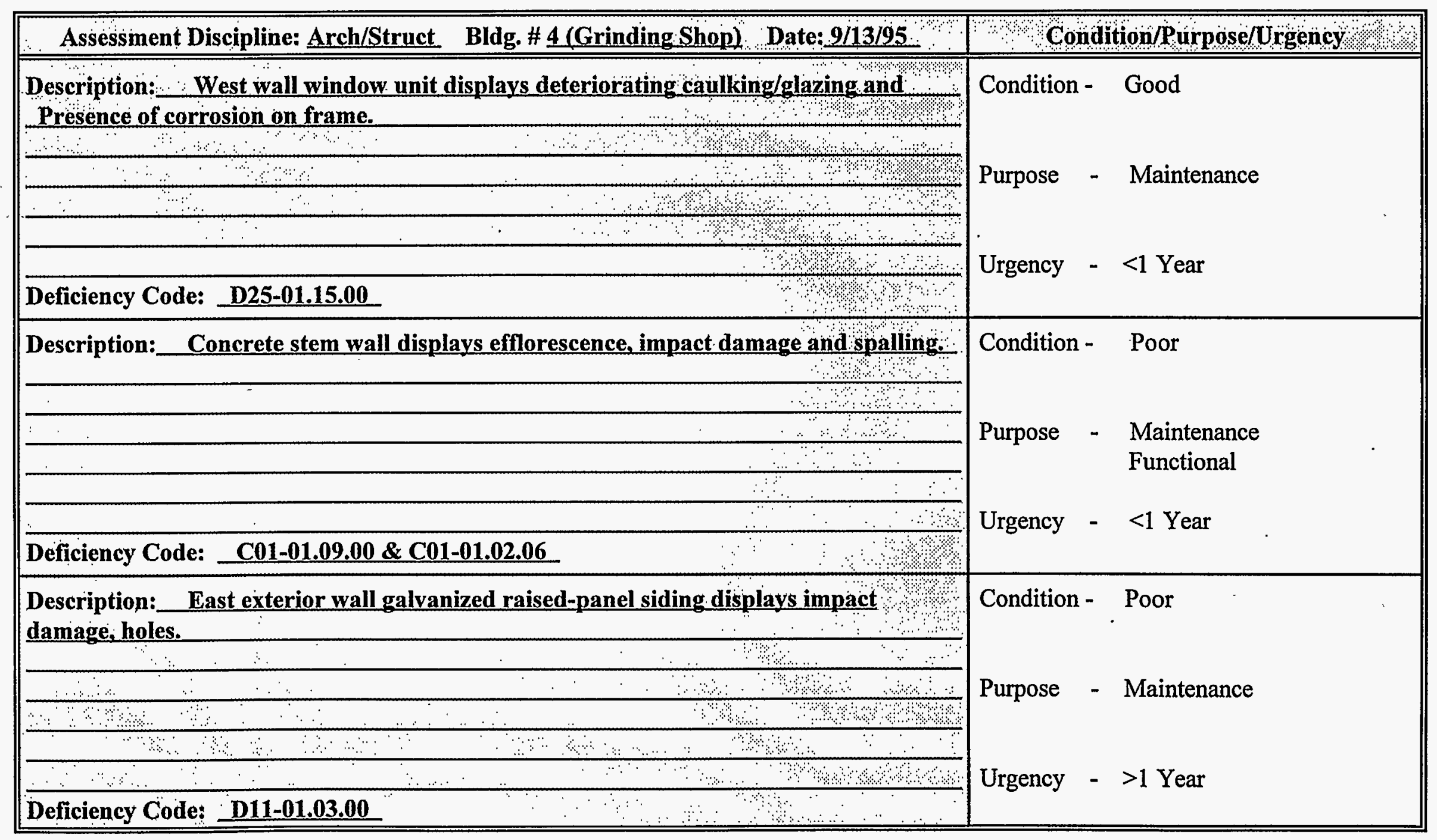




\section{Oxnard Condition Assessment Survey Field Report}

\begin{tabular}{|c|c|c|c|}
\hline Assessment Discipline: Arch/Struct Bldg. \# 4(Grinding Shop) Date: 2/13/95 & \multicolumn{3}{|c|}{ Condition/Purpose/Urgency } \\
\hline Description: East exterior galvanized raised-panel siding has peeling paint. & \multirow{3}{*}{\multicolumn{2}{|c|}{$\begin{array}{l}\text { Condition - } \quad \text { Poor } \\
\text { Purpose - ' Maintenance }\end{array}$}} & \multirow{2}{*}{$\therefore$} \\
\hline & & & \\
\hline & & & \\
\hline$\because$ & \multirow{3}{*}{ Urgency } & & \\
\hline 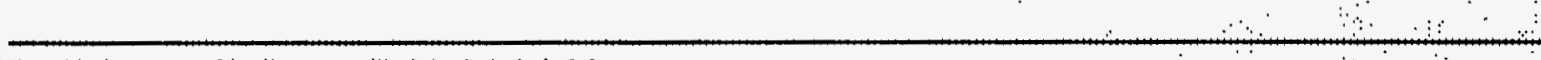 & & \multirow[t]{2}{*}{$->1$ Year } & \\
\hline Deficiency Code: $\quad$ D11-01.04.00 & & & \\
\hline Description: SE Corner concrete stemwall is spalling. & Condition - & Poor & \multirow{6}{*}{$\begin{array}{l}\ddot{\circ} \\
\cdots \\
\cdots\end{array}$} \\
\hline & \multirow{5}{*}{$\begin{array}{l}\text { Purpose } \\
\text { Urgency }\end{array}$} & \multirow{2}{*}{ - Functional } & \\
\hline$\ldots \ldots$ & & & \\
\hline- & & & \\
\hline$\because$ & & \multirow{2}{*}{$-\quad<1$ Year } & \\
\hline Deficiency Code: C01-01.06.00 & & & \\
\hline Description: Interior structureal steel coating is rusting, peeling at all columns and & Condition - & \multirow[t]{2}{*}{ - Poor } & \multirow{3}{*}{ " } \\
\hline Purlins 4W thru 4E.* & \multirow{4}{*}{ Purpose } & & \\
\hline 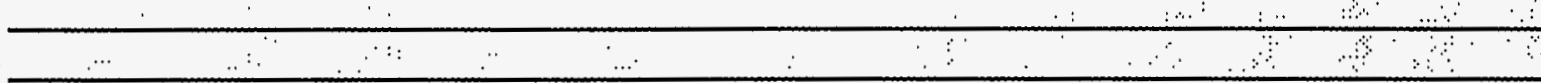 & & \multirow{2}{*}{ - Maintenance } & \\
\hline$\because$ & & & \multirow{3}{*}{ 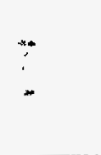 } \\
\hline$\because \quad: \quad=$ & & & \\
\hline Deficiency Code: D11-01.01.00 & Urgency & $>1$ Year & \\
\hline
\end{tabular}




\section{Oxnard Condition Assessment Survey Field Report}

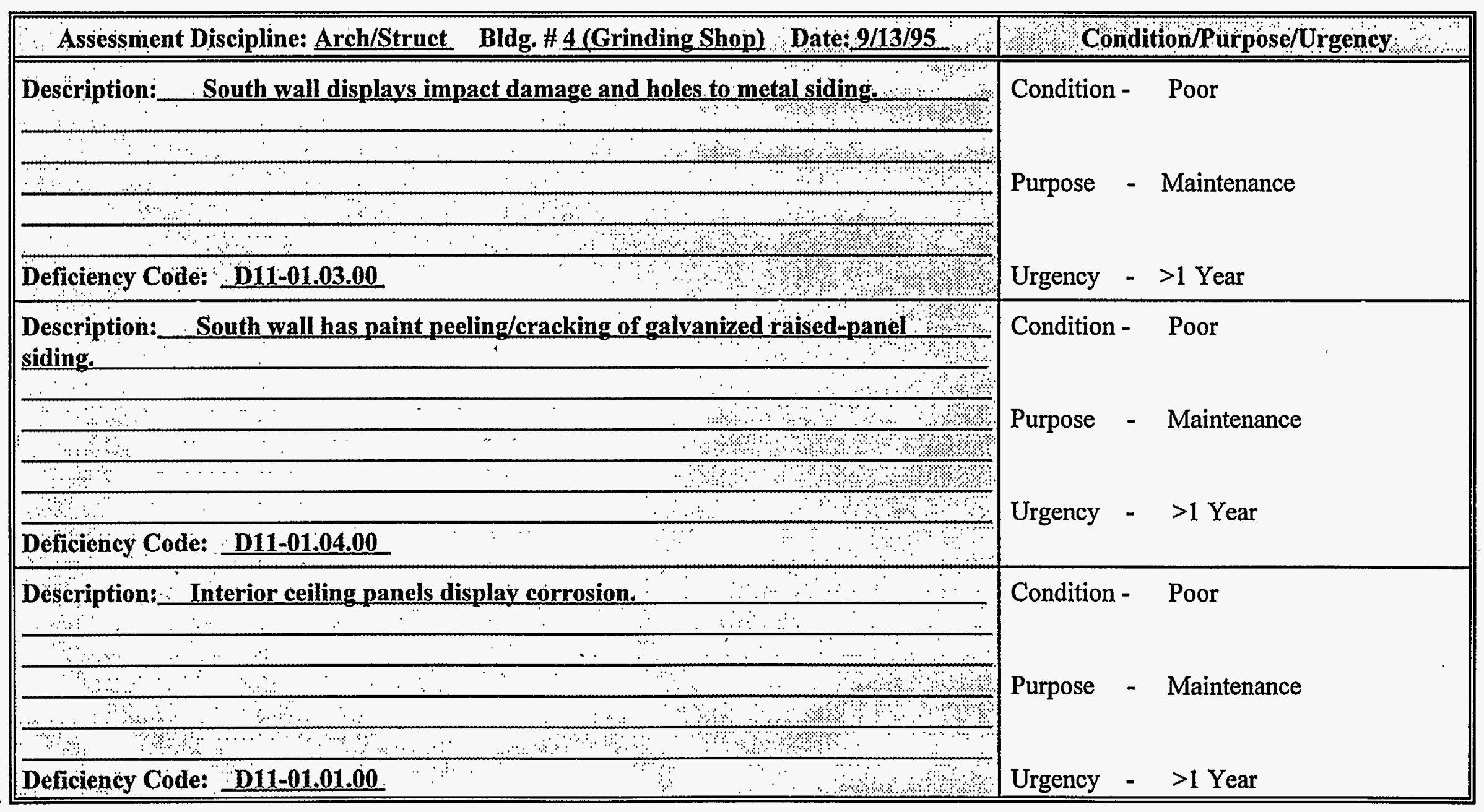




\section{Oxnard Condition Assessment Survey Field Report}

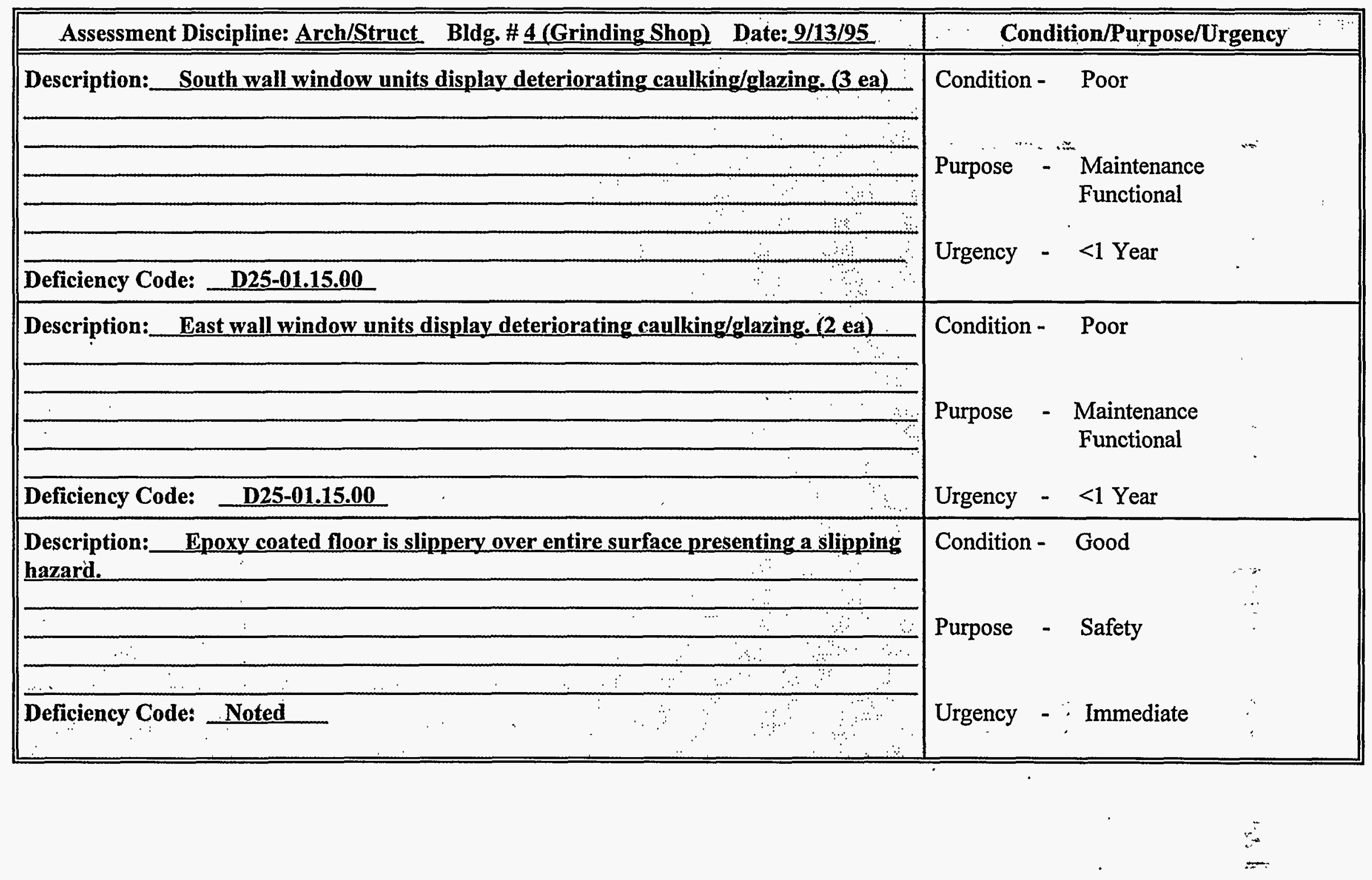




\section{Oxnard Condition Assessment Survey Field Report}

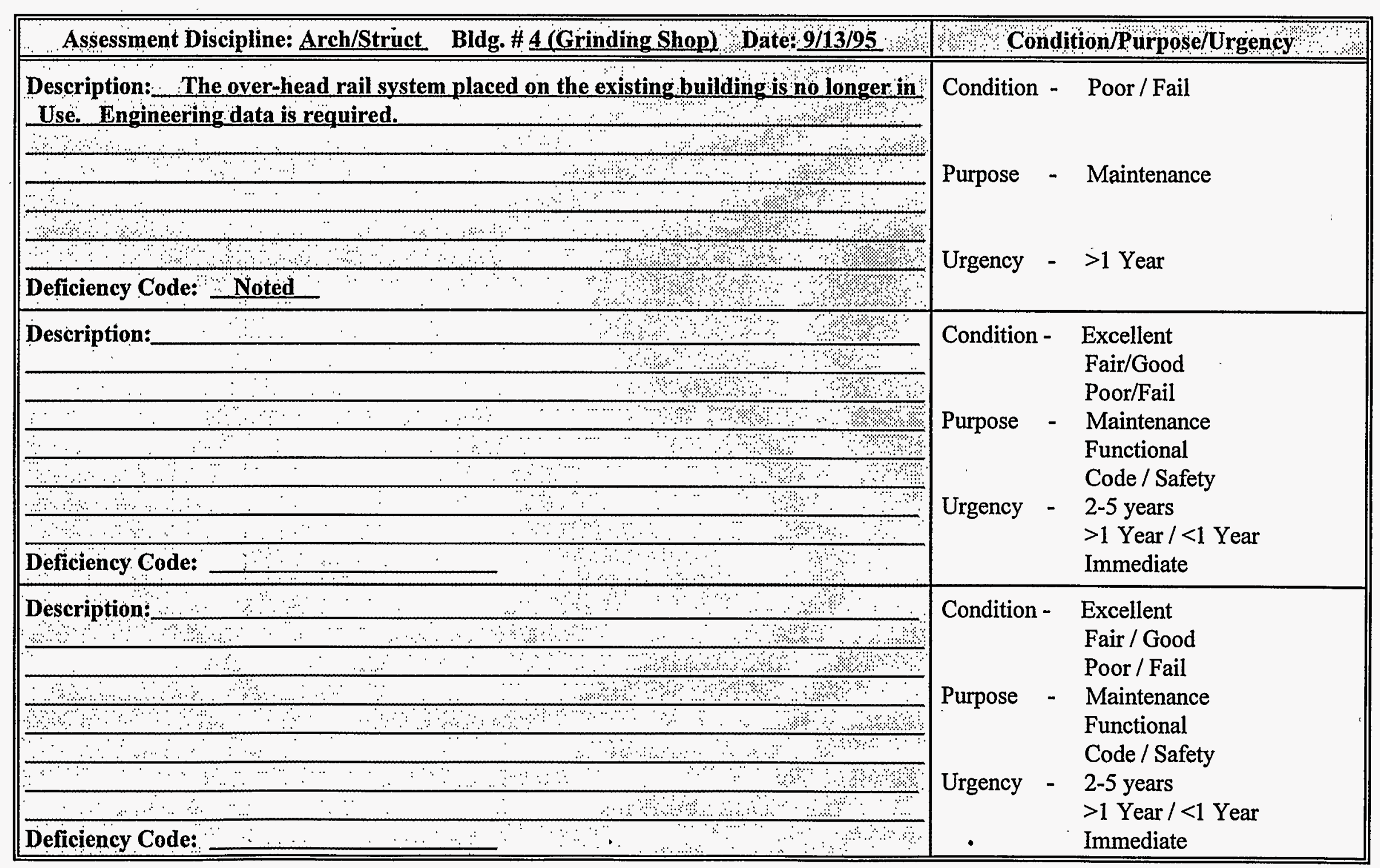




\section{Oxnard Condition Assessment Survey Field Report}

\begin{tabular}{|c|c|c|c|}
\hline Assessment Discipline: Electrical Bldg. \# 4(Grinding Shop) Date: 9/13/95 & \multicolumn{3}{|c|}{ Condition/Purpose/Urgency } \\
\hline $\begin{array}{l}\text { Description: } 2 \text { " EMT South exterior of building - raceway is not adequately } \\
\text { secured to the mounting surface. }\end{array}$ & \multirow{4}{*}{$\begin{array}{l}\text { Condition - } \\
\text { Purpose }\end{array}$} & \multirow{4}{*}{$\begin{array}{ll} & \text { Poor } \\
-\quad \text { Maintenance } \\
\text { Code }\end{array}$} & \multirow{4}{*}{$\cdots$} \\
\hline & & & \\
\hline$\because \quad \because \quad 3$ & & & \\
\hline 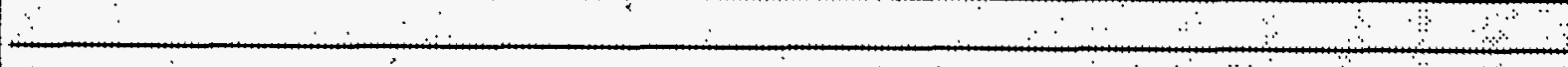 & & & \\
\hline$\therefore \quad \cdots \quad 3 \quad 3 \quad 3$ & \multirow[t]{2}{*}{ Urgency } & \multirow[t]{2}{*}{$<1$ Year } & \multirow{2}{*}{ 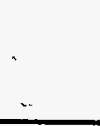 } \\
\hline Deficiency Code: $\quad$ 103-08.02.07 & & & \\
\hline $\begin{array}{l}\text { Description: Various conduits on interior of building - are not adequately secuired to } \\
\text { the mounting surface and/or are not secured within three feet of the box }\end{array}$ & Condition . & Poor & \multirow{6}{*}{$\begin{array}{l}\cdot \\
\cdot \\
\cdot \\
\cdots\end{array}$} \\
\hline 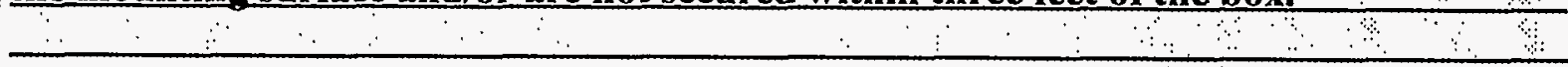 & \multirow{5}{*}{$\begin{array}{l}\text { Purpose } \\
\text { Urgency }\end{array}$} & & \\
\hline 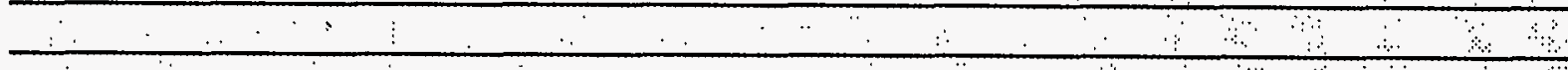 & & \multirow{3}{*}{$\begin{array}{l}\text { Maintenance } \\
\text { Code }\end{array}$} & \\
\hline 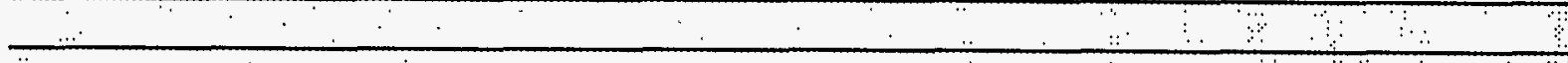 & & & \\
\hline$\because \quad \because \quad \cdots \quad \ddots \quad \because \quad 3 \quad \ddots$ & & & \\
\hline $\begin{array}{llllllll}\text { Deficièncy Code: } & \text { 103-08.02.07 and 08.02.10 } & \ddots & & & & & \\
\end{array}$ & & $-\quad<1$ Year & \\
\hline $\begin{array}{l}\text { Description: Disconnect enclosire displays moderate corrosion at Column } 4 \text { W33, } \\
\text { DP4A \#15. (480V) at north west corner and interlock inoperable. }\end{array}$ & \multirow[t]{2}{*}{ Condition - } & \multirow[t]{2}{*}{ Fair } & \multirow{2}{*}{$\therefore$} \\
\hline 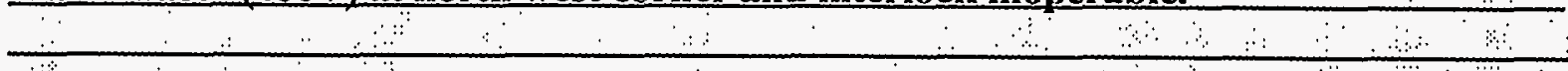 & & & \\
\hline 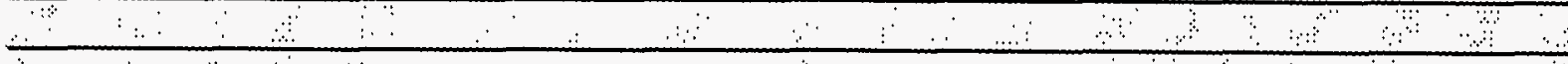 & \multirow{5}{*}{$\begin{array}{l}\text { Purpose } \\
\text { Urgency }\end{array}$} & \multirow[t]{3}{*}{ - Functional } & \multirow{5}{*}{$\begin{array}{c}\ddots \dot{y} \\
\vdots \\
\cdots\end{array}$} \\
\hline$\therefore \quad \therefore \quad \therefore \quad \therefore \quad \therefore$ & & & \\
\hline$\because \quad \because \quad \therefore \quad \therefore \quad \cdots$ & & & \\
\hline$\because \quad \cdots \quad \cdot \quad \cdot \quad \cdot$ & & $->1$ Year & \\
\hline Deficiency Code: I03-03.06.01 \& I03-03.06.03 & & & \\
\hline
\end{tabular}




\section{Oxnard Condition Assessment Survey Field Report}

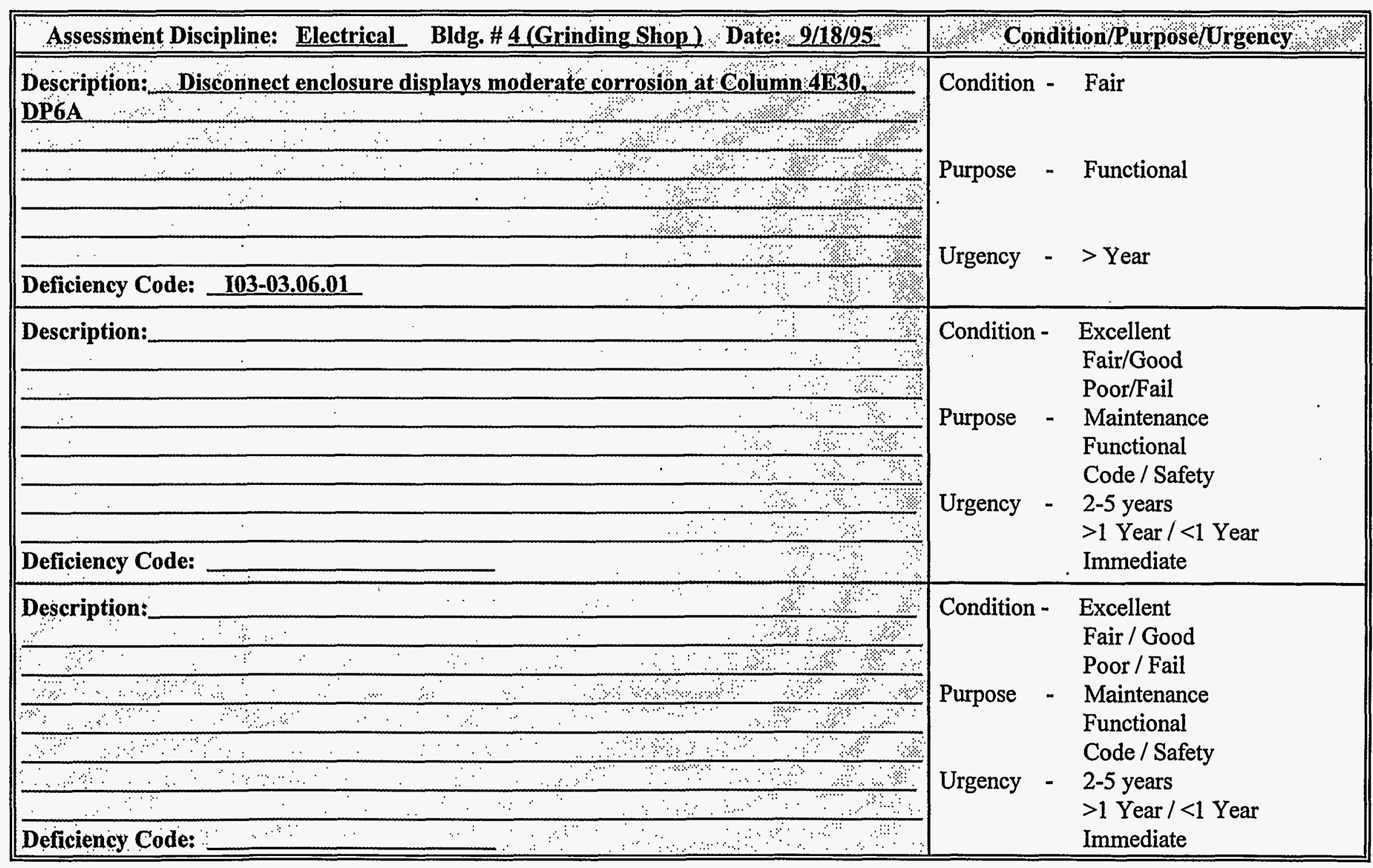




\section{Oxnard Condition Assessment Survey Field Report}

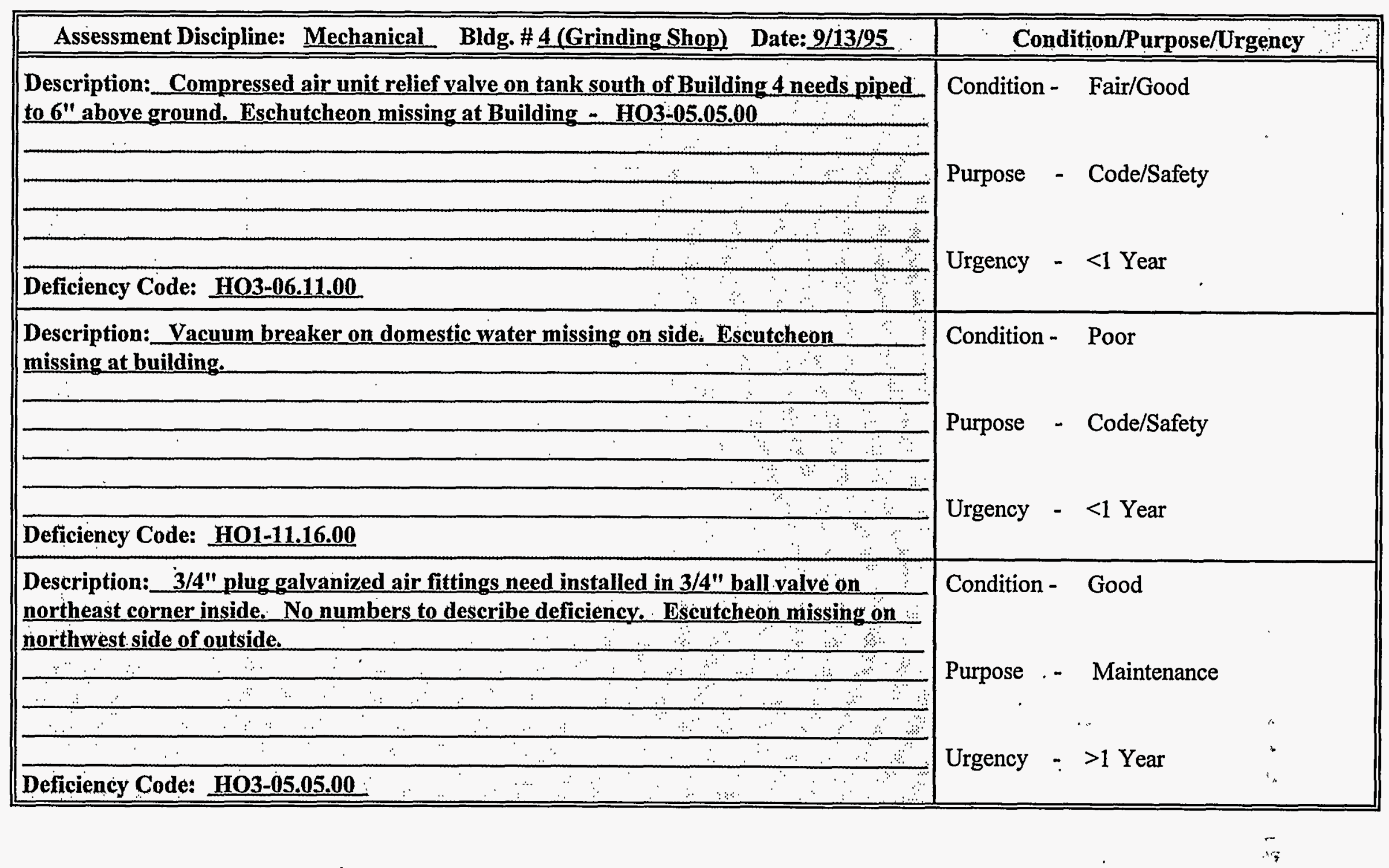




\section{Oxnard Condition Assessment Survey Field Report}

\begin{tabular}{||l|l|l|l|}
\hline Assessment Discipline: Mechanical Bldg. \# 4 (Grinding Shop) Date: 9/13/95 & Condition/Purpose/Urgency \\
\hline \hline Description: Driers and filters on the compressed air unit should not drain to the \\
loor.
\end{tabular}




\section{Oxnard CAS Field Notes}

Building 5 



\section{Oxnard Condition Assessment Survey Field Report}

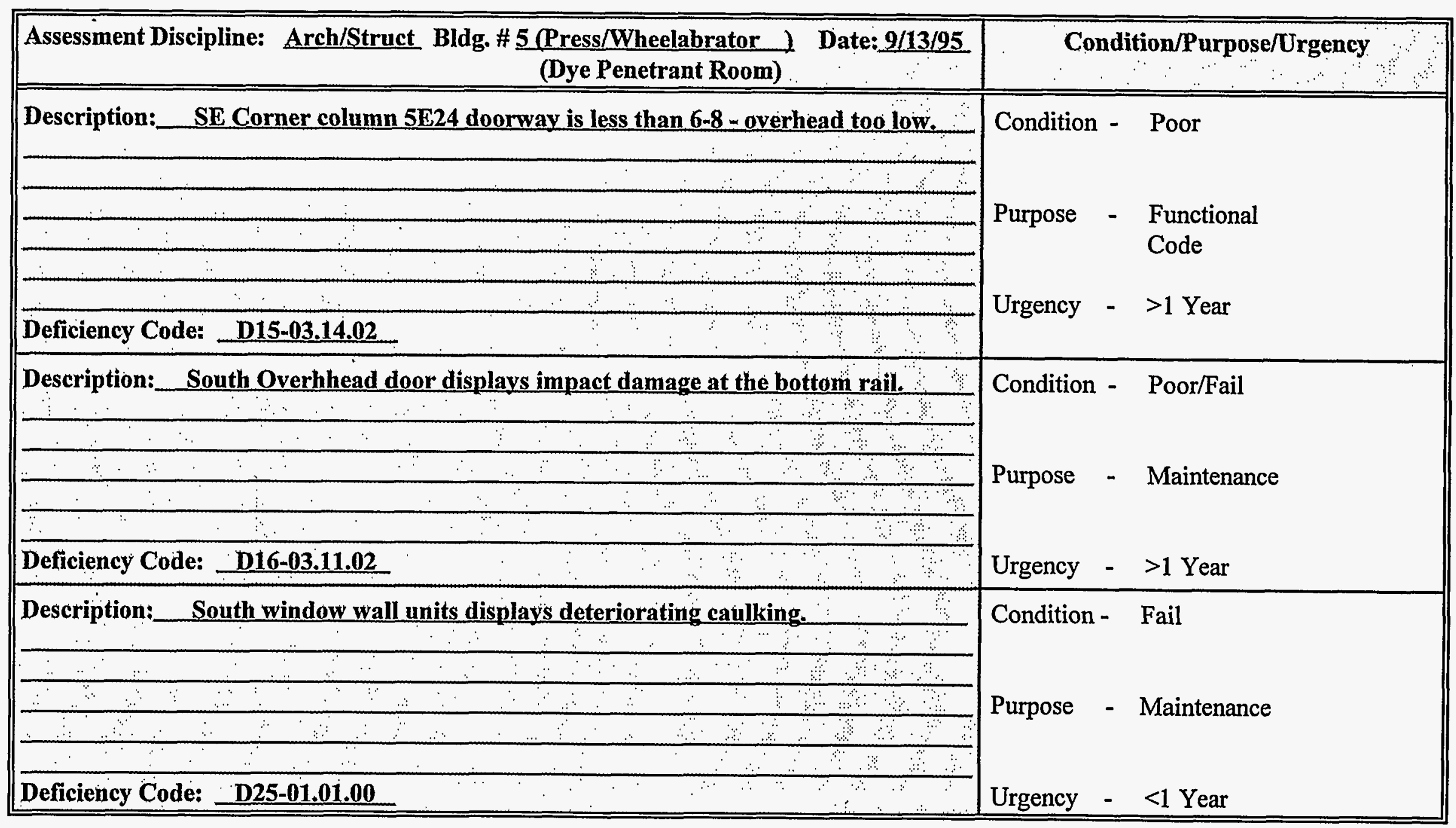




\section{Oxnard Condition Assessment Survey Field Report}

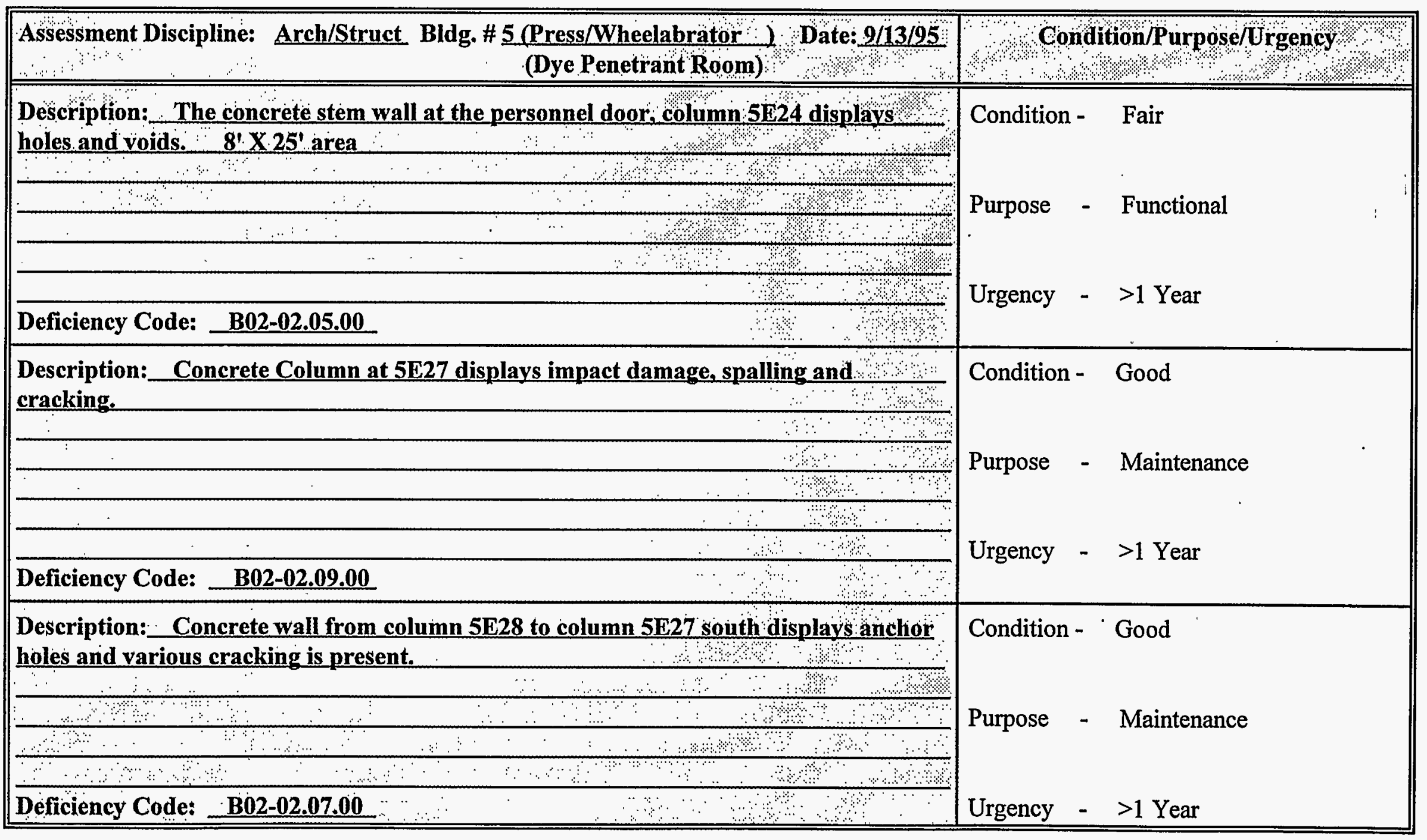




\section{Oxnard Condition Assessment Survey Field Report}

\begin{tabular}{|c|c|c|c|}
\hline Assessment Discipline: Arch/Struct Bldg. \# $\frac{5 \text { (Press/Wheelabrator) }}{\text { (Dye Penetrant Room) }}$ Date: 2/13/95 & \multicolumn{3}{|c|}{ Condition/Purpose/Urgency: } \\
\hline Description: Anchors are missing and concrete is spalling at Column 5E26. & Condition - & \multirow{2}{*}{ Good } & \multirow{5}{*}{-} \\
\hline$\because:$ & \multirow{3}{*}{\multicolumn{2}{|c|}{ Purpose - Maintenance }} & \\
\hline & & & \\
\hline نג & & & \\
\hline Deficiency Code: __ & Urgency & - $>1$ Year & \\
\hline Description: Roof leaking at high area column 5 W26, 5 W27 at stack covering & \multicolumn{2}{|c|}{ Condition - Poor } & \\
\hline 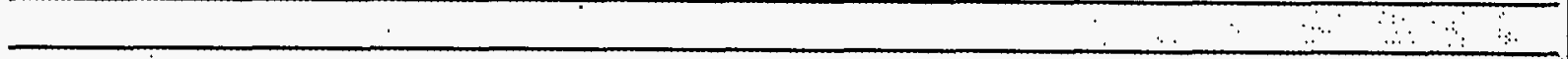 & \multirow{2}{*}{ Purpose } & - Maintenance & \\
\hline$\because \cdots \quad:$ & & & \\
\hline$\because \because \because \quad \because \quad \because$ & \multirow{3}{*}{ Urgency } & & \\
\hline 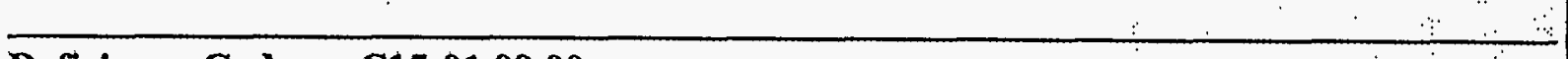 & & \multirow[t]{2}{*}{-. $<1$ Year } & \\
\hline Deficiency Code: $\quad$ C15-01.09.00 & & & \\
\hline Description: Structural steel supports are missing in the steel truss system of this & \multirow[t]{2}{*}{ Condition - } & Poor & , \\
\hline bulloing at column $5 E 26$ thru $5 \mathrm{H} 24$ across entire span. & & & H \\
\hline$\because \quad \because \quad \because$ & \multirow{3}{*}{ Purpose } & \multirow{2}{*}{$\begin{array}{l}\text { - Functional } \\
\text { Code / Safety }\end{array}$} & \\
\hline$\therefore \quad \because \quad \because$ & & & \\
\hline$\because \quad \because$ & & & $:$ \\
\hline$\therefore \quad \therefore \quad \because \quad \because \quad \because$ & \multirow[t]{2}{*}{ Urgency } & \multirow{2}{*}{$-\quad<1$ Year } & $\because$ \\
\hline Deficiency Code: C05-01.04.00 & & & $\because$ \\
\hline
\end{tabular}




\section{Oxnard Condition Assessment Survey Field Report}

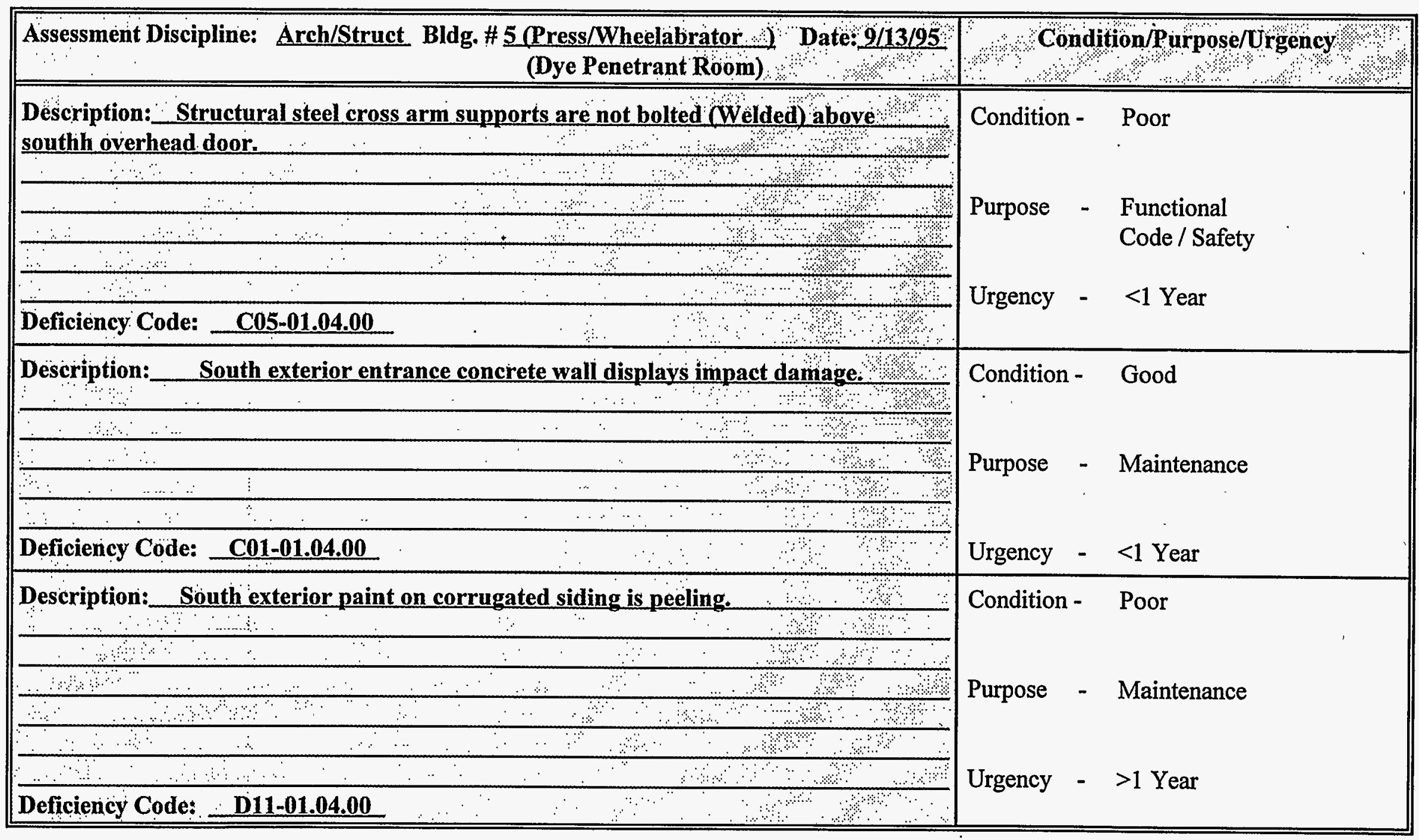




\section{Oxnard Condition Assessment Survey Field Report}

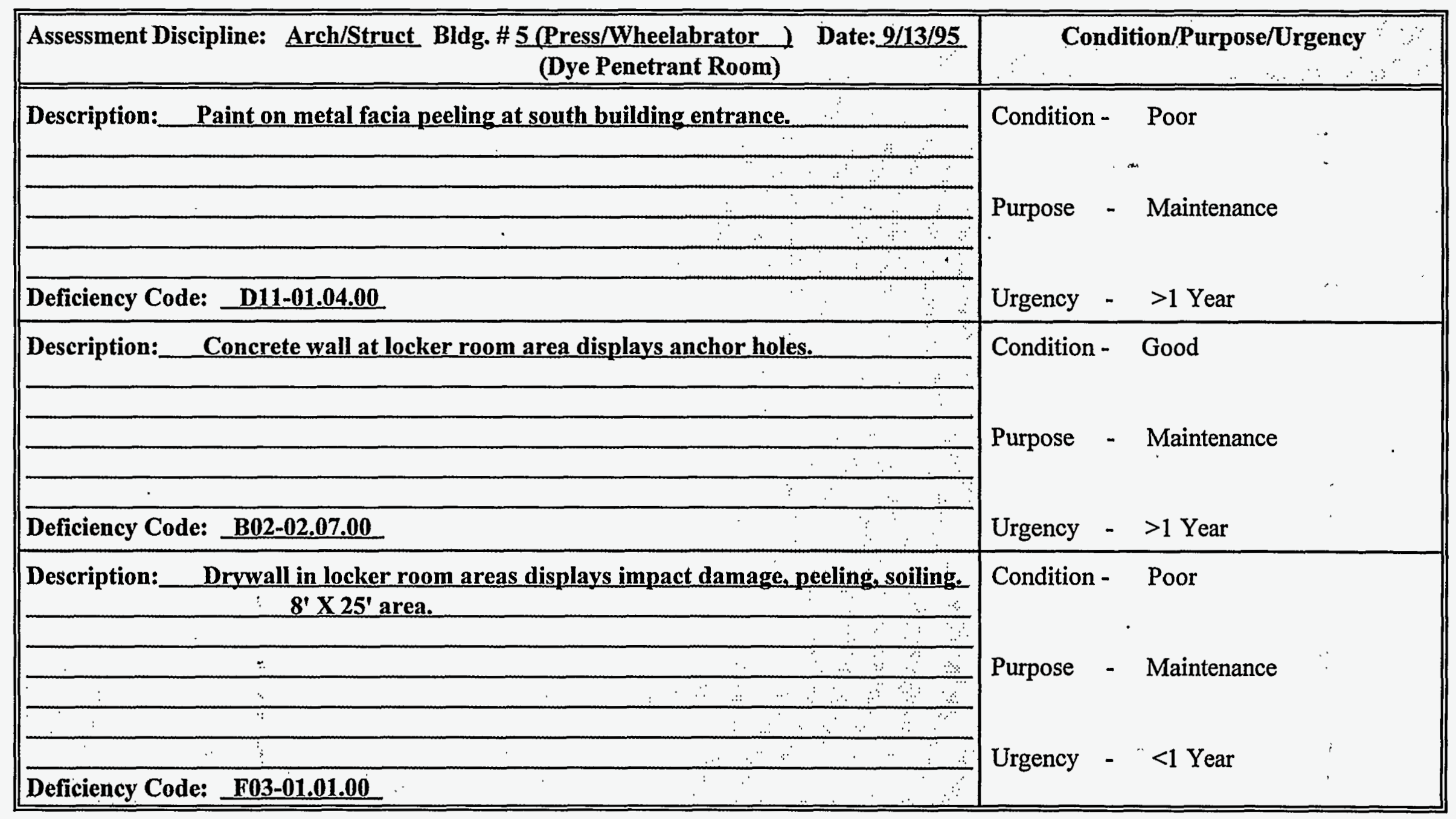




\section{Oxnard Condition Assessment Survey Field Report}

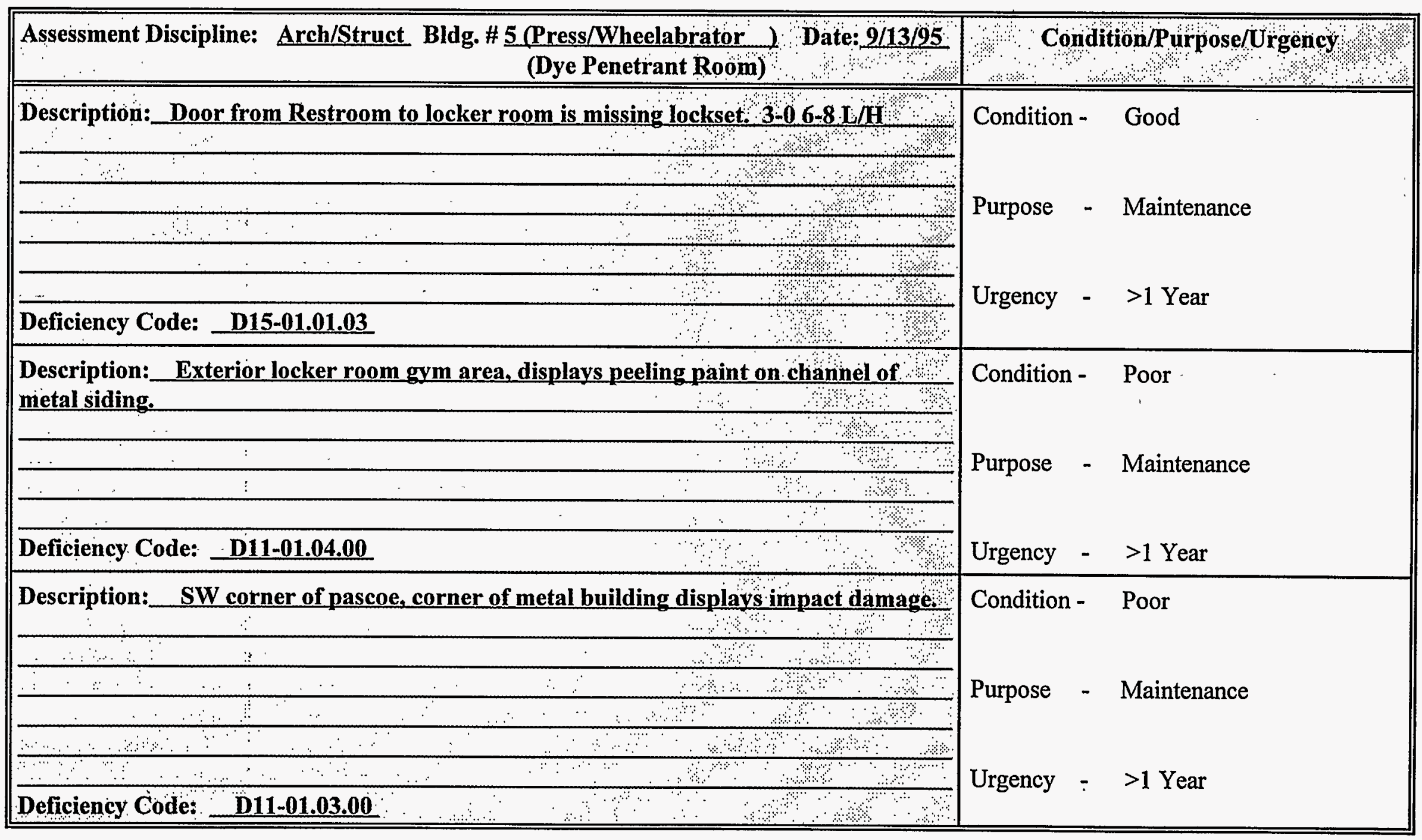




\section{Oxnard Condition Assessment Survey Field Report}

\begin{tabular}{|c|c|c|c|}
\hline 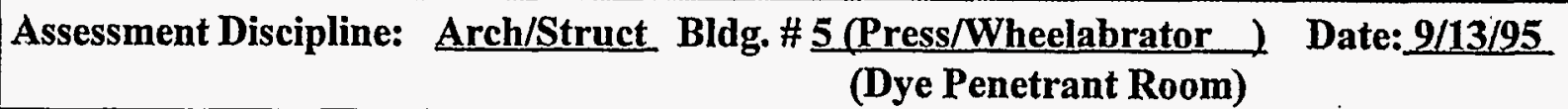 & \multicolumn{3}{|c|}{ Condition/Purpose/Urgency } \\
\hline Description: West exterior sliding door displays impact damage. & \multirow{2}{*}{\multicolumn{2}{|c|}{$\begin{array}{l}\text { Condition - Good } \\
\text { Purpose - Maintenance }\end{array}$}} & \multirow[b]{3}{*}{ - } \\
\hline & & & \\
\hline Deficiency Code: $\quad$ D21-03.11.02 & \multicolumn{2}{|c|}{ Urgency $->1$ Year } & \\
\hline Description: West side exterior is soiled and rusted from cleaner usage. & \multirow{2}{*}{\multicolumn{2}{|c|}{$\begin{array}{l}\text { Condition - Good } \\
\text { Purpose - Maintenance }\end{array}$}} & \\
\hline 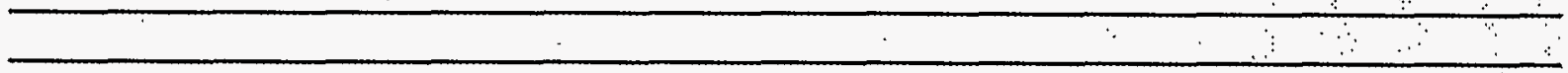 & & & \\
\hline & \multirow{2}{*}{ Urgency } & & \\
\hline Deficiency Code: D11-01.01.00 & & $>1$ Year & \\
\hline Description: Concrete floor throughout is displaying cracks. & \multirow{2}{*}{\multicolumn{2}{|c|}{ Condition - Good }} & . \\
\hline$x$ & & & $\therefore$ \\
\hline$\therefore \quad \therefore \quad 1 \quad$ & \multirow{2}{*}{\multicolumn{2}{|c|}{ Purpose - Functional }} & \\
\hline$\therefore \quad \therefore \quad \therefore \quad$ & & & $\ldots$ \\
\hline Deficiency Code: $\quad$ C10-01.05.06 & Urgency - & $>1$ Year & \\
\hline
\end{tabular}




\section{Oxnard Condition Assessment Survey Field Report}

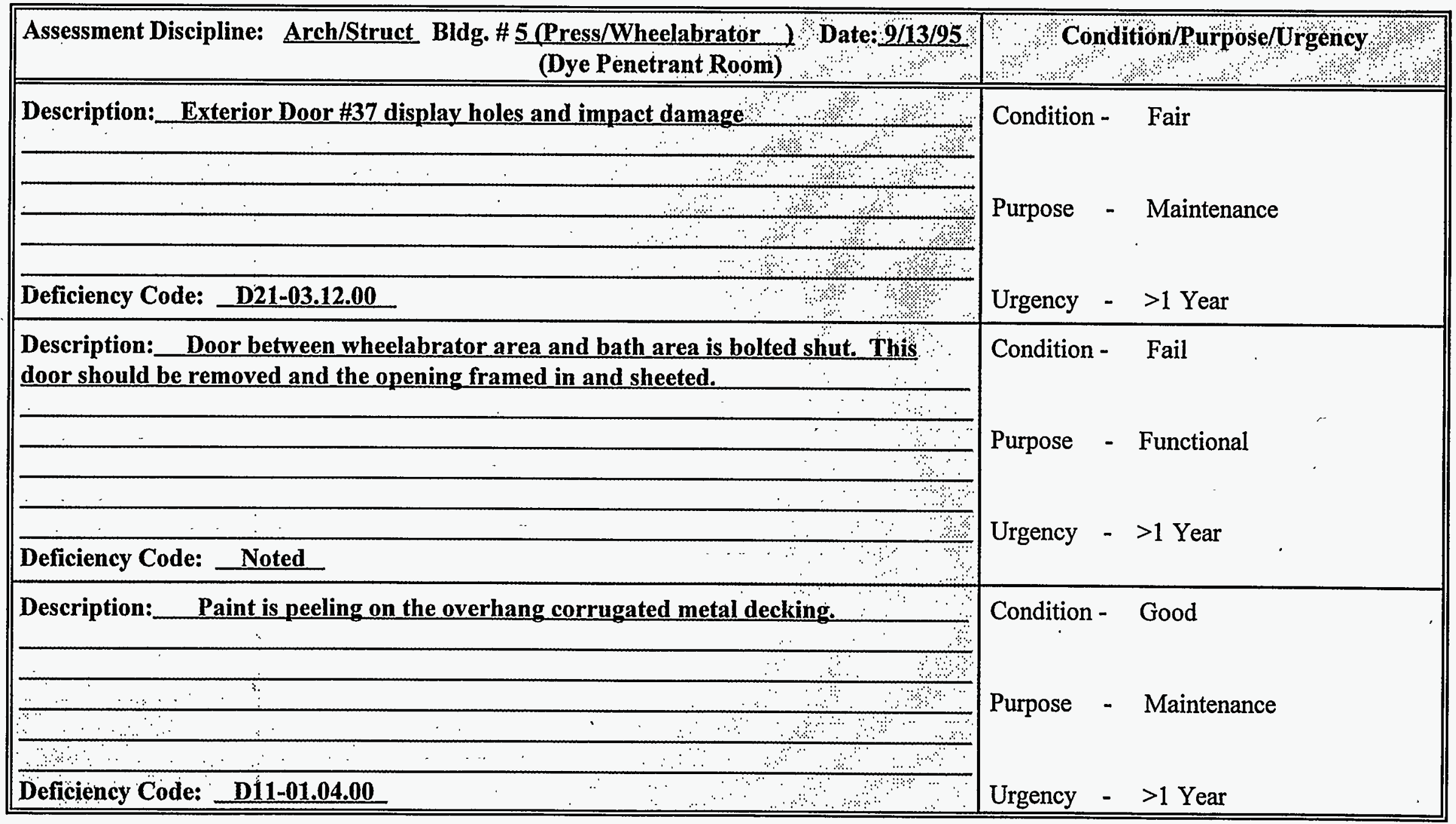




\section{Oxnard Condition Assessment Survey Field Report}

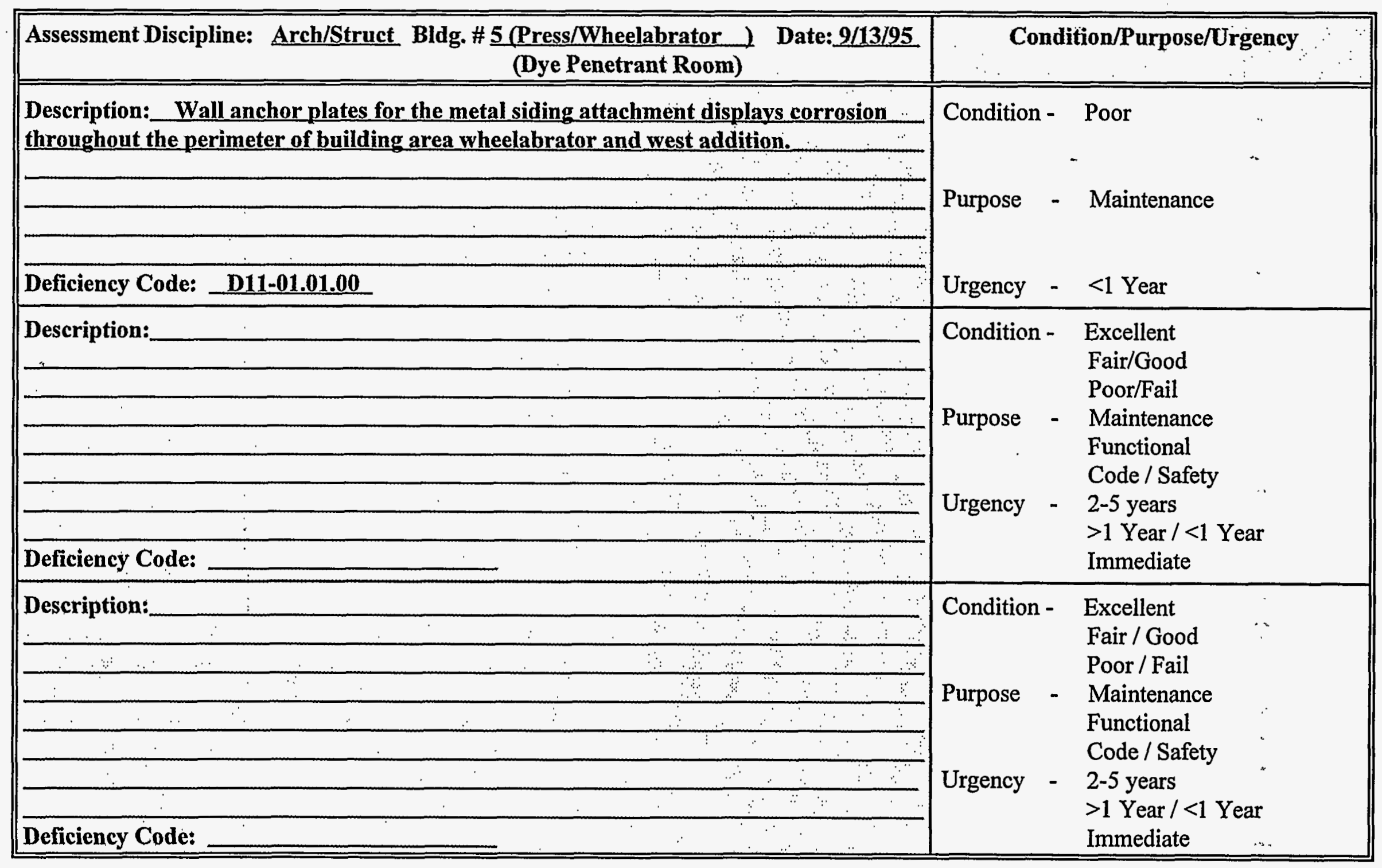




\section{Oxnard Condition Assessment Survey Field Report}

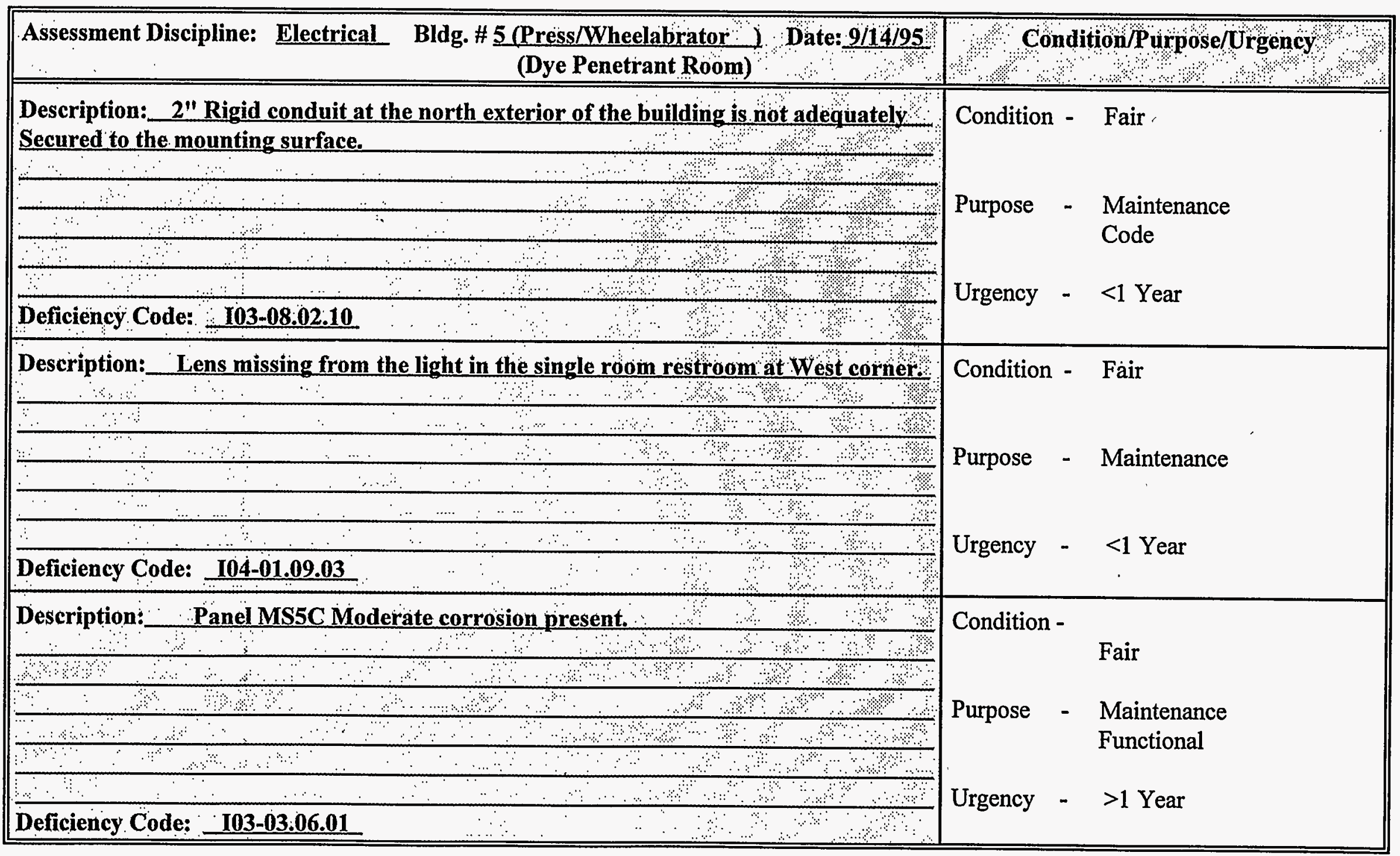




\section{Oxnard Condition Assessment Survey Field Report}

\begin{tabular}{|c|c|c|c|}
\hline Assessment Discipline: Electrical Bldg. \# $\underline{5 \text { (Press/Wheelabrator ) Date: } 9 / 14 / 95}$ & \multicolumn{3}{|c|}{ Condition/Purpose/Urgency } \\
\hline $\begin{array}{l}\text { Description: } 4 \text { " X 4" Gutter is without support and displays heavy corrosion at the } \\
\text { south east awning between buildings } 5 \text { and } 6 \text {. }\end{array}$ & \multirow{2}{*}{\multicolumn{2}{|c|}{$\begin{array}{l}\text { Condition - Fair } \\
\text { Purpose - Maintenance }\end{array}$}} & \multirow[t]{2}{*}{$\cdots$} \\
\hline & & & \\
\hline & \multirow[t]{2}{*}{ Urgency } & \multirow[t]{2}{*}{ - $>1$ Year } & \\
\hline Deficiency Code: $\mathbf{I 0 3 - 0 8 . 0 2 . 0 7}$ & & & \\
\hline $\begin{array}{l}\text { Description: Unit \#1463 at south east side (exterior) under awning, the receptacle } \\
\text { box is not GFCI protected. }\end{array}$ & \multirow{3}{*}{\multicolumn{2}{|c|}{$\begin{array}{l}\text { Condition - Poor } \\
\text { Purpose - Code / Safety }\end{array}$}} & \\
\hline & & & \\
\hline ; & & & \\
\hline 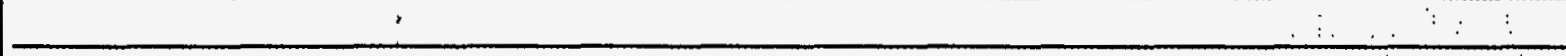 & \multirow[t]{2}{*}{ Urgency } & \multirow[t]{2}{*}{ - $\quad<1$ Year } & \\
\hline Deficiency Code: $\quad$ I16-01.03.00 & & & \\
\hline $\begin{array}{l}\text { Description: South east under awning, } 2 \text { exterior outlets }(6 \mathrm{~B} 10) \text { are without GFCI } \\
\text { Protection. }\end{array}$ & \multirow{3}{*}{$\begin{array}{l}\text { Condition } \\
\text { Purpose } \\
\text { Urgency }\end{array}$} & \multirow{3}{*}{$\begin{array}{l}-\quad \text { Poor } \\
-\quad \text { Code / Safety } \\
-\quad<1 \text { Year }\end{array}$} & \multirow{3}{*}{$\cdots$} \\
\hline & & & \\
\hline Deficiency Code: I16-01.03.00 & & & \\
\hline
\end{tabular}




\section{Oxnard Condition Assessment Survey Field Report}

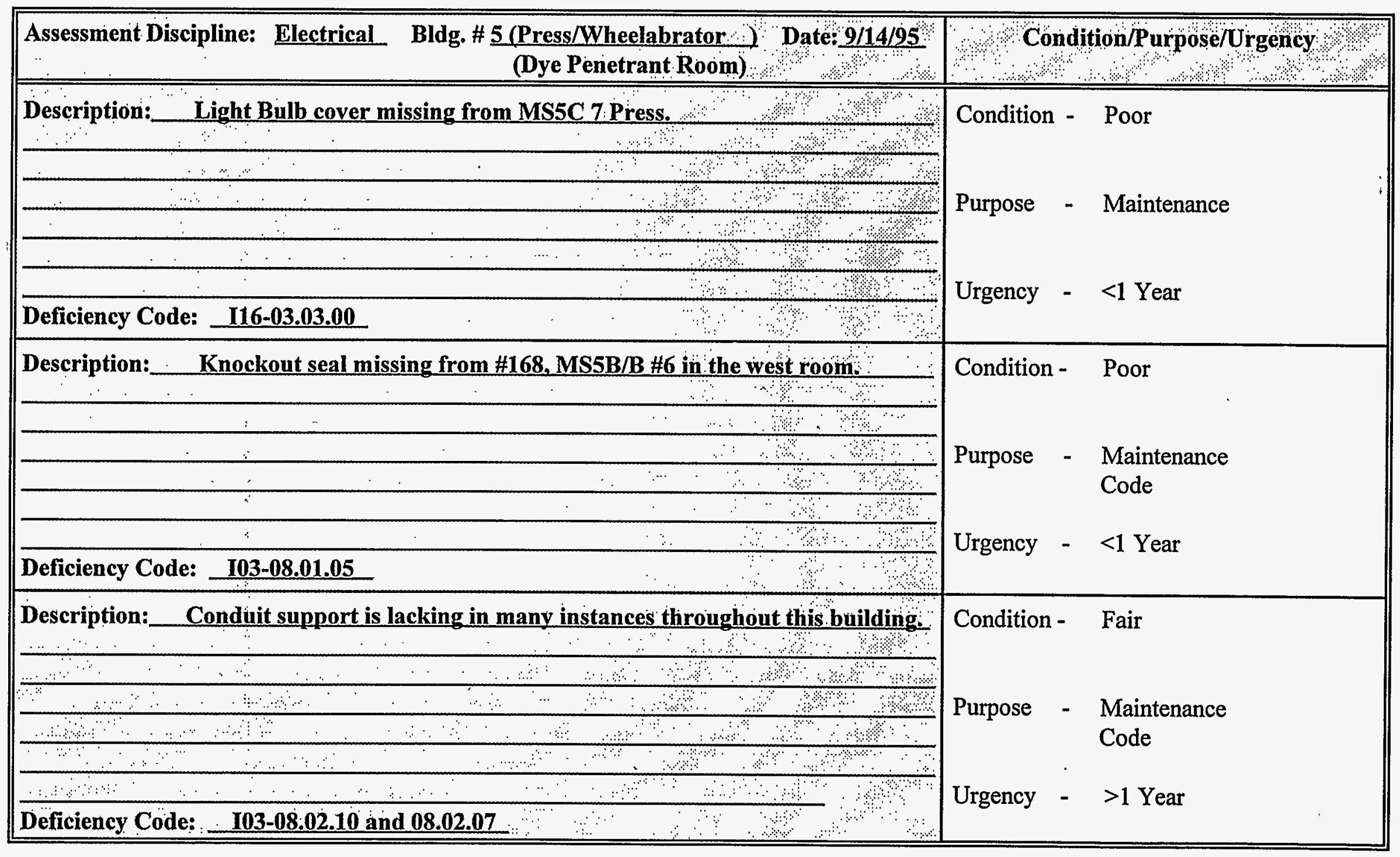




\section{Oxnard Condition Assessment Survey Field Report}

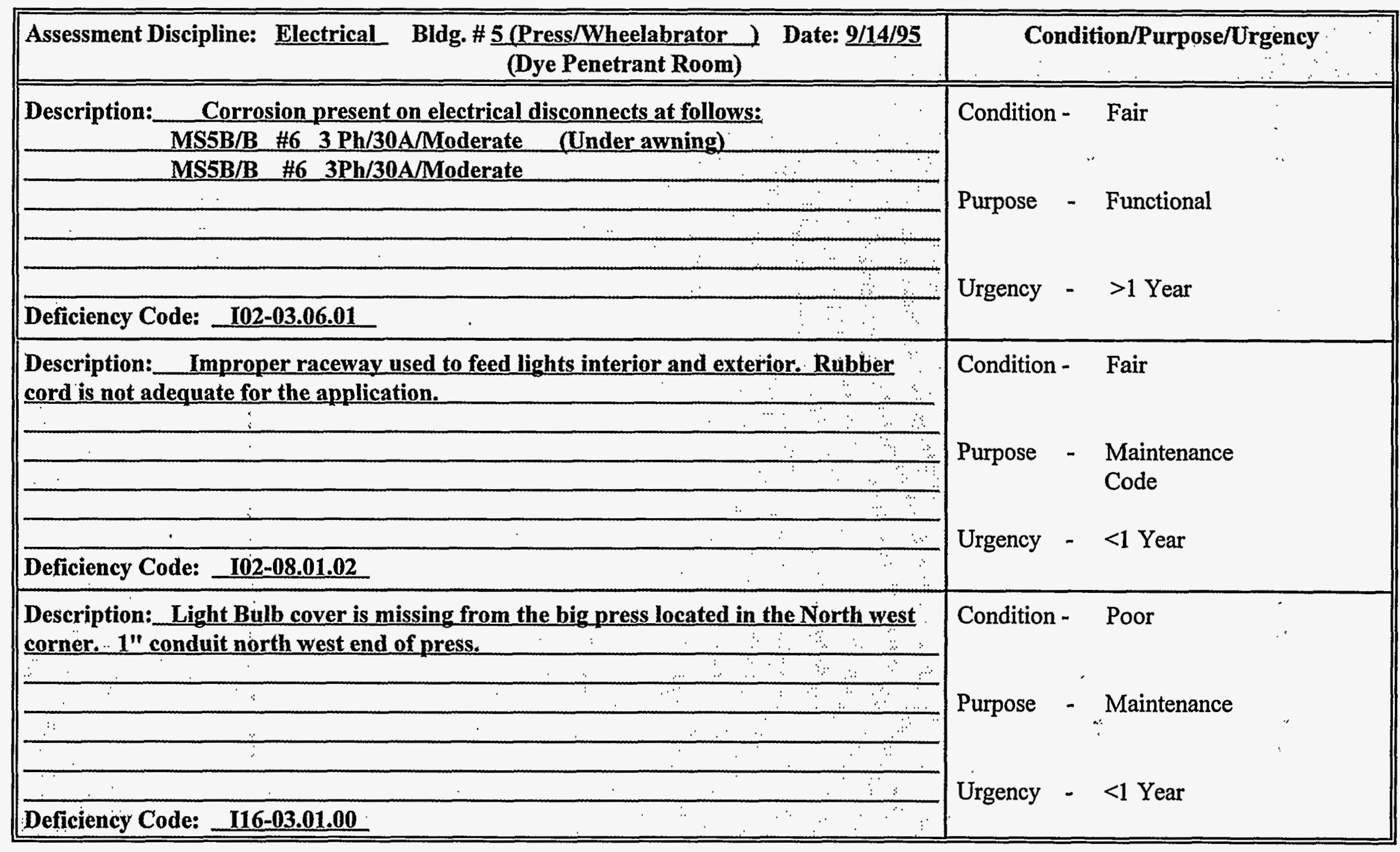




\section{Oxnard Condition Assessment Survey Field Report}

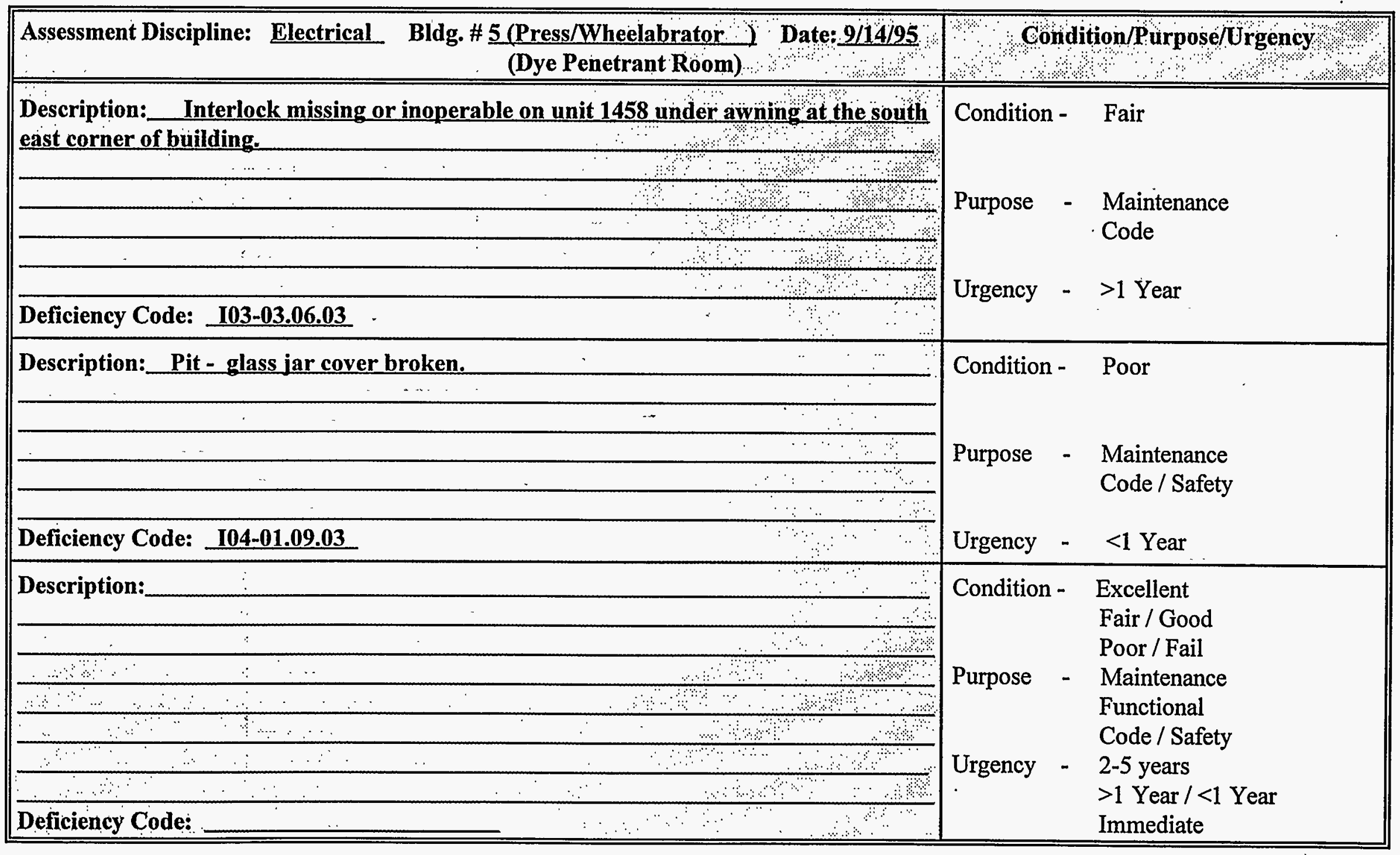




\section{Oxnard Condition Assessment Survey Field Report}

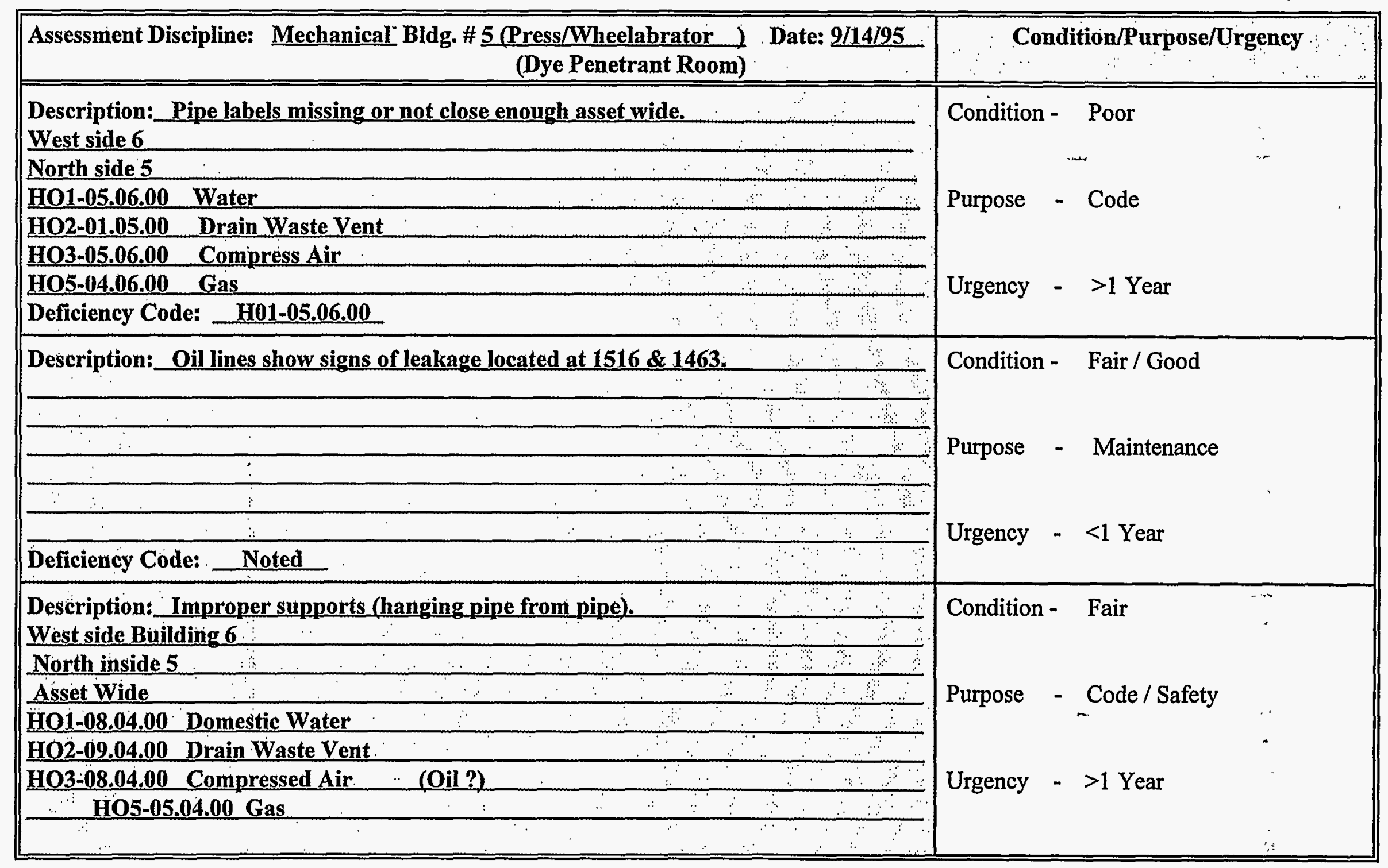




\section{Oxnard Condition Assessment Survey Field Report}

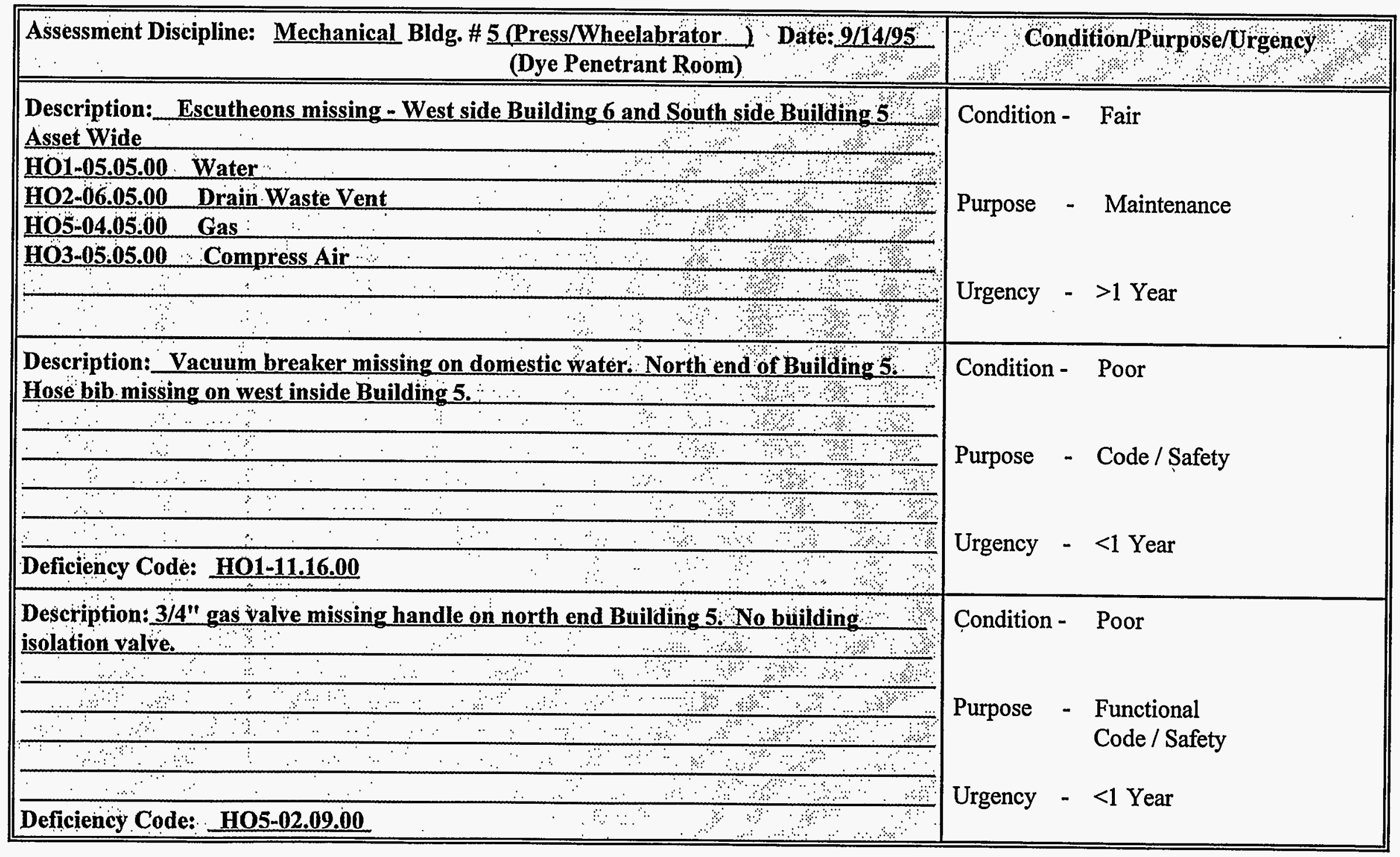




\section{Oxnard Condition Assessment Survey Field Report}

\begin{tabular}{|c|c|}
\hline 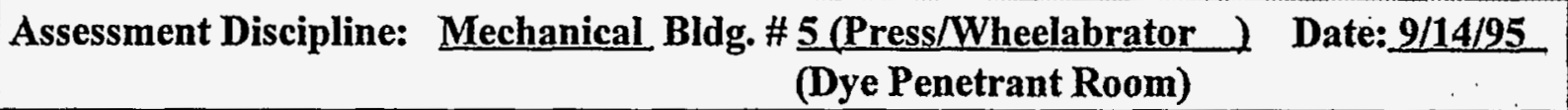 & Condition/Purpose/Urgency \\
\hline $\begin{array}{l}\text { Description: } 11 / 2^{\prime \prime} \text { brass gate valve on compressed air unit missing handle. West wall } \\
\text { inside Building } 5 \text {. }\end{array}$ & \multirow{3}{*}{$\begin{array}{ll}\text { Purpose } & -\begin{array}{l}\text { Functional } \\
\text { Code / Safety }\end{array} \\
\text { Urgency - } & <1 \text { Year }\end{array}$} \\
\hline & \\
\hline Deficiency Code: HO3-06.10.00 & \\
\hline Description:_Compressed air filters and driers drain to floor. & Condition - Fair / Poor \\
\hline & \multirow{3}{*}{$\begin{array}{l}\text { Purpose - Safety } \\
\text { Urgency - }<1 \text { Year }\end{array}$} \\
\hline & \\
\hline Deficiency Code: HO3-04.07.00 & \\
\hline Description: Strong gas smell is sometimes present in this building. & Condition - Poor / Fail \\
\hline$\because \because$ & \multirow{3}{*}{ Purpose - Safety } \\
\hline$\because$ & \\
\hline 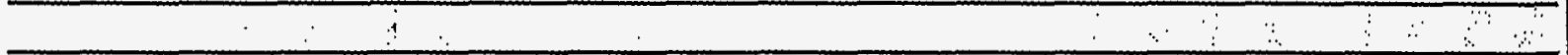 & \\
\hline 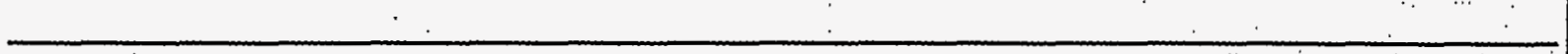 & \multirow{2}{*}{$\begin{array}{ll}\text { Urgency }- & <1 \text { Year } \\
& \text { Immediate } \\
\end{array}$} \\
\hline Deficiency Code: Concern Noted & \\
\hline
\end{tabular}




\section{Oxnard Condition Assessment Survey Field Report}

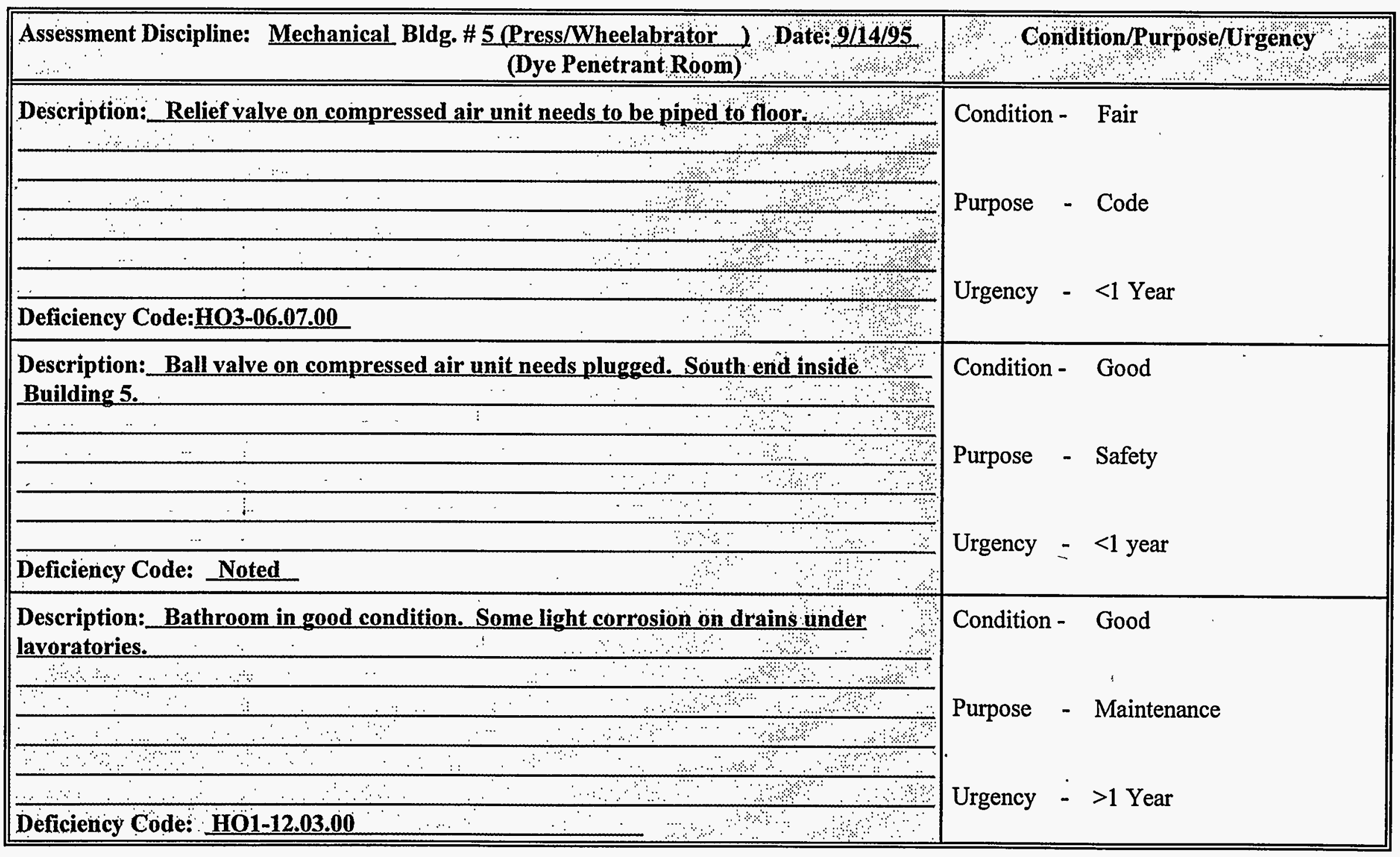


Oxnard CAS Field Notes

Building 6 



\section{Oxnard Condition Assessment Survey Field Report}

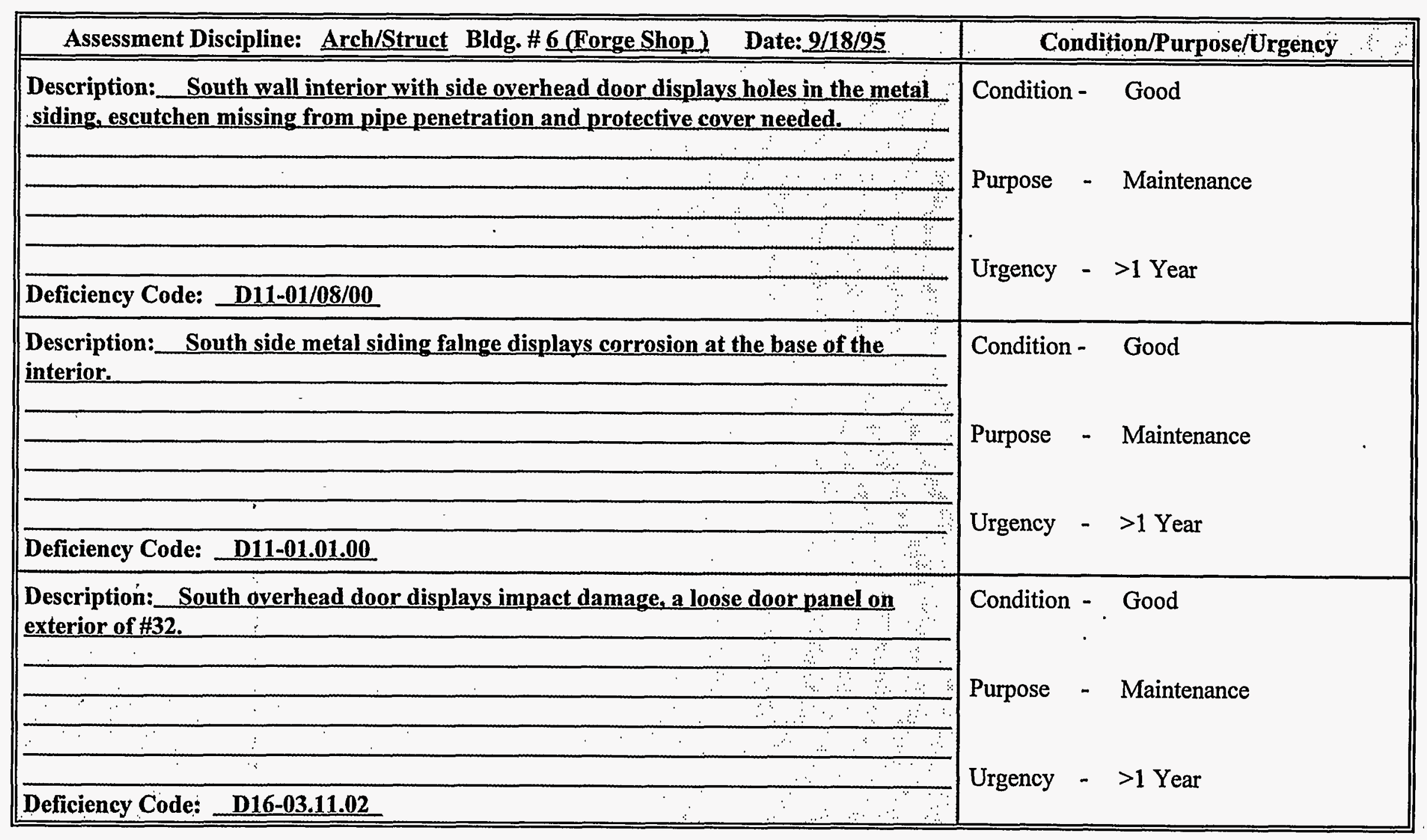




\section{Oxnard Condition Assessment Survey Field Report}

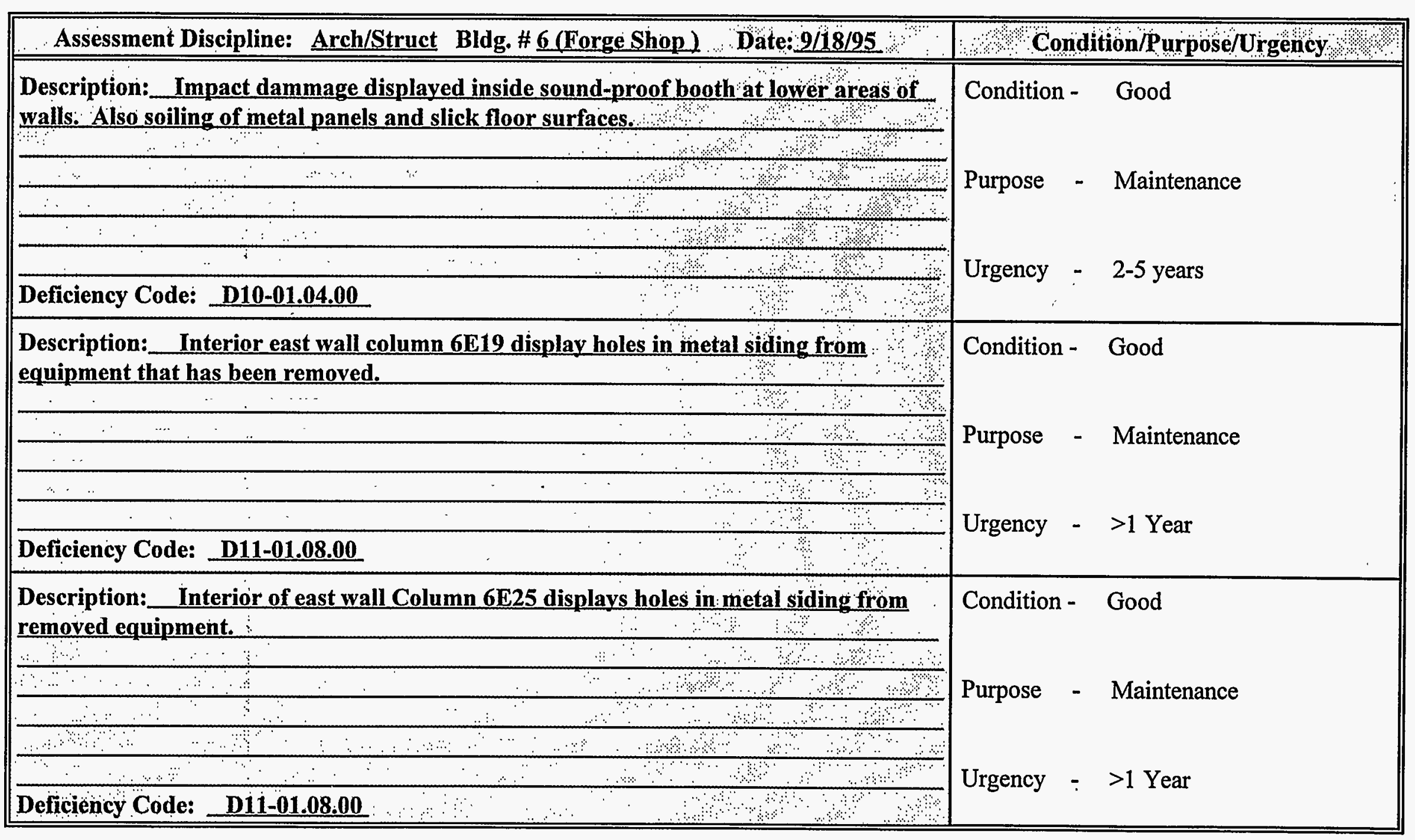




\section{Oxnard Condition Assessment Survey Field Report}

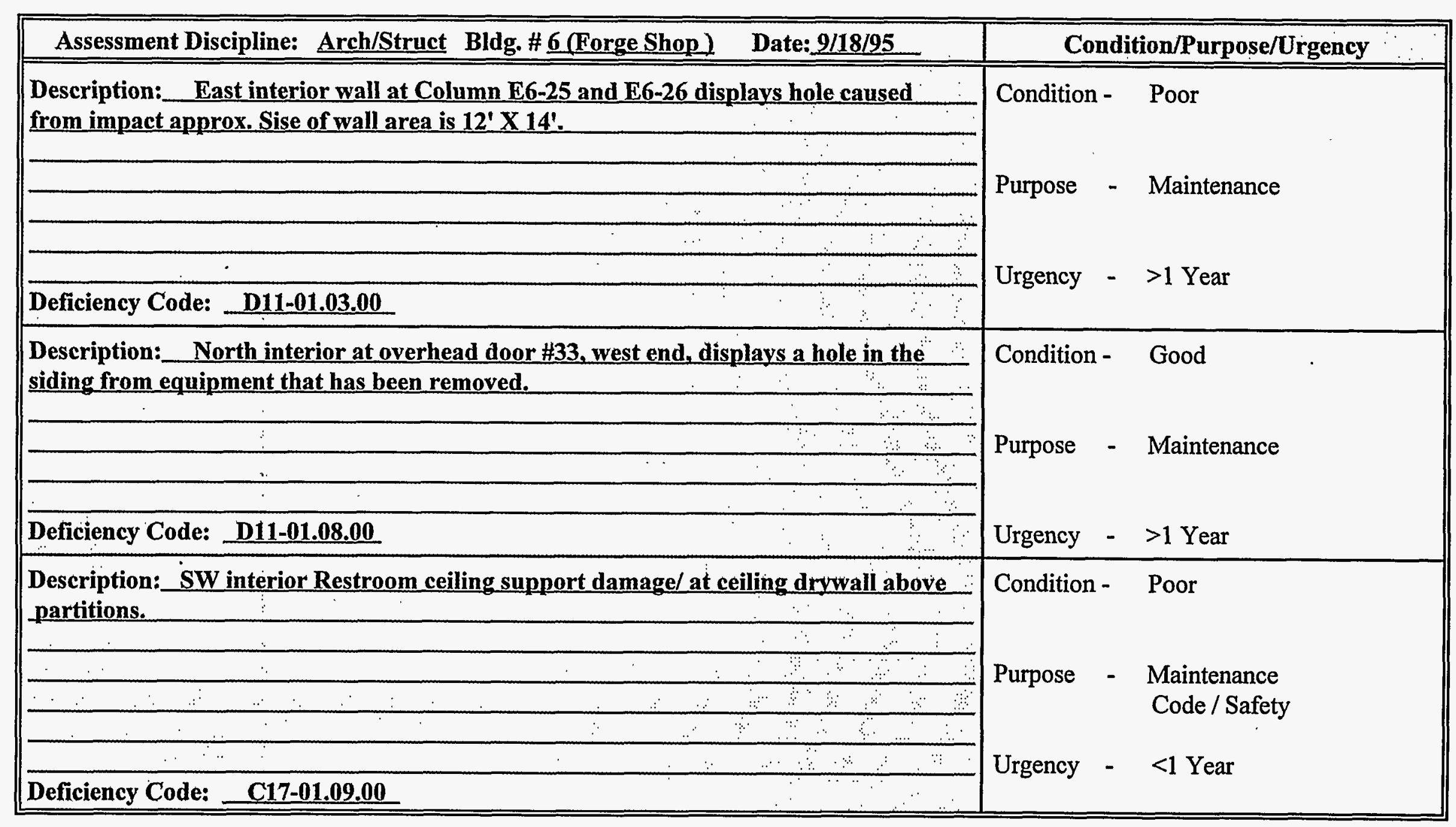




\section{Oxnard Condition Assessment Survey Field Report}

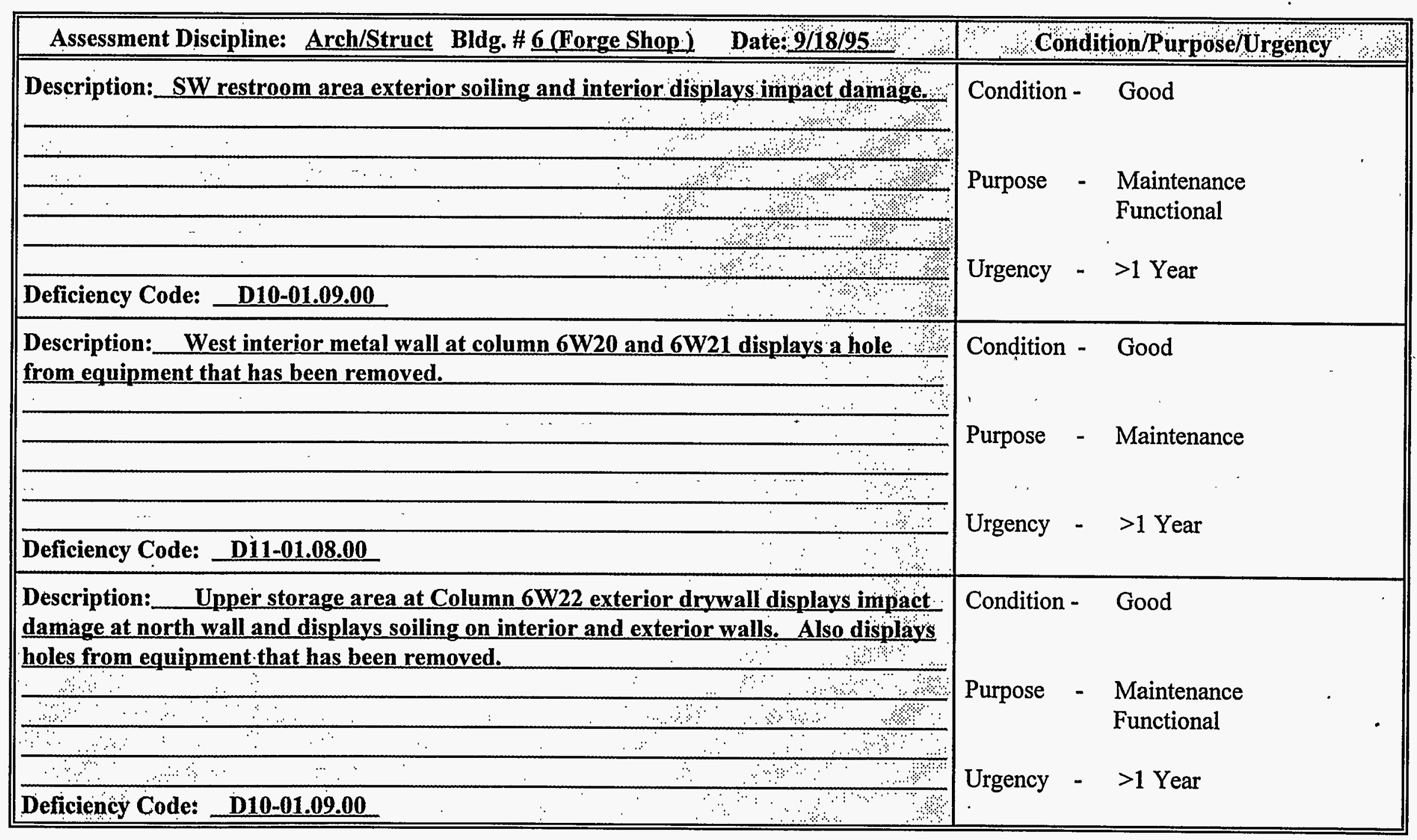




\section{Oxnard Condition Assessment Survey Field Report}

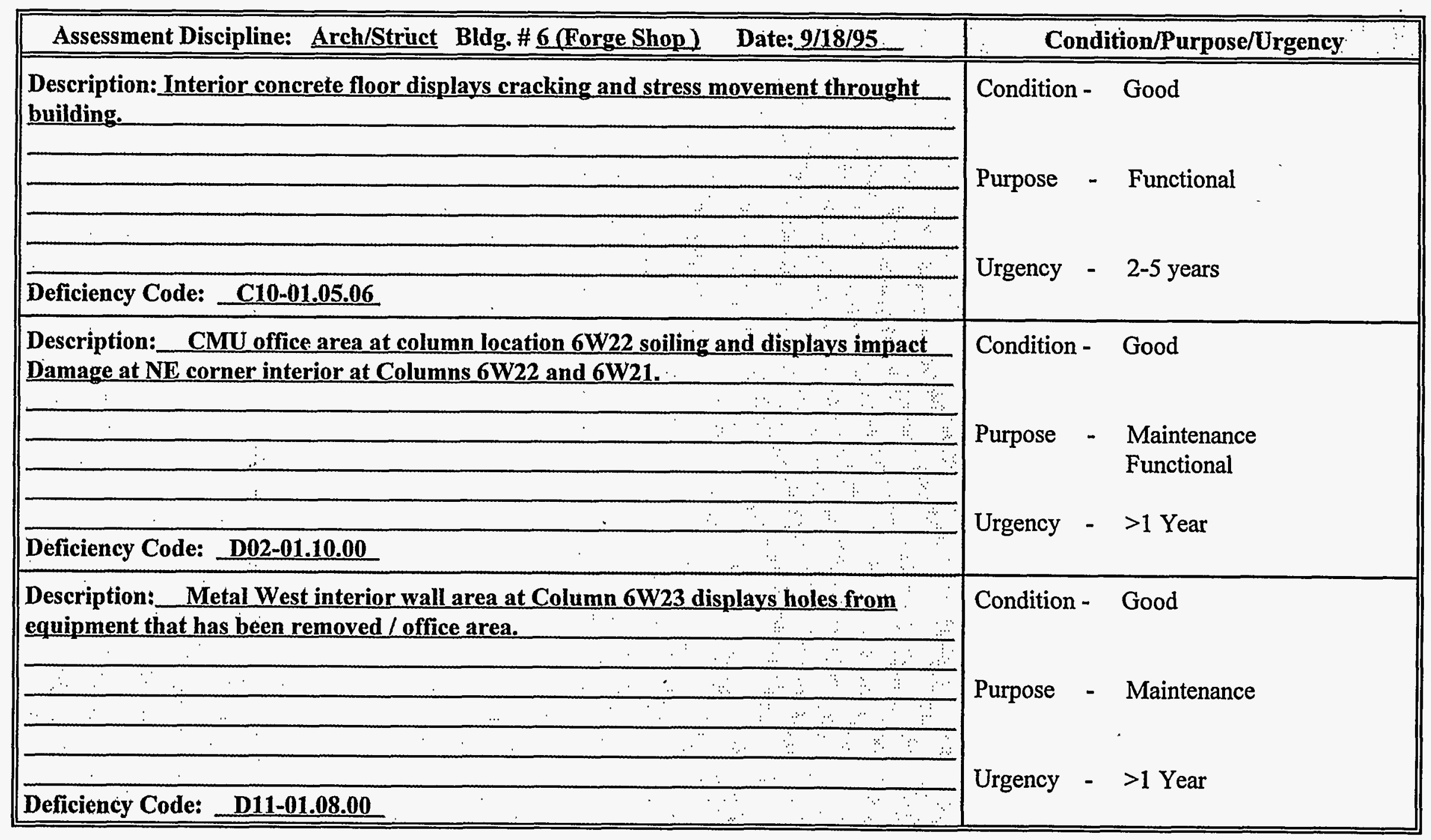




\section{Oxnard Condition Assessment Survey Field Report}

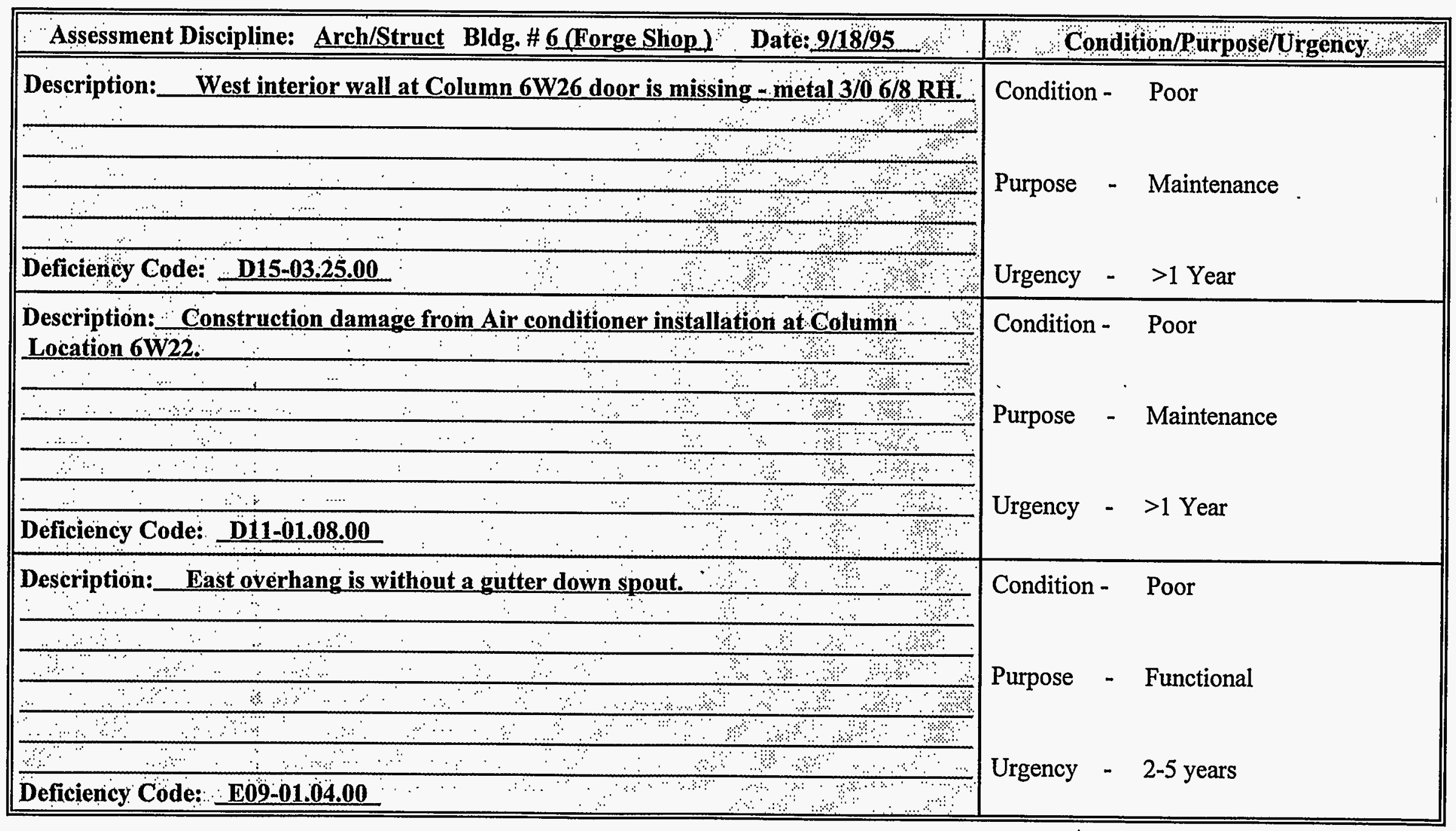




\section{Oxnard Condition Assessment Survey Field Report}

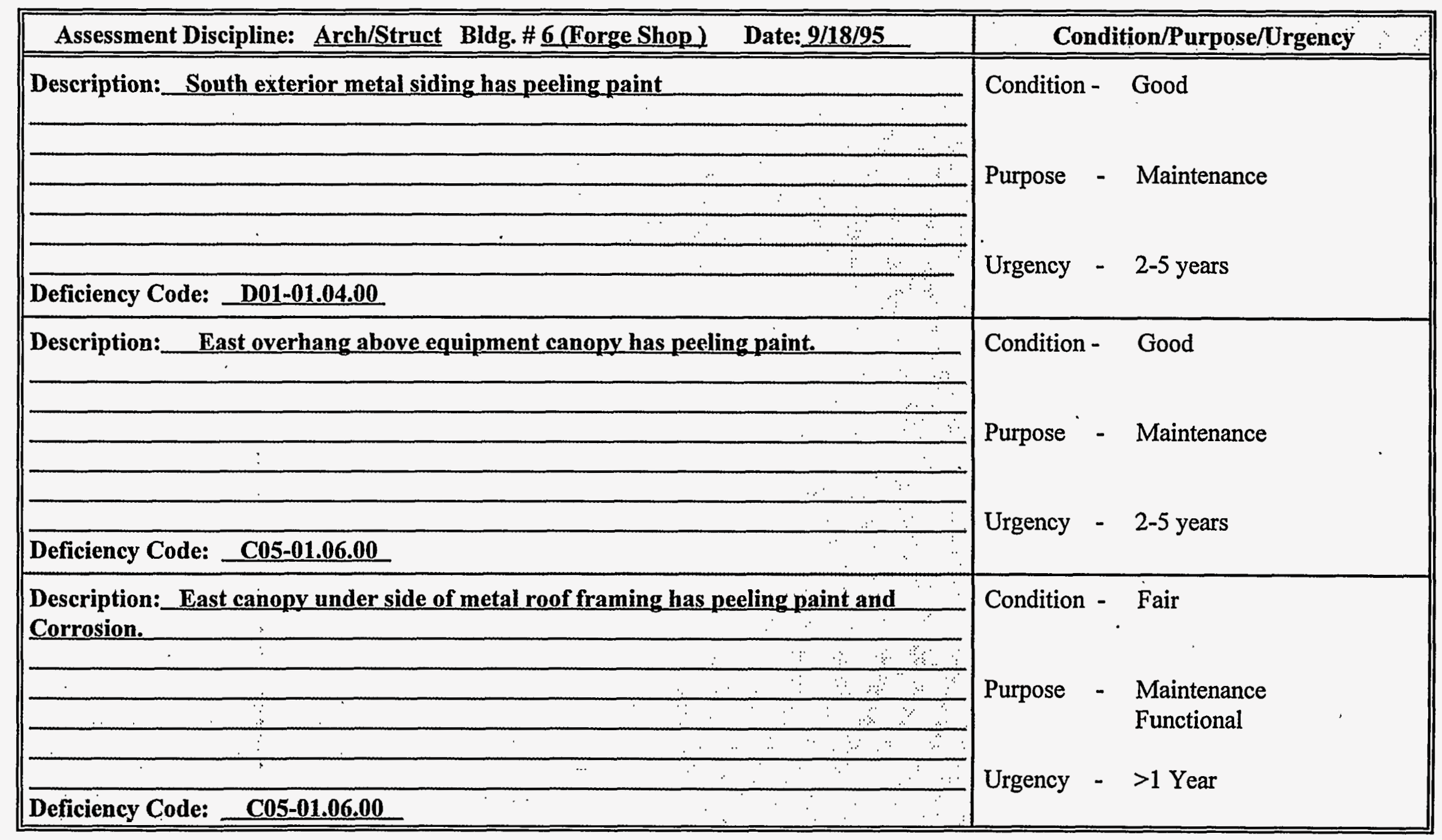




\section{Oxnard Condition Assessment Survey Field Report}

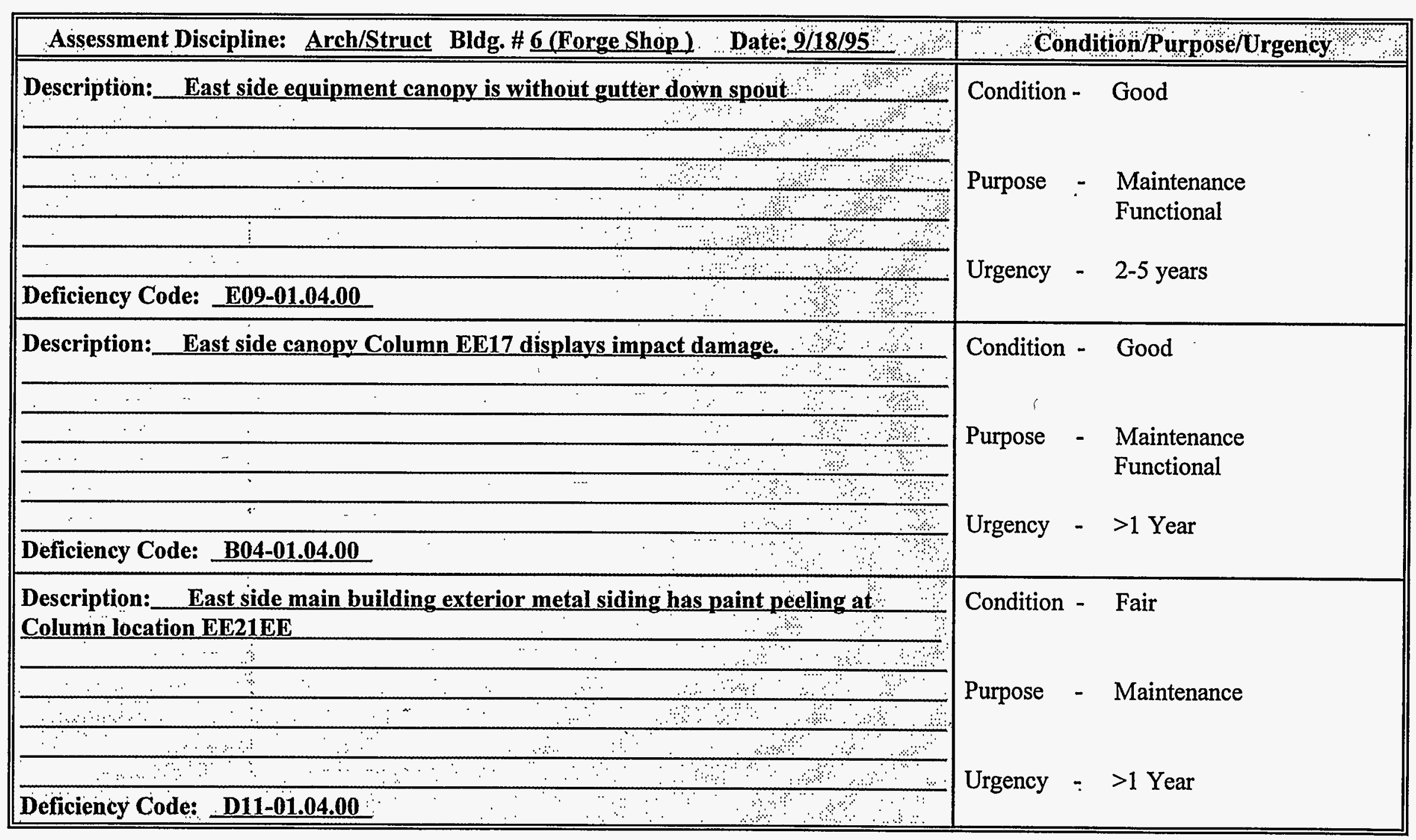




\section{Oxnard Condition Assessment Survey Field Report}

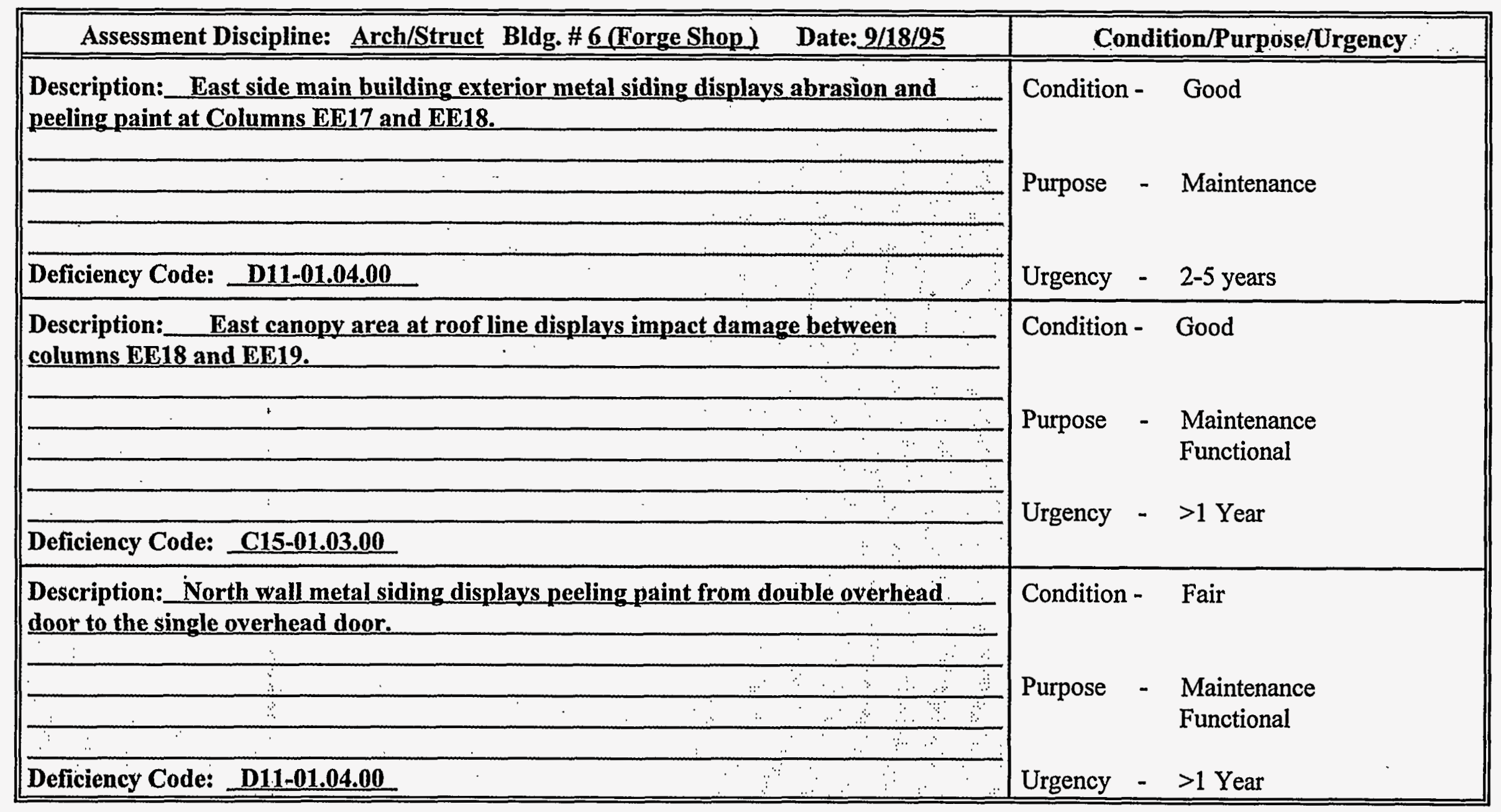




\section{Oxnard Condition Assessment Survey Field Report}

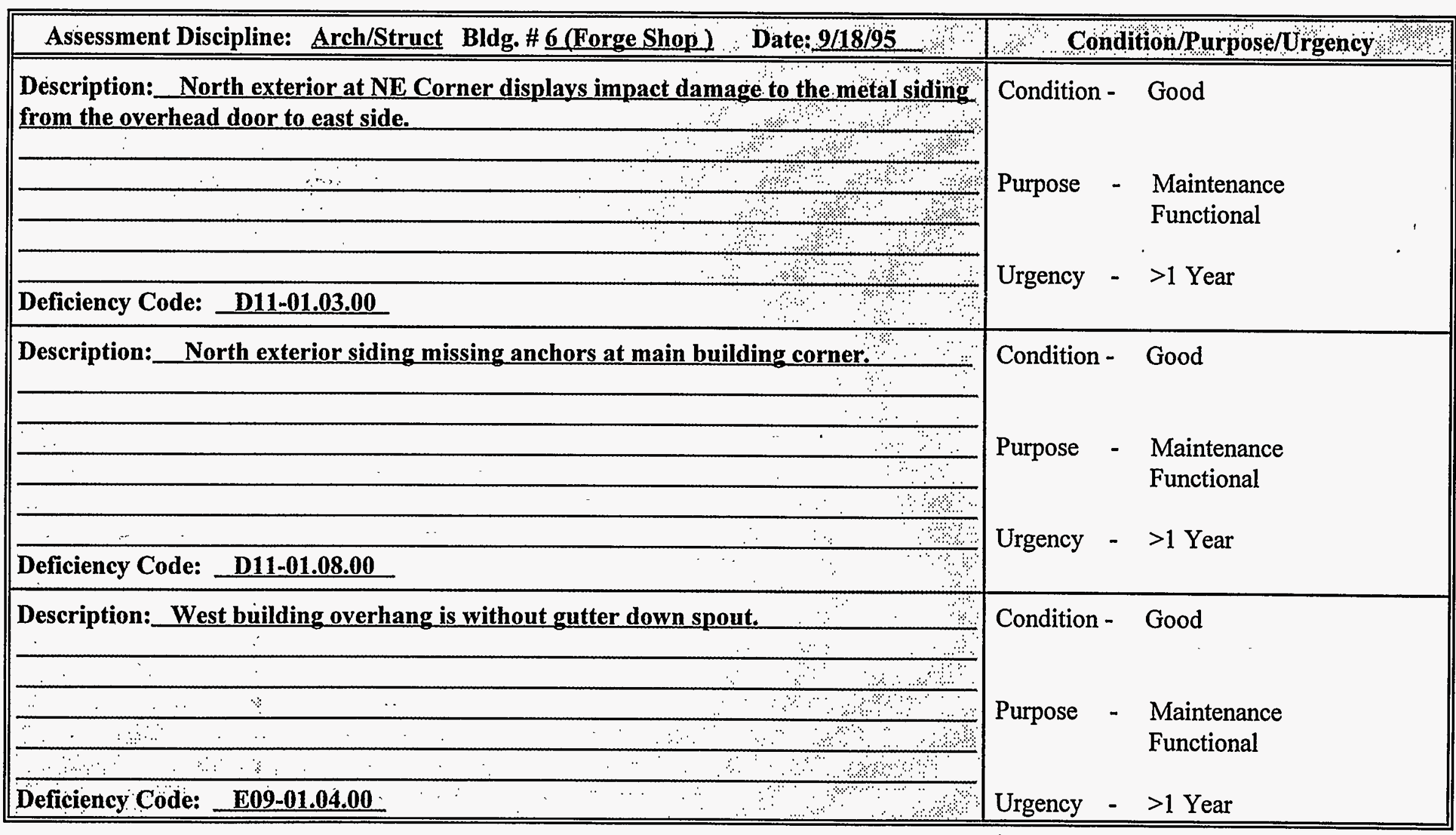




\section{Oxnard Condition Assessment Survey Field Report}

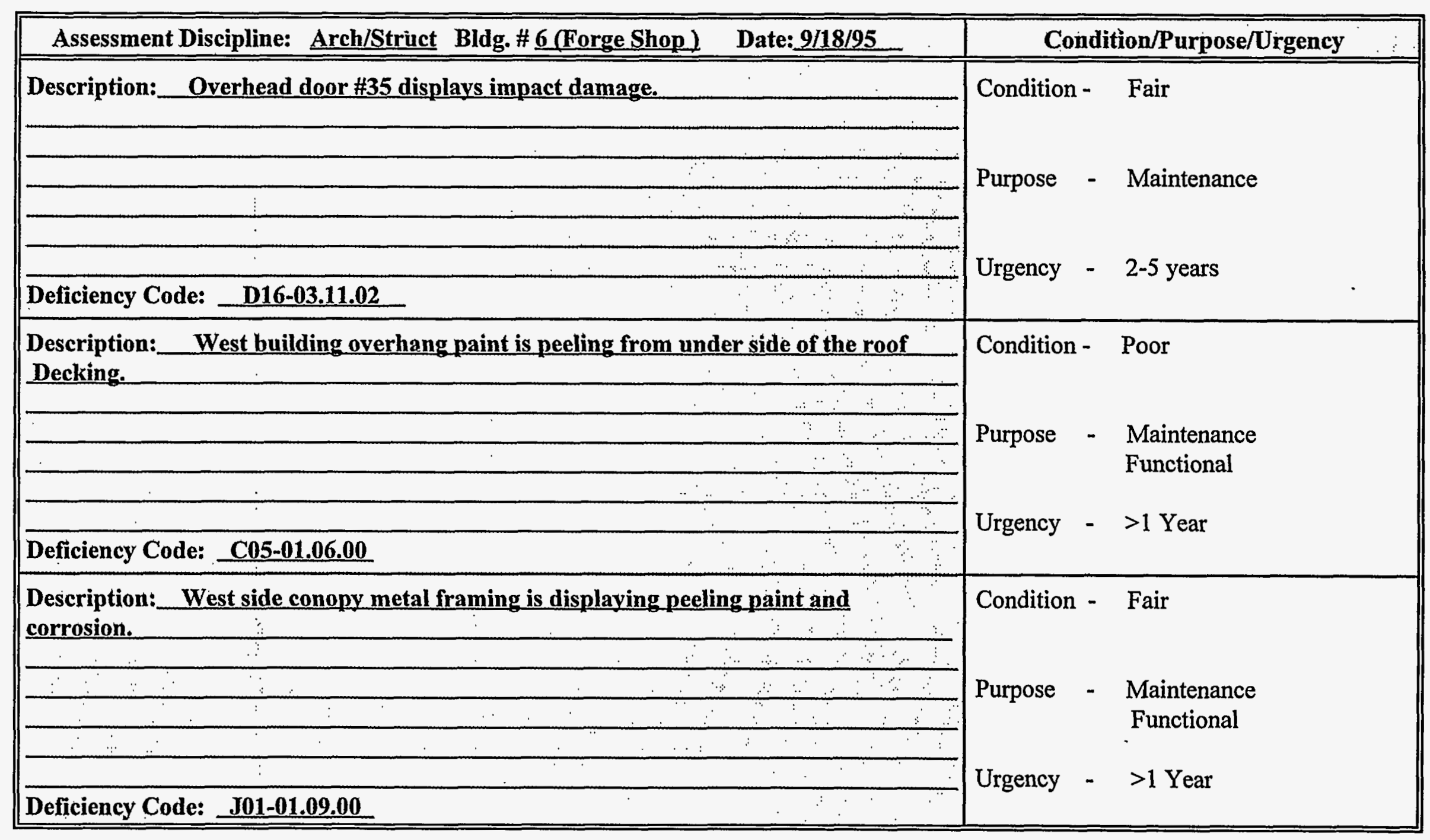




\section{Oxnard Condition Assessment Survey Field Report}

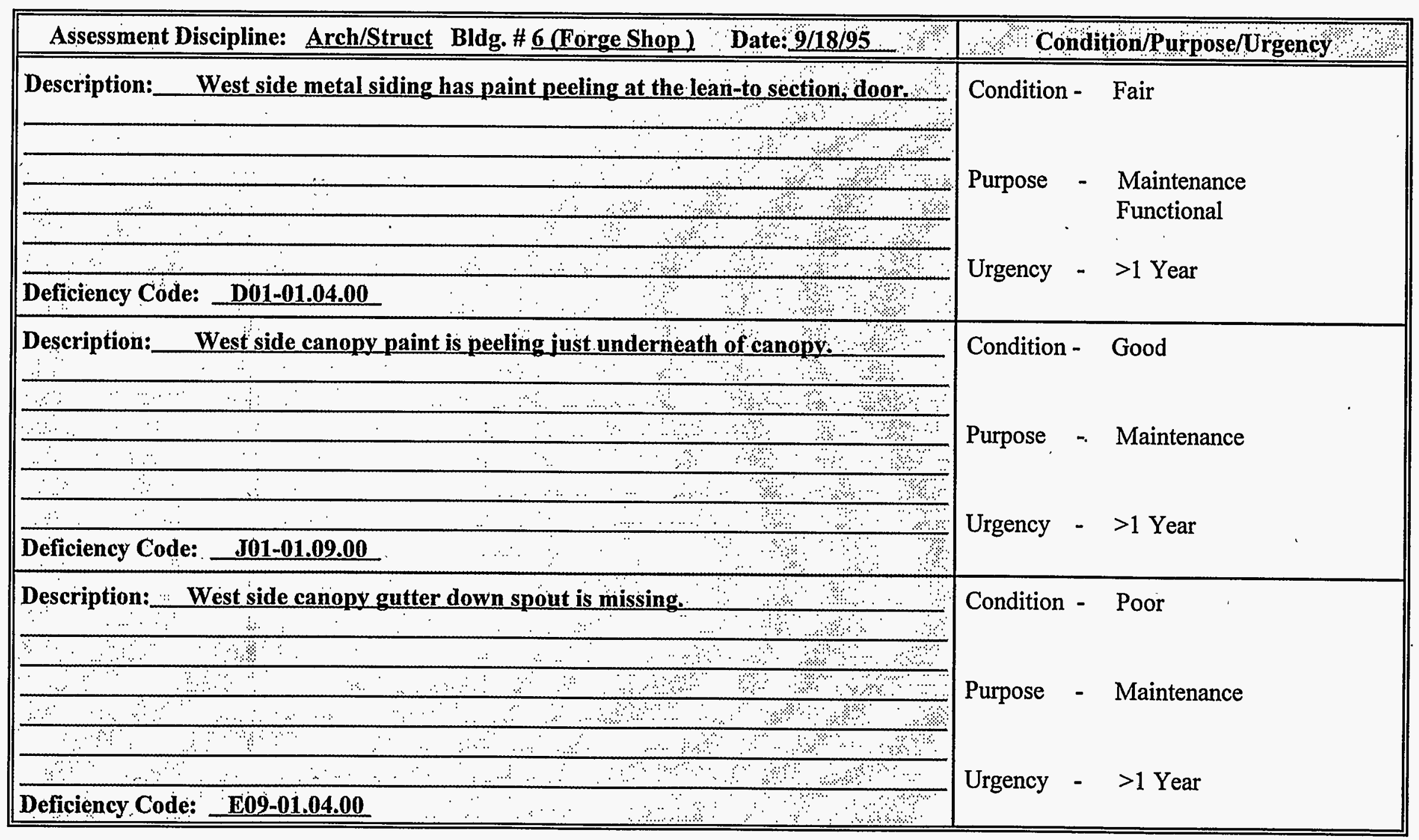




\section{Oxnard Condition Assessment Survey Field Report}

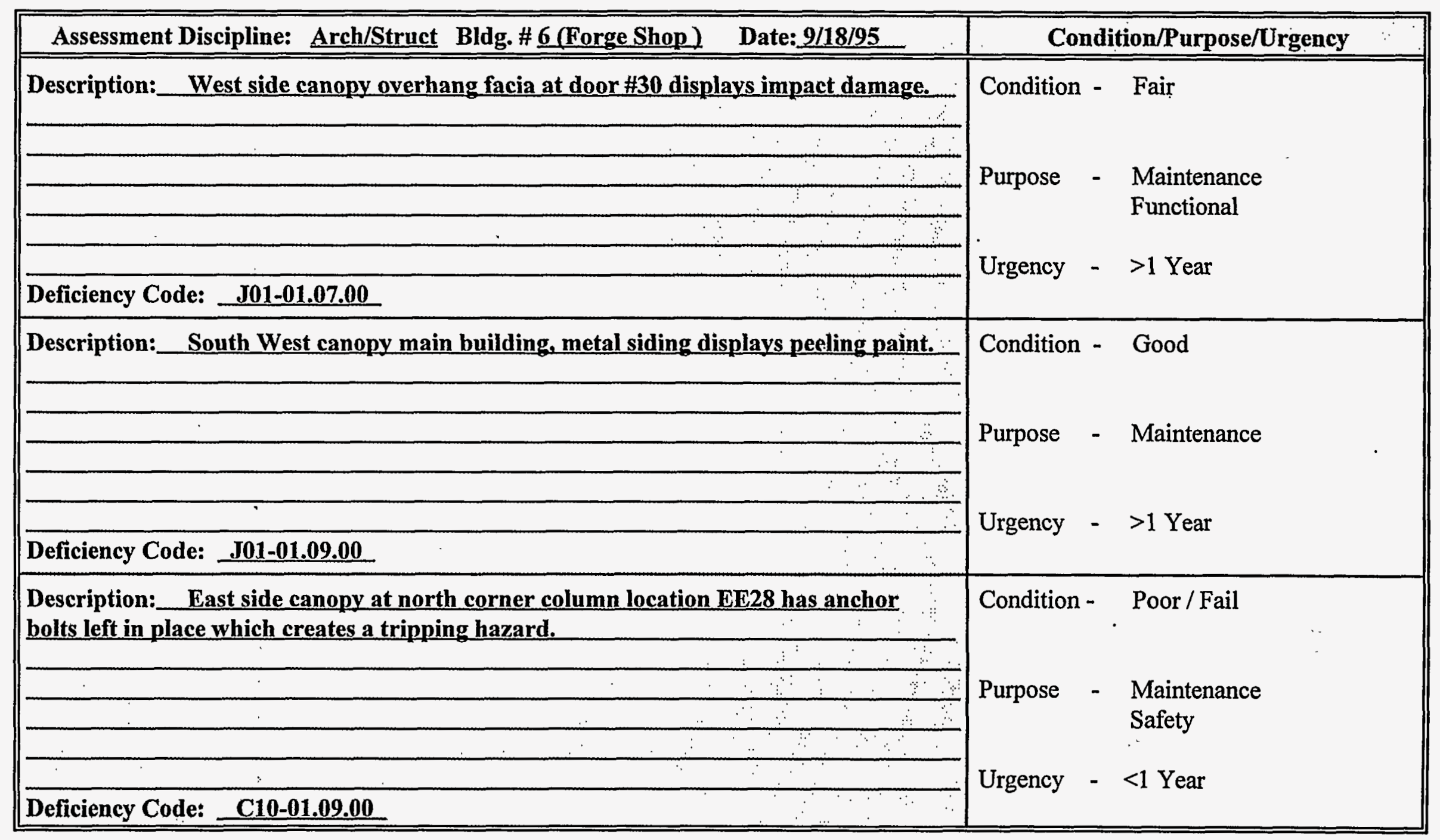




\section{Oxnard Condition Assessment Survey Field Report}

\begin{tabular}{|c|c|c|}
\hline Assessment Discipline: Arch/Struct Bldg. \# 6 (Forge Shop) & \multicolumn{2}{|c|}{ Condition/Purpose/Urgency $\$$ ? } \\
\hline South west canopy overhang displays impact damage at corner facia. & \multirow{2}{*}{\multicolumn{2}{|c|}{ Condition - Good }} \\
\hline$\because \cdots \cdots$ & & \\
\hline 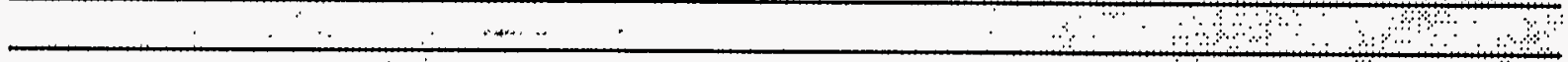 & \multirow{3}{*}{\multicolumn{2}{|c|}{$\begin{array}{ll}\text { Purpose - } & \text { Maintenance } \\
& \text { Functional }\end{array}$}} \\
\hline$\because \quad 3 \quad 3 \quad 3$ & & \\
\hline 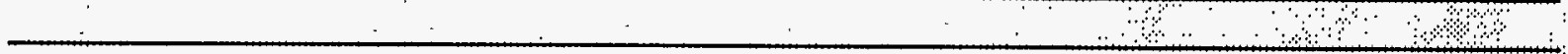 & & \\
\hline$\because \quad \because \quad 1 \%$ & \multirow[t]{2}{*}{ Urgency } & \multirow[t]{2}{*}{$->1$ Year } \\
\hline Deficiency Code: J01-01.07.00 & & \\
\hline Description: East side concrete apron at canopy column location EE-20 is spalling & \multirow{2}{*}{\multicolumn{2}{|c|}{ Condition - Fair }} \\
\hline . 10 - & & \\
\hline$\therefore \quad \because$ & \multirow{3}{*}{\multicolumn{2}{|c|}{$\begin{array}{ll}\text { Purpose - } & \text { Maintenance } \\
& \text { Functional }\end{array}$}} \\
\hline$\therefore \quad \therefore$ & & \\
\hline e. & & \\
\hline$\therefore \quad \because$ & \multirow[t]{3}{*}{ Urgency } & \multirow[t]{3}{*}{$->1$ Year } \\
\hline$\therefore \quad \cdots \quad y_{1}^{\prime} \quad \cdots$ & & \\
\hline Deficiency Code: C10-01.02.00 and C10-01.05.00 & & \\
\hline $\begin{array}{l}\text { Description: North east canopy area at column location EE28 displays concrete } \\
\text { spalling and settlement expansion. }\end{array}$ & \multirow{2}{*}{\multicolumn{2}{|c|}{ Condition - Fair }} \\
\hline 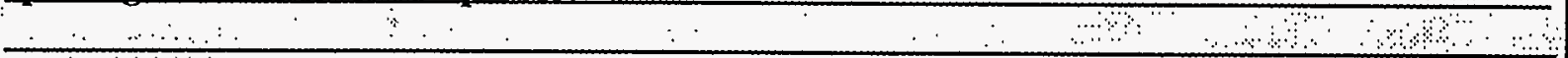 & & \\
\hline 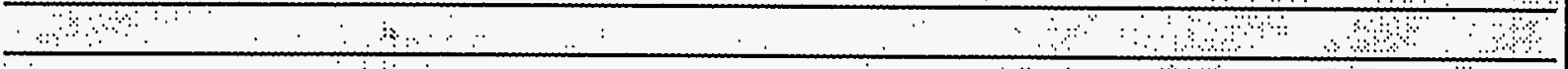 & \multirow[t]{3}{*}{ Purpose } & \multirow{3}{*}{$\begin{array}{l}\text { Maintenance } \\
\text { Functional }\end{array}$} \\
\hline$\therefore \quad \cdots \quad \cdots$ & & \\
\hline . & & \\
\hline$\therefore \quad \therefore \quad \vdots \quad \vdots \quad \cdots$ & \multirow[t]{2}{*}{ Urgency } & \multirow[t]{2}{*}{$-\quad>1$ Year } \\
\hline Deficiency Code: C10-01:05.00 & & \\
\hline
\end{tabular}




\section{Oxnard Condition Assessment Survey Field Report}

\begin{tabular}{|c|c|}
\hline Assessment Discipline: Electrical Bldg. \# 6 (Forge Shop) & Condition/Purpose/Urgency \\
\hline $\begin{array}{l}\text { Description:-Electrical disconnects for pumps \#1557 and } 1515 \text { are not labeled as to } \\
\text { what they operate. Missing or insufficient data. }\end{array}$ & Condition - Fair \\
\hline & Purpose - Maintenance \\
\hline Deficiency Code: & $>1$ Yoon \\
\hline $\begin{array}{l}\text { Description: Moderate corrosion is displayed at pump controller, distribution panel } \\
\text { \#6AE. }\end{array}$ & Condition - Fair \\
\hline & - Functional \\
\hline$-\cdots$ & 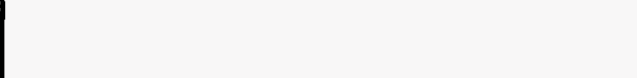 \\
\hline Deficiency Code: $103-07.07 .03$ & - $>1$ Year \\
\hline $\begin{array}{l}\text { Description: Air compressor (\#1498) located at the NE corner exterior - Disconnect } \\
\# 1498,6 \mathrm{EE} / 27,480 \mathrm{~V} \text {, displays heavy corrosion. }\end{array}$ & Condition - Fair \\
\hline$+6 \quad 3$ & Purpose - Functional \\
\hline$\frac{1}{\because} \quad \therefore \quad \therefore \quad s$ & 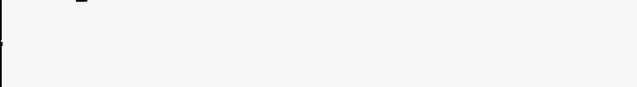 \\
\hline 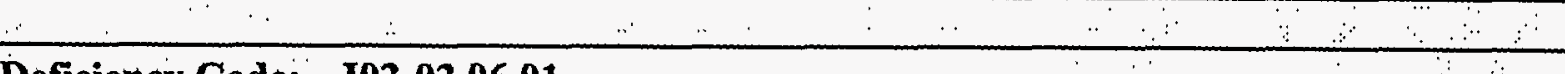 & Urgency - $>1$ Year \\
\hline Deficiency Code: $103-03.06 .01$ & \\
\hline
\end{tabular}




\section{Oxnard Condition Assessment Survey Field Report}

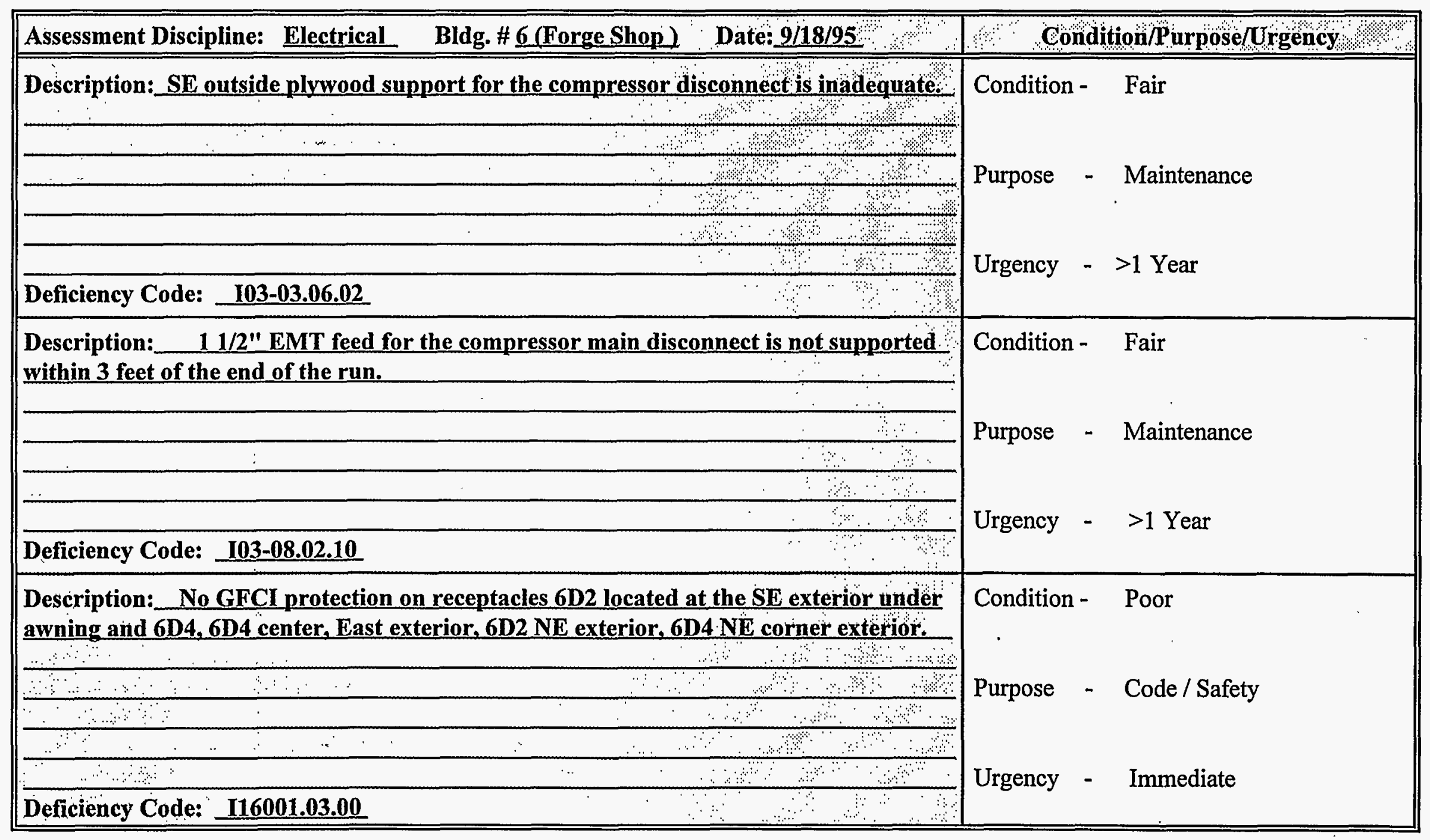




\section{Oxnard Condition Assessment Survey Field Report}

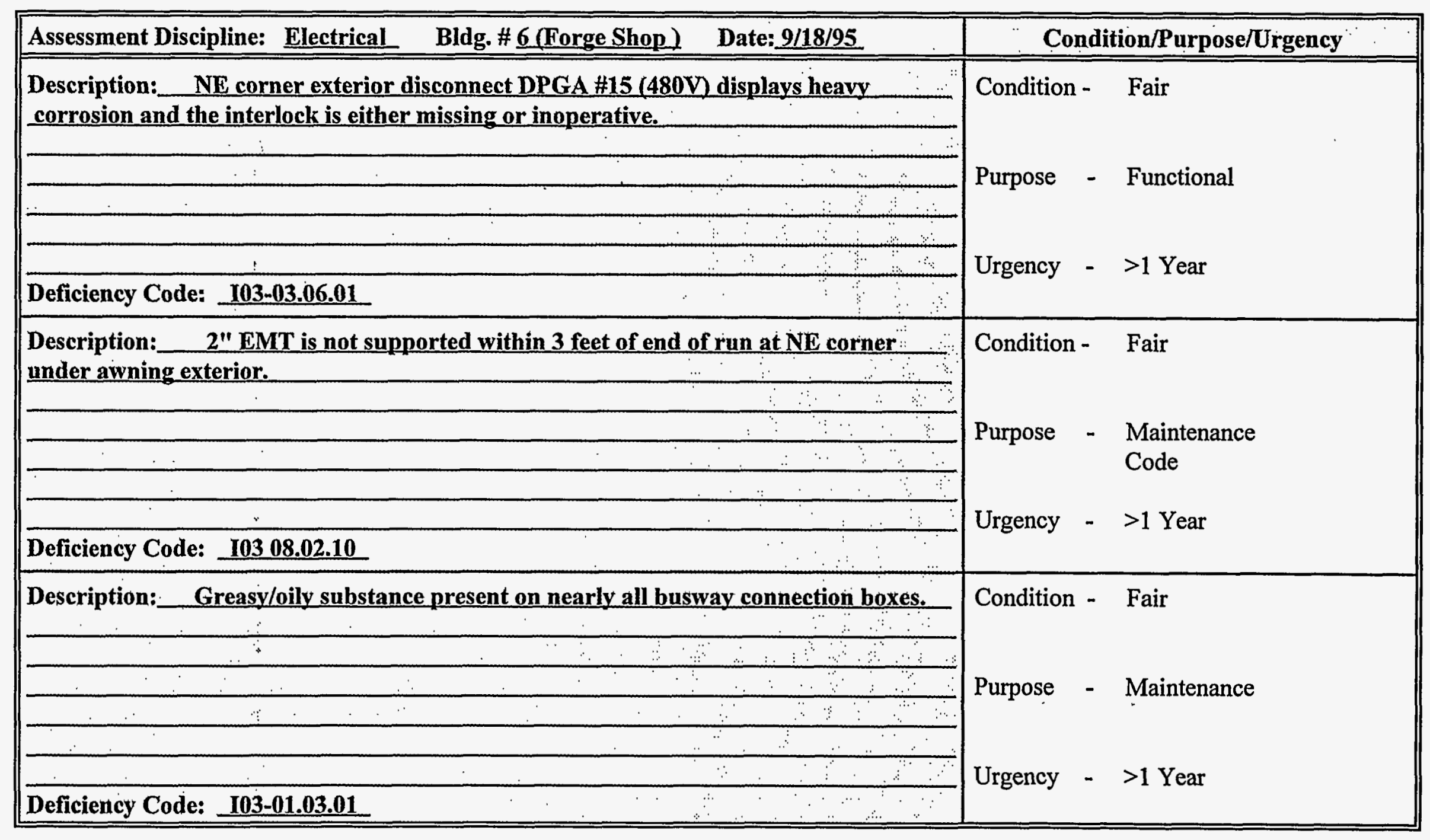




\section{Oxnard Condition Assessment Survey Field Report}

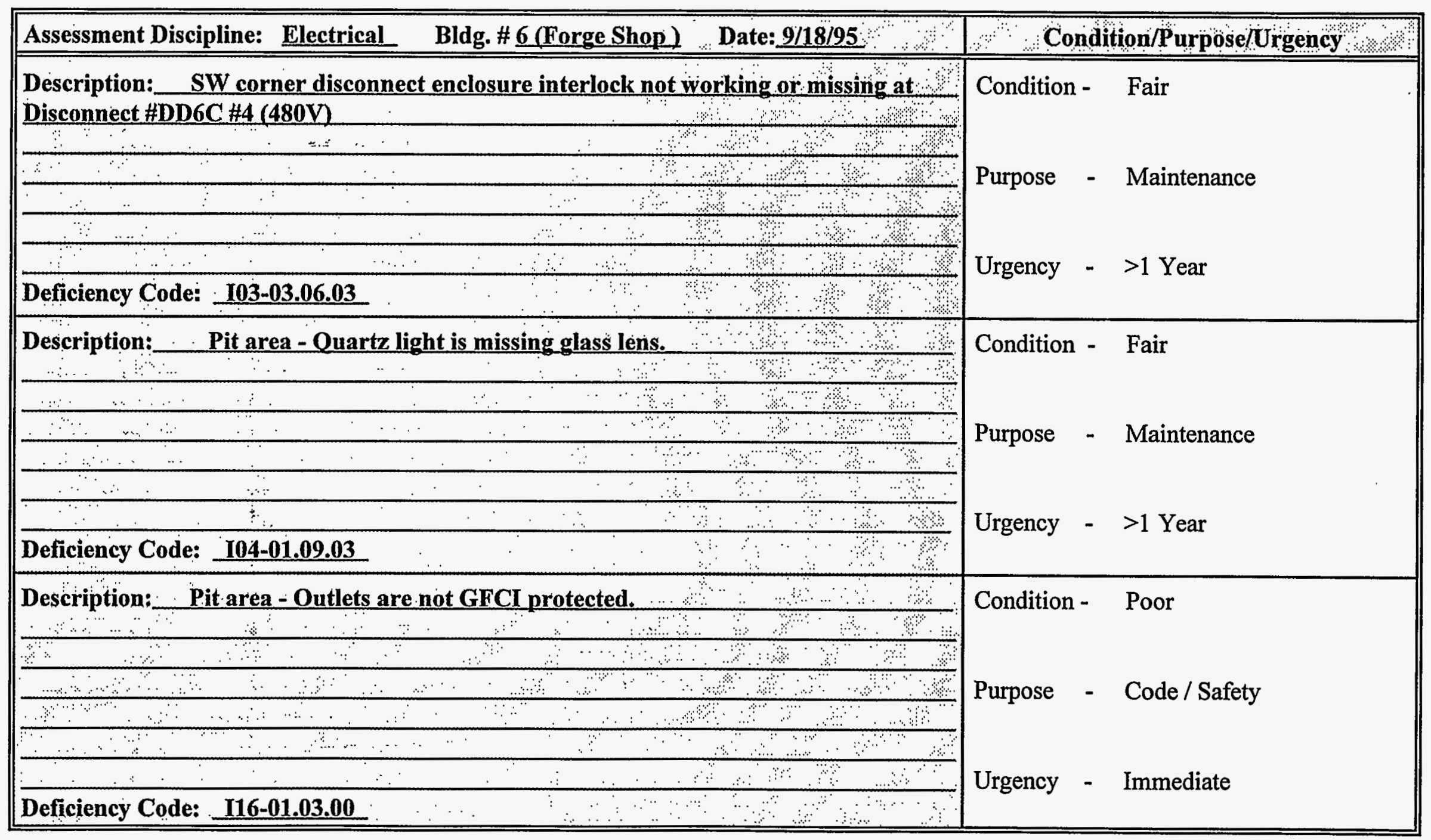




\section{Oxnard Condition Assessment Survey Field Report}

\begin{tabular}{|c|c|}
\hline Assessment Discipline: Mechanical & Condition/Purpose/Urgency \\
\hline $\begin{array}{l}\text { Description: Asset wide - all building penetrations should be caulked and/or have an : } \\
\text { escutcheon. }\end{array}$ & \multirow{2}{*}{$\begin{array}{l}\text { Condition - Good } \\
\text { Purpose - Maintenance }\end{array}$} \\
\hline Interior walls also. & \multirow{4}{*}{ Purpose - Maintenance } \\
\hline$\therefore \quad \therefore$ & \\
\hline HO1-05.05.00 Water & \\
\hline HO2-06.05.00 Drains. & \\
\hline Deficiency Code: NFPA & \\
\hline Description: Pipes being supported by 4" pve pipe outside Building 6 - chilled lines. & \multirow[t]{2}{*}{ Condition - Fair / Poor } \\
\hline Pipes not labeled. & \\
\hline$\therefore$ & \multirow{2}{*}{ Purpose - Code / Safety } \\
\hline i & \\
\hline 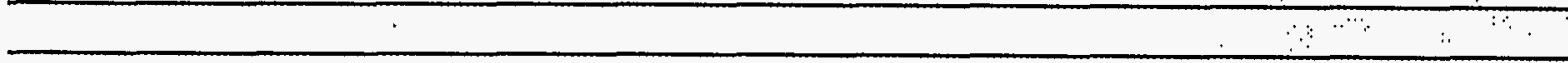 & \multirow[t]{2}{*}{ Urgency - $>1$ Year } \\
\hline Deficiency Code: H30-04.06.00 Labels / H30-07.04.00 & \\
\hline $\begin{array}{l}\text { Description: Relief valves on compressed air unit need piped to code. } \\
\text { 1-1/2" S.E. outside corner }\end{array}$ & \multirow[t]{2}{*}{ Condition - Poor } \\
\hline 1-3/4" S.E. outside corner & \\
\hline $1-3 / 4^{\prime \prime}$ N.E. outside corner & \multirow[t]{2}{*}{ Purpose - Code / Safety } \\
\hline $1-1 / 2 "$ N.E. outside corner/ equipment \#1681, 1499, 1498 & \\
\hline$\therefore \quad \therefore \quad \cdots \quad \vdots$ & \multirow[t]{2}{*}{ Urgency - $<1$ Year } \\
\hline Deficiency Code: HO3-06.11.00 & \\
\hline
\end{tabular}




\section{Oxnard Condition Assessment Survey Field Report}

\begin{tabular}{|c|c|c|}
\hline Assessment Discipline: Mechanical & \multicolumn{2}{|c|}{ Q Condition/Purpose/Urgency } \\
\hline$\frac{2+4}{4}$ & \multirow{2}{*}{\multicolumn{2}{|c|}{ Condition - Fair / Poor }} \\
\hline 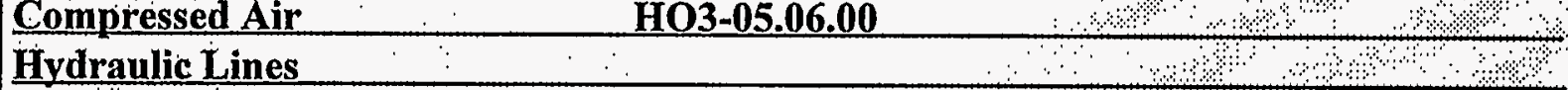 & & \\
\hline 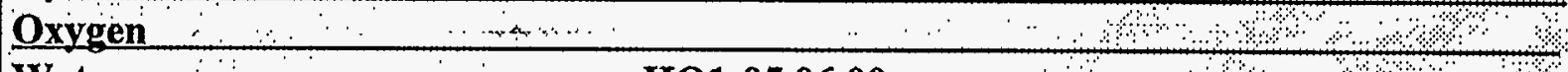 & \multirow{3}{*}{\multicolumn{2}{|c|}{$\begin{array}{cl}\text { Purpose - } & \text { Maintenance } \\
\text { Safety }\end{array}$}} \\
\hline - $\cdot 1 \%$ & & \\
\hline 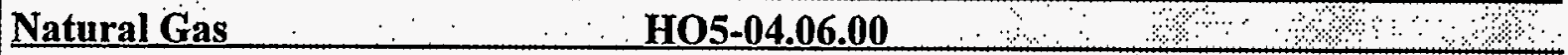 & & \\
\hline$\because: \quad 2 \times \quad$ \% & \multirow{3}{*}{ Urgency } & \multirow{3}{*}{$-\quad<1$ Year } \\
\hline $\begin{array}{ll}\text { Vacuum } & \text { HO4-06.06.00 } \\
\end{array}$ & & \\
\hline Deficiency Code: $\quad$ See above & & \\
\hline $\begin{array}{l}\text { Description: Some pans leaking (liners \#1515, 1557, 1471)/ most pans contain some. } \\
\text { oil - indicating “some leakage" outside Building } 6 .\end{array}$ & \multicolumn{2}{|c|}{ Condition - Fair / Good } \\
\hline Hydraulic pump stations are dirty but appear to be in good over-all condition. & \multirow{2}{*}{\multicolumn{2}{|c|}{ Purpose - Maintenance }} \\
\hline 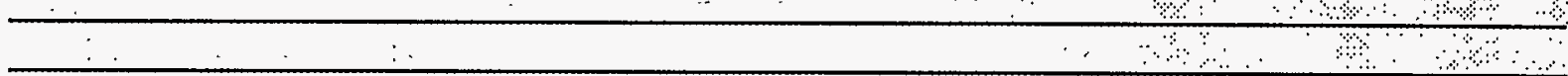 & & \\
\hline क्ष: $: 9:$ & \multirow[t]{2}{*}{ Urgency } & \multirow[t]{2}{*}{ - $>1$ Year } \\
\hline 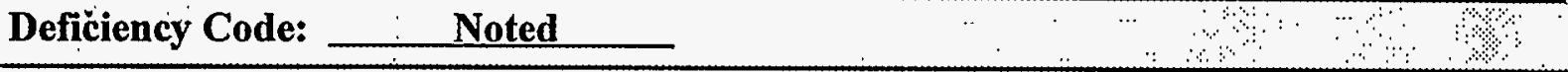 & & \\
\hline Description: Compressor \#1499 leaks oil. Under repair - lock-out-tag-out in effect. & \multirow{2}{*}{\multicolumn{2}{|c|}{ Condition - Good }} \\
\hline 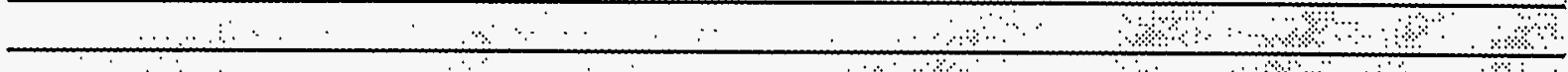 & & \\
\hline $\mathrm{N}, \mathrm{QH}, \mathrm{Q}$ & \multirow{2}{*}{\multicolumn{2}{|c|}{ Purpose - Maintenance }} \\
\hline 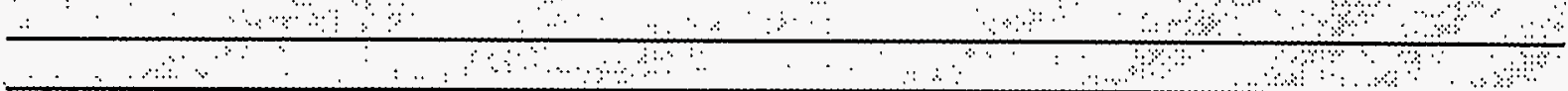 & & \\
\hline Deficiency Codet HO3-01.01.00 $\quad \ldots \ldots$ & Urgency & $<1$ Year \\
\hline
\end{tabular}




\section{Oxnard Condition Assessment Survey Field Report}

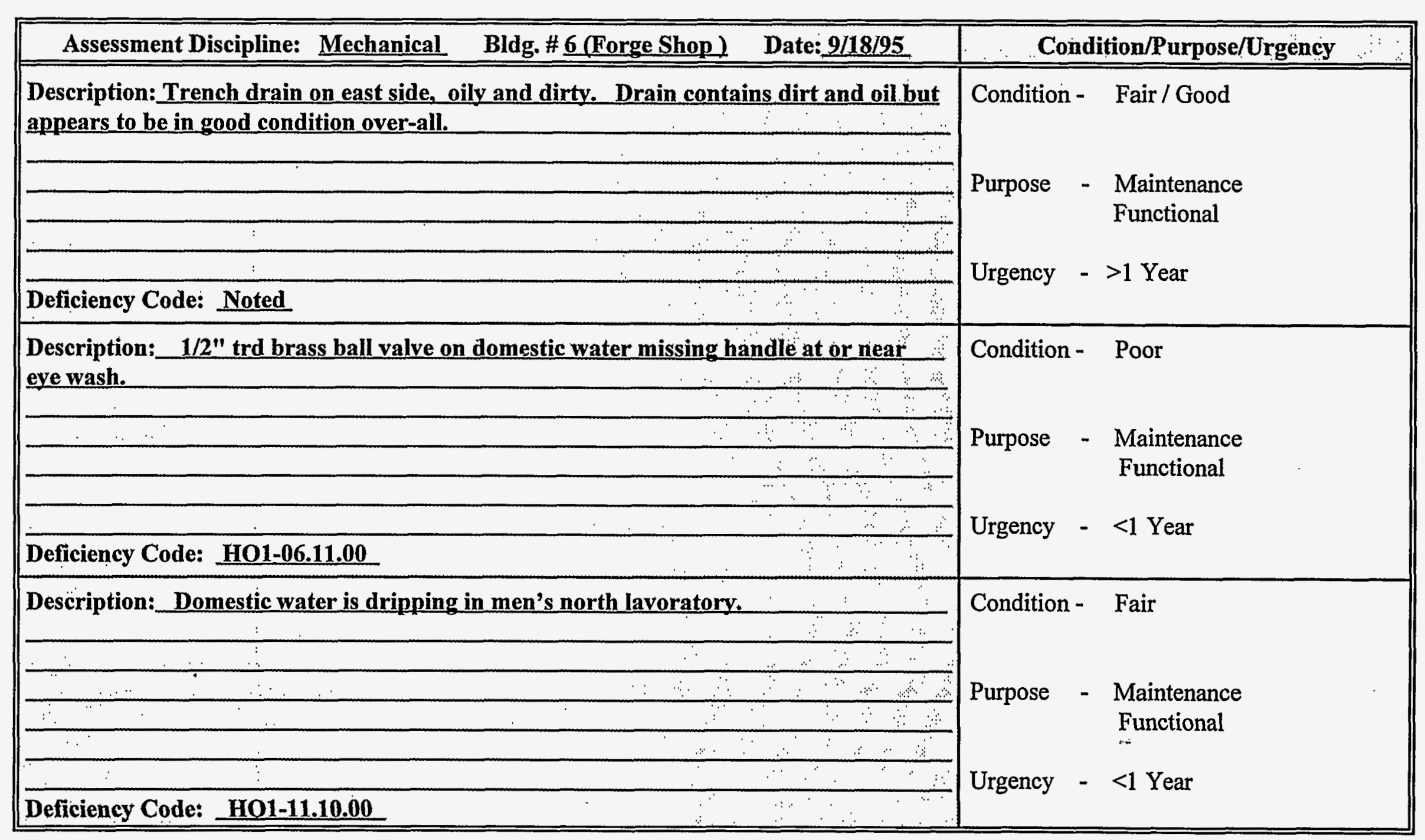




\section{Oxnard Condition Assessment Survey Field Report}

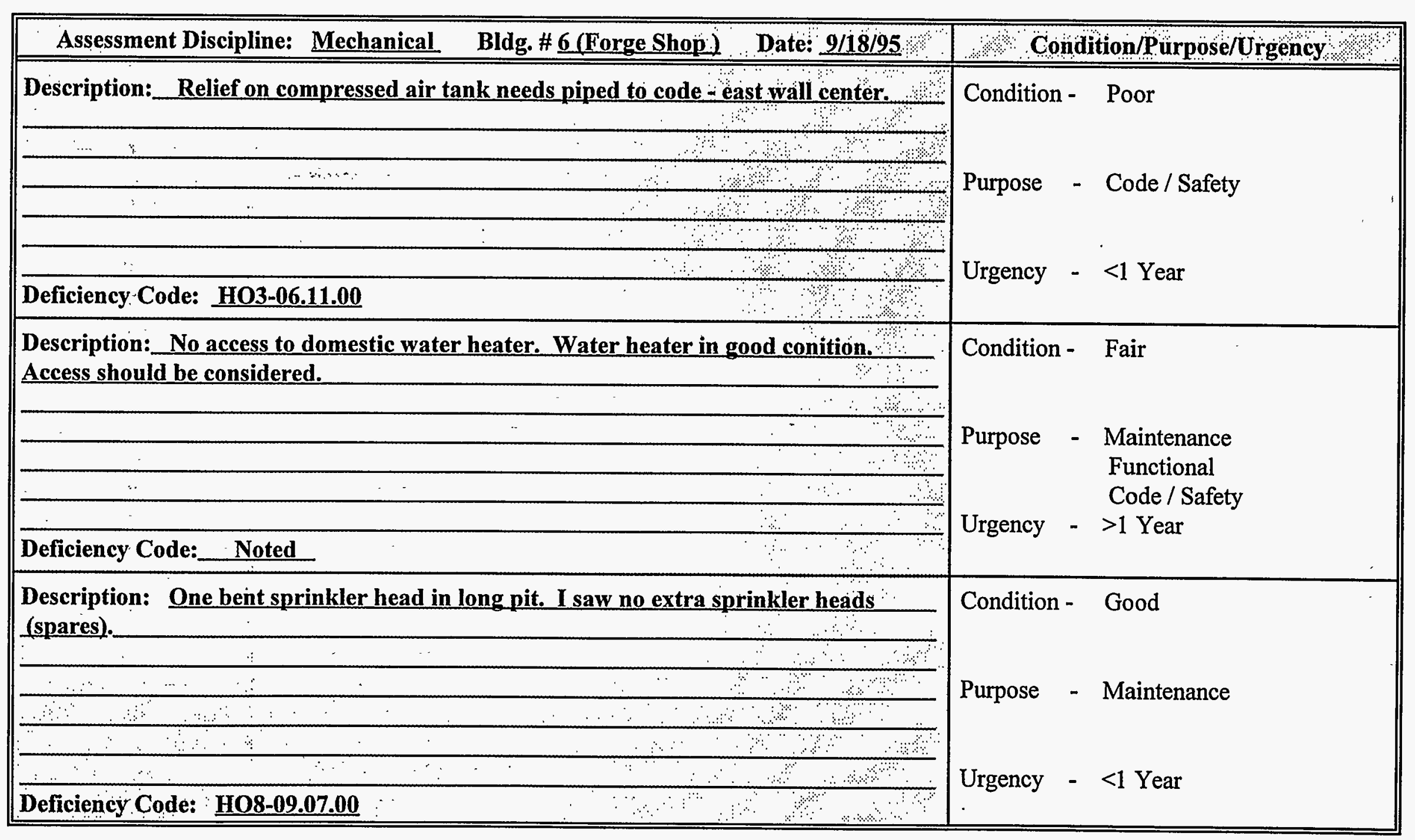




\section{Oxnard Condition Assessment Survey Field Report}

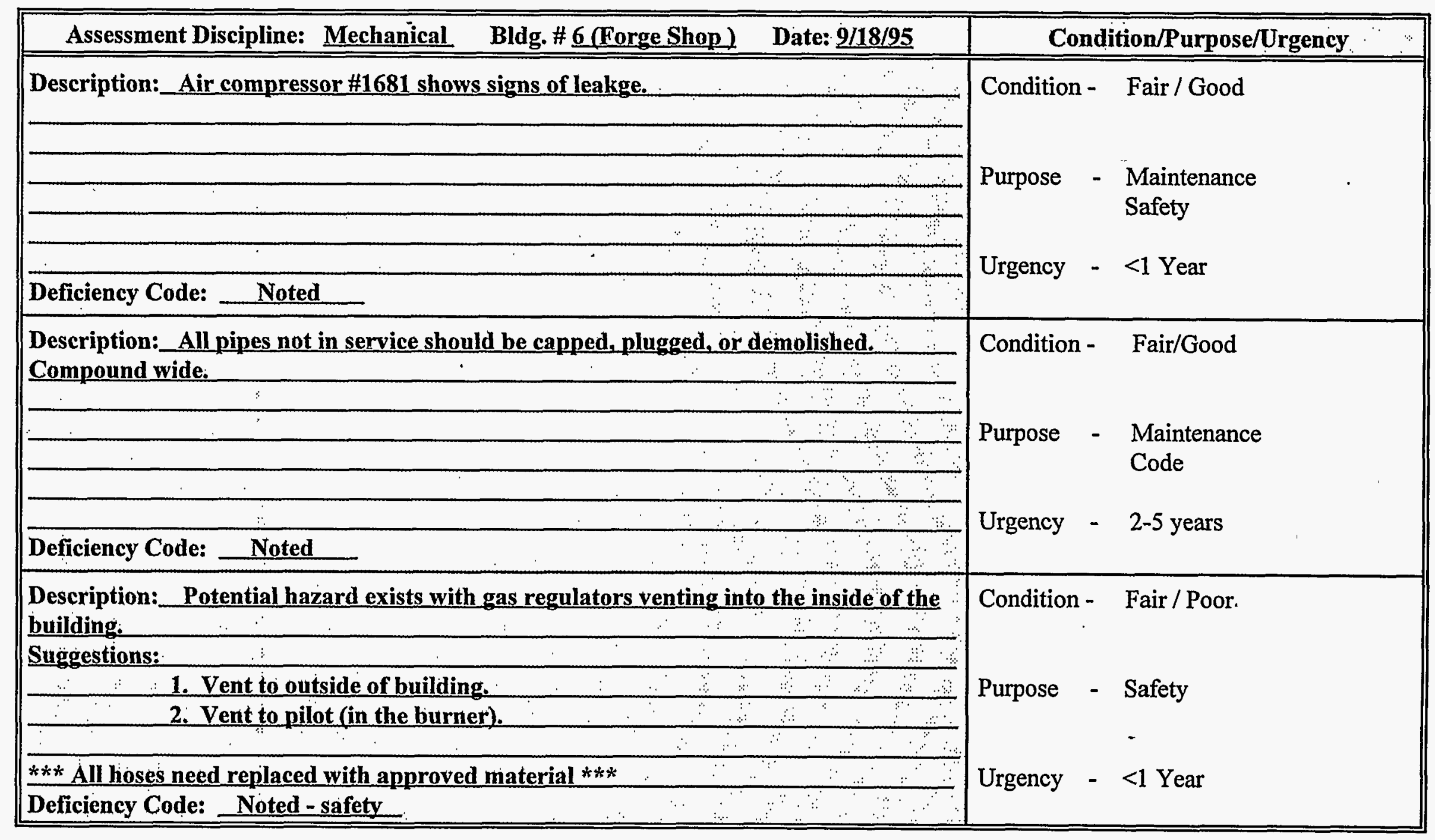





\section{Oxnard CAS Field Notes}

Building 7 



\section{Oxnard Condition Assessment Survey Field Report}

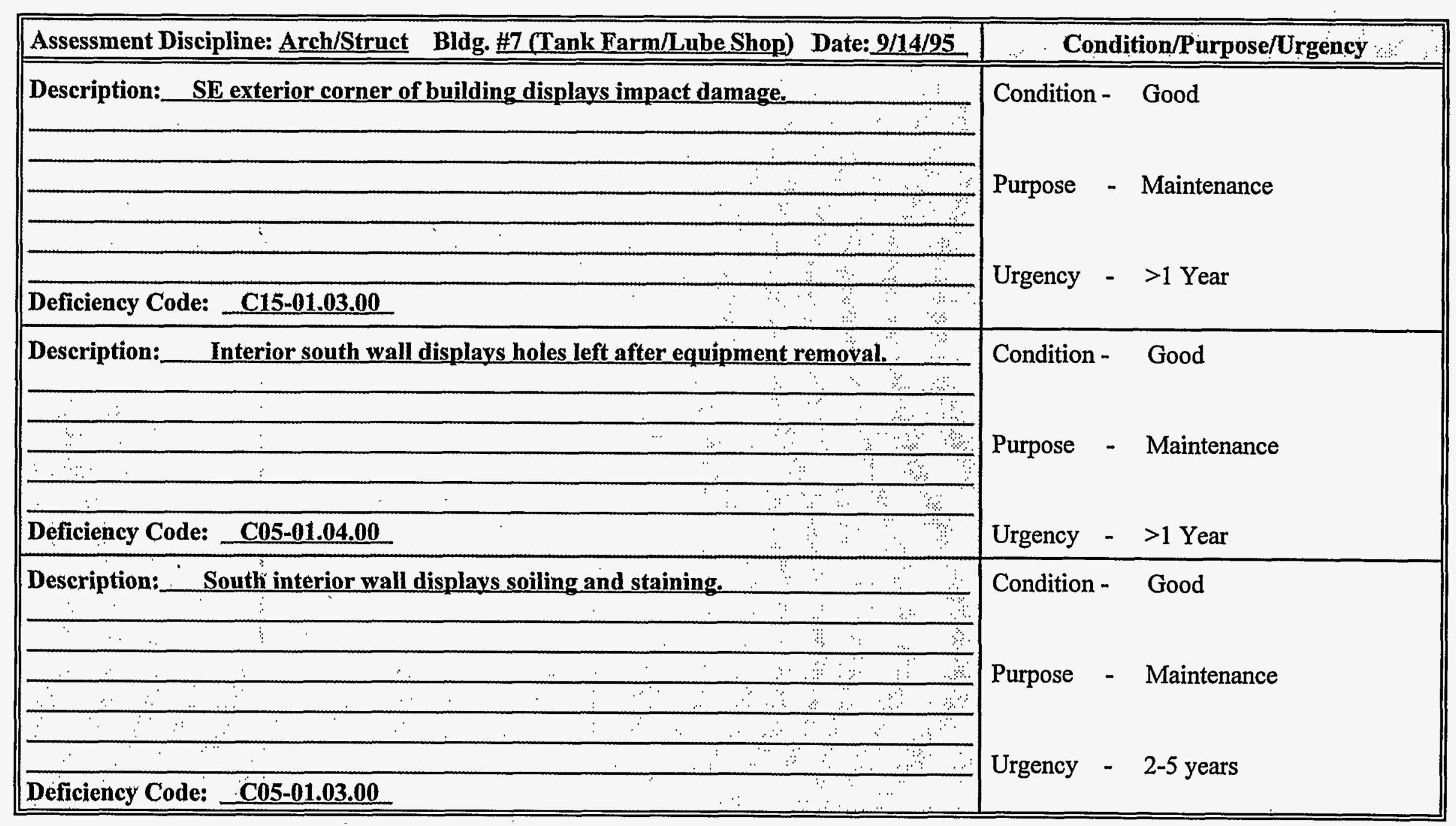




\section{Oxnard Condition Assessment Survey Field Report}

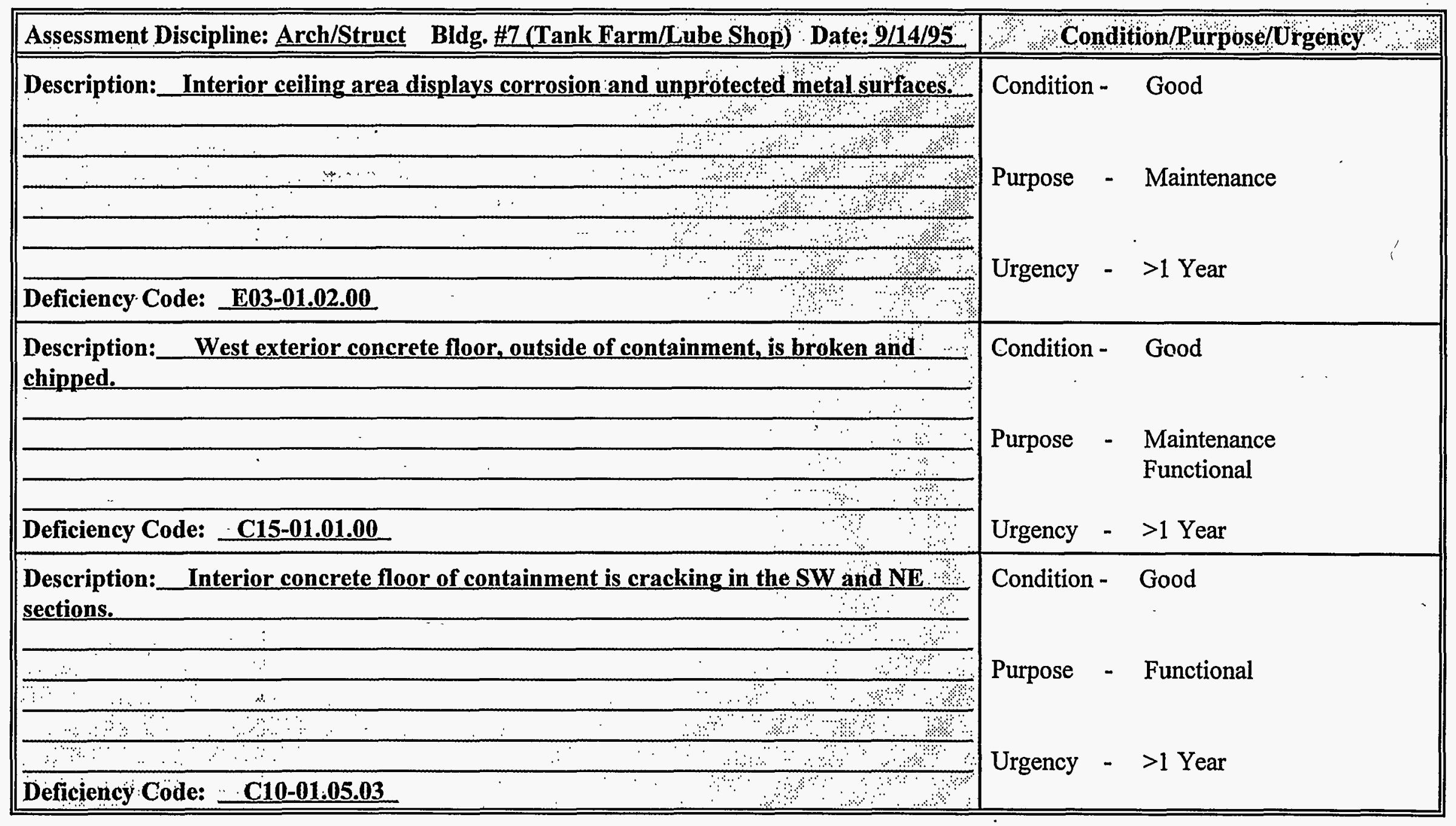




\section{Oxnard Condition Assessment Survey Field Report}

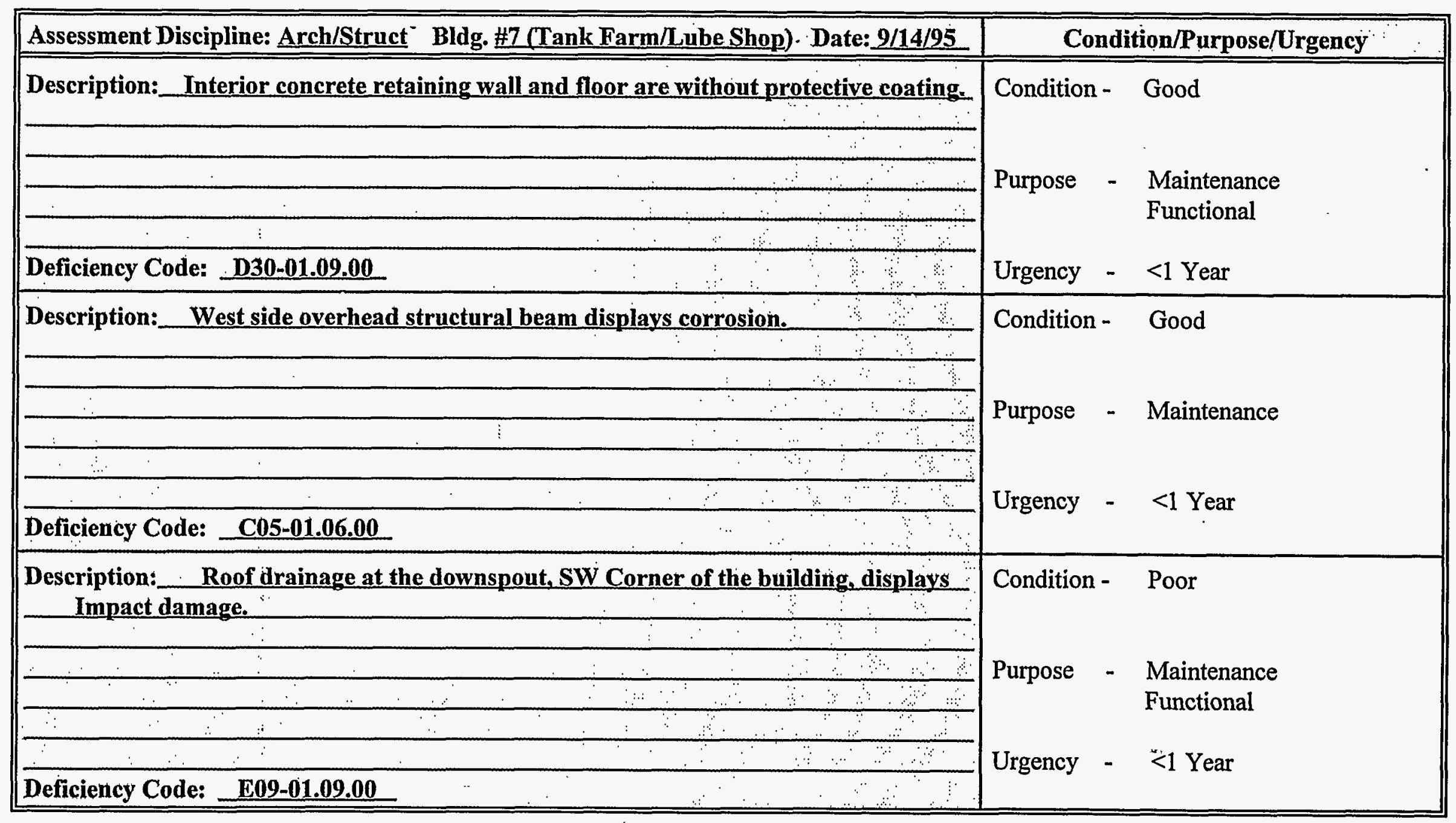




\section{Oxnard Condition Assessment Survey Field Report}

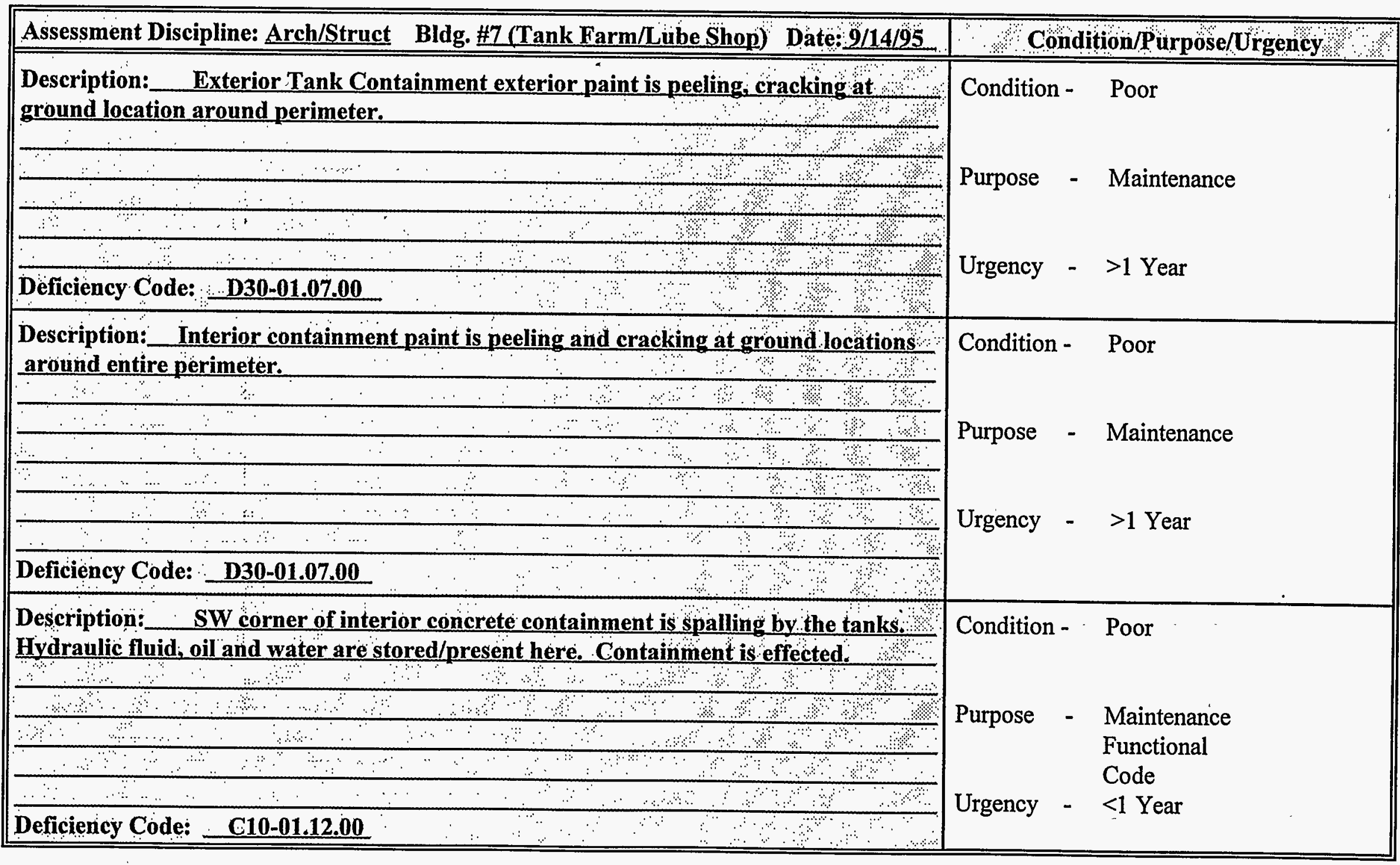




\section{Oxnard Condition Assessment Survey Field Report}

\begin{tabular}{|l|l|l||}
\hline Assessment Discipline: Arch/Struct & Bldg. \#7 (Tank Farm/Lube Shop) Date: 9/14/95 & Condition/Purpose/Urgency \\
\hline \hline Description: $\quad$ The interior tank containment area is ineffective as concrete removal \\
has taken place at the NE corner of the containment floor/wall.
\end{tabular}




\section{Oxnard Condition Assessment Survey Field Report}

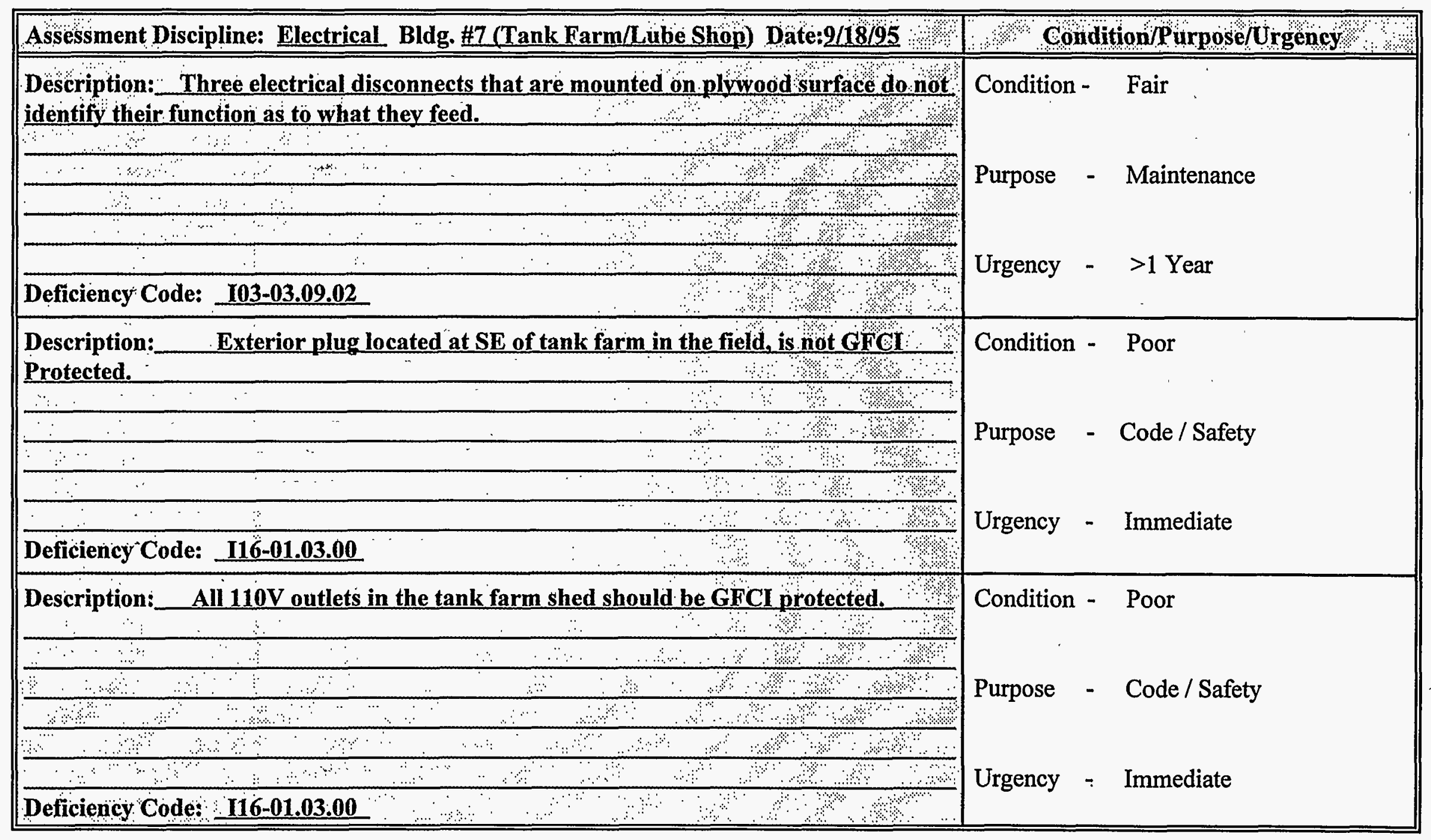




\section{Oxnard Condition Assessment Survey Field Report}

\begin{tabular}{|c|c|}
\hline Assessment Discipline: Electrical Bldg. \#7 (Tank Farm/Lube Shop) Date: 9/18/95 & Condition/Purposè/Urgency \\
\hline $\begin{array}{l}\text { Description: Plywood stand supporting } 3 \text { disconnects and a gutter, is not adequate. } \\
\text { This is located at the SE area of the tank farm and needs to be replaced. }\end{array}$ & Condition - Fair \\
\hline & Maintenance \\
\hline & \multirow{2}{*}{ Urgency - $>1$ Year } \\
\hline Deficiency Code: $\quad$ 103-03.06.02 & \\
\hline $\begin{array}{l}\text { Description: - Outlet under plywood stand (as addressed above) are not GFCI } \\
\text { protected. }\end{array}$ & Condition - Poor \\
\hline & Purpose - Code / Safety \\
\hline & \multirow{2}{*}{ Urgency - Immediate } \\
\hline Deficiency Code: $\mathbf{I 1 6 - 0 1 . 0 3 . 0 0}$ & \\
\hline $\begin{array}{l}\text { Description: } \quad \text { SE Tank Farm area - conduits running into } 4^{\prime \prime} \times 6^{\prime \prime} \times 2^{\prime} \text { gutter are } \\
\text { not supported within } 3 \text { feet of run. There are } 4 \text { ea. } 3 / 4^{\prime \prime} \text { and } 3 \text { ea. } 1 " \text { conduits, all which } \\
\text { are feeding disconnects on board. }\end{array}$ & \multirow{2}{*}{$\begin{array}{l}\text { Condition - Fair } \\
\text { Purpose - } \begin{array}{l}\text { Maintenance } \\
\text { Code }\end{array}\end{array}$} \\
\hline 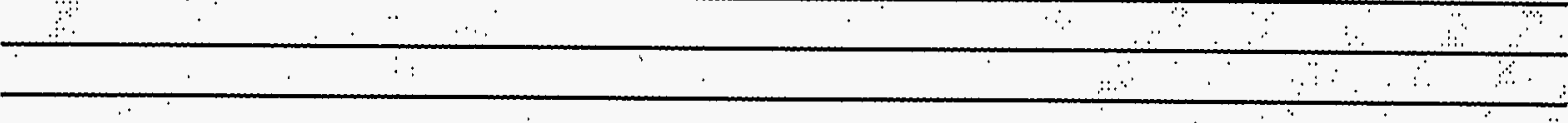 & \\
\hline$\therefore \quad \therefore \quad \cdots$ & \multirow{2}{*}{ Urgency - >1 Year } \\
\hline Deficiency Code: $\quad$ 103-08.02.10 & \\
\hline
\end{tabular}




\section{Oxnard Condition Assessment Survey Field Report}

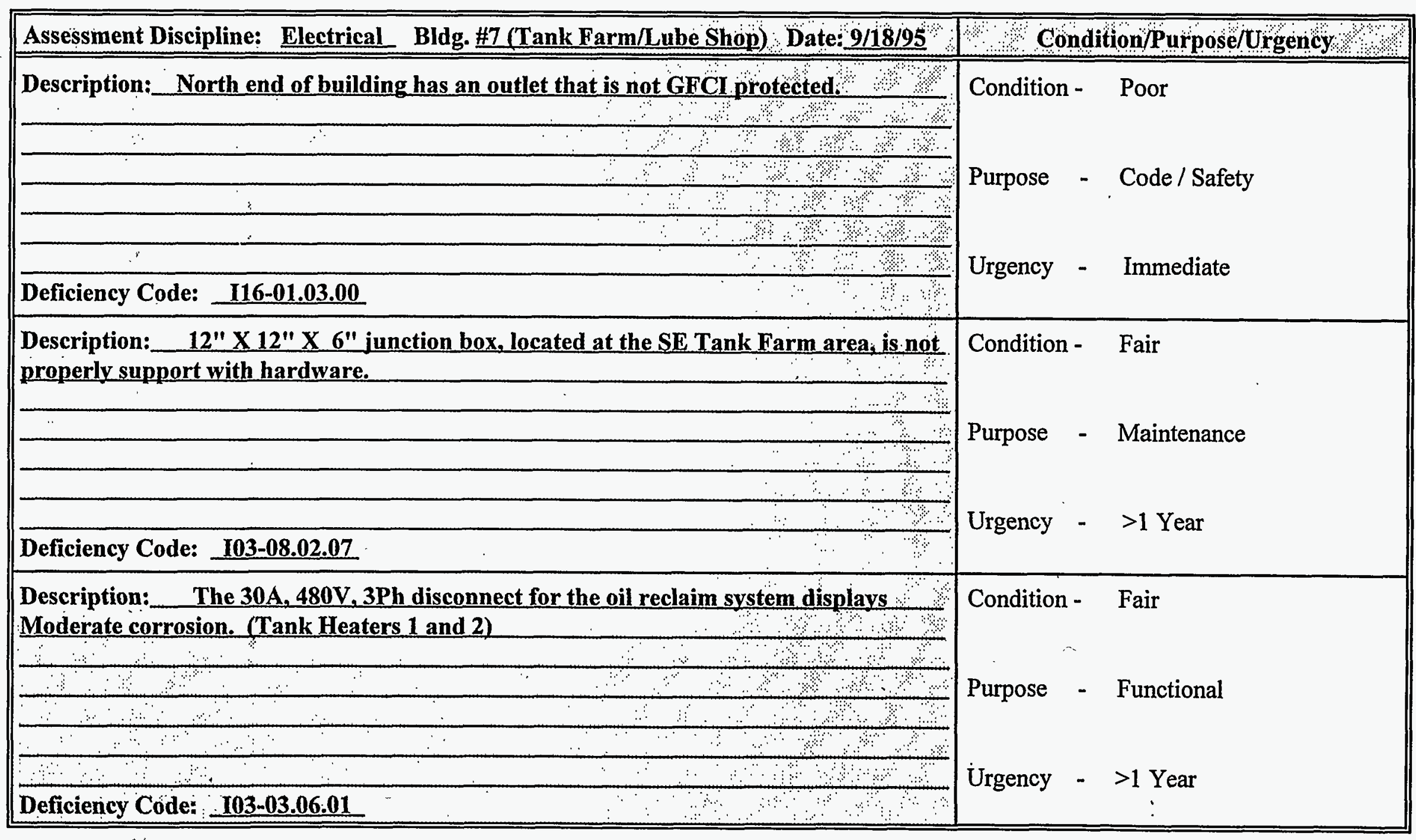




\section{Oxnard Condition Assessment Survey Field Report}

\begin{tabular}{|c|c|}
\hline Assessment Discipline: Mechanicai Bldg. \#7 (Tank Farm/Lube Shop) Date: 9/18/95 & Condition/Purpose/Urgency \\
\hline Description: Domestic water vacuum breaker missing. & \multirow{3}{*}{ Condition - Poor } \\
\hline 2- Hose bibs south outside of Building 7 & \\
\hline 2-Hose bibs north inside Building 7 & \\
\hline 1 - Hose bib south inside Building 7 & \multirow[t]{4}{*}{ Purpose - Code / Safety } \\
\hline $3 \quad 3 \quad 1 \quad+\quad 3$ & \\
\hline$\%$ & \\
\hline Deficiency Code: HO1-11.16.00 & \\
\hline $\begin{array}{l}\text { Description: Two 1" trd domestic water gate valves and two 1" trd ball valves } \\
\text { missing handles. }\end{array}$ & \multirow[t]{2}{*}{ Condition - Fair } \\
\hline $2 \quad \therefore \quad 3$ & \\
\hline 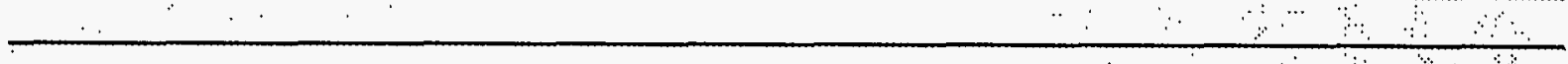 & \multirow{3}{*}{$\begin{array}{c}\text { Purpose - Maintenance } \\
\\
\text { Functional }\end{array}$} \\
\hline$\therefore \quad \because \quad 3 \quad 3$ & \\
\hline$\because \quad \because \quad \because \quad 3$ & \\
\hline$\therefore \quad \therefore \quad 3$ & \multirow{2}{*}{ Urgency - >1 Year } \\
\hline Deficiency Code: HO1-06.11.00 & \\
\hline 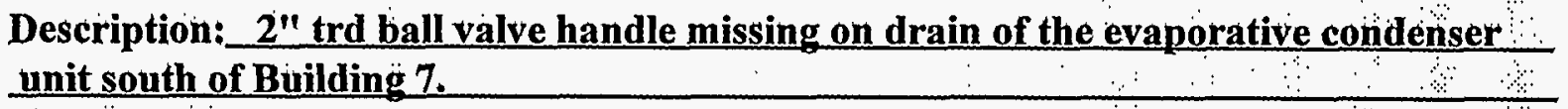 & \multirow[t]{2}{*}{ Condition - Fair } \\
\hline 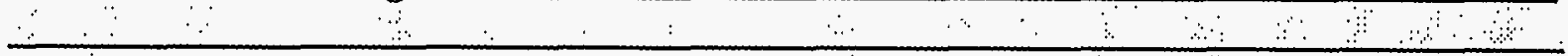 & \\
\hline 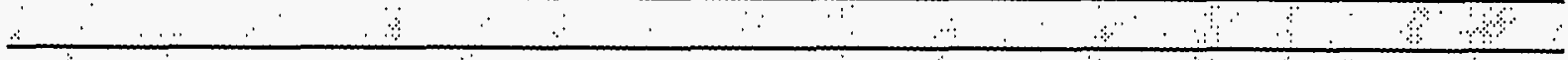 & \multirow{3}{*}{$\begin{array}{cc}\text { Purpose } & - \text { Maintenance } \\
& \text { Functional }\end{array}$} \\
\hline$\ldots-2$ & \\
\hline 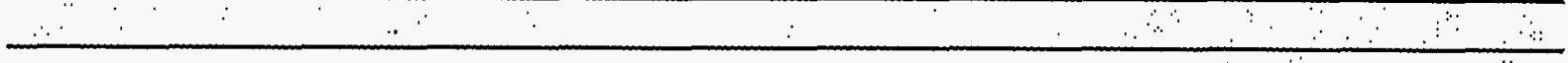 & \\
\hline m. & \multirow[t]{2}{*}{ Urgency $\quad->1$ Year } \\
\hline Deficiency Code: HO2-07.10.00 & \\
\hline
\end{tabular}




\section{Oxnard Condition Assessment Survey Field Report}

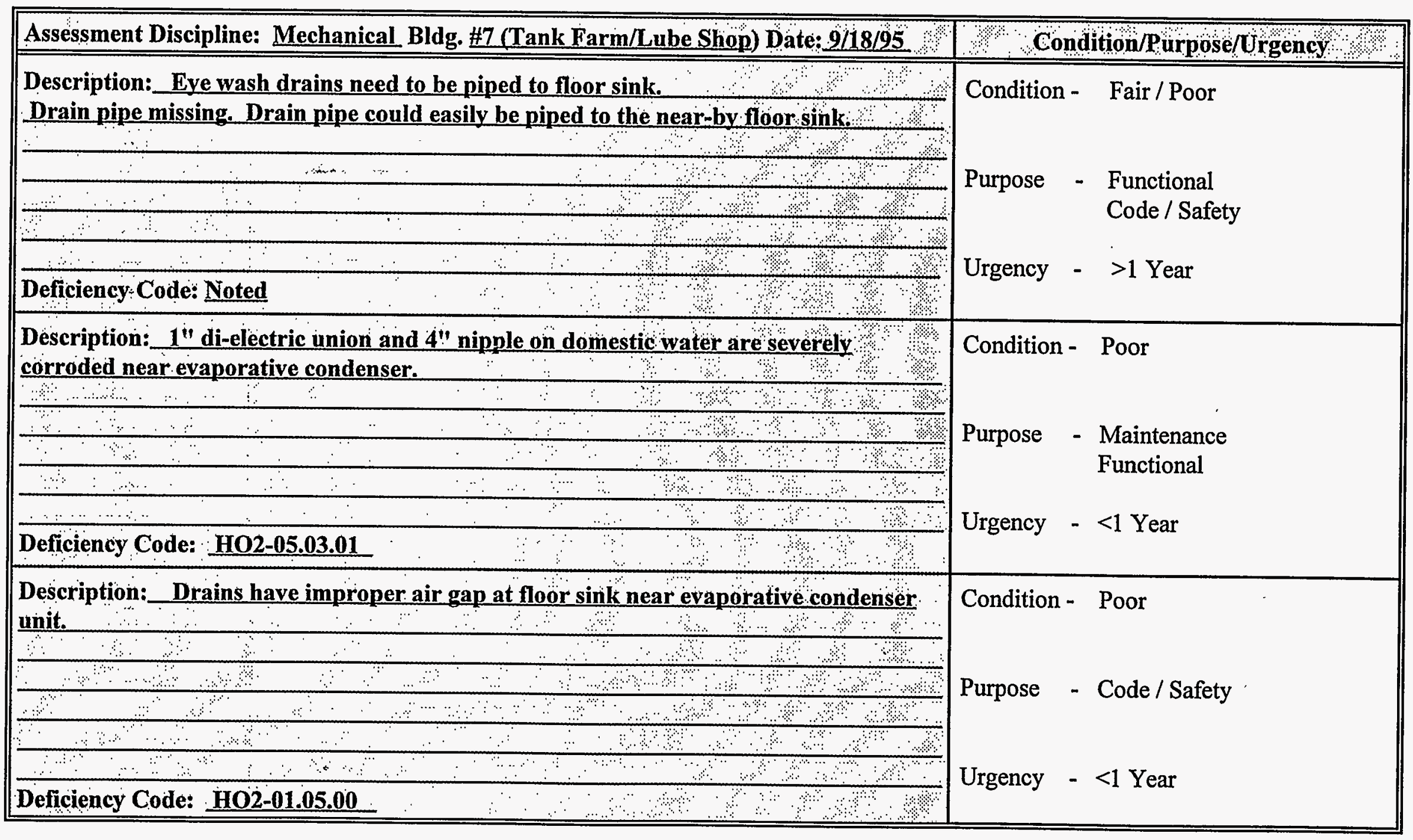




\section{Oxnard Condition Assessment Survey Field Report}

\begin{tabular}{|c|c|}
\hline Assessment Discipline: Mechanical Bldg. \#7 (Tank Farm/Lube Shop) Date: 2/18/95 & Condition/Purpose/Urgency \\
\hline Description: Chilled chemical pot feeders - rusty but good. & \multirow{4}{*}{$\begin{array}{l}\text { Condition - Good } \\
\text { Purpose - Maintenance } \\
\text { Urgency - None }\end{array}$} \\
\hline $\begin{array}{l}\text { Chemical pot feeders belonging to the evaporative condenser are rusty, but in good } \\
\text { over-all condition. }\end{array}$ & \\
\hline & \\
\hline Deficiency Code: Noted & \\
\hline Description: Domestic water hose bib handle broken. & \multirow{4}{*}{$\begin{array}{l}\text { Condition - } \text { Poor } \\
\text { Purpose - } \begin{array}{l}\text { Maintenance } \\
\text { Functional }\end{array} \\
\text { Urgency - }<1 \text { Year }\end{array}$} \\
\hline & \\
\hline & \\
\hline Deficiency Code: HO1-06.11.00 & \\
\hline Description:_All hoses should be replaced by approved piping. & \multirow[t]{2}{*}{ Condition - Poor } \\
\hline - & \\
\hline & Purpose - Code \\
\hline & \multirow[t]{2}{*}{ Urgency - $<1$ Year } \\
\hline Deficiency Code: Code-Noted & \\
\hline
\end{tabular}




\section{Oxnard Condition Assessment Survey Field Report}

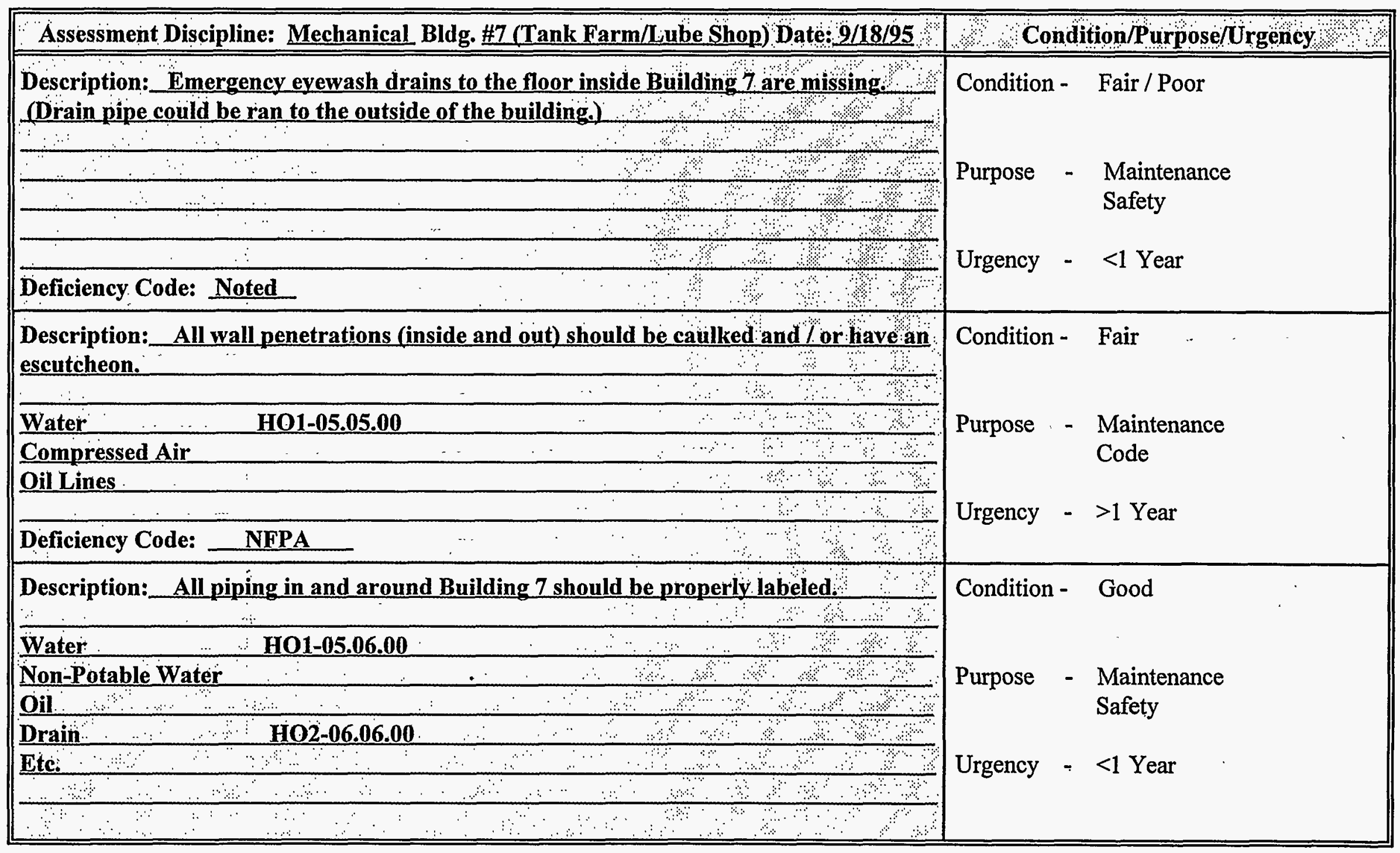

